LIBRARY

U.S. BUREAU OF MINES Westem Field Operation Center East 360 3rd Ave.

Spokane, Washington 99202

FEB 13 :OS

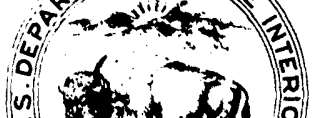

(1)

GEOLOGY OF THE

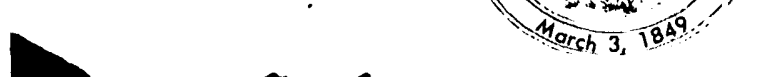

\title{
ARABIAN PENINSULA
}

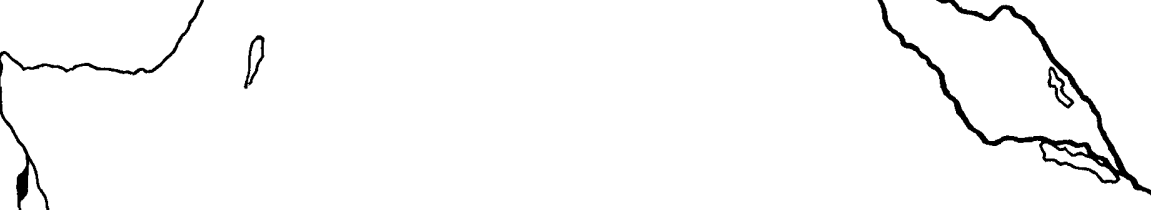

Western Saudi Arabia
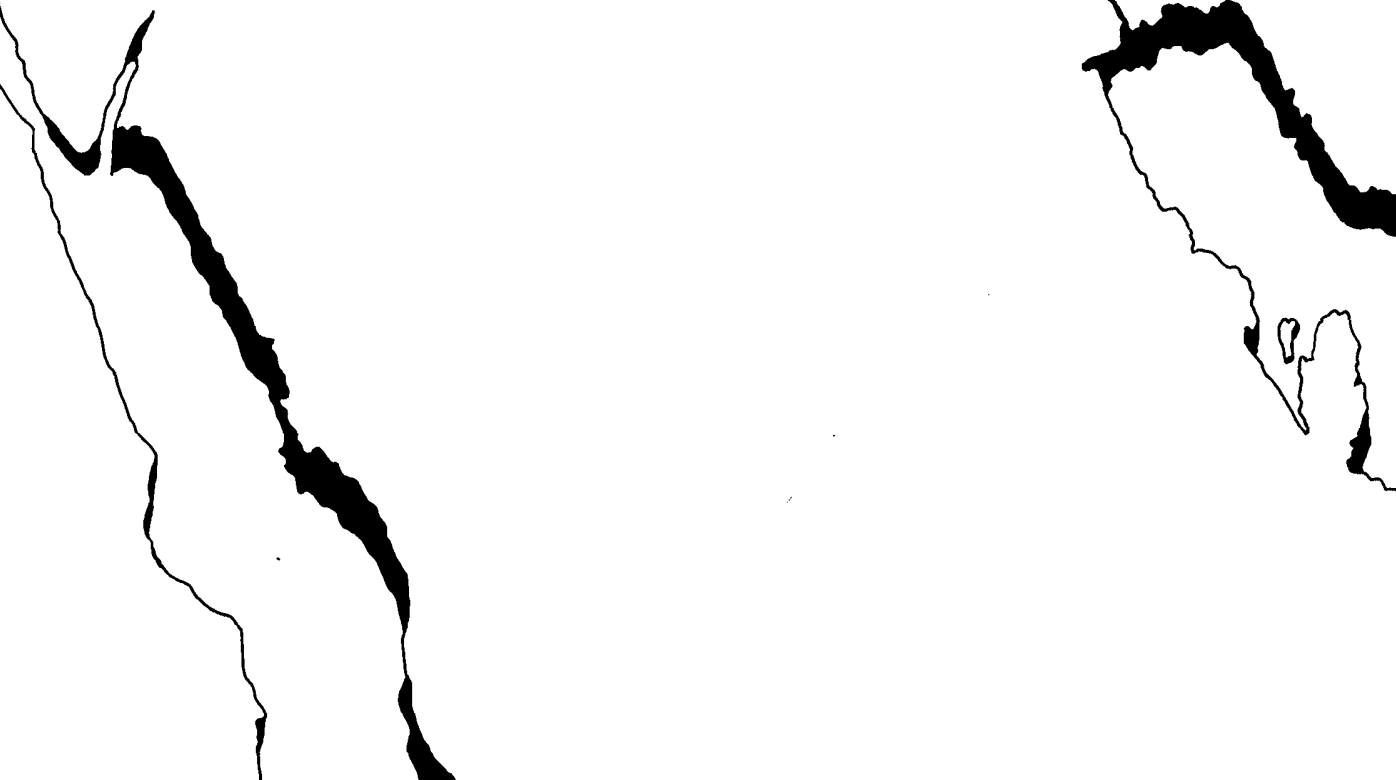

Shield Area of 1 m. 


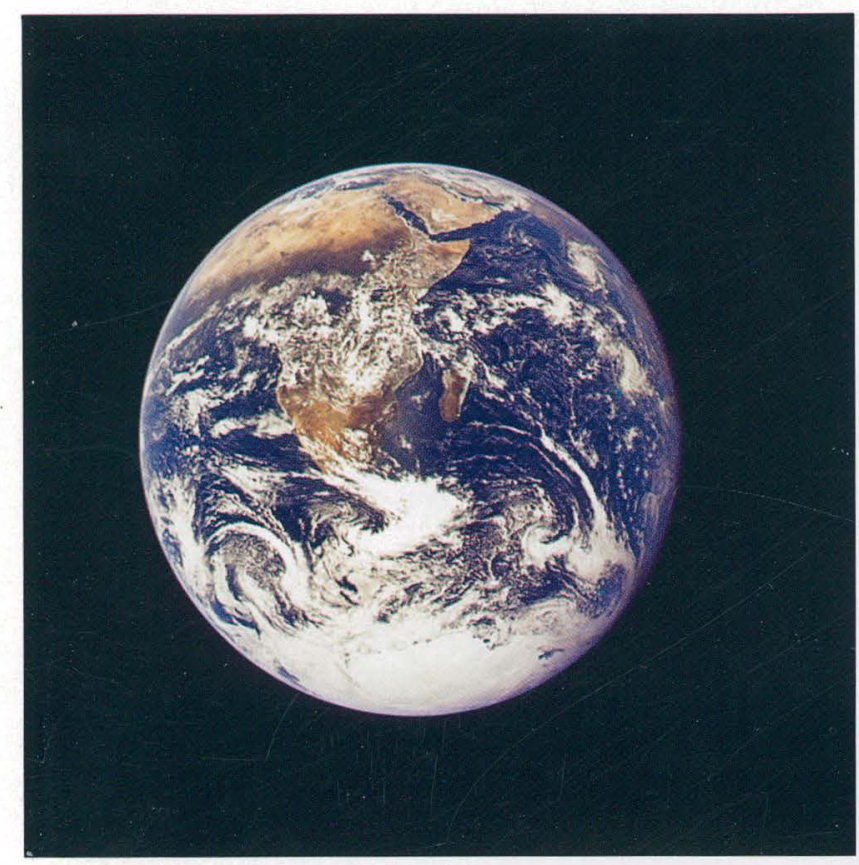

The Earth from space. Africa and Arabia lie north of the Indian and Atlantic Oceans, cloud-draped from the Antarctica Ice Cap to the Equator.

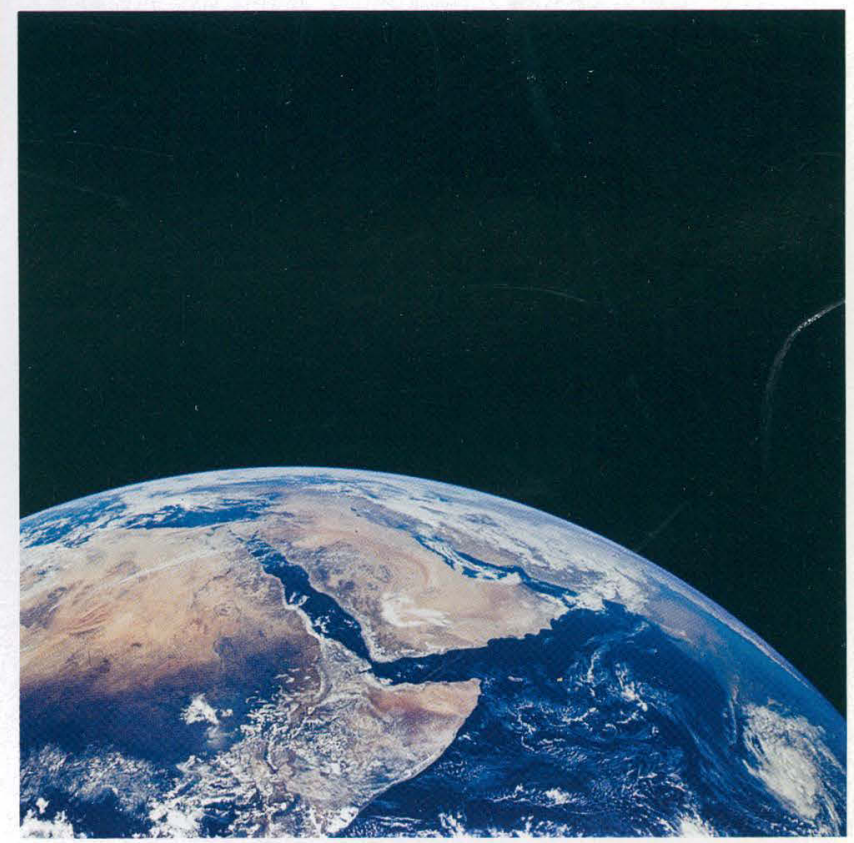

A closer view shows northeast Africa, the Arabian Peninsula, and the Arabian Sea. Photographs from Apollo 17 spacecraft at about 100,000 nautical miles from Earth. 


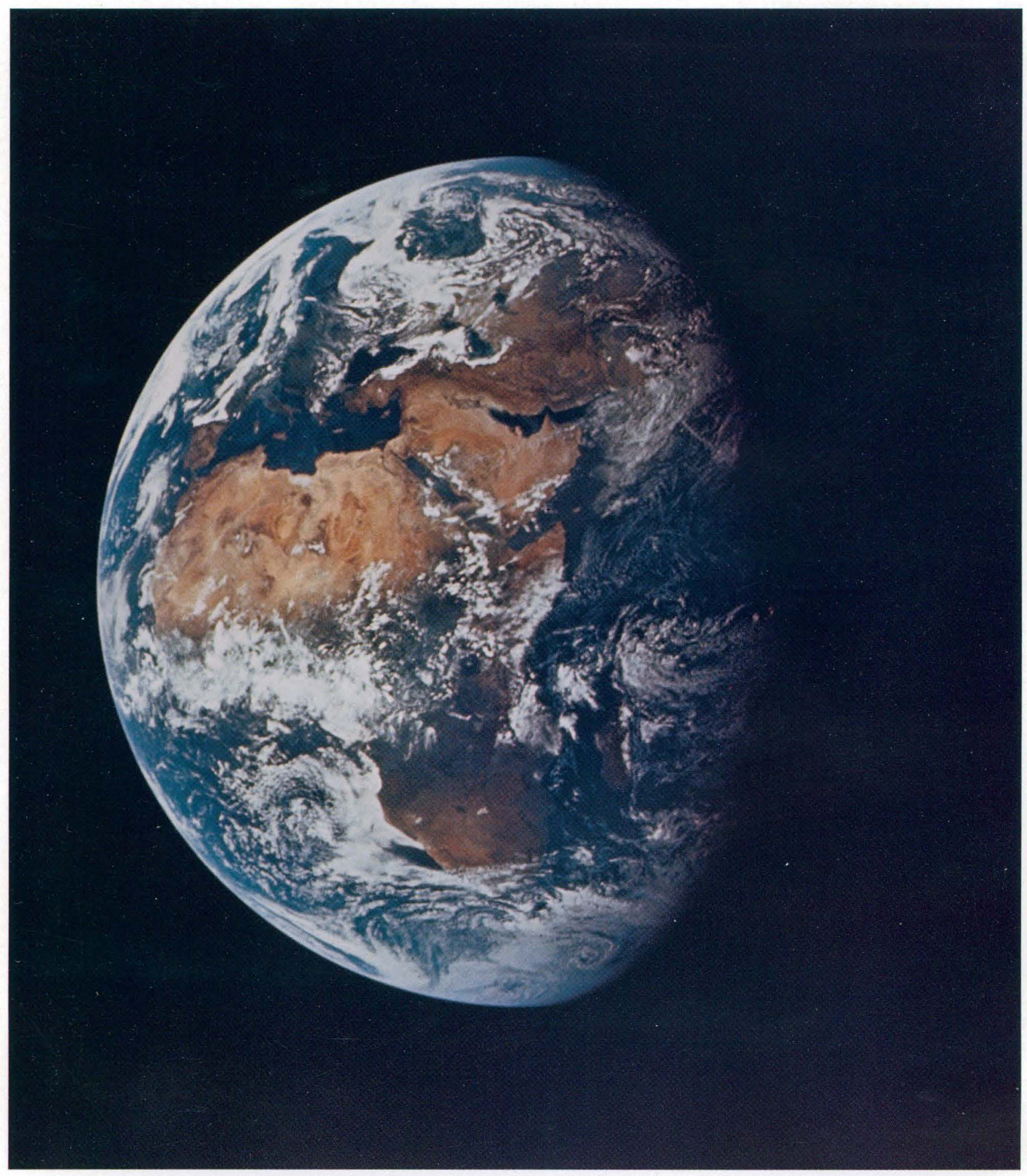

Frontispiece.-The Arabian Shield. As seen from space, the shield appears as a red crescent on the west side of the Arabian Peninsula and as a continuation of the Sahara Desert extending across north Africa. Photograph from Apollo II spacecraft at about 98,000 nautical miles from Earth. 


\section{Geology of the Arabian Peninsula}

\section{Shield Area of \\ Western Saudi Arabia}

By GLEN F. BROWN, DWIGHT L. SCHMIDT, and A. CURTIS HUFFMAN, JR.

U.S. GEOLOGICAL SURVEY PROFESIONAL PAPER 560-A

Prepared in cooperation with the

Ministry of Petroleum and

Mineral Resources, Deputy Ministry

of Mineral Resources, Jiddah,

Kingdom of Saudi Arabia

A review of the geology of western

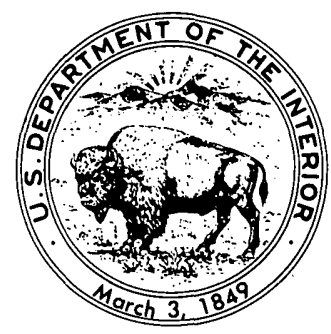

Saudi Arabia as refined from

U.S. Geological Survey Miscellaneous

Geologic Investigations Map I-270A,

"Geologic Map of the Arabian

Peninsula", 1963 


\title{
DEPARTMENT OF THE INTERIOR
}

\author{
MANUEL LUJAN, JR., Secretary
}

\section{U.S. GEOLOGICAL SURVEY}

Dallas L. Peck, Director

Any use of trade, product, or firm names in this publication is for descriptive purposes only and does not imply endorsement by the U.S. Government

Library of Congress Cataloging in Publication Data

Brown, Glen Francis, 1911-

Shield area of Western Saudi Arabia.

(U.S. Geological Survey professional paper ; 560-A)

"A review of the geology of western Saudi Arabia as refined from U.S. Geological Survey miscellaneous geologic investigations map I-270-A, 'Geologic map of the Arabian Peninsula,' 1963."

Bibliography: $p$.

Supt. of Docs. no.: I 19.16:560-A

1. Geology-Saudi Arabia. I. Schmidt, Dwight Lyman, 1926- . II. Huffman, A.C. III. Title. IV. Series: Geological Survey professional paper ; $560-\mathrm{A}$.

\begin{tabular}{llll} 
QE291.S28B76 & 1989 & $555.3^{\prime} 8$ & $87-600038$ \\
\hline
\end{tabular}

For sale by the Books and Open-File Reports Section, U.S. Geological Survey, Federal Center, Box 25425, Denver, CO 80225 


\section{FOREWORD}

This volume, "The Geology of the Arabian Peninsula," is a logical consequence of the geographic and geologic mapping project of the Arabian Peninsula, a cooperative venture between the Kingdom of Saudi Arabia and the Government of the United States. The Arabian-American Oil Co. and the U.S. Geological Survey did the fieldwork within the Kingdom of Saudi Arabia, and, with the approval of the governments of neighboring countries, a number of other oil companies contributed additional mapping to complete the coverage of the whole of the Arabian Peninsula. So far as we are aware, this is a unique experiment in geological cooperation among several governments, petroleum companies, and individuals.

The plan for a cooperative mapping project was originally conceived in July 1953 by the late William E. Wrather, then Director of the U.S. Geological Survey, the late James Terry Duce, then Vice President of Aramco, and the late E.L. deGolyer. George Wadsworth, then U.S. Ambassador to Saudi Arabia, and Sheikh Abdullah Sulaiman, then Minister of Finance of the Government of Saudi Arabia, lent their support to the plan. In November of the following year, 1954, Director Wrather approved the U.S. Geological Survey's participation and designated G.F. Brown responsible for the western Arabian shield region in which he had previously worked under U.S. foreign-aid programs. In January 1955, F.A. Davies, Chairman, Board of Directors, Arabian-American Oil Co., approved Aramco's participation and appointed the late R.A. Bramkamp, chief geologist, responsible for compilation of the area within the Kingdom where the sediments crop out. This responsibility fell to L.F. Ramirez following the death of R.A. Bramkamp in September 1958.

R.A. Bramkamp and G.F. Brown met in New York in February 1955 and planned the program, including scales of maps, areas of responsibility, types of terrain representation, and bilingual names. Thus there was established a cooperative agreement between the Kingdom of Saudi Arabia, the U.S. Department of State, and the Arabian-American Oil Co. to make available the basic areal geology as mapped by Aramco and the U.S. Geological Survey.

The agreement specified publication of a series of 21 maps on a scale of 1:500,000, each map covering an area $3^{\circ}$ of longitude and $4^{\circ}$ of latitude. Separate geologic and geographic versions were to be printed for each of the quadrangles; both versions were to be bilingual-in Arabic and English. A peninsular geologic map on a scale of $1: 2,000,000$ was to conclude the project.

High-altitude photography, on a scale of 1:60,000, of the Kingdom of Saudi Arabia was initiated during 1949 by the Aero Service Corp. and completed in 1959. Both third-order vertical and horizontal control and shoran were utilized in compiling the photography. This controlled photography resulted in highly accurate geographic maps at the publication scale which then served as a base for the geologic overlay. The topography of the sedimentary areas was depicted by hachuring and that of the shield region by shaded relief utilizing the airbrush technique.

The first geographic quadrangle was published in July 1956 and the last in September 1962. While preparation of the geographic sheets was in progress, a need arose for early publication of a 1:2,000,000-scale peninsular geographic map. Consequently, a preliminary edition was compiled and published in both English and Arabic in 1958. The second edition, containing additional photography and considerable new topographic and cultural data, was published in 1963. The first of the geologic map series was published in July 1956 and the final sheet in early 1964 . The cooperative map project was completed in October 1963 with the publication of the 1:2,000,000-scale "Geologic Map of the Arabian Peninsula" (Miscellaneous Geologic Investigations Map I-270 A).

As work on the quadrangles progressed, geologists, companies, and governments working in areas adjacent to the Kingdom of Saudi Arabia were consulted by Aramco and invited to participate in the mapping project. The number of cooperating participants was expanded to 11, which included the operating oil companies in the peninsula and which are identified elsewhere in this text; the Overseas Geological Surveys, London; the Government of Jordan; F. Geukens, who had worked in Yemen; and Z.R. Beydoun, who had studied the Eastern Aden Protectorate. With the close cooperation of the authors, the new data were added to data already plotted on the base map of the Arabian Peninsula.

As the geological coverage of the peninsular map grew, the need for a text to accompany the map became 
apparent to both the U.S. Geological Survey and the Aramco geologists. Exploratory conversations were begun by Aramco with companies working in the other countries of the Arabian Peninsula for their participation in the preparation of a monograph on the geology of the Arabian Peninsula. Each author prepared a description of the geology of the area for which he was responsible, as shown in the sources of geologic compilation diagram on the peninsular map. The U.S. Geological Survey undertook the publishing of the volume as a professional paper, and the Government of Saudi Arabia was to finance its printing. It was early agreed that there would be no effort to confine the contributions to a standard format and that no attempt would be made to work out an overall correlation chart other than shown on the "Geologic Map of the Arabian Peninsula." Thus, the individual style of authors of several nationalities is preserved.
Cooperation and relations have been of the highest order in all phases of the work. The project would not have been possible without the full support of the U.S. Department of State, the Kingdom of Saudi Arabia, and all contributors. In fact, the funds which made publication of this volume possible were contributed by the Saudi Arabian Government.

The data provided by the maps and in the professional paper provide information for an orderly scientific and economic development of a subcontinent.

O. A. SEAGer, Arabian-American Oil Co. (Retired)

W. D. Johnston, JR., Former Chief, Foreign Geology Branch, U.S. Geological Survey (Deceased) 


\section{CONTENTS}

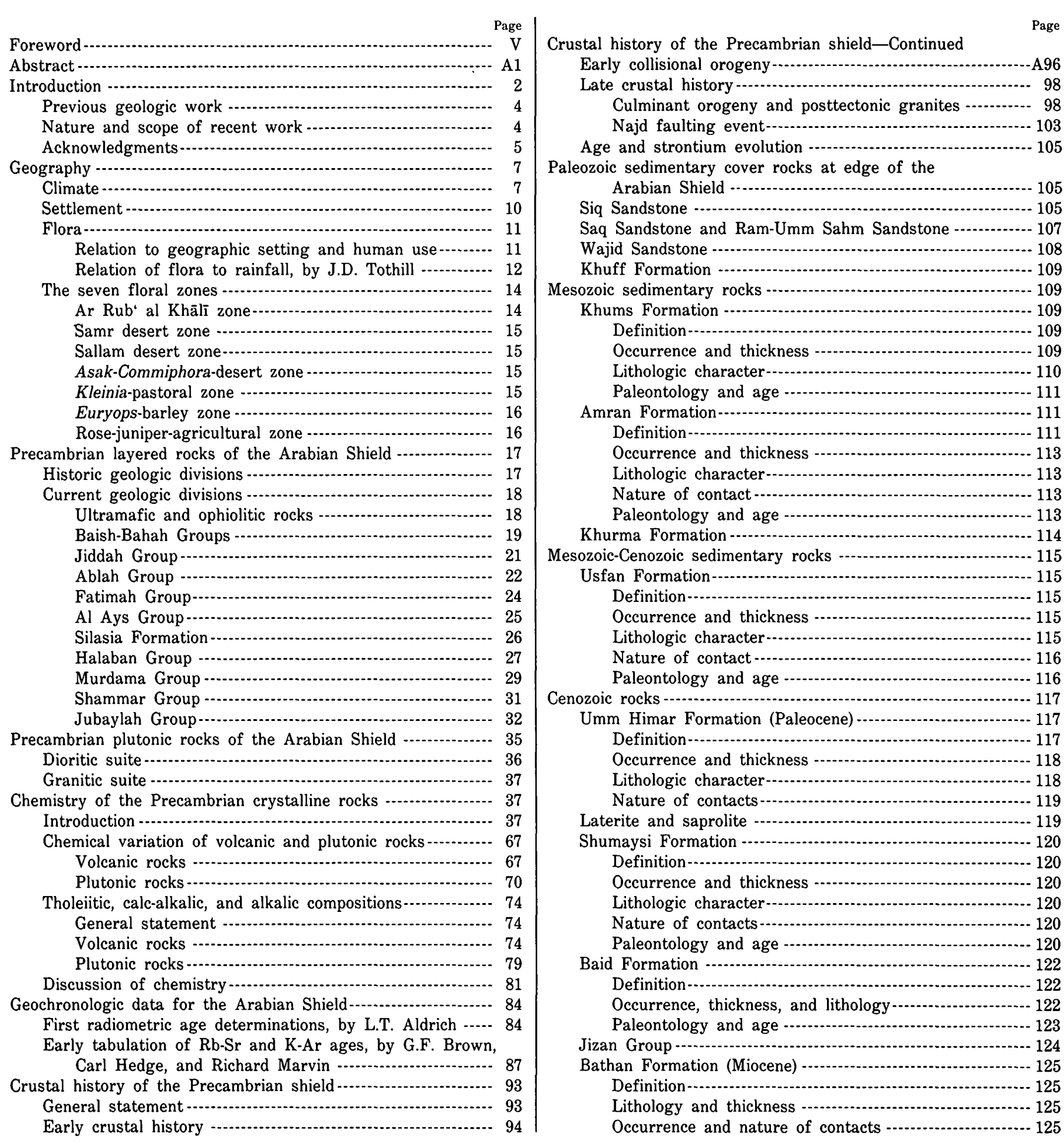


Cenozoic rocks-Continued

Bathan Formation (Miocene)-Continued

Paleontology and age

Raghama Formation (Miocene)

Definition

Occurrence, thickness, and lithology-

Nature of contacts

Paleonotology and age

Cenozoic igneous rocks

Cenozoic basaltic lava flows -................................ 149

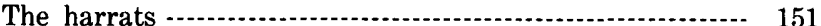

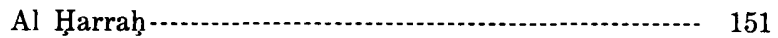

Harrat ar Rabāh-'Uwayrid .................................- 152

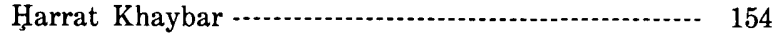

Harrat Lunayyir-.......................................... 155

Ḩarrat I'shara-Khirsāt and Ḩarrat Harairah ------... 156

Harrat Kuramā' -...........................................-.-.- 157

Ḩarrat Rahaţ--......... 157

Harrat al Kishb -............................................. 158

Hुarrat al Hutaymah -............. 158
Cenozoic igneous rocks-Continued

The harrats-Continued

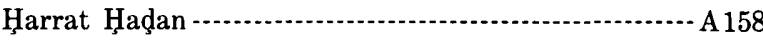

Harrat Nawās̄if-al Buqūm .................................... 161

Ḩarrat ad Damm and Ḩarrat Tuffil (Shamā) -........ 161

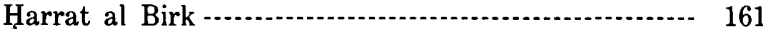

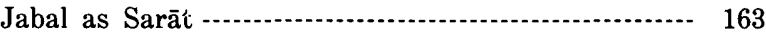

Cenozoic history and evolution of the Red Sea ................. 163

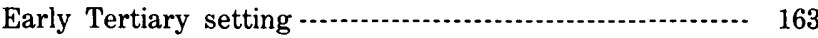

Continental rift-valley stage .............................-.- 163

First-stage sea-floor spreading -......................... 165

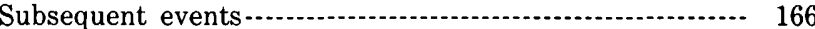

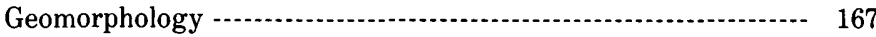

Cycles of erosion -............................................... 167

Arid cycle -.-...........-...- 167

Common desert erosion cycle -...........................- 168

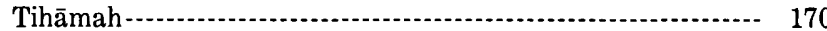

Scarp mountains -............................................... 172

Hejaz-'Asīr and Ḩismā Plateaus -......................... 174

Najd pediplain -................................................................. 175

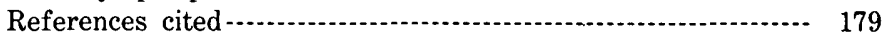

\section{ILLUSTRATIONS}

[Plates are in pocket]

Frontispiece. The Arabian Shield as seen from space.

Plate 1. Geologic map of the Saudi Arabian Shield.

2. Maps of Cenozoic igneous rocks of Saudi Arabia.

3. Physiographic provinces of the Arabian Peninsula.

4. Maps showing details of sharms along the Arabian coast of the Red Sea and the Gulf of 'Aqaba, Saudi Arabia.

FIGURE

1. Index map showing location of the Arabian Shield, Arabian Peninsula .......

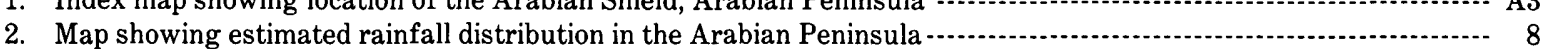

3. Photographs of examples of a few phreatophytes, indicating permanent ground water -.................................. 12

4. Photomicrograph of amygdaloidal metabasalt at the Jabal Ess ophiolite complex

5, 6. Photographs of:

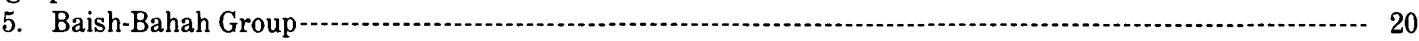

6. Hornblendite interbedded with pink marble of the Jiddah (Samran) Group in Wādĩ Fāţimah, and pillow lava in basalt, Hilwa area, on north wall of Wādi Baysh gorge -............................... 21

7. Oblique aerial photograph showing view to the northwest across the Ablah and Jiddah Groups -...................... 23

8-13. Photographs of:

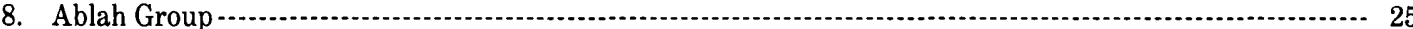

9. Fatimah Group, north Wādī Fāţimah

10. Silasia Formation showing siliceous hematite outcrops intruded by diorite at Wādī Sawāwīn -................ 27

11. View north along the strike of stratabound gossan at Wādī Wassāt in Halaban Group volcaniclastic

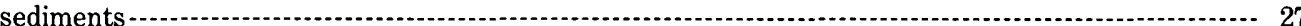

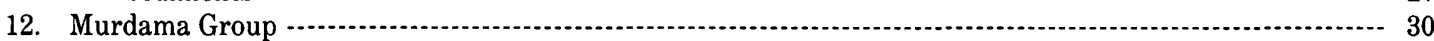

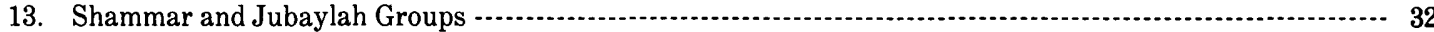

14. Graph showing incremental ${ }^{39} \mathrm{Ar}_{\mathrm{K}}$ of the Jubaylah andesite at the type locality of the Jubaylah Group …............ 34

15. Aerial view to the southeast of Jabal Huassan at the east edge of the shield and photograph of Jabal Shāyi layered gabbro pluton in the southeastern shield near the village of Khaybar -........................................ 35

16. Map showing tectonic belts and regions used to define regional variations and trends in the chemical data examined for this report

17-21. $\mathrm{Na}_{2} \mathrm{O}-\mathrm{CaO}-\mathrm{K}_{2} \mathrm{O}$ diagrams showing:

17. Chemical distribution (molar data) of control samples of metavolcanic rocks -

18. Chemical distribution (molar data) of metavolcanic rocks and a few dike rocks reported in this report in tables 3 and 4 
19. Plutonic-rock chemistry (molar data) of control samples classified by age -.................................. A71

20. Plutonic-rock chemistry (molar data) of samples reported in this report in tables 3 and 4 and classified by age

21. Summary of all the plutonic-rock chemistry (molar data) examined for this report-......

22-25. AFM diagrams showing:

22. Metavolcanic-rock chemistry (weight percent data) of control samples classified by age -..................... 75

23. Chemical (weight percent data) distribution of metavolcanic rocks and a few dike rocks given in tables 3 and 4

24. Chemical (weight percent data) distribution of rocks of the dioritic suite from the Saudi Arabian Shield

25. Chemical (weight percent data) distribution of rocks of the granitic suite from the Saudi Arabian Shield

26-28. Alkali-silica diagrams showing:

26. Metavolcanic-rock chemistry (weight percent data) of control samples of the Baish-Bahah and Jiddah Groups and of the Halaban Group

27. Metavolcanic-rock chemistry (weight percent data) of control samples of the Murdama, Shammar and Jubaylah Groups, and of the metavolcanic rocks analyzed for this report

28. Plutonic-rock chemistry (weight percent data) of dioritic suite for samples of this report and of control set

29. Alkali-silica diagrams showing plutonic-rock chemistry (weight percent data) of granitic suite for samples of control set and of this report, and histogram showing distribution of $\mathrm{K}-\mathrm{Ar}$ and $\mathrm{Rb}-\mathrm{Sr}$ ages for several minerals

31. Oblique aerial photograph showing view to the northwest of orthogneiss dome containing enfolded amygdaloidal metabasalt flows of the Baish Group

32, 33. Photographs showing:

32. Schistose gneiss at Wādī Dhuqiyah $65 \mathrm{~km}$ southeast of Aț Tā'if

33. Aerial view south of the eastern edge of the Khamis Mushayt gneiss dome, and Jabal al Hidab

34. Histograms showing results of geochronologic analyses of Paleozoic and Precambrian rocks

35. Oblique aerial photograph of view to the southeast from lat $23^{\circ} 05^{\prime} \mathrm{N}$., long $45^{\circ} 05^{\prime} \mathrm{E}$. near the east edge of the shield

36, 37. Aerial photographs of:

36. View to the northwest of the Najd fault zone southwest of 'Afif

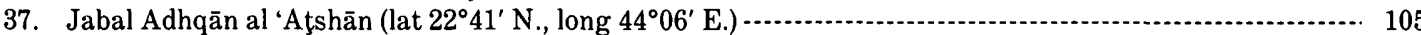

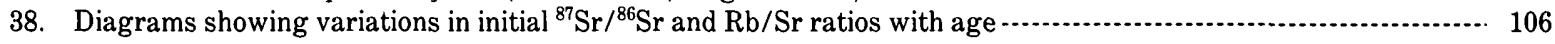

39-41. Photographs of:

39. Siq Sandstone

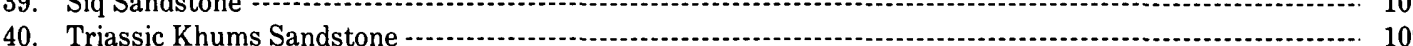

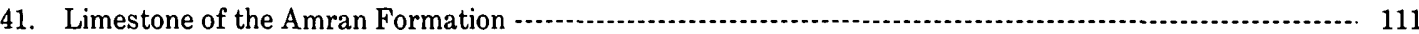

42, 43. Type sections of:

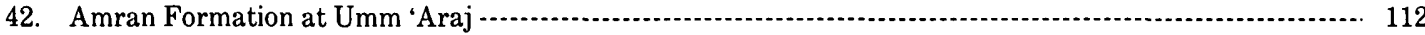

43. Usfan Formation at 'Usfãn Pass -................................................................................ 116

44. Composite columnar section of the Umm Himar Formation and overlying and underlying rocks in the Jabal

Umm Himar area

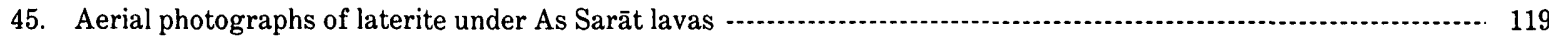

46. Type referenced section slightly modified from locality of the measured Shumaysi Formation ........................ 121

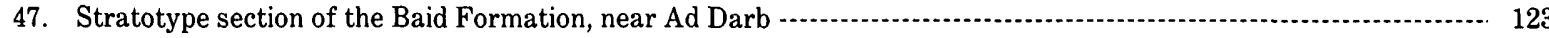

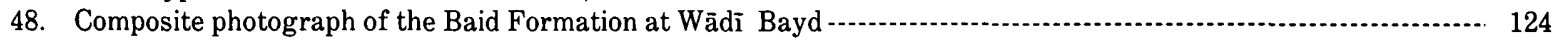

49. Photograph of exposure of the Bathan boulder conglomerate at Wãdi ad Duqah in the Jabal Shada quadrangle -

50. Aerial photograph of the Raghama Formation southeast of Dubā-

51. Histogram showing ages of selected Tertiary igneous rocks and glauconite from around the Red Sea ................ 129

52. Chart showing modal analyses of Tertiary continental dikes plotted on quartz-alkali feldspar-plagioclase diagram

53. Harker diagram showing alkalies plotted against silica f

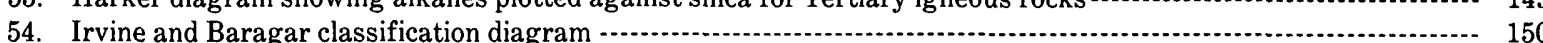

55, 56. Ternary diagrams showing distribution of Tertiary igneous rocks from western Saudi Arabia:

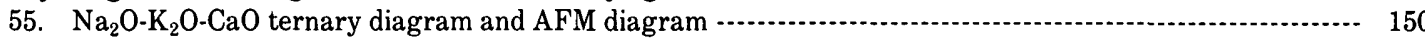

56. Normative albite+nepheline-orthoclase-anorthite diagram and Y-La-Ce ternary diagram -...-.-.........- 151

57-60. Photographs of:

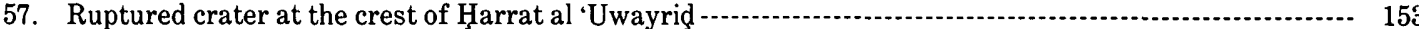

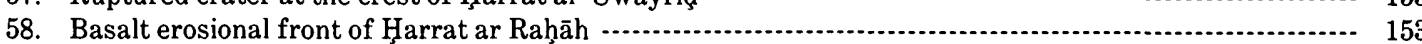

59. Eolian undercutting of Ram-Umm Sahm Sandstone at a Nabatean tomb (65 A.D.) at Madā'in Şālih ...... 154

60. Holocene crater of white rhyolite tuff and lapilli of Abyad wa Ubayyid in Harrat Khaybar-................ 154 
FIGURE 61. Aerial photograph of the tholoid of Jabal Ithnayn, Harrat al Ithnayn $\ldots$

62. Photograph of the crater of Jabal Hibran, Harrat al Ithnayn

63. Oblique aerial photograph of view east-northeast of Ḩarrat al Kishb showing linearity of the craters -............ 159

64, 65. Aerial photographs showing:

64. Al Wahbah phreatic crater from a Holocene eruption at the northwestern corner of Harrat al Kishb … 160

65. Cinder-ash cone, Jabal al Qishr -

66, 67. Diagrams showing:

66. Summary of the geologic history of the southern coastal plain area relative to that of the adjacent

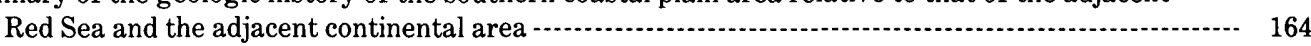

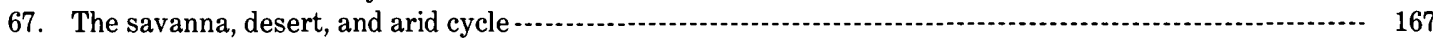

68. Landsat image showing effects of wind erosion, north of Wādī ar Rimah and east of Ḩarrat Khaybar and Harrat Ithnayn -........................ 169

69. Aerial photograph of terrace benches at 6, 22, and $31 \mathrm{~m}$ above the northern Red Sea north of Al Wajh ….......... 170

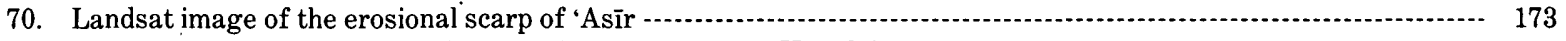

71. Aerial photograph of loessal silt in Wādī Tathlīth above Ḩamḑah $-y_{1}$

72-75. Photographs showing:

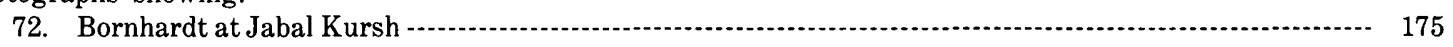

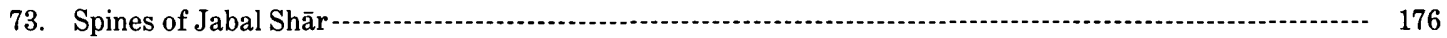

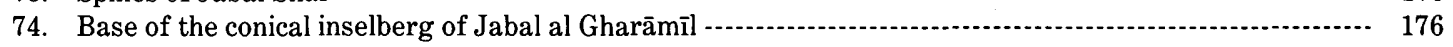

75. Wādī Tharīb (Ash Schism) yardang valley

76. Aerial photograph of yardang troughs following one set of joints parallel to the wind direction to N. $70^{\circ} \mathrm{E}$.

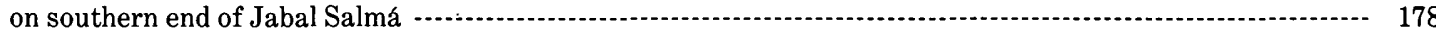

\section{TABLES}

TABLE 1. Air temperatures and relative humidities at selected sites in western. Saudi Arabia -......................................... A9

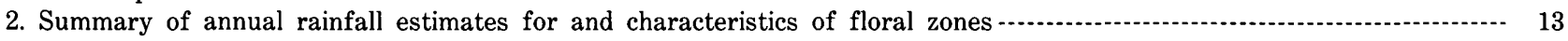

3. Description and classification of crystalline rocks of the Arabian Shield for which chemical analyses are given in table 4

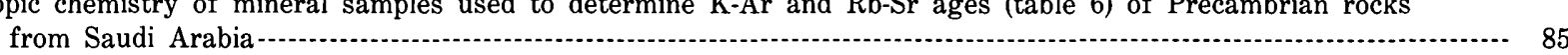

6. Locations, rock types, and ages of mineral samples from Precambrian rocks of Saudi Arabia -........................... 88

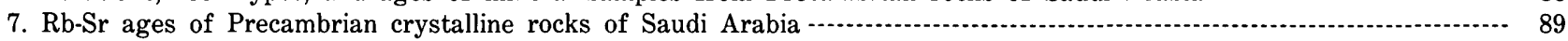

8. K-Ar ages of Precambrian crystalline rocks of Saudi Arabia -

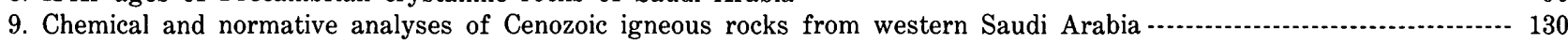

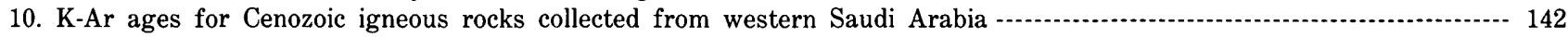

11. Locations and rock types for Cenozoic igneous rocks for which chemical analyses are given in table 9 and isotopic ages in table 10 


\title{
GEOLOGY OF THE ARABIAN PENINSULA
}

\section{SHIELD AREA OF WESTERN SAUDI ARABIA}

\author{
By Glen F. Brown, Dwight L. Schmidt, and A. Curtis Huffman, Jr.
}

\begin{abstract}
"Nay, the very slate beds of Snowdonia have not forced their way up from under the mountain without long and fearful struggles. They are set in places upright on end, then horizontal again, then sunk in an opposite direction, then curled like sea-waves, then set nearly upright once more, and faulted through and through...."
\end{abstract}

\section{ABSTRACT}

Western Arabia lies within the low-latitude desert of north Africa and the Middle East, the core being the Arabian segment of the African Shield. The core of complex basement rocks accounts for about $670,000 \mathrm{~km}^{2}$, or one-third of the Arabian Peninsula. Reconnaissance mapping of these crystalline rocks, together with bordering sedimentary rocks and volcanic flows, begun in 1950, resulted during the next 13 years in a series of geologic and geographic maps without extensive texts. The maps served as general guides for development of natural resources, including water supplies, ore deposits, and building materials. An intensive exploration program that began in 1963 and involved numerous geologists has vastly increased geologic information.

Rainfall in Arabia is meager and episodic, and vegetation is sparse except in isolated copses on the crest of the Hejaz Range. Comparison of flora with similar species in the Sudan, where records of rainfall have long been kept, allows evaluation of mean annual precipitation. Wandering bedouin following fodder created a delicate balance between population and water supply-now disturbed by wells drilled in alluvium and lava fields.

A trapezoidal region of Precambrian crystalline rocks lies along the northeast flank of the Red Sea, with two long prongs extending northwest and southeast for a total of $1,800 \mathrm{~km}$. These basement rocks of the Arabian Shield are well exposed on the uplands, scarp mountains, and coastal pediments where the Phanerozoic cover rocks have been stripped as a result of Paleozoic epeirogeny and Tertiary ramping. The shield outcrops are divided into three tectonic provinces by $\mathrm{N} .45^{\circ} \mathrm{W}$.- trending shear zones of the Najd fault system of latest Proterozoic and possibly earliest Paleozoic time. The southwestern province, the 'Asīi' upland, was sharply uplifted and tilted to the northeast during the Neogene. The northwestern province, consisting of the Ash Shifä'- Hismā upland as well as Jabal Shammar farther

${ }^{1}$ Geographic place names follow, in general, the U.S. Board on Geographic Names "Official Standard Names Gazetteer, Saudi Arabia, 1978," except where new names are introduced and where general usage has anglicized the spelling. Diacritical marks are added to help transliteration into Arabic. Local usage is followed for practicality as comprehended.

Manuscript approved for publication October 30, 1986.
Charles Kingsley, in Town Geology (1873)

east, similarly was uplifted and tilted. These two provinces are separated by the flat-lying median Najd province, which is chiefly bounded by the principal Najd faults.

The outcrops of the shield rocks are of the Late Proterozoic Eonupper Riphean to Vendian or Infracambrian epochs, including the Ediacarian System. The most reliable isotopic ages range from about 900 to 560 m.y., but some Middle Proterozoic rocks may be present in the easternmost shield. The rocks are divided into six lithostratigraphic sequences, two plutonic suites, and an ophiolitic suite. The mafic and ultramafic volcanic and plutonic rocks of the ophiolitic suite everywhere were emplaced tectonically and are probably of different ages in different places. Some ophiolite occurs as obducted blocks, but most is highly deformed and altered to serpentinite in fault zones that mostly define sutures between different tectonic blocks or terranes within the shield.

Three of the lithostratigraphic sequences consist of mafic to silicic volcanic rocks and volcanic-derived clastic rocks which, with their subvolcanic plutonic rocks of a dioritic suite, probably formed in oceanic island arcs during convergent plate tectonism. These rocks make up the primary, or first-formed, crust of the shield. Chemical analyses show that the primary shield rocks, regardless of age, are principally calc-alkalic with some associated tholeiitic varieties. Most of the layered rocks are andesitic, but they range from basalt to dacite and in places contain intercalated pillow basalt, marble, chert, and carbonaceous or graphitic schist. Most of the plutonic rocks of the dioritic suite are dioritic, but they range from gabbro to trondhjemite and rarely contain potassium feldspar. The sequences and an associated dioritic suite become younger toward the eastern shield, that is, the primary crust of the shield youngs toward the east.

Two western sequences consist of the Jiddah (Samran) and BaishBahah Groups and range in radiometric age from about 900 to 800 m.y.; the eastern sequence consists of the Halaban (Hulayfah) Group and ranges from 800 to about 700 m.y. During subsequent orogeny, most of the rocks were intensely deformed and mostly metamorphosed to upper greenschist facies, but rising in places to the almandine-amphibolite facies.

Two other lithostratigraphic sequences with an associated plutonic granitic suite are the products of two mountain-building episodes during which the primary crust was greatly thickened and converted into craton. The two sequences, including largely the Ablah (Al Ays) and Murdama (Shammar) Groups, consist of abundant sedimentary rocks, commonly arkosic, that are the erosional products of the 
orogenic mountains. They are several thousand meters thick. Less abundant calc-alkalic to alkalic volcanic rocks, commonly dacitic and rhyolitic, are intercalated with the sedimentary rocks. The plutonic rocks of the granitic suite in association with both sequences have syntectonic and posttectonic phases, are products of the orogenies, and are the principal new ingredients making up the craton.

Gneiss domes were a significant part of these cratonization orogenies. In association with orogenic crustal heating, some of the low-density, more silicic tonalitic and trondhjemitic rocks of the primary crust rose as gneiss domes. Partial melting in the middle or lower crust below the gneiss domes produced large volumes of granitic magma that intruded the gneiss domes as granodioritic batholiths.

The Ablah Group and the older part of the granitic suite are about 775 to $740 \mathrm{Ma}$ old and are associated with the Ablah orogeny and early cratonization in the western and earlier formed half of the shield. The Murdama (Shammar) Group and the younger part of the granitic suite are about 660 to $580 \mathrm{Ma}$ old and are associated with the culminant orogeny and late cratonization that was shieldwide. The granitic suite during both orogenies consists of early, syntectonic granodiorite batholiths associated with the gneiss domes and late, posttectonic monzogranite plutons. Only during the culminant orogeny, late magmatic evolution produced syenogranite and alkali-feldspar granite commonly in circular and ring-structured plutons and with associated explosive volcanic deposits (Shammar Group); final products, some of which have economic potential, were peralkalic and peraluminous. The late plutonism of the culminant orogeny was distinctly bimodal in that subordinate gabbroic rocks are associated with the granites.

Various building blocks or terranes of the andesitic and dioritic primary crust were collisionally agglomerated during the Ablah orogeny, early cratonization, whereas the entire shield as currently exposed was further collisionally accreted and compressionally consolidated during the culminant orogeny, final cratonization. Thousands of kilometers of oceanic crust had to be subducted in about 300 m.y. to form the large primary crust of the Arabian Shield. The inevitable collisional events during consumption of such a large volume of oceanic crust invariably led to numerous collisional orogenies that collectively encompass the widely known Pan African tectonic episode.

The youngest lithostratigraphic sequence, the Jubaylah Group, is essentially postcratonic, although it is the end product of the collisional culminant orogeny. Final east-west compression of the entire shield from about 580 to 560 m.y. caused the craton to fracture along the large northwest-trending, left-lateral faults and elsewhere along lesser, northeast-trending, right-lateral, conjugate faults of the Najd fault system. Erosional products of this more localized deformation were the sedimentary rocks of the Jubaylah Group, which also includes intercalated andesitic to basaltic volcanic rocks of a mafic alkalic compositional trend.

The collisional edge of an old continental plate (or tectonic fragments thereof), suspected on the eastern edge of the Arabian Shield, has not been shown with certainty to be exposed. Presumably, widespread contamination from such an old continental crust affects $\mathrm{U} / \mathrm{Pb}, \mathrm{Sm} / \mathrm{Nd}, \mathrm{Rb} / \mathrm{Sr}$, and common lead ratios in the young plutonic rocks of the easternmost shield. One mass of anorthosite near Jabal Khidā' on the central eastern edge of the shield may be a fragment of this old continental plate in that associated granodiorite may be as old as 1,600 to $1,800 \mathrm{Ma}$.

Epeirogenic uplift, erosion, and cooling of the uppermost shield during Early and Middle Cambrian time is indicated by an average fission track age of $510 \pm 52$ m.y., on sphene from diorite (hornblende $\mathrm{K}$-Ar age of $615 \pm 12$ m.y.) in the southwestern part of the shield. The hiatus was followed by extensive deposition of the Cambro-Ordovician Saq Sandstone in the north and northeast and the Wajid Sandstone in the southeast and south of the shield. The Cambrian Siq
Sandstone had already been deposited in the northern part. During the middle and late Paleozoic, broad epeirogeny caused further erosion of the shield until marine transgression deposited the Upper Permian Khuff Formation at least in the eastern part of the shield. In the southwestern shield, the nonmarine Upper Triassic Khums Sandstone was deposited variably on Wajid or Precambrian rocks and is overlain by limestone of the middle Upper Jurassic Amran Formation.

Except for shallow marine sandstone of problematic Cretaceous age deposited on the Amran Formation in the southwestern shield and on Precambrian rocks in the northwestern shield, the younger beds on the shield are Paleocene and younger, with the possibility that the lowermost are upper Maestrichtian. The early Tertiary beds contain vertebrate fossils of coastal marine or estuarine environment $250 \mathrm{~km}$ east of the Red Sea in the central shield. Marginal marine sediments were deposited in a western tongue of the latest Tethys Sea as late as Eocene on the western shield and at least as far south as Jiddah.

The great harrats of flood basalt erupted on the western shield during late Oligocene and early Miocene at the same time a 2,000-kmlong continental rift valley developed along the future Red Sea axis. Within this rift valley, Baid freshwater tuffaceous lakebeds were deposited between mafic and silicic volcanoes. During late early Miocene time, the Red Sea opened at a rate of $4.4 \mathrm{~cm} / \mathrm{yr}$ in a firststage movement while continental dikes and swarms of oceanic tholeiitic dikes, gabbro, and granophyre plutonic rocks were intruded into the rift sedimentary and volcanic rocks at the newly formed continental margin. The continental margin was deformed and greatly extended at this time. About 14 or 15 m.y., as the first-stage spreading stopped, the Red Sea Escarpment rose; its erosion caused deposition of coarse conglomerate of the Bathan Formation. About $3,000 \mathrm{~m}$ of evaporite was deposited on the young Red Sea oceanic crust during the late Miocene desiccation crisis.

A second stage of sea-floor spreading about 4-5 m.y. produced the Red Sea axial trough, consisting of oceanic crust, as well as renewed uplift and tilting of the three tectonic provinces in response to compression from counterclockwise rotation against the Dead Sea Rift. This late movement caused widespread major stream capture, especially along the wadis that formerly drained southwesterly or northwesterly, the channels turning westward through narrow gorges to the coastal plain and the Red Sea.

\section{INTRODUCTION}

The mapping of the geology of the Arabian Shield of Saudi Arabia during the period 1950 to 1958, as shown on Miscellaneous Geologic Investigations Map I-270A by the U.S. Geological Survey and the Arabian-American Oil Company (USGS-ARAMCO, 1963), was part of a larger program undertaken by the Kingdom of Saudi Arabia and the Government of the United States (see "Foreword" for details). The geology shown on the geologic map of this report (pl. 1) is a revision of part of Map I-270A and represents a compilation of geologic mapping done as part of the USGS-Saudi Arabian program from 1963 to the present. As chapter A of USGS Professional Paper 560, this report synthesizes and brings up to date a large amount of geologic data gathered by many individuals.

Prior to the inception of USGS fieldwork in 1950, almost no geologic studies of the western part of the Arabian Shield (fig. 1, pl. 1) had been made. This lack 


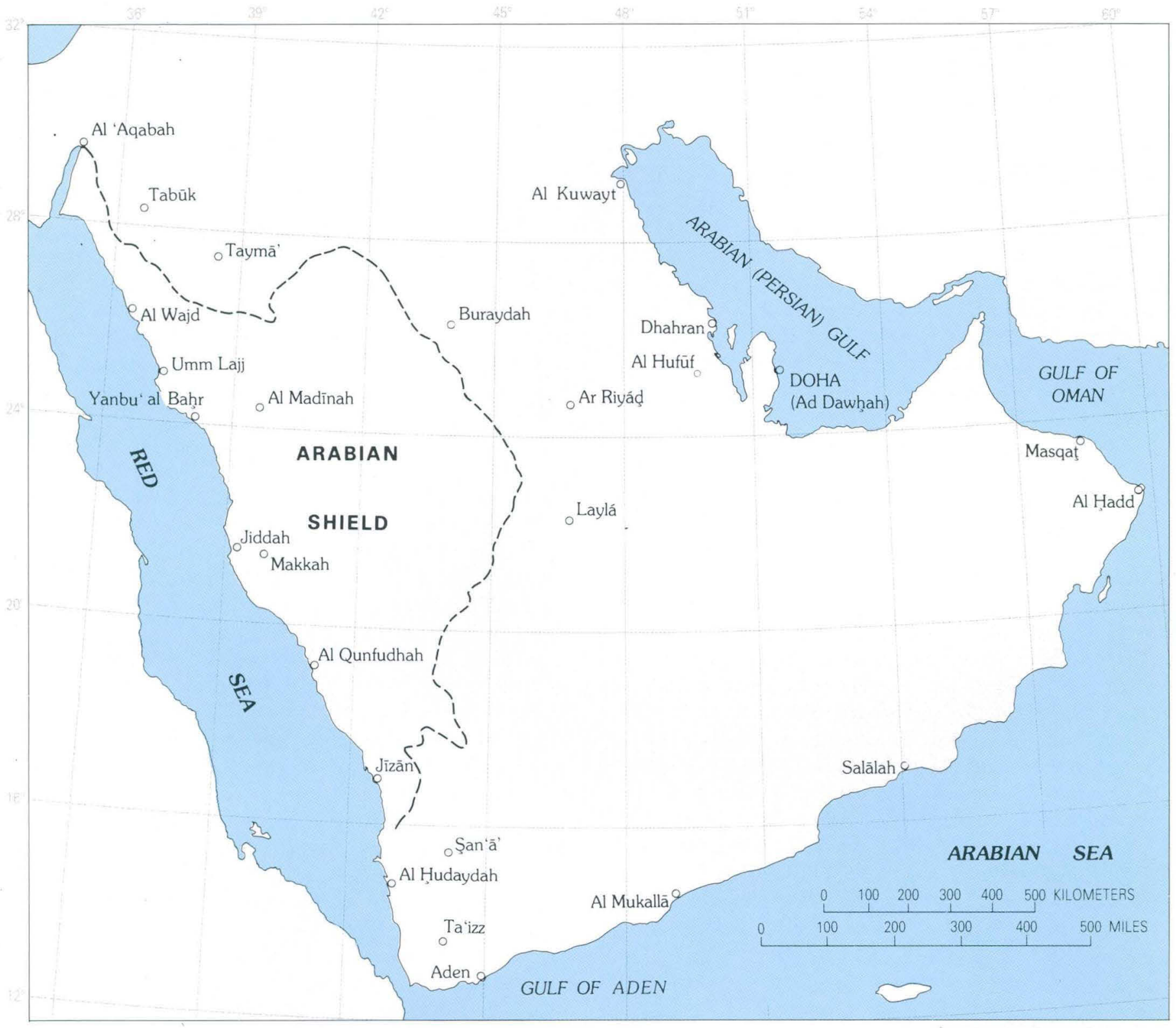

Figure 1.-Location of the Arabian Shield, Arabian Peninsula.

contrasted sharply with the wealth of data available for central and eastern Arabia, where petroleum companies have been actively exploring for nearly 50 years. Fieldwork from 1950 to 1958 contributed information for the western part of the geologic map of the Arabian Peninsula (USGS-ARAMCO, 1963) and for a series of 20 quadrangle maps. As a base for geologic mapping, the USGS field party used aerial photography and necessarily described petrologic units in field terms because it was soon recognized that the shield rocks that could be classified into lithostratigraphic groups ranged from nonmetamorphosed facies through green- schist and amphibolite metamorphic facies. These Precambrian rocks are intricately folded and faulted, and in addition are intruded by numerous hypabyssal and plutonic igneous rocks. Nonetheless, from 1950 to 1963 the USGS laid the groundwork for economic development in the Arabian Peninsula of other than hydrocarbons by completing and publishing reconnaissance maps of $670,000 \mathrm{~km}^{2}$ of complex basement rocks-and also by accomplishing its primary purpose of evaluating the economic, principally hydrologic, possibilities of the region. The resulting maps were published as a series of quadrangle maps at 1:500,000 scale (geologic 
I-200 to I-220A, geographic I-200 to I-220B) and a geologic map of the entire peninsula published at $1: 2,000,000$ scale (I-270A). Beginning in 1963, emphasis was placed on economic mineral resource evaluation of the shield as well as continued mapping of the shield rocks.

The economic boom in Saudi Arabia since 1970 has led to more detailed mapping by many geologists from several nations, besides a growing number of Saudi Arabian geologists, with the result that the quality of geologic knowledge and interpretation of the western part of the shield now approaches that obtained by earlier petroleum exploration in the area of sedimentary cover of the shield. This report describes the layered and massive igneous rocks of the Arabian Shield area in western Saudi Arabia and traces the evolution of the geologic nomenclature from 1950 to the geologic names as they are currently being used (1984). Data from geophysical and geochemical field studies have aided these intensified surveys, as have results of chemical and petrographic analyses prepared in laboratories in Jiddah and abroad. Diamond-drilling at encouraging prospects has added some third dimension. Perhaps the most important studies have been isotope measurements for determining radiometric ages (Baubron and others, 1976; Fleck and others, 1976, 1980; Aldrich and others, 1978; Cooper and others, 1979) which supply the "pegs" on which the Precambrian geologic history hangs, because fossils in the Arabian Shield are limited to stromatolitic and related forms of debatable value as time markers. Fortunately, the Arabian segment of the African megacraton is well exposed, and ideas on the outcrop can and have been challenged. The diversity of geologic concepts from various disciplines and many cultural backgrounds has done much to elucidate the geology of the segment.

Like chapter D of Professional Paper 560 (Powers and others, 1966), this report is essentially a compilation which, without the chronological data and the large volume of work done by many geologists since the preliminary map was published, would be less useful. This report discusses the stratigraphic and tectonic history of the shield area and relates the stratigraphic terminology of the 1963 map to current concepts.

\section{PREVIOUS GEOLOGIC WORK}

The first areal geologic descriptions of western Arabia (fig. 1) in modern times are those of Charles Doughty (1888), who prepared a general geologic map colored in along the routes of his trips during the period 1875 to 1878 and described in "Travels in Arabia Deserta." He separated the granites and traps, most of which crop out in what he called the central plutonic country extending from Jabal Shammar (Ḩā'il) to Makkah, from the overlying sandstone and limestone of the Great Nefud and the Qasim and from the still younger volcanic eruptions, the extensive basaltic lava fields (harrats) of Tertiary and Quaternary age.

Concurrent with Doughty's travels and 4 yr later, though their trails never crossed, Charles Huber traveled in central and northern Arabia as far as Jabal Shammar and Al Qaşim (Huber, 1891). His map, published posthumously, carries scant geologic information, but he collected rock specimens which were later studied by S. Meunier (1891) and P. Lamare (1923), who quotes Meunier. The distribution of alkalic granite plutons and peralkalic rhyolite in the Shammar region was noted by Lamare (1923, 1930a, 1930b).

During the period 1908-1915, Alois Musil (1926) explored and mapped much of northwestern Arabia. L. Kober (1911) accompanied him in 1910 to make geologic observations that gave the first general sequence, mostly of the Phanerozoic, of that part of Arabia.

Although Richard Burton (1978) briefly explored and searched for gold mines in northwestern Arabia along the Red Sea coast from near lat $27^{\circ} 30^{\prime} \mathrm{N}$. to lat $28^{\circ} \mathrm{N}$., the first serious mineral exploration following the expansion period of the Abbasid Caliphates (750-833 A.D.) was that of K.S. Twitchell on behalf of King Abdul Aziz ibn Sa'ud. Twitchell, an American mining engineer, made many reconnaissance trips in search of water supplies and mineral prospects (Twitchell, 1958). His work led to the development of a gold mine $400 \mathrm{~km}$ northeast of Jiddah at Mahd adh Dhahab, which produced 643,817 ounces of gold and 1,003,131 ounces of silver from 1939 to 1954 . Recent work by the USGS has led to the discovery of an extension of the veins (Luce and others, 1976; Worl, 1978). During the development period of this mine, G.A. Dirom and T.P. Larken visited several ancient prospects in the Najd and in the northern Hejaz in the vicinity of $\mathrm{Al}$ Wajh and Yanbu' an Nakhl. Some prospecting was also done in the southern Hejaz south of At Tä'if.

In 1944, Max Steineke and E.L. Berg of the ArabianAmerican Oil Company and Lt. G. Wadsack of the American Military Mission mapped about $10,000 \mathrm{~km}^{2}$ by reconnaissance methods along the Red Sea coast, covering Jiddah, 'Usfān, and Wādī Fāţimah, to choose drilling sites for a water supply for Jiddah (Steineke and others, 1944). The crystalline rocks were not subdivided, but Tertiary and Quaternary sediments and lavas were mapped.

\section{NATURE AND SCOPE OF RECENT WORK}

The USGS began a systematic reconnaissance of western and central Arabia in 1950 at the request of the 
late King Abdul Aziz ibn Sa'ud. R.O. Jackson and G.F. Brown started in the 'Asīr province of southwestern Arabia, assisted by Sharif Kasem and Hisham Farouki, and joined by R.G. Bogue and G.H. Goudarzi in the fall of 1953. D.F. Dougherty spent one year studying the surface-water possibilities. The reconnaissance was extended northward and eastward, with Bogue visiting the northwestern and southeastern portions of the shield.

Because no adequate maps for western Arabia existed, W.E. Wrather, then Director of the U.S. Geological Survey, obtained sufficient funds to contract for aerial photographs covering $55,000 \mathrm{~km}^{2}$ of the 'Asir Province in the southwestern part of the country. These flights were conducted in 1951. Subsequently, the U.S. Foreign Economic Administration contracted for aerial photography covering an additional $68,500 \mathrm{~km}^{2}$ along the Red Sea coast north of the 'Asīr; and finally, in 1955, on the advice of H.E. George Wadsworth, the American Ambassador, H.R.H. Faisal ibn Abdul Aziz, then viceroy of the Hejaz, requested aerial photography of the remainder of the western provinces. This covered about $810,000 \mathrm{~km}^{2}$ and included the western part of a belt of trimetrogon photography taken earlier that extended from Jiddah to Dhahran on the east coast. The aerial photographs served as a base for compilation of the series of geographic and geologic maps, at scales of 1:500,000 and 1:2,000,000, published between 1956 and 1963.

In 1954, under the direction of Dr. F.K. Kabbani, the Saudi Arabian Directorate General of Petroleum and Mineral Affairs began geologic work, much of which was done by D.F. Schaffner and W.H. McLean, assisted by Hashim Shigdar and Ahmed Al-Shanti; G.F. Brown assisted in this work in 1957-58. In 1954, the Bundesanstalt fur Bodenforschung sent four geologists, G. Richter-Bernburg, H.R. von Gaertner, W. Schott, and H. Schurenburg, who made a rapid reconnaissance along the major roads and trails. In 1955, Roman Karpoff of the Societe Lyonnaise des Eaux et de l'Eclairage, Paris, made two trips across the shield and traveled to the vicinity of $\mathrm{Al}$ Madinah and $\mathrm{Al}$ Lith. In 1956-58, G.F. Brown made a reconnaissance of the northeastern part of the shield, and in December 1962 he traveled from Tabūk westward to Wādi 'Ifāl and south to Al Wajh in the company of Dr. F.K. Kabbani.

Following this early geological reconnaissance, more intensive geological surveying was begun in 1963 under the direction of G.F. Brown, USGS, for Sheikh Ahmed Zaki Yamani, Minister of Petroleum and Mineral Resources. The areal work was extended in 1963, in 1965-67, and to the present (1984) to include airborne magnetic and total-gamma radiation measurements. In 1963, the USGS sent W.C. Overstreet, Richard Gold- smith, R.F. Johnson, J.W. Mytton, J.W. Whitlow, C.L. Hummel, and V.A. Trent to carry out reconnaissance and geochemical sampling, and W.E. Davis and R.V. Allen to introduce ground geophysical exploration, all under the direction of G.F. Brown. G.H. Sultan, Abdullah Ankary, Hashim Hakim, and Jamil Kouther, of the Saudi Arabian Directorate General of Mineral Resources, assisted and mapped areas on their own responsibility. The early fieldwork was supported by limited geochronological laboratory work by the Carnegie Institution, Washington, D.C.

Early in 1964 the Japanese Geological Survey sent a team of seven geoscientists to Arabia under the direction of Dr. Shizuka Okumi, and then, in 1966, under Dr. Ken Kirayama. These scientists concentrated their activities on specific mineral occurrences, with emphasis on geochemistry and detailed mapping.

In 1965 the French Bureau des Recherches Geologiques et Minieres (BRGM) began work for the Ministry, consisting mostly of areal mapping and prospecting, when they were given responsibility for a 140,000 $\mathrm{km}^{2}$ region that was subdivided into three areas of the Arabian Shield. Under the direction of Jacques Reneaux, and later under J.J. Altmann, much of the mapping has been directed by G. Eijekelboom, J. Delfour, M. Bertucat, and currently C. Pellaton, as Chief Geologists. Under their direction and with their participation, quadrangle mapping has been accomplished by 25 geologists, supported by K-Ar and Rb-Sr isotopic dating in France (Baubron and others, 1976).

\section{ACKNOWLEDGMENTS}

Thanks are due many members of the Government of Saudi Arabia who helped in the field, financed the work, and made the surveys possible. The work was started under the guidance of the late Sheikh Abdullah Sulaiman, former Minister of Finance, was continued under Sheikh Abdullah Tariki, former Minister of Petroleum and Mineral Resources, and was completed under Sheikh Ahmed Zaki Yamani, former Minister of Petroleum and Mineral Affairs. Dr. Fadil Kabbani, former Deputy Assistant Minister of Petroleum and Mineral Affairs, directed much of the early phases and accompanied G.F. Brown in the field in northwestern Arabia and east of Jiddah. Sheikh Ghazi H. Sultan followed Dr. Kabbani as Deputy Minister, and both he and Sheikh Mohammed Qusai Assad provided assistance vital to the completion of this report. G.H. Sultan, Abdullah Ankary, A.Y. Bagdady, Haskim Hakim, A.M. Helaby, F.M. Kana'an, Ziad-al Koulak, M.M. Mawad, Mohammad Naqui, J.H. Kouther, Ghanum Jeri, Misfir bin Yam, A.O. Ankary, R.G. Bogue, Gus Goudarzi, and Wallace McLain spent many arduous months in the 
desert. K.S. Twitchell kindly made his field notes available, and Daniel Schaffner supplied his mine examination reports and the geologic reports written by members of the Saudi Arabian Mining Syndicate. Dr. Kabbani supplied the reconnaissance reports of the Bundesanstalt fur Bodenforschung, the notes of Sheikh Ahmad Fakhry, who had earlier searched extensively for lost mines with Twitchell, and various unpublished reports prepared by the Directorate of Mineral Affairs.

Viktor Kahr, working for the Ministry of Petroleum and Mineral Affairs, mapped $7,650 \mathrm{~km}^{2}$ in northwestern Arabia and $1,500 \mathrm{~km}^{2}$ along the eastern edge of the shield. In addition, A. Al-Shanti mapped $2,000 \mathrm{~km}^{2}$ of the eastern shield and areas near Jiddah, W.K. Liddicoat mapped $10,000 \mathrm{~km}^{2}$ north of Jiddah, and F.M. Kana'an mapped $2,850 \mathrm{~km}^{2}$ adjacent to Liddicoat's map, as did J. Kouther. Karl Nebert mapped $2,400 \mathrm{~km}^{2}$, also north of Jiddah, and $1,500 \mathrm{~km}^{2}$ at the eastern edge of the shield, and also directed work by students of the Saudi Research Center for Applied Geology along the northern flank of Wādi Faţimah between Jiddah and Makkah. These areas have been incorporated in our compilation, as has later work, where available, from BRGM, Ministry geologists, the Japanese Geologic Survey, and members of the geological department of King Abdulaziz University.

The French oil company AUXERAP studied the coastal strip under the direction of Dr. Michel Gillmann in 1966 and 1967. They made their report available and gave permission for incorporation of the measured stratigraphic section of the Khums and Amran Formations.

P.K. Theobald, Jr., and Charles Thompson, USGS, beginning in 1963 gave support in geochemical prospecting and chemistry. W.J. Dempsey expanded the geophysical program and initiated diamond-drilling. Concurrently in 1963, the Topographic Division, USGS, sent G.W. Harbert, J.S. Crabtree, and T.E. Taylor to prepare the base maps and supervise aerial photography. Later this work was continued by F.G. Lavery, K.S. McLean, R.C. Nixon, G.C. Myers, C.M. Robins, and R.H. Tucker. A special team composed of R.E. Kenfield, G.E. Morrison, Jr., R.C. Nixon, A.A. Shands, W.E. Smith, and D.J. Winstead came to prepare topographic maps of the phosphate-bearing area in Wādī as Sirḩān near the Jordan frontier. More recent topographic map control has been continued by F.J. Fuller and D.J. Faulkender.

Under a 3-yr extension, 1966-1969, of the 1963 agreement, which ended essentially in 1966, and subsequent to the earlier reconnaissance photogeologic mapping and search for mineral deposits, the work became more intensive in areas considered of greatest economic potential. It consisted of geological mapping, mostly at a scale of 1:100,000 (some more recently has been compiled at a scale of 1:250,000), using helicopters and support by geophysical, geochemical, and drilling activities as well as petrographic, chemical, and geochronological support in the laboratory. A second 3-yr extension of the working agreement began in 1969, continuing the work initiated in 1966 under the direction of G.F. Brown. This new extension was directed first by J.J. Norton, later by T.H. Kiilsgaard, followed by F.S. Simons and D.G. Hadley, all assisted by R.O. Jackson. Currently (1984), R.O. Jackson is continuing direction of the field party. The program has continued to the present; the last 9 yr of work has been part of the Saudi Arabian first and second Five Year Development Plans as directed by Sheikhs Ahmed Zaki Yamani and Ghazi Sultan. USGS geologists who contributed to the geologic and economic knowledge during the extended time include D.L. Schmidt, D.B. Stoeser, J.C. Cole, and P.L. Williams as chief geologists, and G.H. Allcott, R.E. Anderson, W.R. Brock, D.A. Brobst, R.W. Bailey (deceased), F.W. Cater, J.C. Cole, R.G. Coleman, H.R. Cornwall, F.C.W. Dodge, J.L. Doebrich, E.A. DuBray, R.L. Earhart, J.E. Elliott, G.M. Fairer, D.J. Faulkender, W.D. Fenton, D.L. Gaskill, Louis Gonzalez, R.C. Greene, W.R. Greenwood, D.G. Hadley, F. Hershey, K.S. Kellogg, R.W. Luce, Conrad Martin, C.R. Meissner, J.S. Pallister, W.C. Prinz, J.S. Ratte, R.J. Roberts, L.F. Rooney, D.L. Rossman, E.G. Sable, R.P. Sheldon, C.W. Smith, J.S. Stuckless, P.K. Theobald, F.V. Tompkins, A.E. Weissenborn, J.W. Whitney, K.L. Wier, and R.G. Worl. R.V. Allen, G.E. Andreasen, H.R. Blank, W.F. Davis, V.J. Flanigan, M.E. Gettings, Andrew Griscom, S.A. Hall, D.L. Hase, D.R. Mabey, and J.A. Pitkin gave geophysical support. R.W. Girdler served as consultant. After L.T. Aldrich's and T.W. Stern's pioneer work in geochronology, C.E. Hedge, R.F. Marvin, H.H. Mehnert, V. Merritt, B.R. Doe, and R.J. Fleck analyzed shield rocks for isotopic ratios and estimates of age. J.A. Cooper and J.S. Stacey analyzed zircon by the $\mathrm{U}$, Th- $\mathrm{Pb}$ method for dating (Cooper and others, 1979). J.F. Sutter measured ${ }^{40} \mathrm{Ar} /{ }^{39} \mathrm{Ar}$ of a sample from the youngest flows in the craton. Chemical analyses were made under the direction of $\mathrm{M}$. Gonshor, and later of W.L. Campbell and K.J. Curry. Petrographic work was first directed by D.H. Johnson, followed by R.W. Luce, F.C.W. Dodge, and J.J. Matzko.

As regards geologic mapping, regional compilation of the geology of the shield, using the 1:100,000-scale geologic maps, was begun in 1977 on a scale of $1: 250,000$. These smaller scale maps were used in the preparation of this report as they became available. Jacques Delfour, Robert Dhellemmes, Yves Gros, John Kemp, J. Letalenet, Claude Pellaton, and Jean-Pierre Prian of the Bureau des Recherches Geologiques et 
Minieres prepared 1:250,000-scale maps in the central and northern part of the shield. H.R. Blank, G.M. Fairer, M.E. Gettings, W.R. Greenwood, R.O. Jackson, W.C. Prinz, and F.S. Simons of the U.S. Geological Survey prepared similar maps of the southern part of the shield. And finally, larger areas at scales of 1:500,000 and 1:1,000,000 were compiled first in 1983 by J.Y. Calvez, C. Aloac, J. Delfour, J. Kemp, and C. Pellaton of the French Bureau for most of the northern part of the shield (Calvez and others, 1983), followed by plutonic rock compilations of the southern part of the shield below lat $20^{\circ} \mathrm{N}$. by D.B. Stoeser of the U.S. Geological Survey at the larger scale (Stoeser, 1985). Stoeser, together with J. Elliott, also prepared a similar maps of the northeastern corner of the shield north of lat $25^{\circ} \mathrm{N}$. (Stoeser and Elliott, 1985).

For this report, Salman Bloch assisted with petrographic work and geologic compilation from March 1975 until April 1977, continuing petrographic work begun earlier by the late Dr. Carl Brodel, R.O. Jackson, G.F. Brown, and R.G. Coleman. Subsequently, Richard Hoeksema recalculated the geochronological data to new constants published by the International Subcommission on Geochronology (Steiger and Jager, 1977) and plotted the histograms. Thanks are due W.C. Overstreet and M.E. Gettings for their technical review and constructive suggestions. Elizabeth J. Tinsley typed and assembled the manuscript, and Audrey G. Schmidt prepared many of the illustrations. Final geologic map preparation was accomplished by Neal Maxfield, Jewel A. Dickson, Jerry M. Russell, and Will R. Stettner.

And finally, it is impossible to give sufficient credit to Wenonah Bergquist, who edited the report and supervised preparation of the illustrations at an early stage. Her sustained counsel and encouragement made completion possible, as did family support during the long years of fieldwork and separation, especially support of Laura Cameron Brown, late wife of the senior author.

\section{GEOGRAPHY}

\section{Climate}

Saudi Arabia lies in the center of the great tradewind desert that extends across northern Africa into Asia. In Saudi Arabia, important modifications of the hot, dry, desert climate are due to the effect of the Red Sea and the flanking mountain ranges of Ash Shifā', Hejaz, and 'Asīr.

Rainfall is meager and episodic, ranging from a minimum mean annual of less than $2 \mathrm{~cm}$ in the north to a maximum of about $30 \mathrm{~cm}$ along the southern crest of the 'Asir range in the southwest. Most of the region probably has an average annual rainfall of $10 \mathrm{~cm}$ (see fig. 2), but systematic records have been kept only in recent years. At Mahd adh Dhahab, in west-central Saudi Arabia, a 14-yr record shows a range from a minimum of $1.2 \mathrm{~cm}$ to a maximum of $13.1 \mathrm{~cm}$, with an average of $6.2 \mathrm{~cm}$. At Jiddah, on the Red Sea coast, where the average is about $8 \mathrm{~cm}$, infrequent rains during the last $15 \mathrm{yr}$ have been as much as $15 \mathrm{~cm}$, falling within a few hours, although there are some years when no rain falls. Indeed, parts of the vast interior region, particularly north of $\mathrm{Al}$ Madinnah and east of the 'Asīr, have had no rain for periods of several years, according to bedouin accounts and as attested by the sparseness of the vegetation. The northern regions, within the belt of westerly planetary winds, have winter precipitation. In the southern region, the monsoon season of late summer is marked by moistureladen winds which, blowing from the southwest against the western rim of the high plateau of Yemen and Hejaz ('Asīr), are forced upward to furnish the greatest precipitation along the crest. Records kept at Şan'â' in the Yemen, east of the crest, show an average of 30.2 $\mathrm{cm}$ (minimum $15.4 \mathrm{~cm}$, maximum $49.5 \mathrm{~cm}$ ), with the heaviest precipitation in July and August and a secondary peak in April. At Ar Riyād, in central Saudi Arabia, a 7-yr record shows an average of $10 \mathrm{~cm}$ (minimum 2.8 $\mathrm{cm}$, maximum $23.8 \mathrm{~cm}$ ), almost all precipitation falling in the winter and spring months.

Even if rainfall had been recorded for many years, the episodic and local nature of most precipitation makes averages of doubtful value. A better guide has been provided by the late Dr. John Tothill's study of the vegetation along the Red Sea coast and in the 'Asir. Drawing on his knowledge of the vegetation in the Sudan, where rainfall records have been kept, he correlated precipitation with various index genera and floral groups common to the Sudan and western Arabia. Figure 2 is based mainly on his work; also included in figure 2 is a record compiled by Ali H. Al-Shalash, University of Riyadh, from 60 stations for a period of 3 to $14 \mathrm{yr}$ (Ministry of Agriculture and Water, written commun., 1974).

Temperatures range from below freezing during the winter months in the mountains and high plateaus to summer maxima of about $120^{\circ} \mathrm{F}\left(44.5^{\circ} \mathrm{C}\right)$. Temperatures have large diurnal ranges (table 1)-sometimes as much as $60^{\circ} \mathrm{F}\left(16^{\circ} \mathrm{C}\right)$-caused by the rapid transmittal of heat from the bare rock surfaces as the desert passes from day to night.

Relative humidity during the day is low away from the seacoast-in the summer about 10 percent and in the winter 45 percent, according to the fragmentary records. The large diurnal range of temperature often produces an early morning dew when the rock surfaces 


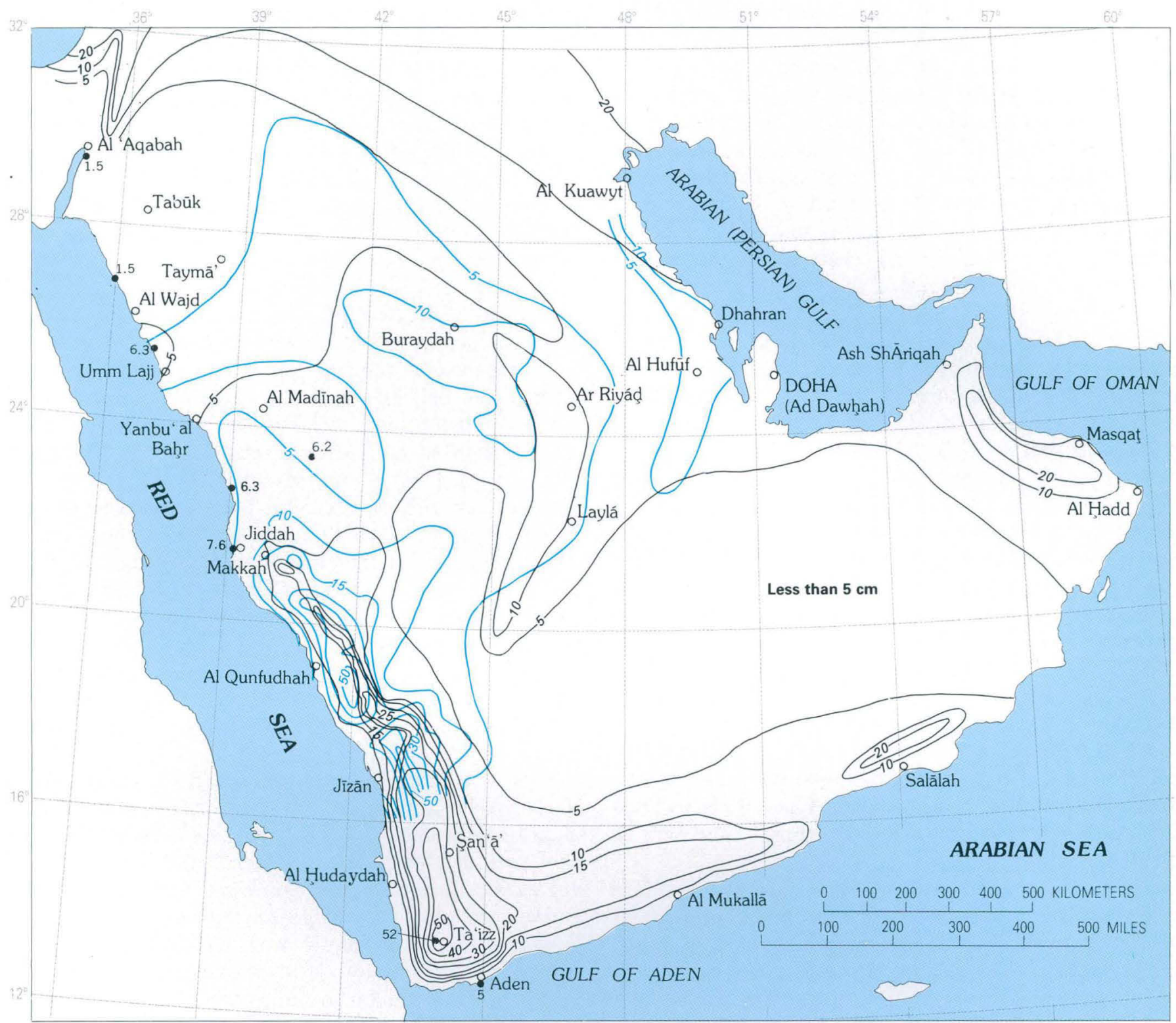

\section{EXPLANATION}

Annual Rainfall

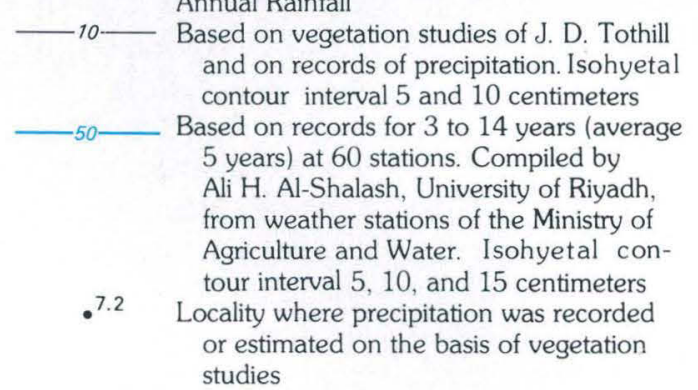

Figure 2.-Estimated rainfall distribution in the Arabian Peninsula. 
TABLE 1.-Air temperatures and relative humidities at selected sites in western Saudi Arabia

[Air temperatures in degrees Celsius, relative humidities in percent. After Saudi Arabian Ministry of Agriculture and Water]

\begin{tabular}{|c|c|c|c|c|c|c|c|c|c|c|c|c|c|c|c|c|}
\hline \multirow{3}{*}{ Month } & \multicolumn{4}{|c|}{$\begin{array}{c}\text { Abha"' } \\
\text { Alt. } 2,200 \mathrm{~m} \text {; lat } 18^{\circ} 13^{\prime} \mathrm{N} \text {., long } 42^{\circ} 29^{\prime} \mathrm{E} .\end{array}$} & \multicolumn{4}{|c|}{$\begin{array}{c}\text { Al Mindak } \\
\text { Alt. } 1,920 \mathrm{~m} \text {; lat } 20^{\circ} 06^{\prime} \mathrm{N} \text {., long } 41^{\circ} 17^{\prime} \mathrm{E} \text {. }\end{array}$} & \multicolumn{4}{|c|}{$\begin{array}{l}\text { Belesmer }{ }^{2} \\
\text { Alt. } 2,250 \mathrm{~m} \text {; lat } 18^{\circ} 46^{\prime} \mathrm{N} \text {., long } 42^{\circ} 13^{\prime} \mathrm{E} .\end{array}$} & \multicolumn{4}{|c|}{$\begin{array}{c}\text { Bitjursh } \vec{r}^{2} \\
\text { Alt. } 2,040 \mathrm{~m} \text {; lat } 19^{\circ} 51^{\prime} \quad \mathrm{N} \text {., long } 41^{\circ} 34^{\prime} \mathrm{E} \text {. }\end{array}$} \\
\hline & \multicolumn{3}{|c|}{ Air temperature } & \multirow{2}{*}{$\begin{array}{l}\text { Mean rel. } \\
\text { hum. }\end{array}$} & \multicolumn{3}{|c|}{ Air temperature } & \multirow{2}{*}{$\begin{array}{l}\text { Mean rel. } \\
\text { hum. }\end{array}$} & \multicolumn{3}{|c|}{ Air temperature } & \multirow{2}{*}{$\begin{array}{l}\text { Mean rel. } \\
\text { hum. }\end{array}$} & \multicolumn{3}{|c|}{ Air temperature } & \multirow{2}{*}{$\begin{array}{l}\text { Mean rel. } \\
\text { hum. }\end{array}$} \\
\hline & Max. & Min. & Avg. & & Max. & Min. & $\overline{\text { Avg. }}$ & & Max. & Min. & Avg. & & Max. & Min. & $\overline{\text { Avg. }}$ & \\
\hline Jan & 22.2 & 1.2 & 11.2 & 68 & 20.2 & -1.2 & 10.9 & 61 & 23.1 & -2.2 & 8.7 & 60 & 21.8 & 2.2 & 12.1 & 50 \\
\hline $\mathrm{Feb}$ & 20.0 & 4.8 & 13.3 & 67 & 22.0 & -5.8 & 11.6 & 63 & 22.8 & -2.0 & 10.5 & 59 & 22.6 & -2.0 & 12.7 & 55 \\
\hline Mar- & 23.0 & 2.8 & 14.8 & 64 & 23.6 & 4.0 & 14.8 & 64 & 21.4 & 0.5 & 11.7 & 66 & 24.0 & 3.8 & 15.8 & 54 \\
\hline $\mathrm{Apr}-$ & 25.8 & 9.2 & 17.8 & 56 & 27.0 & 6.4 & 17.1 & 63 & 22.6 & 4.8 & 13.1 & 66 & 26.0 & $\begin{array}{l}0.0 \\
9.0\end{array}$ & 17.2 & 54 \\
\hline May & 29.6 & 8.8 & 20.3 & 40 & 32.2 & 6.8 & 19.5 & 54 & 27.6 & 5.0 & 14.6 & 64 & 32.1 & 10.0 & 20.0 & 40 \\
\hline Jun- & 31.0 & 13.6 & 22.4 & 34 & 31.2 & 6.5 & 20.9 & 51 & 26.0 & 4.2 & 17.1 & 43 & 31.2 & 10.4 & 23.2 & 35 \\
\hline Jul- & 29.4 & 11.6 & 21.3 & 42 & 33.0 & 11.0 & 22.5 & 54 & 28.2 & 8.1 & 19.0 & 43 & 32.8 & 11.2 & 23.6 & 41 \\
\hline Aug - & 29.4 & 14.0 & 21.7 & 40 & 32.2 & 11.2 & 23.2 & 56 & 27.8 & 9.8 & 19.0 & 51 & 32.6 & 14.6 & 24.1 & 43 \\
\hline Sep- & 29.0 & 10.0 & 19.4 & 40 & 32.0 & 10.2 & 21.7 & 50 & 27.3 & 3.6 & 16.8 & 46 & 31.9 & 11.7 & 22.4 & 43 \\
\hline Oet- & 25.4 & 7.0 & 16.6 & 39 & 28.2 & 5.7 & 18.5 & 50 & 23.0 & 0.4 & 12.6 & 47 & 27.8 & 8.4 & 18.7 & 42 \\
\hline Nov- & 23.0 & 5.0 & 14.0 & 46 & 26.6 & 4.0 & 14.5 & 66 & 22.0 & -0.8 & 10.5 & 71 & 24.0 & 6.4 & 14.7 & 58 \\
\hline Dec & 18.8 & 2.3 & 11.6 & 53 & 22.8 & -1.8 & 11.1 & 62 & 19.8 & -3.0 & 8.0 & $\left({ }^{3}\right)$ & 22.6 & 1.0 & 11.7 & 58 \\
\hline \multirow[t]{2}{*}{ Ann.- } & 31.0 & 1.2 & 17.0 & 49 & 33.0 & -5.8 & 17.2 & 58 & 28.2 & -3.0 & 13.5 & $\left({ }^{3}\right)$ & 32.8 & -2.0 & 18.0 & 48 \\
\hline & \multicolumn{4}{|c|}{$\begin{array}{c}\text { Qal'at Bishah }{ }^{2} \\
\text { Alt. 1,040 m; lat } 20^{\circ} 00^{\prime} \text { N., long } 42^{\circ} 36^{\prime} \mathrm{E} .\end{array}$} & \multicolumn{4}{|c|}{$\begin{array}{c}H_{\tilde{a}^{\prime} \mathrm{i}^{2}} \\
\text { Alt. } 1,010 \mathrm{~m} ; \text { lat } 27^{\circ} 28^{\prime} \mathrm{N} \text {., long } 41^{\circ} 38^{\prime} \mathrm{E} .\end{array}$} & \multicolumn{4}{|c|}{$\begin{array}{c}\mathrm{Jiddah}^{4} \\
\text { Alt. } 17 \mathrm{~m} \text {; lat } 21^{\circ} 30^{\prime} \mathrm{N} \text {., long } 39^{\circ} 12^{\prime} \mathrm{E} \text {. }\end{array}$} & Alt. 648 & $\begin{array}{l}\text { Al Madi } \\
\text { t } 24^{\circ} 31^{\prime}\end{array}$ & ong $39^{\circ}$ & $42^{\prime}$ E. \\
\hline Month & & erature & & & & gerature & & & & gerature & & & & perature & & Mean rel. \\
\hline & Max. & Min. & $\overline{\text { Avg. }}$ & hum. & Max. & Min. & Avg. & hum. & Max. & Min. & Avg. & hum. & Max. & Min. & $\overline{\text { Avg. }}$ & hum. \\
\hline Jan & 30.2 & 3.8 & 16.9 & 45 & 23.6 & -3.0 & 10.1 & 54 & 26.6 & 19.4 & 23.0 & 55.5 & 23.9 & 13.3 & 18.6 & 46 \\
\hline Feb & 34.0 & 5.0 & 19.9 & 43 & 27.0 & -4.4 & 12.0 & 45 & 26.6 & 18.9 & 22.8 & 52.5 & 25.0 & 13.3 & 19.1 & 42 \\
\hline Mar- & 35.0 & 9.0 & 22.4 & 36 & 30.4 & 1.6 & 17.2 & 31 & 28.9 & 20.5 & 24.7 & 52 & 28.9 & 16.1 & 22.5 & 32 \\
\hline $\mathrm{Apr}$ & 36.4 & 13.0 & 24.8 & 41 & 33.1 & 7.0 & 19.3 & 30 & 31.0 & 22.8 & 26.9 & 54.5 & 35.5 & 23.3 & 29.4 & 29 \\
\hline May - & $\begin{array}{l}0.4 \\
39.1\end{array}$ & $\begin{array}{l}10.0 \\
16.8\end{array}$ & $\begin{array}{l}24.0 \\
27.9\end{array}$ & $\begin{array}{l}41 \\
40\end{array}$ & $\begin{array}{l}38.1 \\
38.8\end{array}$ & 12.4 & $\begin{array}{l}15.0 \\
25.2\end{array}$ & 23 & $\begin{array}{l}32.7 \\
32.7\end{array}$ & 24.4 & 28.6 & 54 & 37.7 & 24.4 & 31.1 & 37 \\
\hline Jun-- & 40.0 & 14.8 & 28.0 & 27 & 38.0 & 15.0 & 26.6 & 15 & 34.4 & $\begin{array}{l}24.4 \\
25.5\end{array}$ & $\begin{array}{l}20.0 \\
30.0\end{array}$ & 56 & 40.5 & $\begin{array}{l}24.4 \\
27.8\end{array}$ & $\begin{array}{l}01.1 \\
34.2\end{array}$ & 16 \\
\hline $\mathrm{Jul}$ & 41.0 & 18.0 & 30.3 & 26 & 41.0 & 18.0 & 30.1 & 13 & 34.4 & 27.2 & 30.8 & 53.5 & 37.7 & 26.1 & 31.9 & 26 \\
\hline Aug & 41.1 & 19.4 & 30.8 & 30 & 41.0 & 19.0 & 30.1 & 15 & 35.0 & 27.8 & 31.4 & 55.5 & 40.0 & 27.8 & 33.9 & 22 \\
\hline Sep- & 39.0 & 10.0 & 26.4 & 28 & 40.2 & 13.2 & 27.7 & 13 & 33.9 & 26.6 & 30.3 & 63.5 & 40.5 & 26.1 & $\begin{array}{l}33.3 \\
33.3\end{array}$ & 25 \\
\hline Oct & 32.6 & 6.6 & 20.0 & 23 & 34.2 & 8.0 & 21.6 & 16 & 32.7 & 24.4 & 28.6 & 61.5 & 36.1 & 23.3 & 29.7 & 26 \\
\hline Nov & 33.6 & 5.1 & 20.9 & 37 & 31.0 & 2.2 & 16.4 & 32 & 30.5 & 23.3 & 26.9 & 57 & 28.3 & 27.8 & 28.1 & 43 \\
\hline Dec - & 31.0 & 2.0 & 16.7 & 44 & 27.2 & -5.0 & 9.8 & 45 & 28.3 & 21.1 & 24.7 & 54 & 25.5 & 13.3 & 19.4 & 47 \\
\hline Ann.- & 41.1 & 2.0 & 23.8 & 35 & 41.0 & -5.0 & 20.5 & 28 & 31.1 & 23.3 & 27.2 & 55.5 & 33.3 & 21.1 & 27.2 & 33 \\
\hline & Alt. 2,600 & $\begin{array}{l}\text { An } \mathrm{Nir} \\
\text { at } 19^{\circ} 06\end{array}$ & long 42 & $2^{\circ} 09^{\prime}$ E. & Alt. 600 & $\begin{array}{l}\text { As Sul } \\
\text { it } 20^{\circ} 28\end{array}$ & long 45 & ${ }^{\circ} 34^{\prime}$ E. & Alt. 1,53 & $\begin{array}{l}\text { Ta' if }(\mathrm{f} \\
\text { at } 21^{\circ} 2\end{array}$ & $\begin{array}{l}\text { h) } \\
\text { long } 4 \mathrm{C}\end{array}$ & $0^{\circ} 27^{\prime} \mathrm{E}$. & Alt. 850 & $\begin{array}{r}\text { Tura } \\
\text { at } 31^{\circ} 41^{\prime}\end{array}$ & long 38 & ${ }^{\circ} 40^{\prime}$ E. \\
\hline Month & & serature & & & & perature & & & & perature & & Mean rel. & & perature & & Mean rel. \\
\hline & Max. & Min. & $\overline{\text { Avg. }}$ & hum. & Max. & Min. & $\overline{\text { Avg. }}$ & hum. & Max. & Min. & $\overline{\text { Avg. }}$ & hum. & Max. & Min. & $\overline{\text { Avg. }}$ & hum. \\
\hline Jan & 12.6 & -5.6 & 3.3 & 36 & 27.6 & 2.3 & 13.9 & 47 & 26.6 & 3.2 & 14.2 & 36 & 17.2 & 4.4 & 10.8 & 56 \\
\hline $\mathrm{Feb}$ & 17.0 & -4.4 & 8.6 & 40 & 35.6 & 2.0 & 17.5 & $\begin{array}{l}39 \\
39\end{array}$ & 27.8 & 1.8 & 16.6 & 37 & 15.5 & 3.3 & 9.4 & 49 \\
\hline Mar & 20.2 & 1.8 & 10.8 & 41 & 37.2 & 5.8 & 21.7 & 27 & 28.6 & 9.8 & 19.5 & 42 & 20.0 & 6.7 & $\begin{array}{r}13.4 \\
13.4\end{array}$ & 48 \\
\hline $\mathrm{Apr}$ & 24.0 & 4.0 & 13.2 & $\begin{array}{l}58 \\
58\end{array}$ & $\begin{array}{l}39.2 \\
\end{array}$ & 14.0 & 26.5 & 26 & 30.4 & $\begin{array}{r}10.0 \\
10.0\end{array}$ & 20.2 & 34 & 26.6 & 19.4 & 23.0 & 26 \\
\hline May & 27.8 & 7.2 & 16.4 & 51 & 43.7 & 18.4 & 31.7 & 18 & 36.0 & 8.0 & 22.9 & 29 & 32.7 & 15.4 & 24.1 & 17 \\
\hline Jun- & 27.0 & 6.8 & 18.1 & 40 & 45.0 & 18.4 & 32.5 & 11 & 35.2 & $\begin{array}{r}0.0 \\
14.8\end{array}$ & 26.1 & 15 & 37.7 & 20.0 & 28.9 & 21 \\
\hline Jul & 29.0 & 10.2 & 19.0 & 43 & 45.0 & 22.6 & 34.8 & 10 & 35.8 & 16.8 & 27.2 & 18 & 35.5 & 20.0 & 27.8 & 19 \\
\hline Aug & 28.0 & 12.0 & 19.0 & 41 & 45.0 & 21.1 & 34.5 & 9 & 35.8 & 18.8 & 27.3 & 20 & 37.7 & 20.5 & 29.1 & 18 \\
\hline Sep- & 28.0 & 6.0 & 18.0 & 43 & 44.0 & 14.1 & 30.3 & 10 & 35.2 & $\begin{array}{l}13.0 \\
13.0\end{array}$ & 25.0 & 18 & 32.7 & 16.7 & 24.7 & 16 \\
\hline Oct- & 23.8 & 3.0 & 13.8 & 44 & $\begin{array}{l}37.0 \\
37.0\end{array}$ & 10.0 & 24.0 & 13 & 30.8 & 10.2 & 20.8 & 24 & 28.9 & 14.4 & 21.7 & 26 \\
\hline Nov- & 20.0 & $\begin{array}{l}0.0 \\
1.4\end{array}$ & 10.6 & $\begin{array}{l}44 \\
65\end{array}$ & 35.0 & 6.1 & $\begin{array}{l}2.0 \\
19.5\end{array}$ & $\begin{array}{l}10 \\
26\end{array}$ & $\begin{array}{l}58.0 \\
28.6\end{array}$ & 8.2 & $\begin{array}{l}2.0 \\
18.0\end{array}$ & $\begin{array}{l}24 \\
36\end{array}$ & 18.9 & 9.4 & 14.2 & 41 \\
\hline Dec- & $\begin{array}{l}20.0 \\
19.7\end{array}$ & $\begin{array}{l}1.4 \\
-0.2\end{array}$ & 8.0 & 70 & 29.9 & $\begin{array}{l}0.1 \\
3.6\end{array}$ & 16.6 & $\begin{array}{l}20 \\
35\end{array}$ & $\begin{array}{l}20.0 \\
27.8\end{array}$ & $\begin{array}{r}0.2 \\
-1.4\end{array}$ & $\begin{array}{l}10.0 \\
13.6\end{array}$ & $\begin{array}{l}50 \\
36\end{array}$ & 17.2 & $\begin{array}{l}5.4 \\
4.4\end{array}$ & $\begin{array}{l}10.2 \\
10.8\end{array}$ & $\begin{array}{l}41 \\
53\end{array}$ \\
\hline Ann. & 29.0 & -5.6 & 13.2 & 48 & 45.0 & 2.0 & 27.6 & 25 & 36.0 & -1.4 & 21.0 & 29 & 26.6 & 12.2 & 19.4 & 33 \\
\hline
\end{tabular}


and nearby air have cooled below the dewpoint; however, the low humidity and the first rays of the morning sun quickly evaporate it. The high humidity and heat along the Red Sea cause much discomfort and lethargy, especially as the dampening effect of the sea often holds the air temperature at a high level throughout the night.

Winds range widely in direction and velocity. A prominent northwesterly, the "shimal," blows strongly in spring and early summer in the central and eastern parts of the country. A southwestern wind direction in northwestern Arabia has produced prominent yardang troughs north of lat $24^{\circ} \mathrm{N}$. Sand storms and dust storms are common, and eolian landforms and deposits are conspicuous in the younger terranes (see fig. 68).

\section{SETTLEMENT $^{2}$}

Long the home of wandering bedouin who traditionally follow the rains for forage, Saudi Arabia is changing rapidly as the few larger populated places grow at the expense of nomadic groups and smaller agricultural communities. Makkah, the religious center of the Moslem world, now (1980) has a permanent population of at least 200,000 , and its population increases manyfold during the annual pilgrimage, as does that of Jiddah, the port for Makkah, which has a permanent population of more than 1,300,000 (1980). Al Madinah, the second holy city, had a permanent population of perhaps 40,000 before current expansion but has grown substantially in recent years; it also experiences a very large influx during the annual pilgrimage. At Ta' 'if, the summer capital near the crest of the Hejaz Range east of Makkah, is comparable in size to Al Madinah and is likely to grow at an increasing rate, provided there is sufficient water. The only large inland city is Riyadh, the political capital, which has a population of about 1,000,000 (1980). An important industrial complex is being built near Yanbu' al Bahr at the terminus of the east-west double pipeline for oil and gas from Al Ḩasā.

A series of small agricultural communities extends southward from Aț Tā'if along the mountain crest through the southern Hejaz and the 'Asīr into Yemen. They include the districts of Bilād Ghāmid, 'Asīr, As Sarāt, and Zahran, settlements increasing in size and number southward. Starting from about lat $20^{\circ} \mathrm{N}$., a similar series of villages and cultivated tracts extends toward the south at the seaward base of the range, culminating in several large villages in the Jizan coastal

${ }^{2}$ Demography as of 1980 . plain just north of the Yemen frontier. The topographically higher communities practice terrace cultivation, whereas the Red Sea coastal plain (the Tihāmat) valleys and the inland valleys are cultivated by flood diversion, supplemented in the Jizān area by a modified terrace or basin type of irrigation where there is sufficient rainfall. A new dam on Wādī Jīzān supplies irrigation water to the area around Abū 'Arīsh.

About 90 percent of all cultivated land and most of the predominantly agrarian communities in Saudi Arabia lie within two belts in the southern Hejaz, 'Asīr and the southern Tihāmat; the seaports of Al Lith, Al Qunfudhah, and Jīzān serve as outlets for the area. Going eastward toward the desert, the villages are replaced by seminomadic and nomadic groups.

Small ports and fishing villages are situated along the Red Sea north of Jiddah at Rābigh, Yanbu' al Baḩr, Umm Lajj, Al Wajh, and Dubā, in general decreasing in size and importance northward. The small village of 'Aynūnah is situated at the northeastern corner of the Red Sea, and Maqnā and Haql are on the eastern shore of the Gulf of Aqaba. A string of settlements are in Wādī Fātimah east of Jiddah; several other small communities lie in the larger wadi valleys north of Jiddah. These extend as far north as Yanbu' an Nakhl, directly west of $\mathrm{Al}$ Madinah, beyond which there are almost no permanent settlements except on the coast; the most important of the inland villages near the coast are Madrakah, Khulayş, Buraykah, Haqqāq, Ar Rayyān, Badr Hुunayn, Al Ḩamrā', and Al Musayj'īd. In the upland of the Hुismā, the only settlements of any size are Taymā' and Tabūk, although Khaybar and $\mathrm{Al}$ 'Ulā are on the edge of the upland. Al Bad' in Wādì 'Ifāl, which forms a valley in the Shifā' east of the Gulf of Aqaba in northwesternmost Saudi Arabia, has a small settled community.

Inland on the crystalline desert plateau are palm oases and farms at Qal'at Bīshah, Turabah, Khurmah, and Ranyah, all separated by wide expanses of stony desert. Farther north, in the northern Hejaz, the populated places are smaller and more widely spaced. Of these, Al Muwayhs, Ad Dafĩnah, 'Afíf, Al Qā'ìyah, and Ad Dawādimī lie along the At Tā'if-to-Riyadh road; Hुādhah, Şufaynah, Al Ḩanākīyah, Al Khalf, Al Hुuwayyiţ, Al Hुāyiţ, and Zarghaţ are small villages along the eastern edge of the lava fields (where water is available) that extend from near At Tā'if northward to lat $27^{\circ} \mathrm{N}$. The ancient community of Khaybar occupies a similar position on the western edge of the lava fields north of Al Madinah. East of Qal'at Bīshah, beyond the four small villages that extend northward up the wadi for $40 \mathrm{~km}$, there are no permanently inhabited settlements until the constriction of Wādi ad Dawāsir at the east edge of the crystalline plain, some 
$200 \mathrm{~km}$ distant. Northeastward from Bīshah no villages are found until Şabhâ', some $400 \mathrm{~km}$ away, is reached. To the north beyond Şabhā', several villages-Sanām, Ar Ruwaydah, and 'Arwā'-lie at wadi constrictions, where there are slight local increases in ground-water supply.

Hुă'il, the capital of the Jabal Shammar province, has a population of about 5,000 and is located where dikes and a wadi constriction impound some ground water beneath a grus plain. Toward Ḩẩ'il, in the northeastern corner of the crystalline shield, the most important villages are Darīyah, Miskah, An Nabhānīyah, Al Fawwārah, Samīrah, Tābah, Fayd, Al 'Uqaylāt, Ghazzālah, Mawqaq, and Qufār.

\section{FLORA}

\section{RELATION TO GEOGRAPHIC SETTING AND HUMAN USE}

Acacia, or camel thorn, is the most widespread, and in many places almost the only, shrub and small tree of the western desert. The acacia "samr" (Acacia tortilis (Forsk) Hayne), perhaps the hardiest, may be found growing along the wadis or, where rainfall is slightly higher, on the desert plains as isolated flat-topped trees or copses. The acacia "sallam" (Acacia ehrenbergiana Hayne) is also widespread, growing in clumps or thickets along wadi floors, as it requires more water than the samr. In the high mountains the acacia locally called "talh" (Acacia seyal?) probably includes more than one species; it grows into trees larger than samr or sallam. There are several other species, but none of the acacias is a true phreatophyte, indicating land without a permanent water table; most vegetation away from the mountains and seacoast is mesophytic, growing only when rain falls (Vesey-FitzGerald, 1957).

The most important of the Arabian phreatophytes are the date palm "nakhla" (Phoenix dactylifera L.), the "sidr" (Zisyphus spina-christi L. Willd.; fig. $3 A$ ), with its lacy foliage and haw-shaped fruit rich in vitamins, the two tamarisks "tarfa" and "ithil" (Tamarix orientalis Forsk and Tamarix macrocarpa (Ehrenb.) Bunge; fig. $3 B$ ), the "dom" palm (Hyphaene thebaica (Del.) Mart.), and the "rak" (Salvadora persica L.), or "toothbrush shrub," which forms rings of dense vegetation, often growing on small dunes and maintaining a root system below the sand. The halfa grass (Desmostachya cynosuroides Stapf) is common in the upper stretches of wadis in the southern part of the country.

A distinct flora grows where salty or saline ground water is present, especially along the Red Sea coast, dominant plants being the succulent salt bush Suaeda (represented by two or three species) and the sea lavender (Statice axillaris); the mangrove-reef-fringing copses (Rhizophora sp., Bruguiera sp., or Avicennia marina) are prominent only south of Al Qunfudhah at lat $18^{\circ} 30^{\prime} \mathrm{N}$. (Vesey-FitzGerald, 1955).

In vast stretches of the western desert, where many of the acacias have been burned for charcoal, vegetation is limited to forage shrubs and grass which spring into foliage and flower after rains. The hamdh vegetation is largely Salsola tetrandra Forsk, which grows in saline conditions, but there are also at least five other shrubs. The rimth vegetation is largely Haloxylon schweinfurthii Ascherson, which is favored for camel grazing. Another widely scattered bush is the harmal (Rhazya stricta), or African rue (Peganum harmala). The grasses Panicum turgidum Forsk and Lasiurus hirsutus (Boiss) Monro are widespread, growing in clumps that furnish forage for camels, sheep, and goats and are often gathered for hay.

Many unusual plants and trees grow in the Hejaz and the "Asīr. Of these, the juniper "ar'ar" (Juniperus macropoda Boiss. or Juniperus procera Hochst.) (Mandaville, 1973) is the only important conifer in Arabia. It grows in small forests or groves along the crest of the 'Asīr where rainfall is 12 in $(30.5 \mathrm{~cm})$ or slightly higher. The largest indigenous tree in Saudi Arabia, Acokanthera deflersii Schwein, grows only in the upper foothills east of Jizān (fig. $3 C$ ). The wild fig (Ficus salici folia Vahl), the tamarind (Tamarindus indica L.; fig $3 D$ ), the poinciana (Delonix elata L. Gamble), the dragon's blood tree (Dracaena ombet Kotschy and Peyr), the sandalwood (Osyris compressa Berg.), the aloes, the 'ishr (Coloptropis sp.), with its milky, rubber-producing sap, and the Adenium sp., or poison bush, with its beautiful pink or red flowers, are of special interest. The cactuslike Euphorbia is represented by several species, there being no indigenous cacti on the peninsula, although the prickly pear, Opuntia sp., has been imported for its fruit.

Food crops in Saudi Arabia are grown mostly in the 'Asir where the mountain slopes are terraced to hold the rain, along wadis draining the 'Asīr and the Hejaz, and in scattered isolated oases where ground water sustains the date palm, grains, fruit trees, vegetables, and hay. The most important food crops by far are dates, wheat and barley, the nonsaccharine sorgums (dhura and dukhn), corn, and alfalfa. Fruits besides dates include olive, fig, orange, lemon, lime, pomegranate, grape, cactus apple, apricot, banana, and guava. The vegetables currently cultivated include beans, squash, eggplant, okra, tomato, lettuce, cabbage, onion, carrot, pepper, and potato. Three varieties of truffle grow wild in the desert following fall and winter rains, and several varieties of melons are hauled to the markets in season. 


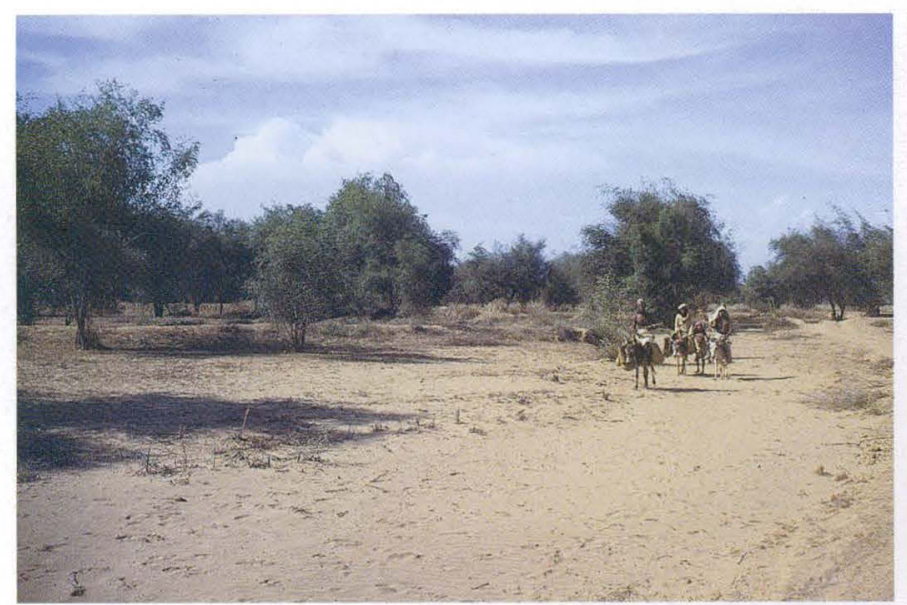

A

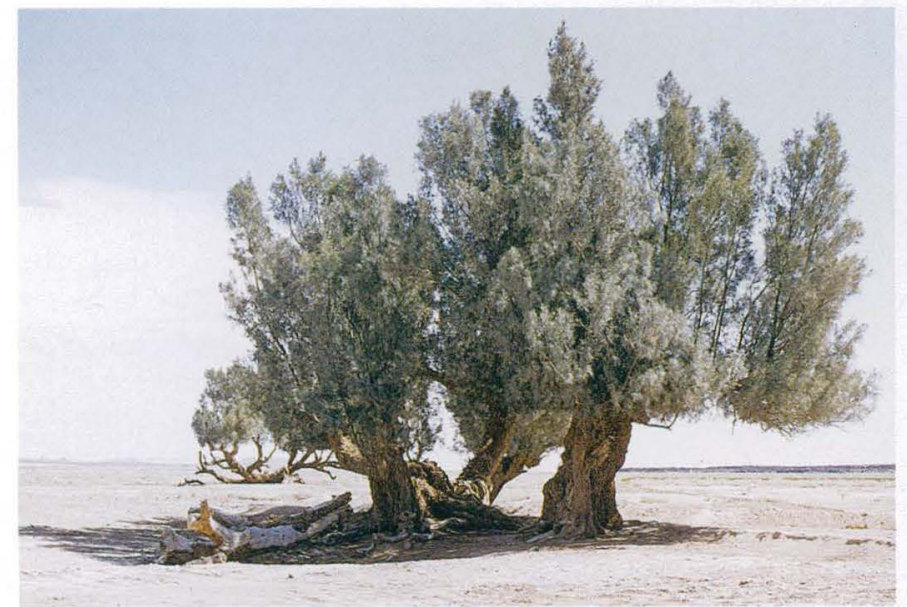

B

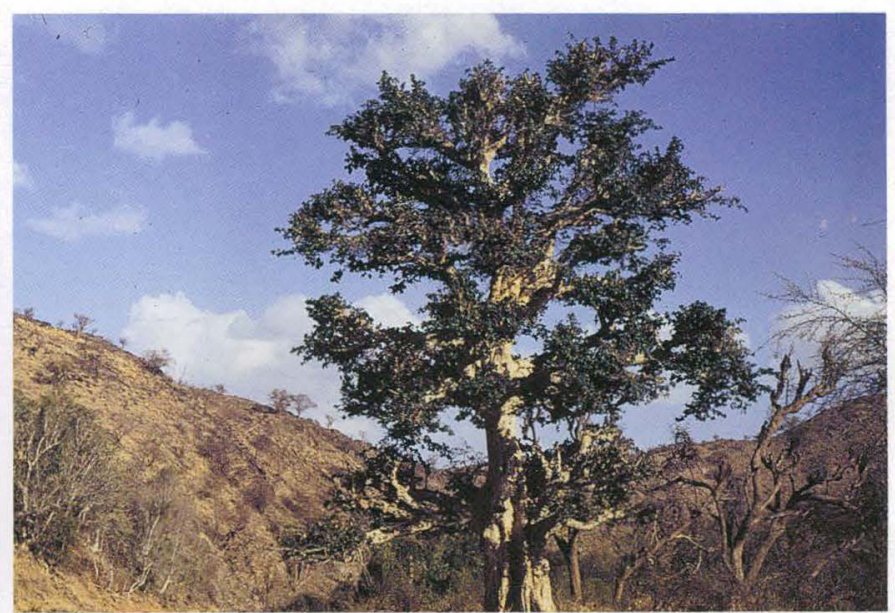

C

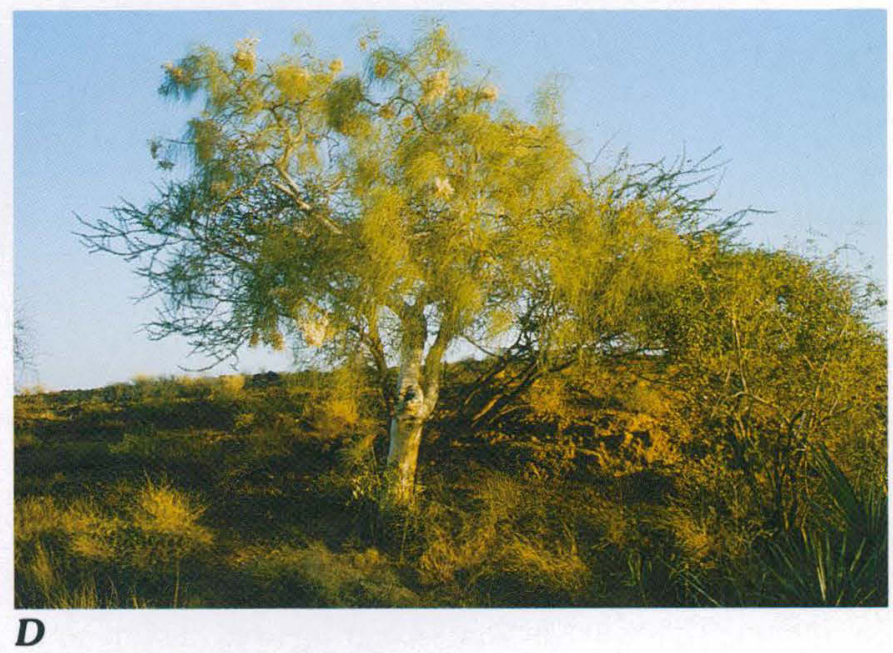

Figure 3.-Examples of a few phreatophytes, indicating permanent ground water. A, Zisyphus spina-christi L. Willd. B, Tamarix macrocarpa (Ehrenb.) Bunge. C, Acokanthera deflersii Schwein., the largest indigenous tree in Saudi Arabia, photographed at the junction of Wādī Qa'ah (Yithrib) and Wādī Shini near Jallat al Mawt. D, Tamarindus indica L.

\section{RELATION OF FLORA TO RAINFALI}

\section{By J.D. Tothill ${ }^{3}$}

Fieldwork for a study of the floral zones of the coastal plain, or Tihāmat, between Jiddah and the Yemen frontier, and of the 'Asīr Mountains was done in 1950 and 1951 when the Food and Agriculture Organization of the United Nations was examining the possibility of supplementing the existing irrigation systems of the coastal plain in Jizzān province. From time immemorial, primitive to well-developed irrigation systems were employed on the principal rivers that rise in the 'Asīr Mountains and form desert deltas on the Tihāmat. With the increase of population and the need for more food, both for humans and for domestic

${ }^{3}$ Deceased. animals, some reinforcements by means of barrages had become necessary.

The size and number of barrages that would be required would depend on rainfall in the catchment areas, and an estimate of rainfall accurate enough to use as a basis for determining the size of the barrage projects was urgently needed. No rainfall records for the area had ever been kept, and it was not practical to wait a decade or longer for meteorological observations to be made. In these circumstances it was decided to test the possibility of defining natural floral zones and using these as the basis for estimating rainfall on the mountains. Seven zones were eventually distinguished, and the rainfall estimate made for each was used for figure 2 (see table 2).

The plan was to make three widely separated traverses down the face of the mountains, to establish as 
TABLE 2.-Summary of annual rainfall estimates for and characteristics of floral zones [Rainfall in inches]

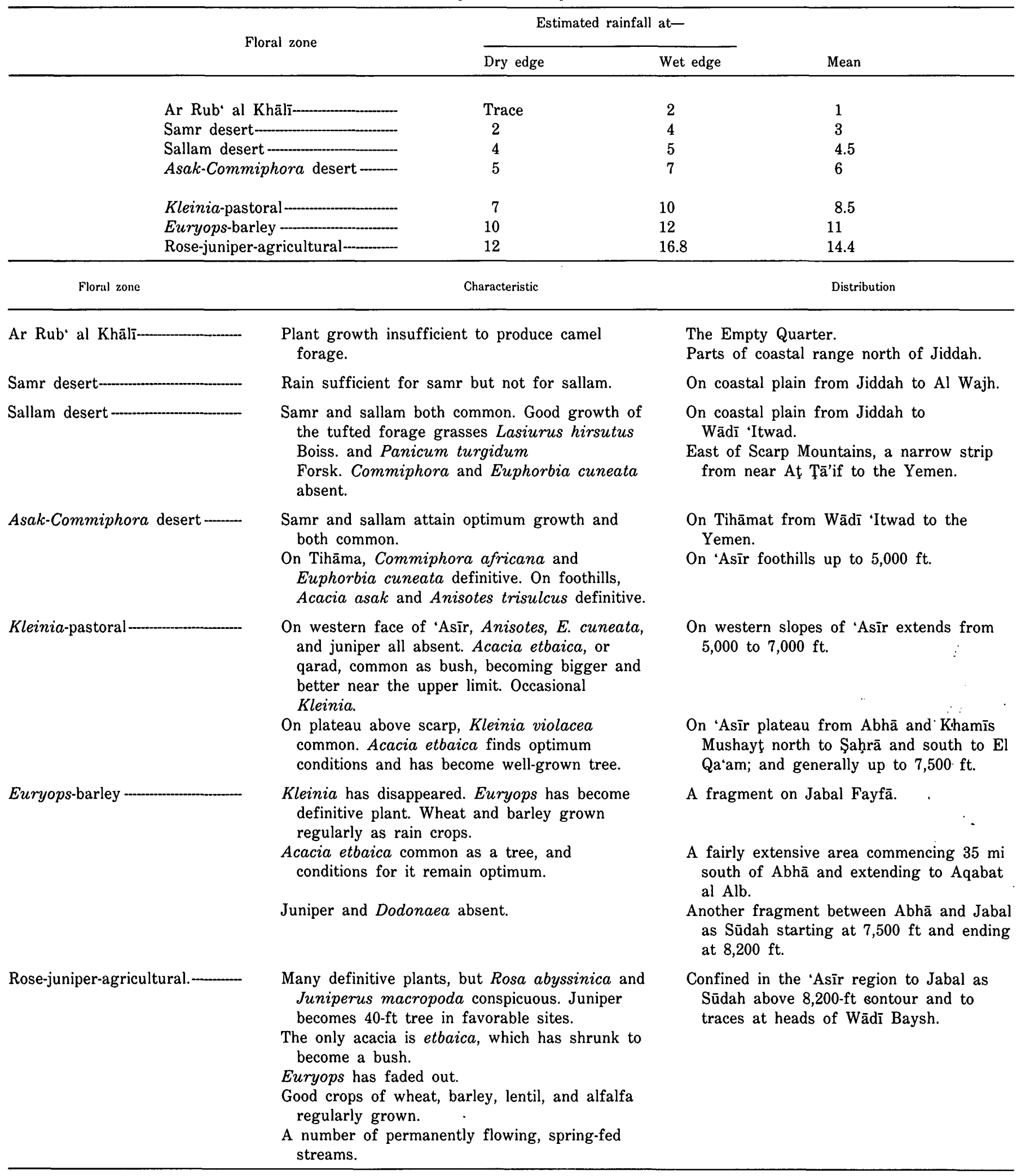


many zones as could be easily recognized by the coming in or disappearance of readily identified definitive, or index, plants, and then to determine rainfall for each zone. The area of each zone would be calculated from an aerial survey map by joining the zone contours for the three traverses.

Despite the wild ruggedness, precipitate steepness, and inaccessibility of the upper $2,000 \mathrm{ft}(610 \mathrm{~m})$ of the western face of the mountains, the plan worked quite well. Seven easily recognized zones were eventually defined; their definitions, extent in the 'Asīr area, and probable rainfall are given below. The project proved to be of considerable practical value, as its findings were used as the rainfall basis for designing the required barrages.

The traverses of the western face of the 'Asir were made by the ascent of Jabal Fayfā, Wādì Baysh, and Wādī 'Itwad.

During the fieldwork on the coastal plain, I had the stimulating companionship of Dr. Van der Plas. For the three traverses of the "Asīr, I was most happily accompanied by Thomas Smallwood", a specialist in water supplies for irrigation purposes. For the journey down Wādī Qa'ah and into the main Wādi Baysh I am deeply indebted to Dr. Glen Brown, a geologist of the U.S. Geological Survey whose friendship and stimulating ideas I came to value greatly.

The Saudi Arabians were both our hosts and our friends, and it is a pleasure to acknowledge the many acts of courtesy and kindness shown us by a long list of local residents, from the late King Abdul Aziz ibn Sa'ud and his sons, the late Kings Saud and Faisal, down through all walks of life to humble folk who helped us in our daily tasks.

Plant specimens were determined through the kindness of Dr. Taylor, then Custodian of the Herbarium and Head of the Botanical Department of the British Natural History Museum. Miss D. Hillcoat did most of the determinations, and to her I owe a special debt of gratitude.

Basic plant collections in southwest Arabia were made by Hugh Scott and E.B. Britton of the expedition sponsored by the British Natural History Museum, and in recent years D.F. Vesey-FitzGerald added important collections from both the coastal plain and the mountains. My own collection of some 170 named species supplements the collections made by Scott, Britton, and Vesey-FitzGerald and was made from the somewhat narrower point of view of discovering and delineating floral zones. In a few cases, material was insufficient or inadequate for sending to the museum and it was necessary to make provisional determinations. This

${ }^{4}$ Deceased. applies particularly to the free form of Acacia etbaica Schweinf. and to Commiphora africana (A. Rich.) Engler.

\section{THE SEVEN FLORAL ZONES}

As there are no names in general use that fit the seven zones, it seemed best to use names that could easily be learned and understood by anyone working in Arabia. The country people of Saudi Arabia are good botanists and have Arabic names for all the index plants used, and they will naturally use these names instead of the few Latin names that have seemed necessary here.

The zone-defining plants were identified as a result of making a collection of every species that was in blossom or in seed or in a recognizable condition of any kind. This was done on all the journeys, and the distribution of each plant was noted. The collection was eventually handed to the Botanical Department of the British Natural History Museum for determination, because its Herbarium is particularly rich in material from Arabia and the Middle East; this proved to be a most happy arrangement.

The rainfall estimates are based partly on the known isohyet limits of definitive plants that also happen to grow in the Sudan, where there is a long history of botanical collecting, culminating in the three fine volumes on "The Flowering Plants of the Sudan" by Dr. F.W. Andrews $(1950,1952,1956)$, and where rainfall statistics have also been compiled for many years.

For the barley zone, the estimates are based on the known minimum requirements for crops in the Soluch area near Bengazi; for the agricultural zone, the estimates represent the writer's personal opinion of the rainfall required to produce regular good yields of barley, wheat, lentil, and alfalfa on that sort of soil and in that climate. In all cases, the definitive plants or crops are supposed to be growing as pure rain crops unblessed by added water from a dry wadi or by runoff water collected naturally or by human wit.

To fit the seven zones into their places, it seems both logical and useful to commence the discussion with three of the driest zones in Arabia, which, however, are not represented on the western face of the 'Asīr Mountains.

\section{AR RUB` AL KHĀLĪ ZONE}

The driest of all zones in Arabia coexists with the geographical Ar Rub` al Khāli, or, literally, The Empty Quarter; as this name is so widely known, it seems proper to use it for the driest of the floral zones. It is too dry to support trees or shrubs, and vegetation is limited to grasses and other plants that spring to life 
after receiving rain from one of the rare and sporadic showers that may occur.

Throughout the zone there are dry wadis, and the large ones may carry ground water for many miles. The best of these intruding tongues support the vegetation of wetter zones; although these tongues occur in the geographical Ar Rub' al Khālī, they are here excluded from the floral zone of that name.

The rainfall for the zone can be estimated to be only a trace in the driest part and ranging to a shade less than 2 in at the boundary with the next zone. The 2-in limit is fixed by the fact that in the northern Sudan the boundary-defining shrub, samr (Acacia tortilis), disappears as one proceeds northward into the Sahara at approximately the 2 -in isohyet.

The zone occurs in many pockets outside Ar Rub` al Khāli of the maps, as for instance in the hills behind the port of $\mathrm{Al}$ Wajh which include the ancient mine workings of $\mathrm{Al}$ Hurayrah.

\section{SAMR DESERT ZONE}

This zone is marked by the fact that the conspicuous bush Acacia tortilis (Forsk) Hayne, known universally by Arabs on both sides of the Red Sea as samr, is dominant. It grows sparingly to abundantly depending on soil, but there is no other bush that remotely resembles it. The zone is extended into wetter areas to the point where the bush Acacia ehrenbergiana Hayne, universally known to Arabs as sallam, puts in an appearance.

In the Sudan the northern limit of distribution of sallam practically coincides with the 4 -in isohyet, so the samr zone extends from the 2-in to the 4-in isohyet. The zone is widespread in Saudi Arabia, but on the coastal plain it does not occur south of Jiddah.

\section{SALLAM DESERT ZONE}

This zone is characterized by the presence of the two well-known acacias samr and sallam, and by the absence of Commiphora and Euphorbia cuneata. The acacias are about equally common.

On the Tihāmah, the dry edge of the zone begins a few miles south of Jiddah, where sallam comes, at first sparingly, onto the scene. For the next $200 \mathrm{mi}(322 \mathrm{~km})$ there are many samr but few sallam, indicating a continuation of the dry edge. Between Wādì Ḩalī and Wādì 'Itwad, however, the rainfall increases so that sallam becomes well grown and as abundant as samr. The zone extends another $10 \mathrm{mi}$ south until it becomes wet enough for Commiphora africana, or qaffal, and Euphorbia cuneata, known locally as maz.

Nowhere in this zone can the grain "dukhn," Pennisetum purpureum, be grown as a rain crop. In the
Sudan 6 in of rain are required for the poorest crop of dukhn that is worth growing, and this affords a basis for assessing the wet boundary of this zone at a little less than 6 in or perhaps 5 in.

\section{ASAK-COMMIPHORA-DESERT ZONE}

The name of this zone calls attention to two of the index plants-Acacia asak, one of the thorn bushes called "hashab" by Arabs, and, on the coastal plain, Commiphora africana, or qaffal. The other two index plants are Anisotes trisulcus of the foothills and the nonprickly Euphorbia cuneata of the Tihāmah, which is widely used for supporting the thatch of village houses.

The two acacias samr and sallam reach their maximum development in this zone, as do the two important tufted grazing grasses of the samr and sallam zones, Lasiurus hirsutus Boiss. and Panicum turgidum Forsk. On the coastal plain this zone commences a little south of Wādī 'Itwad and extends through Jīzān province to beyond the Yemen frontier. As to rainfall, the northern boundary was estimated to receive 5 in.

Nothing in the vegetation indicates the rainfall at the southern boundary, but as one proceeds south through the zone, one finds that the millet "dukhn," P. purpureum, is grown as a rain crop at about the latitude of Port Jīzān, thus indicating a rainfall of 6 in there. As this comes at about the middle of the zone, the southern boundary can be assessed by proportion as having rainfall of $7 \mathrm{in}$.

A check on these estimates is that in the Sudan Commiphora africana first appears as one proceeds south in Andrews' (1950, 1952, 1956) acacia-desertscrub region, fairly close to the northern boundary at about the 5 -in isohyet. This Commiphora is probably the same as, and certainly the ecological representative of, the coastal plain species of the Jīzān.

\section{KLEINIA-PASTORAL ZONE}

This is a well-marked zone commencing on the western escarpment at about $5,000 \mathrm{ft}(1,524 \mathrm{~m})$, varying a little with aspect, where Anisotes and Acacia asak fade out. At this boundary the succulent composite herb Kleinia violacea, locally "thuriya," becomes an index plant, but it is not as common on the steep mountain slopes as on the plateau lands above.

Associated with it, however, is Acacia etbaica, or "qarad" of the Arabs. On the escarpment this takes the form of a vigorous bush or small tree as much as $15 \mathrm{ft}$ high, but on the better lands above the scarp it takes the form of a well-grown tree as much as $30 \mathrm{ft}$ high.

The upper limit of the zone, at about 7,500 ft $(2,287$ m), is marked both by the appearance of Euryops and 
by the point at which barley comes to be grown as a purely rain crop. This zone is important, as it embraces the finest pastoral lands in southwest Saudi Arabia. The rainfall at the upper limit of the zone is that required for growing barley as a rain crop. Statistics are available for the Soluch area of Cyrenaica, Libya, situated on the coastal plain to the west of Bengazi. The precipitation at this place is 7 in, some 5.8 in of which fall during the barley growing season. Barley is here regularly grown as a subsistence crop, and this seems to show that 6 in of rain is the minimum needed for this crop in a Mediterranean climate. In tropical Arabia where barley is similarly grown on marginal lands, the sun is hotter and it seems reasonable to raise the estimate to 8 in.

Barley is grown in this zone only during long rains, and for 8 in of long rains one must add 2 in for short rains, making a total of $10 \mathrm{in.}$ On this basis, the Kleinia-pastoral zone begins at the 7-in isohyet and extends to the 10-in isohyet.

\section{EURYOPS-BARLEY ZONE}

This is preeminently the zone in which barley and wheat, where suitable lands are available, are regularly grown as subsistence crops on true rain lands that receive no additional subsoil or runoff waters. It is also marked by the conspicuous presence of the composite Euryops arabicus Steud, locally "jabur," which can be recognized from a distance even when not in bloom because it bears remarkable resemblance to a 2 -ft-high seedling of Scotch or similar pine. The herb is as stiff as a pine and has a similarly symmetrical arrangement of branches, and it completes the deception by having 2-in long leaves so narrow as to suggest needles. The dominant, and only, acacia, Acacia etbaica Schweinf., finds optimum conditions in this zone and becomes a well-grown(?) tree as much as $30 \mathrm{ft}$ tall.

The upper limit of the zone is sharply marked by the lower altitude limit of the conspicuous and only conifer, Juniperus macropoda Boiss., which is likewise the lower limit for the bush Dodonaea viscosa Jacq.

We came upon fragments of this zone on Jabal Fayfā at what we estimated to be 4,000 to $4,500 \mathrm{ft}$ (1,219 to 1,372 m), at El Qa'am on top of the escarpment $35 \mathrm{mi}$ south of $\mathrm{Abh} \overline{\mathrm{a}}$ at $7,500 \mathrm{ft}(2,286 \mathrm{~m})$ by altimeter, near Aqabat al Alb on the Yemen frontier at 7,700 ft $(2,347 \mathrm{~m})$, and, finally, between Abhā and Jabal as Sūdah, where it began and ended at 7,500 ft $(2,286 \mathrm{~m})$ and $8,200 \mathrm{ft}(2,499 \mathrm{~m})$, respectively.

As to rainfall for the zone, the dry edge coincides with the minimum required for the regular cultivation of wheat and barley as rain crops, which was estimated above to be 10 in annually.
The wet edge is the boundary line for juniper. In the account by Hugh Scott and Everard B. Britton (1941) in the British Natural History Museum's "Expedition to South West Arabia 1937-38," this species is recorded as occurring on Jabal Gelal some $25 \mathrm{mi}$ south of Şan'ā', Yemen. The rainfall at Şan' $\bar{a}$ ' averaged 11.78 in from 1938 to 1947 , inclusive, and this suggests a minimum moisture requirement of 12 in for this bush or tree.

At the same edge the ubiquitous shrub Dodonaea makes its appearance. At Erkowit in the Sudan, this Erkowit privet, as it is there called, makes a first appearance as one approaches from the desert. The Kassala isohyet of $300 \mathrm{~mm}$, or $12 \mathrm{in}$, passes through or very close to Erkowit, which also suggests the 12-in isohyet as being the upper limit for this zone.

ROSE-JUNIPER-AGRICULTURAL ZONE

The rainfall in this zone affects the flow of water to the coastal plain to only a minor extent, both because the zone is so very limited in extent and because the plateau waters from a line usually about half a mile from the edge of the scarp flow eastward to Ar Rub' al Khālī. Wādì Baysh and wadis to the south of it receive practically no water from this zone. Wādī 'Itwad receives a little, and $W \bar{a} d \bar{\imath}$ Ḩalī receives a substantial quantity.

Literally dozens of definitive plants could be used for index purposes, but Rosa abyssinica R.Br. and Juniperus macropoda Boiss. are outstanding and suffice for practical purposes. The zone is confined in the 'Asirr region to the top of Jabal Fayfā, to a trace at the heads of Wādì Qa'ah, and to Jabal as Sūdah from above the $8,200 \mathrm{ft}(2,499 \mathrm{~m})$ contour up to the summit at $9,425 \mathrm{ft}$ $(2,873 \mathrm{~m})$. There is no precise method of estimating rainfall for this zone, but for reasons given above the lower limit can with some degree of confidence be estimated at 12 in.

At an elevation of $9,200 \mathrm{ft}(2,804 \mathrm{~m})$ was a field owned and farmed by Sheikh Mohammad bin Mohammad bin Said, who was a brother of the "naib," or headman, of the village of Sūdah on Jabal as Sūdah. Mohammad gave me the following cropping history for the field, sown in December and harvested in May:

\begin{tabular}{|c|c|c|}
\hline Year & Yield & Average \\
\hline 1952 & Fair & - \\
\hline 1951 -................ Barley & Good & $\begin{array}{l}240 \mathrm{~kg} \text {, or } \\
528 \mathrm{lb}, \\
\text { per feddan, or } \\
\text { acre }\end{array}$ \\
\hline 1950 & Fair & - \\
\hline 1949 & Fair & - \\
\hline 1948 -................. & Good & -- \\
\hline
\end{tabular}


Mohammad stated that during his $50 \mathrm{yr}$ of active farming at the village, there had never been a crop failure, that the crops could be grown only in the long rains, and that in the short, or monsoon, rains moisture was sufficient only for the freshening of forage grasses and for the healthy maintenance of the widely grown alfalfa. The Sheikh also said that when crops of wheat and barley began to deteriorate, it was the custom to alternate with a crop of Lens esculenta, or lentil, as a rejuvenator.

As on Jabal Fayfā, the effectiveness of the rainfall must be substantially enhanced by the regular appearance of moist cloud, or shabura, which we experienced on several occasions during our 10-day visit. The area covered by shabura is as much as a mile wide on the plateau and is marked by festoons of Spanish moss that drape the branches of the juniper trees.

This is a good agricultural area that has very regular rainfall sufficient for the production of a fair to good crop of barley, wheat, lentil, or alfalfa. It is believed that such crops, together with juniper trees, some of which attain a height of $40 \mathrm{ft}$, seem to require rainfall of at least $14 \mathrm{in}$. The village land has an altitude of $9,200 \mathrm{ft}(2,804 \mathrm{~m})$, so the upper limit would have, by proportion, an annual rainfall of 15 in. For total rainfall, one must then add a correction of 12 percent at the Jabal as Sũdah latitude for the summer, or monsoon, rains, making a total annual rainfall of 16.8 in at the top of the mountain.

The error, in my opinion, may not be very great because Mr. Eric Mackinnon, after a very long experience in the Sudan, records for the Blue Nile Province (see Tothill, 1948, p. 804) that, "In general it may be said that the production of successful rain crops requires a rainfall of $400 \mathrm{~mm}, 16 \mathrm{in}$., or more." The hotter sun at the lower elevation of that province, coupled with the absence of shabura (mist), would tend to equate the 16 in with a little less on Jabal as Sūdah.

The estimates of rainfall for the zone are therefore 12 in at the lower boundary and 16.8 in at the top, with an average of $14.4 \mathrm{in}$.

\section{PRECAMBRIAN LAYERED ROCKS OF THE ARABIAN SHIELD}

The rock units mapped in reconnaissance fashion on the geologic map of the Arabian Peninsula (USGS-ARAMCO, 1963) were at that time described mostly in field terms, and the map was based in part on interpretation of aerial photographs. Although the map was planned only as a general guide for ore and water search, at an early stage lithostratigraphic groups were recognized to range from unmetamorphosed sediments and volcanics to greenschist and amphibolite metamor- phic facies, and to change lithologically in short distances along the strike of the volcanogenic and related rocks. Because the resolution of stratigraphic problems would require detailed work over many years, the units were described only in general terms on the map legend. We have attempted to correct and amplify the earlier reconnaissance mapping with current knowledge from numerous contributors (see annotated bibliographies, Ministry of Petroleum and Mineral Resources, 1977, 1980, 1981).

\section{HISTORIC GEOLOGIC DIVISIONS}

In the first published attempts at division of the basement rock, Karpoff $(1955,1957$ a) described two series-the Medina and the Wadi Fatima, in the central part of the shield. We had begun our reconnaissance study 5 yr earlier at the Yemen border and had worked northward. By 1960, general rock assemblages had been extended into the areas described by Karpoff (1960), wherein we tentatively recognized eight units exclusive of plutonic rocks. Of these, six were equivalent to parts of the older Medina Series of Karpoff and two were equivalent to and coordinate with his younger Wadi Fatima Series. By 1963, after mapping seven quadrangles $\left(3^{\circ} \times 4^{\circ}\right.$ at a scale of $\left.1: 500,000\right)$ and segments of three others-about 36 percent of the Kingdom of Saudi Arabia-we compiled a map at 1:2,000,000 scale which was combined with the mapping of the Arabian-American Oil Company (ARAMCO) and various others into the composite map of the Arabian Peninsula (USGS-ARAMCO, 1963). At that time we recognized a total of 33 units among the basement rocks, of which 19 were given rock-term names without formal reference to geographic localities. Of the 14 names based on geographic localities, 2 were regional, namely the Halaban and the Shammar. The Halaban was named for Ḩalabān Ridge and the region around Halabān Pass near the southern end of the ridge in the east-central part of the shield, and the Shammar was named for Jabal Shammar, the regional name for the area around Hầil in the northeastern part of the shield.

Some explanation of the first use of these names is desirable. The first geologist to describe layered rocks near Ḩalabān Ridge was Bogue (1953) of the USGS. He thought that the dioritic and andesitic greenstone and related rocks of the region were probably the same as the "Shawaq volcanics" he had earlier described at Wādĩ Shawãq in the Jabal ash Shifā' region $900 \mathrm{~km}$ northwest of Hुalabān and near the northwestern edge of the shield. We felt that even though the lithology and metamorphism appeared to be similar, the units were separated by too great a distance of unmapped terrain to justify correlation until the areas were 
mapped in more detail. Accordingly, G.F. Brown and R.O. Jackson (1960) chose the name "Halaban Andesite" for Bogue's "Shawaq volcanics"; later the term "Halaban formation" was used, because it was known that rock types other than andesitic greenstone were in the section and that an assemblage of at least group rank would be found if it were mapped in any detail (Eijkelboom, 1969).

Likewise, the name "Shammar rhyolite" was used (Brown and Jackson, 1960) to designate the largely unmetamorphosed and silicic volcanic rocks associated with sedimentary rocks cropping out in the northern part of the shield, to distinguish these younger rocks from older rhyolite and related volcanogenic beds farther south.

The six other names published in 1960 (Brown and Jackson, 1960) were, from oldest to youngest, the Hali Schist, Baish Greenstone, and Lith Complex below the Halaban, and the Murdama, Fatimah, and Ablah Formations above the Halaban and below the Shammar rhyolite. Later we described (USGS-ARAMC0, 1963) the Jiddah Greenstone, which is intruded by the Mecca Granite, the Hibshi Formation, dominantly a clasticvolcanic formation that disconformably overlies a gray biotite granite and underlies at least part of the Murdama phyllite in the Jabal Shammar region, the Hadiyah slate in the northwestern part of the shield, and the iron-bearing Silasia Formation. Units were individually named because field information was insufficient to warrant closer correlation over large areas. Names of large wadis or coastal towns were used to avoid giving a specific type locality until the units could be mapped in detail and the best type sections and localities could be chosen. The Murdama and Hibshi Formations are exceptions; both have excellent sections on the mountains of those names.

\section{GURRENT GEOLOGIC DIVISIONS}

During the past $17 \mathrm{yr}$, more detailed mapping by numerous geologists has begun to elucidate structural complexities, chronologic episodes and sequences, sedimentary and volcanogenic facies changes, and metallogenic epochs. Computer-enhanced imagery from satellite signals supplied a new tool that supplements aerial photographs and standard color composites of satellitederived scenes (Blodget and Brown, 1982). Airborne magnetic and digital gamma-radiation surveys also helped in interpreting the geology.

As a result of the more detailed mapping, some formations of the USGS-ARAMCO map (1963) were raised to group rank (Schmidt and others, 1973) to include various formations named for specific localities and type sections. Also, because many more radiomet- ric ages have been determined and structural control is better understood, the sequence of groups has been changed somewhat. The rock units, as currently (1982) mapped and named, are shown in plate 1 .

\section{ULTRAMAFIC AND OPHIOLITIC ROCKS}

Ultramafic and serpentine rocks and associated mafic volcanic and plutonic rocks, chert, slate, and marble are widespread but are sparsely distributed throughout the shield. Ultramafic rocks associated with the older marine beds of the Baish-Bahah were described by Hadley (1975a) as an ultramafic complex in part "conformably interbedded in the Baish group and in part intrusive into it" (Qunfudhah quadrangle, lat $19^{\circ} 10^{\prime}$ N.). Greenwood (1975b) has mapped a serpentine-talc belt at the base of the Baish Group farther northeast in the Biljurshī' quadrangle at lat $19^{\circ} 55^{\prime} \mathrm{N}$. At Jabal Rayyan (Wask) (Al 'Ays area) a mafic-ultramafic dome has been studied intensely for its chromite potential (Kahr, 1961; Johnson and Trent, 1967; Bakor and others, 1976; Kemp and others, 1980; Kemp, 1981). Samples collected by Viktor Kahr, who recognized the domal rocks as belonging to the ophiolitic suite, were studied by T.P. Thayer, who identified peridotite, alpine gabbro (with alteration to prehnite and other minerals of the rodingite suite), and "....albitized volcanic breccia commonly associated with albite granite of the end member of the alpine mafic magma stem," all identified with ophiolite. Kemp and others (1980) report a $\mathrm{Pb} / \mathrm{U}$ age of $882 \pm 12$ m.y. from zircon in plagiogranite of the ophiolitic complex. Bakor, Gass, and Neary (1976) consider the ophiolite dome to have formed in a backarc environment. As these ultramafic rocks are on the southwest flank of the youngest fault zone (part of the Najd fault system of the shield) and on the northeast flank of the Wādi Kamāl domal complex, it appears that the upper mantle-basal oceanic layers of the Wask ophiolite were exposed either by Najd faulting and subsequent erosion or by uplift of circumferential younger granites-or by some combination of the two processes. This interpretation is strengthened by the fact that the tectonics follow the pattern of equalintensity aeromagnetic lines (Andreasen and Petty, 1974).

Farther north and $38 \mathrm{~km}$ southeast of Al Wajd, black schists are exposed for $1 \mathrm{~km}$ along the track (von Gaertner and Schurenburg, 1954). The carbonaceous schist extends northward at least as far past $\mathrm{Al}$ Wajh as the southeast border of Jabal Libān at lat $26^{\circ} 30^{\prime} \mathrm{N}$., where the older metamorphosed flows and sediments produce a bluish hue on enhanced satellite imagery (Blodget and Brown, 1982). These beds, as mapped by the Japanese Geological Mission (1965), include meta- 
gabbro, metabasalt, metadiabase, cherty slate, and limestone (marble). Serpentinite is exposed along an overthrust fault at the eastern edge of the Wajd segment but may not represent the ultramafic base of a possible Wajd ophiolitic suite (Japanese Geological Mission, 1965), although it is in a structural position similar to that of the ophiolite fragments along sutures displaced from the southeast by Najd transverse faulting.

Other ophiolitic suites, generally incomplete as ophiolite was defined at the Penrose Conference (Anonymous, 1972) as a classical stratigraphic section of ocean floor above mantle rocks, occur in fault zones at junctions considered to be plate or island-arc boundaries within the shield. The most complete but fragmented ophiolite (fig. 4) is at Jabal Ess (Al-Shanti and Roobal, 1982) (lat $26^{\circ} 22^{\prime}$ N., long $37^{\circ} 38^{\prime}$ E.); the largest exposures are around Jabal Rayyan (Wask) within the $\mathrm{Al}$ 'Ays region northwest of Yanbu' at lat $25^{\circ} \mathrm{N}$., long $37^{\circ} 30^{\prime}-38^{\circ} 10^{\prime} \mathrm{E}$. The longest outcrop of ultramafic and related rocks of probable ophiolitic origin follows the Nabitah fault zone from the Yemen border northward to the Najd fault zone at lat $21^{\circ} \mathrm{N}$., where it is offset $120 \mathrm{~km}$ left laterally along the Najd fault zone to the northwest (in various fragments), the northwesternmost outcrop being the Tuluhah tectonic belt north of lat $25^{\circ} 30^{\prime} \mathrm{N}$. (Frisch and Al-Shanti, 1977). The easternmost ophiolitic zone is along the front of the Al AmarIdsas fault near the east edge of the shield. Other than the ophiolite in the $\mathrm{Al}$ 'Ays region, the ages of other ultramafic and serpentinized suites are not known to us.

If these rocks are dismembered ophiolites, they should be structurally, not stratigraphically, related to-and of different ages from-adjoining rocks. If the island-arc accretion model is accepted, the ophiolite rocks might be expected to become progressively younger eastward; they should be somewhat older than the island-arc rocks with which they are structurally associated.

\section{BAISH-BAHAH GROUPS}

The presumed lowermost and oldest rocks originally described in the Arabian Shield were metadiorite, metagabbro, and amphibolite formerly named the Lith Complex and occupying a $1^{\circ}$ square at $\mathrm{Al}$ Lith. A 55$\mathrm{km}$-wide belt of metamorphosed lavas and sedimentary rocks along the banks of Wādì Halī and Wādī Tayyah at lat $18^{\circ} 30^{\prime}-18^{\circ} 44^{\prime} \mathrm{N}$. were named the "Hali Schist." These rocks consist of two units: (1) basalt and andesitic basalt containing local pillow structure, with interbedded marine volcaniclastic and sedimentary wacke, and (2) carbonaceous and graphitic schists, and minor

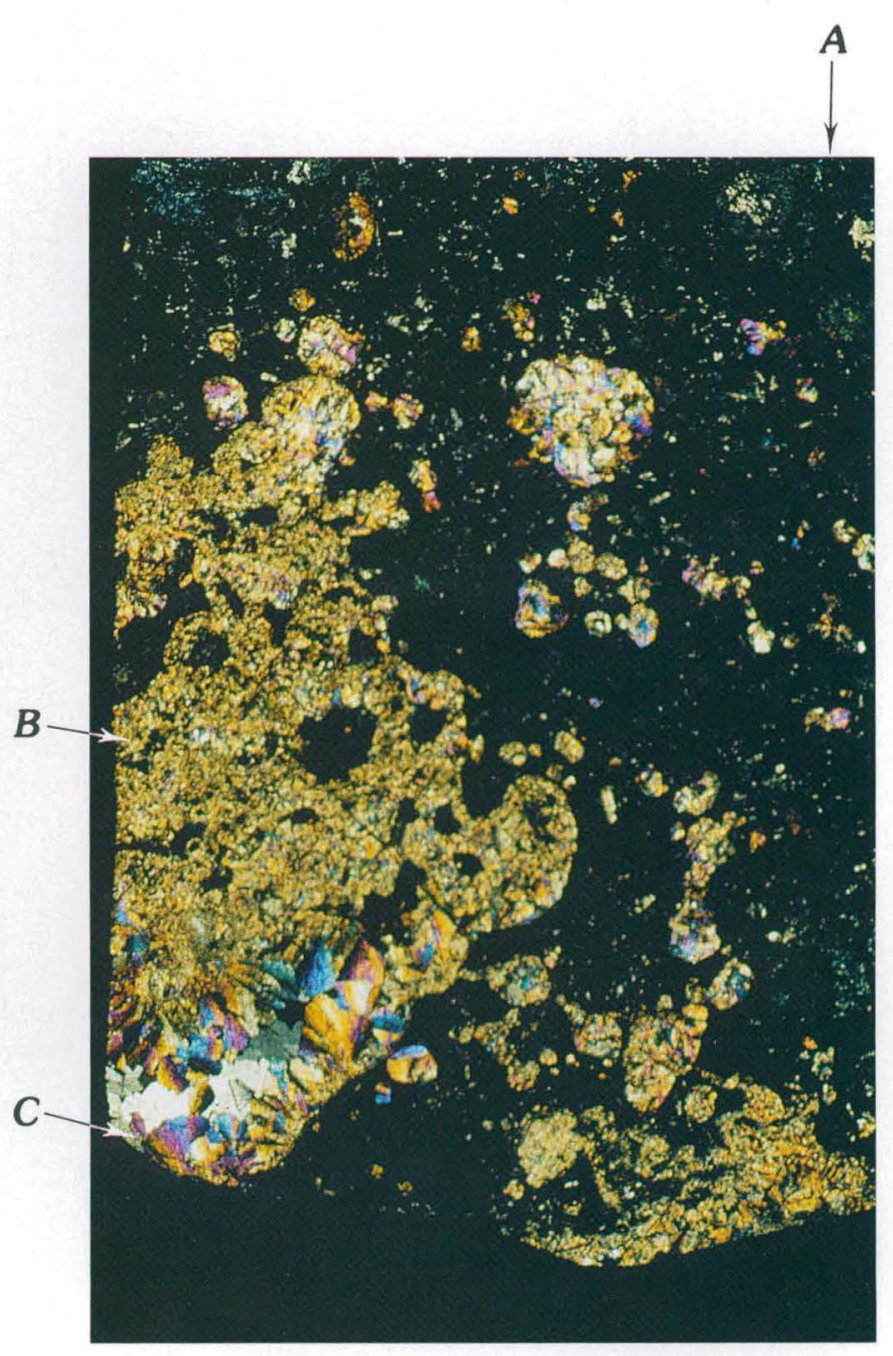

Figure 4.-Photomicrograph of amygdaloidal metabasalt at the Jabal Ess ophiolite complex at lat $26^{\circ} 22^{\prime}$ N., long $37^{\circ} 38^{\prime}$ E. The groundmass is equigranular, completely chloritized clinopyroxene(?) and calcic feldspar $(A)$ with two generations of amygdules, the oldest fine grained and filled with secondary calcite (bent twinning planes), quartz, chlorite, and prehnite. The multimineral vesicle filling $(B)$ was later transected by coarse-grained calcite $(C)$. Described by Salman Bloch. Magnification $\times 10$.

marble and chert, all metamorphosed to the greenschist facies and generally retrograded from the amphibolite facies. Both of these units were originally shown on the geologic map of the Arabian Peninsula (USGS-ARAMCO, 1963). The Hali Schist was later redefined (Schmidt and others, 1973) as a result of more detailed mapping and was described as quartz-biotitegarnet schist interbedded with marble, stretched-pebble conglomerate, and metamorphosed volcanic rocks, including the older, more metamorphosed "Lith Complex." The name "Lith Complex," shown on the 1963 


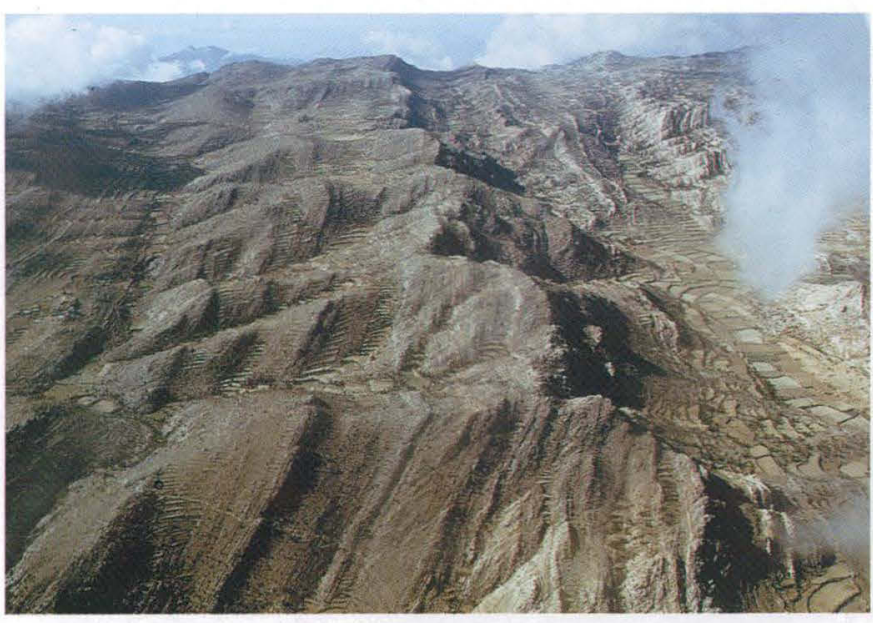

A

Figure 5.-Baish-Bahah Group. $A$, Metasedimentary schists in the Jabal 'Aya quadrangle formerly believed to be of Baish-Bahah age but now believed to belong in the younger Jiddah Group, having been overthrust from the east. View south along the top

map as the basal rock unit of the shield, was abandoned because it included diverse rock types that were later mapped as separate units.

The Baish Greenstone was first described from exposures in cliffs along Wādī Baysh, at lat $17^{\circ} 40^{\prime} \mathrm{N}$. (Brown and Jackson, 1959, 1960), where spilitic pillow lava crops out (fig. 5) as massive greenstone or sericitechlorite schist derived from mafic igneous rocks. Also included in the unit were metadiorite, metagabbo, and amphibolite with subordinate siliceous slate. The thickness as originally measured along the western limb of a syncline was $12,000 \mathrm{~m}$, but more recent work suggests a synclinal structure with $6,700 \mathrm{~m}$ of tholeiitic basalt on one limb (George Simmons, USGS, oral commun., 1979). The unit was originally thought to overlie the Hali schists downstream in the Baysh Canyon, but the structure is complicated by tight folds and faults and the relationships are still not clear.

Schmidt and others (1973) redefined and raised the Baish Greenstone to group rank and placed it immediately above the Hali Group. The upper schistose part was split off from the marine sediments containing metabasalt cobbles above a disconformity (Greenwood, 1975c) to form the Bahah Group, although the lithologic description remained much the same. Baish and Bahah Groups form the "metabasalt graywacke-chert assemblage" of Greenwood (1975c) or the "basaltic assemblage" of Fleck and others (1980).

The two groups have as common components pillow metabasalt, marble, chert, carbonaceous or graphitic schist, and some meta-andesite, but the components are

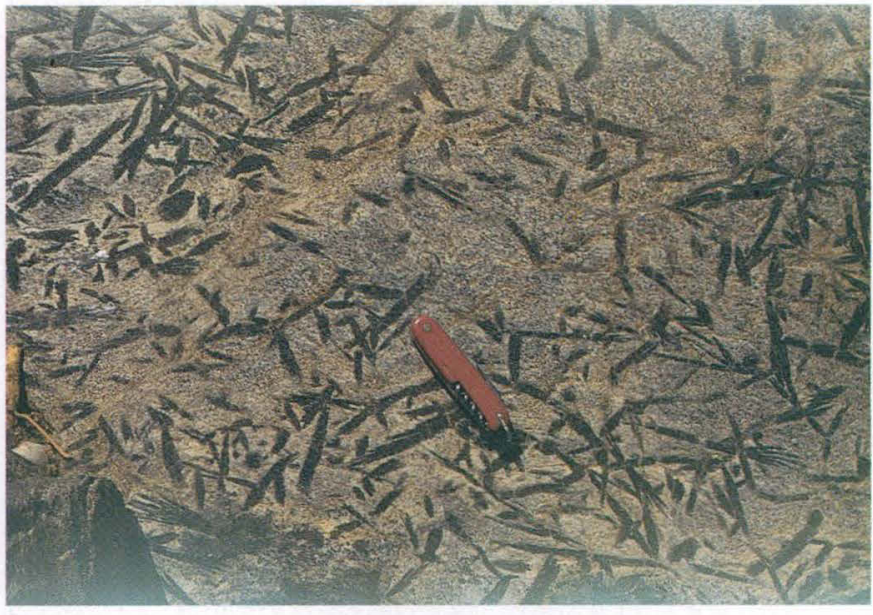

$\boldsymbol{B}$

of the An Nimas escarpment. The beds dip east. $B$, Glaucophane schist near Ad Darb in Bahah Group indicating high pressure in the low-glaucophane schist facies beneath overthrusts from the east. The pocket knife is $9 \mathrm{~cm}$ long.

volumetrically in widely different proportions, hence their separate names. The two groups are quite distinct from the overlying andesitic lavas and volcanogenic sedimentary rocks of the Jiddah and younger groups (Schmidt and others, 1973; Hadley 1975a, 1975c; Greenwood and others, 1976).

The Bahah Group, named from the village of $\mathrm{Al}$ Bāḩah in the Jabal Ibrāhīm quadrangle (Schmidt and others, 1973; Greenwood, 1975c), includes formations considered in this report to be structurally below the Baish Group but stratigraphically above or synchronous with the Baish. The marine Bahah Group includes paraschists of clastic or tuffaceous origin with distinct ferruginous quartzite, metachert, and carbonaceous and arkosic members. Marble, stretched-pebble conglomerate, and metabasalt make up less conspicuous beds. Carbonaceous beds common in both the Baish and Bahah Groups appear to owe the carbon to algal growth in water within the photic zone, probably less than $250 \mathrm{~m}$ depth (Jackaman, 1972; Kiilsgaard and others, 1978). A similar assemblage of rocks including metabasalt, carbonaceous or graphitic schists, and jasper or chert extending intermittently on promontories of the crystalline shield as far north as Al Wajh at lat $26^{\circ} 16^{\prime} \mathrm{N}$. has been questionably remapped as part of a younger epoch. At Wādī Fāţimah, paraschist, jasper, and keratophyre (including minor marble, and epidosite) are intruded by "pyroxene granite" (RichterBernburg and Schott, 1954) and were contact-metamorphosed to amphibolite facies. Northwest of Yanbu' at lat $24^{\circ} 20^{\prime}$ N., gneiss comprising amphibolite, quartzite, 
and leptite is intruded by the Wadi Kamal Complex of norite, orthoamphibolite, anorthosite, and ultramafic (serpentinized) rocks which were tentatively referred to the Hali by Baubron and others (1976).

Three samples of metabasalt near Al Lìth at Wādī al Fagh, lat $20^{\circ} 23^{\prime}$ N., gave an age of $1,165 \pm 110$ m.y. from a whole-rock Rb-Sr isochron (Fleck and others, 1980, p. 31 ), in contrast to more recent work in another laboratory which resulted in Rb-Sr dates of $836 \pm 60$ m.y. and $830 \pm 9$ m.y. for metabasalt in Wādi Sa'diyah in the western part of the Al Lith area, presumably from rocks considered Baish ("Lith") age (Reischmann, 1981). Minimum ages for the layered rocks based on whole-rock $\mathrm{Rb}-\mathrm{Sr}$ isochrons of intrusive quartz diorite near Biljurshì' (lat $19^{\circ} 52^{\prime} \mathrm{N}$.) and $\mathrm{Al}$ Lith are about 850 and 890 m.y., respectively (Fleck and others, 1980, p. 19); farther north, Aldrich (Aldrich and others, 1978) measured a single K-Ar age of 1,190 m.y. for hornblende in amphibolite at the northwestern corner of Jabal Shār, lat $27^{\circ} 20^{\prime}$ N. (sample 11, table 6). Still farther north, carbonaceous slate containing fossil blue-green algae in southeastern Sinai yielded a $\mathrm{Rb}-\mathrm{Sr}$ isochron date of $934 \pm 80$ m.y. (Shimron and Horowitz, 1972; Shimron and Brookins, 1974). However, the Sinai date has been questioned (Halpern, 1980).

\section{JIDDAH GROUP}

First shown on the peninsular geologic map (USGS-ARAMCO, 1963) as the Jiddah Greenstone, the meta-andesite in the foothills east of Jiddah, where it is intruded by the Mecca Granodiorite, was described as andesite and andesite porphyry metamorphosed to the greenschist facies. In many places it is schistose, but it includes some diabase, gray slate, conglomerate, dacite, and marble. The first radiometric dates determined for the Mecca Granodiorite range from 965 to 1,025 m.y. for Rb-Sr ages and from 720 to 760 m.y. for K-Ar ages as determined by Aldrich (in Brown, Jackson, Bogue, and MacLean, 1963; Aldrich and others, 1978; samples 114 and 124, table 6), using the decay rates adopted by the 25th International Geological Congress (1976). For this reason the Jiddah Greenstone was considered to be among the oldest rocks of the shield and was placed beneath the Baish Greenstone and above the Silasia iron formation of northwestern Arabia, as described by Bogue.

Bhutta (1970) recognized two units within the Jiddah Greenstone: a lower series of metamorphosed flows and pyroclastic rocks (andesite, diabase, andesite porphyry, rhyolite, and greenstone) and an upper, younger sequence of slate, metaconglomerate, tuff, quartzite, and marble (figs. $6 A, 6 B$ ). The unit was further defined and named the "Jiddah group" (Schmidt and others,

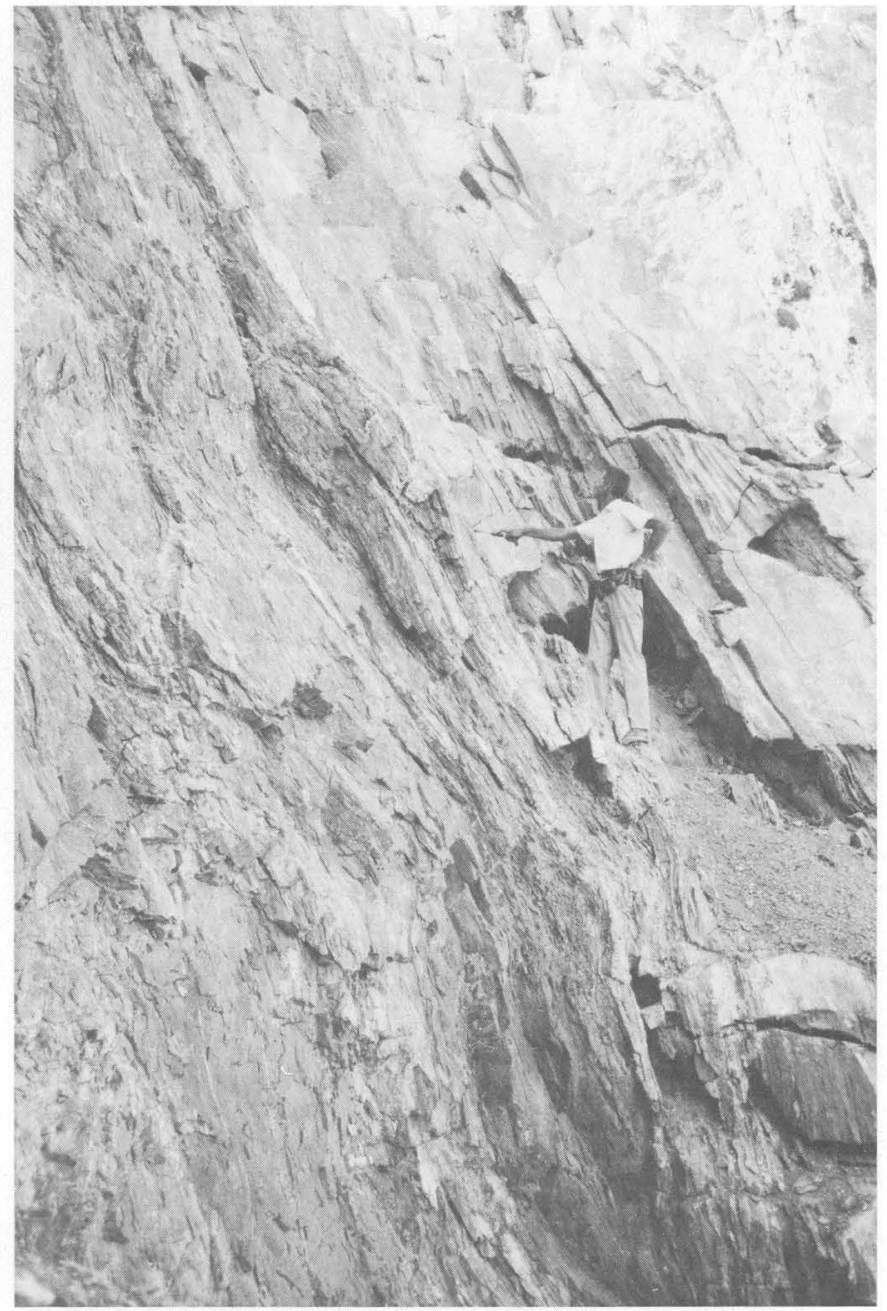

$A$

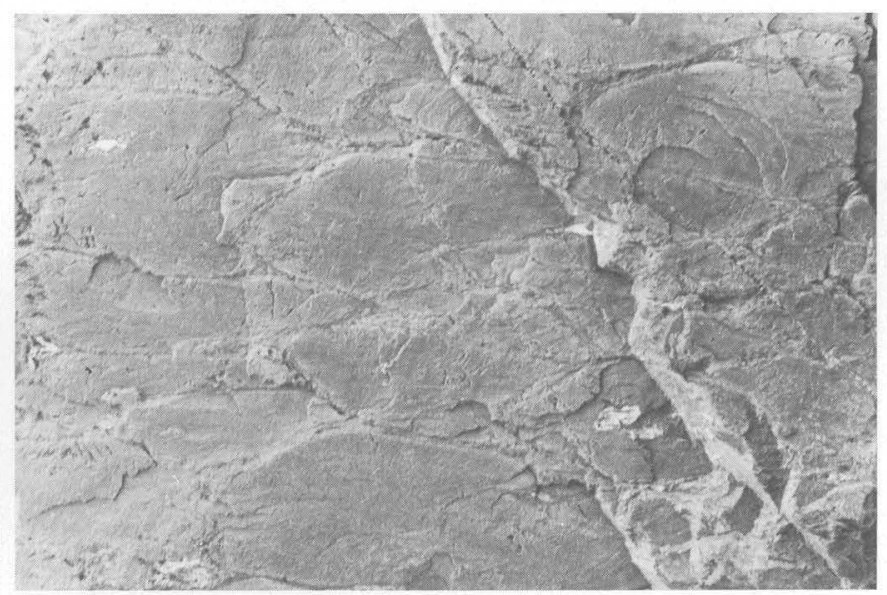

B

Figure 6. $-A$, Hornblendite interbedded with pink marble of the Jiddah (Samran) Group in Wādī Fāţimah. Two periods of folding are apparent. $B$, Pillow lava in basalt, Hilwa area, on north wall of Wādī Baysh gorge, formerly believed to be in the Baish Group but now believed to belong to the Jiddah Group. Lower pillow is about 1 $\mathrm{m}$ long. 
1973) following more intensive fieldwork and an extended field conference that covered eleven $30^{\prime}$ quadrangles in the southern part of the shield. The relationship between the Baish-Bahah and Jiddah Groups is not clear, but possibly the Baish-Bahah Group is slightly older in that metamorphosed clasts of the older rocks were reported in the basal units of the Jiddah Group (Greenwood and others, 1976) and in turn were overlain with angular unconformity by the Ablah Group. This is the stratigraphic position we had earlier assigned to the meta-andesitic and related rocks exposed within the Southern Hejaz quadrangle (Brown, Jackson, Bogue, and MacLean, 1963).

The Jiddah Group consists of a lower volcanic formation, the Qirshah Andesite, and an upper clastic, predominately immature arkosic and volcaniclastic wacke, the Khutnah Formation (Greenwood, 1975b). The Qirshah Andesite, named for Wādi Kirshah, a tributary to Wādī Ranyah at lat $20^{\circ} 10^{\prime} \mathrm{N}$., consists predominantly of andesitic pyroclastic and flow rocks and includes dacite breccia, ignimbrite, marble, and some pillow basalt, suggesting a marginal marine origin similar to the calc-alkaline suite of island-arc volcanics in contrast to the more mafic (calcic) volcanic rocks and deeper marine beds of the Baish-Bahah Groups. Much of the sedimentary and volcaniclastic schist of the Jiddah Group is graphitic and includes beds of chert and minor basalt, thus resembling the upper Bahah Group. Within the 'Aqiq quadrangle and west of the Ablah graben, the Jiddah Group includes spilitic mafic flows, tan and brown marble, andesite with blobs of serpentine, and fine-grained pyroxenite(?), the ultramafic suite of G. Eijkelboom (oral commun.).

More recent work extending northward along the coast indicates that metabasalt, meta-andesite, and graphitic schists that were mapped as Jiddah Greenstone as far north as Al Wajh lithologically resemble the Baish-Bahah (Blodget and Brown, 1982) and are the same beds named "Samran" by Charles Smith and Vicktor Kahr (unpub. data), later adopted by several authors, and finally defined by Skiba (1980). Some metavolcanic and metasedimentary rocks in the southeastern part of the shield that were considered Jiddah group by Schmidt and others (1973) and by Greenwood and others (1980) also resemble the Baish-Bahah Groups, so that on the basis of chemical analyses, as well as on the basis of structural relations and metamorphism, these rocks are shown to be similar to the Hali schist as it was originally mapped (Brown and Jackson, 1959). The most striking evidence of the Bahah-Hali (now Bahah) affinity is the cherty and carbonaceous or graphitic schists that crop out on both flanks of the Khamis Mushayt gneiss complex in the 'Asīr highlands. In contrast, the Jiddah Greenstone as originally mapped was mostly meta-andesite in greenschist facies. The more extensively mapped Jiddah Group, however, does contain cherty and carbonaceous facies, and, in fact, any island-arc terrane, regardless of age, might be expected to contain this facies; the facies is not group diagnostic. The Jiddah Group as shown on plate 1 also includes andesitic rocks mapped as the Samran series in the area of Jabal Samrān (Nebert, 1969).

A tentative reported age for the volcanic rocks of the Jiddah Group comes from a roof pendant on the An Nimas batholith, where Fleck and others (1980) found an apparent age of $912 \pm 76$ m.y. from a whole-rock Rb$\mathrm{Sr}$ isochron. The rocks were considered part of the lower Jiddah Qirshah meta-andesite by Greenwood (1979).

Concurrent with and subsequent to the accumulation of the Jiddah Group, calcic and calc-alkalic plutonic rocks of a comagmatic suite ranging from gabbro through quartz diorite to trondhjemite and granodiorite, but mostly diorite and quartz diorite, were synkinematically intruded into the Baish-Bahah and Jiddah Groups during the period $890 \pm 67$ to $848 \pm 28$ m.y., according to $\mathrm{Rb} / \mathrm{Sr}$ isotopic ratios (Fleck and others, 1980). Some of these plutonic rocks were locally reactivated during the period $797 \pm 15$ to $763 \pm 4$ m.y. (Cooper and others, 1979) into orthogneiss domes ranging in composition from tonalite to granodiorite; thus they define a minimum Jiddah age. The intrusions and the concomitant orogenies have raised locally the metamorphic rank of the older lithostratigraphic assemblages to amphibolite facies.

Recent, more detailed study of the granitoid intrusives in the Jiddah-Makkah area at the type locality of the Jiddah Group gives ages of $763 \pm 159$ m.y. from whole-rock isochrons (Fleck, 1985) of samples including quartz diorite and granodiorite gneiss intruded into the calc-alkaline meta-andesite of the Jiddah Group. If correct, this value establishes a younger minimum age for the group than determined by Aldrich and corresponds in general to the K-Ar ages of biotite, 720 and 760 m.y., and of muscovite, 800 m.y., obtained by Aldrich for the same samples yielding $\mathrm{Rb}-\mathrm{Sr}$ biotite ages of 965 and 1,025 m.y. (table 6). Thus it appears that the major gneiss doming of the subsequent Ablah orogeny at about $763 \mathrm{~m} . \mathrm{y}$. reset the intrusive dates, and the relative ages of the Jiddah (Samran) and BaishBahah Groups remain uncertain.

\section{ABLAH GROUP}

The name "Ablah" was first used for the Ablah Formation (fig. 7) and applied to a belt of sedimentary rocks exposed in a graben extending south-southwest 


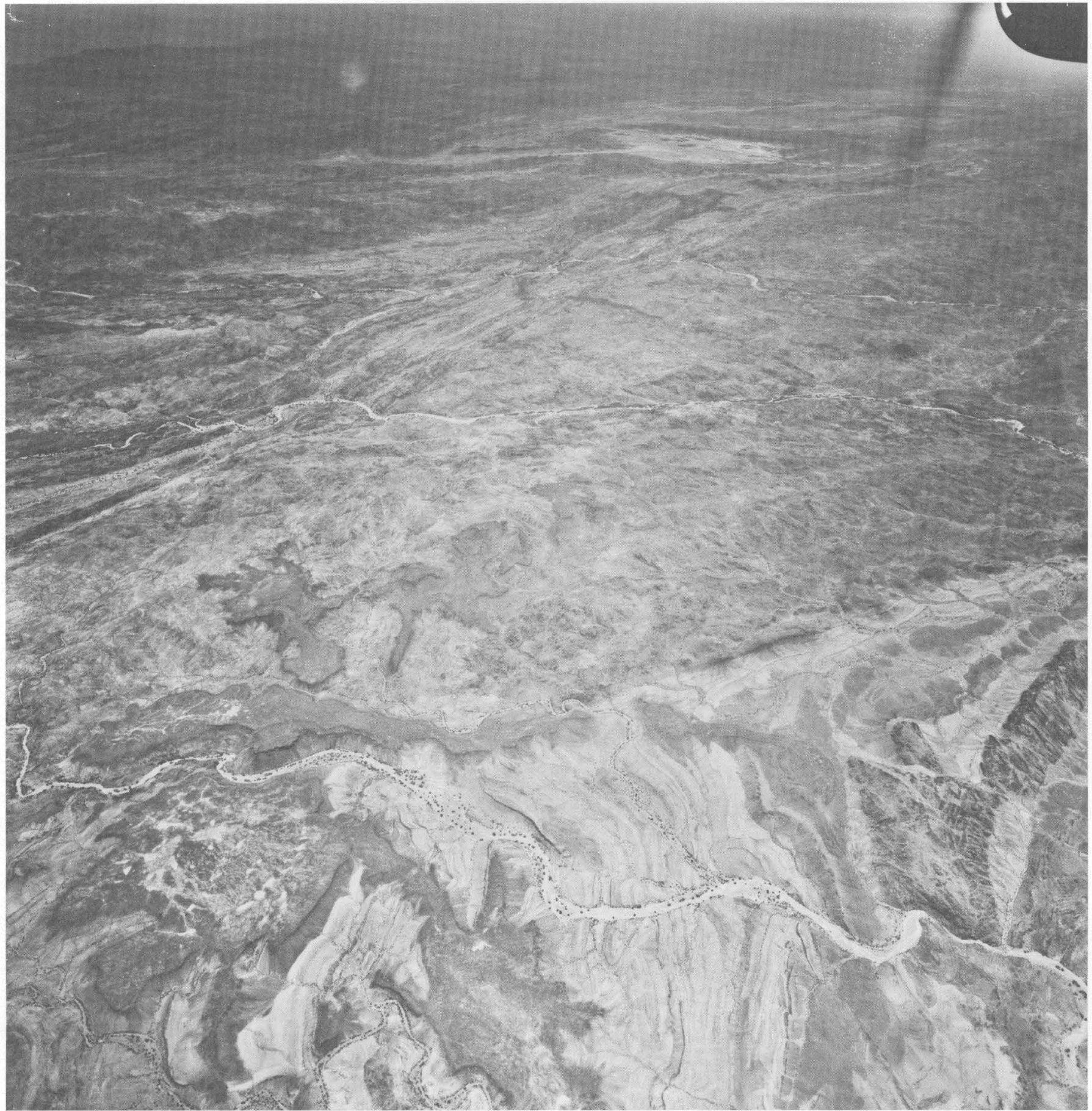

Figure 7.-Oblique aerial view to the northwest across the Ablah and Jiddah Groups. The boundary between the groups is the fault line in the lower third of the scene at the edge of the folded Ablah sediments. Wādī Ranyah flows northeast in the foreground, bisecting Tertiary, probably Pliocene, flood-basalt outli- ers, and the folded and faulted Ablah Group. Wādī. Kirshah in the middle distance and Wādi Thurat beyond flow across andesitic metavolcanic rocks of the Jiddah Group. Older dioritic rocks intrude the volcanogenic rocks in a belt east of the oasis of 'Aqīq, the small light-colored plain in the distance. 
from Jabal Rafā‘ (lat $22^{\circ} 30^{\prime}$ N., long $42^{\circ}$ E.) past a fluorspar pipe at 'Ablah that was mined during the time of the Abbasid Caliphate (Brown and Jackson, 1960). The basal polymict conglomerate, quartzitic wacke, red sandstone, stromatolitic limestone, arkose, and purple shale rest nonconformably on Jiddah Group metavolcanic and plutonic rocks. They are folded into a series of asymmetric anticlines and dip predominantly to the east. Theobald and Thompson (1966), in a detailed study in the vicinity of a native copper occurrence at Jabal "Namar" (Rumur) in the southeastern part of the "Aqīq quadrangle, described a sequence of red laminated siltstone and gray crossbedded sandstone containing some conglomerate lenses on disconformities within the sediments which grade upward into ferruginous wacke and andesitic breccia. Andesite flows and feeder dikes are present in the midsection. Some of the coarser grained sedimentary rocks contain fluorite, seemingly epigenetic and coeval with the Ablah fluorite pipe. The copper is stratabound, possibly syngenetic, and is structurally related to the contemporaneous andesite flows and dikes.

The Ablah rocks extend southward discontinuously from the Ablah mine area in a narrow faulted belt in which the rocks are found as roof pendants in, or as grabens between, the diorite-trondhjemite-granodiorite batholiths of the 'Asīr and Tihāmat ash Shām (Bayley, 1972; Greenwood, 1975a, 1975b; Hadley, 1975c; Anderson, 1979). Thin beds of stromatolitic marble thicken southward from two horizons in the Ablah beds at 'Ablah (calcite and siderite cement are common at several horizons). The beds become metamorphosed progressively southward to a greenschist facies in the northern part of the Wādi Yiba quadrangle and to almandine-amphibolite and sillimanite facies at lat $19^{\circ}$ N. (Bayley, 1972; Hadley, 1975c) in the Wādī Ḩalī quadrangle. At the north end of the infolded graben at 'Ablah, Greenwood (1975a) divided the Ablah Group into three formations: a basal polymict conglomeratewacke-marble series metamorphosed to greenschist, his Rafa Formation; a middle unit composed essentially of calc-alkaline flows but including rhyolite, quartz latite, some basaltic extrusives with pillow structure, marble, and pyroclastic rocks, his Jerub Formation; and the younger beds making up the original Ablah Formation, his Thurat Formation. Greenwood (1975a) gives a thickness of the Thurat as 1,100-1,300 m, but the lower units and their southward extension in the Ablah graben are so folded and faulted as to make thickness estimates unreliable. The Ablah Group appears to extend southward to and across the Yemen border at lat $17^{\circ} 30^{\prime} \mathrm{N}$., mostly as paraschist, marble, conglomerate, and quartzite or slate beds (Anderson, 1979). The basal polymict conglomerate is discontinuously exposed from 'Ablah to Jallat al Mawt on the Yemen border, where the pebbles and cobbles are gray gneissic quartz diorite or trondhjemite, quartzite, and chloritic hornfels.

Hadley (1975c) divided the Ablah Group in the Wādī Ḩali quadrangle into two formations, because the correlation with the three formations defined by Greenwood (1975a) in the 'Aqiq quadrangle is tenuous on the basis of depositional and metamorphic facies changes. Hadley's two formations are the Sarban, mostly paraschist and marble metamorphosed to the amphibolite facies, and the overlying Hadab, likewise amphibolite schist but retrogressively metamorphosed and including gneiss (Hadley, 1975d). However, the Ablah Group comprises a definite sequence of shallowwater and nonmarine beds (fig. 8) overlain by calcalkalic lavas and stromatolitic limestone and marble above a widespread nonconformity. The basal conglomerates contain boulders of the Jiddah trachytoidal and amygdaloidal andesites and related rocks as well as some rocks from the older Baish-Bahah Groups.

\section{FATIMAH GROUP}

The Fatimah Group (fig. 9) exposed in the hills north of Wādī Fāţimah as originally described by Karpoff $(1955,1957 a)$ is remarkably similar to the Ablah Group. It rests nonconformably on the granodiorite of Mecca and includes red and green immature clastics, stromatolitic limestone, tuffaceous sediments, andesite and basalt flows (Goldsmith, 1966; Nebert and others, 1974). However, the uppermost flows come from feeder dikes which transect the lower sediments and are younger than the sedimentary rocks. The thickness as measured by Goldsmith is about $1,000 \mathrm{~m}$ and is comparable to the Thurat Formation at 'Ablah. The age of the Ablah-Fatimah Groups may fall somewhere within the timespan between $816 \pm 3$ m.y. (Kemp and others, 1982) for the Mahd adh Dhahab area and $763 \pm 55$ m.y. for the Bagarah gneiss dome of the Ablah belt (Fleck and others, 1980).

Both the Fatimah and the Ablah are intruded by postorogenic magma; an andesite sill and a basalt flow at the top of the exposed section of sedimentary rocks in Wādī Fāţimah are dated at $592 \pm 23$ and $576 \pm 28$ m.y., respectively, by K-Ar whole-rock analyses (table 6); however, both the andesite and the basalt are hydrothermally altered and the ages could be reset. At 'Ablah an intrusive basalt plug that pierces the upper Ablah-Thurat sedimentary rock is dated at $585 \pm 39$ m.y. (sample 136, table 8). However, stromatolites described by Karpoff are Collenia and Corophyton, which together in general appearance led him to consider the Fatimah Upper Precambrian in accordance with the age assignment in Africa. More recently, a sample of one stromalolite collected by D.L. Schmidt was examined by 


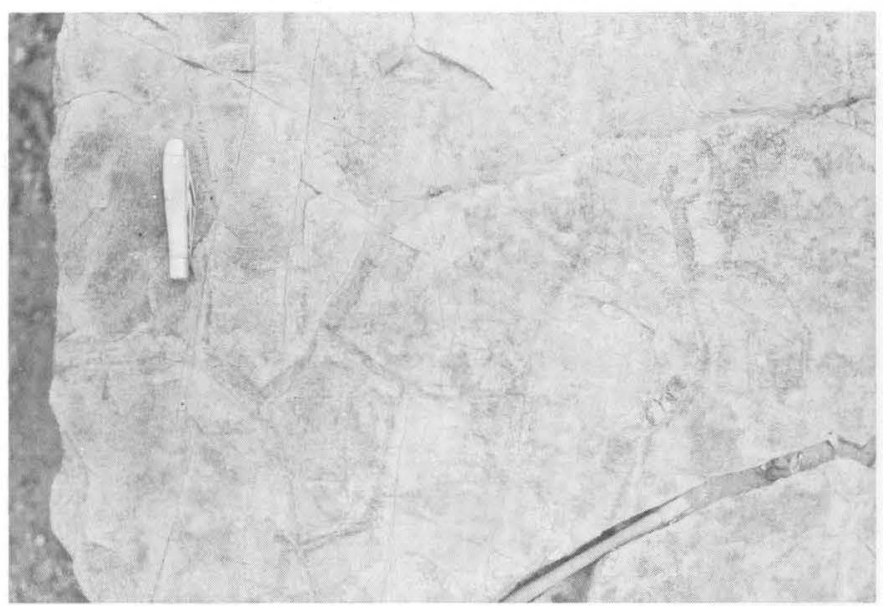

A

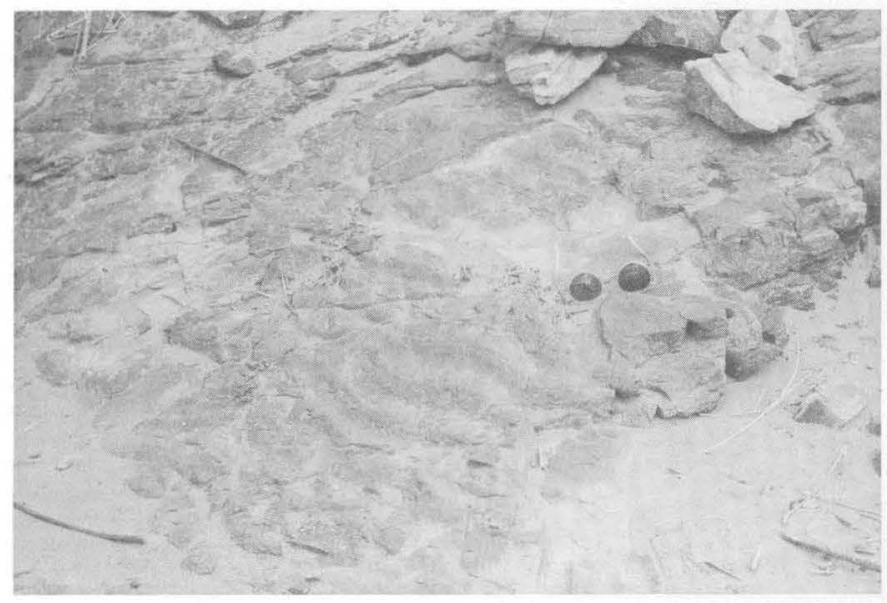

B

Figure 8.-Views of the Ablah Group. $A$, Infilled desiccation cracks in gray siltstone, and $B$, symmetrical ripple marks in associated red siltstone, at type locality of Ablah Group, $7.5 \mathrm{~km}$ upstream from the junction in Wādī Kirshah, Al 'Aqīq quadrangle. C, Small thrust fault in Ablah Group at the type locality. Siltstone

Preston Cloud (USGS) and S.M. Awramik (written commun., 1978), who consider the form to be close to the morphology of Kussiella, a characteristically lower Riphean form of 1,350 to 1,650 m.y. (glauconite age). However, they report at least one record of Kussiella from the upper Riphean or within the timespan 675-950 m.y. (glauconite). Thus, it appears that the Fatimah sedimentary rocks could be coeval with the Ablah. These sedimentary rocks constitute a typical molasse assemblage, in contrast to the underlying Jiddah, Baish, and Bahah Groups, which are predominantly island-arc assemblages.

\section{AL AYS GROUP}

The term "Al Ays Group" has been applied in the western part of plate 1 north of lat $24^{\circ} \mathrm{N}$. for extensive sedimentary and volcanic rocks that are similar to the

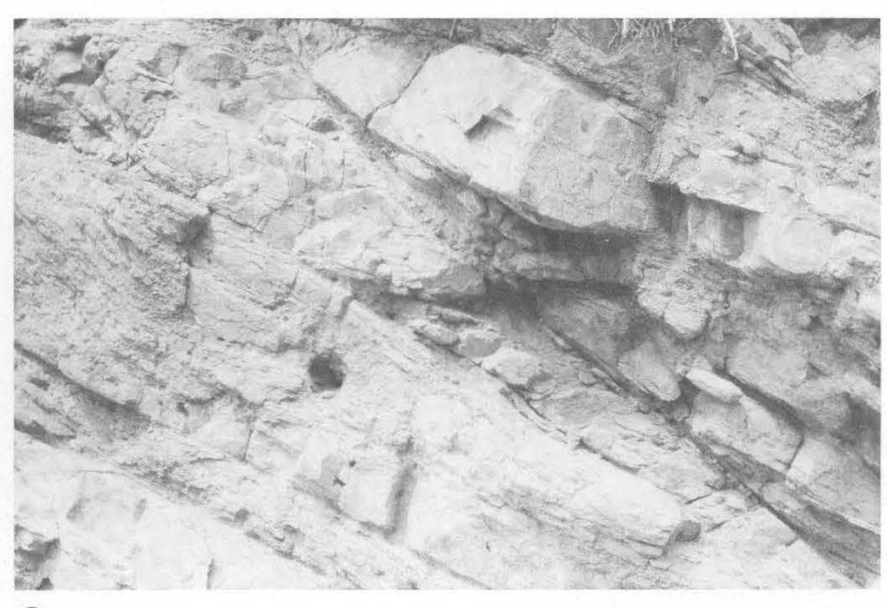

C

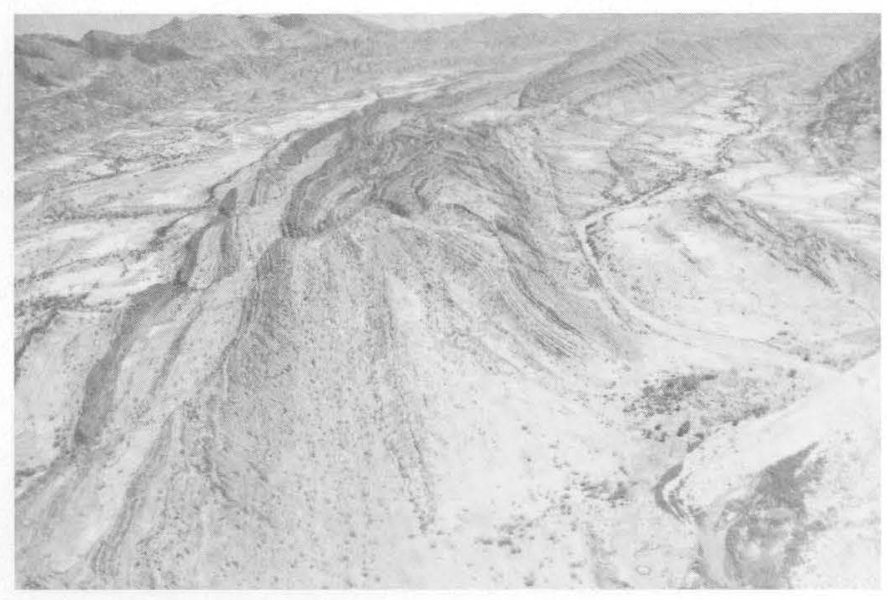

D

at lower left underlies red faulted sandstone. Thrust is from the east at the junction of Wādī Kirshah and Wādī Ranyah, Al 'Aqīq quadrangle. $D$, Parasediments of the Ablah Group intensely folded into a plunging syncline at Wādī Yiba. View northwest.

Ablah Group rocks in the southwestern shield. Many of the predominantly metavolcanic rocks in an eastern belt had been mapped as Halaban andesite, and many of the predominantly sedimentary rocks of volcanic derivation in a western belt had been mapped as sericite and chlorite schist by Brown, Jackson, Bogue, and Elbert (1963). Hadley, mapping in the northern part of these two belts, had assigned both the sedimentary and volcanic rocks to the Halaban Group on the basis of lithology and limited age relationships. On the basis of more extensive mapping, rock description, and more recent age determinations by Kemp (1981), Pellaton (1979), and Kemp and others (1980), we have tentatively correlated the Al Ays Group with the Ablah Group of the southern shield (pl. 1).

The Al Ays Group is well defined by Kemp (1981) in the Wādī al 'Ays quadrangle (lat $25^{\circ} \mathrm{N}$., long $38^{\circ} \mathrm{E}$.), where it is divided into a western facies of predominant- 



Figure 9.-Fatimah Group, north Wādī Fāţimah. Folds in stromatolitic and clastic sedimentary rocks.

ly metasedimentary rocks and an eastern facies of predominantly metavolcanic rocks. The base of the group consists of conglomerate, minor marble, basalt flows, the minor silicic volcanic rocks overlain by thick deep-water graywacke containing some silicic tuffs. Several regressions and transgressions higher in the section resulted in more conglomerate, stromatolitic marble, and shallow-water graywacke containing silicic welded tuffs and basaltic andesite flows. The volcanic eastern facies is bimodal, containing basaltic andesite and silicic tuffs with minor sedimentary rocks (Kemp, 1981).

The age of the $\mathrm{Al}$ Ays Group is not well defined, but the group is underlain by volcanic and plutonic rocks that are probably older than 800 m.y. and is overlain by the Hadiyah Formation of the Murdama Group. Two silicic tuffs within the Al Ays Group have $\mathrm{Rb}$-Sr ages of $743 \pm 12$ (initial strontium ratio 0.7027 ) and $725 \pm 16$ m.y. (initial strontium ratio 0.7046), and the Jabal Salajah tonalite intruding the $\mathrm{Al}$ Ays Group has a U-Pb zircon age of $725 \pm 12$ m.y.

\section{SILASIA FORMATION}

Farther north, near lat $28^{\circ}$ N., the iron-bearing Silasia Formation is associated with spilitic and diabasic greenstone. The formation was named by Richard Bogue (1953) and was described as consisting of mostly shale, locally sandy, calcareous, or conglomeratic, and enclosing thin-bedded limestone and thin alternate beds of jasper and hematite in the upper part with a thickness of not less than $1,400 \mathrm{~m}$ (fig. 10). The typical iron-formation-banded jaspilite-hematite layers, best seen in Wādī Sawāwīn at lat $27^{\circ} 55^{\prime}$ N., are exposed in pods interbedded with slate, limestone, tuffs, agglomerate, and conglomerate, all of which are intruded by finegrained diabasic diorite and intensely deformed. Bogue believed that the spilitic greenstone, which he named 


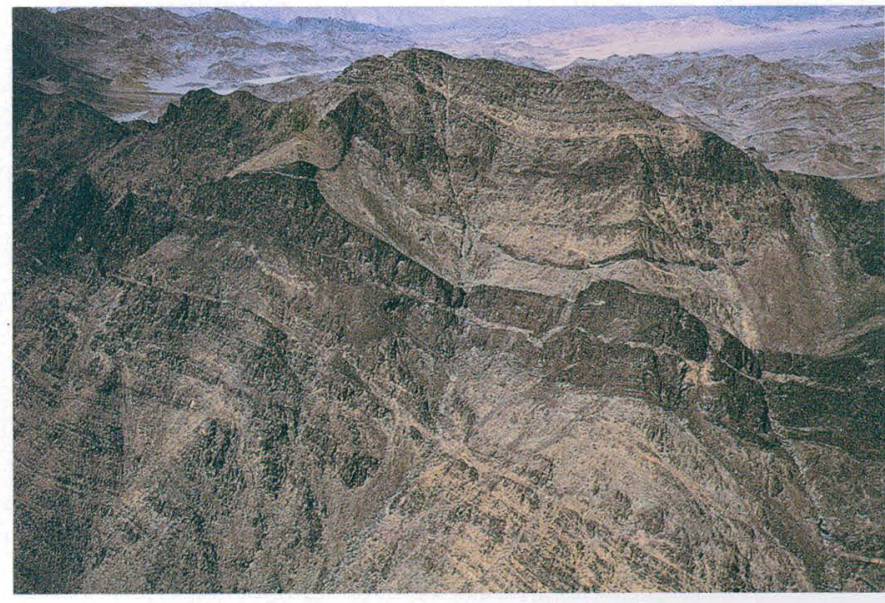

FIGURE 10.- Silasia Formation showing siliceous hematite outcrops intruded by diorite at Wādī Sawāwīn.

the "Shawaq Greenstone" from the ancient town and wadi $80 \mathrm{~km}$ southeast of Wādi Sawāwīn, overlay the iron formation, but he further stated that the ironbearing beds are highly folded and in places are overturned. Later, Johnson and Trent (1967) believed that the greenstone underlay the Silasia clastics. The Japanese Geological Mission in 1967 correlated the beds with the Hali schist, and Liddicoat (1975) measured a thickness of $919 \mathrm{~m}$ for the upper sedimentary section. The Silasia and Shawaq Formations remain enigmatic to this day; on plate 1 the Shawaq Greenstone is mapped as part of the Jiddah Group and the Silasia Formation is retained as a younger volcanogenic metasedimentary unit equivalent to the Ablah type, following the concept that many of the beds formerly named "Hali Schist" are of Ablah age.

\section{HALABAN GROUP}

In the eastern part of the Arabian Shield, Bogue (1953) briefly described "a variety of both intrusive and extrusive rocks... mostly andesite and fine-grained diorite, but gabbro, basalt, and basalt porphyry are not uncommon"-igneous rocks he considered possibly equivalent to the Shawaq volcanics of northwestern Arabia. Our subsequent mapping suggested that the calc-alkaline volcanic rocks and associated plutonic and hypabyssal intrusives in the eastern regions were most likely somewhat younger than the Shawaq volcanics, although both are regionally metamorphosed, the Shawaq to a somewhat higher grade. Accordingly, we proposed the name "Halaban Andesite," choosing a regional name for the area where Bogue described widespread outcrops in the region around Halabān Pass and the water well near the southern end of the

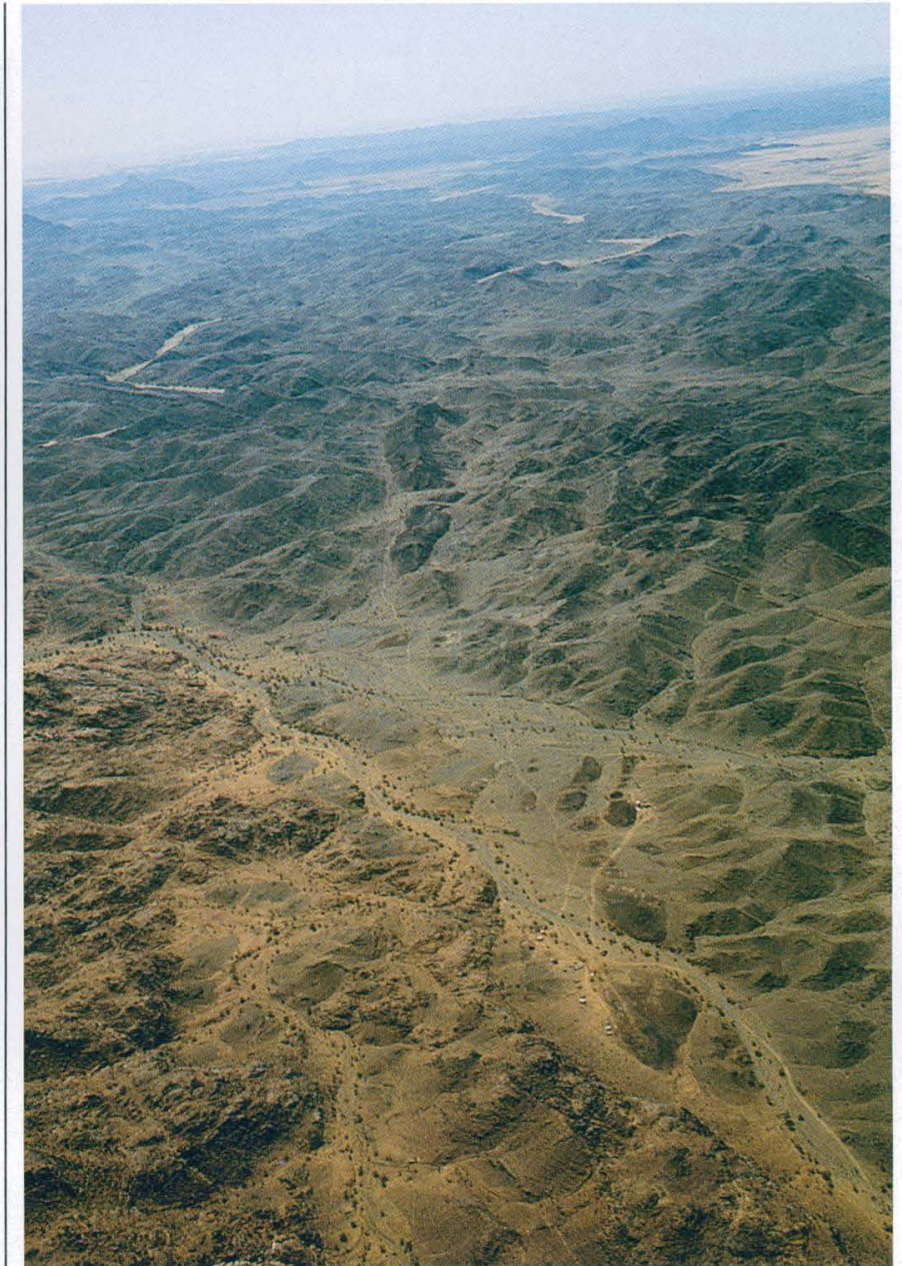

FIGURE 11.-View north along the strike of stratabound gossan at Wādī Wassāt in Halaban Group volcaniclastic sediments cut by late Halaban quartz diorite in foreground. The quartz diorite is locally gneissic from upward movement during the culminant orogeny.

50-km northwest-trending ridge, Samrā’ Ḩalabān, and where Sha'īb Hुalabān, a tributary to Wādī as Sirrah, affords an east-west caravan route. As we could not designate a type section for a formation, it was necessary to use a rock name in accordance with the rules of nomenclature, choosing what Bogue considered the dominant rock in his description (Bogue, 1953). The formation (fig. 11) as mapped includes, besides andesite, agglomerate, quartzite, graywacke, interbedded marble and rhyolite, and a somewhat younger series of epidiorite, diorite, diabase, gabbro, and serpentinite (Brown and Jackson, 1960; Jackson, Bogue, Brown, and Gierhart, 1963). Most of the rocks are metamorphosed to the lower and middle greenschist facies, but higher grades of metamorphism are found locally.

Later, Bureau des Recherches Geologiques et Minieres (BRGM) (Eijkelboom, 1966; Vincent, 1968) mapped 
the Hुalabān region in considerable detail and began to elucidate the lithologic units where we had shown all as Halaban Formation (Jackson and others, 1963). Although BRGM did not assign relative ages and specifically avoided describing a sequence of events, designating only lithologic units, the sequence shown on their maps includes ultramafic rocks, mostly pyroxenite and harzburgite much serpentinized, at the base. As part of an ophiolitic suite, these ultramafic rocks are generally older than the andesite-dioritic basement that they structurally overlie. The calc-alkalic Halaban rocks are variously metamorphosed, mostly in the greenschist facies (chlorite-epidote-zoisite-albite), and are overlain by metamorphic tuffs and sedimentary rock. Some subvolcanic fine-grained diorite intrudes the uppermost metavolcanics and metasedimentary rocks. The structure is complex; in places a melange is present within the section and complicates the stratigraphy, so the suggested sequence might be changed with further study. Vincent raised the Halaban to group status. Eijkelboom separated the volcanic and subvolcanic rocks east of the Al Amar-Idsas fault-the easternmost exposed segment of the Arabian Shield-from those to the west because the flow rocks, namely andesite and dacite, including rhyolite, tuff, agglomerate, volcaniclastic conglomerate, breccia, albitophyre, keratophyre, and some marble and graywacke, are different from the volcanic and subvolcanic rocks west in the vicinity of Ḩalabān.

BRGM later divided the Halaban into four units, including basal ophiolitic melange (BRGM, 1966; Eijkelboom, 1969). Bois (1971) separated the rocks exposed along the northeast edge of Ḩalabān Ridge into ultramafic and gabbroic rocks of an ophiolitic suite, and an overlying unit, the Ar Ridaniyah Formation, consisting of volcaniclastic rocks, chert, and marble beds metamorphosed to the amphibolite facies. Later, the upper units were collectively called the "Pyroclastic Halaban," in contrast to the underlying "Andesitic Halaban" (Bounny, 1973). Still later, the Halaban rocks were formally named the "Afna" and "Nuqrah" Formations (Delfour, 1975) and assigned to the Hulayfah Group, named for Wādi Hulayfah and the town on the wadi where both formations are exposed; the type locality (lat $26^{\circ} \mathrm{N}$.) is midway between $\mathrm{Al}$ Madīnah and Hुă'il. The younger Nuqrah Formation was named for the ancient mining site on the $\mathrm{Al}$ Madinah-Al Qaşìm road 75 $\mathrm{km}$ southeast of Hुulayfah, where it has been studied extensively.

More recently, Delfour (1979a) has recompiled the earlier BRGM mapping of the Hुalabān quadrangle and, with additional fieldwork in 1975 and 1977, reinterpreted the geology. He separates the Halaban as mapped earlier into four units: three groups above an older basement composed of granite, orthogneiss, and diorite. The groups, in ascending order, are as follows: Ajal, composed of biotite schist, gneiss, and amphibolite; Urd, consisting of two formations, a lower ophiolitic complex and upper Abt Schist; and Hulayfah, here consisting of only the upper formation of the group, the Nuqrah. The Nuqrah is composed of silicic volcanics and volcaniclastic sediments as well as andesite, marble, and jasper, the rocks first described by Eijkelboom as the Al Amar-Idsas Formation.

Also more recently, BRGM has recompiled the 'Afif quadrangle (Letalenet, 1979), extending the detailed mapping west from the Ḩalabān region. There the formations originally shown as Halaban are divided into five formations grouped into three units besides intrusive diorite, gabbro, ultramafic rocks, and serpentinite. The oldest unit, mostly of sedimentary origin, of schist, gneiss, amphibolite, quartzite, metarhyolite, and meta-andesite, is shown only along the western edge of the quadrangle and has been dated in xenoliths in diorite at 825 m.y. (Baubron and others, 1976; Letalenet, 1979). The remaining formations above these older rocks are calc-alkalic to silicic flows and volcaniclastic sedimentary rocks in general becoming more alkalic and silicic in the uppermost beds, which seem to be similar to the Hulayfah Group of the Nuqrah quadrangle (Delfour, 1977).

Hadley (1973) followed the BRGM classification but omitted the lower ophiolitic complex, and assigned formation names within the Sahl al Maţrān quadrangle in northwestern Arabia (lat $26^{\circ} 00^{\prime}-26^{\circ} 30^{\prime} \mathrm{N}$., long $38^{\circ} 00^{\prime}$ E.) as follows: the lowermost $2,550 \mathrm{~m}$ of metaclastic rocks and marble he named the "Thaa Formation" from exposures on Wādi Thaa in the southeastern part of the quadrangle; the middle $3,950 \mathrm{~m}$ of metabasalt, meta-andesite, and pyroclastics exposed in the hills around the central Matran plain are the Matran Formation; and the upper $3,900 \mathrm{~m}$ of lavas, mostly alkalic, and associated pyroclastic rocks are the Jizl Formation, named from the exposures on the flanks of the Wādi al Jizl in the southeastern part of the quadrangle.

Subsequently, the ophiolitic suite, the Ar Ridaniyah Formation, and the overlying Abt Schist were taken together to form the Urd Group, named for Jabal al 'Urd at lat $24^{\circ} 05^{\prime}$ N., long $44^{\circ} 50^{\prime}$ E. (Delfour, 1977). Most of the Jabal al 'Urd region is underlain by the Abt Schist, which we now believe to be clastic deposits of the Murdama Group metamorphosed to paraschists by underthrusting along the Al Amar-Idsas fault and by later granitic intrusions. We suggest abandoning the group name.

The southern extension of the Halaban rocks includes two formations in the Bi'r Juqjuq quadrangle at 
lat $21^{\circ} 00^{\prime}-21^{\circ} 30^{\prime} \mathrm{N}$., long $43^{\circ} 30^{\prime}-44^{\circ} 00^{\prime}$ E. (Hadley, 1976): a lower volcanic formation composed of andesite, basalt, and conglomerate, the Juqjuq Formation (from Wādī Juqjuq, a tributary to Wādī ad Dawāsir), and an upper formation, the Arfan, from Jabal Arfan north of Wādi Juqjuq in the northwestern part of the quadrangle. The Juqjuq Formation is more than $13,100 \mathrm{~m}$ thick at the type locality, and the Arfan Formation was estimated to be more than 7,600 m thick (Hadley, 1976; Schmidt and others, 1979). Subsequently, it has been suggested by C.R. Ramsey and N.J. Jackson (oral commun., 1980) that some of the rocks of the Arfan Formation belong to the Murdama Group.

Delfour (1977), in describing the rocks of the Hulayfah Group in the Nuqrah quadrangle, reported 6,500 m of the Afna Formation in two units, a lower $2,500 \mathrm{~m}$ of conglomerate, marble, tuffs, and siltstone and an upper $4,000 \mathrm{~m}$ of predominantly andesite and basalt but including diabase sills and some rhyolitic tuffs and flows at lat $25^{\circ}-26^{\circ} \mathrm{N}$., long $40^{\circ} 30^{\prime}-42^{\circ} \mathrm{E}$. The Nuqrah Formation at the type locality is $4,000 \mathrm{~m}$ thick and is composed of three members - a lower rhyolitic tuff and rhyolite with less abundant andesite, a middle unit of marble, graphitic tuff, and sulphide mineralized breccia, cherty tuffite, bedded chert, jasper, rhyolite, and subordinate andesite, and an upper unit of conglomerate, ignimbrite, rhyolite, and tuffite.

The Halaban Group (Brown and Jackson, 1979) covers large areas in the eastern and northern parts of the shield and was considered a possible supergroup (Brown and Jackson, 1979), but this is deferred, needing further elucidation. The outcrops extend in folded and faulted belts for at least $800 \mathrm{~km}$ in a north or northwesterly direction and extend laterally as much as $40 \mathrm{~km}$ in the type region. The early mapping limited the outcrops almost entirely to the region north and east of the southernmost Najd fault. Geochronologic dates range from about 785 and 775 m.y. for volcanic rocks (Rb-Sr) to 729 (zircon) and 724 m.y. (Rb-Sr) for tonalite intrusive into the volcanic rocks (Aldrich and others, 1978; Cooper and others, 1979; Fleck and others, 1980). Thus, the predominantly calc-alkalic rocks and uppermost beds include a stratigraphic unit that contains rhyolitic flows, ignimbrite, and tuffaceous sedimentary rocks-in all, a typical island-arc assemblage. This assemblage makes up the Halaban Group (Brown, Delfour, and Coleman, 1972; Brown and Jackson, 1979), here comprising the Hulayfah Group, of the northeastern shield, and the Halaban, as widely mapped in recent years in the southeastern shield.

The granite Delfour includes in the older basement extends into and appears to be part of the large granite batholith that extends through the Dawādimi area. It is about $1,400 \mathrm{~km}^{2}$ in area and, according to Al-Shanti
(1976), is composed mostly of two major types, a syntectonic monzogranite in the western part and a late tectonic, evenly grained monzogranite containing xenoliths of the syntectonic granite in the eastern part. A minor part is posttectonic monzogranite and alkalic granite in small stocks and dikes. These phases appear to have one calc-alkaline magmatic source, with granite intrusion beginning during the tectonic phase and continuing after tectonism ceased. As the batholith and related stocks are intruded into the Abt Schist and the invasion culminated at about 570 m.y. according to numerous K-Ar dates (tables 6, 8), consideration of interpreting the Abt Schist coeval with the Murdama Group seems plausible even though regionally metamorphosed in front of the Al Amar-Idsas overthrust fault.

\section{MURDAMA GROUP}

The Murdama Formation was named after Jabal al Murdamah $30 \mathrm{~km}$ southeast of 'Afif, a type locality (fig. 12) suggested by Bogue (1954) after his reconnaissance of the eastern shield area. Slate, phyllite, quartzite, graywacke, and conglomerate were seen in a traverse across the north end of the mountain. The contact with the underlying metavolcanic rocks seemed to be conformable at the northwest corner of the mountain (Brown and Jackson, 1960). Later, when the peninsular map was compiled, it became known from additional information that at least some paraschists cropping out along the Najd faults are of Murdama age. The Hadiyah slate was tentatively correlated by Brown, Jackson, Bogue, and Elberg (1963) with the type section of the Murdama Formation, even though the sandstonesiltstone of the Hadiyah slate was first seen $500 \mathrm{~km}$ northwest of Jabal al Murdamah at the Hadiyah station on the Hejaz Railroad. The correlation was made on the basis of lithologic similarity, thickness, degree of metamorphism, and an apparent stratigraphic position above rocks considered to be Halaban. Subsequently, the original Murdama Formation was measured in detail by J. Letalenet (1974) on the western flank of Jabal al Murdamah, where he found a polygenetic conglomerate resting on andesite of the Halaban and extending 2,700 $m$ upward as graywacke, siltstone, and sandstone to a reddish-brown rhyolite porphyry overlying a polymict conglomerate. Letalenet (1974) considered the rhyolite to be part of the Murdama because the conglomerate beneath the rhyolite did not contain clasts of the underlying clastics of the Murdama. Earlier we had dated the rhyolite by K-Ar whole-rock methods at $561 \pm 25$ and $560 \pm 20$ m.y. (samples 141a, 141b, table 8; Aldrich and others, 1978). The samples are rhyolite crystal tuff and rhyolite porphyry which are deuterically altered (Salman Block, written commun., 1974). More 


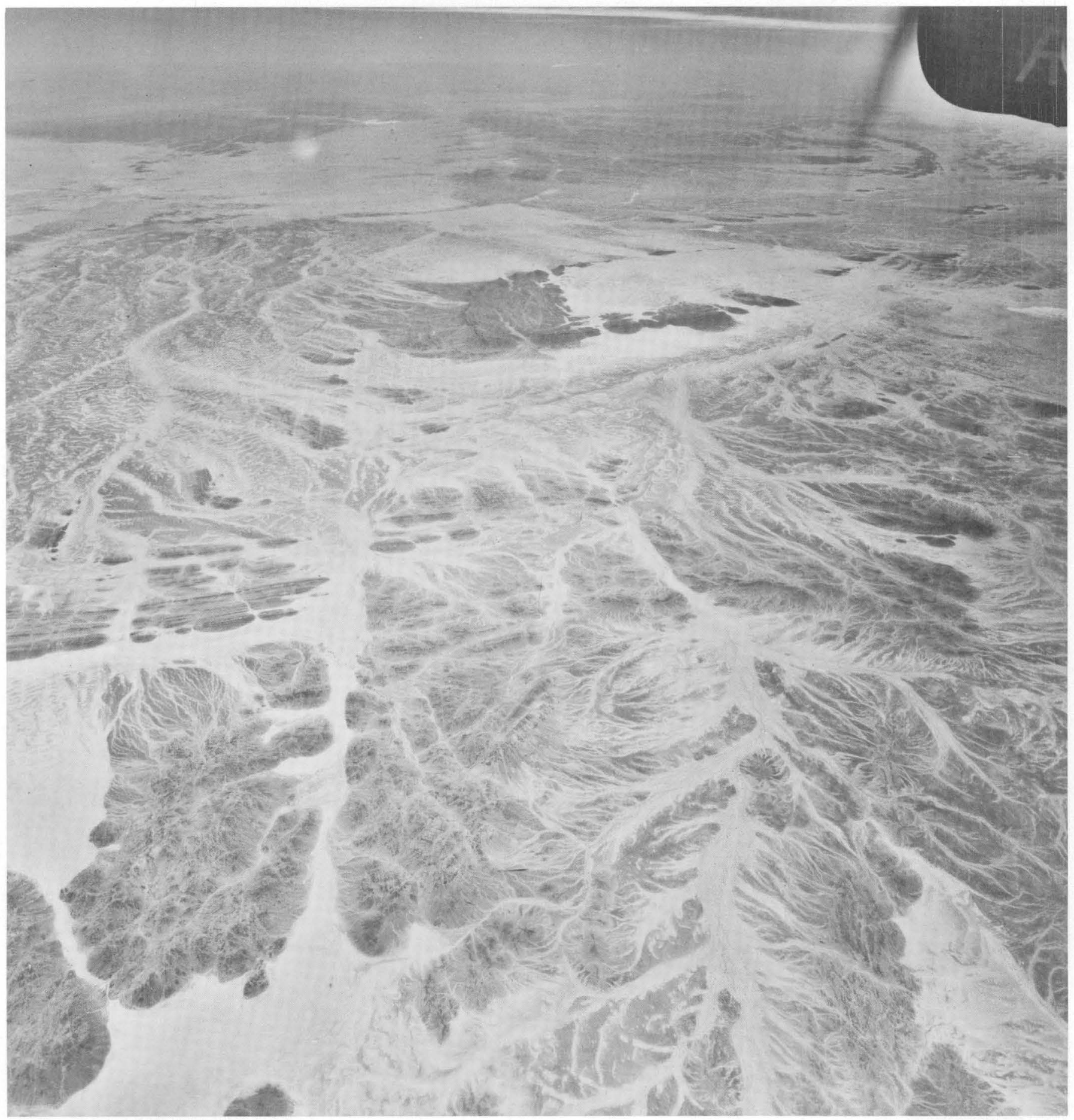

Figure 12A.-Type locality of Murdama Group, view to the southeast. The sediments of the Murdama Group lie in a syncline plunging to the southeast in the middle distance. The syncline rests disconformably on the meta-andesite and metarhyolitic tuffaceous volcanics of the Halaban (Hulayfah) Group exposed in the foreground.

recently, samples from the same locality averaged 544 m.y., using conventional interpretations of K-Ar corrected to the Sydney decay constants (Baubron and others, 1976). Flows southwest of Al Madīnah near the top of a similar thick clastic section gave an age of $633 \pm 15$ m.y. by Rb-Sr whole-rock isochron (samples 94 , 106, table 7). These ages are concordant with those reported by Baubron and others (1976) for the Hibshi 


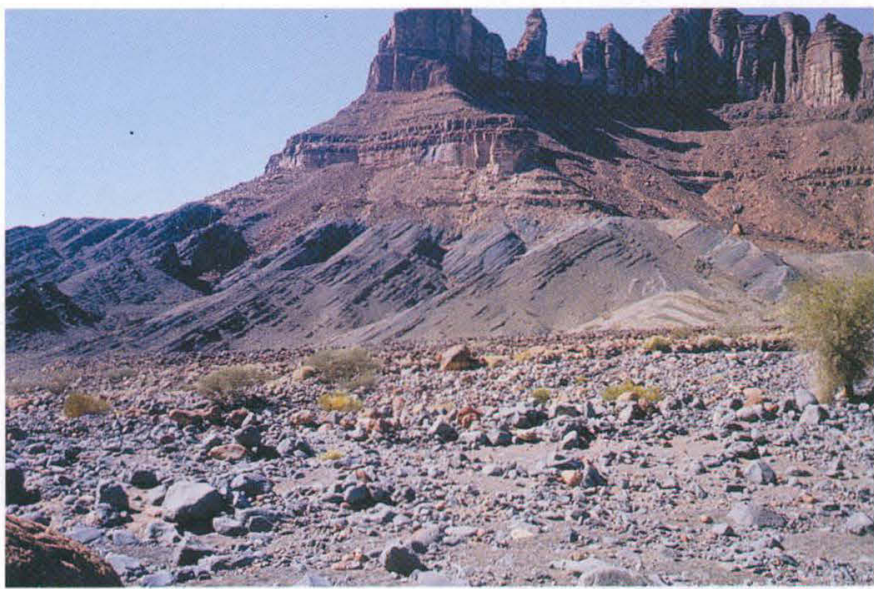

Figure 12B.-Slate and shale of the Hadiyah Group beneath the flaggy Cambrian Siq Sandstone, which in turn underlies the pinnacled Ram-Umm Sahm Sandstone. North edge of the Arabian Shield.

Formation. Baubron and others (1976) considered the younger ages that they obtained for the Murdama to result from rehomogenization of the argon. We are inclined to consider the rhyolite at the center of Jabal al Murdamah disconformable above the Murdama and belonging to the younger Shammar Group, which is dominantly alkalic flows. The Murdama Formation was raised to group rank by Delfour (1977) to include basal conglomerate and flows (Hibshi Formation), limestone and marble (Farida Formation), and the upper sandstone-siltstone facies (Hadiyah Formation).

East of Jabal al Murdamah in Jibāl al 'Alam, the Murdama includes 500-800 m of fine-grained andesite at the core (top) of the Maslum syncline. The syncline is bisected by a posttectonic calc-alkalic granite which, although not isotopically dated, is similar to granites throughout the northeast shield, with $\mathrm{K}-\mathrm{Ar}$ dates of about 600 m.y. The Murdama has schistosity indicating two episodes of folding, an earlier episode resulting in low-grade greenschist facies and a later compression during the Najd faulting at about 570 m.y. A third, possibly older, tectonic epoch involves the Abt paraschists which conformably overlie the Ar Ridaniyah calcareous unit in the Ad Dawādimī district at the eastern edge of the shield (Al-Shanti, 1976). The Abt paraschists and the Ar Ridaniyah unit are combined on the geologic map (pl. 1) as the Abt Schist. The lower part of the Ar Ridaniyah is gneissic and quartzfeldspathic schist which can be correlated with the Hibshi Formation, at least in part, whereas the upper calcareous metasedimentary rocks are most likely the extension of the Farida marble underneath the $\mathrm{Al}$ Amar-Idsas fault. These beds all dip east except where invaded by posttectonic quartz monzonite and granite or where local folds reverse the dip. The Farida marble in outcrop has been squeezed out of a stratigraphic position above basal conglomerate and graywacke, in places transgressing tectonically over the older Halaban crystalline rocks to overlap the Hibshi conglomerate.

Chitinozoan-like microfossils from dolomite at Jabal Rukhām tentatively correlated with the Murdama Group are similar to forms in the upper Riphean of Greenland and suggest that the span 638-600 m.y. for the Murdama Group from K/Ar ratios is reasonable (Vidal, 1979; Binda and Bokhari, 1980).

\section{SHAMMAR GROUP}

The alkalic and peralkalic volcanic rocks of the Shammar Rhyolite and associated gently folded sedimentary beds were considered (Brown and Jackson, $1960)$ to be the youngest Precambrian rocks in the shield (fig. 13A). The sialic, essentially unmetamorphosed volcanic rocks, including flows and tuffs, which are increasingly younger toward the northern edge of the shield, have been divided into two formations (Delfour, 1967). In the northwest Hejaz area, Brown, Jackson, Bogue, and Elberg (1963) later recognized sedimentary rocks interbedded with and above the Shammar, as well as rhyolite and fine-grained granite in dikes and stocks that intrude the Shammar. The younger sedimentary beds, including some flow rocks, were later separated and assigned to the Jubaylah Group by Delfour (1967, 1970), who found the outcrops restricted to long, narrow shear zones or grabens of the northwest-trending Najd fault system.

The radiometric timespan was not closely defined by our early work (Brown and Jackson, 1960). If the age $633 \pm 15$ m.y. obtained by Hedge (samples 94, 106, table 7) is considered the end of the Murdama epoch, the Shammar could represent volcanism from that time until about $555 \pm 25 \mathrm{~m}$.y., the date obtained for the Shammar by Baubron and others (1976, fig. 5) using $\mathrm{Rb} / \mathrm{Sr}$ data. The rhyolite $(561 \pm 25$ and $560 \pm 20$ m.y. whole-rock K-Ar; samples 141a, 141b, table 8) at the center of the Jabal al Murdamah syncline is most likely post-Murdama and a later phase of the Shammar rhyolitic volcanism, but hydrothermal alteration in one sample may have caused argon loss and a deceptively young age. The interval 633 to about 555 m.y. was also a period of widespread felsic plutonic activity. Older components are calc-alkalic monzogranitic batholiths; younger components include transgressive plugs and stocks that increase in alkalinity to peraluminous and peralkalic intrusives, including ring dikes. A comagmatic series has been found to range in age from about 620 


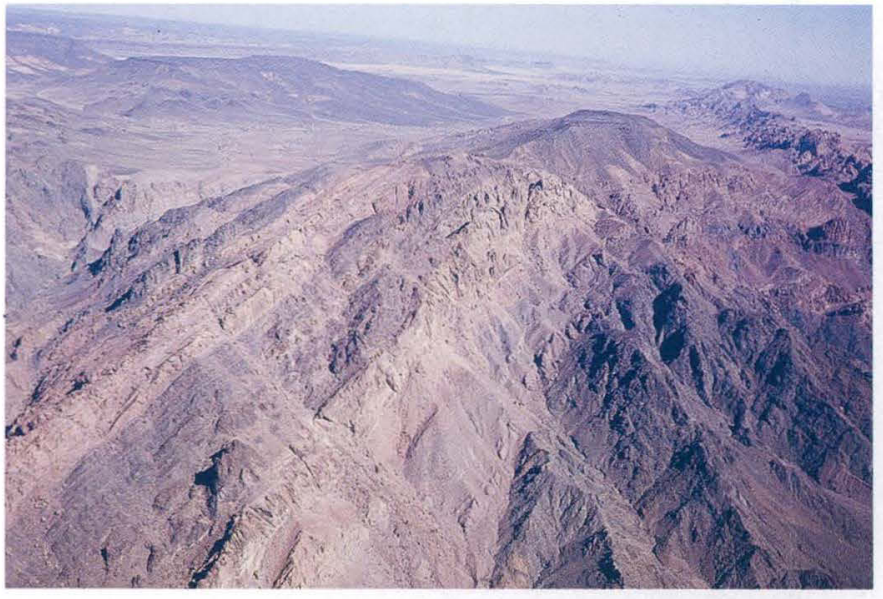

A

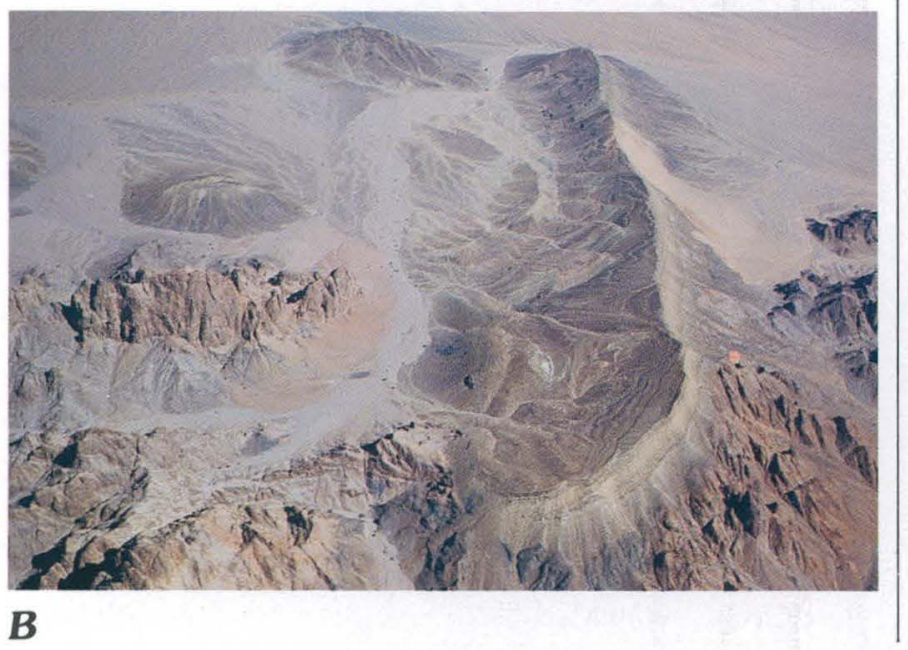

to about 550 m.y. (Fleck and others, 1976; Aldrich and others, 1978; Schmidt and others, 1979). This activity appears to have culminated about 570 m.y.

In the region southwest of Hsâ'il in the Nuqrah quadrangle, Delfour (1977) found two formations in the Shammar-the basal Kuara Formation with a basal conglomerate, upper clastic, rhyolite and andesite; and the upper Malha Formation, mostly rhyolite flows and ignimbrites. The oldest reported age, $621 \pm 25$ m.y., came from the Malha Formation from six $\mathrm{Rb}-\mathrm{Sr}$ measurements (Baubron and others, 1976; Delfour, 1977), thus indicating that the Murdama Group and the older portions of the Shammar Group are essentially the same age, eruptions of Shammar volcanics continuing at least to the end of the Precambrian.

\section{JUBAYLAH GROUP}

Sedimentary beds and flows originally mapped above and interbedded with the upper Shammar rhyolite (Brown, Jackson, Bogue, and Elberg, 1963) were later

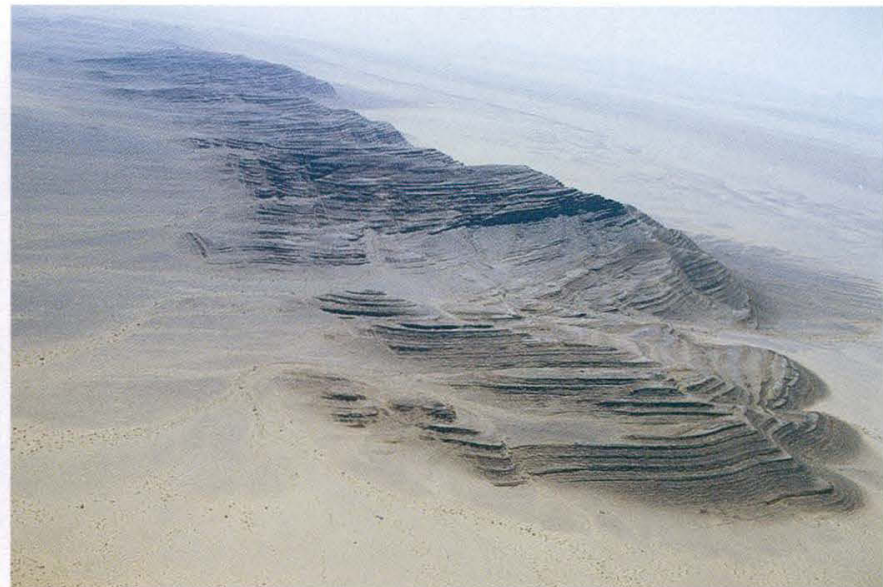

C

Figure 13.-Shammar and Jubaylah Groups. A, Shammar Group. Jabal Garra'ah (lat $27^{\circ} 15^{\prime}$ N., long $36^{\circ} 49^{\prime}$ E.) is composed of lithic tuff and andesite (in foreground); rhyolite dike in background. Jubaylah Group sediments and thin flows overlie these beds on the left (northern) flank. $B$, Jubaylah Group at Jabal Na'adhah, Sahl al Maţrān quadrangle, at lat $26^{\circ} 25^{\prime}$ N., long $38^{\circ} 13^{\prime}$ E.; view southeast. Rhyolite on lower slope, in the Jubaylah, yielded a K-Ar cooling date of $528 \pm 20$ m.y. from biotite (sample 40 , table 8), whereas the rhyolite on the right below the Jubaylah layered rocks yielded an average age of $574 \pm 6.7 \mathrm{~m} . \mathrm{y}$. from biotite for the underlying Shammar volcanics (Fleck and others, 1976). C, The Jubaylah Group at Jabal Antāq near the north end of Ḩalabān ridge and near the east edge of the shield at lat $24^{\circ} \mathrm{N}$. A pebble conglomerate at the base underlies sandstone, siltstone, and shale, here dipping east. Andesite interbedded at the type locality $380 \mathrm{~km}$ northwest is dated at $558 \pm 6 \mathrm{~m} . \mathrm{y} .\left({ }^{40} \mathrm{Ar} /{ }^{39} \mathrm{Ar}\right)$, the end of the Precambrian. Geochronology by J.F. Sutter.

separated and recognized as a distinct unit above an unconformity (Delfour, 1967). They are the youngest rocks involved in the diastrophism of the Arabian Shield (figs. 13A-13C)

Outcrops are along and on top of the three principal Najd fault zones in the northern part of the shield, where underlying schistose rocks have been eroded to form troughs or faulted to form grabens. Subsequent horizontal movement and some vertical movement has folded the Jubaylah beds into taphrogeosynclinoria, in which steeper limbs on the northeast flanks reflect the sinistral movement of the Najd faults (Delfour, 1970; Hadley, 1974). The thicknesses of exposures vary, owing in part to subsequent erosion; they range from $155 \mathrm{~m}$ at Wādi Murdan (lat $27^{\circ} 40^{\prime} \mathrm{N}$.) near the northwest corner of the shield, where the group dips under the Siq Sandstone, to $750-850 \mathrm{~m}$ at the Mashhad area (Hadley, 1974) (fig. 13B ), and to $2,300 \mathrm{~m}$ at Qal'at aş Şawrah (Hadley, 1975b) west of Hुarrat Khaybar. Such great thickness could also be explained by irregular, fault-controlled deposition and the proximity to 
eruptive centers. At the type locality east of Hुarrat Khaybar, the thickness of two formations of the Jubaylah Group is more than 3,300 m (Delfour, 1977). The group extends discontinuously southeast to Jabal Antāq on the west flank of Samrā' Hुalabān at lat $23^{\circ} 50^{\prime} \mathrm{N}$., long $44^{\circ} 10^{\prime} \mathrm{E}$. Along the northeast Najd shear zone and at lat $22^{\circ} 40^{\prime} \mathrm{N}$., long $44^{\circ} \mathrm{E}$., along the middle Najd zone, the thickness is about $320 \mathrm{~m}$.

The Jubaylah Group was divided informally by Delfour (1967) at the type locality east of Hुarrat Khaybar into three units-a basal conglomerate; andesite, basalt, and welded silicic tuff; and a cherty limestone containing stromatolitic structures. Later he named two units, the basal Umm al Aisah and the upper Jifn Formation, but, recognizing that flows and ejecta occurred at various horizons in the section, he did not assign a formal name to the volcanic part (Delfour, 1977). West of Harrat Khaybar, Hadley (1973) found a similar sequence which he named, from base upward, the Rubtayn (conglomerate and fine clastics), Badayi (andesite), and Muraykhah (limestone and shale). West of the Mashhad area described by Hadley (1974) the Jubaylah crops out near Al 'Ulā in small areas along Wādī al Jizl and in Wādī Falqah $32 \mathrm{~km}$ west. The wadi follows a Najd fault zone where the following section is exposed: about $100 \mathrm{~m}$ of polymict conglomerate; andesitic tuff and agglomerate; siliceous beds with calcareous concretions and lithographic limestone; intraformational conglomerate containing clasts of the limestone; and an uppermost tuffaceous green shale. Fifty kilometers farther northwest, in Wādī al Jizl west of Ḩarrat al 'Uwayrid, $85 \mathrm{~m}$ of the lower part of the Jubaylah crops out beneath a rhyolite agglomerate thrust block. The beds are red sandstone and polymict conglomerate. Fifteen kilometers east of the above exposure, the basal conglomerate and sandstone are $64 \mathrm{~m}$ thick below $118 \mathrm{~m}$ of thin-bedded and fetid limestone, paper shale, siltstone, and chert. Ripple and rain-splatter marks in the upper beds suggest a shallow-water and beach environment of deposition.

One hundred fifty kilometers farther northwest, at Wādī Murdan and beyond in Ash Shifā', the beds are polymict conglomerate and trachytoidal andesite porphyry. Along the southwestern flank of Wādi as Sirr in the middle zone of the Najd fault system near where it intersects the Red Sea at lat $27^{\circ} \mathrm{N}$., thick red and green shale and fine-grained sandstone overlie a thick polymict conglomerate wherein are clasts from all the older rocks. Originally mapped as Shammar (Brown, Jackson, Bogue, and Elberg 1963), the strata are probably of Jubaylah age, even though schistosity in some zones approaches that of the underlying Murdama Group.

Toward the southeast end of Jubaylah outcrops, near the eastern edge of the shield at Jabal Antāq $(900 \mathrm{~km}$ southeast of Ash Shifä'), wacke, shale, and siltstone dip $25^{\circ}-30^{\circ} \mathrm{E}$. above gray and red ripple-marked sandstone and a basal conglomerate of white quartz pebbles (fig. $13 C$ ).

The lava flows throughout the exposures of the Jubaylah are petrographically different from older outpourings and are of interest for possible radiometric dating of the Jubaylah Group. They are composed of andesite, dacite, alkalic basalt (mugearite), and rhyolite or lithic tuffs (minor), are generally porphyritic, often with large $(2 \mathrm{~cm})$ plagioclase phenocrysts $\left(\mathrm{An}_{40-60}\right)$, and are amygdaloidal. Amygdule minerals include quartz (cristobalite?) and calcite, with lesser amounts of barite, celestite, and nepheline (Delfour, 1970). Hadley (1974) described devitrified glass and chlorite, chlorophoenicite, epidote, and calcite; opaque iron-rich minerals are common in all sections except the crystal tuffs. Chlorite, epidote, and zeolites are products of hydrothermal alteration in one rock (see sample 67, an altered andesite, tables $3,4,8)$.

Two samples (samples 67, 69, table 8) from Delfour's type locality gave whole-rock K-Ar ages of $299 \pm 11$ m.y. for the lower, hydrothermally altered flow containing deuteric calcite (R. Marvin, written commun., 1972) and $548 \pm 18$ m.y. for the dacite or alkalic andesite flow. The younger apparent age is most certainly unreliable, probably owing to hydrothermal alteration. To check the possible validity of the $548 \pm 18$ m.y. apparent age for the fresh dacite (sample 69, table 8), John Sutter (written commun., 1982) kindly made an ${ }^{40} \mathrm{Ar} /{ }^{39} \mathrm{Ar}$ agespectrum analysis of the dacite (fig. 14) and comments as follows:

${ }^{40} \mathrm{Ar} /{ }^{39} \mathrm{Ar}$ age spectrum plateau age $=558 \pm 6.6 \mathrm{~m} . \mathrm{y}$. where the $6.6 \mathrm{Ma}$ error is reported at the 2 sigma level of confidence ( 95 percent). The ${ }^{40} \mathrm{Ar} /{ }^{39} \mathrm{Ar}$ total gas age is $534 \mathrm{Ma}$ which should be essentially equivalent to the conventional $\mathrm{K} / \mathrm{Ar}$ age for this sample.

The age spectrum indicates loss of ${ }^{40} \mathrm{Ar}$ from the sample by volume diffusion from potassium-bearing mineral phases. I suggest that the major potassium-bearing phase is a feldspar and if so the apparent age of the first two temperature steps on the age spectrum $\left(350-425{ }^{\circ} \mathrm{C}\right.$ ), about $465 \mathrm{Ma}$, represents the last time the sample cooled through $100-150{ }^{\circ} \mathrm{C}$, the temperature range below which feldspars tend to retain most of their radiogenic argon.

A modal analysis of the dacite by one of us (A.C.H.) gave 16 percent potash feldspar.

A whole-rock K-Ar age of $515 \pm 17$ m.y. (sample $22 \mathrm{~b}$, table 8) from a potassium-rich mafic flow could be reliable but could also be low owing to argon loss. The chemical analysis (table 4) shows that the rock is oxidized mugearite (oligoclase-andesine basalt), according to George Phair (USGS, written commun., 1976). At the Mashhad area, Fleck and others (1976) determined $\mathrm{K}$-Ar ages of $567 \pm 6$ and $581 \pm 7$ m.y. for biotite in rhyolite of the Shammar Group ostensibly underlying the Jubaylah Group, although a fault separates the 


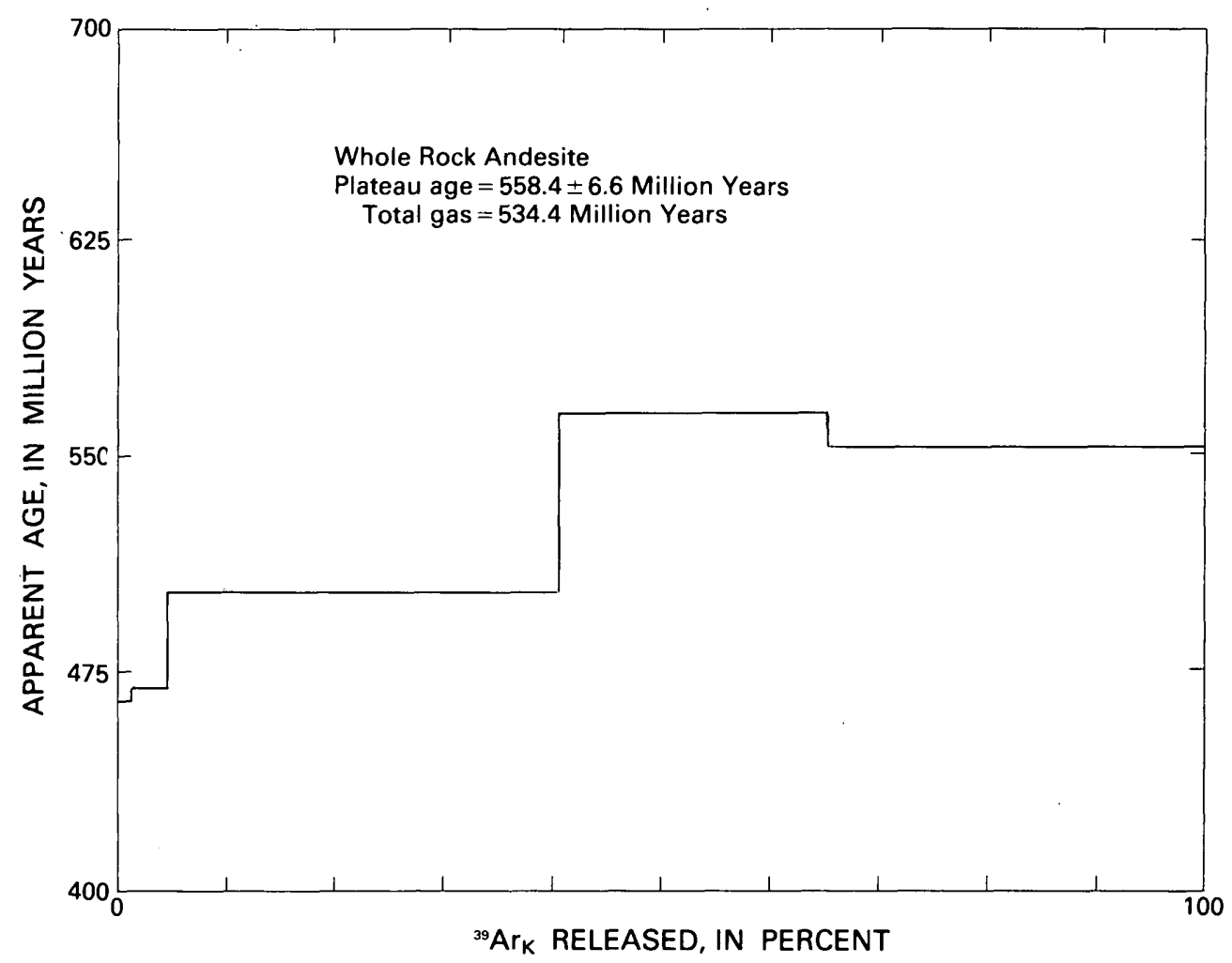

AGE OF SPECTRUM DATA

\begin{tabular}{|c|c|c|c|c|c|c|c|c|}
\hline TEMP & 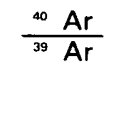 & ${ }^{37} \mathrm{Ar}$ & $\begin{array}{ll}{ }^{36} \mathrm{Ar} \\
{ }^{39} \mathrm{Ar}\end{array}$ & $\begin{array}{c}39 \mathrm{Ar} \\
\% \text { of } \\
\text { TOTAL }\end{array}$ & $\begin{array}{c}{ }^{40} \mathrm{Ar}^{*} \\
\%\end{array}$ & $\begin{array}{c}39 \mathrm{Ar} \\
\text { (mole) }\end{array}$ & $\begin{array}{c}\text { APPARENT } \\
\text { K/Ca } \\
\text { mole } \\
\text { mole }\end{array}$ & $\begin{array}{c}\text { APPARENT } \\
\text { AGE } \\
\text { MILLION YEARS }\end{array}$ \\
\hline $\begin{array}{l}350 \\
425\end{array}$ & $\begin{array}{r}278.124 \\
66.237\end{array}$ & $\begin{array}{l}5.184 \mathrm{E}-01 \\
3.015 \mathrm{E}-01\end{array}$ & $\begin{array}{l}8.234 \mathrm{E}-01 \\
1.048 \mathrm{E}-01\end{array}$ & $\begin{array}{l}1.2 \\
3.4\end{array}$ & $\begin{array}{l}12.5 \\
53.3\end{array}$ & $\begin{array}{l}1.34 E-13 \\
3.67 E-13\end{array}$ & $\begin{array}{l}1.00 E+00 \\
1.72 E+00\end{array}$ & $\begin{array}{l}464.90 \pm 98.90 \\
469.89 \pm 8.64\end{array}$ \\
\hline $\begin{array}{r}500 \\
850 \\
\text { FUSE }\end{array}$ & $\begin{array}{l}45.951 \\
45.840 \\
48.075\end{array}$ & $\begin{array}{l}3.477 E-01 \\
4.055 E-01 \\
1.355 E+00\end{array}$ & $\begin{array}{l}2.669 \mathrm{E}-02 \\
7.577 \mathrm{E}-03 \\
1.898 \mathrm{E}-02\end{array}$ & $\begin{array}{l}35.9 \\
24.7 \\
34.7\end{array}$ & $\begin{array}{l}82.9 \\
95.2 \\
88.5\end{array}$ & $\begin{array}{l}3.90 E-12 \\
2.69 E-12 \\
3.77 E-12\end{array}$ & $\begin{array}{l}1.50 E+00 \\
1.28 E+00 \\
3.84 E-01\end{array}$ & $\begin{array}{l}502.55 \pm 3.14 \\
565.27 \pm 3.25 \\
553.44 \pm 3.31\end{array}$ \\
\hline SAMPLE V & $383 g$ & & & & & & $\begin{array}{r}\text { TOTAL GAS } \\
\text { PLATEAU AGE }\end{array}$ & $\begin{array}{l}534.43 \\
558.37 \pm 3.28\end{array}$ \\
\hline
\end{tabular}

FIGURE 14.-Incremental ${ }^{39} \mathrm{Ar}_{\mathrm{K}}$ of the Jubaylah andesite at the type locality of the Jubaylah Group. Analysis by J.F. Sutter (written commun., 1983).

beds (Hadley, 1973). A sample of biotite from andesite, presumably a flow in the Jubaylah, at the Mashhad area gave a K-Ar age of $528 \pm 20$ m.y., but the stratigraphic position is not certain. Also, a K-Ar age of $532 \pm 15$ m.y. was determined for a whole-rock sample from a basalt dike $41 \mathrm{~km}$ east of the Mashhad area where the dike crops out beneath the unconformity below the Siq Sandstone of probable Late Cambrian age. Baubron, Delfour, and Vialette (1976) measured whole-rock K-Ar ages of 502 and 512 m.y. for flows from the Jubaylah; Delfour suggests that these dates are too young and reflect heating and argon loss during subsequent eruptions. Certainly almost all the samples we have examined show some hydrothermal alteration, but the question of whether this alteration was penecontemporaneous with the eruptions or was related to some later episode remains unanswered, even though much of the evidence points to early hydrothermal metamorphism. The alteration of the lavas studied raises questions about the geological accuracy of the radiometric ages.

The Jubaylah Group as now exposed appears to be of local derivation, even though the sequence in general is similar from basin to basin. Deposition began with 


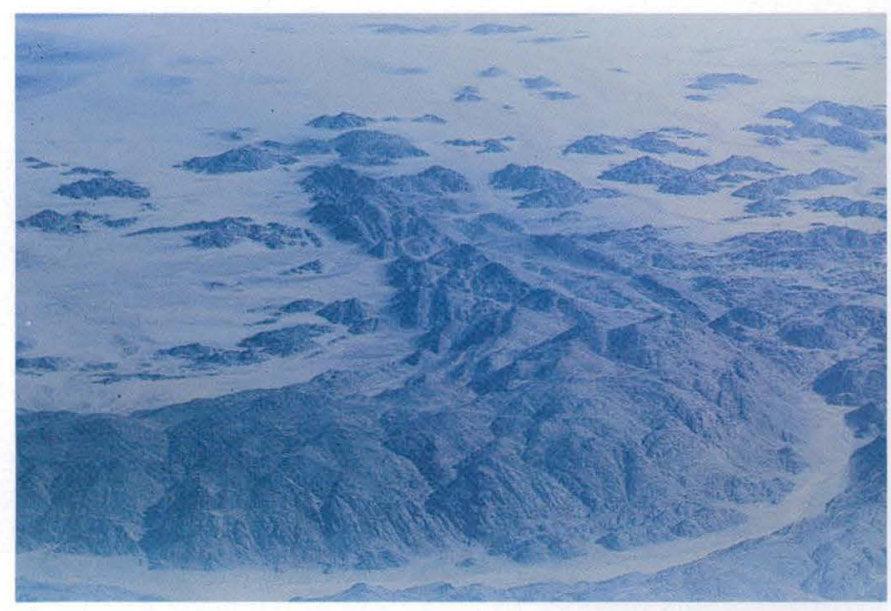

A

FIGURE 15.-A, Aerial view to the southeast of Jabal Huassan (lat $24^{\circ} 01^{\prime} \mathrm{N}$., long $45^{\circ} 08^{\prime}$ E.) at the east edge of the shield. The arcuate antiformal ridge is composed of Halaban metasediments graded through epidote schist to amphibolite around the Al Mizil orthogneiss (tonalite-trondhjemite) which underlies the central plain. The dome probably represents anatectonic reactivation of

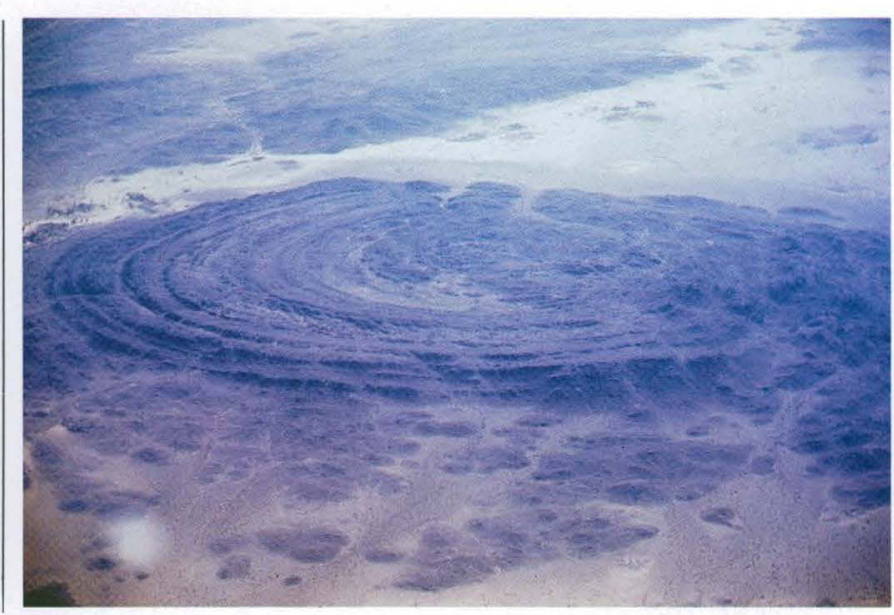

B

Halaban crust at about 600 m.y. ago or possibly later (Fleck and Hadley, 1985). B, Jabal Shãyi layered gabbro pluton in the southeastern shield near the village of Khaybar at lat $18^{\circ} 47^{\prime} \mathrm{N}$., long $42^{\circ} 53^{\prime} \mathrm{E}$. This carefully studied and drilled pluton yielded an age of 616 m.y. from ${ }^{40} \mathrm{Ar} /{ }^{39} \mathrm{Ar}$ in hornblende, with a heating event at about 510 m.y. (Coleman, Ghent, and others, 1977). coarse fluvial clastics, probably fanglomerate, ended with stromatolitic shallow-water marine beds, and was interrupted at various levels by flows of basalt, andesite, and rhyolite or by lithic tuffs. Some mafic small and hypabyssal crystalline intrusives may be of Jubaylah age.

Stromatolitic mats in chert from the Jubaylah Group at Jabal Umm al 'Aisah were studied and found to contain filaments of the blue-green algae Obruchevella parva, Reitlinger, a conical stromatolite, Conophyton, and unicells of uncertain affinity (Cloud and others, 1979). The paucity of fauna suggests that the Jubaylah Group is near the lower boundary of the Phanerozoic or latest Precambrian (Vendian). The disconformable stratigraphic position below the Siq Sandstone, which in turn is subjacent to or part of fossiliferous sandstone considered Upper Cambrian by Seilacher (1970), allows room for the radiometric dates to be true time of deposition and volcanic eruption, but the Ediacarian fauna in the cherty upper beds of the group, together with the hydrothermal alteration and possible argon loss in the volcanic samples, argue for an earlier, possibly earliest Cambrian or latest Precambrian, age. Subsequent to the above-reported age estimates, Pier Binda and C.R. Ramsay (1980) point out from BRGM work (Baubron and others, 1976) that the Jubaylah is disconformable above the granite of Jabal ar Rahadah, which is dated at $577 \pm 15$ m.y. by a seven-point Rb-Sr whole-rock isochron (corrected to the Sydney constants). The granite is intruded into the Murdama Group. Binda (1981) concludes that the Jubaylah Group is probably uppermost Vendian (600-570 m.y.). A minimum age younger than 570 m.y. does not invalidate Cloud's and Binda's conclusions, and the spectral age of $558 \pm 6.6$ m.y. of Sutter (fig. 15) seems most logical from all the evidence.

\section{PRECAMBRIAN PLUTONIC ROCKS OF THE ARABIAN SHIELD}

The plutonic rocks of the Saudi Arabian Shield are divided into an older, pretectonic dioritic suite (fig. $15 \mathrm{~A}$ ) and a younger, syntectonic and posttectonic, mostly granitic suite which includes an early layered gabbroic phase (fig. 15B). Greenwood and Brown (1973, p. 6) estimated that the percentage of granitic to graniticplus-dioritic rocks, that is, granitic rocks to total plutonic rocks, is 32,38 , and 13 , respectively, in the northeast, central, and southwest regions of the shield as mapped on the 1:2,000,000-scale geologic map (USGSARAMCO, 1963).

The percentage of granitic rocks relative to dioritic rocks exposed in different parts of the shield is a function of orogenic intensity and depth of erosion. Greater tectonism and less erosion complement each other in producing and preserving, respectively, more granitic rocks in the northeastern part of the shield. Late major tectonism associated with granite emplacement was most intense in the eastern and northeastern parts of the shield relative to the western and southwestern parts and resulted in a greater abundance of granitic plutons in the northeast. The depth of erosion 
is shallower in the northeastern part of the shield than in other parts (Brown and Jackson, 1960). This is indicated by abundant exposures of the latest Proterozoic rhyolitic Shammar Group; in places, as at Jabal Aja', granitic intrusive rocks are still preserved in direct, subvolcanic contact with the Shammar volcanic rocks (Stoeser and Elliott, 1980). In the central part of the shield, in a belt about $200 \mathrm{~km}$ wide and including the major Najd fault zones, late orogeny was intense and the erosion level is moderately shallow in that many late granitic plutons are exposed with few cogenetic rhyolitic rocks of the Shammar Group. The southern part of the shield is deeply eroded and was less deformed during the late major orogeny, so that this region has the smallest area of granitic plutons and only rare exposures of Shammar-type volcanic rocks.

In addition to the granitic and dioritic suites, a maficultramafic suite of plutonic rocks is present and is characterized by serpentinite in association with ultramafic, gabbroic, basaltic, and diabasic rocks. The maficultramafic suite is confined mostly to narrow belts in large fault zones, is independent of the dioritic-suite rocks, and makes up less than 1 percent of the Precambrian plutonic rocks of the shield. The maficultramafic suite is ophiolite that rarely is complete at any one locality and that probably represents tectonic remnants of oceanic crust of different ages in different places (Bakor and others, 1976; Frisch and Al-Shanti, 1977; Delfour, 1979b; Al-Rehaili and Warden, 1980).

In general, the plutonic rocks of the dioritic and granitic suites are petrographically easily classified into standard rock types. In this report, "granitic" refers to leucocratic plutonic rocks containing more than 5 percent modal potassium feldspar and more than 20 percent modal quartz, thus avoiding the broader term "granitoid." Rock names in this report are those of the classification of plutonic rocks of the International Union of Geological Sciences, Subcommission on the Systematics of Igneous Rocks (Streckeisen, 1973, 1976).

The plutonic rocks are further classified as pretectonic, syntectonic, and posttectonic in reference to a culminant orogeny (dated at about $650 \pm 25$ m.y.), during which most of the rocks of the early (primary) crust were intensely tectonized during the cratonization of the shield (dated at about 675 to 560 m.y.). This tectonic classification in reference to the culminant orogeny is used in spite of the fact that most of the rocks of the primary crust had been variously deformed and metamorphosed prior to the culminant orogeny.

\section{DIORITIC SUITE}

The dioritic suite consists, in order of decreasing abundance, of diorite, quartz diorite, tonalite, trondhjemite, and gabbro. These rocks were emplaced be- tween about 1,000 and 700 m.y. ago (Greenwood and others, 1976; Fleck and others, 1980) and, together with their equivalent volcanic rocks of the andesite assemblage, constitute the primary crust that represents most of the early (precratonization) crust of the shield. Rocks of the dioritic suite are pretectonic in reference to the culminant orogeny.

The plutonic rocks of the dioritic suite are commonly medium grained and hypidiomorphic granular. The diorite contains hornblende with or without varying amounts of biotite and quartz. The quartz diorite and tonalite are quartz-biotite rocks with or without hornblende, and the trondhjemite is a quartz-biotite rock. The gabbro commonly contains clinopyroxene, partly altered to hornblende, and hornblende, partly altered to biotite. In many places, all these plutonic rocks have finer and coarser grained phases and their overall heterogeneous character suggests a shallow level of intrusion. In particular, the diorite commonly has mineral compositions and textures that are variable and heterogeneous even at outcrop and hand-specimen scale, which suggests that some of the diorite was hypabyssal and subvolcanic to overlying andesitic volcanic rocks. Locally, the trondhjemite is graphic or granophyric and contains euhedral quartz phenocysts, which suggests that some trondhjemite is hypabyssal and subvolcanic to overlying dacitic volcanic rocks.

These early rocks were mostly well tectonized, pervasively sheared to a commonly north-trending foliation, and metamorphosed to the greenschist facies before the culminant orogeny and cratonization, when they were tectonically mixed with their volcanic equivalents, the andesitic assemblage, and abundantly intruded by rocks of the granitic suite.

Many of the low-density, more silicic rocks of the dioritic suite, such as the trondhjemites and some tonalites, are found in large gneiss domes, or antiforms, where they had been metamorphosed to amphibolite facies, conspicuously shear foliated, and converted to orthogneisses. These gneiss domes rose gravitationally in response to heating and tectonic thickening during the culminant orogeny. The orthogneisses are tectonically associated with migmatitic and plutonic rocks of the granitic suite.

During past mapping, many granitic gneisses and orthogneissic rock units have been variously depicted as basement rocks older than rocks of the adjoining dioritic suite in any given area. However, none of the isotopic ages of these gneisses has proven to be older than the associated dioritic suite and andestic assemblage rocks. Where well dated, all yield dates related to orogenies that are younger than the dioritic suite in their respective regions. The orthogneisses are in gneiss domes or large fault structures, and most were tectonized and mobilized during the culminant orogeny. 


\section{GRANITIC SUITE}

The granitic suite consists, in order of decreasing abundance, of granodiorite, granite, alkali-feldspar granite, and peraluminous-peralkalic granite, as well as gabbro. The rocks of the granitic suite are exposed throughout the Precambrian shield and are most abundant in the northeastern half of the shield. Alkalifeldspar granite and peralkalic granite have not been found in the southwestern part of the shield (Stoeser and Elliott, 1980); however, several plutons of syenite and shonkinite intrude this part of the shield. Most of these rocks were emplaced during cratonization after about $675 \mathrm{~m} . y$. ago. Many granitic rocks are slightly to extensively tectonized and partly metamorphosed through pervasive cataclastic shear and are classified as syntectonic; these were intruded during the culminant orogeny. Other granitic rocks were intruded in a posttectonic setting from after the culminant orogeny to about the end of the Precambrian. They commonly produce contact metamorphic aureoles in the adjacent wall rock. A relatively few granitic rocks in the western half of the shield were intruded earlier than the culminant orogeny, during more spatially restricted orogenies, for example, about the time of deposition of the Ablah group of rocks.

The syntectonic granitic rocks commonly form large batholiths of predominantly biotite granodioritic composition and are commonly associated with the large gneiss domes of tonalitic and trondhjemitic orthogneiss. The batholithic granodiorite is mostly conspicuously flow foliated as well as cataclastically sheared in response to late orogenic processes.

Early in the posttectonic setting, biotite granite (monzogranite and syenogranite) was intruded as large, irregularly shaped plutons. Presumably with increasing depth of erosion and the resultant ease of brittle fracture at shallow crustal levels, circular plutons of biotite-perthite granite, a few kilometers to as much as $10 \mathrm{~km}$ is diameter, were emplaced somewhat later, about 600 m.y. Many of these are ring structured where they intruded a preexisting structure, or perhaps where some late orogenic forces controlled their emplacement. Some of these circular and elliptical plutons are composed of biotite-perthite alkali-feldspar granite and sodic amphibole peralkalic granite. Some are peraluminous granites. Not all the late, small granitic plutons are circular or layered, but circular layered masses are conspicuous in most parts of the shield.

The posttectonic granitic magmas were bimodal. Some layered granite plutons contain partial concentric layers or irregular intrusive bodies of synchronously intruded basaltic, diabasic, or moderately mafic hybrid rocks. In addition, circular layered plutons of gabbro, commonly leucocratic and from a few kilometers to 10 $\mathrm{km}$ in diameter, are conspicuous throughout the shield (fig. 15B). These gabbroic rocks are late and nonmetamorphosed, and in a few places they can be seen to cut late granite plutons. That the late gabbro and the diabasic intrusions are intricately and synchronously associated with the circular granitic intrusions indicates that immiscible gabbroic magma was associated with granitic magma deep in the crust during cratonization.

The latest and shallowest granitic rocks of the Precambrian shield are granite to alkali-feldspar granite in small plutons or stocks in or adjacent to the Najd fault zone. Those in the Najd fault zone are readily dated as (1) being slightly younger than most Najd faulting where they cut the strongly sheared rocks of the fault zone and commonly form small circular stocks less than several kilometers in diameter, or (2) having been intruded during Najd faulting where the small plutons are greatly elongated (some elongation ratios to about 1:10) in the fault zone and where the granitic rock is highly flow foliated and sheared. These latter, synkinematic rocks are conspicuous granitic gneisses that often in the past were mapped as granitic orthogneisses of an old basement.

Many dikes of diabase and fewer dikes of rhyolite fill conjugate and secondary fractures complementary to the Najd faults in large areas between the Najd faults (Moore, 1979). Large swarms of diabasic dikes are most common in the large granitic batholiths. For example, granodioritic batholiths were systematically fractured, in contrast to the dioritic plutons, in which strains were relieved by ductal yield along preexisting structural grain. A few diabasic, gabbroic, and syenitic plugs less than $1 \mathrm{~km}$ in diameter intruded the Najd fault zones after fault movement had ceased.

\section{CHEMISTRY OF THE PRECAMBRIAN CRYSTALLINE ROCKS}

\section{INTRODUCTION}

The petrography and classification of 199 Precambrian volcanic and plutonic rocks that form the Saudi Arabian Shield and for which chemical analyses have been made are listed in numerical order in table 3 . The analyzed rock samples were collected during reconnaissance geologic mapping, mostly prior to the more detailed 1:100,000-scale mapping, and are from localities (pl. 1) well distributed over the entire shield. The last two samples, numbers 501 and 502, are of Precambrian crystalline rocks from the bottom of two deep drill holes through the Phanerozoic sedimentary rocks in the Eastern province of Saudi Arabia about $400 \mathrm{~km}$ east of the eastern edge of the shield; they are not shown on plate 1 . Chemical and normative analyses of the 199 Precambrian rocks are given in table 4.

Text continues on p. 65 . 
TABLE 3.-Description and classification of crystalline rocks of the Arabian Shield for which chemical analyses are given in table 4

\begin{tabular}{|c|c|c|c|c|c|c|c|c|c|c|c|c|c|c|c|c|}
\hline \multirow[b]{2}{*}{$\begin{array}{l}\text { Sample } \\
\text { (loc. } \\
\text { given } \\
\text { on pl. } \\
\text { 1) }\end{array}$} & \multirow[b]{2}{*}{$\begin{array}{l}\text { Location }{ }^{1} \\
\text { (lat/long) }\end{array}$} & \multirow[b]{2}{*}{$\begin{array}{c}\text { Rock-unit } \\
\text { symbol } \\
\text { on pl. } 1^{2}\end{array}$} & \multicolumn{9}{|c|}{ Petrography } & \multirow[b]{2}{*}{ Remarks" } & \multicolumn{4}{|c|}{ Classification } \\
\hline & & & Color & $\underset{\text { size }^{3}}{\text { Grain }} \mathrm{N}$ & $\begin{array}{l}\text { Normative } \\
\text { color } \\
\text { index }\end{array}$ & $\begin{array}{l}\text { Mafic } \\
\text { content } \\
\text { (percent) }\end{array}$ & $\begin{array}{l}\text { Plagio- } \\
\text { clase } \\
\text { compo- } \\
\text { sition }^{6}\end{array}$ & $\begin{array}{l}\text { Normative } \\
\text { anorthite } \\
\text { (percent) }\end{array}$ & $\begin{array}{l}\text { Potassium- } \\
\text { feldspar }^{\text {fype }} \\
\text { type }\end{array}$ & $\begin{array}{c}\text { Rock } \\
\text { texture- } \\
\text { structure }\end{array}$ & $\begin{array}{c}\text { Rock name } \\
\text { (field or } \\
\text { petrography) }\end{array}$ & & $\underset{\substack{\text { Igneous } \\
\text { type }^{12}}}{\text { In }}$ & $\begin{array}{l}\text { Rock name } \\
\text { from } \\
\text { chemistry }\end{array}$ & $\mathrm{Age}^{14}$ & $\begin{array}{l}\mathrm{Re} \\
\text { gion }^{15}\end{array}$ \\
\hline $1-8712$ & $28 / 35 \mathrm{NW}$. & $\mathrm{mgd}$ & Gray & - & 16 & $3, \mathrm{Bi}$ & Olig & 30 & Or,Pth & $\begin{array}{c}\text { Catacl, } \\
\mathrm{mr}\end{array}$ & Granodio & - & Plutonic & Granodio & $\mathrm{C}, \mathrm{S} ?+$ & $\mathrm{N}$ \\
\hline $3-\mathrm{B} 1813$ & $28 / 35 \mathrm{NW}$. & '[gr](ju) & - & - & 53 & - & - & 58 & - & - & Amphibo & $\begin{array}{l}\text { Jabal Maglah; contact meta- } \\
\text { morphic, volcanic }\end{array}$ & $\begin{array}{l}\text { Metamor } \\
\quad \text { (volcanic) }\end{array}$ & $\begin{array}{r}\text { Amphibo } \\
\text { (basalt) }\end{array}$ & $\mathrm{H}$ & $\mathrm{N}$ \\
\hline $4 a-A L D 3$ & $28 / 35 \mathrm{NW}$. & gr & - & $\mathrm{mg}$ & 2 & $\begin{array}{r}2, \mathrm{Bi} \\
\mathrm{Hb}\end{array}$ & Olig & 11 & Or,Pth & $\begin{array}{l}\text { Minor } \\
\text { catacl }\end{array}$ & Granite & $\begin{array}{l}\text { Near Jabal al Lawz; rock } \\
\text { similar to sample } 4 \mathrm{~b}\end{array}$ & Plutonic & Granite & M+ & $\mathrm{N}$ \\
\hline $4 b-B 1815$ & $28 / 35 \mathrm{NW}$. & gr & - & - & 2 & $2, \mathrm{Bi}$ & Olig & 11 & Or,Pth & $\begin{array}{l}\text { Minor } \\
\text { catacl }\end{array}$ & Granite & $\begin{array}{l}\text { Near Jabal al Lawz; rock } \\
\text { similar to sample } 4 \mathrm{a}\end{array}$ & Plutonic & Granite & M & $\mathrm{N}$ \\
\hline $\begin{array}{c}8-\mathrm{B} 1016 \\
\text { (ALD7) }\end{array}$ & 27/35NE. & gp & - & - & 2 & $1, \mathrm{Hb}$ & - & 0 & $\begin{array}{l}\text { Pth,Mc, } \\
\text { Or }\end{array}$ & - & Granite & $\begin{array}{l}\text { Jabal Harb; } 0.5 \mathrm{~km} \text { inside } \\
\text { west border of pluton }\end{array}$ & Plutonic & $\begin{array}{l}\text { Peralkalic } \\
\text { granite }\end{array}$ & $\mathrm{S}, \mathrm{N} ?+$ & $\mathrm{N}$ \\
\hline $9-\mathrm{B} 1011$ & $27 / 35 \mathrm{NE}$ & {$[j \mathrm{~d}](\mathrm{gp})$} & Red & $\mathrm{mg}$ & 7 & $5, \mathrm{Mt}$ & - & 0 & Pth & - & Granite & $\begin{array}{l}\text { Small satellitic plug related } \\
\text { to pluton of sample } 13\end{array}$ & Plutonic & $\begin{array}{l}\text { Peralkalic } \\
\text { granite }\end{array}$ & $\mathrm{S}, \mathrm{N} ?$ & $\mathrm{NN}$ \\
\hline $12 \mathrm{a}-\mathrm{B} 1009 \mathrm{~A}$ & 27/35SE. & ns & - & - & 3 & - & - & 3 & - & - & Syenite & $\begin{array}{l}\text { Circular pluton in Najd } \\
\text { fault zone }\end{array}$ & Plutonic & $\begin{array}{l}\text { Alkalic } \\
\text { quartz } \\
\text { syenite }\end{array}$ & $\mathrm{N}$ & $\mathrm{NN}$ \\
\hline $12 b-B 1009 B$ & 27/35SE. & $\mathrm{ns}$ & - & - & 1 & - & - & 0 & - & - & Syenite & Same pluton as sample $12 \mathrm{a}$ & Plutonic & $\begin{array}{l}\text { Peralkalic } \\
\text { quartz } \\
\text { syenite }\end{array}$ & $\mathrm{N}$ & NN \\
\hline $13-\mathrm{B} 1022$ & $27 / 35 \mathrm{NE}$ & $\mathrm{gp}$ & Gray & fg-mg & 6 & $\begin{array}{l}20, \mathrm{Hb} \\
\mathrm{Bi}, \mathrm{Mt}\end{array}$ & - & 0 & Pth & $\begin{array}{c}\text { Graphic } \\
\text { mr }\end{array}$ & Granite & $\begin{array}{l}\text { Center of large pluton in } \\
\text { Najd fault zone, Jabal } \\
\text { Shār }\end{array}$ & Plutonic & $\begin{array}{l}\text { Peralkalic } \\
\text { granite }\end{array}$ & $\mathrm{S}, \mathrm{N}$ ? & $\mathrm{NN}$ \\
\hline $14 a-B 1008 C$ & $27 / 35 \mathrm{NE}$ & $\mathrm{gp}$ & Red & - & 7 & - & - & 0 & - & - & Granite & $\begin{array}{l}\text { Near contact, same pluton } \\
\text { as sample } 13 \text {, Jabal Shār }\end{array}$ & Plutonic & $\begin{array}{l}\text { Peralkalic } \\
\text { granite }\end{array}$ & $\mathrm{S}, \mathrm{N} ?$ & $\mathrm{NN}$ \\
\hline $14 \mathrm{~b}-\mathrm{B} 1008$ & $27 / 35 \mathrm{NE}$ & gp & - & fg-mg & 3 & $\begin{array}{l}\text { 3,Bi, } \\
\quad \mathrm{Hb}, \mathrm{Mt}\end{array}$ & - & 0 & Pth,Mc & $\mathrm{Mr}$ & Granite & $\begin{array}{l}\text { Same pluton as sample 13, } \\
\text { Jabal Shār }\end{array}$ & Plutonic & $\begin{array}{l}\text { Peralkalic } \\
\text { granite }\end{array}$ & $\mathrm{S}, \mathrm{N}$ ? & $\mathrm{NN}$ \\
\hline $15-\mathrm{B} 1023$ & $27 / 35 \mathrm{NE}$ & $\mathrm{gp}$ & - & fg & 2 & $2, \mathrm{Mt}$ & - & 0 & Mc,Pth & Microgr & Granite & $\begin{array}{l}\text { Jabal Shār; large sill or lac- } \\
\text { colith on Najd fault }\end{array}$ & Hypabys & $\begin{array}{l}\text { Peralkalic } \\
\text { granite }\end{array}$ & $\mathrm{S}, \mathrm{N}$ ? & $\mathrm{NN}$ \\
\hline $17-\mathrm{B} 1007$ & 27/35NE. & gp & Red & $\mathrm{mg}$ & 1 & $1, \mathrm{Hm}$ & - & 0 & $\begin{array}{l}\text { Mc,Pth, } \\
\text { Or }\end{array}$ & Microgr & Granite & $\begin{array}{l}\text { Jabal Shār; same pluton as } \\
\text { sample } 13\end{array}$ & Plutonic & $\begin{array}{l}\text { Peralkalic } \\
\text { granite }\end{array}$ & $\mathrm{S}, \mathrm{N}$ ? & NN \\
\hline $18-\mathrm{B} 1044$ & $27 / 36 \mathrm{NW}$ & rt & Red & fg & 3 & - & - & 4 & - & Porphyr & $\begin{array}{c}\text { Rhyolite } \\
\text { porph }\end{array}$ & $\begin{array}{l}\text { Intrudes greenstone; intrud- } \\
\text { ed by diabase dikes }\end{array}$ & Hypabys & Rhyolite & $\mathrm{s}$ & $\mathrm{N}$ \\
\hline $19-\mathrm{B} 1046$ & $27 / 36 \mathrm{NW}$ & nj & - & - & 23 & - & - & 37 & - & Trachyt & Andesite & $\begin{array}{l}\text { Associated with Najd fault; } \\
\text { basaltic andesite? }\end{array}$ & Volcanic & $\begin{array}{l}\text { Alkalic ba- } \\
\text { salt }\end{array}$ & $\mathbf{K}$ & $\mathrm{N}$ \\
\hline $20-\mathrm{B} 1052$ & $27 / 36 \mathrm{NW}$. & nj & - & - & 7 & - & - & 32 & - & $\begin{array}{l}\text { Welded } \\
\text { tuff }\end{array}$ & Rhyolite & $\begin{array}{l}\text { Clast in basal conglomerate } \\
\text { on Najd fault }\end{array}$ & Volcanic & K-rhyolite? & $\mathrm{S}$ & $\mathrm{N}$ \\
\hline $21-\mathrm{B} 1047$ & $27 / 36 \mathrm{NW}$ & $\mathrm{gp}$ & $\begin{array}{l}\text { Dark } \\
\text { red }\end{array}$ & $\mathrm{mg}$ & 5 & $\mathrm{Hm}$ & - & 3 & Or,Pth & - & Granite & $\begin{array}{l}\text { Appears disconformable be- } \\
\text { low Jubaylah Group }\end{array}$ & Plutonic & $\begin{array}{l}\text { Alk-feld } \\
\text { granite }\end{array}$ & $\mathrm{s}$ & $\mathrm{N}$ \\
\hline $22 \mathrm{a}-\mathrm{B} 1062 \mathrm{~B}$ & 27/36SE. & $\mathrm{nj}$ & - & - & 2 & $\begin{array}{l}\text { Oxidized } \\
\text { Rieb? }\end{array}$ & - & 6 & - & $\begin{array}{l}\text { Lithic } \\
\text { frag- } \\
\text { mental }\end{array}$ & $\begin{array}{l}\text { Rhyolite } \\
\text { tuff }\end{array}$ & $\begin{array}{l}\text { Flow, } 1 \mathrm{~m} \text { thick, in Jubay- } \\
\text { lah Group }\end{array}$ & Volcanic & $\begin{array}{l}\text { Na-rhyo- } \\
\text { lite? }\end{array}$ & $\mathbf{K}$ & $\mathrm{NN}$ \\
\hline $22 \mathrm{~b}-\mathrm{B} 1062 \mathrm{C}$ & 27/36SE. & $\mathrm{nj}$ & $\begin{array}{c}\text { Dark } \\
\text { grn }\end{array}$ & $\begin{array}{l}\text { Cryp- } \\
\text { to- } \\
\text { cryst }\end{array}$ & 18 & $\begin{array}{l}\text { 26,Px } \\
\quad \text { (alt'd.) }\end{array}$ & $\begin{array}{l}\text { Olig- } \\
\text { And }\end{array}$ & 21 & Or & $\begin{array}{l}\text { Porphyr } \\
\text { amygd }\end{array}$ & Basalt & $\begin{array}{l}\text { Flow in Jubaylah Group; } \\
\text { oxidized mugearite? }\end{array}$ & Volcanic & $\begin{array}{l}\text { Alkalic } \\
\text { basalt }\end{array}$ & $\mathrm{K}+$ & NN \\
\hline $23-B 1059$ & $26 / 36 \mathrm{NW}$. & gb & Gray & - & 10 & $\begin{array}{c}14, \mathrm{Hb} \\
\mathrm{Bi}\end{array}$ & - & 3 & - & - & $\begin{array}{l}\text { Leuco- } \\
\text { gab }\end{array}$ & $\begin{array}{l}\text { Small pluton in Najd fault } \\
\text { zone }\end{array}$ & Plutonic & Trondh & $\mathrm{H}, \mathrm{N}$ ? & WN \\
\hline
\end{tabular}


TABLE 3.-Description and classification of crystalline rocks of the Arabian Shield for which chemical analyses are given in table 4-Continued

\begin{tabular}{|c|c|c|c|c|c|c|c|c|c|c|c|c|c|c|c|c|}
\hline \multirow[b]{2}{*}{$\begin{array}{c}\begin{array}{c}\text { Sample } \\
\text { foc. } \\
\text { given } \\
\text { on pl. } \\
\text { 1) }\end{array} \\
\text { Field } \\
\text { no. } \\
\end{array}$} & \multirow[b]{2}{*}{$\begin{array}{l}\text { Location }{ }^{1} \\
\text { (lat/long) }\end{array}$} & \multirow[b]{2}{*}{$\begin{array}{l}\text { Rock-unit } \\
\text { symbol } \\
\text { on pl. } 1^{2}\end{array}$} & \multicolumn{9}{|c|}{ Petrography } & \multirow[b]{2}{*}{ Remarks" } & \multicolumn{4}{|c|}{ Classification } \\
\hline & & & Color & $\underset{\text { size }^{3}}{\text { Grain }}$ & $\begin{array}{l}\text { Normative } \\
\text { color } \\
\text { index }\end{array}$ & $\begin{array}{c}\text { Mafic } \\
\text { content } \\
{\text { (percent })^{5}}^{5}\end{array}$ & $\begin{array}{l}\text { Plagio- } \\
\text { clase } \\
\text { compo- } \\
\text { sition }^{6}\end{array}$ & $\begin{array}{l}\text { Normative } \\
\text { anorthite } \\
\text { (percent) }\end{array}$ & $\begin{array}{c}\text { Potassium- } \\
\text { feldspar } \\
\text { type }\end{array}$ & $\begin{array}{c}\text { Rock } \\
\text { texture } \\
\text { structure }\end{array}$ & $\begin{array}{c}\begin{array}{c}\text { Rock name } \\
(\text { field or } \\
\text { petrography) }\end{array} \\
\text { (10 }\end{array}$ & & $\begin{array}{l}\text { Igneous } \\
\text { type }^{12}\end{array}$ & $\begin{array}{l}\text { Rock name } \\
\text { from } \\
\text { chemistry }{ }^{13}\end{array}$ & $\mathrm{Age}^{\mathrm{An}}$ & $\begin{array}{l}\text { Re } \\
\text { gion }\end{array}$ \\
\hline $24-\mathrm{B} 1037$ & $26 / 36 \mathrm{NW}$. & ga & Red & $\mathrm{mg}$ & 3 & $\begin{array}{l}6, \mathrm{Bi} \\
\mathrm{Mt}\end{array}$ & - & 8 & Pth,Mc & - & Granite & $\begin{array}{l}\text { Jabal Libān; large granite } \\
\text { pluton; } 1 \% \text { modal fluorite }\end{array}$ & Plutonic & $\begin{array}{l}\text { Alk-feld } \\
\text { granite }\end{array}$ & S+ & $\overline{W N}$ \\
\hline $25 a-B 1823$ & $26 / 37 \mathrm{NW}$. & gr & - & - & 6 & $\begin{array}{c}5, \mathrm{Hb}, \\
\mathrm{Bi}\end{array}$ & Olig & 21 & Or & - & Granodio & $10-\times 20-\mathrm{km}$ elliptical pluton & Plutonic & Granodio & $s$ & NN \\
\hline $25 b-B 1823 A$ & $26 / 37 \mathrm{NW}$. & ${ }^{*}[\mathrm{gr}](\mathrm{nj})$ & - & vfg & 19 & $?, \mathrm{Hb}$ & - & 53 & - & - & Andesite & $\begin{array}{l}\text { Small plug or dike in Najd } \\
\text { fault zone, similar to } \\
\text { sample } 28 \text { a }\end{array}$ & Hypabys & $\begin{array}{l}\text { Quartz an- } \\
\text { desite }\end{array}$ & $\mathrm{K}$ ? & NN \\
\hline $27-B 1824$ & $26 / 37 \mathrm{NW}$. & sr & - & - & 3 & - & - & 3 & - & - & Rhyolite & $\begin{array}{l}\text { Shammar? structurally } \\
\text { above Jubaylah in } \\
\text { Najd fault }\end{array}$ & Volcanic & $\begin{array}{l}\text { Alk-feld } \\
\text { rhyolite }\end{array}$ & $\mathrm{s}$ & NN \\
\hline $28 \mathrm{a}-\mathrm{B} 1822$ & 26/37NW. & ${ }^{*}[\mathrm{mh}](\mathrm{di})$ & - & vfg & 22 & $\mathrm{Cpx}, \mathrm{Hb}$ & And & 41 & - & - & Microdio & $\begin{array}{l}\text { Contact zone of satellite } \\
\text { near sample } 25 \mathrm{~b}\end{array}$ & Hypabys & Diorite & $\mathbf{K}$ ? & NN \\
\hline $28 \mathrm{~b}-\mathrm{B} 1822 \mathrm{~A}$ & $26 / 37 \mathrm{NW}$. & $\begin{array}{l}\text { *[mh] } \\
(\mathrm{gm})\end{array}$ & - & - & 7 & $\underset{\mathrm{Bi}}{10, \mathrm{Hb}}$ & Olig & 24 & Pth & - & Adam & $\begin{array}{l}\text { Small pluton, } 2.5 \mathrm{~km} \\
\text { across, satellitic to } \\
\text { pluton at sample } 25 \mathrm{a}\end{array}$ & Plutonic & Granodio & $\mathrm{s}$ & NN \\
\hline $29-\mathrm{B} 1825$ & $26 / 37 \mathrm{NW}$ & ${ }^{*}[\mathrm{ay}](\mathrm{gp})$ & ) Red & fg & 2 & ?,Hm & - & 4 & Pth,Mc & Microgr & Granite & Granite on Najd fault & Plutonic & $\begin{array}{l}\text { Alk-feld } \\
\text { granite }\end{array}$ & $\mathrm{S}, \mathrm{N} ?$ & NN \\
\hline $30-B 1831$ & $26 / 37 \mathrm{SW}$ & npg & - & - & 2 & $1, \mathrm{Bi}$ & - & 9 & $\begin{array}{l}\text { Mc,Or, } \\
\text { Pth }\end{array}$ & $\begin{array}{l}\text { Hypidio- } \\
\text { morph }\end{array}$ & Granite & In Najd fault zone & Plutonic & Granite & $\mathrm{s}$ & WN \\
\hline $31-\mathrm{B} 1826$ & $26 / 37 \mathrm{SW}$ & $\operatorname{mog}$ & - & - & 3 & ?,Bi & - & 11 & - & $\begin{array}{l}\text { Flaser } \\
\text { gneiss }\end{array}$ & Granite & $\begin{array}{l}\text { Jabal Ghalāl, in Najd fault } \\
\text { zone }\end{array}$ & Gneiss & Trondh & $\mathrm{C}$ & WN \\
\hline $32-\mathrm{B} 1829$ & 26/37SW. & $\begin{aligned}{ }^{*}[\mathrm{ngn}] \\
(\mathrm{gm})\end{aligned}$ & - & fg-mg & 3 & $?, \mathrm{Bi}$ & - & 16 & - & $\begin{array}{l}\text { Hypidio- } \\
\text { morph }\end{array}$ & Adam & $\begin{array}{l}\text { Jabal Ghalāl, in Najd } \\
\text { fault zone }\end{array}$ & Plutonic & Granite & $\mathrm{C}$ & WN \\
\hline $33-\mathrm{B} 1827$ & $26 / 37 \mathrm{SW}$ & $\begin{aligned} & * \text { *npg] } \\
&(\mathrm{gm})\end{aligned}$ & - & $\mathrm{cg}$ & 2 & $\begin{array}{l}3, \mathrm{Bi}, \\
\mathrm{Hb}\end{array}$ & Olig & 8 & Pth,Mc & $\begin{array}{l}\text { Hypidio- } \\
\text { morph }\end{array}$ & Granite & $\begin{array}{l}\text { Jabal Ghalāl, in Najd } \\
\text { fault zone }\end{array}$ & Plutonic & $\begin{array}{l}\text { Alk-feld } \\
\text { granite }\end{array}$ & $\mathrm{s}$ & WN \\
\hline $36-\mathrm{B} 1126$ & 26/37SE & sr & $\begin{array}{r}\text { Dark } \\
\text { red }\end{array}$ & $\mathrm{fg}$ & 10 & $?, \mathrm{Hm}$ & - & 17 & - & Microgr & Felsite & $\begin{array}{l}\text { Related to subvolcanic in- } \\
\text { trusion, Shammar age }\end{array}$ & Dike & Dacite & $\mathrm{s}$ & NN \\
\hline $37-\mathrm{B} 1125$ & $26 / 37 \mathrm{SE}$ & ${ }_{(\mathrm{mgd})}^{*[j q]}$ & - & -- & 6 & $10, \mathrm{Bi}, \mathrm{Hb}$ & b Olig & 22 & Or & $\begin{array}{l}\text { Mod } \\
\text { catacl }\end{array}$ & Adam & $\begin{array}{l}\text { In quartz diorite-diorite } \\
\text { body }\end{array}$ & Plutonic & Granodio & $\mathrm{C}, \mathrm{S} ?$ & NN \\
\hline $38-\mathrm{B} 1805$ & 26/37SE. & nj & - & - & 2 & ?,Bi & - & 19 & Or & $\begin{array}{l}\text { Welded } \\
\text { tuff }\end{array}$ & Rhyolite & $\begin{array}{l}\text { In Jubaylah sedimentary } \\
\text { section }\end{array}$ & Volcanic & K-rhyolite & $\mathrm{K}$ & $\mathrm{NN}$ \\
\hline $39-\mathrm{B} 1806$ & $26 / 37 \mathrm{SE}$ & $\mathrm{nj}$ & - & - & 3 & - & - & 12 & - & Pyroclastic & - & $\begin{array}{l}\text { Highly calcic tuff or calcar- } \\
\text { enite }\end{array}$ & Sediment & Calcic tuff & $\mathrm{K}$ & $\mathrm{NN}$ \\
\hline $41-B 1109$ & 26/38SE. & $\begin{array}{l}\text { *[ay] } \\
\text { (gm) }\end{array}$ & - & - & 5 & - & - & 4 & - & $\begin{array}{l}\text { Minor } \\
\text { catacl }\end{array}$ & Granite & $\begin{array}{l}\text { Qal'at as Sawrah quadran- } \\
\text { gle, unit includes } \\
\text { granophyre }\end{array}$ & Plutonic & $\begin{array}{l}\text { Alk-feld } \\
\text { granite }\end{array}$ & $\mathrm{S}$ & NN \\
\hline $42-\mathrm{B} 1107$ & 26/38SE. & $\begin{array}{l}{ }^{*}[\mathrm{ay}] \\
(\mathrm{agr})\end{array}$ & - & - & 7 & $\begin{array}{l}\text { ?,Bi, } \\
\mathrm{Hm}\end{array}$ & - & 18 & Micropth & - & Granite & $\begin{array}{l}\text { Qal'at as Sawrah } \\
\text { quadrangle, weathered }\end{array}$ & Plutonic & Granite & A & $\mathrm{NN}$ \\
\hline $43-B 1804$ & $26 / 38 \mathrm{SE}$ & $\mathrm{gr}$ & Red & $\mathrm{cg}$ & 2 & $1, \mathrm{Bi}$ & Olig & 10 & - & - & Adam & $\begin{array}{l}\text { Qal'at as Şawrah } \\
\text { quadrangle }\end{array}$ & Plutonic & Granite & $\mathrm{S}$ & NN \\
\hline $45-\mathrm{B} 1106$ & $26 / 38 \mathrm{SE}$ & $\begin{array}{c}\text { "[agr] } \\
(\mathrm{agb})\end{array}$ & - & - & 34 & $\mathrm{Hb}, \mathrm{Bi}$ & - & 41 & - & - & Diorite & $\begin{array}{l}\text { Part of Jabal Abu Safiyah } \\
\text { complex }\end{array}$ & Plutonic & $\begin{array}{l}\text { Quartz } \\
\text { diorite }\end{array}$ & A & NN \\
\hline $46-\mathrm{B} 3906 \mathrm{~A}$ & $26 / 38 \mathrm{SE}$ & agd & - & - & 2 & $1, \mathrm{Bi}$ & $\begin{array}{l}\mathrm{Ab}- \\
\text { Olig }\end{array}$ & 2 & Mc & Catacl & Gran gn & $\begin{array}{l}\text { Qal'at as Sawrah } \\
\text { quadrangle }\end{array}$ & Gneiss & $\begin{array}{l}\text { Alk-feld } \\
\text { granite }\end{array}$ & A? & NN \\
\hline $47-B 1104$ & 26/38SE. & agr & Red & fg & 1 & $\mathrm{Bi}$ & And & 3 & - & Porphyr & Granite & $\begin{array}{l}\text { Qal'at as Sawrah } \\
\text { quadrangle, highly altered }\end{array}$ & Plutonic & $\begin{array}{l}\text { Alk-feld } \\
\text { granite }\end{array}$ & $\mathrm{S}, \mathrm{N} ?$ & NN \\
\hline
\end{tabular}

Footnotes at end of table. 
TABLE 3.-Description and classification of crystalline rocks of the Arabian Shield for which chemical analyses are given in table 4-Continued

\begin{tabular}{|c|c|c|c|c|c|c|c|c|c|c|c|c|c|c|c|c|}
\hline \multirow[b]{2}{*}{$\begin{array}{l}\text { Sample } \\
\text { (loc. } \\
\text { given } \\
\text { on pl. } \\
\text { 1) } \\
\end{array}$} & \multirow[b]{2}{*}{$\begin{array}{l}\text { Location' } \\
\text { (lat/long) }\end{array}$} & \multirow[b]{2}{*}{$\begin{array}{l}\text { Rock-unit } \\
\text { symbol } \\
\text { on pl. } 1^{2}\end{array}$} & \multicolumn{9}{|c|}{ Petrography } & \multirow[b]{2}{*}{ Remarks $^{11}$} & \multicolumn{4}{|c|}{ Classification } \\
\hline & & & Color & $\underset{\text { size }^{3}}{\text { Grain }}$ & $\begin{array}{l}\text { Normative } \\
\text { color } \\
\text { index }\end{array}$ & $\begin{array}{cc}\text { Mafic } & P \\
\text { content } & c \\
\text { (percent) } & \mathrm{c} \\
\mathrm{S}\end{array}$ & $\begin{array}{l}\text { Plagio- } \\
\text { clase } \\
\text { compo- } \\
\text { sition }^{6}\end{array}$ & $\begin{array}{l}\text { Normative } \\
\text { anorthite } \\
\text { (percent) }\end{array}$ & $\begin{array}{l}\text { Potassium- } \\
\text { feldspar }^{\text {type }} \\
\text { type }^{8}\end{array}$ & $\begin{array}{c}\text { Rock } \\
\text { texture- } \\
\text { structure }^{9}\end{array}$ & $\begin{array}{c}\text { Rock name } \\
\text { (field or } \\
\text { petrography) }\end{array}$ & & $\begin{array}{c}\text { Igneous } \\
\text { type }^{12}\end{array}$ & $\begin{array}{l}\text { Rock name } \\
\text { from } \\
\text { chemistry }^{13}\end{array}$ & $\mathrm{Age}^{14}$ & $\begin{array}{l}\text { Re- } \\
\text { gion }^{15}\end{array}$ \\
\hline $48-\mathrm{B} 1840$ & $25 / 37 \mathrm{NW}$. & $\mathrm{gr}$ & - & - & 2 & $2, \mathrm{Bi}$ & Olig & 9 & Pth,Mc & $\begin{array}{l}\text { Hypidio- } \\
\text { morph }\end{array}$ & Adam & $\begin{array}{l}\text { Cavernous weathering; in } \\
\text { Najd fault zone }\end{array}$ & Plutonic & $\begin{array}{l}\text { Alk-feld } \\
\text { granite }\end{array}$ & $\mathrm{s}$ & WN \\
\hline $49-\mathrm{B} 1845$ & $25 / 37 \mathrm{NW}$ & $\mathrm{gr}$ & - & $\mathrm{mg}$ & 2 & $2, \mathrm{Bi}$ & Olig & 0 & Mc,Pth & $\begin{array}{l}\text { Hypidio- } \\
\text { morph }\end{array}$ & Adam & - & Plutonic & Granite & $\mathrm{s}$ & WN \\
\hline $50-\mathrm{B} 1839$ & $25 / 37 \mathrm{NW}$ & $\mathrm{gr}$ & - & $\mathrm{mg}$ & 1 & $1, \mathrm{Bi}$ & Olig & 2 & Pth,Mc & $\begin{array}{l}\text { Hypidio- } \\
\text { morph }\end{array}$ & Adam & Cavernous weathering & Plutonic & Granite & $\mathrm{S}$ & WN \\
\hline $51-\mathrm{B} 1838$ & $25 / 37 \mathrm{NW}$ & *[agd] & Black & - & 27 & $50, \mathrm{Hb}$ & - & 65 & - & Diabasic & Diabase & Wādī al Ḩamḑ & Dike & $\begin{array}{l}\text { Leuco } \\
\text { diabase }\end{array}$ & $\mathrm{N}$ & WN \\
\hline $52-\mathrm{B} 1835$ & 25/37NE. & nog & - & $\mathrm{fg}$ & 2 & $4, \mathrm{Bi}$ & - & 20 & - & Gneissic & Trondh & Basement in Najd fault & Gneiss & Trondh & $\mathrm{J}(\mathrm{N}$ ?) & WN \\
\hline $54-\mathrm{S} 1 \mathrm{GFB}$ & $25 / 38 \mathrm{NW}$. & ngn & - & fg-mg & 4 & $7, \mathrm{Bi}$ & - & 20 & Or & Gneissic & Granite & $\begin{array}{l}\text { Wādī al Jizl, in Najd fault } \\
\text { zone }\end{array}$ & Gneiss & Granodio & $\begin{array}{l}\mathrm{C} ? \\
(\mathrm{~N} ?)+\end{array}$ & NN \\
\hline $55-\mathrm{B} 1123$ & 25/38NE. & agb & - & - & 52 & - & - & 55 & - & - & Gabbro & $\begin{array}{l}\text { Part of Jabal Abu Safiyah } \\
\text { complex }\end{array}$ & Plutonic & Gabbro & $A$ & $\mathrm{NN}$ \\
\hline $56-\mathrm{B} 1843 \mathrm{~B}$ & $25 / 37 \mathrm{SW}$ & jgb & - & - & 7 & ?,Bi & - & 27 & - & - & Qtz dio & - & Plutonic & Trondh & $\mathbf{J}$ & WN \\
\hline $57-\mathrm{B} 1630$ & $24 / 38 \mathrm{SW}$ & jt & - & - & 14 & $\begin{array}{l}20, \mathrm{Hb} \\
\mathrm{Px}, \mathrm{Bi}\end{array}$ & $\mathrm{An}_{30}$ & 46 & - & Gneissic & Qtz dio & Yanbu' & Plutonic & Tonalite & J & WC \\
\hline $58-\mathrm{B} 1312$ & $27 / 41 N W$. & gp & Gray & fg & 8 & $\begin{array}{l}5, \mathrm{Bi} \\
\mathrm{Hb}\end{array}$ & - & 0 & Pth & Microgr & Granite & Jabal Aja', gr-gp batholith & $\begin{array}{c}\text { Plutonic, } \\
\text { gneiss }\end{array}$ & $\begin{array}{c}\text { Peralkalic } \\
\text { granite }\end{array}$ & $\mathrm{s}$ & $\mathrm{NE}$ \\
\hline $61 \mathrm{a}-\mathrm{B} 1310$ & 27/41SW. & $\begin{array}{l}{ }^{*}[\mathrm{gm}] \\
(\mathrm{gp})\end{array}$ & Red & - & 1 & - & - & 10 & - & - & Granite & $\begin{array}{l}\text { Jabal Aja', layered, layer } \\
\text { adjacent to sample } 61 \mathrm{~b} ; \\
\text { altered }\end{array}$ & Plutonic & Granite & S & $\mathrm{NE}$ \\
\hline $61 \mathrm{~b}-\mathrm{B} 1311$ & 27/41SW. & $\begin{array}{r}{ }^{*}[\mathrm{gm}] \\
(\mathrm{gp})\end{array}$ & Red & $\mathrm{mg}$ & 1 & $\begin{array}{l}\mathrm{Hm}, \mathrm{Na}- \\
\text { Amph? }\end{array}$ & ? & 6 & Pth & $\begin{array}{l}\text { Hypidio- } \\
\text { morph }\end{array}$ & Granite & $\begin{array}{l}\text { Jabal Aja', layer adjacent } \\
\text { to sample } 61 \mathrm{a}\end{array}$ & Plutonic & $\begin{array}{l}\text { Alk-feld } \\
\text { granite }\end{array}$ & $\mathrm{S}$ & $\mathrm{NE}$ \\
\hline $62-\mathrm{B} 1308$ & $26 / 41 \mathrm{NE}$ & $\mathrm{gp}$ & - & - & 7 & $\begin{array}{l}\mathrm{Hb}(\mathrm{Na}- \\
\text { Amph? }\end{array}$ & ?) & 3 & Pth & Catacl & Granite & $\begin{array}{l}\text { Jibāl ar Rummān, gp } \\
\text { batholith }\end{array}$ & $\begin{array}{r}\text { Plutonic, } \\
\text { gneiss }\end{array}$ & $\begin{array}{l}\text { Alk-feld } \\
\text { granite }\end{array}$ & $\mathrm{s}$ & $\mathrm{NE}$ \\
\hline $63-$ B1103 & 26/39SW. & ay & - & - & 1 & - & - & $? 73$ & - & Agglom? & Rhyolite? & $\begin{array}{l}\text { Jabal as Safran, not } \\
\text { evaluated }\end{array}$ & Altered? & - & $\mathrm{S} ?$ & NN \\
\hline $64-\mathrm{B} 1325$ & 26/41SE. & $\underset{(\mathrm{gr})}{*}[\mathrm{mgd}]$ & Red & fg & 1 & Mt & - & 6 & - & - & Monz & - & Hypabys & Granite & $\mathrm{s}$ & $\mathrm{NE}$ \\
\hline $65-17,827$ & $25 / 39 \mathrm{NW}$. & $\begin{aligned} *[a y] \\
\text { (jq) }\end{aligned}$ & Gray & - & 17 & $\begin{array}{c}14, \mathrm{Hb} \\
\mathrm{Bi}\end{array}$ & Olig & 36 & Or & - & Qtz dio & Old basement & Plutonic & Tonalite & $\mathrm{J}$ & NN \\
\hline $66-\mathrm{B} 1154$ & $25 / 40 \mathrm{NE}$ & ${ }^{*}[\mathrm{mgd}]$ & - & - & 26 & $\mathrm{Bi}$ & - & 47 & - & - & Phonolite & $\begin{array}{l}\text { Nuqrah quadrangle, under } \\
\text { Jubaylah cgl }\end{array}$ & Volcanic & $\begin{array}{c}\text { Andesitic } \\
\text { basalt }\end{array}$ & $\mathrm{H}, \mathrm{K}$ ? & $\mathrm{HN}$ \\
\hline 67 - B1155 & 25/40NE. & $\mathrm{nj}$ & - & vfg & 30 & 14 & Ab-Olig & 31 & - & Amygd & Andesite & $\begin{array}{l}\text { Nuqrah quadrangle, flow } \\
\text { above Jubaylah cgl }\end{array}$ & Volcanic & $\begin{array}{l}\text { Basaltic } \\
\text { andesite }\end{array}$ & $\mathrm{K}+$ & $\mathrm{HN}$ \\
\hline 68a--- JD1714 & $25 / 40 \mathrm{NE}$ & he & - & - & 8 & - & - & 12 & - & - & Rhyolite & $\begin{array}{l}\text { Nuqrah quadrangle, from } \\
\text { drill core }\end{array}$ & Volcanic & Na-dacite & $\mathrm{H}+$ & $\mathrm{HN}$ \\
\hline $68 \mathrm{~b}--\mathrm{JD} 1713$ & $25 / 40 \mathrm{NE}$ & he & - & - & 6 & - & - & 3 & - & - & Rhyolite & $\begin{array}{l}\text { Nuqrah quadrangle, from } \\
\text { drill core, Nuqrah } \\
\text { formation }\end{array}$ & Volcanic & Na-rhyolite & $\mathrm{H}$ & $\mathrm{HN}$ \\
\hline $69-\mathrm{B} 1157$ & $25 / 40 \mathrm{NE}$ & nj & $\begin{array}{l}\text { Red- } \\
\text { brn }\end{array}$ & fg & 18 & $\begin{array}{l}\text { 35,Hm, } \\
\text { Epi }\end{array}$ & And & 30 & - & - & Andesite & Nuqrah quadrangle, flow & Volcanic & Andesite? & $\mathrm{K}+$ & $\mathrm{HN}$ \\
\hline $70-\mathrm{B} 1145$ & $25 / 40 \mathrm{NE}$ & he & - & - & 9 & $12, \mathrm{Mt}$ & Olig & 12 & - & Porphyr & Rhyolite & $\begin{array}{l}\text { Nuqrah quadrangle, flow in } \\
\text { pyroclastic section }\end{array}$ & Volcanic & Na-dacite & $\mathrm{H}$ & $\mathrm{HN}$ \\
\hline $71-J D 1711$ & $25 / 41 \mathrm{NW}$. & he & - & $\begin{array}{l}\text { Micro- } \\
\text { cryst }\end{array}$ & 7 & Mt? & Olig & 6 & Or & Porphyr & Rhyolite & $\begin{array}{l}\text { Nuqrah quadrangle, from } \\
\text { drill core }\end{array}$ & Volcanic & Na-rhyolite & $\mathrm{H}+$ & $\mathrm{TN}$ \\
\hline
\end{tabular}


TABLE 3.-Description and classification of crystalline rocks of the Arabian Shield for which chemical analyses are given in table 4-Continued

\begin{tabular}{|c|c|c|c|c|c|c|c|c|c|c|c|c|c|c|c|c|}
\hline \multirow[b]{2}{*}{$\begin{array}{c}\text { Sample } \\
\text { (oc. } \\
\text { given } \\
\text { on pl. } \\
\text { 1) } \\
\end{array}$} & \multirow[b]{2}{*}{$\begin{array}{l}\text { Location' } \\
\text { (lat/long) }\end{array}$} & \multirow[b]{2}{*}{$\begin{array}{l}\text { Rock-unit } \\
\text { symbol } \\
\text { on pl. } 1^{2}\end{array}$} & \multirow[b]{2}{*}{ Color } & \multirow[b]{2}{*}{$\begin{array}{l}\text { Grain } \\
\text { size }^{3}\end{array}$} & \multirow[b]{2}{*}{$\begin{array}{c}\text { Normative } \\
\text { color } \\
\text { index }\end{array}$} & \multirow[b]{2}{*}{$\begin{array}{c}\text { Mafic } \\
\text { content } \\
\text { (percent) }\end{array}$} & \multicolumn{2}{|c|}{ Petrography } & \multirow[b]{2}{*}{$\begin{array}{c}\text { Potassium- } \\
\text { feldspar } \\
\text { type }\end{array}$} & \multirow[b]{2}{*}{$\begin{array}{c}\text { Rock } \\
\text { texture } \\
\text { structure }\end{array}$} & \multirow[b]{2}{*}{$\begin{array}{c}\text { Rock name } \\
\text { (field or } \\
\text { petrography) }\end{array}$} & \multirow[b]{2}{*}{ Remarks" } & \multicolumn{4}{|c|}{ Classification } \\
\hline & & & & & & & $\begin{array}{l}\text { Plagio- } \\
\text { clase } \\
\text { compo } \\
\text { sition }^{6} \\
\end{array}$ & $\begin{array}{c}\text { Normative } \\
\text { anorthite } \\
\text { (percent) }^{7}\end{array}$ & & & & & $\begin{array}{l}\text { Igneous } \\
\text { type }\end{array}$ & $\begin{array}{l}\text { Rock name } \\
\text { from } \\
\text { chemistry }{ }^{13}\end{array}$ & Age $^{14}$ & $\begin{array}{l}\text { Re- } \\
\text { gion }^{15}\end{array}$ \\
\hline $73-\mathrm{B} 207 \mathrm{~B}$ & 24/40NE. & $\mathrm{mh}(\mathrm{sr} ?)$ & - & - & 3 & - & - & 25 & - & Breccia & Felsite & Jabal Shidā & Volcanic & K-rhyolite & $\mathrm{S}$ & $\mathrm{HC}$ \\
\hline $74-B 1137$ & 24/40NE. & ${ }^{*}[\mathrm{nj}](\mathrm{ns})$ & - & - & 35 & $\mathrm{Px}, 0 \mathrm{px}$ & Lab & 51 & - & - & Gabbro & $\begin{array}{l}\text { Intrusive into Jubaylah with } \\
\text { granophyre }\end{array}$ & Plutonic & Gabbro & $\mathrm{K}$ & $\mathrm{HC}$ \\
\hline $75-B 1138$ & 24/40NE. & nj & - & - & 19 & - & - & 39 & - & - & Mug & Al H̦anākīyah & Volcanic & Andesite & $\mathrm{K}$ & $\mathrm{HC}$ \\
\hline $76-\mathrm{S} 2 \mathrm{GFB}$ & $24 / 39 \mathrm{SW}$ & gm & - & $\mathrm{mg}$ & 2 & $3, \mathrm{Bi}$ & Olig & 13 & Pth,Mc & Catacl & Granite & $\begin{array}{l}\text { SW. of Al Madinah, } \\
\text { batholith cut by Najd } \\
\text { faults }\end{array}$ & Plutonic & Granite & M & WC \\
\hline $77-\mathrm{S} 3 \mathrm{GFB}$ & 24/39SE. & $\begin{array}{l}{ }^{*}[\mathrm{gm}] \\
\text { (ga) }\end{array}$ & Red & $\mathrm{mg}$ & 3 & $3, \mathrm{Mt}, \mathrm{Bi}$ & Olig & 3 & Pth, Mc & $\begin{array}{l}\text { Minor } \\
\text { catacl }\end{array}$ & Granite & $\begin{array}{l}\text { Al Madinah, from quarry, } \\
\text { small granite body }\end{array}$ & Plutonic & $\begin{array}{l}\text { Alk-feld } \\
\text { granite }\end{array}$ & M+ & $\begin{array}{l}\mathrm{HC} \\
(\mathrm{NN})\end{array}$ \\
\hline $78 a-$ ALD9 & $27 / 42 \mathrm{SW}$. & $\mathrm{gr}$ & Gray & $\mathrm{mg}$ & 1 & $2, \mathrm{Bi}$ & Olig? & 6 & Pth,Mc & $\begin{array}{l}\text { Hypidio- } \\
\text { morph }\end{array}$ & Granite & $\begin{array}{l}\text { Jabal Salmá, near sample } \\
78 \mathrm{~b}\end{array}$ & Plutonic & $\begin{array}{l}\text { Alk-feld } \\
\text { granite }\end{array}$ & S+ & $\mathrm{NE}$ \\
\hline $78 b-B 1316$ & $27 / 42$ SW. & $\mathrm{gr}$ & Red & - & 2 & - & - & 7 & - & - & Granite & $\begin{array}{l}\text { Jabal Salmá, near sample } \\
78 \mathrm{a}\end{array}$ & Plutonic & $\begin{array}{l}\text { Alk-feld } \\
\text { granite }\end{array}$ & $\mathrm{s}$ & $\mathrm{NE}$ \\
\hline $79-\mathrm{B} 1333$ & 26/42SW. & $\mathrm{gm}$ & Gray & - & 12 & - & - & 27 & - & - & Granite & $\begin{array}{l}\text { Granitoid, possibly intrudes } \\
\text { Hibshi Formation }\end{array}$ & Plutonic & $\begin{array}{l}\text { Quartz } \\
\text { monzodior }\end{array}$ & $\begin{array}{l}\text { M } \\
\text { ite }\end{array}$ & $\mathrm{NE}$ \\
\hline $80-B 1339$ & $26 / 42 \mathrm{SW}$ & $\begin{array}{r}{ }^{*}[\mathrm{gm}] \\
(\mathrm{hq})\end{array}$ & Gray & - & 29 & - & Olig? & 36 & - & Gneissic & Qtz dio & Gneiss below. Hibshi cgl & Gneiss & $\begin{array}{l}\text { Quartz } \\
\text { diorite }\end{array}$ & $\mathrm{H}$ & NE \\
\hline $81 \mathrm{a}-\mathrm{B} 1338 \mathrm{~A}$ & $26 / 42 \mathrm{SW}$ & $\begin{array}{l}{ }^{*}[\mathrm{mi}] \\
(\mathrm{gr})\end{array}$ & Gray & $\mathrm{cg}$ & 3 & $\mathrm{Bi}$ & Olig & 19 & - & $\begin{array}{r}\text { Hypidio- } \\
\text { morph }\end{array}$ & Adam & $\begin{array}{l}\text { Jabal Hibshī, granite } \\
\text { boulder from Hibshi } \\
\text { cgl }\end{array}$ & Plutonic & Granite & M & $\mathrm{NE}$ \\
\hline $81 b-B 1338 B$ & $26 / 42 \mathrm{SW}$. & $\begin{array}{l}{ }^{*}[\mathrm{mi}] \\
(\mathrm{gr})\end{array}$ & Red & $\mathrm{fg}$ & 1 & - & - & 11 & - & - & Granite & $\begin{array}{l}\text { Jabal Hibshī, granite } \\
\text { boulder from Hibshi } \\
\text { cgl }\end{array}$ & Plutonic & K-granite & M & $\mathrm{NE}$ \\
\hline $82 a-A L D 10$ & 26/42SW. & $\mathrm{gd}$ & Red & - & 15 & $12, \mathrm{Hb}, \mathrm{Bi}$ & Bi Olig & 26 & Or & $\begin{array}{l}\text { Hypidio- } \\
\text { morph }\end{array}$ & Monz & Jabal Tìn & Plutonic & Granodio & $\mathrm{C}+$ & $\mathrm{NE}$ \\
\hline $82 b-B 1330$ & $26 / 42 \mathrm{SW}$ & gd & - & - & 13 & - & - & 29 & - & - & Granite & Jabal Tìn & Plutonic & Granodio & $\mathrm{C}$ & $\mathrm{NE}$ \\
\hline $83 b-B 1334$ & 26/42SW. & $\mathrm{mi}$ & - & - & 5 & - & - & 15 & - & - & Dacite & $\begin{array}{l}\text { Flow in upper Hibshi } \\
\text { Formation }\end{array}$ & Volcanic & Dacite & $\mathrm{S}$ & $\mathrm{NE}$ \\
\hline $84-B 1301$ & 25/42NW. & ga & White & - & 2 & $\mathrm{Na}_{\mathrm{Amph}}$ & h? & 8 & Pth,Or & - & $\begin{array}{l}\text { Qtz } \\
\text { monz }\end{array}$ & $\begin{array}{l}\text { Jabal Qutn, } 15-\mathrm{km} \text { circular } \\
\text { pluton in Murdama }\end{array}$ & Plutonic & $\begin{array}{c}\text { Alk-feld } \\
\text { granite }\end{array}$ & $\mathrm{M}=\mathrm{S}$ & $\mathrm{NE}$ \\
\hline $85-\mathrm{B} 1302$ & $25 / 42 \mathrm{NW}$ & gd & Gray & $\mathrm{mg}$ & 5 & $\mathrm{Bi}$ & - & 29 & Pth,Mc & - & $\begin{array}{l}\text { Qtz } \\
\text { monz }\end{array}$ & $\begin{array}{l}\text { East of Qutn, } 5-\mathrm{km} \text { circular } \\
\text { pluton in Murdama }\end{array}$ & Plutonic & Granodio & $M=S$ & $\mathrm{NE}$ \\
\hline $86-\mathrm{B} 1317$ & $25 / 42 \mathrm{NW}$. & $\mathrm{gd}$ & Gray & - & 6 & - & - & 29 & - & - & $\begin{array}{r}\text { Hornbl } \\
\text { monz }\end{array}$ & $\begin{array}{l}\text { East of Qutn, } 4-\mathrm{km} \text { circular } \\
\text { pluton in Murdama }\end{array}$ & Plutonic & Granodio & $\mathrm{M}=\mathrm{S}$ & $\mathrm{NE}$ \\
\hline $87-\mathrm{B} 1318$ & 25/42NE. & gd & Gray & $\mathrm{mg}$ & 9 & - & - & 30 & - & - & $\begin{array}{l}\text { Hornbl } \\
\text { qtz dio }\end{array}$ & $\begin{array}{l}\text { East of Qutn, 3-km circular } \\
\text { pluton in Murdama }\end{array}$ & Plutonic & Granodio & $M=S$ & NE \\
\hline $88-\mathrm{B} 1340$ & 25/43SE. & $\begin{array}{l}\text { "[as] } \\
\text { (mgd) }\end{array}$ & - & - & 9 & $7, \mathrm{Bi}$ & - & 34 & - & - & Adam & $\begin{array}{l}\text { Intrudes Murdama } \\
\text { Formation }\end{array}$ & Plutonic & Granodio & S & $\mathrm{NE}$ \\
\hline $89-\mathrm{B} 1341$ & 25/43SE. & mgd & - & - & 10 & $\begin{array}{r}19, \mathrm{Bi} \\
\mathrm{Hb}\end{array}$ & And & 32 & $\mathrm{Mc}, \mathrm{Or}$ & - & Adam & $\begin{array}{l}\text { Intrudes Murdama } \\
\text { Formation }\end{array}$ & Plutonic & Granodio & S+ & $\mathrm{NE}$ \\
\hline $90 \mathrm{a}-\mathrm{ALD} 12$ & 24/43NE. & mgd & - & - & 11 & $\begin{array}{l}11, \mathrm{Bi} \\
\mathrm{Hb}\end{array}$ & And & 28 & Or & Catacl & Granite & $\begin{array}{l}\text { Intrudes Murdama and Abt } \\
\text { Formations }\end{array}$ & Plutonic & Granodio & $S+$ & $\mathrm{NE}$ \\
\hline
\end{tabular}


TABLE 3.-Description and classification of crystalline rocks of the Arabian Shield for which chemical analyses are given in table 4-Continued

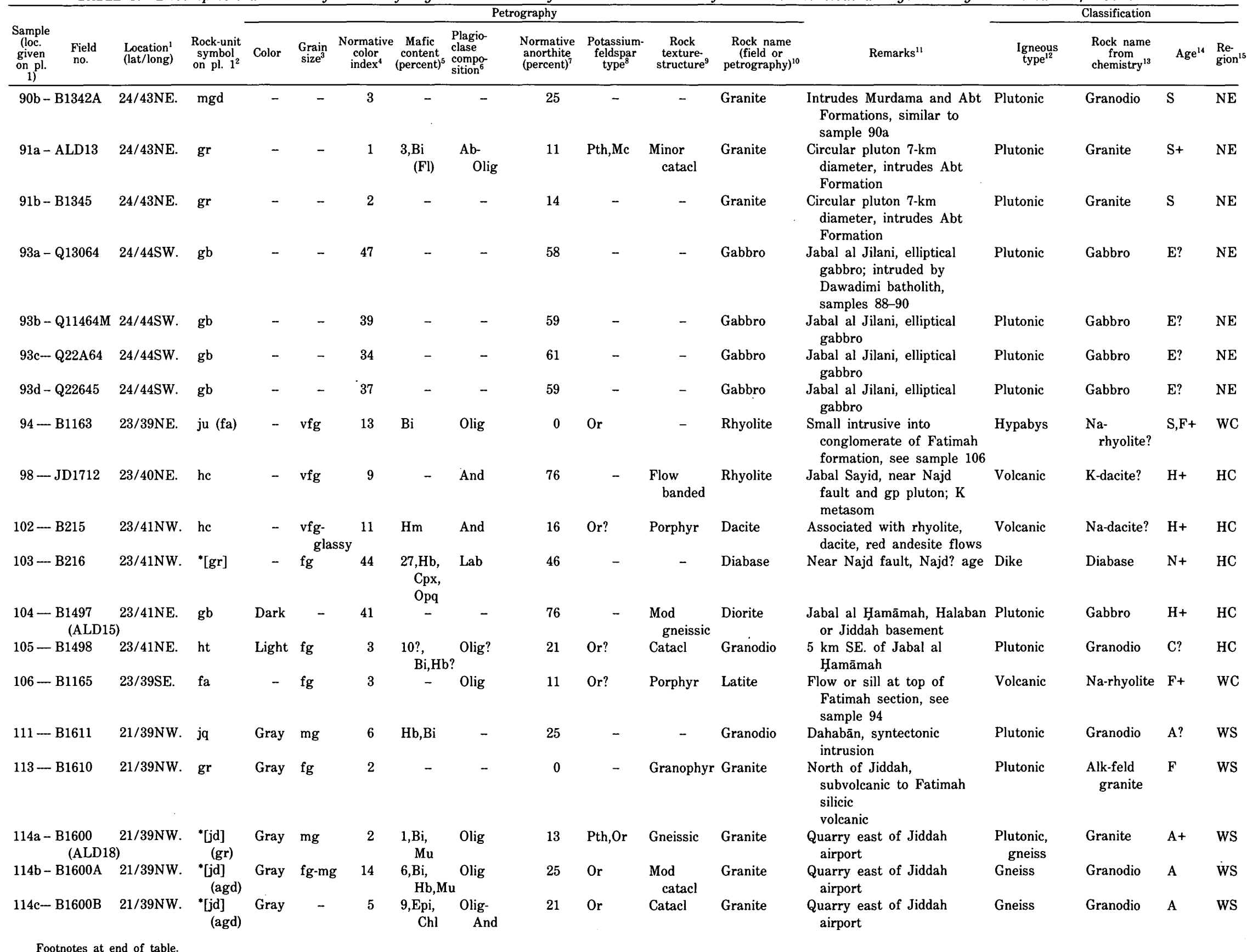


TABLE 3.-Description and classification of crystalline rocks of the Arabian Shield for which chemical analyses are given in table 4-Continued

\begin{tabular}{|c|c|c|c|c|c|c|c|c|c|c|c|c|c|c|c|c|c|}
\hline \multirow[b]{2}{*}{$\begin{array}{l}\text { Sample } \\
\text { (oc. } \\
\text { given } \\
\text { on pl. } \\
\text { 1) }\end{array}$} & \multirow[b]{2}{*}{$\begin{array}{l}\text { Field } \\
\text { no. }\end{array}$} & \multirow[b]{2}{*}{$\begin{array}{l}\text { Location' } \\
\text { (lat/long) }\end{array}$} & \multirow[b]{2}{*}{$\begin{array}{c}\text { Rock-unit } \\
\text { symbol } \\
\text { on pl. } 1^{2}\end{array}$} & \multicolumn{9}{|c|}{ Petrography } & \multirow[b]{2}{*}{ Remarks" } & \multicolumn{4}{|c|}{ Classification } \\
\hline & & & & Color & $\underset{\text { size }}{\text { Grain }}$ & $\begin{array}{c}\text { Normative } \\
\text { color } \\
\text { index }\end{array}$ & $\begin{array}{c}\text { Mafic } \\
\text { content } \\
\text { (percent) })^{5}\end{array}$ & $\begin{array}{l}\text { Plagio- } \\
\text { clase } \\
\text { compo- } \\
\text { sition }^{6}\end{array}$ & $\begin{array}{l}\text { Normative } \\
\text { anorthite } \\
\text { (percent) }\end{array}$ & $\begin{array}{c}\text { Potassium- } \\
\text { feldspar }^{\text {type }} \\
\text { type }\end{array}$ & $\begin{array}{c}\text { Rock } \\
\text { texture } \\
\text { structure }\end{array}$ & $\begin{array}{c}\text { Rock name } \\
\text { (field or } \\
\text { petrography) }\end{array}$ & & 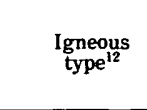 & $\begin{array}{l}\text { Rock name } \\
\text { from } \\
\text { chemistry }{ }^{13}\end{array}$ & $\mathrm{Age}^{14}$ & $\begin{array}{l}\mathrm{Re} \\
\text { gion }^{15}\end{array}$ \\
\hline $115-\mathrm{E}$ & 31601 & $21 / 39 \mathrm{NW}$ & $\begin{array}{l}\text { *[jd] } \\
\text { (agd) }\end{array}$ & - & - & 4 & - & - & 29 & - & Catacl & Granite & East of Jiddah airport & Gneiss & Granodio & A & $\overline{\text { WS }}$ \\
\hline $116-\mathrm{B}$ & $31606 \mathrm{~A}$ & 21/39NW. & ${ }^{*}[j t]$ & Dark & $\mathrm{fg}$ & 24 & - & - & 57 & - & - & Diabase & $\begin{array}{l}\text { North of Jiddah, dike in } \\
\text { metadiorite }\end{array}$ & Dike & $\begin{array}{l}\text { Quartz } \\
\text { diabase }\end{array}$ & F?N? & ws \\
\hline $117 a-E$ & 3900 & 21/39NE. & fa & Dark & - & 42 & $\begin{array}{l}\text { O1?,Px?, } \\
\text { Opq }\end{array}$ & $\begin{array}{l}\text { And- } \\
\quad A b ?\end{array}$ & 3 & - & Porphyr & Basalt & $\begin{array}{l}\text { Jabal Mukassar, 2-m sill, } 10 \\
\text { m below top, upper fa }\end{array}$ & Sill & $\begin{array}{l}\mathrm{Na}- \\
\quad \text { andesite? }\end{array}$ & $\mathrm{F}=\mathrm{S} ?+$ & wS \\
\hline $117 \mathrm{~b}-\mathrm{B}$ & 3901 & 21/39NE. & $\mathrm{fa}$ & Dark & vfg & 18 & $\mathrm{Bi}, \mathrm{Hm}$ & $\begin{array}{l}\text { Olig?- } \\
\text { And }\end{array}$ & 20 & - & Porphyr & Dacite & $\begin{array}{l}\text { Jabal Sidr, } 30-\mathrm{m} \text { sill on top, } \\
\text { intrudes limestone }\end{array}$ & Sill & $\begin{array}{l}\mathrm{Na}- \\
\text { andesite? }\end{array}$ & $\mathrm{F}=\mathrm{S} ?+$ & ws \\
\hline $122-\mathrm{B}$ & 31629 & 21/39SW. & $\begin{array}{l}{ }^{*}[j d] \\
(j u)\end{array}$ & $\begin{array}{r}\text { Dark } \\
\text { grn }\end{array}$ & - & 6 & - & - & 18 & - & - & Dacite & $\begin{array}{l}\text { Greenstone quarry south of } \\
\text { Jiddah }\end{array}$ & Volcanic & Na-dacite & $\mathbf{J}$ & wS \\
\hline $123-\mathrm{B}$ & 31625 & 21/39SW. & $\begin{array}{l}{ }^{*}[\operatorname{agd}] \\
(\text { agr) }\end{array}$ & Red & - & 3 & - & - & 7 & - & - & Granite & $\begin{array}{l}\text { Small pluton east of } \\
\text { Jiddah, north of Makkah } \\
\text { road }\end{array}$ & Plutonic & Na-granite & $\mathrm{F}$ & ws \\
\hline $124 \mathrm{a}-\mathrm{A}$ & LLD19 & 21/39SE. & $\mathrm{jq}$ & Gray & - & 10 & $\begin{array}{c}18, \mathrm{Bi}, \\
\mathrm{Hb}\end{array}$ & And & 39 & - & Catacl & Trondh & $\begin{array}{l}\text { Fresh rock from pipeline } \\
\text { ditch, east of Makkah }\end{array}$ & Gneiss & Tonalite & $\mathrm{J}+$ & WS \\
\hline $124 \mathrm{~b}-\mathrm{B}$ & 31603 & 21/39SE. & $j q$ & - & - & 4 & - & - & 27 & - & - & Trondh & Similar to sample $124 a$ & Gneiss & Trondh & $\mathrm{J}$ & ws \\
\hline $125-\mathrm{A}$ & LLD21 & $21 / 40 \mathrm{SW}$ & agm & - & - & 3 & - & - & 14 & - & - & Granite & $\begin{array}{l}\text { At Tā'if, small gr pluton, } \\
\text { from quarry }\end{array}$ & Plutonic & Granite & St & B \\
\hline $126-\mathrm{A}$ & LL23 & $20 / 41 \mathrm{NW}$. & $\mathrm{jt}$ & - & - & 10 & - & - & 39 & - & - & Qtz dio & $\begin{array}{l}\text { Wādī Shuqub quadrangle, } \\
\text { small outlier to large } \\
\text { batholith }\end{array}$ & Plutonic & Tonalite & $\mathrm{J}+$ & B \\
\hline $127 \mathrm{a}-\mathrm{A}$ & LL22 & $20 / 41 \mathrm{NW}$ & $\mathrm{gp}$ & Pink & $\mathrm{cg}$ & 2 & - & - & 6 & - & - & Granite & $\begin{array}{l}\text { Jabal Qunah pluton, outer } \\
\text { ring granite }\end{array}$ & Plutonic & $\begin{array}{l}\text { Alk-feld } \\
\text { granite }\end{array}$ & S+ & B \\
\hline $127 b-9$ & & $20 / 41 \mathrm{NW}$ & gp & - & - & 2 & - & - & 8 & - & - & Granite & Similar to sample 127 a & Plutonic & $\begin{array}{l}\text { Alk-feld } \\
\text { granite }\end{array}$ & $\mathrm{s}$ & B \\
\hline $133-\mathrm{B}$ & 31702 & 20/41SE. & jd & - & - & 16 & - & - & 64 & - & - & Diorite & $\begin{array}{l}\text { 'Aqīq quadrangle, complex } \\
\text { diorite batholith }\end{array}$ & Plutonic & $\begin{array}{l}\text { Quartz } \\
\text { diorite }\end{array}$ & $\mathbf{J}$ & B \\
\hline $134-\mathrm{B}$ & 31703 & 20/41SE. & $a b$ & - & - & 9 & - & - & 1 & - & - & Rhyolite & $\begin{array}{l}\text { 'Aqīq quadrangle, Ablah } \\
\text { belt, flow near base }\end{array}$ & Volcanic & Na-rhyolite & $\mathrm{A}$ & $\mathrm{J}$ \\
\hline $138-B$ & 31725 & 20/41SE. & $a b$ & - & - & 24 & - & - & 28 & - & - & Andesite & $\begin{array}{l}\text { 'Aqīq quadrangle, Ablah } \\
\text { belt, sill near gr stock }\end{array}$ & Sill & Andesite & A & $J$ \\
\hline $139-\mathrm{B}$ & 31726 & 20/41SE. & ${ }^{*}(\mathrm{ab})[\mathrm{jc}]$ & - & - & 2 & - & - & 7 & - & - & Rhyolite & $\begin{array}{l}\text { Aqiq quadrangle, Ablah } \\
\text { belt, } 10-\mathrm{m} \text {-thick sill }\end{array}$ & Sill & $\begin{array}{l}\text { Alk-feld } \\
\text { rhyolite }\end{array}$ & $\mathrm{S}$ & $\mathrm{J}$ \\
\hline $141 a-B$ & $3230 \mathrm{~A}$ & $23 / 43 \mathrm{NW}$ & $\mathrm{mu}$ & Dark & $\begin{array}{l}\text { ultra- } \\
\text { fg }\end{array}$ & 13 & $\mathrm{Bi}, \mathrm{Cpx}$ & And & 23 & - & $\begin{array}{l}\text { Flow, } \\
\text { porphyr }\end{array}$ & Dacite & $\begin{array}{l}\text { Jabal al Murdamah; flows } \\
\text { above upper cgl, } \\
\text { =Shammar }\end{array}$ & Volcanic & $\begin{array}{l}\text { Dacite- } \\
\text { andesite? }\end{array}$ & S-M+ & TN \\
\hline $141 \mathrm{~b}-\mathrm{B}$ & 3230 & $23 / 43 \mathrm{NW}$ & $\mathrm{mu}$ & $\begin{array}{c}\text { Red- } \\
\text { brn }\end{array}$ & - & 3 & $\mathrm{Cpx}, \mathrm{Hb}$ & Olig & 14 & $\begin{array}{l}\text { Or, } \\
\text { Micro- } \\
\text { pth }\end{array}$ & $\begin{array}{l}\text { Flow, } \\
\text { porphyr }\end{array}$ & Rhyolite & $\begin{array}{l}\text { Jabal al Murdamah; flows } \\
\text { above upper cgl, } \\
\text { =Shammar }\end{array}$ & Volcanic & Rhyolite & S-M+ & $\mathrm{TN}$ \\
\hline $141 c-B$ & 3229 & $23 / 43 N W$ & $\mathrm{mu}$ & $\begin{array}{c}\text { Red- } \\
\text { brn }\end{array}$ & $\begin{array}{l}\text { ultra- } \\
\text { vfg }\end{array}$ & 3 & $\mathrm{Hb} ?, \mathrm{Bi}$ & Olig & 18 & Or & $\begin{array}{l}\text { Flow, } \\
\text { porphyr }\end{array}$ & Rhyolite & $\begin{array}{l}\text { Jabal al Murdamah; flows } \\
\text { above upper cgl, } \\
\text { =Shammar }\end{array}$ & Volcanic & Na-dacite? & S-M & TN \\
\hline $141 d-B$ & $31368 \mathrm{~B}$ & $23 / 43 \mathrm{NW}$ & $\mathrm{mu}$ & $\begin{array}{c}\text { Red- } \\
\text { brn }\end{array}$ & - & 3 & - & - & 5 & - & $\begin{array}{l}\text { Flow, } \\
\text { porphyr }\end{array}$ & Rhyolite & $\begin{array}{l}\text { Jabal al Murdamah; flows } \\
\text { above upper cgl, } \\
\text { =Shammar }\end{array}$ & Volcanic & Rhyolite & S-M & $\mathrm{TN}$ \\
\hline $142-\mathrm{B}$ & $3233 \mathrm{~A}$ & 23/43NE. & $\mathrm{mu}$ & - & vfg & 10 & - & - & 29 & - & - & Dacite & $\begin{array}{l}\text { 'Afif quadrangle, flow } \\
\text { above Murdama, } \\
\text { =Shammar }\end{array}$ & Volcanic & Dacite & S-M & $\mathrm{TN}$ \\
\hline
\end{tabular}


TABLE 3.-Description and classification of crystalline rocks of the Arabian Shield for which chemical analyses are given in table 4-Continued

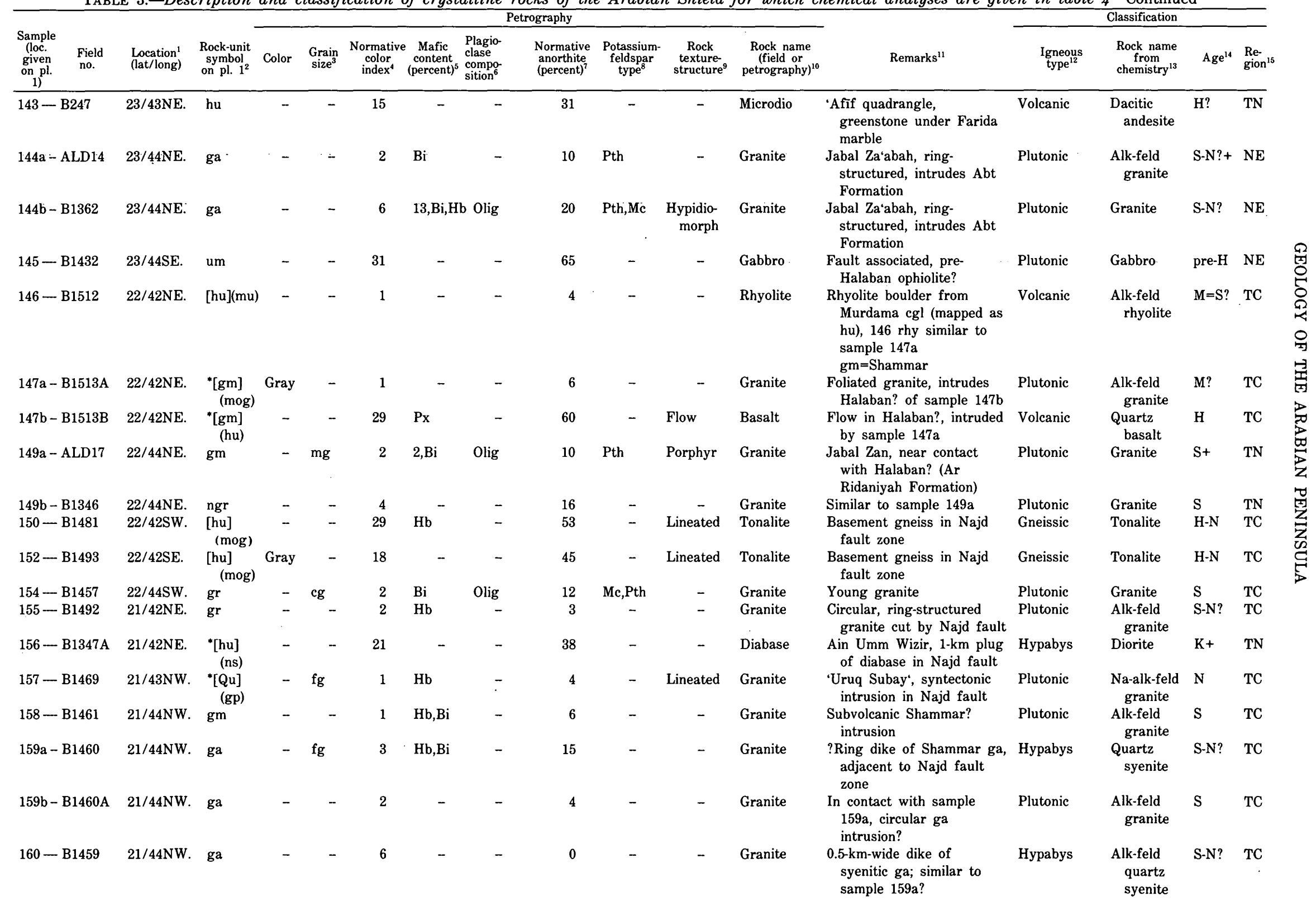


TABLE 3.-Description and classification of crystalline rocks of the Arabian Shield for which chemical analyses are given in table 4-Continued

\begin{tabular}{|c|c|c|c|c|c|c|c|c|c|c|c|c|c|c|c|c|c|}
\hline \multirow[b]{2}{*}{$\begin{array}{c}\text { Sample } \\
\text { loc. } \\
\text { given } \\
\text { on pl. } \\
1) \\
\end{array}$} & \multirow[b]{2}{*}{$\begin{array}{l}\text { Field } \\
\text { no. }\end{array}$} & \multirow[b]{2}{*}{$\begin{array}{l}\text { Location' } \\
\text { (lat/long) }\end{array}$} & \multirow[b]{2}{*}{$\begin{array}{l}\text { Rock-unit } \\
\text { symbol } \\
\text { on pl. } 1^{2}\end{array}$} & \multicolumn{9}{|c|}{ Petrography } & \multirow[b]{2}{*}{ Remarks" } & \multicolumn{4}{|c|}{ Classification } \\
\hline & & & & Color & $\begin{array}{l}\text { Grain } \\
\text { size }^{3}\end{array}$ & $\begin{array}{l}\text { Normative } \\
\text { color } \\
\text { index }^{4}\end{array}$ & $\begin{array}{c}\text { Mafic } \\
\text { content } \\
\text { (percent) }\end{array}$ & $\begin{array}{l}\text { Plagio- } \\
\text { clase } \\
\text { compo- } \\
\text { sition }\end{array}$ & $\begin{array}{l}\text { Normative } \\
\text { anorthite } \\
\text { (percent) })^{7}\end{array}$ & $\begin{array}{l}\text { Potassium- } \\
\text { feldspar }^{\text {tape }} \\
\text { type }^{8}\end{array}$ & $\begin{array}{c}\text { Rock } \\
\text { texture } \\
\text { structure }\end{array}$ & $\begin{array}{c}\text { Rock name } \\
\text { (field or } \\
\text { petrography) }\end{array}$ & & $\begin{array}{l}\text { Igneous } \\
\text { type }^{12}\end{array}$ & $\begin{array}{l}\text { Rock name } \\
\text { from } \\
\text { chemistry }^{13}\end{array}$ & $\mathrm{Age}^{14}$ & $\begin{array}{l}\text { Re- } \\
\text { gion }\end{array}$ \\
\hline $161-1$ & B1458 & $21 / 44 \mathrm{NW}$ & $\mathrm{mgd}$ & - & - & 3 & $\mathrm{Hb}, \mathrm{Bi}$ & - & 22 & Or & Gneissic & Granite & Late syntectonic batholith & Plutonic & Granodio & $\mathrm{C}$ & $\mathrm{TC}$ \\
\hline $162-$ & B1487 & 21/42SE. & $\mathrm{gp}$ & - & - & 5 & - & - & 2 & - & - & Granite & $\begin{array}{l}\text { North of Ranyah, large } \\
\text { north-trending red gr } \\
\text { batholith }\end{array}$ & Plutonic & $\begin{array}{l}\text { Alk-feld } \\
\text { granite }\end{array}$ & $\mathrm{s}$ & HS \\
\hline $163-$ & B1488 & 21/42SE. & $\mathrm{gm}$ & - & - & 4 & - & - & 3 & - & - & Granite & $\begin{array}{l}\text { Similar to sample } 162 \text {, near } \\
\text { contact with orthogneiss }\end{array}$ & Plutonic & $\begin{array}{l}\text { Alk-feld } \\
\text { granite }\end{array}$ & $S$ & HS \\
\hline $164-1$ & B1489 & 21/43SW. & $\mathrm{mgd}$ & - & - & 23 & $\mathrm{Hb}$ & - & 32 & - & Migmatitic & Gneiss & $\begin{array}{l}\text { Migmatized basement, } \\
\text { reoriented in Najd fault } \\
\text { zone }\end{array}$ & Gneissic & $\begin{array}{l}\text { Quartz } \\
\text { monzo- } \\
\text { diorite }\end{array}$ & $\begin{array}{l}\mathrm{H} \\
\quad \text { (C?, } \\
\mathrm{N} ?)\end{array}$ & HS \\
\hline $166-1$ & B1462 & 21/44SW. & ga & Red & - & 3 & - & - & 8 & - & - & Granite & $\begin{array}{l}10 \mathrm{~km} \text { east of Jabal Khidā, } \\
\text { large pluton }\end{array}$ & Plutonic & $\begin{array}{l}\text { Alk-feld } \\
\text { granite }\end{array}$ & $\mathrm{S}$ & $\mathrm{TC}$ \\
\hline $167 a-1$ & B1463 & $21 / 44 \mathrm{SE}$ & an & Gray & - & 27 & - & - & 67 & - & - & Anortho & $\begin{array}{c}\text { Jabal Mahail, } 11 \times 30 \mathrm{~km} \text {, } \\
\text { intrusive vs. tectonic? }\end{array}$ & Plutonic & $\begin{array}{l}\text { Leuco- } \\
\text { gabbro- } \\
\text { norite }\end{array}$ & $\mathrm{E} ?, \mathrm{C} ?$ & $\mathrm{TC}$ \\
\hline $\begin{array}{c}167 \mathrm{~b}- \\
\mathrm{h} .\end{array}$ & $\begin{array}{l}11666- \\
11670 \\
11679\end{array}$ & $21 / 44 \mathrm{SE}$ & an & Gray & - & $\begin{array}{r}3 \text { to } \\
10\end{array}$ & $\begin{array}{l}\mathrm{Bi}, \mathrm{Hb}, \\
\mathrm{Px} ?\end{array}$ & Lab & $\begin{array}{r}51 \text { to } \\
65\end{array}$ & - & $\begin{array}{l}\text { Some } \\
\text { catacl }\end{array}$ & Anortho & $\begin{array}{l}7 \text { analyses on same } \\
\text { anorthosite body as } \\
\text { sample } 167 \text { a }\end{array}$ & Plutonic & Anorthosite & $\mathrm{E} ?, \mathrm{C} ?$ & $\mathrm{TC}$ \\
\hline $170 \mathrm{a}-1$ & B1463A & $21 / 44 \mathrm{SE}$ & gm & Red & - & 3 & $7, \mathrm{Bi}$ & Olig & 22 & $\mathrm{Mc}, \mathrm{Or}$ & $\begin{array}{r}\text { Hypidio- } \\
\text { morph }\end{array}$ & Granite & $\begin{array}{l}\text { Intrusive into anorthosite } \\
\text { body sample } 167 \text {, near } \\
\text { contact }\end{array}$ & Plutonic & Granite & M & $\mathrm{TC}$ \\
\hline $171-$ & ALD25 & $20 / 42 \mathrm{SW}$ & [jd](agd) & Gray & $\mathrm{mg}$ & 4 & $\mathrm{Bi}$ & - & 15 & - & Lineated & Granodio & $\begin{array}{l}\text { Thaniyah quadrangle, An } \\
\text { Nimas batholith complex }\end{array}$ & Plutonic & $\begin{array}{l}\text { Na-grano- } \\
\text { diorite }\end{array}$ & $\mathrm{A}, \mathrm{C} ?+$ & $J$ \\
\hline $172-$ & 1724 & $20 / 42 \mathrm{SW}$. & [jd](agd) & Gray & $\mathrm{mg}$ & 3 & $\mathrm{Bi}$ & - & 24 & - & Gneissic & Granodio & $\begin{array}{l}\text { Thaniyah quadrangle, An } \\
\text { Nimas batholith complex }\end{array}$ & Plutonic & $\begin{array}{l}\text { Na-grano- } \\
\text { diorite }\end{array}$ & $\mathrm{A}, \mathrm{C} ?$ & $\mathbf{J}$ \\
\hline $174-1$ & $\mathrm{~B} 1700$ & $20 / 42 \mathrm{SW}$ & [jd](agd) & - & - & 7 & - & - & 20 & - & - & Qtz dio & $\begin{array}{l}\text { Thaniyah quadrangle, An } \\
\text { Nimas batholith complex }\end{array}$ & Gneissic & $\begin{array}{l}\text { Na-grano- } \\
\text { diorite }\end{array}$ & $\mathrm{A}, \mathrm{C} ?$ & $\mathrm{~J}$ \\
\hline $175-1$ & 8528 & $20 / 42 \mathrm{SW}$. & $\mathrm{gb}$ & Dark & $\mathrm{mg}$ & 43 & $\begin{array}{l}37, \mathrm{Cpx}, \\
01, \mathrm{Opx}\end{array}$ & $x^{\text {Lab(And) }}$ & 36 & - & - & Gabbro & $\begin{array}{l}\text { Thaniyah quadrangle, } 5-\mathrm{km} \\
\text { diameter, layered } \\
\text { intrusion }\end{array}$ & Plutonic & $\begin{array}{l}\text { Olivine } \\
\text { gabbro }\end{array}$ & $\mathrm{J} ?, \mathrm{~S} ?$ & $\mathrm{~J}$ \\
\hline $177-1$ & B-DS1 & 20/42SE. & ${ }^{*}[\mathrm{mu}]$ & $\begin{array}{l}\text { Red- } \\
\text { brn }\end{array}$ & vfg & 2 & $\begin{array}{l}\mathrm{Px} \\
\text { Amph }\end{array}$ & - & 3 & Or & Spherul & Rhyolite & $\begin{array}{l}\text { Rhyolite from mixed } \\
\text { rhyolite-diabase, in } \\
\text { Murdama }\end{array}$ & Dike & Rhyolite & $\mathrm{S} ?, \mathrm{~N} ?$ & HS \\
\hline $178-1$ & B-DS8 & 20/42SE. & $\mathrm{gb}$ & Black & $\mathrm{mg}$ & 33 & - & - & 75 & - & - & Gabbro & $\begin{array}{l}\text { Jabal Sidun, } 4 \times 10 \mathrm{~km} \text {, } \\
\text { elliptical, layered gabbro }\end{array}$ & Plutonic & $\begin{array}{l}\text { Leuco- } \\
\text { olivine } \\
\text { norite }\end{array}$ & $S$ & HS \\
\hline $182-1$ & 81439 & 24/44SE. & $\mathrm{gm}$ & - & - & 22 & - & - & 37 & - & - & Granodio & $\begin{array}{l}\text { Jabal al Hasraj, small gr } \\
\text { pluton, intrudes Abt } \\
\text { Formation }\end{array}$ & Plutonic & $\begin{array}{l}\text { Quartz } \\
\text { monzo- } \\
\text { diorite }\end{array}$ & S & $\mathrm{NE}$ \\
\hline $186-1$ & $\begin{array}{l}31456 \\
\text { (ALD16) }\end{array}$ & $23 / 45 \mathrm{SW}$ & ${ }^{*}[\mathrm{hu}](\mathrm{hj})$ & Gray & - & 5 & $5, \mathrm{Bi}$ & And & 34 & Or & Catacl & Granite & $\begin{array}{l}\text { Old basement from east of } \\
\text { Al Amar-Idsas fault }\end{array}$ & Gneissic & Trondh & E+ & $\mathbf{E}$ \\
\hline $194-1$ & & 19/41NE. & jt & $\begin{array}{r}\text { Dark } \\
\text { grn }\end{array}$ & - & 30 & $\begin{array}{r}24, \mathrm{Hb} \\
\mathrm{Cpx} \\
\mathrm{Opq}\end{array}$ & Lab & 50 & - & $\begin{array}{l}\text { Hypidio- } \\
\text { morph }\end{array}$ & Gabbro & $\begin{array}{l}\text { Diorite basement, } \\
\text { augitehypersthene quartz } \\
\text { diorite }\end{array}$ & Plutonic & $\begin{array}{l}\text { Quartz } \\
\text { leuco- } \\
\text { gabbro- } \\
\text { norite }\end{array}$ & $\mathrm{J}+$ & $\mathrm{J}$ \\
\hline $195-1$ & & 19/41NE. & jt & Gray & - & 9 & - & - & 47 & - & Lineated & Tonalite & $\begin{array}{l}\text { Biljurshī' quadrangle, late } \\
\text { diorite basement rock }\end{array}$ & Gneissic & Trondh & $\mathbf{J}$ & $J$ \\
\hline $196-1$ & & 19/41NE. & jt & Gray & - & 5 & $\mathrm{Hb}, \mathrm{Bi}$ & - & 27 & - & - & Qtz dio & $\begin{array}{l}\text { Biljurshi' quadrangle, part } \\
\text { of An Nimas batholith }\end{array}$ & Plutonic & Trondh & A & $\mathbf{J}$ \\
\hline
\end{tabular}


TABLE 3.-Description and classification of crystalline rocks of the Arabian Shield for which chemical analyses are given in table 4-Continued

\begin{tabular}{|c|c|c|c|c|c|c|c|c|c|c|c|c|c|c|c|c|}
\hline & \multirow[b]{2}{*}{$\begin{array}{l}\text { Location'1 } \\
\text { (lat/long) }\end{array}$} & \multirow[b]{2}{*}{$\begin{array}{l}\text { Rock-unit } \\
\text { symbol } \\
\text { on pl. } 1^{2}\end{array}$} & \multirow[b]{2}{*}{ Color } & \multirow[b]{2}{*}{$\begin{array}{l}\text { Grain } \\
\text { size }^{3}\end{array}$} & \multirow[b]{2}{*}{$\begin{array}{l}\text { Normative } \\
\text { color } \\
\text { index }\end{array}$} & \multicolumn{3}{|c|}{ Petrography } & \multirow[b]{2}{*}{$\begin{array}{l}\text { Potassium- } \\
\text { feldspar }^{\text {type }} \\
\text { type }^{8}\end{array}$} & \multirow[b]{2}{*}{$\begin{array}{c}\text { Rock } \\
\text { texture- } \\
\text { structure }^{9}\end{array}$} & \multirow[b]{2}{*}{$\begin{array}{c}\text { Rock name } \\
\text { (field or }^{\text {or }} \\
\text { petrography) })^{10}\end{array}$} & \multirow[b]{2}{*}{ Remarks" } & \multicolumn{4}{|c|}{ Classification } \\
\hline $\begin{array}{cl}\begin{array}{c}\text { Sample } \\
\text { (loc. }\end{array} & \text { Field } \\
\text { given } \\
\text { on pl. } \\
\text { 1) }\end{array}$ & & & & & & $\begin{array}{c}\text { Mafic } \\
\text { content } \\
\text { (percent) }^{5}\end{array}$ & $\begin{array}{l}\text { Plagio- } \\
\text { clase } \\
\text { compo- } \\
\text { sition }^{6}\end{array}$ & $\begin{array}{l}\text { Normative } \\
\text { anorthite } \\
\text { (percent) }\end{array}$ & & & & & $\begin{array}{l}\text { Igneous } \\
\text { type } e^{12}\end{array}$ & $\begin{array}{l}\text { Rock name } \\
\text { from } \\
\text { chemistry }{ }^{13}\end{array}$ & $\mathrm{Age}^{14}$ & $\begin{array}{l}\text { Re- } \\
\text { gion }^{15}\end{array}$ \\
\hline $197-\mathrm{B} 10$ & 19/41SE. & agd & Pink & $\mathrm{mg}$ & 2 & $\mathrm{Bi}$ & - & 8 & Mc & $\begin{array}{l}\text { Some } \\
\text { foliation }\end{array}$ & Granite & $\begin{array}{l}\text { Jabal Tharbān, intrudes } \\
\text { Bagarah gneiss }(763 \mathrm{Ma})\end{array}$ & Plutonic & $\begin{array}{l}\text { Alk-feld } \\
\text { granite }\end{array}$ & $\mathrm{S}$ & $\mathrm{J}$ \\
\hline $198-\mathrm{B} 33$ & 19/41SE. & sy & Dark & - & 7 & - & - & 10 & - & - & Syenite & $\begin{array}{l}\text { Jabal Tawi (Lakathah), } \\
\text { outer circular pluton, } \\
\text { 10-km diameter }\end{array}$ & Plutonic & Syenite & $\mathrm{S}, \mathrm{A} ?$ & $\mathrm{~J}$ \\
\hline $199-\mathrm{B} 950$ & 19/41SE. & $\mathrm{gb}$ & Black & - & 40 & ol & - & 59 & - & - & Gabbro & $\begin{array}{l}\text { Jabal Tawi (Lakathah), core } \\
\text { of circular pluton, 6-km } \\
\text { diameter }\end{array}$ & Plutonic & $\begin{array}{l}\text { Leuco- } \\
\text { gabbro }\end{array}$ & $\mathrm{S}, \mathrm{A} ?$ & $\mathrm{~J}$ \\
\hline 200 - Birk-2 & $18 / 41 \mathrm{NE}$ & $\begin{aligned} *[a b] \\
(b a)\end{aligned}$ & - & - & 40 & - & - & 73 & - & - & Amphibo & - & Metamor & $\begin{array}{l}\text { Olivine } \\
\text { norite? }\end{array}$ & B & B \\
\hline $201 \mathrm{a}-\mathrm{B} 1705$ & $19 / 42 \mathrm{NW}$ & jt & Gray & - & 18 & $\mathrm{Bi}, \mathrm{Hb}$ & And & 42 & - & Gneissic & Qtz dio & $\begin{array}{l}\text { Wādi Tarj quadrangle, } \\
\text { An Nimas batholith }\end{array}$ & Gneissic & Tonalite & A, & $\mathrm{J}$ \\
\hline $201 b-B 1706$ & 19/42NW. & $\mathrm{jt}$ & $\begin{array}{l}\text { Light } \\
\text { gray }\end{array}$ & $\mathrm{mg}$ & 2 & $\mathrm{Bi}$ & Olig & 24 & - & Gneissic & Trondh & $\begin{array}{l}\text { Wādĩ Tarj quadrangle, } \\
\text { An Nimas batholith }\end{array}$ & Gneissic & Trondh & A, & $\mathrm{J}$ \\
\hline $202-\mathrm{B} 1704$ & $19 / 42 \mathrm{NW}$ & $\begin{array}{r}{ }^{*}[\mathrm{aog}] \\
(\mathrm{gb})\end{array}$ & $\begin{array}{c}\text { Dark } \\
\text { gray }\end{array}$ & $\mathrm{mg}$ & 39 & $\begin{array}{l}\mathrm{Cpx}, \mathrm{Ol} \\
\mathrm{Hb}\end{array}$ & Lab & 62 & - & Subophitic & Gabbro & $\begin{array}{l}\text { Jabal Uthaynat, 1-km- } \\
\text { diameter layered gabbro, } \\
\text { outer layer }\end{array}$ & Plutonic & $\begin{array}{l}\text { Olivine } \\
\text { norite }\end{array}$ & $\begin{array}{l}\text { S,A?, } \\
\text { late }\end{array}$ & $J$ \\
\hline $203 a-B 1708$ & $19 / 43 \mathrm{NW}$ & hd & Gray & fg & 2 & $\mathrm{Bi}(\mathrm{Ga})$ & Olig & 29 & $\mathrm{Mc}, \mathrm{Or}$ & Catacl & Granodio & $\begin{array}{l}\text { Part of large gneiss dome } \\
\text { complex, orthogneiss }\end{array}$ & Gneissic & Trondh & $\mathrm{H}(\mathrm{C})$ & HS \\
\hline $203 b-B 1709$ & 19/43NW. & $\begin{array}{l}\text { [hd] } \\
\text { (mgd) }\end{array}$ & Gray & fg & 3 & - & - & 14 & - & Catacl & Granodio & $\begin{array}{l}\text { Same locality as sample } \\
\text { 203a, migmatitic gneiss }\end{array}$ & Gneissic & Granodio & $\mathrm{C}$ & HS \\
\hline $204-\mathrm{B} 1722$ & 19/43NW. & [gd](ga) & Red & fg & 1 & $\mathrm{Bi}$ & - & 7 & Mc & $\begin{array}{l}\text { Hypidio- } \\
\text { morph }\end{array}$ & Granite & $\begin{array}{l}\text { Center, Jabal al Ḩaşī } \\
\text { elliptical layered pluton }\end{array}$ & Plutonic & $\begin{array}{l}\text { Alk-feld } \\
\text { granite }\end{array}$ & $\mathrm{s}$ & HS \\
\hline $205-\mathrm{B} 1723$ & 19/43NW. & ga & Red & $\mathrm{cg}$ & 1 & $\mathrm{Bi}$ & Olig & 5 & Pth,Mc & $\begin{array}{l}\text { Hypidio- } \\
\text { morph }\end{array}$ & Granite & $\begin{array}{l}\text { Outer ring, Jabal al Hasiir } \\
\text { elliptical layered pluton }\end{array}$ & Plutonic & $\begin{array}{l}\text { Alk-feld } \\
\text { granite }\end{array}$ & S & HS \\
\hline $206-B 1710$ & $19 / 43 \mathrm{NW}$ & $\operatorname{mog}$ & - & - & 3 & - & - & 26 & - & Catacl & Granodio & $\begin{array}{l}\text { Part of large gneiss dome } \\
\text { complex, orthogneiss }\end{array}$ & Gneissic & Trondh & $\mathrm{H}(\mathrm{C})$ & HS \\
\hline $207-\mathrm{B} 1716$ & 19/43NE. & $\mathrm{mgd}$ & - & - & 4 & - & - & 26 & - & Lineated & Granodio & $\begin{array}{l}\text { Large syntectonic batholith, } \\
\text { complex }\end{array}$ & Gneissic & Granodio & $\mathrm{C}$ & TS \\
\hline $208-\mathrm{B} 1713$ & 19/43NE. & hd & - & - & 28 & - & - & 47 & - & - & Qtz dio & $\begin{array}{l}\text { Diorite basement of } \\
\text { Halaban age }\end{array}$ & Plutonic & $\begin{array}{l}\text { Quartz } \\
\text { diorite }\end{array}$ & $\mathrm{H}$ & TS \\
\hline $209-\mathrm{B} 1721$ & 19/43SW. & ga & Red & $\mathrm{cg}$ & 4 & $\mathrm{Hb}, \mathrm{Bi}$ & - & 7 & Mc,Pth & $\begin{array}{l}\text { Hypidio- } \\
\text { morph }\end{array}$ & Granite & $\begin{array}{l}\text { Outer ring, Jabal al Ḩaşī } \\
\text { elliptical layered pluton }\end{array}$ & Plutonic & $\begin{array}{l}\text { Alk-feld } \\
\text { granite }\end{array}$ & $S$ & HS \\
\hline $210-\mathrm{B} 34$ & 19/41SE. & $\begin{array}{l}*[j c] \\
(\mathrm{gr})\end{array}$ & - & - & 2 & - & - & 11 & - & - & Granite & $\begin{array}{l}\text { Granite, west of Lakathah, } \\
\text { Wãdi Yiba quadrangle }\end{array}$ & Plutonic & Granite & $\mathrm{A}, \mathrm{S} ?$ & $\mathrm{~J}$ \\
\hline $212-\mathrm{B} 1719$ & 19/43SE. & $g b$ & - & - & 48 & - & - & 68 & - & - & Gabbro & $\begin{array}{l}\text { Jibāl al 'Ashsha, SW. end, } \\
\text { elliptical layered gb } \\
\text { complex }\end{array}$ & Plutonic & $\begin{array}{l}\text { Olivine } \\
\text { gabbro }\end{array}$ & $\mathrm{s}$ & TS \\
\hline $214-\mathrm{B} 1720$ & 19/43SE. & $\begin{array}{l}{[\mathrm{gb}]} \\
\quad(\mathrm{um})\end{array}$ & - & - & 94 & Ol & - & 90 & - & - & Serpen & $\begin{array}{l}\text { Jibāl al 'Ashsha, center, } \\
\text { elliptical layered gb } \\
\text { complex }\end{array}$ & Plutonic & Hartzburgite & & TS \\
\hline $215-\mathrm{B} 1718$ & 19/43SE. & $\begin{array}{c}{ }^{*}[\text { Oew }] \\
\text { (hq) }\end{array}$ & - & - & 10 & - & - & 38 & - & - & Qtz dio & $\begin{array}{l}\text { Basement rock outlier in } \\
\text { Wajid sandstone }\end{array}$ & Plutonic & $\begin{array}{l}\text { Quartz } \\
\text { diorite }\end{array}$ & $\mathrm{H}$ & TS \\
\hline BWP1 & 18/42NW. & jv & - & - & 43 & - & - & 56 & - & - & Basalt & On Wädĩ Tayyah road & Volcanic & $\begin{array}{l}\text { Quartz } \\
\text { basalt }\end{array}$ & $\mathrm{J}$ & $\mathrm{J}$ \\
\hline $218-B W P 1$ & $18 / 42 \mathrm{NW}$ & jc & - & - & 46 & - & - & 62 & - & - & Schist & On Wādĩ Tayyah road & Vol & Basalt & $\mathrm{J}$ & $J$ \\
\hline $223 a-B 1707 E$ & 18/43NE. & $\begin{array}{l}{[\mathrm{gb}]} \\
(\mathrm{mog})\end{array}$ & - & $\mathrm{mg}$ & 8 & $\mathrm{Hyp}, \mathrm{Hb}$ & And & 39 & Or & - & Enderbite & $\begin{array}{l}\text { Granulite-facies rock along } \\
\text { Najd fault zone }\end{array}$ & Metamor & Trondh? & $\mathrm{H}(\mathrm{N})$ & TS \\
\hline
\end{tabular}

Footnotes at end of table. 
TABLE 3.-Description and classification of crystalline rocks of the Arabian Shield for which chemical analyses are given in table 4-Continued

\begin{tabular}{|c|c|c|c|c|c|c|c|c|c|c|c|c|c|c|c|c|c|}
\hline \multirow[b]{2}{*}{$\begin{array}{c}\text { Sample } \\
\text { (loc. } \\
\text { given } \\
\text { on pl. } \\
\text { 1) } \\
\end{array}$} & \multirow[b]{2}{*}{$\begin{array}{l}\text { Field } \\
\text { no. }\end{array}$} & \multirow[b]{2}{*}{$\begin{array}{l}\text { Location' } \\
\text { (lat/long) }\end{array}$} & \multirow[b]{2}{*}{$\begin{array}{l}\text { Rock-unit } \\
\text { symbol } \\
\text { on pl. } 1^{2}\end{array}$} & \multicolumn{9}{|c|}{ Petrography } & \multirow[b]{2}{*}{ Remarks ${ }^{11}$} & \multicolumn{4}{|c|}{ Classification } \\
\hline & & & & Color & $\underset{\text { size }^{3}}{\text { Grain }}$ & $\begin{array}{l}\text { Normative } \\
\text { color } \\
\text { index }\end{array}$ & $\begin{array}{c}\text { Mafic } \\
\text { content } \\
\text { (percent) }\end{array}$ & $\begin{array}{l}\text { Plagio- } \\
\text { clase } \\
\text { compo- } \\
\text { sition }^{6}\end{array}$ & $\begin{array}{l}\text { Normative } \\
\text { anorthite } \\
\text { (percent) }\end{array}$ & $\begin{array}{c}\text { Potassium } \\
\text { feldspar }^{\text {type }} \\
\text { type }\end{array}$ & - $\quad \begin{array}{c}\text { Rock } \\
\text { texture- } \\
\text { structure }\end{array}$ & $\begin{array}{c}\text { Rock name } \\
\text { (field or } \\
\text { petrography) }\end{array}$ & & $\underset{\substack{\text { Igneous } \\
\text { type }^{1 / 2}}}{ }$ & $\begin{array}{l}\text { Rock name } \\
\text { from } \\
\text { chemistry }\end{array}$ & $\mathrm{Age}^{14}$ & $\begin{array}{l}\mathrm{Re} \\
\text { gion }^{15}\end{array}$ \\
\hline $223 b-B$ & $1707 \mathrm{~W}$ & 18/43NE. & $\begin{array}{l}{[\mathrm{gb}]} \\
(\mathrm{mog})\end{array}$ & - & - & 64 & - & - & 70 & - & - & Charnock & $\begin{array}{l}\text { Granulite-facies rock along } \\
\text { Najd fault zone }\end{array}$ & Metamor & Metabasalt? & $\mathrm{H}(\mathrm{N})$ & TS \\
\hline $223 c-B$ & SW402 & 18/43NE. & $\begin{array}{l}{[\mathrm{gb}]} \\
(\mathrm{mog})\end{array}$ & - & - & 4 & - & - & 19 & - & - & Charnock & $\begin{array}{l}\text { Granulite-facies rock along } \\
\text { Najd fault zone }\end{array}$ & Metamor & Metagranite? & $? \mathrm{H}(\mathrm{N})$ & TS \\
\hline $224-\mathrm{G}$ & FB151 & 18/42SW. & $\mathrm{gm}$ & - & - & 1 & $1, \mathrm{Bi}$ & Olig & 16 & Mc & Foliated & Granite & $\begin{array}{l}\text { Young, large granite } \\
\text { pluton }\end{array}$ & Plutonic & Granite & C-A?+ & $\mathrm{J}$ \\
\hline $227-B$ & & 18/42SE. & $\operatorname{mog}$ & $\begin{array}{r}\text { Dark } \\
\text { grn }\end{array}$ & $\mathrm{mg}$ & 34 & $\mathrm{Hb}, \mathrm{Bi}$ & - & 37 & - & - & Diorite & $\begin{array}{l}\text { On Abhā-Khamis } \\
\text { Mushayt road, basement } \\
\text { diorite }\end{array}$ & Plutonic & $\begin{array}{l}\text { Quartz } \\
\text { diorite }\end{array}$ & H & HS \\
\hline $237-B$ & & 17/42NE. & $\mathrm{mgd}$ & Gray & - & 6 & $\mathrm{Bi}$ & - & 29 & - & $\begin{array}{l}\text { Some } \\
\text { gneissic }\end{array}$ & Granite & $\begin{array}{l}\text { Elliptical pluton, east of } \\
\text { Wâdi Baysh }\end{array}$ & Plutonic & Granodio & C-A? & HS \\
\hline $241-\mathrm{B}$ & & 17/43SW. & aog & Gray & $\mathrm{mg}$ & 7 & - & - & 30 & - & Gneissic & Granite & $\begin{array}{l}\text { Jabal al Harīsī, syntectonic } \\
\text { granitoid complex }\end{array}$ & Gneissic & Granodio & $\mathrm{C}-\mathrm{A}$ ? & HS \\
\hline $501^{16}$ & - & $?$ & $?$ & - & - & - & - & - & - & - & - & Gray? & $\begin{array}{l}\text { Eastern Province, Shagar } \\
\# 1 \text {, drill core, top of } P \epsilon\end{array}$ & Sediment? & $\begin{array}{l}\text { Basaltic } \\
\text { andesite? }\end{array}$ & $?$ & $\mathrm{EE}$ \\
\hline $502^{16}$ & - & $?$ & $?$ & - & - & - & - & - & - & - & - & Gray? & $\begin{array}{l}\text { Eastern Province, } \\
\text { Shamasiyah \#1, drill core, } \\
\text { top of } \mathrm{P} \epsilon\end{array}$ & Sediment? & - & $?$ & $\mathrm{EE}$ \\
\hline \multicolumn{11}{|c|}{ 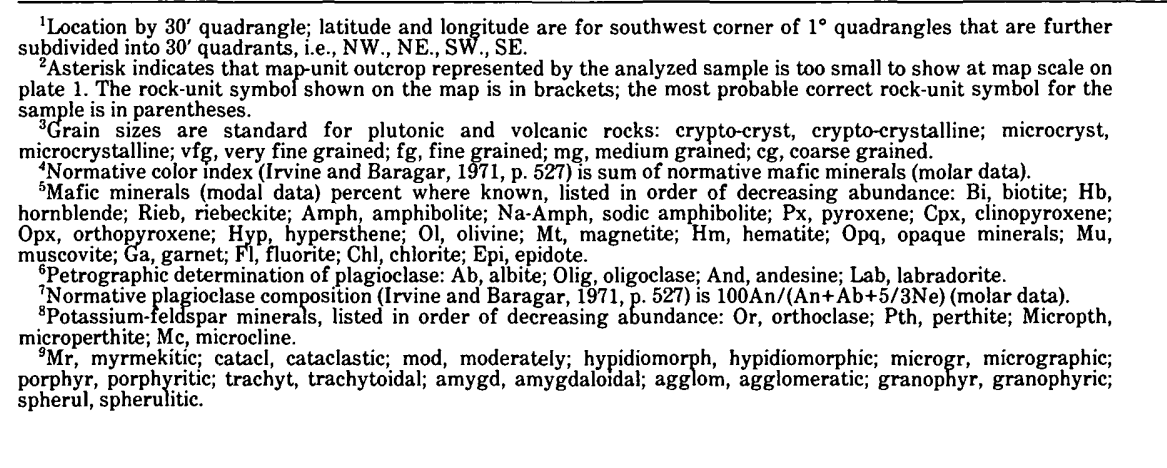 } & \multicolumn{7}{|c|}{ 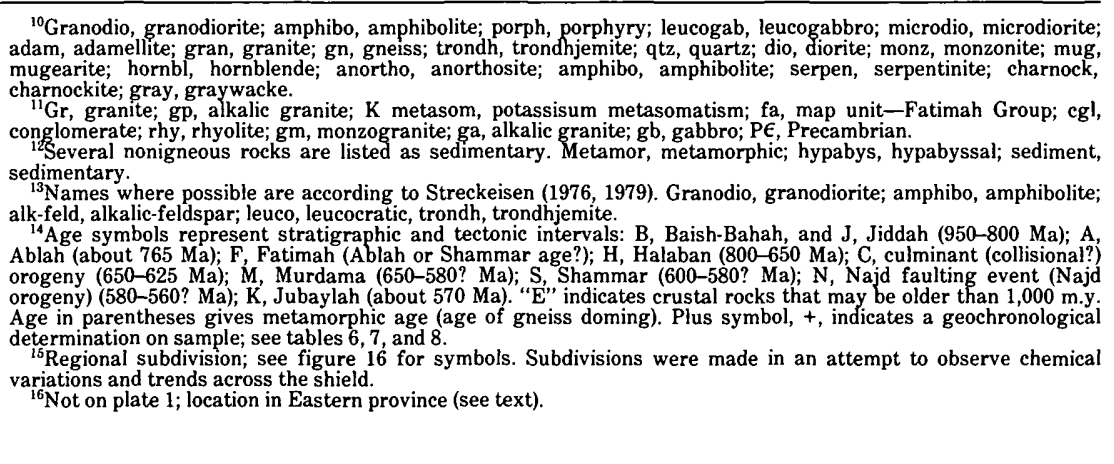 } \\
\hline
\end{tabular}


TABLE 4.-Chemical and normative analyses of crystalline rocks of the Arabian Shield

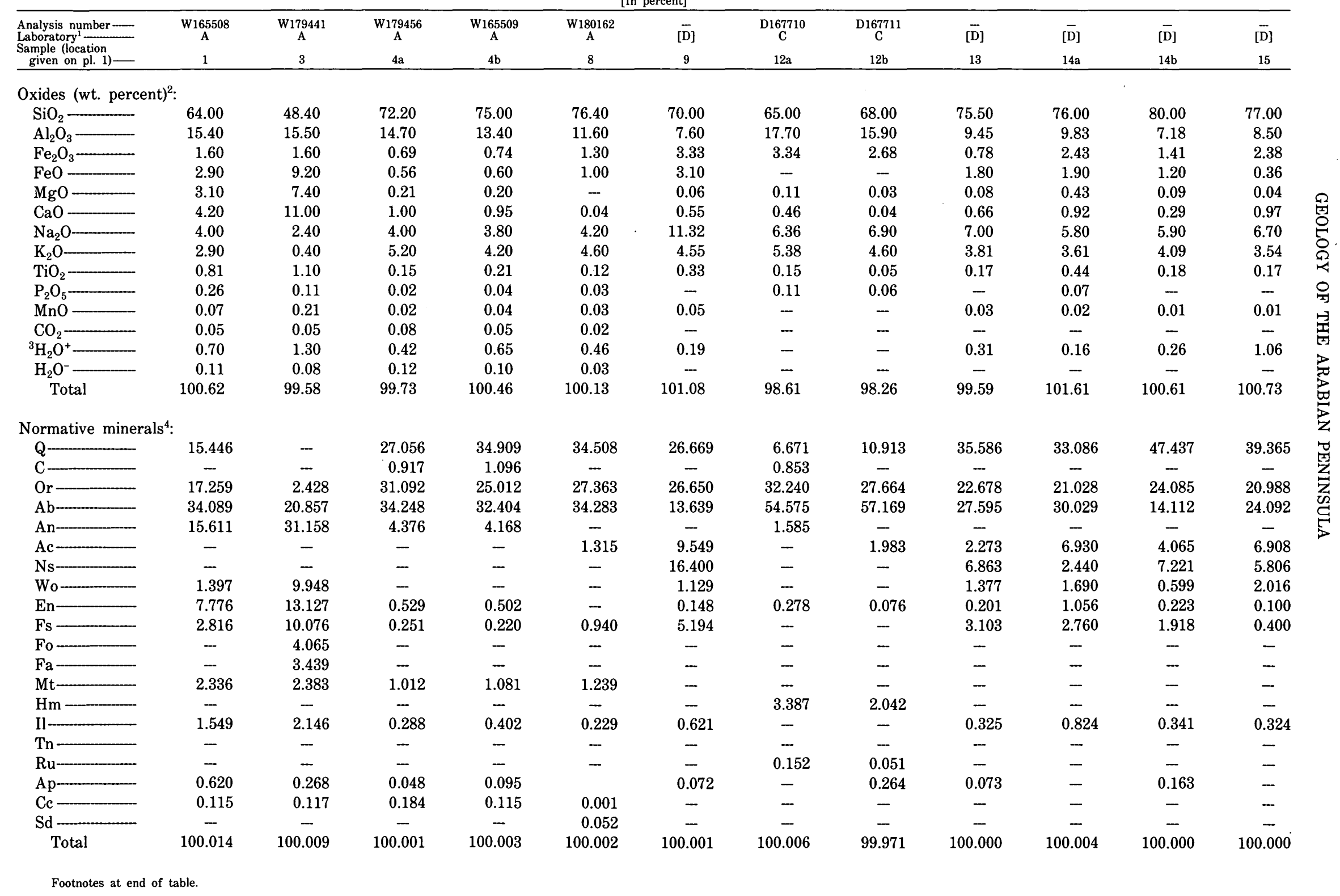


TABLE 4.-Chemical and normative analyses of crystalline rocks of the Arabian Shield-Continued

\begin{tabular}{|c|c|c|c|c|c|c|c|c|c|c|c|c|}
\hline $\begin{array}{l}\text { Analysis number - } \\
\text { Laboratory' } \\
\text { Sample (location } \\
\text { given on pl. 1) } \\
\end{array}$ & $\begin{array}{c}{[\bar{D}]} \\
17\end{array}$ & $\begin{array}{c}\text { W1 } 80420 \\
\mathrm{~A} \\
18 \\
\end{array}$ & $\begin{array}{c}\text { W180027 } \\
\text { B } \\
19 \\
\end{array}$ & $\begin{array}{c}\bar{D} \\
20 \\
\end{array}$ & $\begin{array}{l}{[\overline{\mathrm{D}}]} \\
21\end{array}$ & $\begin{array}{r}\bar{D} \\
22 a \\
\end{array}$ & $\begin{array}{c}\text { W } 180029 \\
\text { B } \\
22 \mathrm{~b} \\
\end{array}$ & $\begin{array}{l}\bar{D} \\
23 \\
\end{array}$ & $\begin{array}{c}\text { W180023 } \\
\text { B } \\
24 \\
\end{array}$ & $\begin{array}{c}\text { W179460 } \\
\mathrm{A} \\
25 \mathrm{a} \\
\end{array}$ & $\begin{array}{c}W_{179461} \\
A \\
25 b \\
\end{array}$ & $\begin{array}{c}\underset{\text { W179462 }}{\mathrm{A}} \\
27 \\
\end{array}$ \\
\hline \multicolumn{13}{|c|}{ Oxides (wt. percent) ${ }^{2}$ : } \\
\hline $\mathrm{SiO}_{2} \longrightarrow$ & 80.00 & 70.70 & 48.40 & 77.00 & 73.50 & 82.40 & 48.00 & 74.00 & 76.90 & 66.20 & 59.40 & 70.40 \\
\hline $\mathrm{Al}_{2} \mathrm{O}_{3} \longrightarrow$ & 8.58 & 15.30 & 17.00 & 7.18 & 10.25 & 8.50 & 15.40 & 12.00 & 12.20 & 17.10 & 16.70 & 12.60 \\
\hline $\mathrm{Fe}_{2} \mathrm{O}_{3}$ & 2.27 & 2.10 & 10.50 & 6.22 & 3.08 & 1.70 & 13.50 & 1.18 & 0.70 & 0.40 & 2.10 & 3.60 \\
\hline $\mathrm{FeO}-$ & 0.40 & 0.12 & 1.50 & 0.72 & 0.68 & 0.80 & 0.84 & 1.60 & 1.00 & 2.00 & 4.70 & 0.08 \\
\hline $\mathrm{MgO}-$ & 0.05 & 0.38 & 4.20 & 0.36 & 0.17 & 0.09 & 2.50 & 0.60 & 0.15 & 1.00 & 3.60 & 0.15 \\
\hline $\mathrm{CaO}=$ & 0.28 & 0.70 & 6.00 & 0.53 & 0.82 & 0.42 & 4.00 & 2.00 & 0.70 & 2.70 & 6.00 & 1.60 \\
\hline $\mathrm{Na}_{2} \mathrm{O}$ & 3.20 & 4.90 & 4.00 & 0.50 & 2.90 & 3.83 & 4.80 & 6.20 & 4.10 & 4.60 & 2.80 & 3.80 \\
\hline $\mathrm{K}_{2} \mathrm{O} \longrightarrow$ & 3.91 & 3.80 & 2.40 & 4.58 & 4.82 & 0.34 & 3.10 & 1.01 & 4.00 & 3.90 & 0.66 & 4.70 \\
\hline $\mathrm{TiO}_{2} \longrightarrow$ & 0.16 & 0.35 & 3.10 & 0.47 & 0.47 & 0.10 & 3.10 & 0.42 & 0.10 & 0.51 & 0.96 & 0.25 \\
\hline $\mathrm{P}_{2} \mathrm{O}_{5}$ & - & 0.17 & 0.38 & 0.08 & 0.06 & - & 0.74 & 0.04 & - & 0.22 & 0.19 & 0.01 \\
\hline $\mathrm{MnO}$ & 0.02 & - & 0.13 & 0.18 & 0.11 & - & 0.20 & 0.06 & - & 0.05 & 0.16 & 0.06 \\
\hline $\mathrm{CO}_{2}$ & - & 0.06 & - & - & - & - & - & - & - & 0.11 & 0.02 & 1.10 \\
\hline${ }^{3} \mathrm{H}_{2} \mathrm{O}^{+}$ & 0.52 & 0.78 & 2.55 & 1.42 & 2.06 & 0.57 & 3.81 & 0.69 & 0.39 & 0.64 & 1.10 & 0.39 \\
\hline $\mathrm{H}_{2} \mathrm{O}^{-}$ & - & 0.36 & - & - & - & - & - & - & - & 0.16 & 0.34 & 0.24 \\
\hline Total & 99.39 & 100.53 & 100.16 & 99.24 & 98.92 & 98.75 & 99.99 & 99.80 & 100.24 & 100.12 & 99.71 & 99.45 \\
\hline \multicolumn{13}{|c|}{ Normative minerals ${ }^{4}:$} \\
\hline$Q$ & 47.668 & 26.611 & - & 56.346 & 38.183 & 58.858 & - & 30.315 & 35.663 & 17.191 & 20.970 & 30.188 \\
\hline $\mathrm{C}-$ & - & 2.434 & - & 0.642 & -- & 1.087 & - & - & - & 1.198 & 0.999 & 0.940 \\
\hline Or - & 23.369 & 22.779 & 14.530 & 27.668 & 29.406 & 2.046 & 19.046 & 6.022 & 23.673 & 23.328 & 4.009 & 28.240 \\
\hline $\mathrm{Ab}-$ & 22.620 & 42.060 & 34.676 & 4.325 & 25.334 & 33.009 & 42.229 & 52.934 & 34.745 & 39.401 & 24.353 & 32.694 \\
\hline $\mathrm{An}$ & - & 2.011 & 21.866 & 2.154 & 0.739 & 2.122 & 11.768 & 1.947 & 3.076 & 11.400 & 29.189 & 0.934 \\
\hline Ac- & 4.199 & - & - & - & - & - & - & - & - & - & - & - \\
\hline Wo- & 0.587 & - & 0.626 & - & 1.276 & - & - & 3.257 & 0.168 & -- & - & - \\
\hline En- & 0.126 & 0.960 & 7.933 & 0.917 & 0.437 & 0.228 & 2.319 & 1.508 & 0.374 & 2.521 & 9.216 & 0.380 \\
\hline $\mathrm{Fs}$ & - & - & - & - & - & - & - & 1.394 & 1.095 & & 2.625 & 5.764 \\
\hline Fo - & - & - & 1.951 & - & - & - & 2.911 & - & - & - & - & - \\
\hline Mt-___ & 0.901 & - & - & 1.580 & 1.227 & 2.331 & - & 1.726 & 1.016 & 0.587 & 3.130 & - \\
\hline $\mathrm{Hm} \cdots$ & 0.223 & 2.130 & 10.757 & 5.269 & 2.334 & 0.124 & 14.036 & - & - & - & - & 3.660 \\
\hline $\mathrm{Il} \longrightarrow$ & 0.307 & 0.257 & 3.531 & 0.913 & 0.922 & 0.193 & 2.289 & 0.805 & 0.190 & 0.980 & 1.874 & 0.302 \\
\hline $\mathrm{Tn} \longrightarrow$ & - & - & 3.232 & - & - & - & 2.704 & - & - & - & - & - \\
\hline $\mathrm{Ru}-$ & - & 0.220 & - & - & - & - & 0.916 & - & - & - & - & 0.095 \\
\hline $\mathrm{Ap} \longrightarrow$ & - & 0.408 & 0.922 & 0.194 & 0.147 & - & 1.822 & 0.096 & - & 0.527 & 0.463 & 0.024 \\
\hline $\mathrm{Cc} \longrightarrow$ & - & 0.138 & - & - & - & - & - & - & - & 0.253 & 0.047 & 2.544 \\
\hline Total & 100.000 & 100.009 & 100.022 & 100.007 & 100.005 & 100.000 & 100.042 & 100.003 & 100.000 & 100.012 & 100.012 & 100.001 \\
\hline
\end{tabular}

Footnotes at end of table. 
TABLE 4.-Chemical and normative analyses $\underset{\text { [In percent] }}{\text { crystalline rocks of the Arabian Shield-Continued }}$

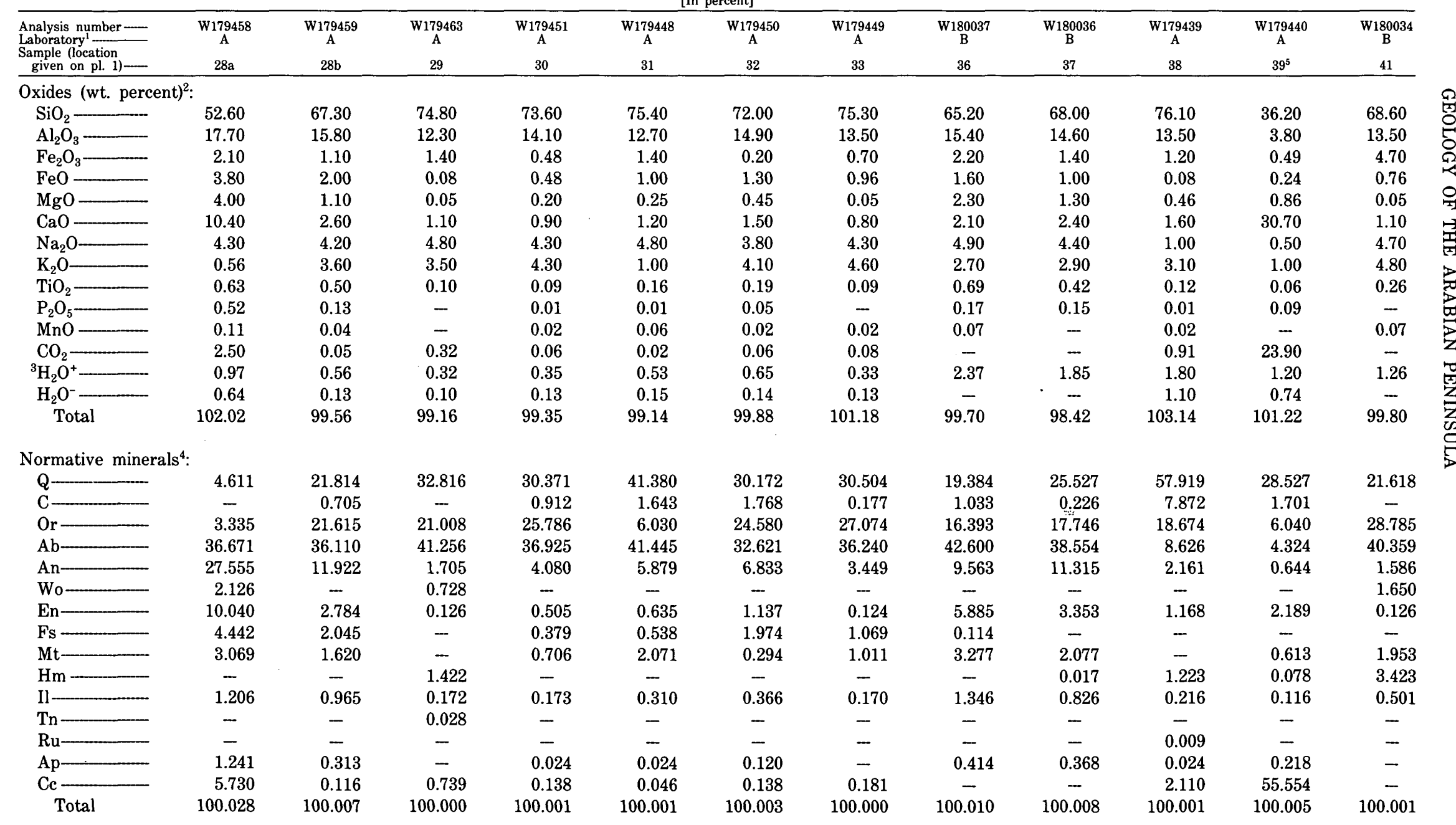

Footnotes at end of table. 
TABLE 4.-Chemical and normative analyses of crystalline rocks of the Arabian Shield-Continued

\begin{tabular}{|c|c|c|c|c|c|c|c|c|c|c|c|c|}
\hline $\begin{array}{l}\text { Analysis number } \\
\text { Laboratory' } \\
\text { Sample (location } \\
\text { given on pl. 1) }\end{array}$ & $\begin{array}{c}\text { W180033 } \\
\mathrm{B} \\
42 \\
\end{array}$ & $\begin{array}{c}\text { W179458 } \\
\text { A } \\
43 \\
\end{array}$ & $\begin{array}{c}\text { W180032 } \\
\text { B } \\
45 \\
\end{array}$ & $\begin{array}{c}\text { W179437 } \\
\text { A } \\
46\end{array}$ & $\begin{array}{c}\text { W180031 } \\
\text { B } \\
47 \\
\end{array}$ & $\begin{array}{c}\text { W179442 } \\
\text { A } \\
48\end{array}$ & $\begin{array}{c}\text { W179445 } \\
\mathrm{A} \\
49 \\
\end{array}$ & $\begin{array}{c}\text { W179455 } \\
\text { A } \\
50\end{array}$ & $\begin{array}{c}\text { W179454 } \\
\text { A } \\
51 \\
\end{array}$ & $\begin{array}{c}\text { W179453 } \\
\text { A } \\
52\end{array}$ & $\begin{array}{c}\text { W165083 } \\
\mathbf{A} \\
54\end{array}$ & $\begin{array}{l}\text { W180035 } \\
\quad \text { B } \\
55\end{array}$ \\
\hline \multicolumn{13}{|c|}{ Oxides (wt. percent) } \\
\hline $\mathrm{SiO}_{2}-\longrightarrow$ & 73.80 & 75.10 & 55.20 & 73.70 & 77.60 & 73.20 & 73.30 & 72.40 & 49.20 & 77.90 & 70.11 & 45.30 \\
\hline $\mathrm{Al}_{2} \mathrm{O}_{3}$ & 9.30 & 14.00 & 15.70 & 13.70 & 12.50 & 14.50 & 14.10 & 14.80 & 19.20 & 12.00 & 15.57 & 14.30 \\
\hline $\mathrm{Fe}_{2} \mathrm{O}_{3}$ & 7.80 & 0.71 & 0.21 & 2.00 & 0.78 & 0.69 & 0.22 & 0.34 & 5.50 & 0.73 & 0.89 & 5.10 \\
\hline $\mathrm{FeO}-$ & 0.48 & 0.20 & 7.00 & - & 0.16 & 0.56 & 0.80 & 0.40 & 4.60 & 0.52 & 1.20 & 10.10 \\
\hline $\mathrm{MgO}=$ & 0.08 & 0.29 & 4.60 & 0.20 & 0.10 & 0.22 & 0.24 & 0.18 & 4.80 & 0.23 & 0.66 & 6.20 \\
\hline $\mathrm{CaO}-$ & 0.70 & 0.82 & 7.40 & 0.25 & 0.20 & 1.00 & 1.70 & 1.00 & 9.20 & 2.00 & 2.64 & 9.20 \\
\hline $\mathrm{Na}_{2} \mathrm{O}-$ & 1.80 & 3.60 & 3.70 & 4.30 & 4.10 & 4.80 & 4.00 & 4.60 & 2.30 & 4.20 & 4.67 & 2.40 \\
\hline $\mathrm{K}_{2} \mathrm{O}$ & 3.30 & 4.70 & 1.10 & 4.70 & 3.90 & 4.40 & 3.60 & 4.40 & 1.00 & 0.66 & 3.17 & 0.80 \\
\hline $\mathrm{TiO}_{2}$ & 0.26 & 0.09 & 1.60 & 0.19 & 0.15 & 0.14 & 0.10 & 0.03 & 0.55 & 0.14 & 0.39 & 4.70 \\
\hline $\mathrm{P}_{2} \mathrm{O}_{5}$ & - & 0.01 & 0.30 & 0.02 & - & 0.02 & 0.02 & - & 0.24 & 0.01 & 0.29 & 0.22 \\
\hline $\mathrm{MnO}$ & 0.04 & 0.01 & 0.13 & 0.27 & - & 0.04 & 0.04 & 0.02 & 0.17 & 0.02 & 0.04 & 0.58 \\
\hline $\mathrm{CO}_{2}-$ & - & 0.04 & - & 0.02 & - & 0.06 & 0.56 & 0.08 & 0.06 & 0.06 & 0.14 & - \\
\hline${ }^{3} \mathrm{H}_{2} \mathrm{O}^{+}$ & 1.01 & 0.49 & 1.57 & 0.54 & 0.52 & 0.37 & 0.64 & 0.49 & 1.40 & 0.29 & 0.39 & 1.08 \\
\hline $\mathrm{H}_{2} \mathrm{O}^{-}$ & - & 0.31 & - & 0.28 & - & 0.07 & 0.12 & 0.24 & 0.32 & 0.11 & 0.08 & - \\
\hline Total & 98.57 & 100.96 & 98.51 & 100.76 & 100.01 & 100.35 & 99.93 & 99.50 & 99.67 & 99.15 & 100.55 & 99.98 \\
\hline \multicolumn{13}{|c|}{ Normative minerals ${ }^{4}$ : } \\
\hline $\mathrm{Q}-$ & 50.310 & 34.269 & 5.634 & 30.175 & 38.445 & 26.151 & 33.742 & 26.882 & 5.628 & 47.158 & 25.023 & 0.421 \\
\hline $\mathrm{C}$ & 1.532 & 1.623 & - & 1.186 & 1.176 & 0.211 & 1.903 & 0.852 & - & 0.917 & 0.677 & - \\
\hline $\mathrm{Or}-$ & 19.988 & 27.894 & 6.705 & 27.955 & 23.164 & 26.097 & 21.558 & 26.464 & 6.103 & 3.961 & 18.776 & 4.780 \\
\hline $\mathrm{Ab}-$ & 15.612 & 30.594 & 32.297 & 36.623 & 34.871 & 40.767 & 34.300 & 39.617 & 20.101 & 36.091 & 39.607 & 20.534 \\
\hline An & 3.560 & 3.766 & 23.707 & 0.990 & 0.997 & 4.468 & 4.827 & 4.535 & 40.396 & 9.625 & 10.341 & 26.171 \\
\hline Wo- & - & - & 5.069 & - & - & - & - & - & 1.976 & - & - & 7.735 \\
\hline $\mathrm{En} \longrightarrow$ & 0.204 & 0.725 & 11.818 & 0.501 & 0.250 & 0.550 & 0.606 & 0.456 & 12.347 & 0.582 & 1.648 & 15.613 \\
\hline $\mathrm{Fs}$ & - & - & 10.605 & - & - & 0.303 & 1.213 & 0.449 & 3.420 & 0.160 & 0.901 & 7.736 \\
\hline $\mathrm{Mt}-$ & 0.947 & 0.418 & 0.314 & 0.333 & 0.081 & 1.004 & 0.323 & 0.502 & 8.236 & 1.075 & 1.293 & 7.477 \\
\hline $\mathrm{Hm} \longrightarrow$ & 7.342 & 0.425 & - & 1.784 & 0.728 & - & - & - & - & - & - & - \\
\hline $\mathrm{Il} \longrightarrow$ & 0.506 & 0.172 & 3.135 & 0.363 & 0.286 & 0.267 & 0.192 & 0.058 & 1.079 & 0.270 & 0.742 & 9.026 \\
\hline $\mathrm{Ap}-$ & - & 0.024 & 0.733 & 0.048 & - & 0.048 & 0.048 & - & 0.587 & 0.024 & 0.688 & 0.527 \\
\hline $\mathrm{Cc} \longrightarrow$ & - & 0.091 & - & 0.046 & - & 0.137 & 1.291 & 0.185 & 0.141 & 0.139 & 0.319 & - \\
\hline Total & 100.001 & 100.001 & 100.018 & 100.005 & 100.000 & 100.002 & 100.002 & 100.000 & 100.015 & 100.001 & 100.016 & 100.019 \\
\hline
\end{tabular}


TABLE 4.-Chemical and normative analyses of crystalline rocks of the Arabian Shield-Continued

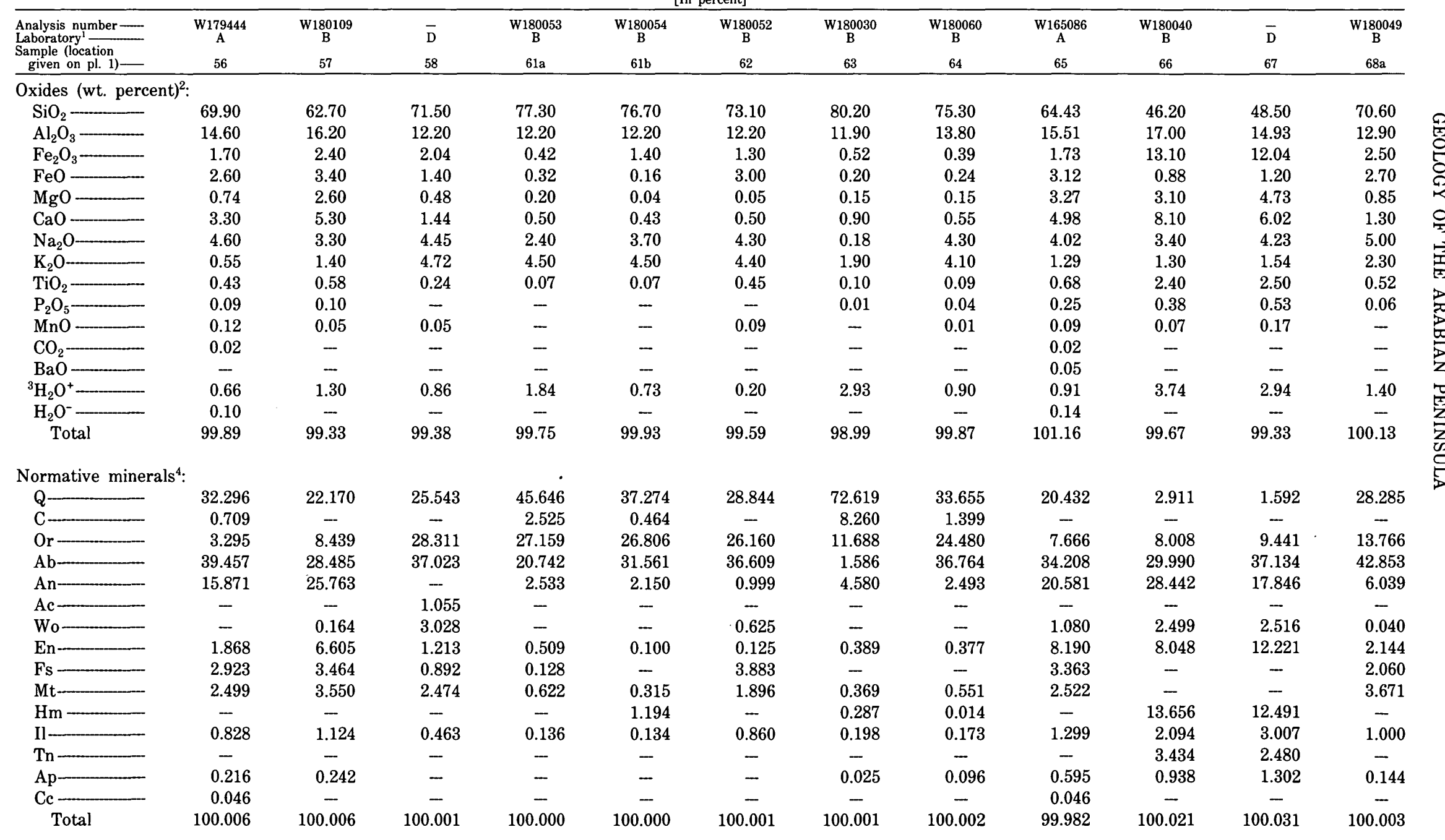

Footnotes at end of table. 
TABLE 4.-Chemical and normative analyses of crystalline rocks of the Arabian Shield-Continued

\begin{tabular}{|c|c|c|c|c|c|c|c|c|c|c|c|c|}
\hline $\begin{array}{l}\text { Analysis number } \\
\text { Laboratory } 1 \\
\text { Sample (location } \\
\text { given on pl.1) }\end{array}$ & $\begin{array}{c}\text { W180048 } \\
\text { B } \\
68 \mathrm{~b}\end{array}$ & $\begin{array}{c}\text { W180042 } \\
\text { B } \\
69\end{array}$ & $\begin{array}{c}\text { W180044 } \\
\text { B } \\
70\end{array}$ & $\begin{array}{c}\text { W180046 } \\
\text { B } \\
71\end{array}$ & $\begin{array}{c}\underset{\text { W }}{\mathrm{A} 67118} \\
73\end{array}$ & $\begin{array}{c}\overline{\mathrm{D}} \\
74\end{array}$ & $\begin{array}{c}\text { W180039 } \\
\text { B } \\
75\end{array}$ & $\begin{array}{l}\underset{\mathrm{W}}{\mathrm{W} 165085} \\
\mathrm{~A} \\
76\end{array}$ & $\begin{array}{l}\text { W165084 } \\
\text { A } \\
77\end{array}$ & $\begin{array}{c}\underset{\mathrm{A}}{\mathrm{W} 165310} \\
78 \mathrm{a}\end{array}$ & $\begin{array}{c}\text { W180059 } \\
\text { B } \\
78 \mathrm{~b}\end{array}$ & $\begin{array}{c}\bar{D} \\
79\end{array}$ \\
\hline \multicolumn{13}{|c|}{ Oxides (wt. percent) ${ }^{2}$} \\
\hline $\mathrm{SiO}_{2} \longrightarrow$ & 71.30 & 54.40 & 65.80 & 75.60 & 76.10 & 47.50 & 53.50 & 72.80 & 69.20 & 74.20 & 73.90 & 62.40 \\
\hline $\mathrm{Al}_{2} \mathrm{O}_{3} \longrightarrow$ & 13.10 & 14.00 & 13.90 & 11.90 & 11.80 & 17.19 & 15.50 & 14.11 & 14.47 & 13.80 & 13.70 & 17.53 \\
\hline $\mathrm{Fe}_{2} \mathrm{O}_{3}-$ & 3.30 & 12.60 & 4.00 & 0.40 & 2.10 & 4.54 & 10.90 & 0.95 & 1.29 & 0.44 & 1.10 & 1.64 \\
\hline $\mathrm{FeO}-$ & 2.50 & 1.50 & 2.30 & 1.30 & 0.24 & 5.80 & 1.50 & 0.82 & 1.25 & 0.60 & 0.56 & 2.30 \\
\hline $\mathrm{MgO}-$ & 0.32 & 1.00 & 1.00 & 1.70 & 0.38 & 4.31 & 1.20 & 0.21 & 0.01 & 0.10 & 0.23 & 1.91 \\
\hline $\mathrm{CaO}-$ & 0.23 & 5.60 & 1.80 & 0.65 & 0.72 & 9.09 & 7.80 & 1.43 & 1.77 & 0.63 & 0.50 & 4.20 \\
\hline $\mathrm{Na}_{2} \mathrm{O}$ & 3.60 & 3.90 & 5.60 & 5.30 & 1.00 & 3.12 & 3.70 & 4.18 & 4.60 & 4.80 & 3.90 & 4.74 \\
\hline $\mathrm{K}_{2} \mathrm{O}$ & 1.70 & 2.00 & 1.90 & 0.21 & 5.60 & 1.45 & 1.40 & 4.44 & 4.41 & 4.50 & 5.10 & 3.60 \\
\hline $\mathrm{TiO}_{2}$ & 0.38 & 2.40 & 0.76 & 0.16 & 0.25 & 2.60 & 3.00 & 0.27 & 0.48 & 0.07 & 0.22 & 0.50 \\
\hline $\mathrm{P}_{2} \mathrm{O}_{5}$ & 0.04 & 1.10 & 0.11 & - & 0.04 & 0.47 & 1.00 & 0.20 & 0.20 & 0.02 & - & 0.24 \\
\hline $\mathrm{MnO}-$ & - & 0.10 & 0.11 & 0.02 & 0.04 & 0.11 & 0.08 & 0.03 & 0.05 & 0.06 & - & 0.04 \\
\hline $\mathrm{CO}_{2} \longrightarrow$ & - & - & - & - & 0.05 & - & - & 0.04 & 0.98 & 0.05 & - & - \\
\hline${ }^{3} \mathrm{H}_{2} \mathrm{O}^{+}$ & - & 0.92 & 1.32 & 1.41 & 0.80 & 2.25 & 1.07 & 0.35 & 0.83 & 0.47 & 0.86 & 0.58 \\
\hline $\mathrm{H}_{2} \mathrm{O}^{-}$ & - & - & - & - & 0.14 & - & - & 0.06 & 0.13 & 0.10 & - & - \\
\hline Total & 96.47 & 99.52 & 98.60 & 98.65 & 99.86 & 98.43 & 100.65 & 100.15 & 100.28 & 100.21 & 100.07 & 99.68 \\
\hline \multicolumn{13}{|c|}{ Normative minerals ${ }^{4}:$} \\
\hline $\mathrm{Q}-$ & 43.987 & 15.141 & 21.733 & 40.321 & 47.796 & 0.876 & 13.288 & 28.790 & 25.081 & 27.620 & 30.525 & 9.728 \\
\hline $\mathrm{C}-$ & 5.199 & - & - & 1.822 & 3.047 & - & - & 0.402 & 1.682 & 0.052 & 0.862 & - \\
\hline Or & 10.413 & 11.986 & 11.542 & 1.276 & 33.657 & 8.909 & 8.308 & 26.374 & 26.400 & 26.787 & 30.377 & 21.467 \\
\hline $\mathrm{Ab}$ & 31.577 & 33.469 & 48.711 & 46.120 & 8.606 & 27.449 & 31.440 & 35.555 & 39.433 & 40.915 & 33.263 & 40.473 \\
\hline An & 0.912 & 14.997 & 7.380 & 3.316 & 3.046 & 29.754 & 21.641 & 5.564 & 1.296 & 2.698 & 2.500 & 16.068 \\
\hline Wo & - & 1.546 & 0.443 & - & - & 5.821 & 2.637 & - & - & - & - & 1.409 \\
\hline $\mathrm{En} \longrightarrow$ & 0.826 & 2.526 & 2.560 & 4.354 & 0.963 & 11.160 & 3.001 & 0.526 & 0.025 & 0.251 & 0.577 & 4.800 \\
\hline Fs - & 1.282 & - & - & 1.882 & - & 2.923 & - & 0.333 & 0.537 & 0.740 & - & 2.137 \\
\hline $\mathrm{Mt}-$ & 4.960 & - & 5.725 & 0.596 & 0.183 & 6.844 & - & 1.385 & 1.895 & 0.643 & 1.176 & 2.399 \\
\hline $\mathrm{Hm} \longrightarrow$ & - & 12.779 & 0.164 & - & 2.010 & - & 10.946 & - & - & - & 0.297 & - \\
\hline $\mathrm{Il}$ & 0.748 & 3.430 & 1.484 & 0.312 & 0.483 & 5.134 & 3.353 & 0.515 & 0.924 & 0.134 & 0.421 & 0.958 \\
\hline $\mathrm{Tn}$ & - & 1.541 & - & - & - & - & 3.060 & - & - & - & - & - \\
\hline$A p-$ & 0.098 & 2.642 & 0.268 & - & 0.096 & 1.157 & 2.379 & 0.476 & 0.480 & 0.048 & - & 0.574 \\
\hline $\mathrm{Ce}-$ & - & - & - & - & 0.116 & - & - & 0.091 & 2.258 & 0.115 & - & - \\
\hline Total & 100.002 & 100.059 & 100.007 & 100.000 & 100.003 & 100.027 & 100.053 & 100.011 & 100.011 & 100.002 & 100.000 & 100.013 \\
\hline
\end{tabular}


TABLE 4.-Chemical and normative analyses of crystalline rocks of the Arabian Shield-Continued

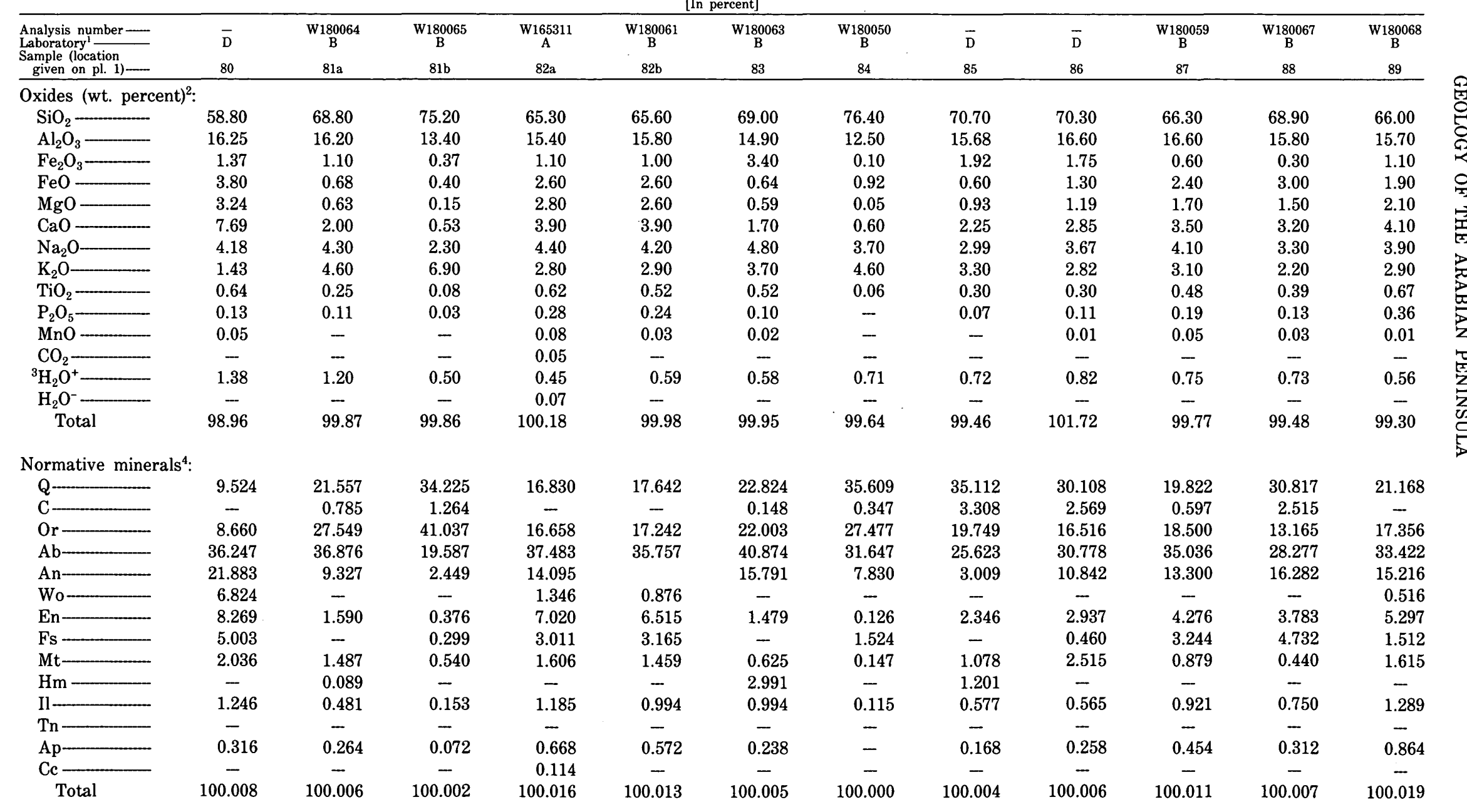

Footnotes at end of table. 
TABLE 4.-Chemical and normative analyses of crystalline rocks of the Arabian Shield-Continued

\begin{tabular}{|c|c|c|c|c|c|c|c|c|c|c|c|}
\hline $\begin{array}{l}\text { Analysis number - } \\
\text { Laboratory' } \\
\text { Sample (location } \\
\text { given on pl. 1)- } \\
\end{array}$ & $\begin{array}{c}\text { W165512 } \\
\text { A } \\
90 \mathrm{a} \\
\end{array}$ & $\begin{array}{c}\text { W180070 } \\
\text { B } \\
90 \mathrm{~b} \\
\end{array}$ & $\begin{array}{c}\text { W165313 } \\
\mathrm{A} \\
91 \mathrm{a} \\
\end{array}$ & $\begin{array}{c}\text { W180071 } \\
\text { B } \\
91 \mathrm{~b} \\
\end{array}$ & $\begin{array}{c}\text { W163663 } \\
\mathrm{A} \\
93 \mathrm{a} \\
\end{array}$ & $\begin{array}{c}W_{163062} \\
\mathrm{~A} \\
93 \mathrm{~b} \\
\end{array}$ & $\begin{array}{c}\text { W163661 } \\
\mathrm{A} \\
93 \mathrm{c} \\
\end{array}$ & $\begin{array}{c}\text { W163660 } \\
\mathrm{A} \\
93 \mathrm{~d} \\
\end{array}$ & $\begin{array}{l}\bar{D} \\
94\end{array}$ & $\begin{array}{c}\text { W180047 } \\
\text { B } \\
98 \\
\end{array}$ & $\begin{array}{c}\text { W167119 } \\
\mathrm{A} \\
102 \\
\end{array}$ \\
\hline \multicolumn{12}{|c|}{ Oxides (wt. percent) } \\
\hline $\mathrm{SiO}_{2} \longrightarrow$ & 66.20 & 71.80 & 76.20 & 75.20 & 53.20 & 50.80 & 50.20 & 48.30 & 74.00 & 73.20 & 62.10 \\
\hline $\mathrm{Al}_{2} \mathrm{O}_{3} \longrightarrow$ & 15.60 & 15.40 & 13.00 & 13.60 & 16.70 & 18.20 & 20.10 & 18.40 & 6.23 & 12.40 & 14.00 \\
\hline $\mathrm{Fe}_{2} \mathrm{O}_{3}$ & 0.87 & 0.40 & 0.23 & 1.17 & 1.40 & 2.50 & 0.60 & 2.30 & 2.35 & - & 5.20 \\
\hline $\mathrm{FeO}-$ & 2.30 & 1.00 & 0.70 & 0.84 & 5.30 & 5.30 & 6.00 & 6.80 & 1.30 & 2.00 & 2.30 \\
\hline $\mathrm{MgO} \longrightarrow$ & 2.20 & 0.54 & 0.10 & 0.15 & 8.20 & 7.40 & 8.30 & 8.70 & 0.76 & 1.90 & 1.50 \\
\hline $\mathrm{CaO}=$ & 3.80 & 2.40 & 0.85 & 1.30 & 10.80 & 9.80 & 9.80 & 9.40 & 2.00 & 3.10 & 4.10 \\
\hline $\mathrm{Na}_{2} \mathrm{O}$ & 4.30 & 3.90 & 3.50 & 3.80 & 2.70 & 2.80 & 2.90 & 2.80 & 5.55 & 0.55 & 5.40 \\
\hline $\mathrm{K}_{2} \mathrm{O}$ & 2.80 & 3.00 & 4.60 & 4.80 & 0.14 & 0.22 & 0.27 & 0.34 & 0.78 & 2.60 & 0.97 \\
\hline $\mathrm{TiO}_{2} \longrightarrow$ & 0.74 & 0.23 & 0.08 & 0.06 & 0.42 & 0.70 & 0.67 & 1.40 & 0.01 & 0.08 & 1.40 \\
\hline $\mathrm{P}_{2} \mathrm{O}_{5}$ & 0.34 & 0.01 & 0.02 & 0.03 & 0.06 & 0.11 & 0.09 & 0.52 & 0.01 & - & 0.59 \\
\hline $\mathrm{MnO}-$ & 0.10 & - & 0.03 & - & 0.11 & 0.13 & 0.07 & 0.13 & - & 0.03 & 0.15 \\
\hline $\mathrm{CO}_{2}$ & 0.05 & - & 0.05 & - & 0.08 & 0.09 & 0.11 & 0.08 & - & - & 1.20 \\
\hline${ }^{3} \mathrm{H}_{2} \mathrm{O}^{+}$ & 0.63 & 0.79 & 0.55 & 0.53 & 0.44 & 1.50 & 0.73 & 0.60 & 6.44 & 3.07 & 1.40 \\
\hline $\mathrm{H}_{2} \mathrm{O}^{-}$ & 0.07 & - & 0.03 & - & 0.17 & 0.32 & 0.14 & 0.16 & - & - & 0.18 \\
\hline Total & 100.44 & 99.47 & 100.29 & 101.48 & 100.15 & 101.06 & 100.54 & 100.44 & 99.43 & 98.93 & 101.48 \\
\hline \multicolumn{12}{|c|}{ Normative minerals ${ }^{4}:$} \\
\hline $\mathrm{Q}-$ & 19.444 & 31.619 & 36.228 & 31.369 & 2.565 & 1.503 & - & - & 45.996 & 51.052 & 21.129 \\
\hline $\mathrm{C}-$ & - & 1.416 & 0.887 & - & - & - & - & - & - & 3.176 & 0.814 \\
\hline $\mathrm{Or}$ & 16.663 & 17.965 & 27.358 & 28.098 & 0.835 & 1.326 & 1.610 & 2.026 & 4.957 & 16.028 & 5.795 \\
\hline$A b-$ & 36.642 & 33.442 & 29.807 & 31.852 & 23.052 & 24.164 & 24.759 & 23.891 & 29.791 & 4.855 & 46.197 \\
\hline$A n-$ & 15.101 & 11.999 & 3.794 & 5.820 & 33.331 & 37.167 & 41.398 & 36.940 & - & 16.043 & 8.998 \\
\hline $\mathrm{Ac}-$ & -- & - & - & - & - & - & - & - & 7.311 & - & - \\
\hline $\mathrm{Ns} \longrightarrow$ & - & - & - & - & - & - & - & - & 2.889 & - & - \\
\hline Wo- & 0.555 & - & - & 0.156 & 8.277 & 4.637 & 2.656 & 2.567 & 4.426 & - & - \\
\hline $\mathrm{En} \longrightarrow$ & 5.518 & 1.363 & 0.251 & 0.370 & 20.606 & 18.796 & 12.114 & 13.356 & 2.035 & 4.936 & 3.777 \\
\hline Fs - & 2.486 & 1.141 & 1.026 & 0.472 & 8.159 & 6.887 & 5.594 & 5.250 & 2.549 & 3.752 & - \\
\hline Fo & - & - & - & - & - & - & 6.127 & 5.951 & - & - & - \\
\hline $\mathrm{Fa} \longrightarrow$ & - & - & - & - & - & - & 3.118 & 2.578 & - & - & - \\
\hline Mt- & 1.270 & 0.588 & 0.336 & 1.680 & 2.048 & 3.697 & 0.878 & 3.363 & - & - & 3.887 \\
\hline $\mathrm{Hm} \longrightarrow$ & - & - & - & - & - & - & - & - & - & - & 2.576 \\
\hline $\mathrm{Il} \longrightarrow$ & 1.415 & 0.443 & 0.153 & 0.113 & 0.805 & 1.356 & 1.284 & 2.681 & 0.020 & 0.158 & 2.688 \\
\hline $\mathrm{Ap}-$ & 0.811 & 0.024 & 0.048 & 0.070 & 0.143 & 0.266 & 0.215 & 1.242 & 0.025 & - & 1.413 \\
\hline $\mathrm{Cc}=$ & 0.115 & - & 0.114 & - & 0.184 & 0.209 & 0.252 & 0.183 & - & - & 2.759 \\
\hline Total & 100.019 & 100.001 & 100.001 & 100.002 & 100.005 & 100.007 & 100.006 & 100.029 & 100.001 & 100.000 & 100.033 \\
\hline
\end{tabular}

Footnotes at end of table 
TABLE 4.-Chemical and normative analyses of crystalline rocks of the Arabian Shield-Continued

\begin{tabular}{|c|c|c|c|c|c|c|c|c|c|c|c|c|}
\hline $\begin{array}{l}\text { Analysis number- } \\
\text { Laboratory' } \\
\text { Sample (location } \\
\text { given on pl. 1) } \\
\end{array}$ & $\begin{array}{c}\text { W167120 } \\
\text { A } \\
103 \\
\end{array}$ & $\begin{array}{c}\text { W180094 } \\
\text { B } \\
104 \\
\end{array}$ & $\begin{array}{c}\text { W180095 } \\
\text { B } \\
105 \\
\end{array}$ & $\begin{array}{c}\text { W180045 } \\
\text { B } \\
106 \\
\end{array}$ & $\begin{array}{c}\text { W180106 } \\
\text { B } \\
111 \\
\end{array}$ & $\begin{array}{c}\text { W180105 } \\
\text { B } \\
113 \\
\end{array}$ & $\begin{array}{c}\mathrm{W} 165316 \\
\mathrm{~A} \\
114 \mathrm{a} \\
\end{array}$ & $\begin{array}{c}\bar{D} \\
114 \mathrm{~b}\end{array}$ & $\begin{array}{c}\bar{D} \\
114 \mathrm{c}\end{array}$ & $\begin{array}{c}\text { W180101 } \\
\mathbf{B} \\
115 \\
\end{array}$ & $\begin{array}{c}\text { W180104 } \\
\text { B } \\
116 \\
\end{array}$ & $\begin{array}{c}\text { W174515 } \\
\mathrm{A} \\
117 \mathrm{a} \\
\end{array}$ \\
\hline \multicolumn{13}{|c|}{ Oxides (wt. percent) } \\
\hline $\mathrm{SiO}_{2} \longrightarrow$ & 52.50 & 46.70 & 72.60 & 75.40 & 70.50 & 75.50 & 75.20 & 66.50 & 69.50 & 73.30 & 52.20 & 50.70 \\
\hline $\mathrm{Al}_{2} \mathrm{O}_{3}$ & 15.30 & 20.70 & 14.90 & 13.70 & 14.10 & 12.90 & 13.70 & 13.87 & 14.00 & 13.80 & 18.70 & 16.40 \\
\hline $\mathrm{Fe}_{2} \mathrm{O}_{3}$ & 2.60 & 1.70 & 1.00 & 0.70 & 1.30 & 1.30 & 0.43 & 0.59 & 0.75 & 1.30 & 3.40 & 1.90 \\
\hline $\mathrm{FeO}$ & 6.80 & 5.90 & 1.20 & 0.52 & 1.80 & 0.36 & 0.70 & 1.80 & 0.76 & 1.00 & 5.70 & 8.30 \\
\hline $\mathrm{MgO}$ & 5.80 & 9.40 & 0.42 & 0.32 & 1.00 & 0.08 & 0.40 & 0.72 & $0.3^{n}$ & 0.73 & 4.10 & 9.40 \\
\hline $\mathrm{CaO}-$ & 9.50 & 12.60 & 1.80 & 1.50 & 2.20 & 0.08 & 1.20 & 7.56 & 7.63 & 2.40 & 8.00 & 1.20 \\
\hline $\mathrm{Na}_{2} \mathrm{O}$ & 3.40 & 1.70 & 3.60 & 6.10 & 3.60 & 4.40 & 4.10 & 4.32 & 4.40 & 3.50 & 3.00 & 2.10 \\
\hline $\mathrm{K}_{2} \mathrm{O} \ldots$ & 0.27 & 0.20 & 4.10 & 1.10 & 3.50 & 3.60 & 3.10 & 1.80 & 2.64 & 2.80 & 0.55 & 1.40 \\
\hline $\mathrm{TiO}_{2}-$ & 1.50 & 0.16 & 0.22 & 0.11 & 0.55 & 0.14 & 0.19 & 0.28 & 0.13 & 0.24 & 1.10 & 1.60 \\
\hline $\mathrm{P}_{2} \mathrm{O}_{5} \longrightarrow$ & 0.24 & 0.14 & 0.05 & - & 0.03 & 0.28 & 0.04 & 0.01 & 0.09 & 0.06 & 0.24 & 0.19 \\
\hline $\mathrm{MnO}-$ & 0.09 & 0.10 & 0.01 & 0.03 & 0.01 & - & 0.05 & 0.04 & - & - & 0.10 & 0.03 \\
\hline $\mathrm{CO}_{2}$ & 0.06 & - & - & - & - & - & 0.05 & - & - & - & - & 0.64 \\
\hline${ }^{3} \mathrm{H}_{2} \mathrm{O}^{+}$ & 1.70 & 0.89 & 0.78 & 1.50 & 0.66 & 0.66 & 0.73 & 0.83 & 0.61 & 0.59 & 1.48 & 5.99 \\
\hline $\mathrm{H}_{2} \mathrm{O}^{-}-$ & 0.13 & - & - & - & - & - & 0.04 & - & - & - & - & - \\
\hline Total & 101.01 & 100.19 & 100.68 & 100.98 & 99.25 & 99.30 & 100.39 & 98.32 & 100.81 & 99.72 & 98.57 & 99.85 \\
\hline \multicolumn{13}{|c|}{ Normative minerals ${ }^{4}$ : } \\
\hline $\mathrm{Q}-$ & 4.163 & - & 31.189 & 32.220 & 29.856 & 36.507 & 36.554 & 21.931 & 22.707 & 36.314 & 8.029 & 14.682 \\
\hline $\mathrm{C}-$ & - & - & 1.388 & - & 0.468 & 1.789 & 1.643 & -- & - & 0.799 & - & 11.918 \\
\hline Or & 1.627 & 1.190 & 24.252 & 6.534 & 20.978 & 21.567 & 18.474 & 10.911 & 15.569 & 16.691 & 3.348 & 8.814 \\
\hline $\mathrm{Ab}-$ & 29.339 & 14.486 & 30.493 & 51.886 & 30.898 & 37.745 & 34.987 & 37.496 & 37.157 & 29.876 & 26.146 & 18.932 \\
\hline $\mathrm{An}-$ & 26.196 & 48.600 & 8.612 & 6.787 & 10.872 & - & 5.421 & 13.476 & 10.632 & 11.615 & 37.011 & 0.710 \\
\hline Wo- & 8.301 & 5.607 & - & 0.290 & - & - & - & 10.408 & 11.089 & - & 0.940 & - \\
\hline En- & 14.731 & 8.224 & 1.047 & 0.801 & 2.526 & 0.202 & 1.005 & 1.839 & 0.746 & 1.834 & 10.517 & 24.942 \\
\hline Fs & 8.188 & 3.285 & 1.034 & 0.252 & 1.361 & - & 0.715 & 2.493 & 0.560 & 0.369 & 6.208 & 11.811 \\
\hline$F_{0}$ & - & 10.758 & - & - & - & - & - & - & - & - & - & - \\
\hline $\mathrm{Fa}$ & - & 4.736 & - & - & - & - & - & - & - & - & - & - \\
\hline Mt - & 3.844 & 2.482 & 1.451 & 1.020 & 1.912 & 0.765 & 0.629 & 0.877 & 1.085 & 1.901 & 5.077 & 2.935 \\
\hline $\mathrm{Hm}-$ & - & - & - & - & - & 0.790 & - & - & - & - & - & - \\
\hline $\mathrm{Il}$ & 2.905 & 0.306 & 0.418 & 0.210 & 1.060 & 0.270 & 0.364 & 0.545 & 0.246 & 0.460 & 2.152 & 3.238 \\
\hline $\mathrm{Ap}-$ & 0.580 & 0.334 & 0.119 & - & 0.072 & 0.146 & 0.096 & 0.024 & 0.213 & 0.143 & 0.585 & 0.479 \\
\hline $\mathrm{Cc}$ & 0.139 & - & - & - & - & - & 0.115 & - & - & - & - & 1.551 \\
\hline Total & 100.014 & 100.009 & 100.003 & 100.000 & 100.002 & 99.781 & 100.003 & 100.001 & 100.005 & 100.003 & 100.014 & 100.011 \\
\hline
\end{tabular}

Footnotes at end of table. 
TABLE 4.-Chemical and normative analyses of crystalline rocks of the Arabian Shield-Continued

\begin{tabular}{|c|c|c|c|c|c|c|c|c|c|c|c|c|}
\hline $\begin{array}{l}\text { Analysis number } \\
\text { Laboratory' } \\
\text { Sample (location } \\
\text { given on pl. 1) }\end{array}$ & $\begin{array}{c}\underset{A}{W} 174516 \\
117 \mathrm{~b}\end{array}$ & $\begin{array}{c}\text { W180108 } \\
\text { B } \\
122\end{array}$ & $\begin{array}{c}\mathrm{W} 180107 \\
\mathbf{B} \\
123\end{array}$ & $\begin{array}{c}\mathrm{W} 165317 \\
\mathrm{~A} \\
124 \mathrm{a}\end{array}$ & $\begin{array}{c}\mathrm{W} 180102 \\
\mathrm{~B} \\
124 \mathrm{~b}\end{array}$ & $\underset{\mathrm{A}}{\mathrm{W} 165318}$ & $\underset{\mathrm{A}}{\mathrm{W} 165320}$ & $\begin{array}{c}\mathrm{W} 165319 \\
\mathrm{~A} \\
129\end{array}$ & $\begin{array}{c}\text { W165977 } \\
\text { A }\end{array}$ & $\underset{A}{\mathrm{~W} 180170}$ & $\underset{A}{W 180171}$ & $\underset{A}{W 180157}$ \\
\hline \multicolumn{13}{|c|}{ Oxides (wt. percent) } \\
\hline $\mathrm{SiO}_{2}$ & 59.90 & 67.80 & 73.40 & 63.40 & 72.40 & 73.40 & 66.70 & 74.30 & 75.20 & 60.40 & 65.70 & 54.20 \\
\hline $\mathrm{Al}_{2} \mathrm{O}_{3}-$ & 15.60 & 14.90 & 13.30 & 17.40 & 15.70 & 14.50 & 16.20 & 13.50 & 13.00 & 17.50 & 15.30 & 14.30 \\
\hline $\mathrm{Fe}_{2} \mathrm{O}_{3}$ & 2.80 & 3.30 & 1.70 & 1.20 & 0.30 & 0.53 & 0.92 & 0.17 & 0.90 & 2.80 & 3.80 & 3.80 \\
\hline $\mathrm{FeO} \ldots$ & 5.50 & 1.70 & 0.76 & 3.90 & 1.60 & 0.70 & 2.80 & 0.60 & 0.82 & 4.10 & 1.40 & 7.30 \\
\hline $\mathrm{MgO}-$ & 2.90 & 0.55 & 0.31 & 1.00 & 0.60 & 0.50 & 2.10 & 0.10 & 0.13 & 2.90 & 1.60 & 3.50 \\
\hline $\mathrm{CaO} \longrightarrow$ & 3.20 & 2.30 & 0.83 & 5.20 & 3.20 & 1.20 & 4.60 & 0.65 & 0.77 & 6.60 & 0.86 & 3.80 \\
\hline $\mathrm{Na}_{2} \mathrm{O}$ & 5.20 & 5.60 & 4.80 & 4.00 & 4.50 & 3.70 & 3.80 & 4.30 & 4.00 & 2.00 & 6.60 & 4.10 \\
\hline $\mathrm{K}_{2} \mathrm{O}-$ & 0.57 & 1.40 & 2.30 & 1.20 & 0.76 & 4.20 & 1.30 & 5.10 & 4.30 & 0.58 & 1.10 & 2.00 \\
\hline $\mathrm{TiO}_{2}=$ & 1.20 & 0.44 & 0.21 & 0.50 & 0.13 & 0.19 & 0.42 & 0.09 & 0.16 & 0.42 & 0.81 & 2.10 \\
\hline $\mathrm{P}_{2} \mathrm{O}_{5}$ & 0.42 & 0.04 & 0.13 & 0.36 & 0.13 & 0.06 & 0.10 & - & 0.03 & 0.14 & 0.23 & 0.58 \\
\hline $\mathrm{MnO}$ & 0.13 & 0.07 & 0.05 & 0.20 & 0.02 & 0.03 & 0.10 & 0.06 & 0.06 & 0.17 & 0.09 & 0.21 \\
\hline $\mathrm{CO}_{2}$ & 0.20 & - & - & 0.05 & - & 0.05 & 0.05 & 0.05 & 0.05 & 0.02 & 0.30 & 0.08 \\
\hline${ }^{3} \mathrm{H}_{2} \mathrm{O}^{+}$ & 2.19 & 1.23 & 1.59 & 1.00 & 0.50 & 0.46 & 0.72 & 0.24 & 0.45 & 2.30 & 1.20 & 2.80 \\
\hline $\mathrm{H}_{2} \mathrm{O}^{-}$ & - & - & - & 0.05 & - & 0.05 & 0.26 & 0.05 & 0.02 & 0.05 & 0.10 & 0.27 \\
\hline Total & 99.81 & 99.33 & 99.38 & 100.09 & 99.84 & 99.89 & 100.75 & 99.40 & 100.17 & 101.36 & 99.89 & 100.93 \\
\hline \multicolumn{13}{|c|}{ Normative minerals ${ }^{4}$ : } \\
\hline $\mathrm{Q}-$ & 15.642 & 24.703 & 35.591 & 21.804 & 35.019 & 32.835 & 25.521 & 28.345 & 33.773 & 26.967 & 20.801 & 8.252 \\
\hline $\mathrm{C}-$ & 2.130 & 0.088 & 1.755 & 1.061 & 1.981 & 1.963 & 0.538 & - & 0.556 & 2.011 & 3.000 & 0.058 \\
\hline Or & 3.450 & 8.433 & 13.898 & 7.206 & 4.521 & 25.054 & 7.753 & 30.466 & 25.558 & 3.511 & 6.647 & 12.315 \\
\hline $\mathrm{Ab}-$ & 45.074 & 48.303 & 41.534 & 34.394 & 38.331 & 31.605 & 32.450 & 36.783 & 34.044 & 17.334 & 57.109 & 36.150 \\
\hline $\mathrm{An} \cdots$ & 12.156 & 11.365 & 3.342 & 23.503 & 15.126 & 5.295 & 22.052 & 2.499 & 3.327 & 32.471 & 0.887 & 15.168 \\
\hline Wo & - & - & - & - & - & - & - & 0.184 & - & - & - & - \\
\hline $\mathrm{En}-$ & 7.399 & 1.396 & 0.790 & 2.531 & 1.504 & 1.257 & 5.278 & 0.252 & 0.326 & 7.398 & 4.075 & 9.083 \\
\hline $\mathrm{Fs}-$ & 6.194 & - & - & 5.809 & 2.529 & 0.595 & 3.910 & 0.934 & 0.613 & 4.956 & - & 7.490 \\
\hline Mt - & 4.159 & 4.518 & 2.049 & 1.768 & 0.438 & 0.776 & 1.346 & 0.249 & 1.313 & 4.158 & 2.514 & 5.741 \\
\hline $\mathrm{Hm} \longrightarrow$ & - & 0.248 & 0.325 & - & - & - & - & - & - & - & 2.152 & - \\
\hline $\mathrm{Il} \longrightarrow$ & 2.335 & 0.852 & 0.408 & 0.965 & 0.249 & 0.364 & 0.805 & 0.173 & 0.306 & 0.817 & 1.573 & 4.156 \\
\hline$A p-$ & 1.019 & 0.097 & 0.315 & 0.866 & 0.310 & 0.143 & 0.239 & - & 0.071 & 0.340 & 0.557 & 1.431 \\
\hline $\mathrm{Cc}-$ & 0.466 & - & - & 0.116 & - & 0.115 & 0.115 & 0.115 & 0.114 & 0.047 & 0.698 & 0.190 \\
\hline Total & 100.024 & 100.003 & 100.007 & 100.021 & 100.007 & 100.003 & 100.006 & 100.001 & 100.002 & 100.010 & 100.013 & 100.034 \\
\hline
\end{tabular}


TABLE 4.-Chemical and normative analyses of crystalline rocks of the Arabian Shield-Continued

\begin{tabular}{|c|c|c|c|c|c|c|c|c|c|c|c|c|}
\hline $\begin{array}{l}\text { Analysis number- } \\
\text { Laboratory' } \\
\text { Sample (location } \\
\text { given on pl. 1) } \\
\end{array}$ & $\begin{array}{c}\text { W } 180158 \\
\text { A } \\
139 \\
\end{array}$ & 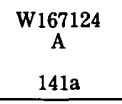 & $\begin{array}{c}\begin{array}{c}\text { W167122 } \\
\mathrm{A}\end{array} \\
141 \mathrm{~b} \\
\end{array}$ & $\begin{array}{c}\underset{A}{W} 167121 \\
141 \mathrm{c} \\
\end{array}$ & $\begin{array}{c}\text { W180074 } \\
\text { B } \\
141 \mathrm{~d} \\
\end{array}$ & $\begin{array}{c}\text { W167123 } \\
\text { A } \\
142 \\
\end{array}$ & $\begin{array}{c}\text { W167125 } \\
\mathrm{A} \\
143 \\
\end{array}$ & $\begin{array}{c}\text { W165314 } \\
\text { A } \\
144 a \\
\end{array}$ & $\begin{array}{c}\text { W180073 } \\
\text { B } \\
144 \mathrm{~b} \\
\end{array}$ & $\begin{array}{c}\text { W180075 } \\
\text { B } \\
145 \\
\end{array}$ & $\begin{array}{c}\text { W180096 } \\
\text { B } \\
146 \\
\end{array}$ & $\begin{array}{c}\text { W180097 } \\
\text { B } \\
147 \mathrm{a} \\
\end{array}$ \\
\hline \multicolumn{13}{|c|}{ Oxides (wt. percent) ${ }^{2}$ : } \\
\hline $\mathrm{SiO}_{2}$ & 76.10 & 57.70 & 73.20 & 75.70 & 71.00 & 66.00 & 63.70 & 74.70 & 70.00 & 49.70 & 76.10 & 75.80 \\
\hline $\mathrm{Al}_{2} \mathrm{O}_{3}$ & 11.90 & 17.50 & 13.60 & 12.30 & 14.25 & 15.00 & 15.50 & 13.30 & 14.50 & 21.30 & 12.70 & 13.80 \\
\hline $\mathrm{Fe}_{2} \mathrm{O}_{3}$ & 1.50 & 4.90 & 1.80 & 1.70 & 1.82 & 2.90 & 2.20 & 0.21 & 0.41 & 0.50 & 1.40 & 0.52 \\
\hline $\mathrm{FeO}-$ & 0.60 & 1.20 & 0.36 & 0.20 & 0.52 & 1.10 & 2.40 & 0.90 & 2.60 & 3.80 & 0.20 & 0.36 \\
\hline $\mathrm{MgO}-$ & 0.05 & 1.90 & 0.54 & 0.58 & 0.53 & 2.40 & 2.20 & 0.10 & 0.55 & 10.20 & 0.06 & 0.02 \\
\hline $\mathrm{CaO}-$ & 1.00 & 4.80 & 1.40 & 2.00 & 0.60 & 3.00 & 4.70 & 0.78 & 2.00 & 9.80 & 0.36 & 0.55 \\
\hline $\mathrm{Na}_{2} \mathrm{O}$ & 4.60 & 5.30 & 3.80 & 3.80 & 4.61 & 3.60 & 4.00 & 3.60 & 3.80 & 2.70 & 4.20 & 4.60 \\
\hline $\mathrm{K}_{2} \mathrm{O}$ & 2.90 & 3.20 & 3.20 & 1.50 & 4.14 & 3.60 & 2.90 & 5.20 & 3.80 & 0.12 & 4.30 & 4.20 \\
\hline $\mathrm{TiO}_{2} \longrightarrow$ & 0.10 & 0.89 & 0.33 & 0.15 & 0.32 & 0.44 & 0.63 & 0.11 & 0.38 & 0.22 & 0.16 & 1.00 \\
\hline $\mathrm{P}_{2} \mathrm{O}_{5}$ & 0.01 & 0.32 & 0.16 & 0.04 & 0.10 & 0.12 & 0.31 & - & 0.19 & 0.04 & 0.04 & 0.03 \\
\hline $\mathrm{MnO} \ldots$ & 0.02 & 0.11 & 0.10 & 0.04 & 0.04 & 0.06 & 0.12 & 0.04 & 0.01 & 0.02 & - & 0.11 \\
\hline $\mathrm{CO}_{2} \longrightarrow$ & 0.08 & 0.66 & 0.05 & 0.34 & - & 0.15 & 0.05 & 0.05 & - & - & - & - \\
\hline${ }^{3} \mathrm{H}_{2} \mathrm{O}^{+}$ & 0.89 & 1.30 & 0.57 & 0.66 & 0.29 & 1.00 & 1.10 & 0.57 & 0.31 & 0.21 & 0.80 & 0.69 \\
\hline $\mathrm{H}_{2} \mathrm{O}^{-}$ & 0.11 & 0.13 & 0.13 & 0.07 & - & 0.08 & 0.07 & 0.05 & - & - & - & - \\
\hline Total & 100.49 & 100.79 & 99.70 & 99.53 & 98.22 & 100.11 & 100.59 & 99.99 & 98.55 & 98.61 & 100.32 & 101.68 \\
\hline \multicolumn{13}{|c|}{ Normative minerals ${ }^{4}$ : } \\
\hline$Q-[-$ & 36.940 & 4.945 & 36.162 & 44.481 & 27.110 & 22.391 & 17.539 & 31.873 & 27.527 & - & 34.633 & 31.535 \\
\hline $\mathrm{C}-$ & - & - & 1.866 & 1.700 & 1.362 & 0.368 & - & 0.452 & 0.971 & - & 0.581 & 0.751 \\
\hline Or & 17.335 & 19.202 & 19.190 & 9.013 & 24.982 & 21.626 & 17.361 & 31.042 & 22.858 & 0.721 & 25.532 & 24.576 \\
\hline $\mathrm{Ab}-$ & 39.373 & 45.539 & 32.631 & 32.694 & 39.833 & 30.967 & 34.289 & 30.773 & 32.731 & 23.218 & 35.711 & 38.542 \\
\hline $\mathrm{An}-$ & 3.295 & 14.733 & 5.667 & 7.637 & 2.372 & 13.369 & 15.980 & 3.590 & 8.836 & 46.387 & 1.532 & 2.508 \\
\hline Wo- & 0.478 & 1.289 & - & - & - & -- & 2.200 & - & - & 1.151 & - & - \\
\hline En & 0.126 & 4.805 & 1.365 & 1.469 & 1.348 & 6.076 & 5.551 & 0.252 & 1.394 & 12.053 & 0.150 & 0.049 \\
\hline Fs & - & - & - & - & - & - & 1.796 & 1.386 & 3.895 & 2.960 & - & - \\
\hline$F_{0}$ & - & - & - & - & - & - & - & - & - & 9.645 & - & - \\
\hline $\mathrm{Fa}-$ & - & - & - & - & - & - & - & - & - & 2.610 & - & - \\
\hline $\mathrm{Mt}-$ & 1.729 & 1.673 & 0.538 & 0.346 & 0.898 & 2.507 & 3.231 & 0.308 & 0.605 & 0.737 & 0.182 & - \\
\hline $\mathrm{Hm} \longrightarrow$ & 0.325 & 3.822 & 1.456 & 1.490 & 1.239 & 1.219 & - & - & - & - & 1.281 & 0.515 \\
\hline $\mathrm{Il} \longrightarrow$ & 0.192 & 1.716 & 0.636 & 0.290 & 0.621 & 0.850 & 1.212 & 0.211 & $0.735 \cdots$ & $0: 425$ & 0.305 & 0.986 \\
\hline $\mathrm{Ru} \longrightarrow$ & - & - & - & - & - & - & - & - & - & - & - & 0.471 \\
\hline $\mathrm{Ap}-$ & 0.024 & 0.770 & 0.385 & 0.096 & 0.242 & 0.289 & 0.744 & - & 0.458 & 0.096 & 0.095 & \\
\hline $\mathrm{Cc}-$ & 0.184 & 1.524 & 0.115 & 0.786 & - & 0.347 & 0.115 & 0.115 & - & - & - & - \\
\hline Total & 100.001 & 100.018 & 100.010 & 100.003 & 100.006 & 100.007 & 100.018 & 100.000 & 100.010 & 100.002 & 100.002 & 100.003 \\
\hline
\end{tabular}

Footnotes at end of table. 
TABLE 4.-Chemical and normative analyses of crystalline rocks of the Arabian Shield-Continued

\begin{tabular}{|c|c|c|c|c|c|c|c|c|c|c|c|c|}
\hline $\begin{array}{l}\text { Analysis number- } \\
\text { Laboratory' } \\
\text { Sample (location } \\
\text { given on pl. 1) }\end{array}$ & $\begin{array}{c}\text { W180098 } \\
\text { B } \\
147 \mathrm{~b} \\
\end{array}$ & $\begin{array}{c}\mathrm{W} 165315 \\
\mathrm{~A} \\
149 \mathrm{a} \\
\end{array}$ & $\begin{array}{c}\text { W180072 } \\
\text { B } \\
149 \mathrm{~b} \\
\end{array}$ & 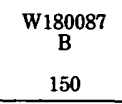 & 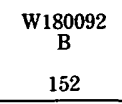 & $\begin{array}{c}\text { W180078 } \\
\text { B } \\
154 \\
\end{array}$ & 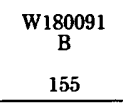 & $\begin{array}{c}\text { W179446 } \\
\mathrm{A} \\
156 \\
\end{array}$ & $\begin{array}{c}\text { W180086 } \\
\text { B } \\
157 \\
\end{array}$ & 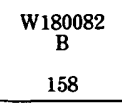 & $\begin{array}{c}\text { W180081 } \\
\text { B } \\
159 \mathrm{a} \\
\end{array}$ & $\begin{array}{c}\text { W180081a } \\
\text { B } \\
159 \mathrm{~b} \\
\end{array}$ \\
\hline \multicolumn{13}{|c|}{ Oxides (wt. percent) ${ }^{2}$ : } \\
\hline $\mathrm{SiO}_{2}$ & 52.20 & 75.50 & 74.70 & 57.00 & 65.40 & 74.90 & 76.30 & 56.80 & 74.80 & 76.50 & 66.00 & 73.00 \\
\hline $\mathrm{Al}_{2} \mathrm{O}_{3}$ & 18.30 & 13.50 & 13.20 & 16.10 & 15.00 & 13.70 & 12.50 & 15.70 & 13.20 & 12.80 & 17.70 & 14.40 \\
\hline $\mathrm{Fe}_{2} \mathrm{O}_{3}$ & 6.50 & 0.29 & 0.30 & 3.08 & 2.40 & 0.80 & 1.10 & 2.10 & 0.25 & 0.39 & 0.94 & 1.10 \\
\hline $\mathrm{FeO}-$ & 3.60 & 1.00 & 1.10 & 6.40 & 4.00 & 0.44 & 0.60 & 6.70 & 0.24 & 0.40 & 1.40 & 0.80 \\
\hline $\mathrm{MgO}-$ & 5.10 & 0.20 & 0.39 & 3.80 & 2.50 & 0.23 & 0.10 & 3.00 & 0.06 & 0.04 & 0.32 & 0.04 \\
\hline $\mathrm{CaO}-$ & 9.60 & 0.78 & 1.60 & 7.40 & 5.80 & 1.10 & 0.76 & 5.10 & 1.20 & 0.50 & 1.90 & 0.63 \\
\hline $\mathrm{Na}_{2} \mathrm{O}$ & 2.80 & 3.20 & 3.50 & 2.80 & 3.10 & 3.90 & 4.10 & 3.50 & 5.60 & 4.10 & 5.30 & 4.70 \\
\hline $\mathrm{K}_{2} \mathrm{O}$ & 0.12 & 4.60 & 4.80 & 1.10 & 1.30 & 4.40 & 4.30 & 2.70 & 2.90 & 4.30 & 5.10 & 4.70 \\
\hline $\mathrm{TiO}_{2} \ldots$ & 0.08 & 0.12 & 0.20 & 0.44 & 0.60 & 0.31 & 0.12 & 1.80 & 0.09 & 0.06 & 0.69 & 0.15 \\
\hline $\mathrm{P}_{2} \mathrm{O}_{5}$ & 0.34 & 0.02 & 0.08 & 0.15 & 0.18 & 0.09 & 0.38 & 0.74 & 0.02 & 0.01 & 0.16 & 0.24 \\
\hline $\mathrm{MnO}-$ & 0.03 & 0.04 & - & 0.20 & 0.09 & - & - & 0.12 & - & - & 0.07 & 0.02 \\
\hline $\mathrm{CO}_{2}$ & - & 0.10 & - & - & - & - & - & 0.02 & - & - & - & - \\
\hline${ }^{3} \mathrm{H}_{2} \mathrm{O}^{+}$ & 0.50 & 0.51 & 0.22 & - & 0.71 & 0.20 & 0.68 & 0.49 & - & 0.05 & 0.30 & - \\
\hline $\mathrm{H}_{2} \mathrm{O}^{-}$ & - & 0.07 & - & - & - & - & - & 0.15 & - & - & - & - \\
\hline Total & 99.17 & 100.30 & 100.09 & 98.47 & 101.08 & 100.07 & 100.94 & 99.35 & 98.36 & 99.15 & 99.88 & 99.78 \\
\hline \multicolumn{13}{|c|}{ Normative minerals: } \\
\hline $\mathrm{Q}-$ & 9.652 & 37.214 & 31.851 & 13.222 & 25.540 & 32.974 & 35.199 & 9.714 & 29.730 & 35.268 & 11.247 & 26.843 \\
\hline $\mathrm{C}-$ & - & 2.132 & - & - & - & 0.738 & 0.627 & - & - & 0.521 & 0.391 & 1.012 \\
\hline Or & 0.719 & 27.360 & 28.401 & 6.601 & 7.654 & 26.035 & 25.344 & 16.234 & 17.423 & 25.641 & 30.264 & 27.835 \\
\hline $\mathrm{Ab}-$ & 24.012 & 27.255 & 29.655 & 24.061 & 26.135 & 33.044 & 34.603 & 30.134 & 48.176 & 35.008 & 45.036 & 39.858 \\
\hline $\mathrm{An}-$ & 37.509 & 3.127 & 6.139 & 28.550 & 23.089 & 4.875 & 1.284 & 19.489 & 2.354 & 2.437 & 8.416 & 1.561 \\
\hline Wo- & 3.552 & - & 0.537 & 3.230 & 1.840 & - & - & 0.504 & 1.489 & - & - & - \\
\hline $\mathrm{En}-$ & 12.873 & 0.501 & 0.973 & 9.611 & 6.203 & 0.574 & 0.248 & 7.602 & 0.152 & 0.101 & 0.800 & 0.100 \\
\hline $\mathrm{Fs}$ & 1.180 & 1.483 & 1.444 & 8.991 & 4.522 & - & - & 7.956 & 0.087 & 0.316 & 0.788 & 0.351 \\
\hline $\mathrm{Mt}-$ & 9.551 & 0.423 & 0.436 & 4.535 & 3.467 & 0.520 & 1.582 & 3.098 & 0.369 & 0.571 & 1.369 & 1.598 \\
\hline $\mathrm{Hm}-$ & - & - & - & - & - & 0.442 & 0.006 & - & - & - & - & - \\
\hline $\mathrm{Il} \longrightarrow$ & 0.154 & 0.229 & 0.380 & 0.849 & 1.135 & 0.590 & 0.227 & 3.478 & 0.174 & 0.115 & 1.316 & 0.286 \\
\hline $\mathrm{Ap}-$ & 0.816 & 0.048 & 0.190 & 0.361 & 0.425 & 0.213 & 0.898 & 1.783 & 0.048 & 0.024 & 0.381 & 0.570 \\
\hline $\mathrm{Cc}$ & - & 0.229 & - & - & - & - & - & 0.046 & - & - & - & - \\
\hline Total & 100.018 & 100.002 & 100.004 & 100.010 & 100.010 & 100.005 & 100.020 & 100.040 & 100.001 & 100.000 & 100.009 & 100.013 \\
\hline
\end{tabular}

Footnotes at end of table. 
TABLE 4.-Chemical and normative analyses of crystalline rocks of the Arabian Shield-Continued

\begin{tabular}{|c|c|c|c|c|c|c|c|c|c|c|c|c|}
\hline $\begin{array}{l}\text { Analysis number - } \\
\text { Laboratory } \\
\text { Sample (location } \\
\text { given on pl. 1) }\end{array}$ & $\begin{array}{l}{[\bar{D}]} \\
160\end{array}$ & $\begin{array}{c}\text { W180079 } \\
\text { B } \\
161 \\
\end{array}$ & $\begin{array}{c}\text { W180088 } \\
\text { B } \\
162 \\
\end{array}$ & $\begin{array}{c}\text { W180089 } \\
\text { B } \\
163 \\
\end{array}$ & $\begin{array}{c}\text { W180090 } \\
\text { B } \\
164 \\
\end{array}$ & $\begin{array}{c}\text { W180083 } \\
\text { B } \\
166 \\
\end{array}$ & $\begin{array}{c}\text { W180084 } \\
\text { B } \\
167 \mathrm{a} \\
\end{array}$ & $\begin{array}{c}\bar{E} \\
167 \mathrm{~b} \\
\end{array}$ & $\begin{array}{c}\bar{E} \\
167 c \\
\end{array}$ & $\begin{array}{c}\bar{E} \\
167 d\end{array}$ & $\begin{array}{c}\bar{E} \\
167 \mathrm{e} \\
\end{array}$ & $\begin{array}{c}\bar{E} \\
167 \mathrm{f} \\
\end{array}$ \\
\hline \multicolumn{13}{|c|}{ Oxides (wt. percent) } \\
\hline $\mathrm{SiO}_{2} \ldots$ & 65.50 & 71.40 & 73.70 & 74.30 & 62.60 & 73.30 & 51.70 & 48.00 & 50.00 & 49.10 & 42.50 & 48.80 \\
\hline $\mathrm{Al}_{2} \mathrm{O}_{3}$ & 15.11 & 15.20 & 13.20 & 13.10 & 15.70 & 12.90 & 21.00 & 28.30 & 28.40 & 29.44 & 17.00 & 28.20 \\
\hline $\mathrm{Fe}_{2} \mathrm{O}_{3} \ldots$ & 4.19 & 0.76 & 1.80 & 1.94 & 1.60 & 1.60 & 0.94 & 5.04 & 2.12 & 2.20 & 9.34 & 3.23 \\
\hline $\mathrm{FeO}-$ & 0.36 & 0.76 & 1.00 & 1.40 & 3.10 & 0.92 & 5.90 & - & - & - & - & - \\
\hline $\mathrm{MgO}-$ & 0.35 & 0.55 & 0.33 & 0.06 & 4.10 & 0.23 & 4.40 & 3.32 & 0.49 & 1.16 & 12.82 & 2.82 \\
\hline $\mathrm{CaO} \longrightarrow$ & 0.70 & 2.30 & 1.20 & 0.60 & 4.90 & 0.83 & 10.40 & 10.35 & 11.47 & 12.03 & 10.98 & 11.94 \\
\hline $\mathrm{Na}_{2} \mathrm{O}$ & 4.42 & 4.20 & 4.70 & 4.50 & 3.80 & 4.30 & 2.50 & 3.60 & 4.70 & 4.40 & 1.04 & 3.76 \\
\hline $\mathrm{K}_{2} \mathrm{O} \ldots$ & 7.20 & 3.40 & 4.70 & 4.90 & 3.30 & 4.20 & 0.35 & 0.61 & 0.54 & 0.47 & 0.11 & 0.33 \\
\hline $\mathrm{TiO}_{2}$ & 0.37 & 0.30 & 0.24 & 0.20 & 0.61 & 0.25 & 0.56 & - & - & - & - & - \\
\hline $\mathrm{P}_{2} \mathrm{O}_{5}$ & 0.07 & 0.12 & - & 0.03 & 0.02 & 0.03 & 0.04 & - & - & - & - & - \\
\hline $\mathrm{MnO}$ & - & - & 0.02 & -- & 0.06 & 0.03 & 0.06 & - & - & - & - & - \\
\hline${ }^{3} \mathrm{H}_{2} \mathrm{O}^{+} \ldots$ & 0.15 & 0.04 & - & 0.55 & 1.24 & 0.55 & 0.89 & 1.40 & 1.90 & 1.80 & 3.60 & 1.40 \\
\hline $\mathrm{H}_{2} \mathrm{O}^{-}$ & -- & - & - & - & - & - & - & - & - & - & - & - \\
\hline Total & 98.42 & 99.03 & 100.89 & 101.58 & 101.03 & 99.14 & 98.74 & 100.62 & 99.62 & 100.60 & 97.39 & 100.48 \\
\hline \multicolumn{13}{|c|}{ Normative minerals ${ }^{4}:$} \\
\hline$Q-$ & 11.231 & 28.715 & 26.141 & 27.874 & 11.513 & 30.699 & 4.329 & - & - & - & - & - \\
\hline $\mathrm{C}-$ & - & 0.723 & - & - & - & - & - & 2.922 & - & - & - & - \\
\hline $\mathrm{Or}=$ & 43.296 & 20.297 & 27.529 & 28.660 & 19.542 & 25.174 & 2.114 & 3.633 & 3.265 & 2.811 & 0.693 & 1.968 \\
\hline $\mathrm{Ab}-$ & 38.059 & 35.902 & 39.419 & 37.690 & 32.222 & 36.906 & 21.619 & 30.702 & 31.495 & 26.902 & 9.383 & 27.695 \\
\hline An $\longrightarrow$ & 0.127 & 10.735 & 1.030 & 1.063 & 16.069 & 3.543 & 46.034 & 51.750 & 56.078 & 59.910 & 44.134 & 59.643 \\
\hline $\mathrm{Ne}-{ }_{-}$ & - & - & - & - & - & - & - & - & 4.986 & 5.841 & - & 2.393 \\
\hline Wo & 1.228 & - & 2.034 & 0.705 & 3.407 & 0.181 & 2.683 & - & 0.899 & 0.207 & 5.823 & 0.059 \\
\hline $\mathrm{En} \longrightarrow$ & 0.887 & 1.384 & 0.815 & 0.148 & 10.233 & 0.581 & 11.199 & 0.246 & 0.777 & 0.179 & 20.565 & 0.051 \\
\hline Fs & - & 0.275 & - & 0.631 & 3.482 & 0.011 & 9.448 & - & - & - & - & - \\
\hline Fo- & - & - & - & - & - & - & - & 5.668 & 0.331 & 1.924 & 9.444 & 4.931 \\
\hline Mt- & 0.089 & 1.113 & 2.570 & 2.784 & 2.325 & 2.353 & 1.393 & - & - & - & - & - \\
\hline $\mathrm{Hm} \longrightarrow$ & 4.202 & - & 0.012 & - & - & - & - & 5.080 & 2.169 & 2.227 & 9.958 & 3.260 \\
\hline $\mathrm{Il} \longrightarrow$ & 0.715 & 0.576 & 0.452 & 0.376 & 1.161 & 0.482 & 1.087 & - & - & - & - & - \\
\hline$A p-$ & 0.169 & 0.287 & - & 0.070 & 0.047 & 0.072 & 0.097 & - & - & - & - & - \\
\hline Total & 100.004 & 100.006 & 100.000 & 100.002 & 100.002 & 100.002 & 100.003 & 100.000 & 100.000 & 100.000 & 100.000 & 100.000 \\
\hline
\end{tabular}

Footnotes at end of table. 
TABLE 4.-Chemical and normative analyses of crystalline rocks of the Arabian Shield-Continued

\begin{tabular}{|c|c|c|c|c|c|c|c|c|c|c|c|c|}
\hline $\begin{array}{l}\text { Analysis number- } \\
\text { Laboratory' } \\
\text { Sample (location } \\
\text { given on pl. 1)- }\end{array}$ & $\begin{array}{c}\overline{\mathrm{E}} \\
167 \mathrm{~g}\end{array}$ & $\begin{array}{c}\overline{\mathbf{E}} \\
167 \mathrm{~h}\end{array}$ & $\begin{array}{c}\text { W180085 } \\
\text { B } \\
170 \mathrm{a} \\
\end{array}$ & $\begin{array}{c}\text { W165321 } \\
\mathrm{A} \\
171 \\
\end{array}$ & $\begin{array}{c}\text { W180156 } \\
\mathrm{A} \\
172 \\
\end{array}$ & $\begin{array}{c}\text { W180169 } \\
\mathrm{A} \\
174 \\
\end{array}$ & $\begin{array}{c}\text { W180010 } \\
\text { B } \\
175 \\
\end{array}$ & $\begin{array}{c}\text { W180150 } \\
A \\
177 \\
\end{array}$ & $\begin{array}{c}\text { W180151 } \\
\mathbf{A} \\
178 \\
\end{array}$ & $\begin{array}{c}\text { W180076 } \\
\text { B } \\
182\end{array}$ & $\begin{array}{c}\text { W180077 } \\
\text { B } \\
186 \\
\end{array}$ & $\begin{array}{c}\underset{\mathrm{A}}{\mathrm{W} 165976} \\
194 \\
\end{array}$ \\
\hline \multicolumn{13}{|c|}{ Oxides (wt. percent) } \\
\hline $\mathrm{SiO}_{2} \longrightarrow$ & 49.50 & 48.80 & 73.70 & 72.20 & 73.40 & 71.10 & 47.70 & 75.20 & 44.80 & 62.20 & 69.90 & 54.60 \\
\hline $\mathrm{Al}_{2} \mathrm{O}_{3}$ & 30.80 & 31.40 & 14.20 & 14.60 & 14.50 & 13.60 & 16.30 & 13.00 & 22.10 & 16.00 & 15.80 & 17.00 \\
\hline $\mathrm{Fe}_{2} \mathrm{O}_{3}$ & 2.24 & 3.23 & 0.20 & 1.30 & 1.00 & 1.80 & 3.20 & 0.49 & 1.90 & 1.00 & 1.20 & 3.00 \\
\hline $\mathrm{FeO}-$ & - & - & 1.00 & 1.00 & 0.72 & 2.50 & 7.30 & 1.20 & 2.80 & 3.80 & 1.60 & 5.40 \\
\hline $\mathrm{MgO}-$ & 0.76 & 0.65 & 0.43 & 0.80 & 0.38 & 0.92 & 7.40 & 0.12 & 12.40 & 4.20 & 0.85 & 4.80 \\
\hline $\mathrm{CaO}-$ & 9.54 & 11.75 & 1.80 & 1.80 & 2.90 & 2.20 & 8.90 & 0.36 & 9.80 & 5.80 & 3.80 & 8.50 \\
\hline $\mathrm{Na}_{2} \mathrm{O}-$ & 5.03 & 3.49 & 3.40 & 5.00 & 4.40 & 4.50 & 4.20 & 3.80 & 1.80 & 4.10 & 4.00 & 3.40 \\
\hline $\mathrm{K}_{2} \mathrm{O} \cdots$ & 1.01 & 0.28 & 4.10 & 1.70 & 1.40 & 1.40 & 1.50 & 4.60 & 0.08 & 1.30 & 1.20 & 0.31 \\
\hline $\mathrm{TiO}_{2}$ & - & - & 0.18 & 0.41 & 0.14 & 0.49 & 2.40 & 0.14 & 0.12 & 0.51 & 0.25 & 1.10 \\
\hline $\mathrm{P}_{2} \mathrm{O}_{5}$ & - & - & 0.06 & 0.12 & 0.06 & 0.13 & 0.59 & 0.03 & 0.04 & 0.27 & 0.08 & 0.43 \\
\hline $\mathrm{MnO} \longrightarrow$ & - & - & - & 0.14 & 0.04 & 0.14 & 0.14 & 0.03 & 0.07 & 0.05 & 0.06 & 0.08 \\
\hline $\mathrm{CO}_{2} \longrightarrow$ & - & - & - & 0.05 & 0.23 & 0.01 & - & 0.08 & 0.07 & - & - & 0.05 \\
\hline${ }^{3} \mathrm{H}_{2} \mathrm{O}^{+}$ & 1.40 & 0.30 & - & 0.47 & 0.65 & 0.85 & - & 0.77 & 3.60 & 0.67 & 0.60 & 1.30 \\
\hline $\mathrm{H}_{2} \mathrm{O}^{-}$ & - & - & -- & 0.22 & - & 0.06 & - & 0.02 & 0.31 & - & - & 0.02 \\
\hline Total & 100.28 & 99.90 & 99.07 & 100.30 & 100.20 & 100.25 & 99.63 & 100.31 & 102.29 & 99.90 & 99.34 & 100.76 \\
\hline \multicolumn{13}{|c|}{ Normative minerals ${ }^{4}$ : } \\
\hline $\mathrm{Q}-$ & - & 1.286 & 33.591 & 32.162 & 36.615 & 33.113 & - & 34.434 & - & 14.308 & 32.517 & 9.019 \\
\hline $\mathrm{C}-$ & 4.133 & 4.008 & 1.050 & 1.680 & 1.160 & 1.029 & - & 1.386 & 1.555 & - & 1.219 & - \\
\hline Or & 6.036 & 1.661 & 24.455 & 10.135 & 8.342 & 8.374 & 8.897 & 27.443 & 0.493 & 7.742 & 7.182 & 1.857 \\
\hline $\mathrm{Ab}-\ldots$ & 32.822 & 29.650 & 29.040 & 42.684 & 37.543 & 38.544 & 24.861 & 32.463 & 15.869 & 34.962 & 34.279 & 29.158 \\
\hline $\mathrm{An} \longrightarrow$ & 47.864 & 58.526 & 8.618 & 7.899 & 12.646 & 10.124 & 21.272 & 1.095 & . & 49.921 & 21.580 & 18.563 \\
\hline $\mathrm{Ne}-$ & 5.538 & - & - & - & - & - & 5.856 & - & - & - & - & - \\
\hline Wo- & - & - & - & - & - & - & 8.007 & - & - & 2.355 & - & 3.738 \\
\hline En & - & 1.625 & 1.081 & 2.010 & 0.954 & 2.319 & 5.358 & 0.302 & 10.541 & 10.541 & 2.144 & 12.116 \\
\hline Fs & - & -- & 1.387 & 0.349 & 0.342 & 2.586 & 2.052 & 1.639 & 1.196 & 5.445 & 1.666 & 5.848 \\
\hline Fo & 1.341 & - & - & - & - & - & 9.208 & - & 15.161 & - & - & - \\
\hline $\mathrm{Fa} \cdots$ & - & - & - & - & - & - & 3.887 & - & 1.896 & - & - & - \\
\hline $\mathrm{Mt} \longrightarrow$ & - & - & 0.293 & 1.902 & 1.462 & 2.642 & 4.657 & 0.717 & 2.870 & 1.461 & 1.762 & 4.408 \\
\hline $\mathrm{Hm} \longrightarrow$ & 2.265 & 3.243 & - & - & - & - & - & - & - & - & - & - \\
\hline $\mathrm{Il} \longrightarrow$ & - & - & 0.345 & 0.786 & 0.268 & 0.942 & 4.575 & 0.268 & 0.237 & 0.976 & 0.481 & 2.117 \\
\hline $\mathrm{Ap} \longrightarrow$ & - & - & 0.143 & 0.287 & 0.143 & 0.312 & 1.403 & 0.072 & 0.099 & 0.644 & 0.192 & 1.032 \\
\hline $\mathrm{Cc}-$ & - & - & - & 0.115 & 0.527 & 0.023 & - & 0.184 & 0.166 & - & - & 0.115 \\
\hline Total & 100.000 & 100.000 & 100.003 & 100.008 & 100.004 & 100.009 & 100.032 & 100.002 & 100.003 & 100.015 & 100.005 & 100.024 \\
\hline
\end{tabular}

Footnotes at end of table. 
TABLE 4.-Chemical and normative analyses of crystalline rocks of the Arabian Shield-Continued

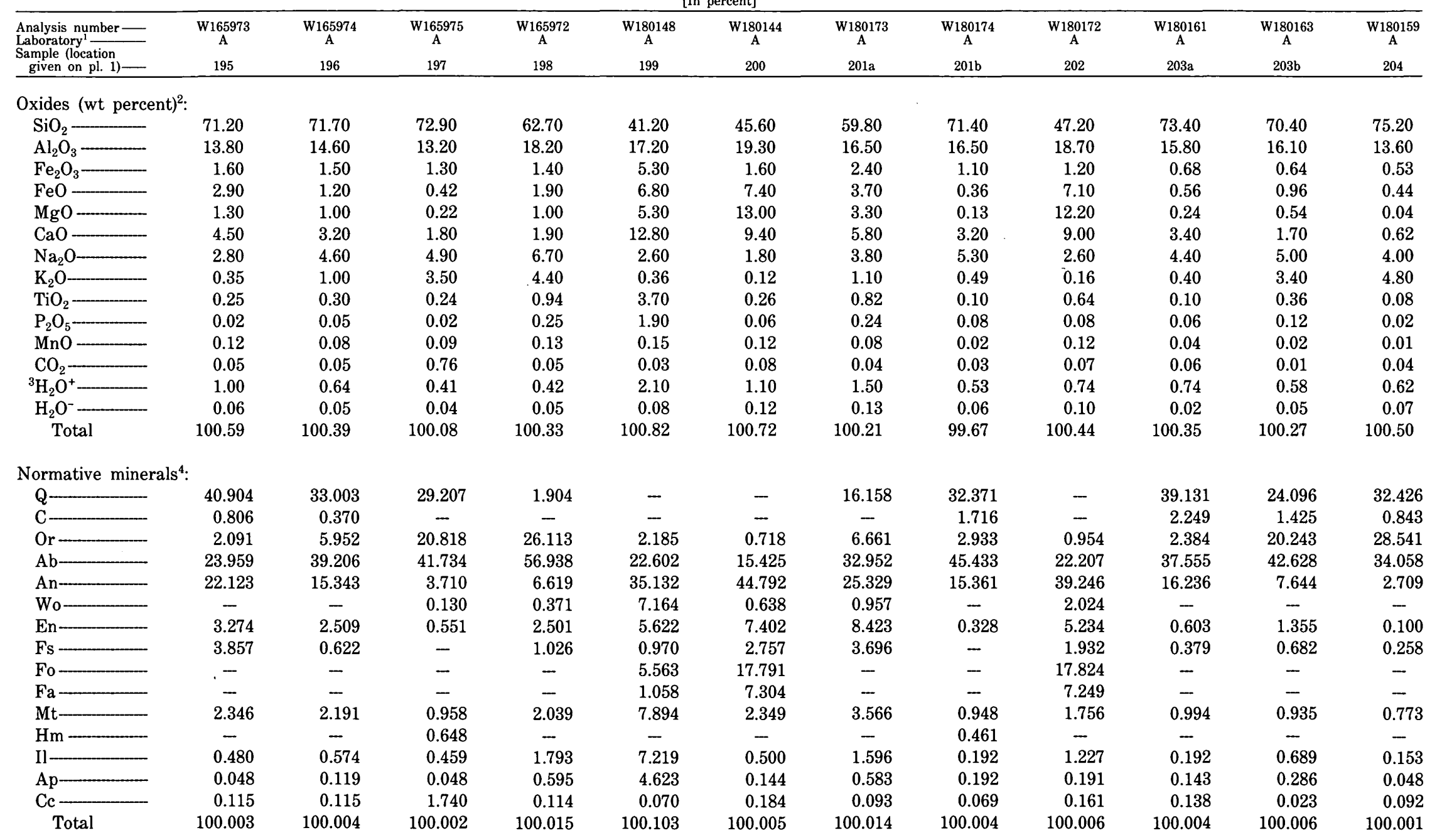

Footnotes at end of table 


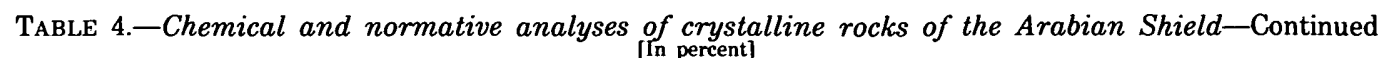

\begin{tabular}{|c|c|c|c|c|c|c|c|c|c|c|c|c|}
\hline $\begin{array}{l}\text { Analysis number- } \\
\text { Laboratory' } \\
\text { Sample (location } \\
\text { given on pl. 1) }\end{array}$ & $\begin{array}{c}\text { W180160 } \\
\text { A } \\
205\end{array}$ & $\underset{\mathrm{A}}{\mathrm{W}} \mathbf{1 8 0 1 6 4}$ & $\underset{A}{W 180166}$ & $\underset{A}{W 180165}$ & $\underset{A}{W 180155}$ & $\underset{\mathrm{A}}{\mathrm{W} 180168}$ & $\underset{\mathrm{A}}{\mathrm{W}} \mathbf{1 8 0 1 5 4}$ & $\underset{A}{W 180167}$ & $\underset{\mathrm{A}}{\mathrm{W}} 180152$ & $\underset{\mathrm{A}}{\mathrm{W}} \mathbf{1 8 0 1 5 3}$ & $\underset{A}{W 180175}$ & $\underset{\mathrm{A}}{\mathrm{W}}$ \\
\hline given on pl. 1$)-$ & 200 & 200 & 200 & 208 & 209 & 212 & 214 & 210 & 211 & 218 & $225 a$ & 2230 \\
\hline \multicolumn{13}{|c|}{ Oxides (wt percent) ${ }^{2}$ : } \\
\hline $\mathrm{SiO}_{2}$ & 75.50 & 70.80 & 70.80 & 58.70 & 72.90 & 49.00 & 35.30 & 63.30 & 51.40 & 46.70 & 68.00 & 49.40 \\
\hline $\mathrm{Al}_{2} \mathrm{O}_{3}$ & 13.10 & 16.60 & 14.70 & 15.20 & 12.60 & 18.40 & 1.40 & 17.70 & 15.20 & 15.20 & 14.50 & 13.70 \\
\hline $\mathrm{Fe}_{2} \mathrm{O}_{3}$ & 1.00 & 0.75 & 0.88 & 3.10 & 1.70 & 1.00 & 11.70 & 0.67 & 3.00 & 1.70 & 3.60 & 2.70 \\
\hline $\mathrm{FeO}-$ & 0.40 & 0.68 & 1.20 & 4.20 & 1.80 & 5.20 & 2.80 & 3.30 & 6.80 & 9.00 & 2.40 & 8.70 \\
\hline $\mathrm{MgO} \longrightarrow$ & - & 0.44 & 0.77 & 4.60 & 0.17 & 10.70 & 33.20 & 1.70 & 7.50 & 10.30 & 0.88 & 9.80 \\
\hline $\mathrm{CaO} \longrightarrow$ & 0.48 & 3.20 & 3.30 & 6.90 & 0.62 & 12.00 & 1.40 & 5.60 & 8.20 & 7.40 & 4.40 & 11.50 \\
\hline $\mathrm{Na}_{2} \mathrm{O}$ & 4.10 & 4.90 & 3.80 & 3.00 & 3.80 & 2.10 & 0.02 & 4.50 & 2.40 & 2.00 & 3.30 & 1.40 \\
\hline $\mathrm{K}_{2} \mathrm{O}$ & 4.30 & 1.00 & 2.30 & 1.40 & 5.00 & 0.16 & 0.12 & 1.00 & 1.10 & 0.92 & 0.28 & 0.24 \\
\hline $\mathrm{TiO}_{2}$ & 0.10 & 0.12 & 0.31 & 0.54 & 0.32 & 0.37 & 0.08 & 0.67 & 0.90 & 1.10 & 0.38 & 0.37 \\
\hline $\mathrm{P}_{2} \mathrm{O}_{5}$ & - & 0.08 & 0.14 & 0.27 & 0.04 & 0.05 & 0.08 & 0.39 & 0.22 & 0.26 & 0.13 & 0.08 \\
\hline $\mathrm{MnO}$ & 0.01 & - & 0.02 & 0.16 & 0.09 & 0.10 & 0.17 & 0.08 & 0.18 & 0.20 & 0.06 & 0.21 \\
\hline $\mathrm{CO}_{2}$ & 0.04 & 0.01 & 0.56 & 0.07 & 0.07 & 0.07 & 0.76 & 0.04 & 0.05 & 0.08 & 0.30 & 0.07 \\
\hline${ }^{3} \mathrm{H}_{2} \mathrm{O}^{+}$ & 0.55 & 0.49 & 0.82 & 1.40 & 0.64 & 0.82 & 9.99 & 0.77 & 2.40 & 3.80 & 0.82 & 1.30 \\
\hline $\mathrm{H}_{2} \mathrm{O}^{-}$ & 0.04 & 0.02 & 0.06 & 0.08 & 0.07 & 0.05 & 0.86 & 0.10 & 0.06 & 0.23 & 0.07 & 0.10 \\
\hline Total & 99.98 & 99.39 & 100.20 & 100.51 & 100.26 & 100.55 & 106.64 & 100.37 & 100.86 & 101.32 & 99.67 & 100.42 \\
\hline \multicolumn{13}{|c|}{ Normative minerals ${ }^{4}$ : } \\
\hline$Q-$ & 34.612 & 31.462 & 33.553 & 15.170 & 29.950 & - & - & 18.093 & 3.798 & - & 38.442 & 0.912 \\
\hline $\mathrm{C}-$ & 0.930 & 1.880 & 1.612 & - & 0.068 & - & 0.740 & 0.060 & - & - & 1.807 & - \\
\hline Or & 25.659 & 5.994 & 13.759 & 8.430 & 29.812 & 0.954 & 0.815 & 5.972 & 6.705 & 5.731 & 1.684 & 1.445 \\
\hline $\mathrm{Ab}$ & 35.033 & 42.060 & 32.552 & 25.866 & 32.443 & 17.922 & 0.194 & 38.482 & 20.947 & 17.840 & 28.427 & 12.067 \\
\hline $\mathrm{An}$ & 2.149 & 15.510 & 12.064 & 24.326 & 2.393 & 40.653 & 1.860 & 25.246 & 28.317 & 31.394 & 19.426 & 30.955 \\
\hline Wo & - & - & - & 3.468 & - & 7.772 & - & - & 4.942 & 2.081 & - & 10.930 \\
\hline En- & - & 1.112 & 1.941 & 11.674 & 0.427 & 13.387 & 35.645 & 4.279 & 19.267 & 17.220 & 2.231 & 24.862 \\
\hline Fs & - & 0.437 & 1.014 & 4.644 & 1.554 & 4.168 & - & 4.597 & 9.136 & 9.181 & 0.934 & 13.777 \\
\hline Fo & - & - & - & - & - & 9.453 & 41.599 & - & - & 6.883 & - & - \\
\hline $\mathrm{Fa}-$ & - & - & - & - & - & 3.244 & - & - & - & 4.045 & - & - \\
\hline $\mathrm{Mt} \longrightarrow$ & 1.042 & 1.103 & 1.292 & 4.580 & 2.487 & 1.462 & 10.739 & 0.982 & 4.487 & 2.598 & 5.314 & 3.988 \\
\hline $\mathrm{Hm}$ & 0.291 & - & - & - & - & - & 6.037 & - & - & - & - & - \\
\hline $\mathrm{Il}$ & 0.192 & 0.231 & 0.596 & 1.045 & 0.613 & 0.709 & 0.175 & 1.286 & 1.763 & 2.202 & 0.735 & 0.716 \\
\hline Ap $\longrightarrow$ & - & 0.192 & 0.336 & 0.652 & 0.096 & 0.119 & 0.218 & 0.934 & 0.537 & 0.649 & 0.313 & 0.193 \\
\hline $\mathrm{Cc} \longrightarrow$ & 0.092 & 0.023 & 1.289 & 0.162 & 0.161 & 0.161 & 1.986 & 0.092 & 0.117 & 0.192 & 0.695 & 0.162 \\
\hline Total & 100.000 & 100.004 & 100.008 & 100.016 & 100.003 & 100.004 & 100.007 & 100.021 & 100.014 & 100.017 & 100.008 & 100.007 \\
\hline
\end{tabular}


TABLE 4.-Chemical and normative analyses of crystalline rocks of the Arabian Shield-Continued

\begin{tabular}{|c|c|c|c|c|c|c|c|}
\hline \multirow[b]{2}{*}{$\begin{array}{l}\text { Analysis number } \\
\text { Laboratory } \\
\text { Sample (location } \\
\text { given on pl. 1)- } \\
\end{array}$} & \\
\hline & $\begin{array}{c}\text { W180419 } \\
\text { A } \\
223 \mathrm{c}\end{array}$ & $\begin{array}{c}\text { W167131 } \\
\text { A } \\
224\end{array}$ & $\begin{array}{c}\mathrm{W} 167130 \\
\mathrm{~A} \\
227\end{array}$ & $\begin{array}{c}\text { W167129 } \\
\text { A } \\
237\end{array}$ & $\begin{array}{c}\mathrm{W} 167128 \\
\mathrm{~A} \\
241\end{array}$ & $\begin{array}{c}\text { D167712 } \\
\text { C } \\
501^{5}\end{array}$ & $\begin{array}{l}\text { D167713 } \\
\text { C } \\
502^{5}\end{array}$ \\
\hline \multicolumn{8}{|c|}{ Oxides (wt percent) } \\
\hline $\mathrm{SiO}_{2}$ & 69.70 & 74.90 & $: 55.70$ & 69.20 & 65.30 & 51.60 & 38.70 \\
\hline $\mathrm{Al}_{2} \mathrm{O}_{3}$ & 15.30 & 14.40 & 15.10 & 15.70 & 17.30 & 21.30 & 17.80 \\
\hline $\mathrm{Fe}_{2} \mathrm{O}_{3}$ & 0.84 & 0.29 & 1.50 & 1.00 & 1.00 & 9.86 & 19.10 \\
\hline $\mathrm{FeO} \longrightarrow$ & 1.80 & 0.32 & 5.10 & 1.60 & 1.80 & - & - \\
\hline $\mathrm{MgO}-$ & 0.41 & 0.17 & 5.40 & 0.96 & 1.20 & 3.10 & 7.55 \\
\hline $\mathrm{CaO}-$ & 2.00 & 1.50 & 7.70 & 3.50 & 3.90 & 7.02 & 0.62 \\
\hline $\mathrm{Na}_{2} \mathrm{O}$ & 4.10 & 4.00 & 3.90 & 4.40 & 4.40 & 4.76 & 0.08 \\
\hline $\mathrm{K}_{2} \mathrm{O}-$ & 4.70 & 3.00 & 1.20 & 2.20 & 2.80 & 1.25 & 4.04 \\
\hline $\mathrm{TiO}_{2}$ & 0.50 & 0.07 & 1.70 & 0.41 & 0.66 & 0.50 & 3.50 \\
\hline $\mathrm{P}_{2} \mathrm{O}_{5}$ & 0.15 & 0.05 & 0.58 & 0.11 & 0.36 & 0.26 & 0.73 \\
\hline $\mathrm{MnO}$ & - & 0.05 & 0.10 & 0.04 & 0.07 & -- & - \\
\hline $\mathrm{CO}_{2} \longrightarrow$ & 0.07 & 0.05 & 0.24 & 0.05 & 0.05 & - & - \\
\hline${ }^{3} \mathrm{H}_{2} \mathrm{O}^{+}$ & 0.49 & 0.41 & 0.92 & 0.77 & 0.60 & - & 7.50 \\
\hline $\mathrm{H}_{2} \mathrm{O}^{-}$ & 0.05 & 0.11 & 0.08 & 0.10 & 0.06 & - & - \\
\hline Total & 100.44 & 99.67 & 99.83 & 100.59 & 99.91 & 99.65 & 99.62 \\
\hline \multicolumn{8}{|c|}{ Normative minerals ${ }^{4}$ : } \\
\hline $\mathrm{Q}-$ & 22.868 & 37.262 & 6.527 & 26.234 & 19.533 & 0.215 & 12.506 \\
\hline $\mathrm{C}-$ & 0.355 & 2.106 & -. & 0.097 & 0.929 & - & 14.433 \\
\hline Or & 27.894 & 17.943 & 7.220 & 13.109 & 16.740 & 7.413 & 25.916 \\
\hline$A b-$ & 34.843 & 34.258 & 33.599 & 37.543 & 37.669 & 40.419 & 0.735 \\
\hline $\mathrm{An}-$ & 8.536 & 6.881 & 20.517 & 16.465 & 16.876 & 33.177 & - \\
\hline Wo- & - & - & 5.417 & - & - & - & - \\
\hline $\mathrm{En}-$ & 1.026 & 0.429 & 13.693 & 2.411 & 3.024 & 7.748 & 20.412 \\
\hline Fs & 1.793 & 0.329 & 5.605 & 1.522 & 1.537 & - & - \\
\hline Mt & 1.223 & 0.426 & 2.214 & 1.462 & 1.467 & - & - \\
\hline $\mathrm{Hm}-$ & - & - & - & - & -- & 9.895 & 20.734 \\
\hline Il & 0.954 & 0.135 & 3.287 & 0.785 & 1.268 & - & - \\
\hline $\operatorname{Tn}-$ & - & - & -- & - & - & 0.047 & - \\
\hline $\mathrm{Ru}$ & - & - & - & - & - & 0.482 & 3.799 \\
\hline Ap- & 0.357 & 0.120 & 1.399 & 0.263 & 0.863 & 0.618 & 1.210 \\
\hline $\mathrm{Cc}$ & 0.160 & 0.115 & 0.556 & 0.115 & 0.115 & - & - \\
\hline Total & 100.008 & 100.003 & 100.032 & 100.006 & 100.020 & 100.013 & 99.745 \\
\hline
\end{tabular}

'Laboratory and analytical method: A-USGS, Washington, D.C., rapid rock analysis, single-solution method
(Shapiro 1967) Analysts: Paul Elmore Sam Botts and Lowell Artis (Nov. 1964) S.M. Berthold (July 1965): Paul Elmore, Sam Botts, Lowell Artis, H. Smith, John Glenn, G. Chloe, and D. Taylor (Dec. 1965, Jan. (1966, Aug.; 1966); Lowell Artis (June 1973); and Paul Elmore (Sept. 1973). B-USGS, Washington, D.C., rapid rock analysis, single-

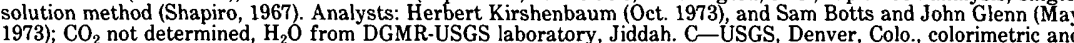
atomic absorption analyses, Claude Huffman, Jr., supervisor. Analysts: G.T. Burrow and Wayne Mountjoy (July 194). D-DGMr.

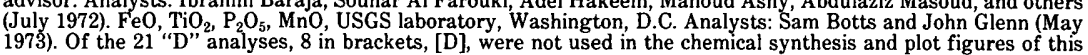

report. E-DGMR-USGS, Jiddah, atomic absorption, volumetric, and gravimetric methods, W.L. Campbell, The chemical analyses of samples $9,13,14 \mathrm{a}, 14 \mathrm{~b}, 15,17,20,21,22 \mathrm{a}, 63,223 \mathrm{a}, 223 \mathrm{~b}$, and $223 \mathrm{c}$ are nondefinitive as standard igneous rocks, presumably because of metamorphism, alteration, or analytical problems, and have not ${ }^{3}$ Where only one value for $\mathrm{H}_{2} \mathrm{O}$ is given, the amount is for total water as loss on ignition. ${ }^{4}$ Normative minerals: $Q$, quartz; $\mathrm{C}$, corundum; Or, orthoclase; $\mathrm{Ab}$, albite; An, anorthosite; Ne, nepheline; $\mathrm{Ac}$ acmite; $\mathrm{Ns}$, sodium metasilicate; Wo, wollastonite; En, enstatite; $\mathrm{Fs}$, ferrosilite; $\mathrm{Fo}$, forsterite; $\mathrm{Fa}$, fayalite; $\mathrm{Mt}$, "Not on plate 1; location in Eastern province (see text). Samples 39, 501, and 502 are sedimentary(?) and have 
Most of the analyses were made in the U.S. Geological Survey laboratory, Washington, D.C., under the direction of F.J. Flanagan and Leonard Shapiro, using the single-solution, rapid-rock method of analysis (Shapiro, 1967). A very few of the analyses reported in table 4 were made by other methods in Jiddah at the Saudi Arabia Directorate General of Mineral Resources-USGS laboratory.

The normative analyses were calculated on the USGS Multex System (Honeywell 6880 computer) using a graphic normative analysis program (Bowen, 1971) as modified by Stuckless and VanTrump (1979). The computer liaison was done by George VanTrump, Jr. The analyses were not corrected for the effects of hydration, oxidation, and introduction of $\mathrm{CO}_{2}$ (Irvine and Baragar, 1971). For most samples for which $\mathrm{CO}_{2}$ and $\mathrm{H}_{2} \mathrm{O}$ are given, these effects can be discounted without significantly affecting the results.

In table 3 , petrographic data are given and each sample is classified by igneous type, name, age, and tectonic region in order to compare the chemistry of the various categories of rocks. The rock names given in the classification part of the table are those recommended by the International Union of Geological Sciences (IUGS) Subcommission on the Systematics of Igneous Rocks (Streckeisen, 1973, 1976, 1979). Each rock name is a consensus or a best compromise of the available petrography and of the normative mineralogy, but for consistency the naming strongly emphasizes the classification schemes of the IUGS subcommission and of Irvine and Baragar (1971). Where naming proved difficult, a further check was made by using weight-percent chemical data directly, in the classification of Church (1975).

The samples used for whole-rock analyses are biased toward granitic rocks, which are represented by 96 analyses, compared with 53 analyses of dioritic and gabbroic rocks, 42 analyses of volcanic rocks, and 8 analyses of dike rocks. Most of the rocks have calcalkalic compositions that fit normal distributions in any of the three classification schemes used. A majority of the rocks were classified preliminarily on the Q-Or$(A n+A b)$ ternary diagram using the IUGS classification. On this diagram, compositions of granite and granodiorite generally separate well. The subdivision of the rocks of granite composition constitutes a major problem (the feldspar problem of normative analyses; Irvine and Baragar, 1971; Le Maitre, 1976) in determining the amount of normative Ab-molecule to allocate to normative anorthite and potassium feldspar.

Peralkalic granite is defined by molar data where the $\left(\mathrm{Na}_{2} \mathrm{O}+\mathrm{K}_{2} \mathrm{O}\right) / \mathrm{Al}_{2} \mathrm{O}_{3}$ ratio is greater than 1 ; the norm of these granites contains acmite and (or) sodium silicate.
The separation of alkali-feldspar granite and granite on the basis of chemical data is not defined in any of the classifications named above; the allocation of albite in the normative analysis makes it impossible to classify on either the Q-Or-(An+Ab) or the Q-(Or+Ab)-An ternary diagram. For this report, alkali-feldspar granite is arbitrarily separated from granite on the basis of molar data in which the $\left(\mathrm{Na}_{2} \mathrm{O}+\mathrm{K}_{2} \mathrm{O}\right) / \mathrm{Al}_{2} \mathrm{O}_{3}$ ratio is greater than 0.850 . This separation of alkali-feldspar granite is well demonstrated on the ternary diagram $\mathrm{Al}_{2} \mathrm{O}_{3}-\mathrm{CaO}$ $\left(\mathrm{Na}_{2} \mathrm{O}+\mathrm{K}_{2} \mathrm{O}\right)$, which also separates the peralkalic and peraluminous rocks. The granitic compositions are well separated on the Q-OrT-AnT diagram, where T equals $(\mathrm{Or}+\mathrm{Ab}+\mathrm{An}) /(\mathrm{Or}+\mathrm{An})$ (Le Maitre, 1976), and on the QOr-An diagram, but statistical boundaries for naming the granitic rocks on these diagrams are not available.

For purposes of synthesizing the chemistry of the 199 rock analyses from widely scattered localities here first published, all the rocks, including those reported elsewhere, were divided into the categories of region, igneous rock type, and age (table 3). Throughout the chemical examination, a fourth category, quality of the chemical analysis (listed in table 3), was constantly considered. The regional divisions are tectonic provinces shown in figure 16. Because the rocks were collected with a shieldwide distribution, it is reasonable to look for regional chemical differences in rocks of the same type and age.

All the rocks were classified as either plutonic or volcanic. A few are of other types (table 3), but after examination of field descriptions, petrography, and chemistry, they were studied as part of either the plutonic or the volcanic category. Nine rocks from dikes and sills were best examined under the volcanic category, and nine rocks from hypabyssal intrusive bodies were examined under the plutonic category. The 23 samples of gneiss appear to be orthogneiss and migmatite and were classified as plutonic. Five highly metamorphic rocks were classified as either plutonic or volcanic depending on the available data. Three sedimentary rocks were not included in the chemical study.

All the rocks were further classified according to an assigned age category (table 3 ). Each age category is a stratigraphic interval with the exception of age categories that correspond to the culminant orogeny and the Najd faulting event. From oldest to youngest, the age categories are Baish-Bahah, Jiddah, Ablah, Fatimah, Halaban, culminant orogeny, Murdama, Shammar, Najd faulting event, and Jubaylah. These age categories relate to stratigraphy discussed earlier in this report and in various reports summarizing the stratigraphy, plutonism, tectonism, and cratonization of the Arabian Shield (for example, Schmidt and others, 1973; 


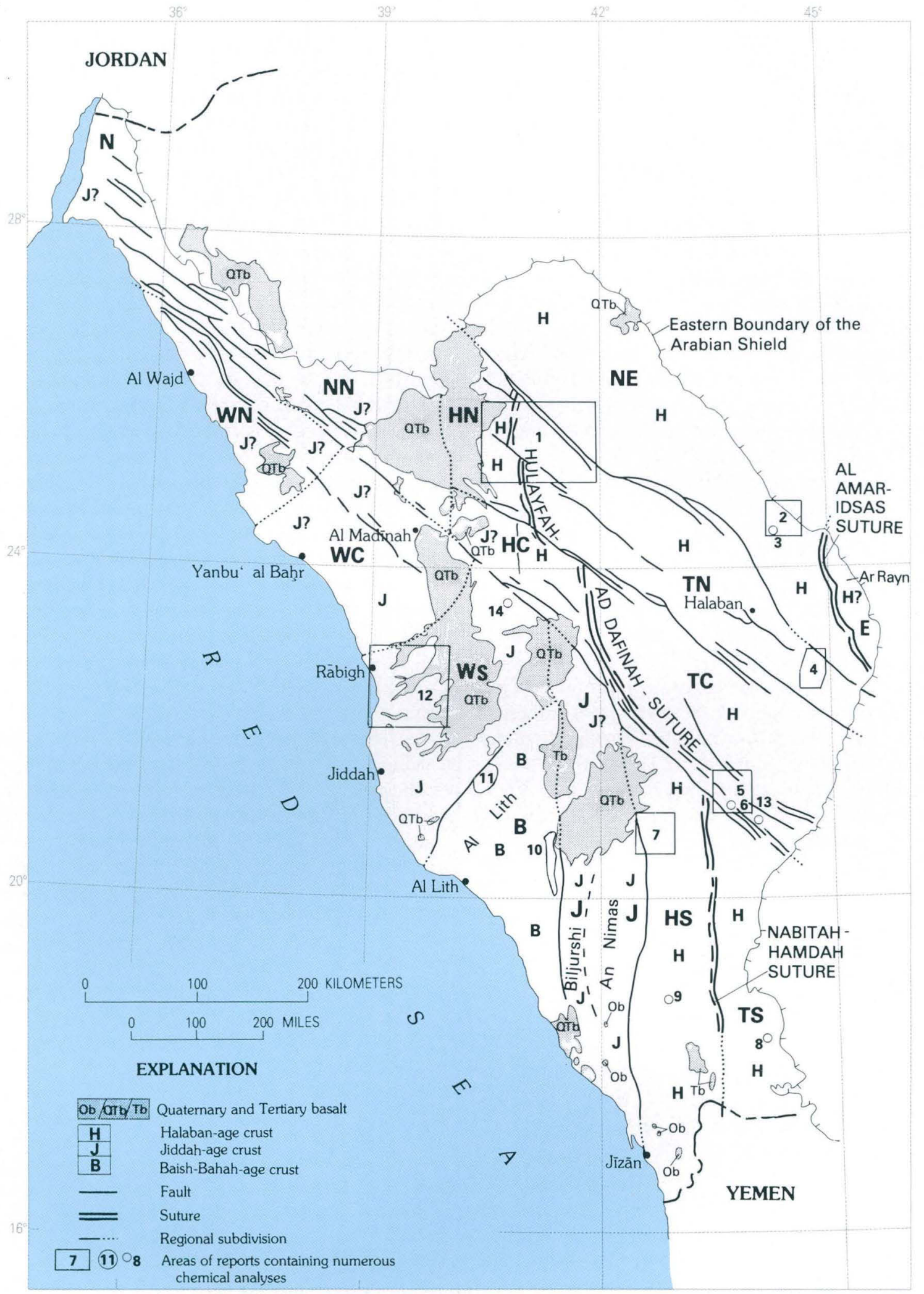


Schmidt and others, 1979; Greenwood and others, 1980) and, with some changes in terminology and nomenclature, to the summary report of Delfour (1979b).

The Baish-Bahah age category includes primitive volcanic rocks from the Wādī Bidah region (region B, fig. 16) as defined by Schmidt and others (1973) and modified by Greenwood and others (1980); Baish-Bahah Groups have been defined in the Wādī Bidah-Bahah area by Greenwood (1975c) even though the name "Baish" is from Wādi Baysh in the southwestern part of the shield, where the "Baish" rocks of Wādī Baysh have been subsequently mapped as Jiddah rocks (pl. 1). The Jiddah age category includes volcanic and plutonic rocks of the andesite assemblage and dioritic suite from the Biljurshī'-An Nimās region (region J, fig. 16). Jiddah rocks were originally named by Brown (USGS-ARAMCO, 1963) for rocks in the vicinity of the city of Jiddah, but the rocks of the Jiddah Group have been described subsequently in more detail in the Biljurshī'-Aqīq area east of Wādī Bidah by Greenwood (1975b, 1975c), and the Jiddah-age rocks (region J, fig. 16) are defined herein in the Biljursh' $\vec{\imath}$ and 'Aqīq quadrangles. The Ablah age category includes rocks of the Bidah and Biljurshī'-An Nimās regions (regions B and $\mathrm{J}$, fig. 16) that are younger than the Jiddah-age rocks but are older than the culminant orogeny. The Halaban age category includes andesitic-assemblage rocks and dioritic-suite rocks from the Nuqrah quadrangle (Delfour, 1977) (area 1, fig. 16) and the Bi'r Juqjuq quadrangle (Hadley, 1976; Dodge and others, 1979) (area 5, fig. 16) from the eastern half of the shield.

The culminant-orogeny age category includes the gneiss domal and batholithic granodiorite rocks of the Jabal al Qarah and Junaynah quadrangles (Schmidt 1981a, 1985) (Bishah area). These syntectonic rocks are

FIGURE 16.-Tectonic belts and regions used to define regional variations and trends in the chemical data examined for this report. Broad tectonostratigraphic belts (small capital letters) are defined by the age of the underlying crust (from pl. 1). The Al Lith belt and the Biljurshi and An Nimas subbelts are located. Large boldface capital letters identify regions used to areally subdivide the chemical data of this report (see table 3). Numbers indicate quadrangles and areas from which published chemical analyses were used for control of the chemical synthesis of this report: 1, Nuqrah quadrangle (Delfour, 1977); 2 and 3, Ad Dawādimī district (Al-Shanti, 1974, 1976); 4, Jabal al Hुawshah (Kanaan, 1979); 5, Bi'r Juqjuq quadrangle (Dodge and others, 1979); 6, Bi'r Juqjuq area (Kroner and others, 1979); 7, Wādī al Miyah (Schmidt, 1980, unpub. data); 8, Wādī Wassā̄t (Jackaman, 1972); 9, Jabal Shāyi` (Coleman, Ghent, and others, 1977); 10, Wādī Bidah (Jackaman, 1972); 11, Aţ Tā'if (Nasseef and Gass, 1977); 12, Khulays quadrangle (W.J. Skiba and C.F. Gilboy, written commun., 1975; Skiba, 1980); 13, Jabal Yafikh (Schmidt, 1981b); 14, Mahd adh Dhahab district (R.J. Roberts, written commun., 1980). well represented throughout the eastern half of the shield and are younger than Halaban rocks but are synchronous with early Murdama and Shammar rocks. Many large gneiss domes (antiforms) formed during the culminant orogeny, but these orthogneisses are tectonized older plutonic rocks, and for chemical comparison they are classified according to their intrusive age rather than the age of tectonism (their tectonic age is generally shown in parentheses after their plutonic age in table 3). In contrast, these orthogneisses are mapped on plate 1 according to their tectonic age.

The Murdama-Shammar age category includes posttectonic plutonic, hypabyssal, and volcanic rocks that intruded at least some Murdama rocks or are stratigraphically within the Murdama and Shammar Groups. The Murdama-Shammar rocks are designated as either Murdama age or Shammar age depending on local stratigraphy; in general, rocks classified as Shammar age are slightly younger than those classified as Murdama age. The Jubaylah-Najd age category includes volcanic, dike, and hypabyssal rocks stratigraphically within or intrusive into the Jubaylah Group in the Najd fault system and intrusive rocks associated with or slightly postdating the Najd faulting event.

Because the 199 chemically analyzed rocks of this report are widely distributed (diluted in space) and are divisible into many age categories (diluted in time), it is desirable to control or compare the chemistry of rocks of this report with the published chemistry of rocks from local areas where stratigraphy and age relations are well known. Fourteen such control areas are shown in figure 16. About 300 control analyses have been examined using the same computed normative program and plots as were used for the 199 analyses reported herein.

\section{CHEMICAL VARIATION OF VOLCANIC AND PLUTONIC ROCKS}

\section{VOLCANIC ROCKS}

The $\mathrm{Na}_{2} \mathrm{O}-\mathrm{CaO}-\mathrm{K}_{2} \mathrm{O}$ (NCK) ternary diagram shows the spread or chemical separation of the analyzed rocks as well as or better than many of the other ternary diagrams examined. For the normative data, the nearly comparable Ab-An-Or diagram does almost as well. Figure 17 shows the distribution of the metavolcanic rocks of the shield used for control. Figure $17 \mathrm{~A}$ shows the distribution of the low- $\mathrm{K}_{2} \mathrm{O}$, mafic volcanic rocks of Baish-Bahah age from Wādī Bidah (area 10, fig. 16). The consistently low $\mathrm{K}_{2} \mathrm{O}$ rocks of wide compositional range, from basalt to dacite and sodic rhyolite (at least some quartz keratophyre), of the Samran Group from the Rābigh area (area 12) have been mapped on plate 1 as Jiddah-age rocks. Figure $17 B$ shows the distribution 


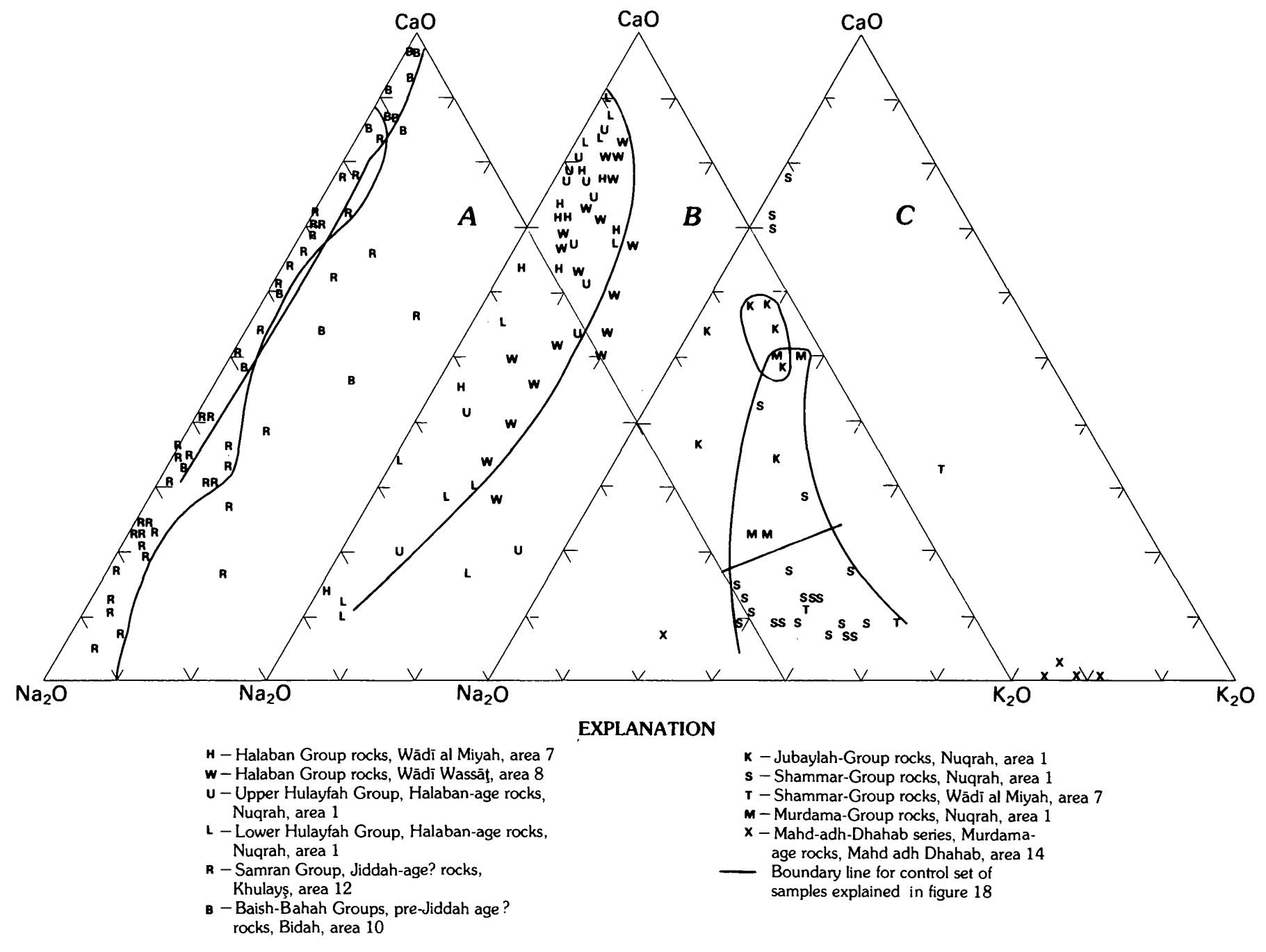

FIGURE 17.- $\mathrm{Na}_{2} \mathrm{O}-\mathrm{CaO}-\mathrm{K}_{2} \mathrm{O}$ diagrams showing the chemical distribution (molar data) of control samples of metavolcanic rocks from the following groups of rocks: Baish-Bahah, Jiddah, Halaban, Murdama, Shammar, and Jubaylah. The samples are from local areas where these stratigraphic units have been described and

chemically analyzed (see fig. 16). The chemistry of rocks of the Baish-Bahah and Jiddah (Samran) Groups is shown in $A$; of the Halaban Group, $B$; and of the Murdama, Shammar, and Jubaylah Groups, $C$. of Halaban rocks. Many Halaban rocks are low in $\mathrm{K}_{2} \mathrm{O}$, but overall they differ from the Baish-Bahah and Jiddah rocks of figure $17 A$ in that many Halaban rocks range to higher $\mathrm{K}_{2} \mathrm{O}$. These Halaban control samples are from the Nuqrah quadrangle, (area 1), the Wādi al Miyah quadrangle (area 7), and Wadi Wassāţ (area 8).

Figure $17 C$ shows the distinctly high- $\mathrm{K}_{2} \mathrm{O}$, low- $\mathrm{CaO}$, dacitic to rhyolitic rocks of the Murdama and Shammar Groups as well as the less potassic, more mafic rocks of the Jubaylah Group, from the Nuqrah quadrangle (area 1) or the Wādī al Miyah quadrangle (area 7). In figure $17 C$ Murdama, Shammar, and Jubaylah rocks separate with little compositional overlap.

Figure 18 shows the metavolcanic rocks analyzed for this report along with the rock-group boundary lines drawn from the control samples of figure 17. In figure 18 , the Shammar and Murdama volcanic rocks are clearly separated by their high $\mathrm{K}_{2} \mathrm{O}$ content from the older volcanic rocks. The Jubaylah volcanic rocks form a cluster that extends toward a composition that is more calcic than the control set, but this is emphasized partly by three diabasic dike rocks (symbol N, fig. 18) of Najd age. The 10 Halaban-age rocks shown scatter widely between the $\mathrm{CaO}$ and $\mathrm{Na}_{2} \mathrm{O}$ corners and have varying $\mathrm{K}_{2} \mathrm{O}$ content within the range of the Halaban control rocks (fig. 17B). Three Jiddah-age rocks are as potassic as the most potassic of the Jiddah control group (fig. 17A).

Three analyses of Fatimah rocks from the JiddahRābigh region (region WS, fig. 16) are on the potassic 


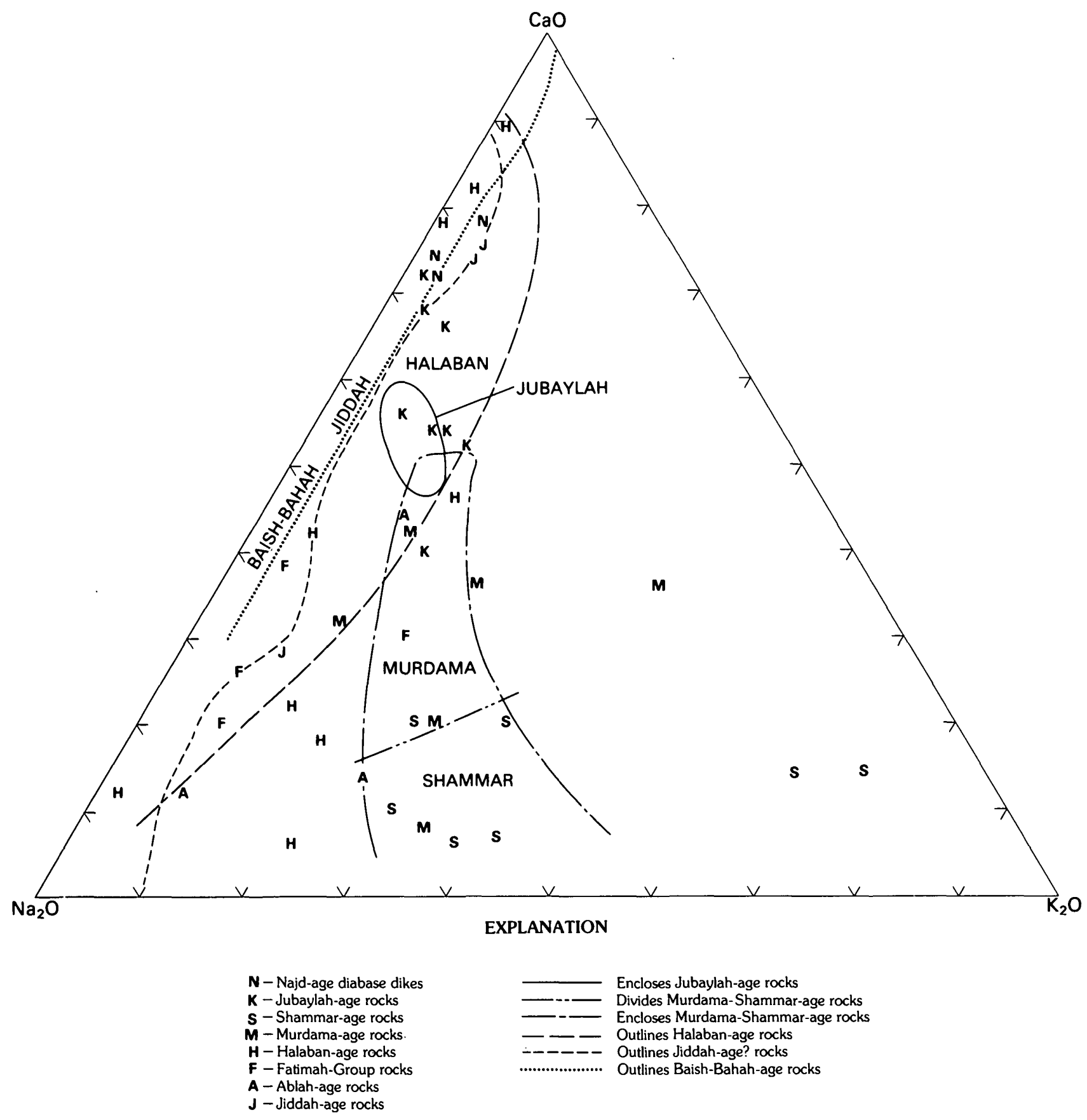

FIGURE 18.- $\mathrm{Na}_{2} \mathrm{O}-\mathrm{CaO}-\mathrm{K}_{2} \mathrm{O}$ diagram showing chemical distribution (molar data) of metavolcanic rocks and a few dike rocks reported in this report in tables 3 and 4 . Boundary lines are those drawn in figure 17 for control set of samples.

side of the Jiddah control rocks from the Rābigh area (area 12), whereas one is as potassic as the Shammar rocks. The Fatimah rocks rest unconformably on rocks of the Samran (Jiddah?) Group of the Rābigh area and are distinctly younger (Skiba, 1980). Three Ablah-age rocks have highly varying potassic contents and plot on the sodic side of the diagram (fig. 18). These few samples of Fatimah and Ablah rocks distinctly suggest rock chemistry that is more mature than that of the calc-alkalic rocks of the Jiddah and Halaban Groups. The scant chemistry does not distinguish between Fatimah and Ablah but does strongly suggest that neither is correlative with either the Murdama or the Shammar rocks.

The consistently low $\mathrm{K}_{2} \mathrm{O}$ content of the Baish-Bahah rocks (Wādī Bidah area, area 10) and the Samran rocks 
(Jiddah Group?, Rābigh area, area 12) in figure $17 \mathrm{~A}$ suggests an origin in an intraoceanic (ensimatic) islandarc environment. The Halaban Group rocks in figure $17 B$ are presumably of similar origin, but the Halaban rocks in this report are restricted to the eastern half of the shield, a part of the shield that was intruded by late granitic plutons much more abundantly than the western half as represented by control samples of the Rābigh area (area 12) and the Wādī Bidah area (area 10). The inference made here is that many more Halaban rocks have been altered by late granitic plutonism and, hence, the Halaban rocks show a much larger spread toward $\mathrm{K}_{2} \mathrm{O}$ enrichment.

A second factor suggests that late potassic hydrothermal metasomatism has altered many of the Halaban rocks because many of the samples, especially the control samples, were collected in mineralized districts, for example, the Nuqrah and Wādī Wassāţ districts. This argument is less forceful, however, because hydrothermal alteration may also be prevalent in parts of the Wādī Bidah and Rābigh areas. In this regard, six analyses of samples (R.J. Roberts, written commun., 1980) from the Mahd adh Dhahab mineralized district (area 14) are plotted in figure $17 C$. Two analyses are of pyroclastic rocks from near the Mahd adh Dhahab mine workings and are probably of Murdama (or Shammar) age, and two are from a rhyolitic plug within the mine workings. These four are highly hydrothermally metasomatized, especially in silica and potassium, as indicated by petrography as well as by the chemistry in figure $17 C$. A fifth sample from a rhyolite dike $2.5 \mathrm{~km}$ northeast of the mine is little altered, has a quartz trachyte composition, and is probably a subvolcanic intrusive of Murdama age. A sixth sample is highly sodic. These Mahd adh Dhahab analyses are presented as an extreme case of known potassium hydrothermal metasomatism of one set of igneous rocks.

A third factor suggests that more samples of siliceous and potassic volcanic rocks are represented in the Halaban samples than in the Baish-Bahah and Jiddah samples because the Halaban Group is younger and less deformed and therefore is less eroded than the Baish-Bahah and Jiddah Groups.

\section{PLUTONIC ROCKS}

The plutonic rocks from the Arabian Shield, both the control samples (fig. 19) and the samples analyzed for this report (fig. 20), are well separated on the $\mathrm{Na}_{2} \mathrm{O}$ $\mathrm{CaO}-\mathrm{K}_{2} \mathrm{O}$ diagram. Figure 19 shows the chemistry of the control samples from the control areas shown in figure 16. In this sampling, analyses of the pretectonic rocks of the dioritic suite, especially of rocks of diorite composition, are sparse. Five analyses of amphibolite gneiss from the Aţ Tà'if area (area 11) (Nasseef and
Gass, 1977) are gabbroic and of very similar composition and may be orthogabbros; they are classified herein as Baish-Bahah age. Three analyses of the ophiolitic suite from the Nuqrah quadrangle (area 1) are given. The Halaban-age trondhjemite from area 6 (Kroner and others, 1979) forms a tight cluster in figure 19 despite its conversion to orthogneiss during Najd faulting. Two samples (symbols $\mathrm{HN}$ and N, fig. 19) of this set are from migmatitic layers in the trondhjemite orthogneiss; one (HN) is a mixture of trondhjemite (Halaban age) and granite (Najd age), and the other $(\mathrm{N})$ is probably entirely granite of Najd age. The mostly granodioritic rocks of large batholiths from areas 1, 4, 7, and 11 (fig. 19) are syntectonic rocks classified in this report as having been intruded during the culminant orogeny. The rocks of granite composition and posttectonic age are classified as Murdama or Shammar age, according to the geologists working in the areas from which the samples were reported. As a generalization, regardless of area, the granitic rocks assigned a Murdama age by different geologists are distinctly more calcic in composition than the Shammarage rocks, even though a distinction between Murdamaage and Shammar-age plutonic rocks is difficult during mapping. The chemical difference between Murdamaage granite and Shammar-age granite is likely that of an early, less evolved granite in contrast to a late, more evolved one. The elliptical layered gabbroic pluton of Jabal al Jilani in the Ad Dawādimī district (area 3) (AlShanti, 1974) is herein considered to be Shammar (or Murdama) age and to be comagnetic with Shammar (or Murdama) granitic rocks.

Figure 20 shows the chemistry of samples analyzed for this report. The distribution of the samples according to age is similar to that in figure 19. Many more primary rocks of the Halaban crust and a few of the Jiddah crust are represented in figure 20 , in contrast to the few in figure 19. These pretectonic, primary, crustal rocks of trondhjemitic, tonalitic, and gabbroic composition are consistently low in $\mathrm{K}_{2} \mathrm{O}$ and lie between the $\mathrm{Na}_{2} \mathrm{O}$ and $\mathrm{CaO}$ corners of the diagram. Seven analyses (samples $167 \mathrm{~b}-\mathrm{h}$, table 3 ) form a tight cluster of anorthositic rocks from Jabal Mahail, an anorthosite body about $10 \mathrm{~km}$ wide by $30 \mathrm{~km}$ long located about 90 km north of Wādī ad Dawāsir. These rocks are associated with crustal rocks of Halaban age on plate 1, but they are coded " $E$ " in figure 20 as they may represent an older suite of rocks such as the rocks from the crustal block east of the Al Amar-Idsas fault (region E, fig. 16).

Syntectonic, granodioritic rocks of the culminant orogeny are well represented in figure 20 , as in figure 19. However, granodioritic rocks classified as Ablah age form a scatter of points that is distinctly more sodic than rocks associated with the culminant orogeny. 


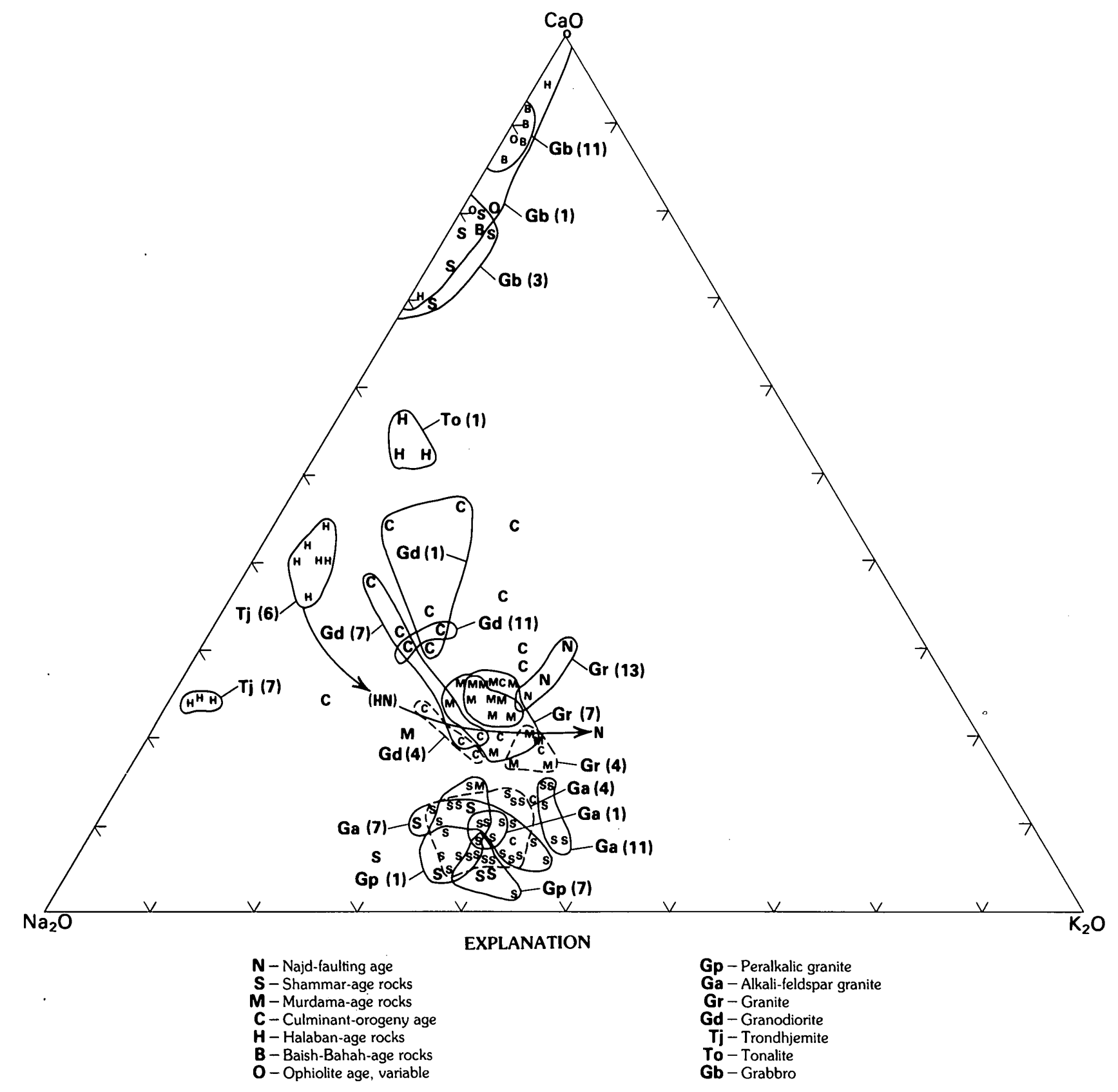

Figure 19.- $\mathrm{Na}_{2} \mathrm{O}-\mathrm{CaO}-\mathrm{K}_{2} \mathrm{O}$ diagram showing plutonic-rock chemistry (molar data) of control samples classified by age. Lines enclose representative samples of rocks of different composition and age from specific areas (areas, identified by numbers in parentheses, correspond to areas shown in fig. 16). The cluster of

These granodiorites are presumably associated with a post-Jiddah-age orogeny of about Ablah age (Ablah-age orogeny). The lower $\mathrm{K}_{2} \mathrm{O}$ content of both the volcanic and plutonic rocks of Ablah age suggests an earlier orogeny in a crust slightly more primitive, thinner, and less cratonized than the crust at the time of the culminant orogeny.

trondhjemite of Halaban age from area $6(\mathrm{Tj}(6))$ consists of orthogneiss containing migmatitic layers (HN) of mixed Halaban-age and Najd-age rock and granitic layers $(\mathrm{N})$ of Najd age. Samples $\mathrm{HN}$ and $\mathrm{N}$ are joined by arrowed tie lines showing compositional trend of migmatization.

The posttectonic plutonic granites of Murdama and Shammar age show a distribution similar to that of the control samples. The greater overlapping distribution of rocks of Murdama and Shammar age in figure 20 may result from greater subjectivity in the age classification of the rocks analyzed for this report compared with the control set. Only one Najd-age granitic rock is 


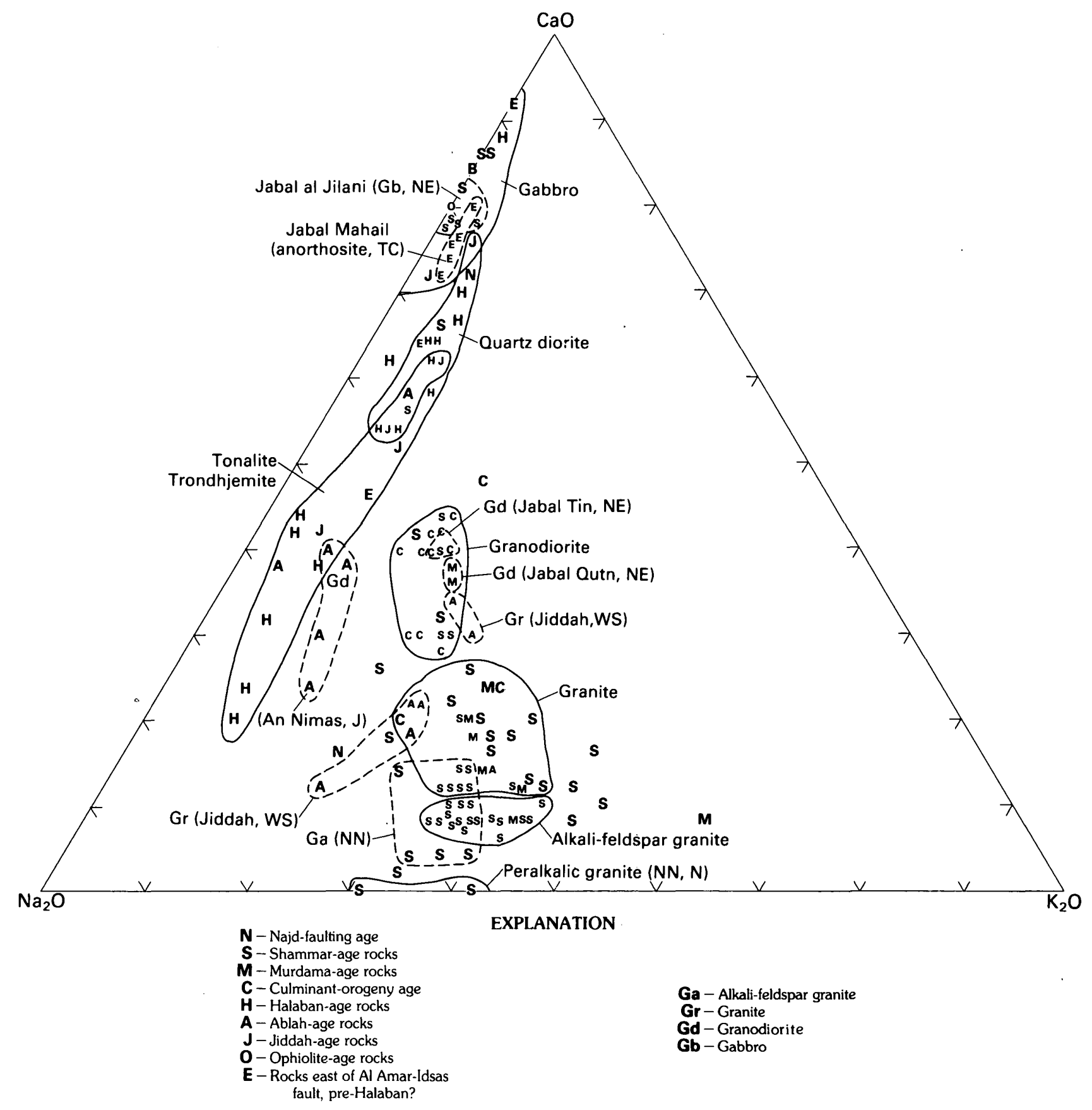

FIGURE 20.- $\mathrm{Na}_{2} \mathrm{O}-\mathrm{CaO}-\mathrm{K}_{2} \mathrm{O}$ diagram showing plutonic-rock chemistry (molar data) of samples reported in this report in tables 3 and 4 and classified by age. Lines enclose representative samples of rocks of different types. Samples from several specific localities (for region code, see fig. 16) are enclosed by dashed lines.

shown in figure 20 in spite of the fact that other granitic rocks are suggested in table 3 to be of possible Najd age. Field criteria are not available to adequately classify these youngest Precambrian rocks, and on this diagram preference is given to a Shammar age.

Granodioritic batholithic rocks (samples 88, 89, 90a, and $90 \mathrm{~b}$, table 3) from the Ad Dawādimī area are classified as youngest Shammar age because they intrude the schist of the Abt Formation as well as rocks of the Murdama Group. Large volumes of granodioritic rocks as young as Shammar age are unusual in the shield where batholithic granodioritic rocks are associated consistently with the culminant orogeny. We infer that youthful granodioritic as well as granitic pluton- 


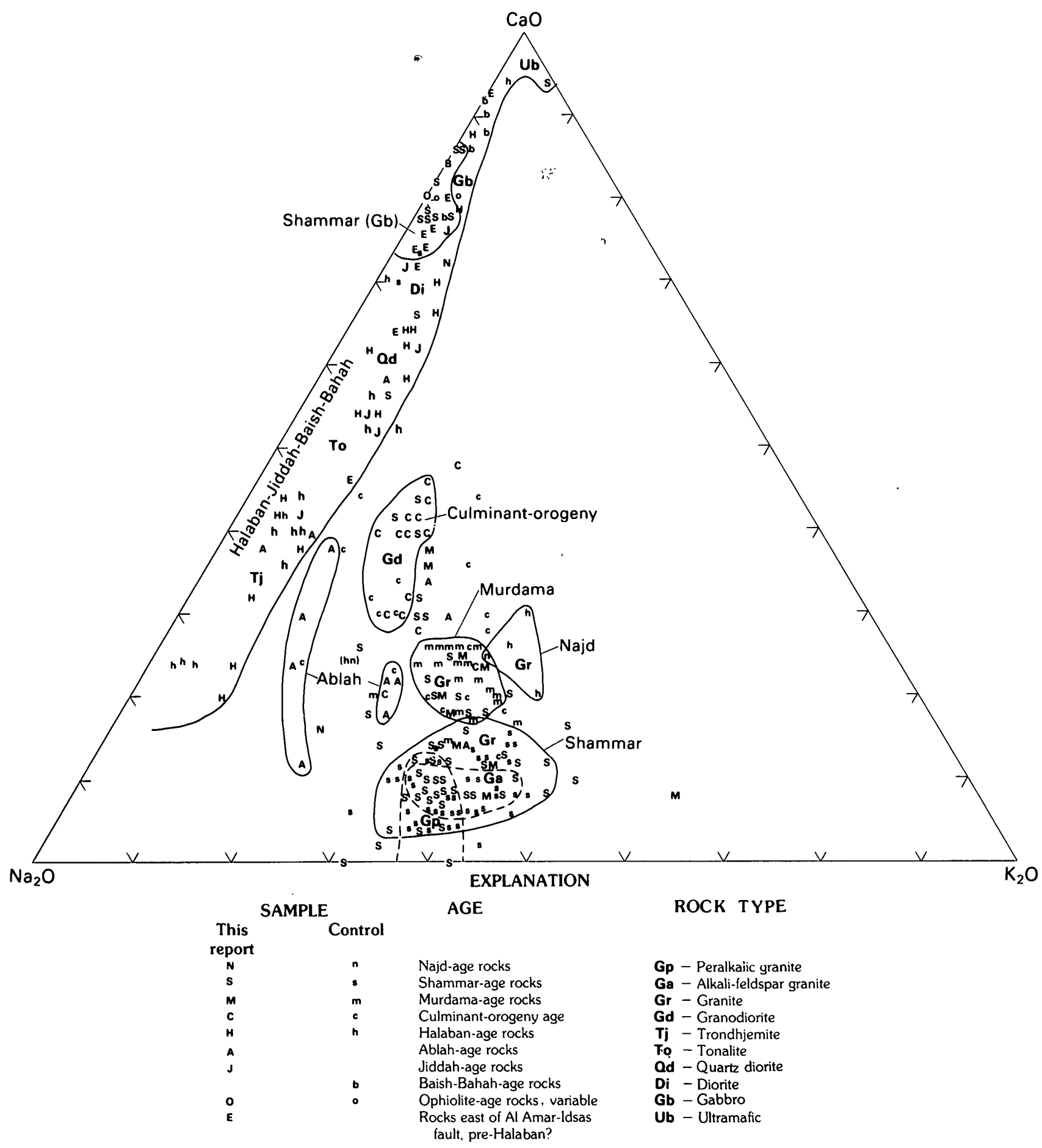

FIGURE 21.- $\mathrm{Na}_{2} \mathrm{O}-\mathrm{CaO}-\mathrm{K}_{2} \mathrm{O}$ diagram summarizing all the plutonicrock chemistry (molar data) examined for this report (combination of figs. 19 and 20). Each sample is classified according to an assigned age. Solid lines enclose clusters of rocks of different age. Dashed lines enclose tight clusters of plutonic rocks of alkali-feldspar granite $(\mathrm{Ga})$ and peralkalic granite $(\mathrm{Gp})$ compositions within the sample cluster of rocks of Shammar age. ism in the large area west of the $\mathrm{Al} \mathrm{Amar-Idsas} \mathrm{fault} \mathrm{is}$ an exception in an area of especially intensive and perhaps longer lasting tectonism.
Figure 21 summarizes the distribution of all plutonicrock analyses classified by age on the $\mathrm{Na}_{2} \mathrm{O}-\mathrm{CaO}-\mathrm{K}_{2} \mathrm{O}$ diagram. Consistently, the pretectonic, dioritic-suite 
plutonic rocks of the primary crusts of Baish-Bahah, Jiddah, and Halaban ages have low $\mathrm{K}_{2} \mathrm{O}$ compositions that range from gabbro to trondhjemite. They have a similar distribution in figure 21 , as do the volcanic rocks of the same ages in figures 17 and 18. These plutonic and volcanic rocks together constitute the primary crusts of Baish-Bahah, Jiddah, and Halaban ages. In general, the syntectonic, granodioritic batholithic rocks of the granitic suite and of culminantorogeny age form a distinct cluster that signifies the first abundance of $\mathrm{K}_{2} \mathrm{O}$ in plutonic rocks of the shield. Equivalent volcanic rocks of culminant-orogeny age are sparse or nonexistent (fig. $17 C$ ). An exception to this generalization, that culminant-orogeny rocks are the first to contain abundant potassium, is that the less abundant granodioritic rocks of Ablah age, of course, contain moderate amounts of potassium and are older than the culminant orogeny. The Ablah granodiorites represent an earlier, more restricted orogeny.

The youthful, posttectonic, granitic-suite plutonic rocks form distinct clusters of Murdama and Shammar age. A comparable distribution of volcanic rocks of the same age is seen in figures $17 C$ and 18 . The alkalifeldspar granites form a tight cluster exclusively within the Shammar-age field. The peralkalic granites form a similarly tight cluster that overlaps the alkali-feldspar granite cluster within the Shammar-age field. These peralkalic granite rocks from control areas 1 and 7 (fig. 16) are representative of a broad belt of peralkalic granite plutons that parallels the HulayfahAd Dafinah-Nabitah-Hamdah serpentinite-bearing fault zone. Many of the alkalic and peralkalic granite plutons of the shield (Stoeser and Elliott, 1980) are associated with this zone of tectonic ophiolite (Frisch and AlShanti, 1977; Delfour, 1979b; Schmidt and others, 1979).

The Shammar-age magmatism was bimodal, and a distinct cluster of Shammar-age gabbro occurs in the gabbroic field of the diagram (fig. 21). These Shammar gabbros are from concentrically layered plutons that are associated in age with the widespread circular granitic plutons of the shield. The few analyses of Najd-age granite form a distinctive high- $\mathrm{K}_{2} \mathrm{O}$ cluster in figure 21.

\section{THOLEIITIC, CALC-ALKALIC, AND ALKALIC COMPOSITIONS}

\section{GENERAL STATEMENT}

The $\left(\mathrm{Na}_{2} \mathrm{O}+\mathrm{K}_{2} \mathrm{O}\right)-\mathrm{FeO}^{*}-\mathrm{MgO}\left(\mathrm{AFM} ; \mathrm{FeO}^{*}\right.$ is total iron as $\mathrm{FeO}$ ) ternary diagram (Irvine and Baragar, 1971; Miyashiro, 1974) and the $\left(\mathrm{Na}_{2} \mathrm{O}+\mathrm{K}_{2} \mathrm{O}\right)-\mathrm{SiO}_{2}$ (alkali-silica) linear diagram (Kuno, 1966; Irvine and Baragar, 1971) have been widely used to distinguish between volcanic rocks of tholeiitic, calc-alkalic, and alkalic composition as related to genesis in oceanic or continental environments. Both types of diagrams have been used in the literature on the Arabian Shield to show the chemical distribution of rocks from some of the local areas listed in figure 16. The reports for these local areas indicate that the bulk of the shield rocks (andesite assemblages and plutonic rocks of Greenwood and others, 1980; dioritic-suite and most granitic-suite rocks of this report) are of calc-alkalic composition, for example, Delfour (1977, Nuqrah quadrangle, area 1, fig. 16) and Dodge and others (1979, Bi'r Juqjuq quadrangle, area 5). A small proportion of the shield rocks, the mafic metavolcanic rocks (basalt assemblage, Greenwood and others, 1980), are in part tholeiitic, as are some rocks of the Baish-Bahah Group (Jackaman, 1972, Wādī Bidah area, area 10). The widespread, but overall smallvolume, ophiolitic rocks (Delfour, 1979b, Nuqrah quadrangle, area 1) are entirely tholeiitic. A few of the latest Precambrian rocks of the shield, rocks of Shammar age and Najd age, are alkalic and peralkalic (Stoeser and Elliott, 1980).

A composite of the published analyses of rocks from the shield (herein the control set of about 300 analyses) and the analyses of this report (about 200 analyses) affirm that the shieldwide, bulk composition is calcalkalic and that only small volumes of the shield rocks are tholeiitic and alkalic (figs. 22 through 29). During the current study, all the analyses were examined on other diagrams used in genetic classifications of volcanic rocks, but for an overview of shieldwide rocks, little additional information is gained by doing so and the results are not reported here. In particular, these other diagrams include those of $\mathrm{SiO}_{2}, \mathrm{FeO}^{*}$, and $\mathrm{TiO}_{2}$ versus $\mathrm{FeO}^{*} / \mathrm{MgO}$ (Miyashiro, 1974), as used, respectively, by Delfour (1977, Nuqrah quadrangle, area 1) and by Greenwood and others (1980, Wādī Bidah area, area 10, and Wādī Wassāţ area, area 8).

\section{VOLCANIC ROCKS}

The metavolcanic rocks of the Baish-Bahah, Jiddah (Samran of control area 12), and Halaban (Hulayfah) Groups for the control data on the AFM diagram are shown in figure 22 . The chemical distribution scatters widely within the calc-alkalic field (Irvine and Baragar, 1971) but is confined within the outer bounds of the composition of the calc-alkalic rocks of the Cascade Mountains (USA; outer dotted line, fig. 22). These metavolcanic rocks of the andesitic and basaltic assemblages completely overlap in distribution, and all range well into the tholeiitic field on the basalt side of the diagram. The only within-group stratigraphic data available are for the Halaban rocks, referred to as the "Hulayfah" by Delfour (1977), where chemistry for 


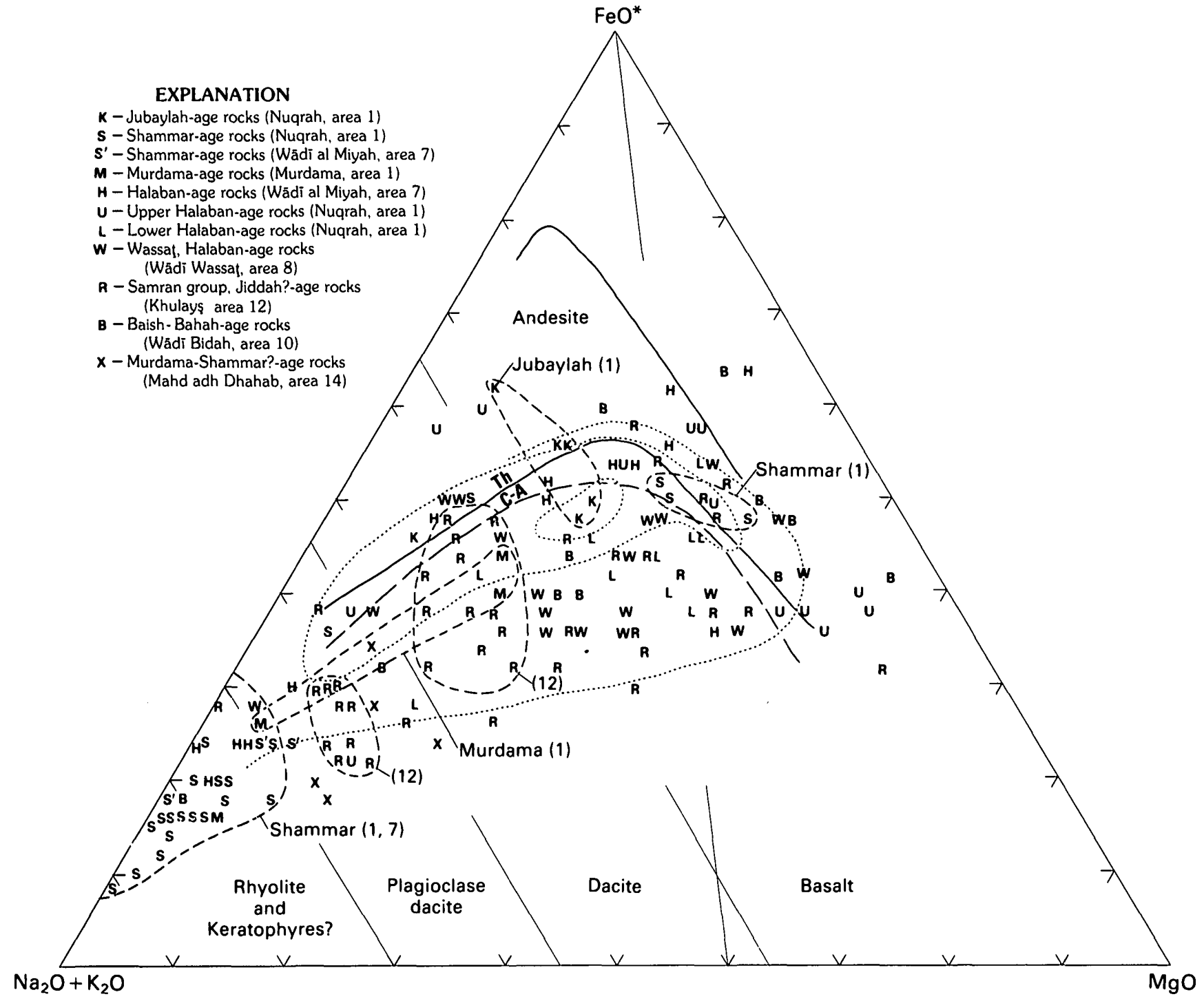

Figure 22.-AFM diagram showing metavolcanic-rock chemistry (weight percent data) of control samples classified by age. Samples are from local control areas shown in figure 16. Lower solid line separates tholeitic compositions (above line) and calcalkalic compositions (below line) according to Irvine and Baragar (1971, p. 528); upper solid line is the Skaergaard liquid trend. Two inner dotted lines are approximate contours drawn on plotted data of calc-alkalic volcanic rocks from the Aleutians (Irvine and Baragar, 1971, p. 528). Outer dotted line encloses calc-alkalic volcanic rocks from the Cascade Mountains in northwestern North America (Irvine and Baragar, 1971, p. 528). Long-dashed line separates upper Hulayfah Group rocks (upper Halaban; above line) from lower Halayfah Group rocks (lower Halaban; below line) from the Nuqrah quadrangle (area 1). Short-dashed lines enclose some specific age-area sets, as labeled (numbers in parentheses correspond to numbered areas in fig. 16). Rock-type classification (approximate) is shown by diagonal lines intersecting the sides of the diagram. upper and lower units are reported (Delfour, 1977). However, the upper Hulayfah rocks consistently straddle the tholeiitic-calc-alkalic line, whereas the lower rocks consistently lie below the line on the calc-alkalic side. This is opposite of what would be expected, and we wonder, because of structural complications, whetrer these rocks can be mapped stratigraphically in the field.
The alkali-silica diagrams, figures $26 A$ and $26 B$, show a similar wide distribution for the metavolcanic rocks of the control set. On these two diagrams, the rocks of the Baish-Bahah and Jiddah (Samran) Groups are distinctly more tholeiitic than the rocks of the Halaban Group, but overlap of some rocks of each group provides a nearly complete intermixing of the data. The rocks of the Halaban (Hulayfah) Group range from tholeiitic to 


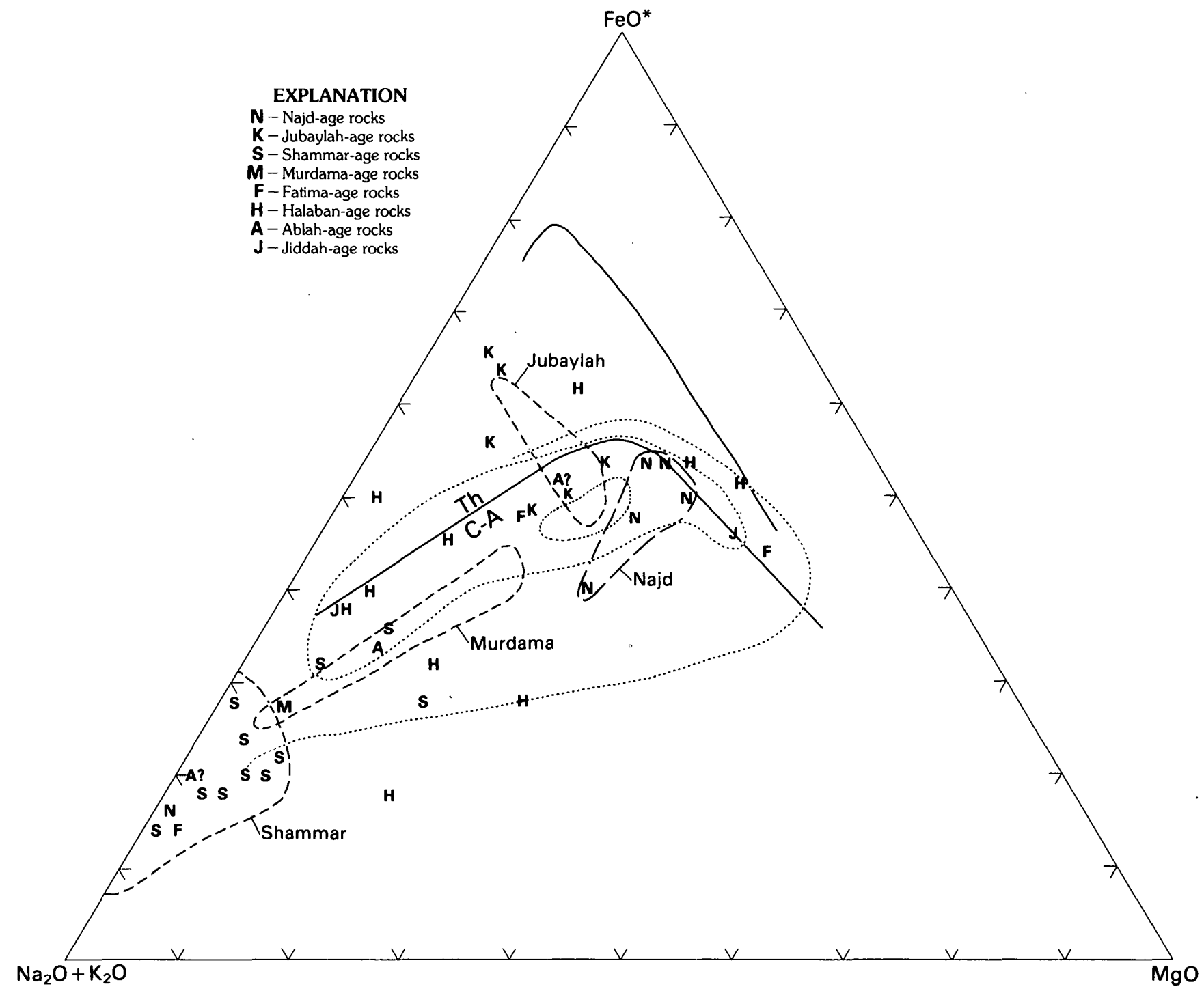

FIGURE 23.-AFM diagram showing chemical (weight percent data) distribution of metavolcanic rocks and a few dike rocks given in tables 3 and 4 . Solid and dotted lines are as in figure 22. Shortdashed lines enclose specific age-area sets of control samples (from fig. 22). Long-dashed line encloses andesitic and diabasic rocks in dikes and small plug intrusions associated with Najd faulting. Lower solid line separates tholeiitic (Th) and calc-alkalic (C-A) compositions according to Irvine and Baragar (1971), p. 528. alkalic by the classification of Kuno (1966). The Wādī Wassāţ (Halaban) rocks in particular tend to be more alkalic. Rocks of all three groups tend to separate into more tholeiitic clusters and more alkalic clusters for rocks of andesitic and basaltic compositions.

The Jiddah and Halaban metavolcanic rocks analyzed for this report are shown in figures 23 and $27 B$. The data show a wide distribution in the calc-alkalic field on the AFM diagram, figure 23; the wide distribution is similar to that of the control-set data in figure 22 , even with the small number of data points available. On the alkali-silica diagram, figure $27 B$, the Jiddah and Halaban rocks mostly form a linear array of calc-alkalic composition. These metavolcanic rocks of the basaltic and andesitic assemblages have an overall average calcalkalic composition that extends into the tholeiitic field The younger volcanic rocks are distinctly segregated on the AFM and alkali-silica diagrams (figs. 22, 23, 27 A, $27 B$ ). The volcanic rocks of the Shammar Group, both the control set and those analyzed for this report, are well segregated as rhyolites. The Shammar volcanics of the control set are distinctly alkalic, by the classification of Kuno (1966), in figure $27 \mathrm{~A}$. A few mafic volcanic rocks of the Shammar Group from the Nuqrah quadrangle (area 1) make a calc-alkalic cluster that trends into the tholeiitic field (figs. 22, 27A). The samples of 

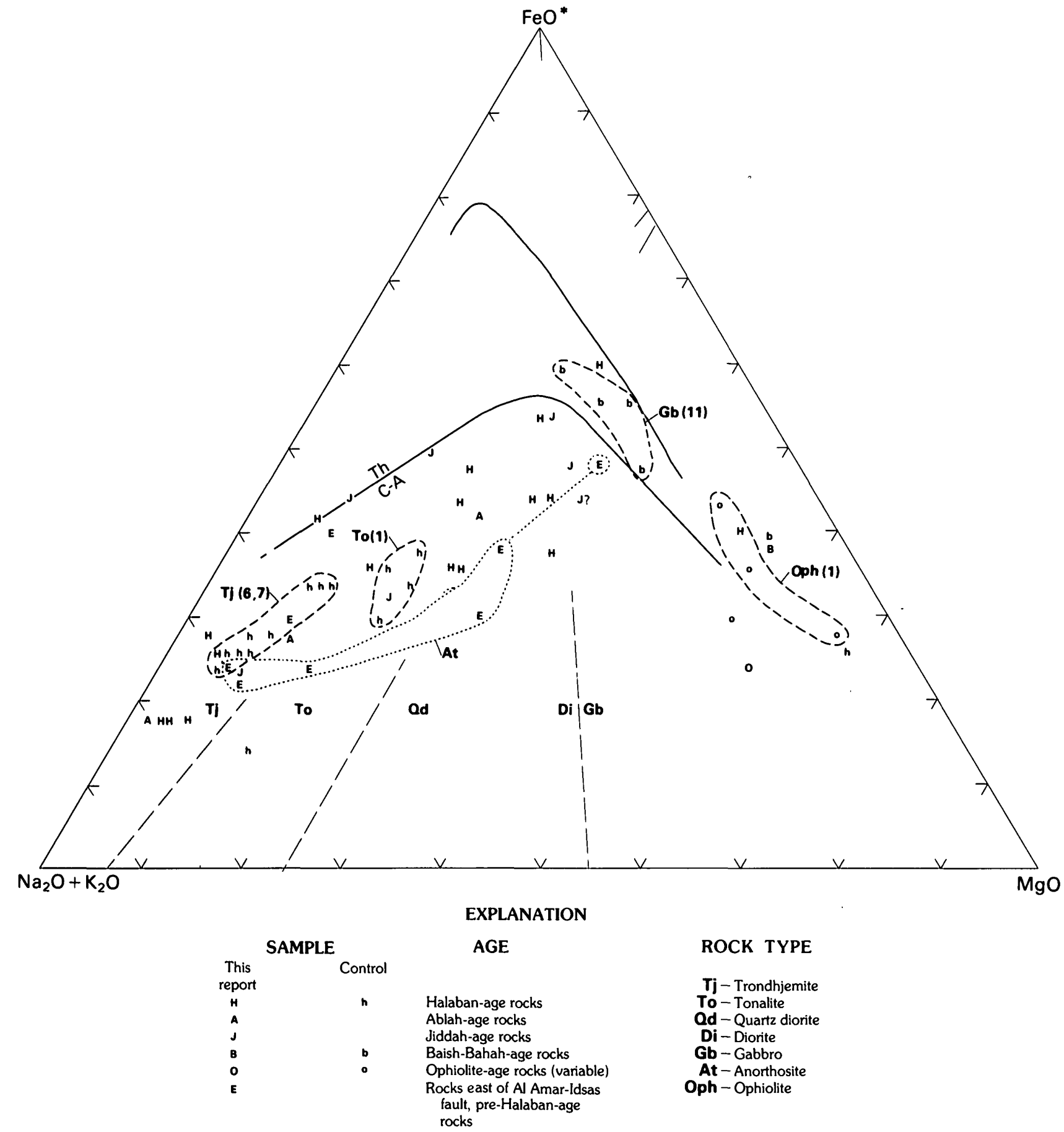

ROCK TYPE

Tj-Trondhjemite

To - Tonalite

Qd - Quartz diorite

Di-Diorite

$\mathbf{G b}$ - Gabbro

At - Anorthosite

Oph-Ophiolite
Figure 24.-AFM diagram showing chemical (weight percent data) distribution of rocks of the dioritic suite from the Saudi Arabian Shield. Analyses are classified by age; single lower case letters represent control analyses; single upper case letters represent analyses of this report. Lower solid line separates tholeiitic (Th) and calc-alkalic (C-A) compositions according to Irvine and
Baragar (1971, p. 528); upper solid line is the Skaergaard liquid trend. Short-dashed lines enclose specific control samples; rock type and source area (number in parentheses; see fig. 16) are given. Dotted line encloses anorthositic rocks (sample 167, table 3). Long-dashed lines approximately divide diagram into principal rock types of the dioritic suite. 


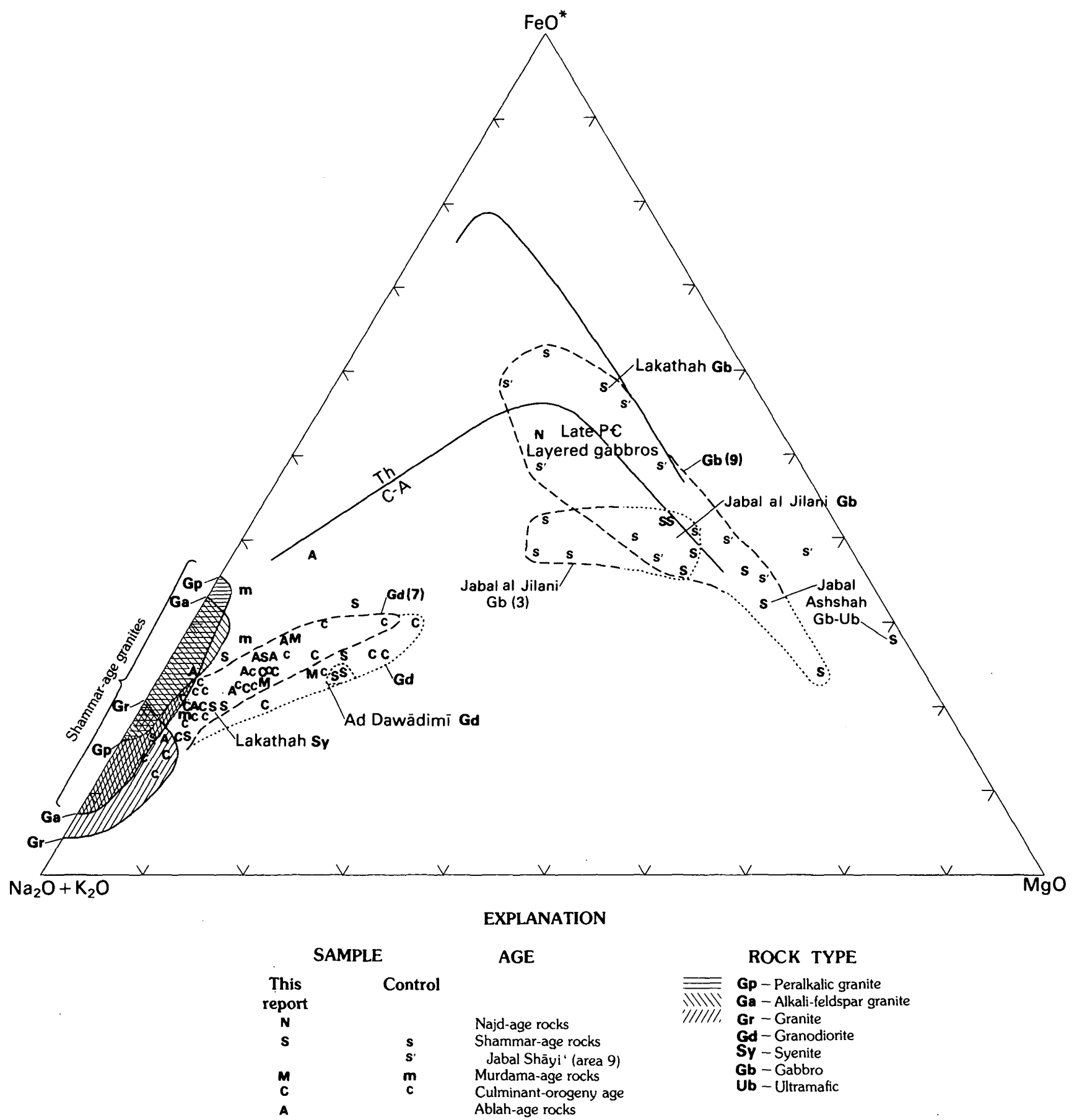

FIGURE 25.-AFM diagram showing chemical (weight percent data) distribution of rocks of the granitic suite from the Saudi Arabian Shield. Analyses are classified by age; single lower case letters represent control analyses; single upper case letters represent analyses of this report. Solid lines are as in figure 24. Shortdashed lines enclose specific sets of control samples; rock type and source area (number in parentheses; see fig. 16) are given. Dotted lines enclose specific sample sets of this report. 
the Murdama Group available (figs. 22, 23, 27 $A, 27 B$ ) are mostly calc-alkalic dacites but are too few to satisfactorily define the trend suggested in figures 22 and $27 \mathrm{~A}$. Rocks of the Jubaylah Group of both the control set and the set of this report make a distinctive cluster that straddles the tholeiitic and calc-alkalic line for mafic to intermediate compositions (figs. 22, 23, $27 A, 27 B)$. Diabasic dike rocks of Najd age form a cluster mostly close to the tholeiitic line but range to calc-alkalic compositions (fig. 23).

Limited data for metavolcanic rocks of the Ablah and Fatimah Groups are available only for analyses of this report (figs. $23,27 B$ ). In figure $27 B$, the few Fatimah rocks form a distinct calc-alkalic trend ranging from andesitic to rhyolite composition and the few Ablah rocks follow a similar trend.

The effects of alteration, such as spilitization, nonisochemical metamorphism, and late hydrothermal alteration, are little known for the metavolcanic rocks herein studied. Increase in $\mathrm{NaO}_{2}$ caused by spilitization may be expected, particularly for the rocks of the BaishBahah Group (basaltic assemblage), and mobility of $\mathrm{NaO}_{2}$ may be expected during the widespread greenschist metamorphism. Such alteration of the alkali content in the rocks of the basaltic and andesitic assemblages may account for some of the spread of the analyses across the alkalic-to-tholeiitic fields on the AFM and alkali-silica diagrams (figs. 22, 23, 26A, 26B). As described above, alkalic alteration may be expected to be more intense in the Halaban metavolcanic rocks because late granitic intrusion and late hydrothermal alteration were more intense in the northeastern half of the shield. Therefore, on the AFM and alkali-silica diagrams, as well as on the NCK diagram, the Halabanrock chemistry does have an alkali range that is wider than that of the Baish-Bahah and Jiddah rocks of the southwestern half of the shield.

Late hydrothermal alteration is widespread in the shield, and many of the analyzed samples, particularly samples from the control set, came from mineralized districts where more detailed studies have been concentrated. It must be assumed, however, that the samples were collected as representive of the least altered rocks. An extreme example of the effects of hydrothermal alteration is shown by five samples from the Madh adh Dhahab district (area 14, fig. 16; R.J. Roberts, written commun., 1980) in figures 22 and $27 \mathrm{~A}$. These volcanic and dike samples of possibly Murdama or Shammar(?) age were collected in the vicinity of the Madh adh Dhahab gold-quartz-vein deposit, and petrographic study and chemical analyses (R.J. Roberts, oral commun., 1980) document well that hydrothermal alteration was intense in all but one sample; figure $27 \mathrm{~A}$ suggests an extreme increase in $\mathrm{SiO}_{2}$ with variable change in alkalies, whereas figure 22 suggests a large increase in alkalies relative to total iron as $\mathrm{FeO}\left(\mathrm{FeO}^{*}\right)$.

\section{PLUTONIC ROCKS}

The plutonic rocks of the dioritic suite follow a welldefined calc-alkalic trend on the AFM diagram (fig. 24); most samples are quartz diorite, tonalite, and trondhjemite of Halaban age. Few diorite and gabbro samples are represented. The mafic rocks of ophiolitic association, several Baish-Bahah rocks, and two Halaban rocks are tholeiitic. As might be expected, none of the rocks. suggest a trend toward iron enrichment of the Skaergaard trend.

On the alkali-silica diagram (fig. 28), the dioritic-suite plutonic rocks systematically increase in alkalies with increased silica to $\mathrm{SiO}_{2}$ contents of about 70 percent; as silica increases further, alkali content decreases. This trend of the plutonic rocks (fig. 28) is similar to that of the volcanic rocks (figs. 26A, 26B) and suggests a comagmatic relationship between the volcanic rocks of the andesitic and basaltic assemblages and the plutonic rocks of the dioritic suites. Specific classification of these plutonic rocks as calc-alkalic or tholeiitic is not possible because the compositional boundaries of Kuno (1966) are defined for volcanic rocks. However, in figure 28 the dioritic-suite rocks of Jiddah age tend to be less alkalic than equivalent rocks of Halaban age, although most samples overlap on the diagram. The anorthositic rocks of sample 167 (tables 3, 4; fig. 28) distinctly lie outside the plutonic rock trend of the dioritic suite. The ophiolitic rocks of the Nuqrah quadrangle (area 1; fig. 28) are tholeiitic and lie within the general dioritic-suite trend but are restricted to the mafic end of the trend. As expected, on the AFM diagram (fig. 24) the ophiolitic rocks form a distinct cluster of high-MgO, tholeiitic composition.

The plutonic rocks of the granitic suite are distinctly bimodal in composition in figures $25,29 A$, and $29 B$. The gabbroic rocks, characteristic of the small, circular, layered plutons of youthful Precambrian (Shammar) age, tend toward distinct iron enrichment but much less so than on the Skaergaard trend. On the alkali-silica diagrams (figs. 29 $A, 29 B$ ) the Shammar gabbros spread across the alkalic to tholeiitic fields of the volcanic classifications of Kuno (1966) and Irvine and Barager (1971). The granodiorite and granite rocks of culminantorogeny age and Shammar age follow a broken calcalkalic trend that is considerably enriched in total alkalies (figs. 25, 29A, 29B) relative to the trend of the dioritic-suite rocks on similar diagrams (figs. 24, 28). The late alkali-feldspar granite and peralkalic granite samples follow a distinctly different trend of total iron enrichment (fig. 25) and of alkali enrichment (figs. $29 \mathrm{~A}$, $29 B)$. 


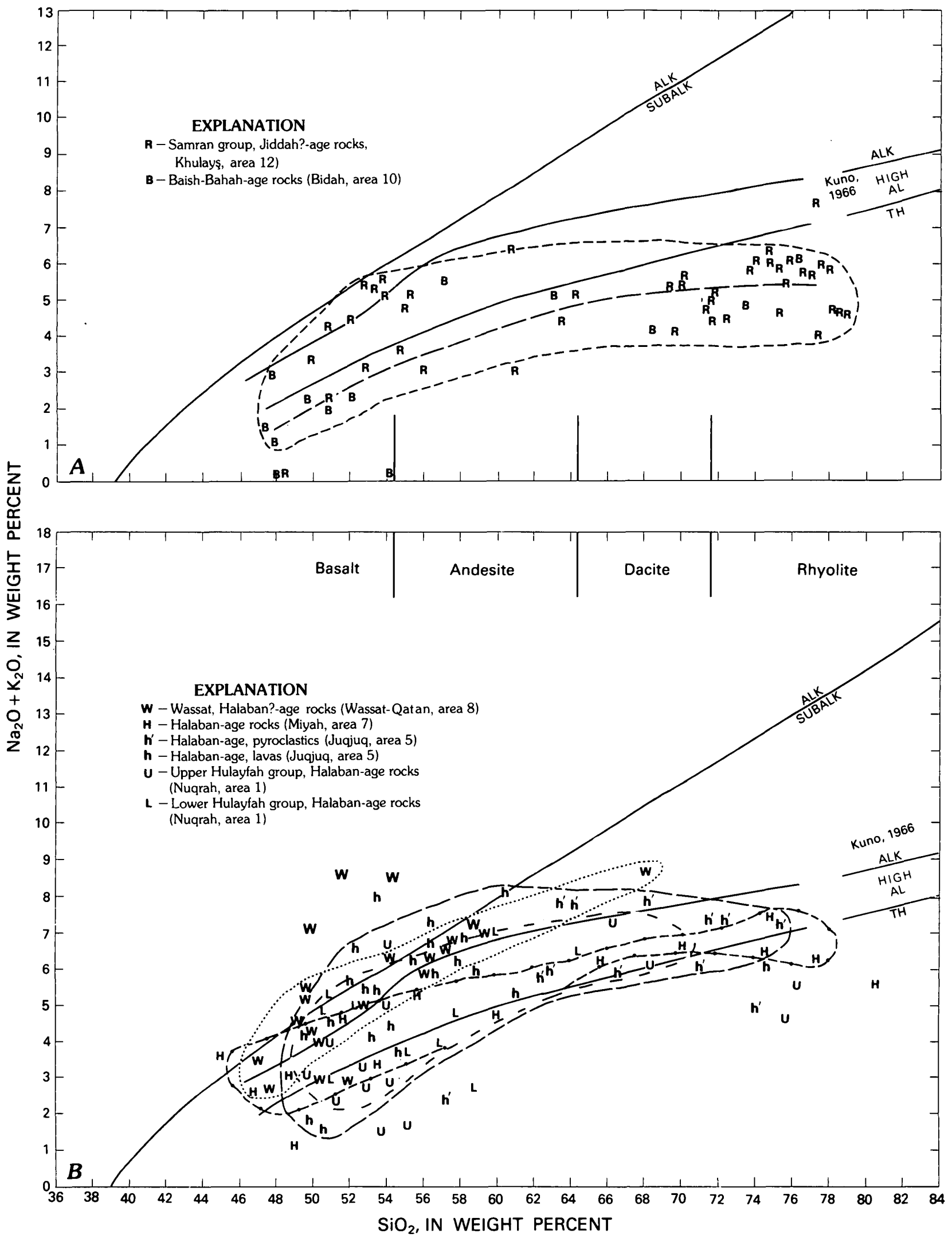


The few analyses of Ablah plutonic rocks, mostly granodiorite and granite, follow the calc-alkalic trend of rocks of culminant-orogeny age on the AFM diagram (fig. 25) but are distinctly less alkalic than rocks of culminant-orogeny age on the alkali-silica diagram (fig. $29 B$ ). The low total-alkalic content of the Ablah rocks is caused by a low $\mathrm{K}_{2} \mathrm{O}$ content that is much lower than in younger granodiorite and granite rocks from elsewhere in the shield. In general, this is characteristic of the early, precratonization granitic rocks of the western part of the shield. This is more strikingly so if the control set of granodioritic gneisses from the At Tā'if area (area 11, fig. 16) of the western shield in figure $29 \mathrm{~A}$ is considered to be Ablah age rather than culminant-orogeny age. We have no age data that dispute such a reclassification of these rocks. The distinctive alkalic cluster of syenitic and quartz monzonitic rocks in figures $29 A$ and $29 B$ are common, but not abundant, for rocks of Shammar age from widely separated parts of the shield. They suggest a trimodal distribution for plutonic rocks of Shammar age.

\section{DISCUSSION OF CHEMISTRY}

The chemical variation of the volcanic and plutonic rocks of the Saudi Arabian Shield has been examined regionally by Greenwood and Brown (1973), Greenwood and others (1976), Greenwood and others (1980), and Delfour (1979b) and locally by Jackaman (1972), Delfour (1977), Al-Shanti (1974, 1976), Kanaan (1979), Dodge and others (1979), Nasseef and Gass (1977), Skiba (1980), and others. This report contributes the fact that the chemical evolution of these rocks is systematic with age in any given region and is similar for different regions throughout the shield regardless of age. The chemical data presented above, as well as this discussion of the chemistry, have been summarized in a review paper by Schmidt and Brown (1984).

FIGURE 26.-Alkali-silica diagram showing metavolcanic-rock chemistry (weight percent data) of control samples of the Baish-Bahah and Jiddah Groups $(A)$ and of the Halaban Group $(B)$. Rocks are classified by age. Upper solid line divides alkaline and subalkaline fields for Hawaiian data (Irvine and Baragar, 1971), and two lower solid lines divide tholeiite (TH), high-alumina basalt (HIGH AL), and alkali-olivine basalt according to Kuno (1966). Approximate rock-type divisions are based on $\mathrm{SiO}_{2}$ content. In $A$, long-dashed line shows trend of Baish-Bahah rocks and short-dashed line encloses total distribution of Baish-Bahah and Jiddah rocks. In $B$, long-dashed line encloses most Halaban rocks of the Bi'r Juqjuq quadrangle (area 5, fig. 16), short-dashed line encloses most Halaban rocks of the Nuqrah quadrangle (area 1), dash-dot line encloses most Halaban rocks of the Wādī al Miyah area (area 7), and dotted line encloses most Halaban rocks of the Wãdi Wassāt area (area 8).
The major-element chemistry is readily divisible into major categories of age (stratigraphy) that are related directly to the chemical evolution and systematic genesis of the shield. Chemical evolutionary trends are summarized on NCK diagrams-metavolcanic rocks in figure 18 and plutonic rocks in figure 21 . The evolutionary trend fits especially well two major categories of crustal development: (1) a primary crust of early, multiple, intraoceanic island arcs broadly categorized agewise (stratigraphically) as Baish-Bahah, Jiddah, and Halaban, and (2) late cratonization crust categorized agewise (stratigraphically and structurally) as the culminant orogeny, Murdama-Shammar, and JubaylahNajd. The chemistry of the early crustal rocks of different ages (Baish-Bahah, Jiddah, and Halaban) is similar regardless of age and is not distinctly subdivided within itself. The chemistry of the late cratonization rocks is more distinctly and more readily subdivided within itself.

The primary crust (that is, the first or early crust) of the shield is composed of calc-alkalic metavolcanic rocks and equivalent calc-alkalic plutonic rocks. Most of the metavolcanic rocks are classified as an andesitic assemblage that ranges in composition from basalt to sodic dacite but whose calc-alkalic composition varies little areally or agewise across the shield. The abundant plutonic rocks are classified in a dioritic suite that ranges in composition from gabbro to trondhjemite and, again, whose calc-alkalic composition varies little with area or age across the shield. Calc-alkalic volcanic and plutonic rocks of such large volume as is found in the Arabian Shield are characteristic of volcanic-magmatic arcs and might be expected to have more mafic and tholeiitic compositions in their early, immature stages of development and more silicic and calc-alkalic compositions in their later, more mature stages of development (Miyashiro, 1974). This is suggested in the chemical data of the major elements (figs. $26 \mathrm{~A}, 26 \mathrm{~B}, 28,22$, 24). However, the Arabian Shield is tectonically complex and deeply eroded, and to date, distinctively immature and mature parts of individual volcanicmagmatic arcs have only been proposed and suggested; more detailed mapping, chemistry, and dating are needed. The sparse age data allow divisions into large regions of primary crustal rocks that have been designated Baish-Bahah, Jiddah, and Halaban ages, but these large regions are in themselves far too large and complex to encompass single volcanic-magmatic arcs. It is likely that each age group actually encompasses several independent arcs that have been collisionally combined at different stages of shield development.

The metavolcanic rocks of the Baish-Bahah, Jiddah, and Halaban Groups do contain some tholeiitic rocks (figs. 26A, 26B, 27B). A slight chemical discrimination 

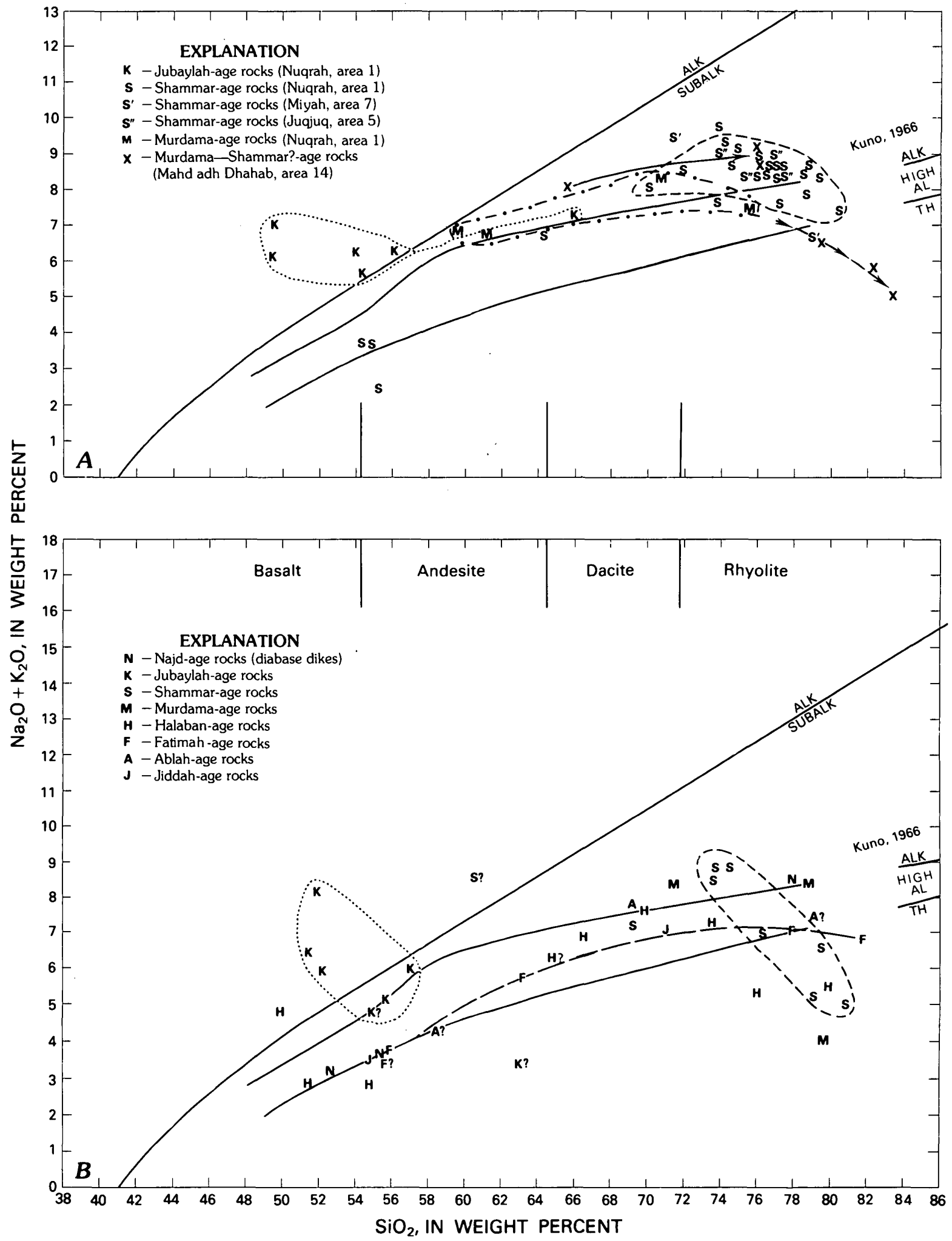
toward a more tholeiitic composition for the metavolcanic rocks of the Baish-Bahah Group compared with the Jiddah Group can be seen in figure $26 \mathrm{~A}$, and a slight discrimination toward a less calc-alkalic composition can be seen for the Baish-Bahah and Jiddah Groups compared with the Halaban Group (figs. 26A, 26B). However, sampling is localized and limited in amount, considering the tectonic complexity of the shield, and the older age and deeper erosion of the Baish-Bahah and Jiddah rocks may simply mean that more rocks of an immature, lower part of the Baish-Bahah and Jiddah have been sampled in comparison with the Halaban. Also, the Halaban rocks of the eastern part of the shield have been much more intruded by young granitic rocks and may have been subjected to more alteration subsequent to their emplacement.

Syntectonic plutonic rocks of the granitic suite of culminant-orogeny age mostly form large granodioritic batholiths of calc-alkalic composition (figs. 25, $29 \mathrm{~A}$, $29 B$ ). Within their calc-alkalic composition, these rocks are distinctly more alkalic than are plutonic rocks of similar $\mathrm{SiO}_{2}$ content of the dioritic suite (figs. 24, 28). Volcanic equivalents of these syntectonic plutonic rocks have not been recognized in the field, although the few analyses of volcanic rocks of the Murdama Group are chemically similar to the granodiorite of culminantorogeny age. In general, the Murdama-age volcanic rocks in their association with molassic sedimentary rocks are classified as posttectonic and early Shammar equivalents, but a sharp distinction between late syntectonic and posttectonic ages of molassic sedimentary rocks cannot be made, especially because this age boundary probably varies slightly from region to region across the shield.

The plutonic rocks of culminant-orogeny age are deep-seated rocks commonly spatially associated with the orthogneisses of large gneiss domes (antiforms),

FIGURE 27.-Alkali-silica diagram showing metavolcanic-rock chemistry (weight percent data) of control samples of the Murdama, Shammar, and Jubaylah Groups $(A)$ and of the metavolcanic rocks analyzed for this report $(B)$. Rocks are classified by age. Solid lines are published alkalic and subalkalic divisions of volcanic rocks, as in figure 26. Approximate rock-type divisions are based on $\mathrm{SiO}_{2}$ content. In $A$, short-dashed line encloses most rocks of the Shammar Group, dash-dot line encloses rocks of the Murdama Group, and dotted line encloses rocks of the Jubaylah Group. Solid arrow suggests direction of intense hydrothermal alteration of "rhyolite porphyry" in dikes and a plug in the Madh adh Dhahab district (area 14, fig. 16), and dashed arrow, for pyroclastic rocks in the same area. In $B$, short-dashed line encloses rocks of the Shammar Group, long-dashed line shows trend of rocks of the Fatimah Group, and dotted line encloses rocks of the Jubaylah Group. and it seems likely that little magma was erupted at the paleosurface at that time. This syntectonic granodiorite formed during tectonic thickening of the shield when the shield in many places was hot enough and thick enough for some old trondhjemitic and tonalitic masses to rise gravitationally as gneiss domes. The thickened crust seems definitely related to this first appearance of large volumes of potassic magma within the shield.

The potassic magmatism continued in a posttectonic cratonic environment, as represented by the plutonic, granitic-suite rocks of Shammar age and their volcanic equivalents of the Shammar Group. These plutonic and volcanic rocks have a distinctive major-element chemistry that is bimodal in granite-rhyolite and gabbro-basalt (figs. 22, 25, 27A, 27B). Their chemistry is distinctly more potassic than that of the plutonic and volcanic rocks of the dioritic suite and the andesite assemblage. The Shammar rocks formed from calc-alkalic magmas that in part evolved to alkalic magmas (figs. 27, 29A).

Synorogenic potassic volcanic and plutonic rocks that are older than the culminant orogeny are those of the Ablah and Fatimah Groups from the southwestern shield regions (regions J, B, and WS, fig. 16). These rocks are distinctly much less potassic than the later culminant-orogeny rocks and are exemplified by the An Nimas batholith and the batholithic rocks of the JiddahMakkah area. The Ablah rocks seem related to an earlier orogeny in a restricted part of the shield that had a thinner, less mature crust than that associated with the later, shieldwide culminant orogeny. On the basis of chemistry, the Fatimah rocks of the JiddahMakkah area (region WS, fig. 16) are similar to the Ablah rocks and formed in a similar crustal environment, but on a different crustal block and perhaps at a different time.

The Jubaylah Group volcanic rocks form a distinctive assemblage of mafic to intermediate rocks that range from calc-alkalic to alkalic in composition (figs. $27 A$, $27 B$ ). They are the youngest rocks of the Arabian Shield, and their composition is suggestive of the magmatism of a continental rift system. However, no characteristic rift existed at Najd faulting time. The Jubaylah volcanic rocks erupted during the compressional, transcurrent faulting of Najd age (Moore, 1979), but they erupted only in restricted, tensional parts of the large-displacement, sinuous faults in the complex Najd fault system.

Granite of Najd age together with minor gabbro and syenite in plugs and sills is penecontemporaneous and bimodal with Jubaylah volcanic rocks (fig. 29A). The Najd-age granite is exposed in the Najd fault zones at different places than the Jubaylah volcanic rocks. The chemical analyses of the Najd granite are too few for satisfactory comparison, but the composition seems 


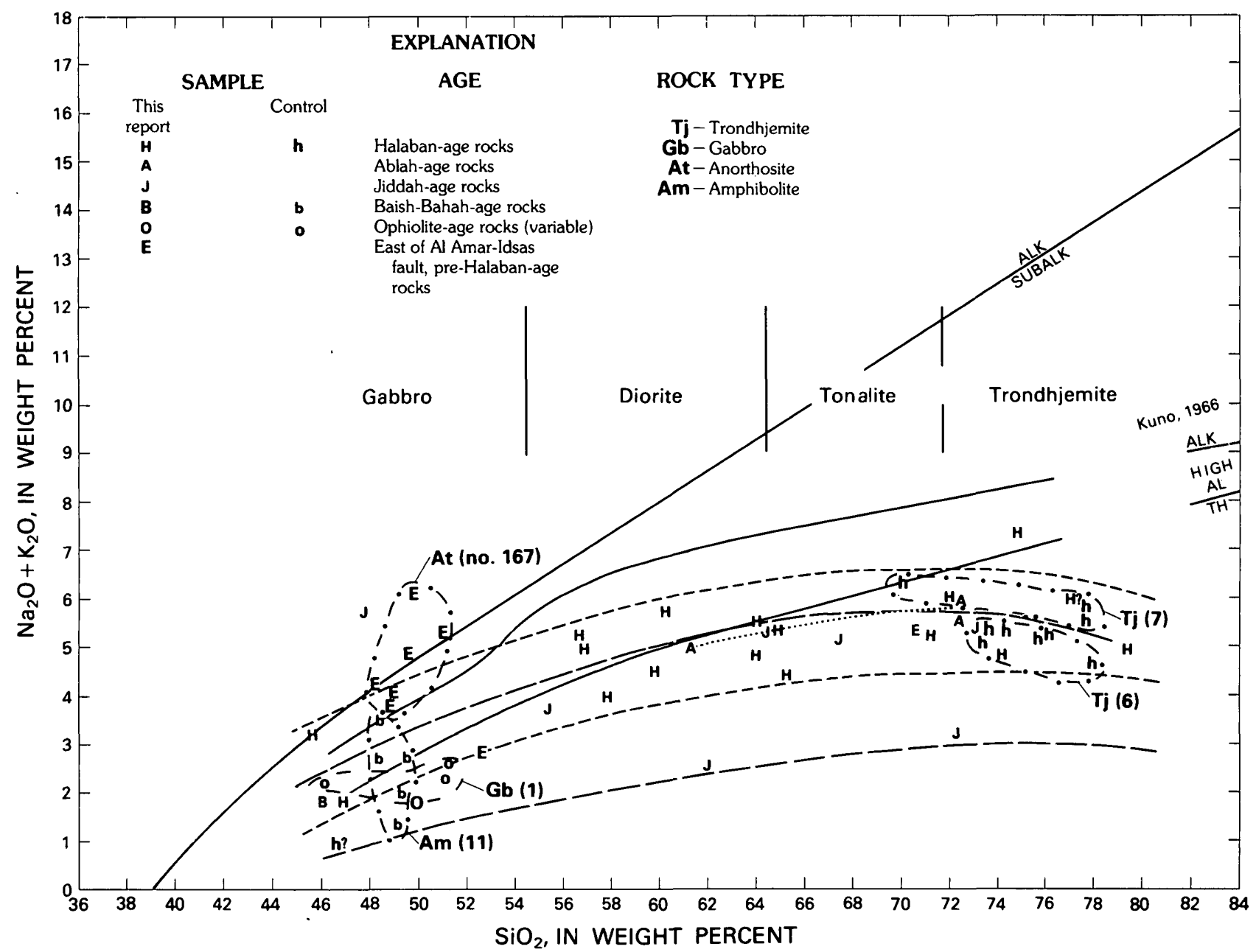

FIGURE 28.-Alkali-silica diagram showing plutonic-rock chemistry (weight percent data) of dioritic suite for samples of this report and of control set. Rocks are classified by age. Solid lines are alkalic-subalkalic divisions of volcanic rocks from the literature, as in figure 26. Approximate rock-type divisions are based on $\mathrm{SiO}_{2}$ content. Long-dashed lines enclose Baish-Bahah-age and

distinctly alkalic and probably in part overlaps that of the Shammar field.

\section{GEOCHRONOLOGIC DATA FOR THE ARABIAN SHIELD}

\section{FIRST RADIOMETRIC AGE DETERMINATIONS}

By L.T. Aldrich

The radiometric ages reported below were determined during the period 1957-63. The rock samples were provided and examined petrographically by Glen F. Brown and his associates at the U.S. Geological Survey. The $25 \mathrm{Rb}-\mathrm{Sr}$ ages of biotites and feldspars and
Jiddah-age rocks, short-dashed lines enclose Halaban-age rocks, dotted line connects rocks of Ablah age from the An Nimas batholith, and dash-dot lines enclose sample sets from specific area (numbers in parentheses correspond to numbered areas in fig. 16; no. 167 from table 3 ).

the $25 \mathrm{~K}-\mathrm{Ar}$ ages of biotites, hornblendes, and totalrock samples constituted the initial suite of ages for the Arabian Shield. Zircons in the quantity required for analysis were not found in any of the rocks examined. The sample locations are distributed over the entire Arabian Shield; the locations, the sample numbers, and the rock types as determined by petrographic examination of thin sections are given in table 6 . Of the 50 dates, 31 have been published without supporting chemical data on U.S. Geological Survey geologic maps I-200A, I-204A, I-206A, I-210A, and I-211A (Bramkamp, Brown, and others, 1963; Bramkamp, Ramirez, and others, 1963; Brown, Jackson, Bogue, and Elberg, 1963; Brown, Jackson, Bogue, and MacLean, 1963; Jackson, Bogue, Brown, and Gierhart, 1963). Table 5 
SHIELD AREA OF WESTERN SAUDI ARABIA

TABLE 5.-Isotopic chemistry of mineral samples used to determine $K \cdot A r$ and Rb-Sr ages (table 6) of Precambrian rocks from Saudi Arabia

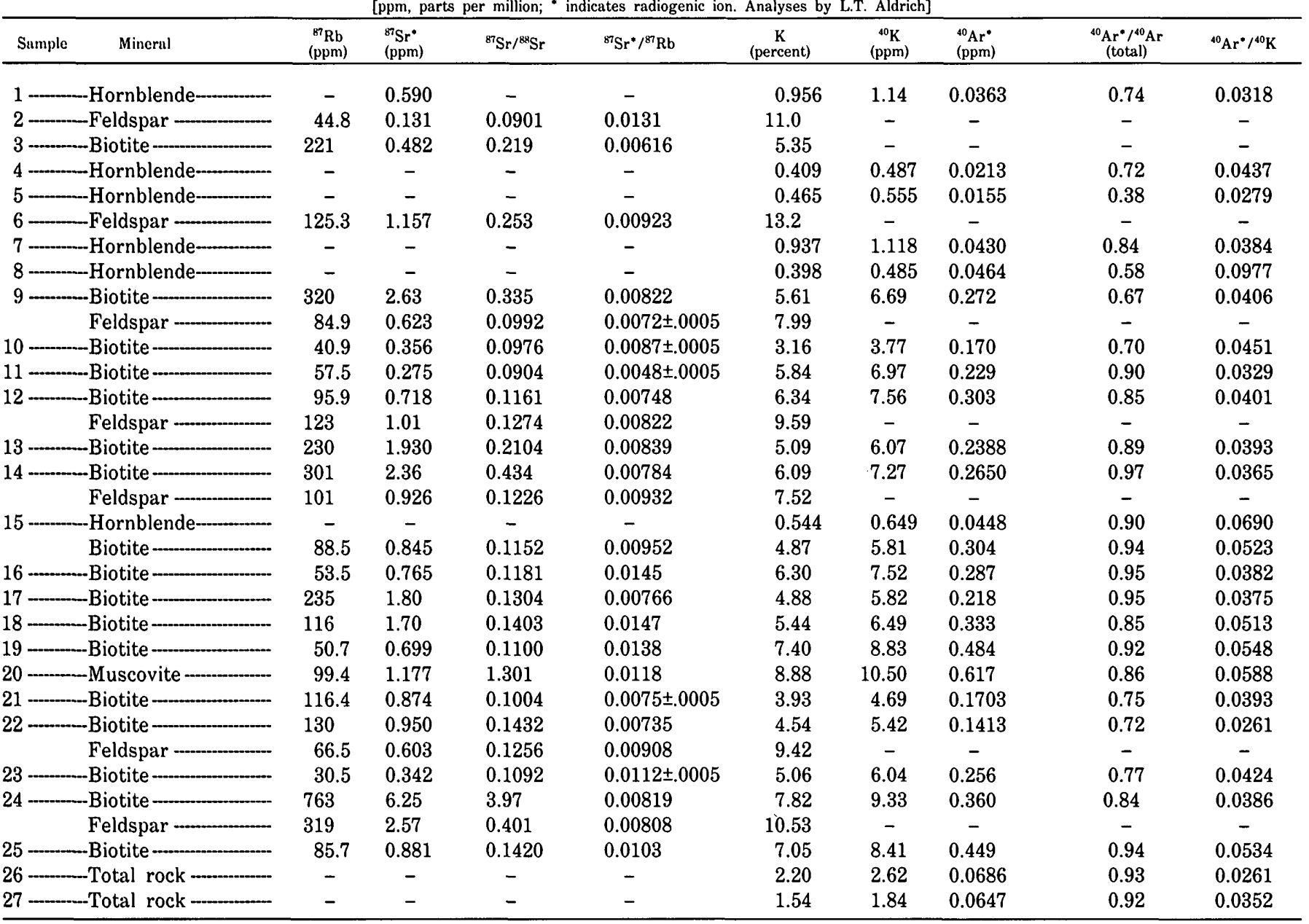

includes the minerals analyzed and the analytical data obtained. The sample numbers correspond to those in table 6 . All the analyses were made using stable isotope dilution procedures described (Aldrich, 1956; Aldrich and others, 1956). The precision of the ratios ${ }^{87} \mathrm{Sr} /{ }^{87} \mathrm{Rb}$ is better than 5 percent for all samples having a ratio ${ }^{87} \mathrm{Sr} /{ }^{88} \mathrm{Sr}>0.11$. For cases in which $0.085<{ }^{87} \mathrm{Sr} /$ ${ }^{88} \mathrm{Sr}<0.11$, the error in the ratio ${ }^{87} \mathrm{Sr}^{*} /{ }^{87} \mathrm{Rb}$ is indicated. The precision of the ratio ${ }^{87} \mathrm{Sr} /{ }^{88} \mathrm{Sr}$ is 0.3 percent or less and is independent of the assumption of the ratio $86 / 88$ to the first order. The absolute ratio ${ }^{87} \mathrm{Sr} /{ }^{88} \mathrm{Sr}$ may be in error as much as 0.5 percent owing to processes in thermal ionization and to errors in thermal calibrations which have not yet been discovered.

The precision of the argon determinations is better than 1.0 percent. This upper limit is set by the variations in the ratios ${ }^{36} \mathrm{Ar} /{ }^{40} \mathrm{Ar}$ in atmospheric argon observed over the years during which it has been measured at the Carnegie Institution of Washington. The ${ }^{40} \mathrm{~K}$ determination has an error of 3.0 percent or less based on reproducibility of results on the same rock sample and the results of mixing standard potassium solutions of different isotopic composition. The errors in the ratios ${ }^{40} \mathrm{Ar} /{ }^{40} \mathrm{~K}$ are less than 4 percent.

Table 6 gives the ages derived from the analytical measurements of table 5 based on the assumptions as to the isotopic abundances and decay constants stated.

Figure $29 C$ is a histogram of the distribution of ages found by the different methods applied to hornblende, biotite, and feldspar-muscovite. The first result of these measurements is the indication that the last event affecting the ages occurred over most of the Arabian Shield about 500 m.y. ago. Secondly, remnants of minerals as old as 1,100 m.y. have been found in several places on the shield. The $\mathrm{Rb}-\mathrm{Sr}$ ages of potassium feldspars and muscovites have been found to be consistently resistant to alteration by metamorphic events which do affect the biotite ages (Aldrich and others, 1965). The six feldspars and the muscovite (see table 5) do not give a pattern basically different from 


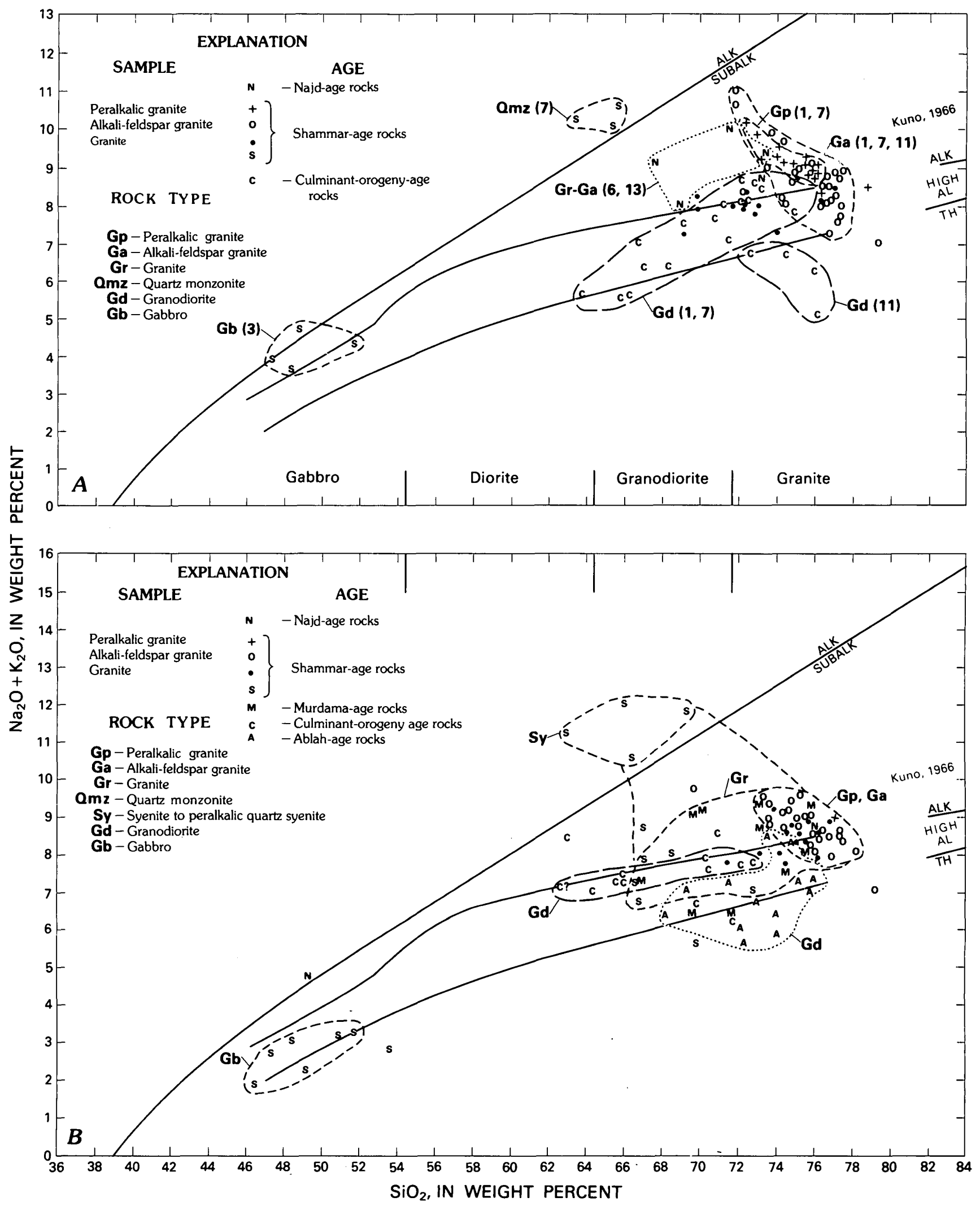




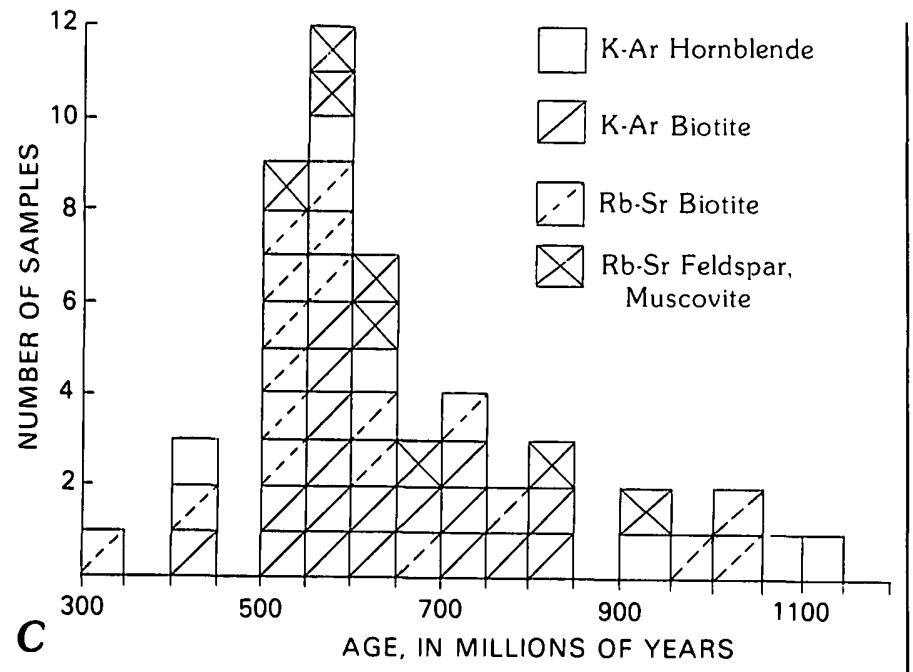

Figure 29C.-Histogram showing distribution of K-Ar and Rb-Sr ages for several minerals.

that of the biotites by themselves or from that seen in all of the Rb-Sr ages measured. The pattern of hornblende $\mathrm{K}$-Ar ages is also similar to that obtained for the other minerals. The simplest generalized picture of the Arabian Shield from these data is that of a large area about 1,000-1,200 m.y. old on which has been superposed an event culminating about 550 m.y. ago. A particular sequence of samples with geographic proximity is that starting at Jiddah with sample 18 and continuing southeast about $300 \mathrm{~km}$. Samples 18, 19, 20, 23 , and 25 (table 6 ) have an age pattern similar to that observed for Precambrian rocks in the Appalachiansgenerally discordant and ranging down from 1,050 m.y. Samples 21, 22, and 24 are relatively concordant and may represent younger rocks associated with the event that lowered the age of the other. rocks.

These data should be compared with the K-Ar ages obtained in eastern Egypt in the area between long $33^{\circ}$ and $35^{\circ}$ E. as published by Higazy and El-Ramly (1960).

FIGURE 29.-Alkali-silica diagrams showing plutonic-rock chemistry (weight percent data) of granitic suite: $A$, Samples of control set; $B$, Samples of this report. Rock are classified by age. Solid lines are alkalic-subalkalic divisions of volcanic rocks from the literature, as in figure 26. Approximate rock-type divisions are based on $\mathrm{SiO}_{2}$ content. In $A$, lines enclose control samples from specific areas (numbers in parentheses correspond to numbered areas in fig. 16): long-dashed lines enclose two sets of granodiorite of culminantorogeny age, short-dashed lines enclose Shammar-age rocks of different compositions, and dotted line encloses Najd-age granitic rocks. In $B$, lines enclose samples of this report of different composition and age: long-dashed line encloses rocks of culminantorogeny age, short-dashed lines enclose rocks of Shammar age of various compositions, and dotted line encloses rocks of Ablah age.
The total-rock argon ages of samples 26 and 27 from the 'Asir are presented not as a measure of their true age or even an approximation of the time of any "event" in this area, but rather as an indication of the minimum age that may be ascribed to these rocks. It must be pointed out that these samples could be older than those in the northern part of the shield and still have this young apparent age, owing to argon leakage from the potassium feldspars.

\section{EARLY TABULATION OF RB-SR AND K-AR AGES}

By G.F. Brown, Carl Hedge, and Richard Marvin

During the course of initial geologic fieldwork by the U.S. Geological Survey in the Arabian Shield, 101 radiometric ages were determined from rocks in the Precambrian-Cambrian basement. These ages were determined between 1963 and 1970 after the pioneering work of L.T. Aldrich (see preceding section) but before the extensive dating of the rocks of the Precambrian Shield after 1972. A tabulation of these Rb-Sr and K-Ar ages is presented recognizing the limitations of $\mathrm{Rb}-\mathrm{Sr}$ and $\mathrm{K}$-Ar ages.

Thirty samples were analyzed, using the Rb-Sr method, by Carl Hedge (table 7) in the Denver laboratory, U.S. Geological Survey. Eight of the $80 \mathrm{~K}-\mathrm{Ar}$ ages listed in table 8 were determined by Richard Marvin, Harald Mehnert, and Violet Merritt in the Denver laboratory. Thirty-one K-Ar ages were determined by Geochron Laboratories, Kruger Enterprises, Inc., Cambridge, Mass., and $41 \mathrm{~K}-\mathrm{Ar}$ ages by Teledyne Isotopes, Westwood, N.J.

Of the $30 \mathrm{Rb}$-Sr age determinations by Hedge (table 7), 19 were used to plot seven isochron diagrams. The samples used for the plots range from gneissic quartz monzonite to aegirine granite and porphyritic felsite. $\mathrm{Rb}-\mathrm{Sr}$ isochron ages range downward from $731 \mathrm{~m} . \mathrm{y}$. for the gneissic quartz monzonite to 572 m.y. for the porphyritic felsite. Samples from the aegirine granite gave an isochron age of $590 \mathrm{~m} . \mathrm{y}$. Where they could be precisely determined, the initial ${ }^{87} \mathrm{Sr} /{ }^{86} \mathrm{Sr}$ ratios were relatively low-0.703-0.705. An initial ratio of 0.704 was therefore used to calculate the individual ages reported in table 7. Rhyolite sills or flows in the upper beds of the Murdama Group gave an age of 633 m.y., whereas rhyolite near the top of the underlying and older Hulayfah (Halaban) Group gave a minimum age of 658 m.y. (sample 68 , table 7). The latter is especially significant as the rhyolite samples came from drill cores in stratabound ore deposits syngenetically deposited above the middle Hulayfah (Halaban) andesite and in the lower part of the uppermost rhyolite of the Halaban Group. 
TABLE 6.-Locations, rock types, and ages of mineral samples from Precambrian rocks of Saudi Arabia

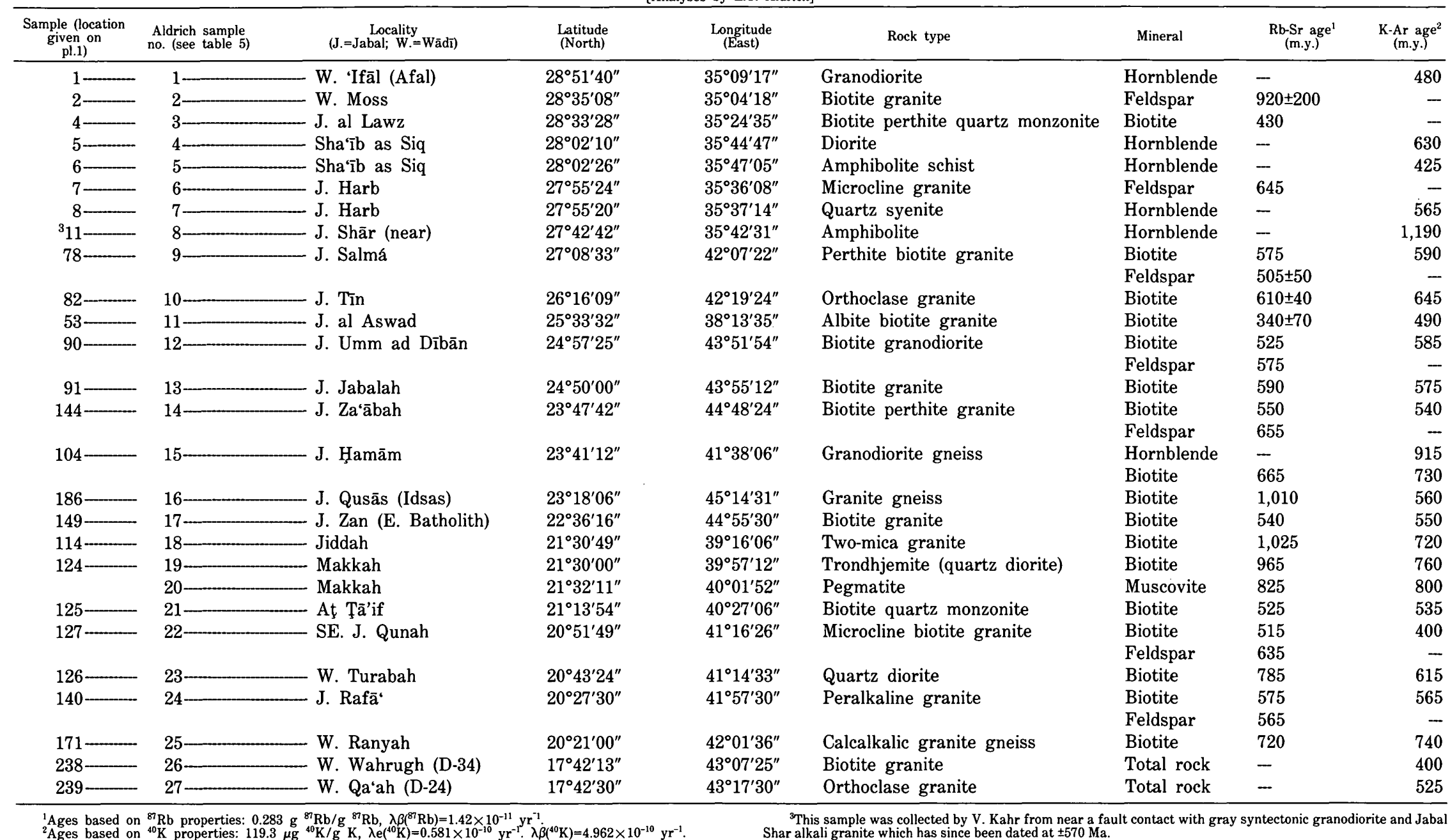


TABLE 7.-Rb-Sr ages of Precambrian crystalline rocks of Saudi Arabia

\begin{tabular}{|c|c|c|c|c|c|c|c|c|c|}
\hline \multirow{2}{*}{$\begin{array}{l}\text { Sumple (location } \\
\text { shown on pl. 1) }\end{array}$} & \multirow{2}{*}{$\begin{array}{c}\text { Age' } \\
\text { (m.y.) }\end{array}$} & \multirow{2}{*}{$\begin{array}{c}\mathrm{Sr} \\
(\mathrm{ppm})\end{array}$} & \multirow{2}{*}{${ }^{87} \mathrm{Rb} /{ }^{86} \mathrm{Sr}^{2}$} & \multirow{2}{*}{${ }^{87} \mathrm{Sr} /{ }^{86} \mathrm{Sr}$} & \multirow{2}{*}{$\begin{array}{r}\text { Material } \\
\text { analyzed }\end{array}$} & \multirow{2}{*}{ Rock } & \multirow{2}{*}{$\begin{array}{l}\text { Collected } \\
\text { by }\end{array}$} & \multicolumn{2}{|c|}{ Location } \\
\hline & & & & & & & & Lat $\mathrm{N}$. & Long E. \\
\hline $59 a-\ldots$ & $192 \pm 15-229$ & 16.4 & 41.60 & 0.9968 & Whole rock & Granite & Petty & $27^{\circ} 42^{\prime}$ & $41^{\circ} 25^{\prime}$ \\
\hline $68 \ldots$ & $58 \pm 40^{*}-29.9$ & 58.1 & 1.491 & 0.7174 & do. & Rhyolite & Delfour & $25^{\circ} 45^{\prime}$ & $40^{\circ} 45^{\prime}$ \\
\hline 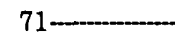 & $558 \pm 40^{*} \ldots$ & 157 & 0.098 & 0.7052 & do. & do. & do. & $25^{\circ} 36^{\prime}$ & $41^{\circ} 26^{\prime}$ \\
\hline 98 & $558 \pm 40^{*} \ldots \quad 45.8$ & 61.3 & 2.167 & 0.7245 & do. & do. & do. & $23^{\circ} 51^{\prime}$ & $40^{\circ} 56^{\prime}$ \\
\hline 94 & $333 \pm 15^{*}-----124$ & 82.6 & 4.364 & 0.7440 & do. & Rhyolite & do. & $23^{\circ} 42^{\prime}$ & $39^{\circ} 40^{\prime}$ \\
\hline 106 & $333 \pm 15^{*}-\ldots \quad 44.7$ & 107 & 1.211 & 0.7149 & do. & Latite & do. & $23^{\circ} 23^{\prime}$ & $39^{\circ} 44^{\prime}$ \\
\hline 95 & $820 \pm 15 \ldots$ & 53.2 & 44.61 & 1.0202 & Biotite & Granite & Goldsmith & $23^{\circ} 36^{\prime}$ & $40^{\circ} 32^{\prime}$ \\
\hline $96 \ldots$ & $572 \pm 35^{*}-\ldots$ & 111 & 0.263 & 0.7068 & Whole rock & Porphyritic felsite & do. & $23^{\circ} 30^{\prime}$ & $40^{\circ} 50^{\prime}$ \\
\hline 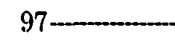 & $572 \pm 35^{*} \ldots 85.3$ & 141 & 1.753 & 0.7184 & do. & do. & do. & $23^{\circ} 32^{\prime}$ & $40^{\circ} 54^{\prime}$ \\
\hline 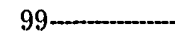 & $731 \pm 15 \ldots 13.7$ & 924 & 0.53 & 0.7046 & do. & Gneissic qtz. monzonite & do. & $23^{\circ} 26^{\prime}$ & $40^{\circ} 59^{\prime}$ \\
\hline $100 a \ldots$ & $692 \pm 15^{*} \ldots 72.1$ & 5.5 & 38.38 & 1.077 & do. & Granite & do. & $23^{\circ} 32^{\prime}$ & $41^{\circ} 06^{\prime}$ \\
\hline $100 \mathrm{~b} \cdots$ & 123 & 5.1 & 69.25 & 1.383 & K-feldspar & do. & do. & $23^{\circ} 32^{\prime}$ & $41^{\circ} 06^{\prime}$ \\
\hline $101 a \ldots$ & $590 \pm 10^{*}-\ldots-\ldots 2$ & 21.9 & 22.05 & 0.8821 & do. & do. & do. & $23^{\circ} 32^{\prime}$ & $41^{\circ} 10^{\prime}$ \\
\hline 101b-............. & $590 \pm 10^{*} \ldots 659$ & 86.5 & 50.60 & 1.1390 & do. & do. & do. & $23^{\circ} 32^{\prime}$ & $41^{\circ} 10^{\prime}$ \\
\hline $101 c$ & $590 \pm 10^{*}----320$ & 1.9 & 494.9 & 4.857 & do. & do. & do. & $23^{\circ} 32^{\prime}$ & $41^{\circ} 10^{\prime}$ \\
\hline 108 & $555 \pm 15-535$ & 43.5 & 35.62 & 0.9699 & Biotite & Granite & do. & $23^{\circ} 27^{\prime}$ & $41^{\circ} 21^{\prime}$ \\
\hline $135 a \ldots$ & $17 \pm 10^{*}----\quad 98.5$ & 48.2 & 5.94 & 0.7587 & Whole rock & Granodiorite & Brown & $20^{\circ} 02^{\prime}$ & $41^{\circ} 51^{\prime}$ \\
\hline $135 \mathrm{~b}-\ldots$ & $817 \pm 10^{*}-88.9$ & 24.2 & 10.73 & 0.7980 & do. & do. & do. & $20^{\circ} 02^{\prime}$ & $41^{\circ} 51^{\prime}$ \\
\hline 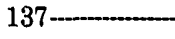 & 64.5 & 20.5 & 9.32 & 0.7827 & do. & Qtz. monzonite & do. & $20^{\circ} 11^{\prime}$ & $41^{\circ} 52^{\prime}$ \\
\hline 169 & 496 - $490 \pm 20$--ב-בי & 140 & 10.33 & 0.7807 & $\mathrm{~K}$-feldspar & Granite gneiss & do. & $20^{\circ} 58^{\prime}$ & $43^{\circ} 49^{\prime}$ \\
\hline 176 & $576 \pm 25-188$ & 78.8 & 6.896 & 0.7603 & Whole rock & Granite & Whitlow & $20^{\circ} 55^{\prime}$ & $44^{\circ} 23^{\prime}$ \\
\hline 193 & $583 \pm 15-184$ & 49.5 & 10.77 & 0.7930 & do. & do. & Trent & $19^{\circ} 33^{\prime}$ & $41^{\circ} 44^{\prime}$ \\
\hline $224-\ldots$ & 212 & 131 & 4.625 & 0.7421 & do. & do: & Brown & $17^{\circ} 57^{\prime}$ & $42^{\circ} 11^{\prime}$ \\
\hline 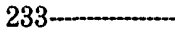 & $586 \pm 35$ - 148 - & 118 & 3.629 & 0.7361 & do. & Rhyolite & Overstreet & $18^{\circ} 20^{\prime}$ & $44^{\circ} 15^{\prime}$ \\
\hline $234 \ldots$ & 387 -----. & 9.3 & 121.0 & 1.755 & Microcline & Pegmatite & do. & $18^{\circ} 07^{\prime}$ & $44^{\circ} 14^{\prime}$ \\
\hline 235 & $550 \pm 20$-.--- 122 & 29.2 & 12.06 & 0.8040 & Whole rock & Granite porphyry & do. & $18^{\circ} 08^{\prime}$ & $44^{\circ} 15^{\prime}$ \\
\hline 240 & 129 & 60.6 & 6.185 & 0.7653 & do. & Gneissic granite & Brown & $17^{\circ} 26^{\prime}$ & $42^{\circ} 54^{\prime}$ \\
\hline
\end{tabular}

'Asterisk indicates isochron age.

${ }^{2}$ Decay constant for ${ }^{87} \mathrm{Rb}: \lambda=1.42 \times 10^{-11} / \mathrm{yr}$.

The K-Ar ages range from 299 to 932 m.y. (table 8). The ages younger than about 500 m.y. undoubtedly reflect some severe resetting caused by late Precambrian hydrothermal alteration and some resetting caused by uplift and deep erosion. In places, some resetting may have been caused by heating by Tertiary volcanism associated with the formation of the Red Sea rift. Hornblende ages, which are least susceptible to resetting (argon loss), cluster around 550 and 570 m.y. and from 590 to $660 \mathrm{~m} . \mathrm{y}$. These ages represent events in the Pan African orogeny. Older hornblende ages, 710-778 m.y., are from calc-alkaline syngenetic, generally gneissic, quartz monozonite, granodiorite, and granite bodies which are usually exposed in domes. Ages in the span 805-932 m.y. were obtained mostly from diorite, quartz diorite, and granodiorite plutons exposed in the scarps and foothills east of the Red Sea and in the southern part of the Arabian Shield.

Following the earlier geochronological work (tables $6,7,8)$, an intensive study was begun in 1972 , with geochronologists working in the field parties. Early analyses of the intensive program were limited to K-Ar determinations (Fleck and others, 1976), but when analytical work became more accurate for low rubidium values, attention was directed to $\mathrm{Rb}-\mathrm{Sr}$ determinations (Fleck and others, 1980). It became apparent that many $\mathrm{K}$-Ar analyses-especially of the biotite and feldspar fractions-gave ages that coincided with dates when magmas cooled below the point at which the daughter argon was retained in the rock. Thus, many K-Ar dates at about 550 m.y. in schist belts of the Najd fault zones suggest that an important period of fault movement occurred at or near the end of cratonization. A later cooling event, possibly representing erosion of cover rocks and upper crust of the shield during Middle to Late Cambrian, was recorded at 520-535 m.y. ago (Fleck and others, 1976). This event was further verified by a "fission track" age on four grains of sphene from a diorite in southwestern Arabia (C.W. Naeser, written commun., 1969), which gave an average of $510 \pm 52$ m.y. as a cooling date, that is, the date when the tracks stopped annealing. The K-Ar age of the hornblende from the diorite was $615 \pm 12$ m.y.

Later work using zircon and measuring daughter leads from uranium and thorium decay in zircons has helped define the two earlier major orogenies when the 
TABLE 8.-K-Ar ages of Precambrian crystalline rocks of Saudi Arabia

\begin{tabular}{|c|c|c|c|c|c|c|c|c|c|c|}
\hline Sample & K & ${ }^{40} \mathrm{Ar} \times 10^{-10}$ & ${ }^{40} \mathrm{Ar}$ & ${ }^{40} \mathrm{Ar}{ }^{40} \mathrm{~K}$ & Analyzed by & Material ana- & Rock & Colonted by & & tion \\
\hline $\begin{array}{l}\text { (location } \\
\text { given on pl. 1) }\end{array}$ & (percent) & (moles/g) & (percent) & $\mathrm{Ar} / \mathrm{K}$ & Analyzed by & lyzed & Rock & Collected by & Lat $\mathbf{N}$. & Long $\bar{E}$. \\
\hline $12 b-487 \pm 17$ & 5.06 & 40.71 & 97 & 0.0318 & USGS & $\begin{array}{r}\text { Whole } \\
\text { rock }\end{array}$ & Syenite & Brown & $27^{\circ} 28^{\prime}$ & $35^{\circ} 07^{\prime}$ \\
\hline $22 \mathrm{~b}-\mathbf{-}-515 \pm 17$ & 2.63 & 27.33 & 94 & 0.0346 & USGS $^{1}$ & do. & Oxidized $^{2}$ & do. & $27^{\circ} 45^{\prime}$ & $36^{\circ} 10^{\prime}$ \\
\hline & 2.67 & & & & & & $\begin{array}{l}\text { mugear- } \\
\text { ite }\end{array}$ & & & \\
\hline - $591 \pm 18$ & 6.29 & 75.81 & 96 & 0.0406 & Geochron $^{3}$ & Biotite & Granite & Trent & $26^{\circ} 47^{\prime}$ & $37^{\circ} 05^{\prime}$ \\
\hline & 6.34 & 77.31 & 89 & & & & & & & \\
\hline - $605 \pm 18$ & 6.68 & 82.07 & 98 & 0.0418 & do. & do. & do. & do. & $26^{\circ} 13^{\prime}$ & $37^{\circ} 30^{\prime}$ \\
\hline & 6.72 & 85.07 & 96 & & & & & & & \\
\hline $40-528 \pm 20$ & 6.53 & 68.91 & 99 & 0.0356 & USGS & do. & Rhyolite & Brown & $26^{\circ} 28^{\prime}$ & $38^{\circ} 13^{\prime}$ \\
\hline & 6.43 & & & & & & & & & \\
\hline $44-532 \pm 15$ & 1.895 & 20.19 & 63 & 0.0360 & Geochron & Whole & Andesite & do. & $26^{\circ} 20^{\prime}$ & $38^{\circ} 37^{\prime}$ \\
\hline & 1.872 & 20.24 & 91 & & & rock & & & & \\
\hline 54 & 6.52 & 74.06 & 97 & 0.0387 & do. & Biotite & Gneiss(?) & do. & $25^{\circ} 48^{\prime}$ & $38^{\circ} 22^{\prime}$ \\
\hline & 6.36 & 74.81 & 98 & & & & & & & \\
\hline 85 & 0.385 & 7.061 & 75 & 0.0609 & do. & Hornblende & $\begin{array}{l}\text { Quartz } \\
\text { diorite }\end{array}$ & do. & $25^{\circ} 48^{\prime}$ & $39^{\circ} 13^{\prime}$ \\
\hline & 0.401 & 7.231 & 81 & & & & & & & \\
\hline $67-299 \pm 11$ & 1.30 & 7.393 & 83 & 0.0189 & USGS & Whole & Andesite & do. & $25^{\circ} 33^{\prime}$ & $40^{\circ} 43^{\prime}$ \\
\hline & 1.32 & & & & & rock & $\begin{array}{l}\text { (some al- } \\
\text { teration) }\end{array}$ & & & \\
\hline $69-548 \pm 18$ & 1.70 & 18.95 & 95 & 0.0372 & do. & do. & Andesite & do. & $25^{\circ} 33^{\prime}$ & $40^{\circ} 45^{\prime}$ \\
\hline & 1.71 & & & & & & & & & \\
\hline $72-525 \pm 16$ & 1.532 & 16.11 & 81 & 0.0354 & Geochron & do. & do. & do. & $24^{\circ} 52^{\prime}$ & $39^{\circ} 11^{\prime}$ \\
\hline & 1.500 & 15.91 & 82 & & & & & & & \\
\hline 89 & 7.27 & 89.07 & 98 & 0.0395 & do. & Biotite & Granite & Mytton & $25^{\circ} 01^{\prime}$ & $43^{\circ} 48^{\prime}$ \\
\hline & 7.29 & 82.57 & 97 & & & & & & & \\
\hline $92-595 \pm 12$ & 6.12 & 75.31 & 95 & 0.0409 & do. & do. & do. & do. & $24^{\circ} 30^{\prime}$ & $43^{\circ} 19^{\prime}$ \\
\hline & 6.10 & 74.06 & 97 & & & & & & & \\
\hline $102-566 \pm 30$ & 1.095 & 13.24 & 30 & 0.0386 & do. & Whole & Dacite & Brown & $23^{\circ} 29^{\prime}$ & $41^{\circ} 23^{\prime}$ \\
\hline & 1.126 & 12.38 & 80 & & & rock & & & & \\
\hline $103-506 \pm 15$ & 2.180 & 22.19 & 83 & 0.0339 & do. & do. & Diabase & do. & $23^{\circ} 50^{\prime}$ & $41^{\circ} 25^{\prime}$ \\
\hline & 2.198 & 22.17 & 92 & & & & dike & & & \\
\hline $112-585 \pm 12$ & 0.50 & 5.959 & 86 & 0.0402 & Isotopes $^{4}$ & Hornblende & Schist & Hanford & $22^{\circ} 24^{\prime}$ & $39^{\circ} 24^{\prime}$ \\
\hline & 0.49 & 5.915 & 86 & & & & & & & \\
\hline $117 a-592 \pm 23$ & 1.247 & 15.11 & 88 & 0.0407 & Geochron & Whole & Altered & Brown & $21^{\circ} 35^{\prime}$ & $39^{\circ} 38^{\prime}$ \\
\hline & 1.222 & 14.91 & 82 & & & rock & basalt & & & \\
\hline $117 \mathrm{~b}-\mathrm{-}-576 \pm 28$ & 0.550 & 6.280 & 69 & 0.0394 & do. & do. & Andesite & do. & $21^{\circ} 35^{\prime}$ & $39^{\circ} 38^{\prime}$ \\
\hline & 0.518 & 6.280 & 68 & & & & & & & \\
\hline $119 \ldots-549 \pm 20$ & 6.65 & 71.56 & 74 & 0.0373 & do. & Biotite & Grantite & Goldsmith & $21^{\circ} 43^{\prime}$ & $40^{\circ} 27^{\prime}$ \\
\hline & 6.48 & 74.81 & 91 & & & & & & & \\
\hline 120 & 7.10 & 82.32 & 84 & 0.0395 & do. & do. & Quartz & do. & $21^{\circ} 41^{\prime}$ & $40^{\circ} 40^{\prime}$ \\
\hline & 7.03 & 84.32 & 96 & & & & $\begin{array}{l}\text { monzo- } \\
\text { nite }\end{array}$ & & & \\
\hline 121 & 7.15 & 83.82 & 97 & 0.0390 & do. & do. & Granite & do. & $21^{\circ} 41^{\prime}$ & $40^{\circ} 41^{\prime}$ \\
\hline & 7.22 & 83.32 & 98 & & & & & & & \\
\hline $128-827 \pm 16$ & 0.32 & 5.829 & 82 & 0.0609 & Isotopes & Hornblende & Diorite & Brown & $20^{\circ} 32^{\prime}$ & $41^{\circ} 25^{\prime}$ \\
\hline & 0.33 & 5.995 & 73 & & & $\begin{array}{l}\text { with } 5 \% \\
\text { impuri- } \\
\text { ties }\end{array}$ & & & & \\
\hline $129 a-932 \pm 46$ & 0.489 & 9.858 & 82 & 0.0709 & Geochron & Hornblende & do. & do. & $20^{\circ} 32^{\prime}$ & $41^{\circ} 31^{\prime}$ \\
\hline & 0.440 & 9.808 & 86 & & & & & & & \\
\hline $821 \pm 16$ & 0.49 & 8.865 & 95 & 0.0604 & Isotopes & Hornblende & do. & do. & $20^{\circ} 32^{\prime}$ & $41^{\circ} 31^{\prime}$ \\
\hline & 0.50 & 8.994 & 95 & & & $\begin{array}{l}\text { with } 5 \% \\
\text { impuri- }\end{array}$ & & & & \\
\hline & & & & & & ties & & & & \\
\hline $129 b-912 \pm 18$ & 0.44 & 9.240 & 91 & 0.0689 & do. & do. & Quartz & do. & $20^{\circ} 32^{\prime}$ & $41^{\circ} 31^{\prime}$ \\
\hline & 0.45 & 9.070 & 90 & & & & diorite & & & \\
\hline
\end{tabular}


TABLE 8.-K-Ar ages of Precambrian crystalline rocks of Saudi Arabia-Continued

\begin{tabular}{|c|c|c|c|c|c|c|c|c|c|c|}
\hline \multirow{2}{*}{$\begin{array}{c}\begin{array}{c}\text { Sample } \\
\text { (loention } \\
\text { given on pl. 1) }\end{array} \\
\end{array}$} & \multirow{2}{*}{$\begin{array}{c}\mathbf{K} \\
\text { (percent) }\end{array}$} & \multirow{2}{*}{$\begin{array}{l}{ }^{40} \mathrm{Ar} \times 10^{-10} \\
(\text { moles } / \mathrm{g})\end{array}$} & \multirow{2}{*}{$\begin{array}{c}{ }^{40} \mathrm{Ar} \\
\text { (percent) }\end{array}$} & \multirow{2}{*}{${ }^{40} \mathrm{Ar} /{ }^{40} \mathrm{~K}$} & \multirow{2}{*}{ Analyzed by } & \multirow{2}{*}{$\begin{array}{c}\text { Material ana- } \\
\text { lyzed }\end{array}$} & \multirow{2}{*}{ Rock } & \multirow{2}{*}{ Collected by } & \multicolumn{2}{|c|}{ Location } \\
\hline & & & & & & & & & Lat N. & Long $\mathrm{E}$. \\
\hline $130-805 \pm 16$ & 0.25 & 4.301 & 90 & 0.0590 & do. & Amphibole & Quartz & do. & $20^{\circ} 26^{\prime}$ & $40^{\circ} 26^{\prime}$ \\
\hline \multirow[t]{2}{*}{$131 \ldots 595 \pm 12$} & 3.72 & 44.86 & 90 & 0.0410 & do. & Muscovite & Paraschist & do. & $20^{\circ} 26^{\prime}$ & $40^{\circ} 49^{\prime}$ \\
\hline & 3.52 & 43.75 & 97 & & & & & & & \\
\hline \multirow[t]{2}{*}{$132-717 \pm 18$} & 0.42 & 6.312 & 90 & 0.0512 & do. & Hornblende & Gneiss & do. & $20^{\circ} 28^{\prime}$ & $40^{\circ} 56^{\prime}$ \\
\hline & 0.42 & 6.517 & 94 & & & & & & & \\
\hline \multirow[t]{2}{*}{136 - $585 \pm 39$} & 0.43 & 5.150 & 87 & 0.0401 & USGS & Whole & Basalt & do. & $20^{\circ} 10^{\prime}$ & $41^{\circ} 52^{\prime}$ \\
\hline & 0.43 & & & & & rock & plug & & & \\
\hline \multirow[t]{2}{*}{$141 \mathrm{a}-561 \pm 25$} & 3.646 & 42.78 & 97 & 0.0382 & Geochron & do. & Andesite & do. & $23^{\circ} 39^{\prime}$ & $43^{\circ} 08^{\prime}$ \\
\hline & 3.635 & 40.28 & 98 & & & & & & & \\
\hline \multirow[t]{2}{*}{$141 b-560 \pm 20$} & 2.162 & 24.92 & 94 & 0.0382 & do. & do. & Dacite(?) & do. & $23^{\circ} 39^{\prime}$ & $43^{\circ} 08^{\prime}$ \\
\hline & 2.277 & 25.67 & 94 & & & & & & & \\
\hline 148-574_-מיב-י12 & 5.24 & 62.62 & 99 & & & $\begin{array}{l}(+60 \\
\text { mesh) }\end{array}$ & & & & \\
\hline \multirow[t]{2}{*}{$585 \pm 12$} & 5.10 & 60.08 & 99 & 0.0401 & do. & biotite & do. & do. & $22^{\circ} 53^{\prime}$ & $44^{\circ} 56^{\prime}$ \\
\hline & 4.98 & 60.66 & 99 & & & $\begin{array}{l}(-60 \\
\text { mesh })\end{array}$ & & & & \\
\hline \multirow[t]{2}{*}{ 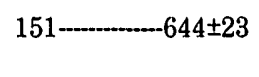 } & 6.44 & 86.65 & 95 & 0.0450 & USGS & Biotite & Gabbro & Hanford & $22^{\circ} 19^{\prime}$ & $42^{\circ} 24^{\prime}$ \\
\hline & 6.47 & & & & & & & & & \\
\hline \multirow[t]{2}{*}{$153 \ldots 12$} & 0.48 & 4.721 & 76 & 0.0331 & Isotopes & Plagioclase & Granite & Brown & $22^{\circ} 25^{\prime}$ & $43^{\circ} 48^{\prime}$ \\
\hline & 0.49 & 4.876 & 26 & & & $\begin{array}{l}\text { and } \\
\text { quartz }\end{array}$ & & & & \\
\hline $156-513 \pm 17$ & 2.66 & 22.73 & 95 & 0.0345 & USGS & $\begin{array}{l}\text { Whole } \\
\text { rock }\end{array}$ & $\begin{array}{l}\text { Gabbro } \\
\text { chilled } \\
\text { rim }\end{array}$ & do. & $22^{\circ} 28^{\prime}$ & $44^{\circ} 34^{\prime}$ \\
\hline $156 b-158 \pm 15$ & 5.87 & 44.09 & 93 & 0.0303 & do. & Biotite & Gabbro & do. & $22^{\circ} 28^{\prime}$ & $44^{\circ} 34^{\prime}$ \\
\hline $165 \ldots$ & 0.69 & 8.485 & 77 & 0.0410 & do. & Hornblende & Quartz & do. & $21^{\circ} 15^{\prime}$ & $43^{\circ} 57^{\prime}$ \\
\hline & 0.71 & 8.664 & 87 & & & $\begin{array}{l}\text { with } 20 \% \\
\text { impuri- } \\
\text { ties }\end{array}$ & $\begin{array}{l}\text { monzo- } \\
\text { nite }\end{array}$ & & & \\
\hline $168 a-778 \pm 16$ & 0.090 & 1.530 & 72 & 0.0565 & do. & Whole & Troctolite & do. & $20^{\circ} 32^{\prime}$ & $42^{\circ} 32^{\prime}$ \\
\hline & 0.092 & 1.538 & 72 & & & rock & & & & \\
\hline $168 b-710 \pm 16$ & 0.092 & 1.418 & 63 & 0.0506 & do. & Whole & Syenite & do. & $20^{\circ} 32^{\prime}$ & $42^{\circ} 32^{\prime}$ \\
\hline & 0.096 & 1.421 & 70 & & & rock & & & & \\
\hline 539 & 0.38 & 4.034 & 29 & 0.0365 & do. & Plagioclase & Gneiss & do. & $20^{\circ} 58^{\prime}$ & $43^{\circ} 49^{\prime}$ \\
\hline & 0.36 & 4.022 & 31 & & & $\begin{array}{l}\text { and } \\
\text { quartz }\end{array}$ & & & & \\
\hline $173 \ldots 16$ & 4.18 & 74.99 & 98 & 0.0608 & do. & Biotite & Diorite? & do. & $20^{\circ} 17^{\prime}$ & $42^{\circ} 08^{\prime}$ \\
\hline & 4.21 & 77.31 & 97 & & & $\begin{array}{l}\text { with } 10 \% \\
\text { impuri- } \\
\text { ties }\end{array}$ & & & & \\
\hline $173 a-711 \pm 30$ & 0.39 & 5.762 & 27 & 0.0506 & do. & Hornblende & do. & do. & $20^{\circ} 17^{\prime}$ & $42^{\circ} 08^{\prime}$ \\
\hline & 0.36 & 5.564 & 24 & & & & & & & \\
\hline 175 & 0.12 & 1.119 & 78 & 0.0323 & do. & Whole & Leuco- & do. & $20^{\circ} 16^{\prime}$ & $42^{\circ} 28^{\prime}$ \\
\hline & 0.11 & 1.097 & 79 & & & rock & gabbro & & & \\
\hline $180 a-598 \pm 12$ & 5.11 & 63.07 & 99 & 0.0412 & do. & Biotite & Granite & do. & $23^{\circ} 58^{\prime}$ & $45^{\circ} 01^{\prime}$ \\
\hline & 5.03 & 61.51 & 99 & & & & & & & \\
\hline $180 \mathrm{~b}-584 \pm 12$ & 6.48 & 77.00 & 99 & 0.0401 & do. & Biotite & do. & do. & $23^{\circ} 58^{\prime}$ & $45^{\circ} 01^{\prime}$ \\
\hline & 6.40 & 77.17 & 99 & & & & & & & \\
\hline $181-597 \pm 12$ & 6.49 & 80.17 & 99 & 0.0411 & do. & Biotite & Granodio- & do. & $23^{\circ} 58^{\prime}$ & $45^{\circ} 05^{\prime}$ \\
\hline & 6.54 & 79.67 & 99 & & & & rite & & & \\
\hline $183-597 \pm 12$ & 7.75 & 94.63 & 99 & 0.0411 & do. & Biotite & do. & do. & $23^{\circ} 41^{\prime}$ & $45^{\circ} 08^{\prime}$ \\
\hline & 7.81 & 96.41 & 99 & & & & & & & \\
\hline
\end{tabular}


TABLE 8.-K-Ar ages of Precambrian crystalline rocks of Saudi Arabia-Continued

\begin{tabular}{|c|c|c|c|c|c|c|c|c|c|c|}
\hline \multirow{2}{*}{$\begin{array}{c}\begin{array}{c}\text { Sample } \\
\text { (location } \\
\text { given on pl. 1) }\end{array} \\
\end{array}$} & \multirow{2}{*}{$\begin{array}{c}\mathbf{K} \\
\text { (percent) }\end{array}$} & \multirow{2}{*}{$\begin{array}{l}{ }^{40} \mathrm{Ar} \times 10^{-10} \\
(\mathrm{moles} / \mathrm{g})\end{array}$} & \multirow{2}{*}{$\begin{array}{c}{ }^{40} \mathrm{Ar} \\
\text { (percent) }\end{array}$} & \multirow{2}{*}{${ }^{40} \mathrm{Ar} /{ }^{40} \mathrm{~K}$} & \multirow{2}{*}{ Analyzed by } & \multirow{2}{*}{$\begin{array}{c}\text { Material ana- } \\
\text { lyzed }\end{array}$} & \multirow{2}{*}{ Rock } & \multirow{2}{*}{ Collected by } & \multicolumn{2}{|c|}{ Location } \\
\hline & & & & & & & & & Lat $N$. & Long E. \\
\hline \multirow[t]{2}{*}{$184-583 \pm 12$} & 6.51 & 77.71 & 98 & 0.0400 & do. & Impure & do. & do. & $23^{\circ} 5 \dot{3}^{\prime}$ & $45^{\circ} 07^{\prime}$ \\
\hline & 6.51 & 77.62 & 98 & & & biotite & & & & \\
\hline \multirow[t]{2}{*}{$546 \pm 5$} & 0.50 & 5.580 & 89 & 0.0370 & do. & Hornblende & Granite & do. & $23^{\circ} 53^{\prime}$ & $45^{\circ} 07^{\prime}$ \\
\hline & 0.51 & 5.580 & 89 & & & & & & & \\
\hline \multirow{2}{*}{$187-589 \pm 12$} & 7.88 & 95.88 & 99 & 0.0405 & do. & Biotite & do. & do. & $23^{\circ} 01^{\prime}$ & $45^{\circ} 29^{\prime}$ \\
\hline & 7.97 & 95.57 & 99 & & & & & & & \\
\hline $188-619 \pm 12$ & 5.40 & 67.94 & 99 & & & & & & & \\
\hline \multirow[t]{2}{*}{$629 \pm 12$} & 1.38 & 18.04 & 95 & 0.0437 & do. & Hornblende & do. & do. & $23^{\circ} 01^{\prime}$ & $45^{\circ} 33^{\prime}$ \\
\hline & 1.40 & 18.23 & 98 & & & & & & & \\
\hline \multirow[t]{2}{*}{$189-632 \pm 12$} & 4.96 & 63.87 & 97 & 0.0440 & do. & Biotite & Quartz & do. & $22^{\circ} 36^{\prime}$ & $45^{\circ} 07^{\prime}$ \\
\hline & 4.92 & 65.79 & 99 & & & $\begin{array}{l}\text { with } \\
\text { minor } \\
\text { horn- } \\
\text { blende }\end{array}$ & diorite & & & \\
\hline $611 \pm 12$ & 0.74 & 9.373 & 92 & & & & & & & \\
\hline $190-759 \pm 20$ & 7.41 & 123.1 & 98 & 0.0548 & Geochron & Biotite & Granite & Trent & $19^{\circ} 55^{\prime}$ & $41^{\circ} 33^{\prime}$ \\
\hline & 7.55 & 121.6 & 98 & & & & & & & \\
\hline $191 \mathrm{a}-646 \pm 12$ & 0.86 & 11.62 & 89 & 0.0451 & Isotopes & Hornblende & Granite & Brown & $19^{\circ} 53^{\prime}$ & $41^{\circ} 37^{\prime}$ \\
\hline & 0.87 & 11.69 & 80 & & & $\begin{array}{l}\text { with } 10 \% \\
\text { impuri- } \\
\text { ties }\end{array}$ & gneiss & & & \\
\hline $191 b-759 \pm 31$ & 0.931 & 15.71 & 91 & 0.0548 & Geochron & Hornblende & do. & do. & $19^{\circ} 53^{\prime}$ & $41^{\circ} 37^{\prime}$ \\
\hline & 0.978 & 15.54 & 93 & & & & & & & \\
\hline $694 \pm 14$ & 1.03 & 15.09 & 98 & 0.0492 & Istopes & Hornblende & do. & do. & $19^{\circ} 53^{\prime}$ & $41^{\circ} 37^{\prime}$ \\
\hline & 1.01 & 14.88 & 97 & & & $\begin{array}{l}\text { with } 20 \% \\
\text { impuri- } \\
\text { ties }\end{array}$ & & & & \\
\hline 607士12 & 0.88 & 10.88 & 96 & 0.0419 & do. & Hornblende & Gneiss & do. & $19^{\circ} 52^{\prime}$ & $41^{\circ} 43^{\prime}$ \\
\hline & 0.88 & 11.15 & 97 & & & & & & & \\
\hline $194-676 \pm 28$ & 0.408 & 5.830 & 84 & 0.0476 & Geochron & Plagioclase & Quartz & do. & $19^{\circ} 35^{\prime}$ & $41^{\circ} 53^{\prime}$ \\
\hline & 0.10 & 1.148 & 60 & & & rock & & & & \\
\hline $213 \mathrm{~b}-516 \pm 10$ & 0.084 & 0.8735 & 77 & 0.0347 & do. & do. & do. & do. & $19^{\circ} 09^{\prime}$ & $43^{\circ} 48^{\prime}$ \\
\hline & 0.083 & 0.8548 & 77 & & & & & & & \\
\hline $219 a-656 \pm 14$ & 0.090 & 1.319 & 87 & 0.0460 & do. & do. & Norite & do. & $18^{\circ} 45^{\prime}$ & $42^{\circ} 53^{\prime}$ \\
\hline & 0.102 & 1.318 & 79 & & & & & & & \\
\hline $219 b-484 \pm 10$ & 0.15 & 1.443 & 86 & 0.0323 & do. & do. & do. & do. & $18^{\circ} 45^{\prime}$ & $42^{\circ} 53^{\prime}$ \\
\hline & 0.15 & 1.448 & 83 & & & & & & & \\
\hline $221 \mathrm{a}-494 \pm 12$ & 0.073 & 0.7258 & 88 & 0.0330 & do. & do. & do. & do. & $18^{\circ} 56^{\prime}$ & $43^{\circ} 02^{\prime}$ \\
\hline & 0.070 & 0.6820 & 57 & & & & & & & \\
\hline $221 \mathrm{~b}-422 \pm 8$ & 0.12 & 0.9806 & 92 & 0.0276 & do. & do. & do. & do. & $18^{\circ} 56^{\prime}$ & $43^{\circ} 02^{\prime}$ \\
\hline & 0.12 & 0.9967 & 92 & & & & & & & \\
\hline $225 a-\cdots+655 \pm 12$ & 7.14 & 95.38 & 99 & 0.0458 & do. & Biotite & Diorite & do. & $18^{\circ} 13^{\prime}$ & $42^{\circ} 32^{\prime}$ \\
\hline & 6.98 & 97.80 & 98 & & & & & & & \\
\hline $615 \pm 12$ & 0.98 & 12.73 & 91 & 0.0425 & do. & Hornblende & do. & do. & $18^{\circ} 13^{\prime}$ & $42^{\circ} 32^{\prime}$ \\
\hline & 1.02 & 12.67 & 92 & & & & & & & \\
\hline $225 b-686 \pm 14$ & 4.86 & 68.34 & 98 & 0.0485 & do. & Biotite & do. & do. & $18^{\circ} 13^{\prime}$ & $43^{\circ} 32^{\prime}$ \\
\hline & 4.70 & 70.03 & 98 & & & $\begin{array}{l}\text { with } 20 \% \\
\text { impuri- } \\
\text { ties }\end{array}$ & & & & \\
\hline $228 \mathrm{a}-649 \pm 23$ & 6.72 & 90.83 & 97 & 0.0453 & USGS & Biotite & Gabbro & do. & $18^{\circ} 24^{\prime}$ & $42^{\circ} 42^{\prime}$ \\
\hline & 6.70 & & & & & & & & & \\
\hline $228 \mathrm{~b}-615 \pm 12$ & 8.50 & 106.9 & 93 & 0.0426 & Isotopes & Muscovite & Pegmatite & do. & $18^{\circ} 24^{\prime}$ & $42^{\circ} 42^{\prime}$ \\
\hline & 8.49 & 109.2 & 94 & & & & & & & \\
\hline $229 a--588 \pm 17$ & 1.970 & 24.17 & 95 & 0.0404 & Geochron & Whole & (Drill core) & do. & $18^{\circ} 08^{\prime}$ & $44^{\circ} 07^{\prime}$ \\
\hline & 2.008 & 23.82 & 95 & & & rock & & & & \\
\hline
\end{tabular}


TABLE 8.-K-Ar ages of Precambrian crystalline rocks of Saudi Arabia-Continued

\begin{tabular}{|c|c|c|c|c|c|c|c|c|c|c|}
\hline \multirow{2}{*}{$\begin{array}{c}\text { Sumple } \\
\text { (location } \\
\text { given on pl. 1) } \\
\end{array}$} & \multirow{2}{*}{$\begin{array}{c}\mathrm{K} \\
\text { (percent) }\end{array}$} & \multirow{2}{*}{$\begin{array}{c}{ }^{40} \mathrm{Ar} \times 10^{10} \\
(\mathrm{moles} / \mathrm{g})\end{array}$} & \multirow{2}{*}{ (percent) } & \multirow{2}{*}{${ }^{40} \mathrm{Ar} /{ }^{40} \mathrm{~K}$} & \multirow{2}{*}{ Analyzed by } & \multirow{2}{*}{$\begin{array}{c}\text { Material ana- } \\
\text { lyzed }\end{array}$} & \multirow{2}{*}{ Rock } & \multirow{2}{*}{ Collected by } & \multicolumn{2}{|c|}{ Location } \\
\hline & & & & & & & & & Lat $\mathrm{N}$. & Long E. \\
\hline \multirow[t]{2}{*}{$229 b-577 \pm 16$} & 2.17 & 25.40 & 94 & 0.0395 & do. & do. & Andesite & do. & $18^{\circ} 08^{\prime}$ & $44^{\circ} 07^{\prime}$ \\
\hline & 2.13 & 25.27 & 90 & & & & $\begin{array}{l}\text { (drill } \\
\text { core) }\end{array}$ & & & \\
\hline \multirow[t]{2}{*}{$229 \mathrm{c}-572 \pm 15$} & 2.15 & 24.37 & 94 & 0.0391 & do. & do. & do. & do. & $18^{\circ} 08^{\prime}$ & $44^{\circ} 07^{\prime}$ \\
\hline & 2.07 & 24.92 & 94 & & & & & & & \\
\hline $229 d-571 \pm 15$ & 2.61 & 30.80 & 94 & 0.0390 & do. & do. & do. & do. & $18^{\circ} 08^{\prime}$ & $44^{\circ} 07^{\prime}$ \\
\hline \multirow[t]{2}{*}{$229 \mathrm{e} \ldots \ldots-\ldots 5 \pm 18$} & 2.179 & 26.87 & 76 & 0.0409 & do. & do. & do. & do. & $18^{\circ} 08^{\prime}$ & $44^{\circ} 07^{\prime}$ \\
\hline & 2.194 & 26.57 & 83 & & & & & & & \\
\hline \multirow[t]{2}{*}{ 230 } & 3.282 & 36.18 & 90 & 0.0369 & do. & do. & Andesite & Overstreet & $18^{\circ} 24^{\prime}$ & $44^{\circ} 11^{\prime}$ \\
\hline & 3.192 & 35.18 & 85 & & & & & & & \\
\hline \multirow[t]{2}{*}{$231 a-\ldots-\ldots 19$} & 3.608 & 36.65 & 93 & 0.0350 & do. & do. & do. & do. & $18^{\circ} 22^{\prime}$ & $44^{\circ} 14^{\prime}$ \\
\hline & 3.503 & 37.56 & 93 & & & & & & & \\
\hline 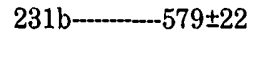 & 0.485 & 5.454 & 68 & 0.0396 & do. & do. & do. & do. & $18^{\circ} 22^{\prime}$ & $44^{\circ} 14^{\prime}$ \\
\hline $232-575 \pm 15$ & 2.204 & 26.52 & 87 & & & & & & & \\
\hline \multirow[t]{2}{*}{234} & 1.087 & 13.09 & 83 & 0.0414 & do. & do. & Diorite & do. & $18^{\circ} 06^{\prime}$ & $44^{\circ} 15^{\prime}$ \\
\hline & 1.015 & 12.86 & 83 & & & & & & & \\
\hline \multirow[t]{2}{*}{$236-565 \pm 16$} & 3.624 & 42.48 & 96 & 0.0385 & do. & do. & Felsite & do. & $18^{\circ} 07^{\prime}$ & $44^{\circ} 15^{\prime}$ \\
\hline & 3.701 & 41.73 & 95 & & & & dike & & & \\
\hline
\end{tabular}

Constants, ${ }^{40} \mathrm{~K} \lambda \epsilon=0.581 \times 10^{-10} / \mathrm{yr} . \lambda \beta=4.962 \times 10^{-10} / \mathrm{yr}$. Atomic abundance, ${ }^{40} \mathrm{~K}=1.167 \times 10^{-4}$.

zircons were palingenetically new-the Ablah and culminant orogenies at 797-763 m.y. and 660-666 m.y. (Cooper and others, 1979). Also, in recent years the French (BRGM) have made numerous analyses (more than 360 samples) in their laboratory at Orleans (Baubron and others, 1976). Likewise, analyses have been reported from the University of Leeds (Kroner and others, 1979) and at the Department of Geology, Nottingham, and the Institute of Geological Sciences, London (Duyverman and others, 1982).

\section{CRUSTAL HISTORY OF THE PRECAMBRIAN SHIELD}

\section{GENERAL STATEMENT}

The evolutionary history of the Precambrian Arabian Shield must be evaluated across a structural width of more than $1,000 \mathrm{~km}$, or a total of more than $1,500 \mathrm{~km}$ if the Nubian Shield of Egypt and Sudan is included. Across these widths, the crustal rocks of the Arabian Shield and the Nubian Shield are grossly similar in petrology, chemistry, structure, and age. Across both shields, the crustal rocks were made and cratonized in about 450 m.y., from about 1,000 m.y. to about 550 m.y.

No continental crust older than about $1 \mathrm{Ga}$ has been reported in the Arabian Shield despite a concerted effort to find "old" continental crustal rocks. In the easternmost part of the shield, a 2,100-m.y.-old continental crust may be nearby, as recent lead-isotope studies indicate that some of the youthful Precambrian granitoid rocks there contain old leads (Stacey and others, 1981). It is not resolved whether the crustal block of the Ar Rayn region east of the Al Amar-Idsas fault (region E, fig. 16) is actually part of this old crust or whether the old crust is still farther east beneath the Phanerozoic sedimentary rocks. ${ }^{5}$

The shield has been subdivided (fig. 16) on the basis of the early crustal stratigraphic groups, the BaishBahah, Jiddah, and Halaban. The actual rock boundaries between these groups are not easily mapped because the overall lithologies of each group are similar. The boundaries in figure 16 are drawn largely along major north-trending structures that bound early crustal volcanic and plutonic rocks of similar age. The north-trending stratigraphic belts are strongly offset by the Najd faults of youngest Precambrian or oldest Cambrian age. The volcanic and plutonic rocks of the late cratonization history are superimposed on the early crustal rocks and, hence, do not affect the subdivisions in figure 16.

The Wādī Bidah region (region B, fig. 16) contains the type reference area of the Baish-Bahah Groups (basaltic assemblage). In the Biljurshī' region (region J, fig. 16), early crustal rocks of the Jiddah Group (andesitic assemblage) are described and dated at between 900 and about 850 m.y. This region also

\footnotetext{
${ }^{5}$ Since this was written, evidence for original emplacement in early Proterozoic time has been established at about 1,630 m.y. ago, with subsequent metamorphism or remobilization at about $660 \mathrm{Ma}$ (see Stacey and Hedge, 1984). We consider the anorthosite at Jabal Mahail east of Jabal Khidā' to be original crust surviving later orogeny.
} 
contains the type locality of a younger group, the Ablah Group, that is dated at between 800 ? and about 750 m.y. The type area for the Halaban Group is centered around Ḩalabān in region TN (fig. 16). Correlatives of the Halaban rocks are well described as the Hulayfah Group in region $\mathrm{HN}$ in the Nuqrah quadrangle (Delfour, 1977) and as the Halaban Group in region TC in the Bi'r Juqjuq quadrangle. The Tathlith regions, TN, $\mathrm{TC}$, and TS, and the northeast region, NE, are largely underlain by crust of Halaban age about 800 to 725 m.y. old, and their western boundary is the long serpentinite fault zone of Hulayfah-Ad Dafinah-Nabitah-Hamdah. Regions HS, HC, and HN form a western belt that at least in large part, perhaps entirely, consists of Halaban crustal rocks.

The early crustal rocks in the western and northern regions have not been named consistently in the literature or well dated. The initial type area of the Jiddah Group is in the vicinity of the townsite of Jiddah, and the available dates suggest that regions WS, WC. and perhaps WN may be underlain by crustal rocks of Jiddah age. A Jiddah age is not denied by the available dates from the Eastern Desert of Egypt (Hashad, 1980), which was an integral part of the Arabian Shield. Region NN is highly faulted by the convergence of the Najd fault system, and, for the time being, the early crust of this northern Najd fault region (NN) and of the northern region $(\mathrm{N})$ is also considered Jiddah age. Region $\mathrm{E}$, east of the Al Amar-Idsas fault, has been most commonly mapped as consisting of Halaban rocks, but it may be older, as suggested earlier in this chapter.

The primary crustal rocks of the shield, that is, the first formed rocks or initial crustal rock at any given locality in the shield, consist of metavolcanic and metasedimentary rocks of a basaltic assemblage and an andesitic assemblage and of plutonic rocks of a dioritic suite; in addition, subordinate tectonic remnants of ophiolitic rocks are scattered in linear belts throughout much of the shield. These rock groupings are based on petrology, petrography, and chemistry (Greenwood and others, 1976; Delfour, 1979b; Dodge and others, 1979) and on $\mathrm{Rb}-\mathrm{Sr}$ and $\mathrm{Pb}$ isotopic studies (Baubron and others, 1976; Fleck and others, 1980; Stacey and others, 1981). Most of the rocks are of the andesitic assemblage and dioritic suite that formed in intraoceanic island arcs as volcanic-magmatic arcs. The basaltic assemblage of the Baish-Bahah Group as sampled in the Wādī Bidah area has a distinctly more tholeiitic composition than the andesitic-assemblage rocks and may have an oceanic crust affinity. More likely the Baish-Bahah rocks are simply an early and basal, more tholeiitic part of the Jiddah andesitic assemblage and of Jiddah age because the basaltic assemblage rocks across regions $J$ and $B$ (fig. 16) are interspersed with andesitic-assemblage rock of the Jiddah Group and dioritic-suite rock that consistently date between 900 and $800 \mathrm{~m} . \mathrm{y}$.

The problem of the age and origin of the Baish-Bahah rock clearly points out the fact that the study of the Arabian Shield has barely begun to decipher individual volcanic-magmatic arcs as well as other specific constructional blocks that form the composite early crust as it is known today. Each of the early stratigraphic groups is broadly bounded by a meaningful age range, but each likely consists of more than a single constructional block within that age range.

The following early and late crustal history of the shield outlines one possible sequence of events that in each event might be expected to be much more complex than described. This crustal history has been summarized in a review paper by Schmidt and Brown (1982).

\section{EARLY CRUSTAL HISTORY}

The early, primary crust of the Saudi Arabian Shield is a composite of several intraoceanic island arcs and subordinate remnants of oceanic crust (ophiolite). These primary constructional blocks have been combined at different times in different places during several subduction and collisional events from after 900 to about 650 m.y. Especially the older constructional blocks have been tectonized, and the combined crust has been tectonically and magmatically thickened during these collisional events. Throughout most parts of the shield, compressional structures are consistently north-trending, and it is presumed that the original island arcs trended generally northward and that subduction may have been both westward and eastward under different arcs at different times. Deep erosion during the late crustal history, discussed below, means that, for the most part, we see today the deep magmatic parts of the volcanic-magmatic arcs. Most of the metavolcanic and metasedimentary rocks exposed adjacent to these deeply eroded magmatic arcs were originally deposited marginally to the volcanic-magmatic arcs. Therefore, the early layered rocks exposed throughout the shield contain a large proportion of pyroclastic and immature clastic metasedimentary rocks derived from the islandarc volcanic rocks. The original andesitic volcanic piles in the upper part of the magmatic arcs are less commonly preserved, except where they have been thrust into adjacent basins prior to the deep erosion of the volcanic arc.

The early, primary crust consists predominantly of an andesitic assemblage of metavolcanic and metasedimentary rocks and comagmatic plutonic equivalents of the dioritic suite. These primary crustal rocks have been well dated in the southern part of the shield at 
between about 900 and 700 m.y. (Fleck and others, 1980). In a broad western belt about $250 \mathrm{~km}$ wide between Al Lìth and Qal'at Bīshah (regions B and J, fig. 16), rocks of the dioritic suite as well as those of the andesitic assemblage are between 900 and $800 \mathrm{Ma}$ and are mapped mostly as Jiddah Group as characterized in the Biljurshì' quadrangle (Greenwood, 1975b). Rocks of similar composition are dated at between 800 and 700 m.y. in a broad eastern belt, about $200 \mathrm{~km}$ wide, between Qal'at Bishah and the eastern edge of the exposed shield (regions HS and TS, fig 16) and are mapped mostly as Halaban rocks as characterized in the Bi'r Juqjuq quadrangle (Hadley, 1976). Both of these belts, one of early primary crust of Jiddah age and the other of late primary crust of Halaban age, are tectonically complex, and undoubtedly each is composed of more than one constructional block of intraoceanic, island-arc materials.

The Jiddah-age crust in regions $\mathrm{B}$ and $\mathrm{J}$ can be further subdivided by age along the $42 \mathrm{~d}$ meridian. To the east of the $42 \mathrm{~d}$ meridian, dioritic-suite rocks of the An Nimas batholith (fig. 30) are dated at between 850 and 800 m.y. The An Nimas batholithic complex (Anderson, 1977) consists of plutons of diorite, quartz diorite, tonalite, and trondhjemite as well as subordinate gabbro and small screens of metavolcanic rocks. The complex is about $70 \mathrm{~km}$ wide and extends $150 \mathrm{~km}$ from lat $19^{\circ} 00^{\prime}$ northward to $20^{\circ} 30^{\prime}$ to where it is covered by the Tertiary flood basalt Harrat al Buqūm. The An Nimas batholithic complex is probably the deeply eroded core of a volcanic-magmatic arc and is just one integral constructional block within the Jiddah-age crust. At one locality within the batholithic complex, meta-andesitic and metadacitic rocks are dated at $912 \pm 76$ m.y. (initial ${ }^{87} \mathrm{Sr} /{ }^{86} \mathrm{Sr}=0.7024$, Fleck and others, 1980), which is about 60 to $100 \mathrm{~m} . \mathrm{y}$. older than the age of the comparable quartz dioritic and trondhjemitic rocks; the 912 m.y. date may be too old and must be substantiated by further study.

To the west of the $42 \mathrm{~d}$ meridian for about $200 \mathrm{~km}$, the plutonic rocks of the dioritic suite are dated consistently at between 900 and 850 m.y. and are interspersed with andesitic-assemblage rocks mapped as Jiddah Group as represented in the Biljursh $\mathbf{1}$ quadrangle (Greenwood, 1975b) and with basaltic-assemblage rocks mapped as Baish-Bahah Group as represented in the Jabal Ibrāhīm quadrangle (Greenwood, 1976c). The basaltic-assemblage rocks of the Baish-Bahah Group are possibly a more tholeiitic, basal, and immature part of the predominantly calc-alkalic andesitic assemblage of the Jiddah Group rather than remnants of oceanic crust, as has been suggested in the literature. The rocks are folded, faulted, and probably thrust faulted as well. On the west side near Al Lith, metabasalts mapped as Baish Group have been dated at $1,165 \pm 110$ m.y. (initial ${ }^{87} \mathrm{Sr} /{ }^{86} \mathrm{Sr}=0.7029$, Hadley and Fleck, 1980a), yet they are intruded by quartz dioritic rocks dated at $895 \pm 173$ m.y. (initial ${ }^{87} \mathrm{Sr} /{ }^{86} \mathrm{Sr}=0.7025$ ). Again, as in the An Nimas batholithic complex, the metavolcanic rocks seem to date too old for the presumably comparable plutonic rocks. An alternative, less likely, explanation is that the volcanic rocks of an older constructional block are tectonically mixed with the Jiddah-age crustal rocks.

The andesitic-assemblage rocks in the vicinity of Jiddah townsite, the original type area for the Jiddah Greenstone, are possibly Jiddah age (as defined in the Biljurshì quadrangle) on the basis of some dated plutonic rocks (table 6). Fifty to $100 \mathrm{~km}$ north of Jiddah and east of Rābigh (region WS, fig. 16), andesiticassemblage rocks of the Samran Group of Skiba (1980) are calc-alkalic and trend toward a tholeiitic composition (fig. 26A). These rocks may be correlated tentatively with those at Jiddah, but ages have not been determined. Still farther northwestward, along the western edge of the shield, ages of early crustal rocks also have not been determined. Support for early crustal rocks of Jiddah age can be extrapolated from dates on the dioritic-suite rocks from the southern part of the Eastern Desert of Egypt where six Rb-Sr wholerock ages range from 987 to 830 m.y. (Hashad, 1980, p. 41). The areal distribution of these rocks in Egypt extrapolates to Saudi Arabia from south of Yanbu' al Bahrr to north of Al Wajh.

The Halaban-age crust in regions HS and TS (fig. 16) are subdivided structurally by the serpentinite belt of Hulayfah-Ad Dafinah-Nabitah-Hamdah into at least two constructional blocks. The belt of Halaban crust west of the serpentinite belt, region HS, contains andesitic-assemblage rocks that are dated at between $786 \pm 96$ m.y. (initial ${ }^{87} \mathrm{Sr} /{ }^{86} \mathrm{Sr}=0.7025$ ) and $746 \pm 16$ m.y. (initial ${ }^{87} \mathrm{Sr} /{ }^{86} \mathrm{Sr}=0.7021$ ) and quartz diorite to tonalite plutonic rocks of the dioritic suite that are dated at $723 \pm 107$ (initial ${ }^{87} \mathrm{Sr} /{ }^{86} \mathrm{Sr}=0.7025$ ) and $724 \pm 93$ m.y. (initial ${ }^{87} \mathrm{Sr} /{ }^{86} \mathrm{Sr}=0.7027$ ) (Fleck and others, 1980). The belt of Halaban east of the serpentinite belt (region TS) contains Halaban andesitic-assemblage and dioriticsuite rocks that have not been satisfactorily dated but suggest ages of between 800 and 700 m.y. These two belts of Halaban-age crust can be extended, using the serpentinite belt, northward across the shield through the type area of the Hulayfah Group (Halaban equivalent; Delfour, 1977) and through the Halaban type area in the vicinity of Halabān Ridge.

In summary, the early, primary crust of the shield is a combination of several constructional blocks, of which each is an intraoceanic island arc consisting of an andesitic assemblage of metavolcanic rocks and a 


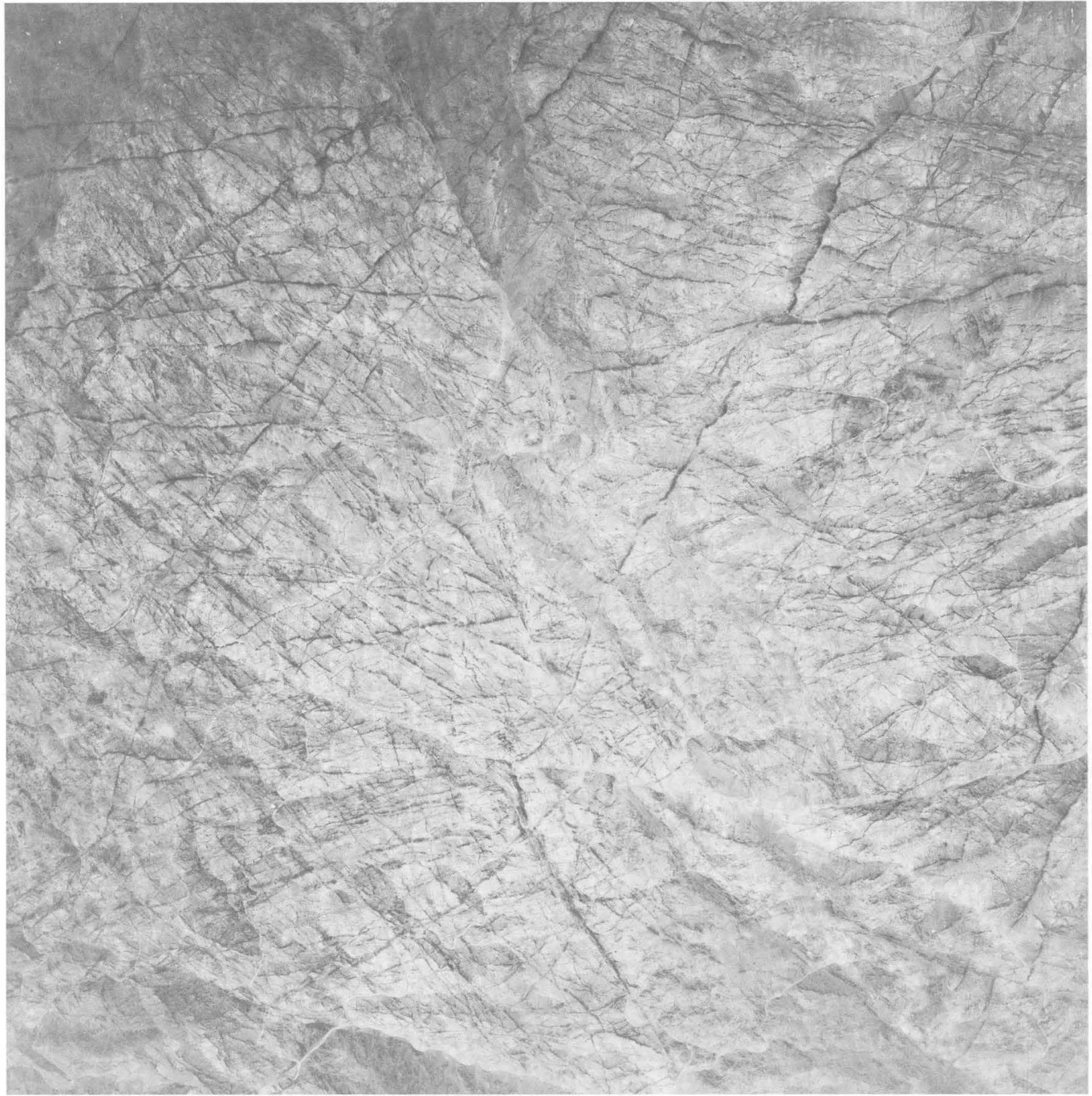

FIGURE 30.-Vertical aerial photograph of the northern portion of the An Nimas batholith, representative parts of which are dated at $816 \pm 4$ m.y. (Cooper and others, 1979) and $837 \pm 50$ m.y. (Fleck and others, 1980). The syntectonic batholith ranges in composition from diorite to tonalite, with much interlayered quartz diorite.
The darker areas represent diorite or mixed rocks. The terrain is crisscrossed with metabasalt and meta-andesite dikes following lineations-faults and joints from at least two and possibly three orogenic episodes. At least some conjugate dikes were formed during the Najd orogeny. (Geology after Green, 1983.) dioritic suite of plutonic rocks. For the convenience of discussion, these rocks are lumped into a primary crust of Jiddah age (900 to 800 m.y.) and a primary crust of Halaban age (800 to 700 m.y.).

\section{EARLY COLLISIONAL OROGENY}

In the southern part of the shield, several widely spaced gneiss domes of tonalitic-trondhjemitic ortho- 


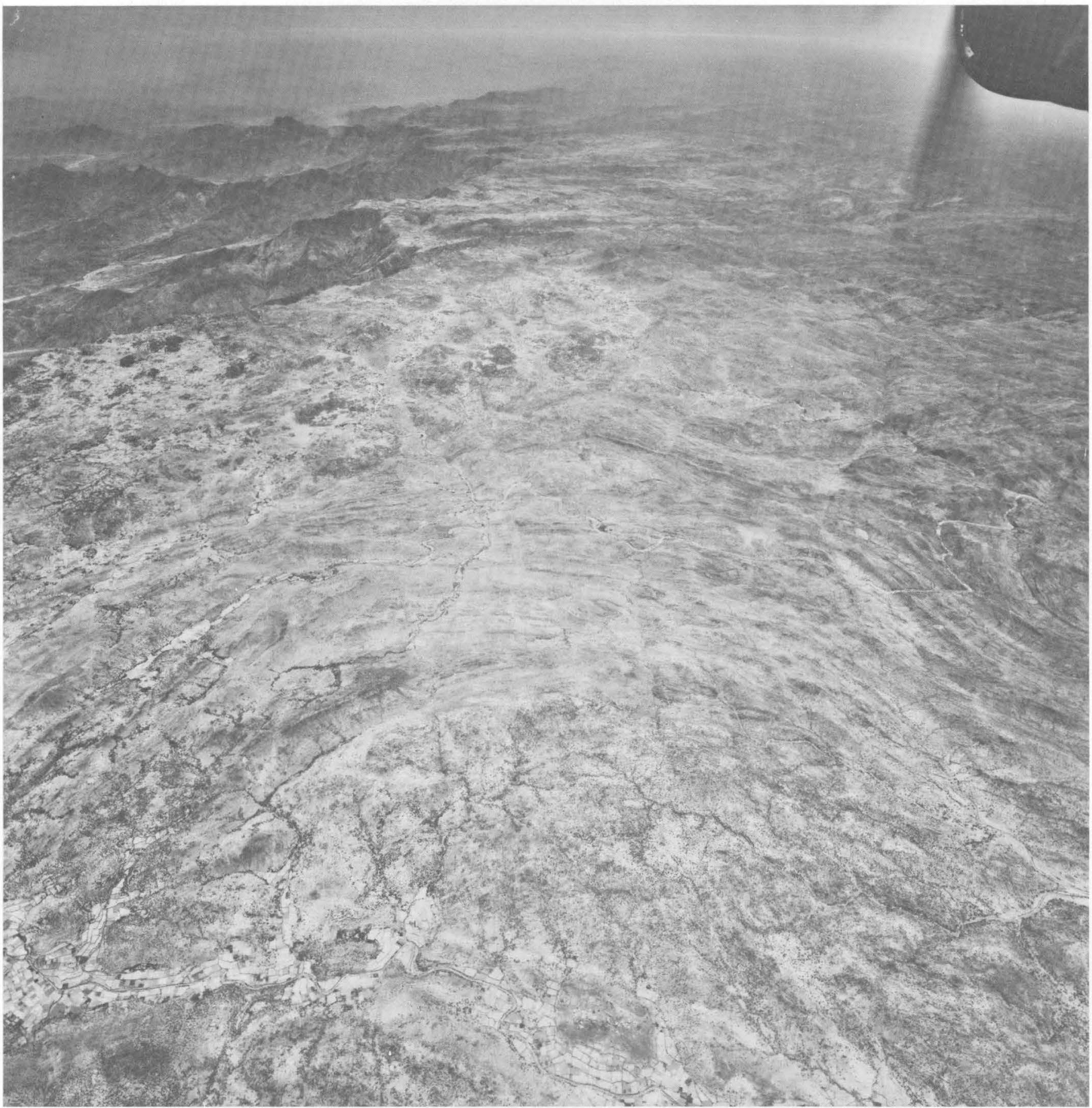

FIGURE 31.-Oblique aerial view to the northwest of orthogneiss dome containing enfolded amygdaloidal metabasalt flows of the Baish Group. K-Ar age of hornblende in the gneiss yielded a KAr age estimate of $759 \pm 31$ m.y. (table 8 , sample 191b), which compares to a total fusion, ${ }^{40} \mathrm{Ar} /{ }^{39} \mathrm{Ar}$ age of $782 \pm 35$ m.y. (Fleck and others, 1976.) The town of Biljurshī' rests on the northwestern flank of the arch, which includes quartz diorite (K-Ar age of
$759 \pm 20$ m.y.; table 8, sample 190) and overlooks the 'Asīr escarpment. The Biljursh' intrusive yielded $\mathrm{Rb} / \mathrm{Sr}$ isochron ages of $848 \pm 282$ m.y. and $890 \pm 87$ m.y. (Fleck and others, 1980, p. 19). Thus the dome represents rejuvenation of quartz diorite estimated to have first solidified at $890 \pm 67 \mathrm{Ma}$. (Geology after Greenwood, 1975b.) gneisses are indicative of crustal heating, tectonism, metamorphism, and the gravitational rise of less dense plutonic parts of the primary crust of Jiddah age (fig.
31). Syntectonic intrusion of granodioritic batholiths accompanied the rise of orthogneisses and represents the first large volumes of potassic plutonic rocks in the 


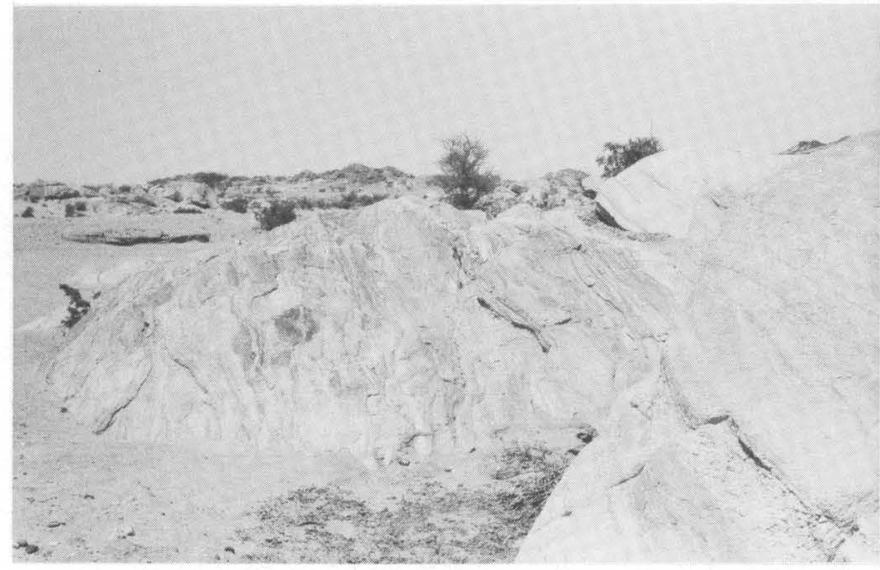

$\boldsymbol{A}$

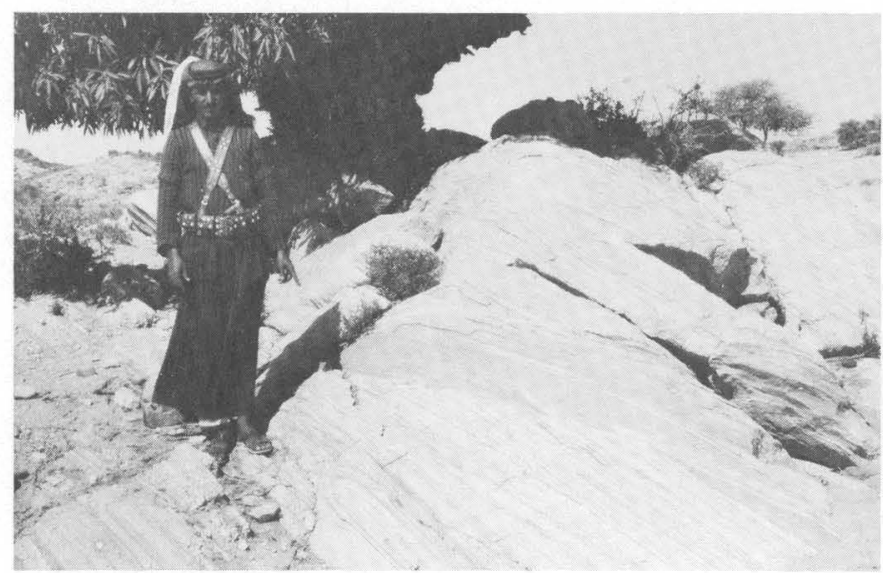

B

early crust at any locality. The best documented example is the Baqarah gneiss dome in the southern end of the An Nimas batholith, where tonalitic and trondhjemitic orthogneisses are accompanied by synkinematic granodioritic gneissic batholithic rocks that are dated at $763 \pm 53$ m.y. (initial ${ }^{87} \mathrm{Sr} /{ }^{86} \mathrm{Sr}=0.7032$; Fleck and others, 1980) and $763 \pm 4$ m.y. by the U-Pb zircon method (Cooper and others, 1979). A similar gneiss domal complex at the northern end of the An Nimas batholith gives less reliable K-Ar dates of between 740 and 711 m.y. (sample 171, table 6; sample 173, table 8). Interpretation suggests that while the early, primary, Halabanage crust was forming somewhere to the east, the older Jiddah-age crust was subjected to combining and consolidation by early collisional tectonism involving tectonic and magmatic crustal thickening, gneiss doming, and granodiorite intrusion.

The orogenic mountains were eroded and resulted in deposition of molassic sedimentary rocks that along the west side of the An Nimas batholith are mapped as the metasedimentary rocks of the Ablah Group. These voluminous graywacke deposits of the Ablah Group are commonly quartz bearing and even contain some

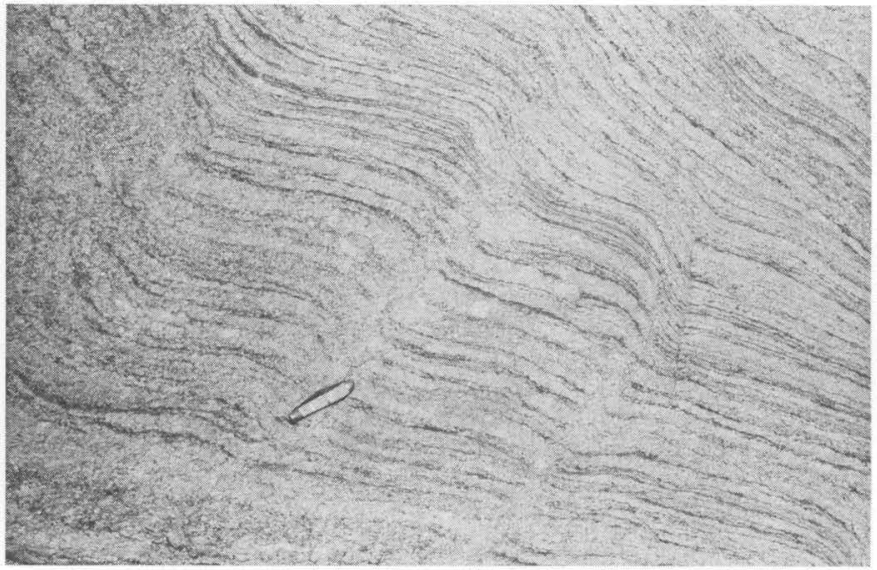

C

FigurE 32.-Schistose gneiss at Wādī Dhuqiyah $65 \mathrm{~km}$ southeast of Aţ Tā'if. The lineation dips east. $A$, Biotite albite xenolithic fragment above pegmatite stringers; $B$, Detailed drag folds in the gneissose limestone, N. $20^{\circ}$ E. sinistral movement; $C$, Gneiss considered to have been formed by the doming at the end of the Ablah cycle (near \pm 760 m.y. ago), the crenulation later during Najd time (about \pm 570 m.y. ago).

quartz sandstones, indicating derivation from large volumes of quartz diorite, tonalite, and trondhjemite in the Jiddah crust. These quartzose sedimentary rocks, together with thick marble deposits, indicate deposition from a deeply eroded crust and in a semistable basin, and this is indicative of a nearly continental environment. However, the generally low- $\mathrm{K}_{2} \mathrm{O}$, calc-alkalic volcanism associated with the Ablah rocks indicates that the continental crust had not uniformly established full thickness.

Other gneiss domes and molassic metasedimentary rocks superposed elsewhere on Jiddah-age crust in the western shield may be related to the same early collisional orogeny and may be correlatives of the Baqarah orthogneiss and Ablah Group rocks or may represent other early collisional orogenies of slightly different age and place. Another example is the metasedimentary rocks mapped as Ablah Group west of a large gneiss domal complex in the adjoining quadrangle of Jabal 'Afaf (Hadley and Fleck, 1980b) and Jabal Ibrāhīm (Greenwood 1975c) (fig. 32). The Fatimah Group and abundant adjacent orthogneisses and intrusive granodiorites, dated at the Jiddah airport at $763 \pm 159$ m.y. (initial ${ }^{87} \mathrm{Sr} /{ }^{86} \mathrm{Sr}=0.7026$; Fleck and others, 1980), are of Ablah age and may represent the same or perhaps a different early collisional orogeny.

\section{LATE CRUSTAL HISTORY}

CULMINANT OROGENY AND POSTTECTONIC GRANITES

The late crustal history of the Arabian Shield began sometime after 700 m.y. ago and involved the final 


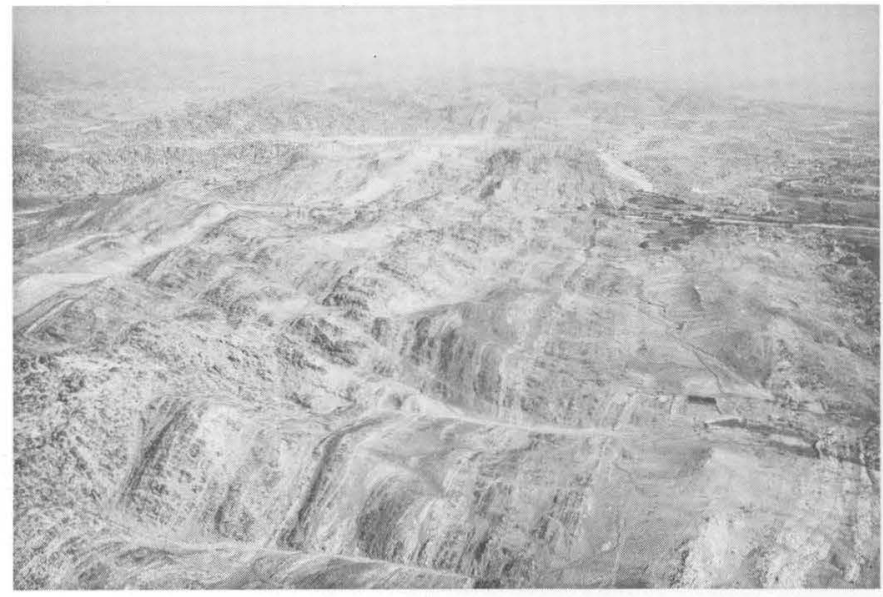

$\boldsymbol{A}$

Figure 33,- A, Aerial view south of the eastern edge of the Khamis Mushayt gneiss dome flanked by paragneiss which in turn has subsequently been intruded by the monzogranite at Tindahah. The Khamis Mushayt gneiss dome of $664 \pm 9$ m.y. (Rb-Sr age) has metamorphosed volcaniclastic sediment of Halaban age (785-665 m.y.) or possibly of Jiddah age (850-780 m.y.). Subsequent to the doming, the Tindahah monzogranite batholith was posttectonically intruded on the eastern flank (K-Ar cooling age about 563

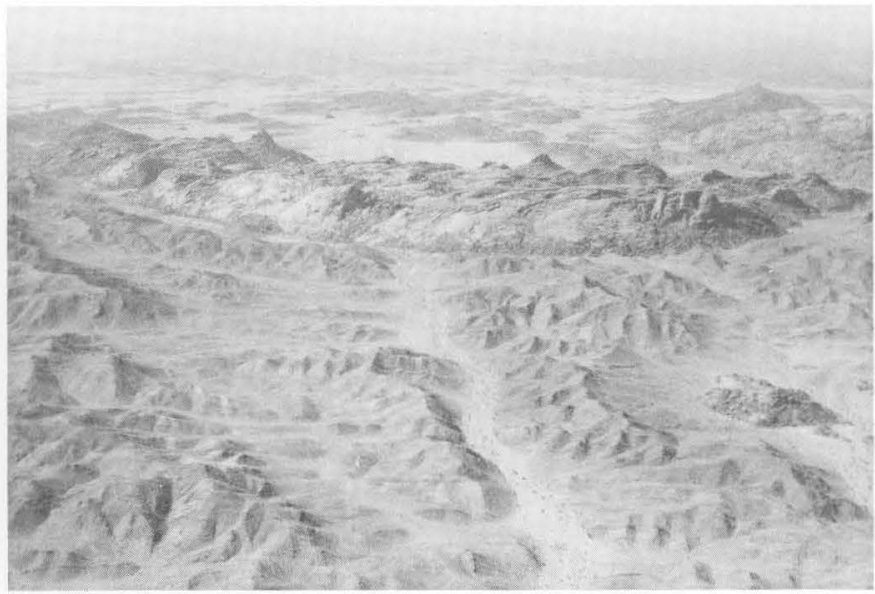

B

m.y.) (Fleck and others, 1976; Fleck and others, 1980). The village of Tindahah is in middle distance on right. $B$. Jabal al Hidab at lat $19^{\circ} 40^{\prime} \mathrm{N}$., long $42^{\circ} 45^{\prime} \mathrm{E}$. Alkalic red granite forming hills in the middle distance is intruded into paragneiss and amphibolite gneiss dome composed of Halaban rocks in a syntectonic dome formed during culminant orogeny. The alkalic granite is posttectonic, with a biotite K-Ar age of $595 \pm 9$ m.y. (Fleck and others, 1976) combining, consolidation, and cratonization of the shield as a whole. The major orogeny associated with this late crustal history is rightly called the culminant orogeny; it was the ultimate orogeny involved in the cratonization of the entire shield. The late crustal history is characterized by many gneiss domes (gneiss antiforms), granodioritic batholiths, and granite plutons that in general became progressively more potassic with decreasing age (fig. 33). The syntectonic and posttectonic granodioritic and granitic rocks constitute about 50 percent of the eastern part of the shield and diminish in abundance westward across the western shield.

The culminant orogeny probably began slightly before $650 \mathrm{~m} . \mathrm{y}$. and extended to about $620 \mathrm{~m} . \mathrm{y}$. The histograms of rock ages in figure 34 show a distinct secondary mode between 650 and 620 m.y. ago. One late, syntectonic granodiorite batholith associated with the large gneiss dome east of Qal'at Bīshah in the Jabal al Qarah quadrangle (Schmidt, 1981a) gives a highquality Rb-Sr isochron age of $623 \pm 18$ m.y. (initial ${ }^{87} \mathrm{Sr} /$ ${ }^{86} \mathrm{Sr}=0.7033$; Fleck and others, 1980).

During the culminant orogeny, widespread crustal heating caused amphibolite-facies metamorphism in the primary crustal rocks at intermediate crustal depths. Under these conditions, large, low-density bodies of trondhjemite and tonalite became gravitationally unstable adjacent to denser diorites and meta-andesit- ic-assemblage rocks, and the trondhjemites and some tonalites rose as orthogneisses in elongated gneiss domes along north-trending axes. $\mathrm{Rb}$-Sr dating of these massive orthogneisses at amphibolite facies indicates that many of them retained their original plutonic age (Halaban or Jiddah age). Large volumes of granodioritic magma with associated granodioritic migmatite accompanied or shortly followed the rise of the orthogneiss and was emplaced at about the same crustal level as the gneiss domes. The granodioritic magma, however, originated presumably from a crustal source that was deeper than the crustal level from which the trondhjemite orthogneiss rose because the orthogneisses rarely show but the slightest evidence of having been partially melted. The granodiorite magma represents partial melting of deep, lower crustal rocks of Halaban and Jiddah age, because the initial strontium ratios (for example, 0.7033) of the granodiorite would be expected from primitive crustal rocks whose ${ }^{87} \mathrm{Rb}$ had evolved over 100 m.y. ago or at most less than several hundred million years.

The very widespread, culminant orogeny is believed to have been caused by collision between the Halaban crust and an older continental crust at the east edge of-or somewhat farther east of-the exposed shield. Such collisional orogeny resulted in the final combining of the various constructional blocks of the primary crust (of Jiddah and Halaban age) and in the final 


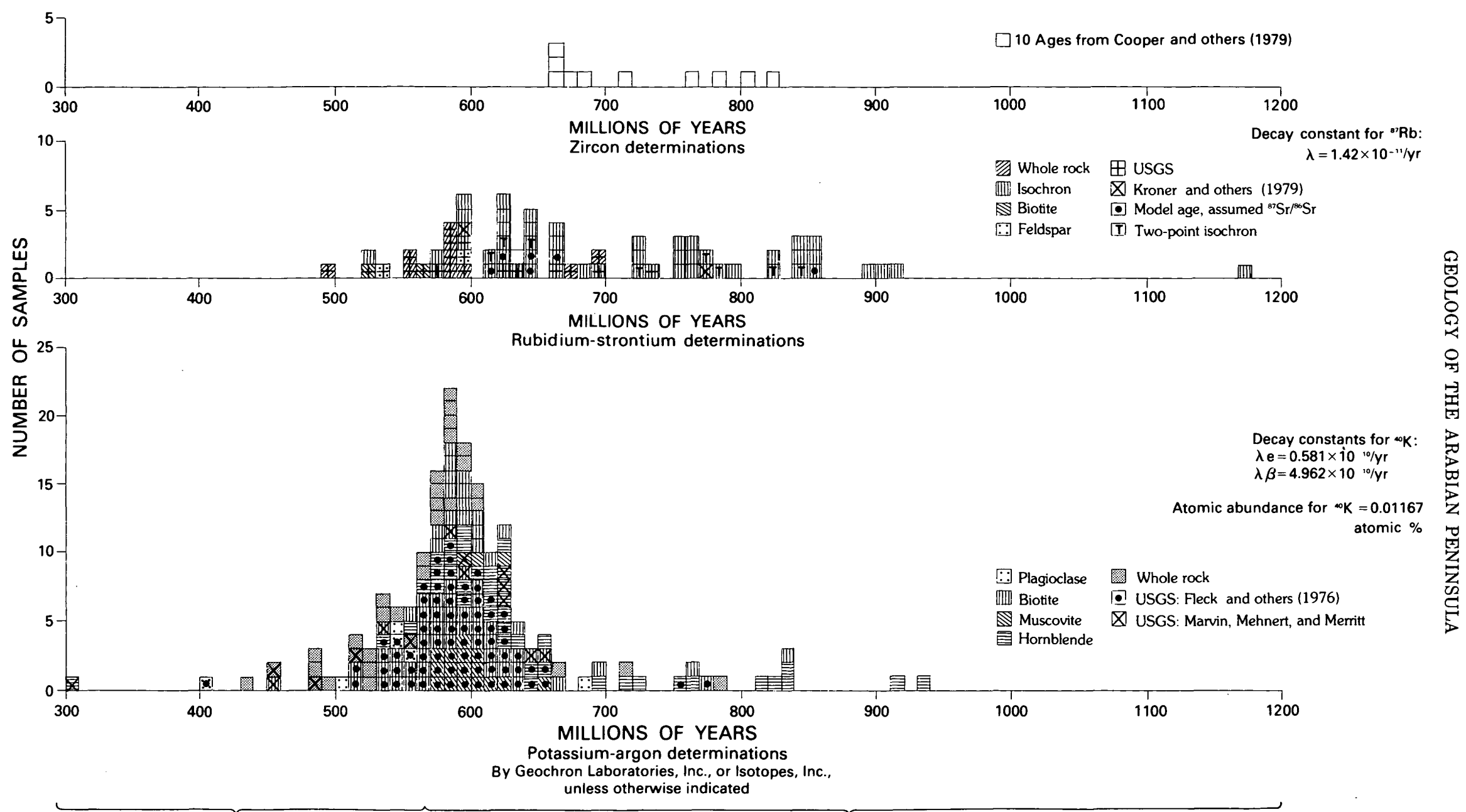

PALEOZOIC

PRECAMBRIAN

Compiled by R. C. Hoeksema, with G. F. Brown, 1980

FIGURE 34.-Histograms showing results of geochronologic analyses of Paleozoic and Precambrian rocks. 
tectonic thickening of the combined crust, such that potassic granitic magma was produced in the lower crust and was intruded at shallow crustal levels in both syntectonic and posttectonic settings.

Production of granitic magma was greatest near the collisional zone in the eastern shield and along other older structures where tectonic thickening was concentrated during the culminant orogeny. Examples are along the Hulayfah-Ad Dafinah-Nabitah-Hamdah serpentinite belt and also, perhaps, in the Egyptian crust where late granitic intrusives are particularly abundant.

The large volume of posttectonic granite was intruded mostly in circular or, more commonly, elliptical plutons oriented northward parallel to the old structural grain. A major mode for Rb-Sr ages of shield rocks suggests maximum emplacement of posttectonic granites at about $600 \mathrm{~m} . \mathrm{y}$. (fig. 34). The potassic magma of about $600 \mathrm{~m}$.y. was intruded commonly as ring-structured plutons near the paleosurface and was voluminously erupted at the surface as rhyolitic rocks of the Shammar Group. Much of this granitic rock is alkalic and has a peralkalic or peraluminous composition. It was most abundantly intruded in the northernmost part of the shield, as at Jabal Aja' and Jabal Salmá, and in a zone about $100 \mathrm{~km}$ wide to the west of the Hulayfah-Ad Dafinah-Nabitah-Hamdah serpentinite belt (Stoeser and Elliott, 1980), where prior tectonic thickening of the crust during the culminant orogeny may have been particularly significant. In contrast, only small volumes of alkalic granite are found dispersed elsewhere across the shield.

The posttectonic granitic magma was bimodal with associated gabbroic magma deep in the crust. Gabbro, commonly fractionated and leucocratic, was intruded at high crustal levels as circular, layered plutons that are conspicuous but not abundant in the eastern half of the shield. Some circular, ring-structured granite plutons (the deep roots of calderas) contain partial layers and pods of gabbro and diabase (Schmidt, 1980), well indicative of bimodal magmatism.

The posttectonic granites seem to be partial melts of primitive lower crustal rocks (Dodge, 1979; Stoeser and Elliott, 1980) that fractionated in time with increased $\mathrm{K}_{2} \mathrm{O}$ content. The bimodal gabbroic magmas, originating in the mantle, likewise fractionated within the crust and were a major contributor of heat to the crust during the late crustal history of the shield. The two magmas did not mix, except subordinately where they mutually produced a few intrusive breccias of mixed rhyolite-diabase and a few intrusive hybrid rocks of metasomatized rhyolite or diabase (Schmidt, 1981b).

The major mountain building of the culminant orogeny resulted in extensive erosion and deposition of molassic deposits of the Murdama Group. The basal Murdama at any given locality contains granitic boulders and cobbles, indicating that everywhere Murdama deposition followed some early granite plutonism. Some granite plutons also intrude the Murdama. The Murdama sedimentary deposits in many places contain a few silicic volcanic units, many of which may have been ignimbrites that flowed considerable distances from late, eruptive granite plutons.

The Murdama molassic deposits filled basins between orogenic mountains, and as the mountains were eroded the Murdama sediments transgressed across them. The thickest and most extensive deposits are in the eastern part of the shield. Extensive but generally thinner deposits are in the western shield. Only remnants of the youngest transgressive basal Murdama appear in the wide zone along the Hulayfah-Ad Dafinah-NabitahHamdah serpentinite belt. This emphasizes that the highest orogenic mountains, along zones of most intensive orogeny, were adjacent to this serpentinite belt and adjacent to the $\mathrm{Al}$ Amar-Idsas fault zone. Gneiss domes, which are the roots of the orogenic mountains, are particularly abundant on either side of the serpentinite belt. Along the margins of the domes, only small, thin, remnant, conglomeratic deposits of Murdama are found. These deposits are commonly younger than the youngest granite plutons in the area; Murdama deposits within the orogenic mountains were scant and late. Thick marble deposits are found in thick sections of the Murdama Group that accumulated in basins adjacent to the orogenic mountains, and are indicative of an already stabilized continental crust.

The quartz-feldspar-biotite and quartz-sericite-chlorite schists of the Abt Formation (and the Ar Ridaniyah Formation) are most probably of Murdama age and constitute a thick, fine-grained, eastern facies of the Murdama Group. The Abt sedimentary rocks were subjected to especially intense late tectonism in the vicinity of the Al Amar-Idsas fault. Alternatively, the Abt Schist may be a continental-marginal sedimentary rock that was associated with the crustal block east of the Al Amar-Idsas fault (region E, fig. 16) and was tectonically emplaced during the collision between this east block and the Halaban crust (fig. 35).

The large batholithic complex in the Ad Dawādimī area is composed largely of granodioritic and subordinate granitic rocks that intrude the Abt deposits (AlShanti, 1976). It has been suggested that the highly tectonized and metamorphosed Abt Schist (and the Ar Ridaniyah Formation, as well) is older than Murdama and the culminant orogeny. However, $100 \mathrm{~km}$ northeast, similar syntectonic granodioritic batholithic rocks intrude Murdama deposits. It seems likely that either the Murdama and Abt metasedimentary rocks of this 


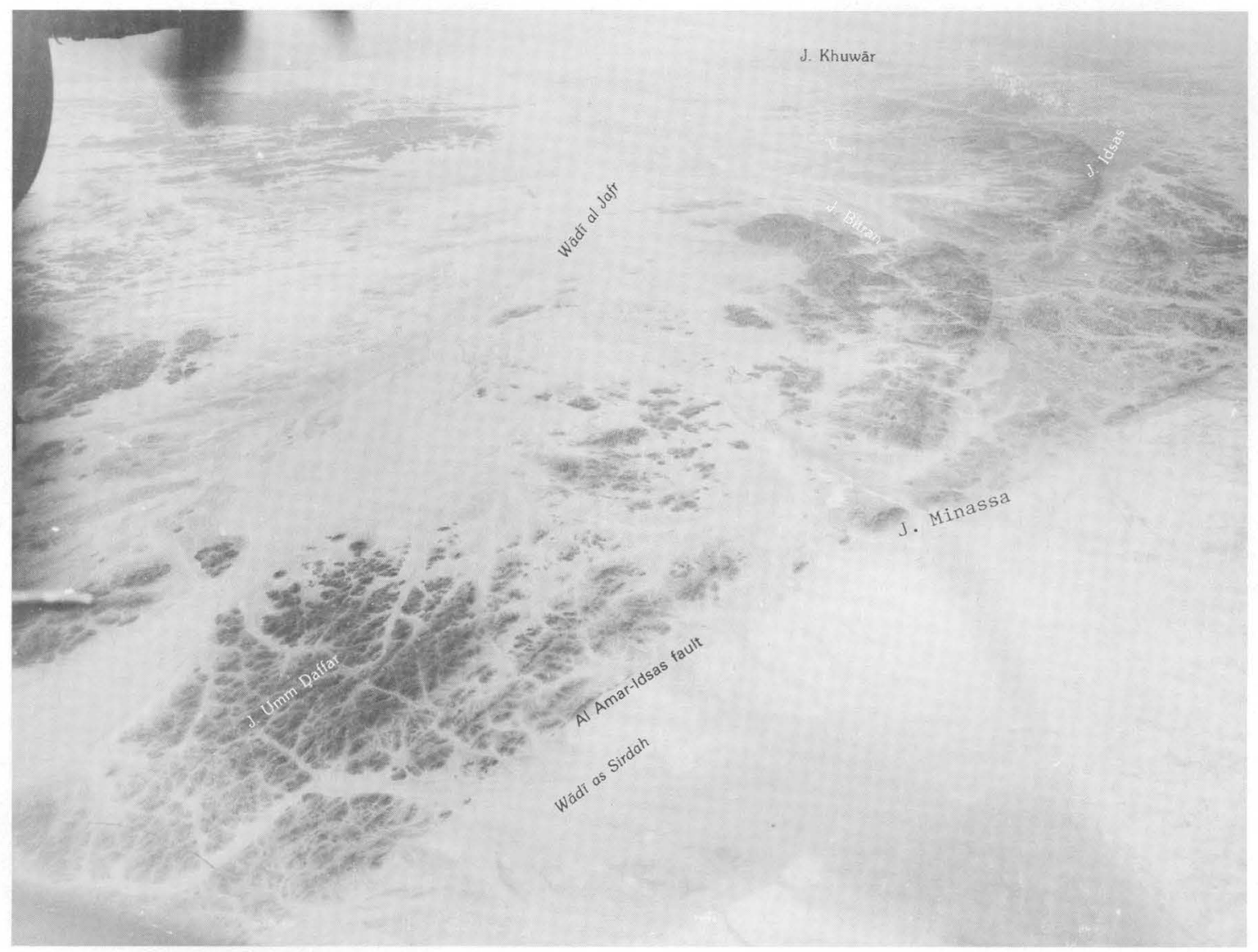

FIGURE 35.-Oblique aerial photograph of view to the southeast from lat $23^{\circ} 05^{\prime} \mathrm{N}$., long $45^{\circ} 05^{\prime} \mathrm{E}$. near the east edge of the shield. The $\mathrm{Al}$ Amar-Idsas fault bounds the east side of the Wādi as Sirdah plain, which is underlain by the Abt Schist ( $\mathrm{pl}$.1). The lobate overthrust fault front is apparent along the southeastern extension. An ophiolite melange marks the fault zone, including a

area are of early culminant-orogeny age or the broad area west of the Al Amar-Idsas fault was orogenically active later, possibly as late as 600 m.y. ago or even just prior to the Najd faulting. If the tectonism of the culminant orogeny did persist in some areas to $600 \mathrm{~m} . \mathrm{y}$. or slightly later, then the large volumes of granodiorite in the Ad Dawādimī area were unusually late in comparison to the rest of the shield, where posttectonic, high- $\mathrm{K}_{2} \mathrm{O}$ granites were being intruded at that time. We cannot determine the time variability of the culminant orogeny because Murdama deposition was not everywhere the same age but was within a timespan of about 50 m.y.

Tectonic remnants of ultramafic rocks and serpentinite, gabbro, sheeted dike complexes, tholeiitic metaba- southern bifurcation south of Jabal Minassa. The outcrops east of the fault zone make up the Ar Rayn granitoid province of figure 16 (Al-Shanti and Gass, 1983) and include voleanic facies in Jabal Umm Daffar in left foreground and west of calc-alkaline to alkalic plutonic rocks.

saltic rocks, and ocean-floor metasedimentary rocks occur in varying combinations in many places in the shield. Many of these have been described as tectonized and deeply eroded ophiolites of either oceanic or backare crustal origin (Bakor and others, 1976; Frisch and Al-Shanti, 1977; Delfour, 1979b; Al-Rehaili and Warden, 1980).

Remnants of ophiolites are to be expected, considering that most or all of the shield is a composite of many primary constructional blocks originally separated by oceanic crust. Further, it is likely that the different dismembered ophiolites in different parts of the shield are of different ages because different constructional blocks were combined at different times and involved different oceanic crusts and (or) back-arc basinal crusts. 
Most of the exposed ophiolites of the shield are small remnants because erosion has been deep and only those remnants that had been structurally placed deep in the crust by tectonism have been preserved. All ophiolites should be associated with specific sutures between different constructional blocks, but the direct relationship is obscured in many places.

The Hulayfah-Ad Dafinah-Nabitah-Hamdah serpentinite belt is a highly tectonized zone that was especially orogenically active during the culminant orogeny. This deeply eroded belt is most likely a suture zone in which in most places mobile serpentine is the only remaining component of the former oceanic crust (Frisch and Al-Shanti, 1977; Schmidt and others, 1979). The much better preserved dismembered ophiolite at Bi'r Umq west of the serpentinite belt in the central shield may be an ophiolite that was obducted (Al-Rehaili and Warden, 1980) before the suture closed during the collisional, culminant orogeny. The broad zone of major faults containing much tectonized serpentinite in the vicinity of-and within - the Al Amar-Idsas fault zone is also very likely a major tectonized suture zone (AlShanti and Mitchell, 1976; Schmidt and others, 1979).

\section{NAJD FAULTING EVENT}

The Najd faulting event or Najd orogeny of Brown (1972) was the final tectonism and final cratonization event of the Arabian Shield. East-west compressional forces acted one last time and the by-then noncompressible, thick, cratonized shield was fractured along a few great shears (fig. 36) rather than by more penetrative internal compression as before. A northwest-trending, left-lateral, transcurrent fault system (Brown and Jackson, 1960; Moore, 1979) prevailed over the conjugate northeast-trending, right-lateral, transcurrent fracturing.

The shield was displaced left laterally more than 250 $\mathrm{km}$ (Brown, 1972), mostly along three major northwesttrending fault zones, each 5 to $10 \mathrm{~km}$ wide. This major, large fault movement on the Najd fault system is in a region $300 \mathrm{~km}$ wide by $1,100 \mathrm{~km}$ long, but the effects of the Najd stresses can be found throughout the shield. At least some, and possibly most, of the northeasttrending structures between Jiddah and Wādi Suwass in the Qal'at as Sawrah quadrangle (regions B, WS, and WC, fig. 16) may be as young as Najd age. Within this large strained region, many older faults were activated and some were rotated toward and into a northwestern direction. These reactivated and rotated older faults give the unwarranted impression that the age of the Najd faulting event began long before 600 m.y., but we cannot agree with this interpretation.

Each of the three major northwest-trending fault zones is in part sinuous, braided, en echelon, and branching, and the whole movement system is convergent to the northwestern part of the shield and into the area of the ancestral Gulf of Suez. The sinuous and branching geometry of the fault zones resulted in a few localized extensional segments consisting of grabens filled with taphrogeosynclinal sedimentary and volcanic rocks of the Jubaylah Group and a few ultracompressional segments in which elongated gneiss antiforms (domes) rose (fig. 37).

The andesitic basalt volcanism of the Jubaylah Group in the taphrograben structures (Delfour, 1970; Hadley, 1974) trends from calc-alkalic to strongly alkalic in composition (figs. 23, 27B), similar to some of the volcanism associated with large continental rifts. For the Najd system, however, these are small, localized continental pseudorifts. The Najd "grabens" formed in a crust that was fully continental and along newly formed, deep-crustal shear structures that tapped magma generated in the mantle.

The gneiss antiforms in the few ultracompressional segments of the Najd fault system are exemplified in the Jabal Yafikh quadrangle (Schmidt, 1981b) where trondhjemitic orthogneiss, $3 \mathrm{~km}$ wide by more than 60 $\mathrm{km}$ long, rose within the fault zone at almandineamphibolite-facies temperatures. The initial plutonic trondhjemite was part of the primary crust of Halaban age (dated $766 \pm 26 \mathrm{~m} . \mathrm{y}$., initial ${ }^{87} \mathrm{Sr} /{ }^{86} \mathrm{Sr}=0.7030$; Kroner and others, 1979; area 6, fig. 16). The trondhjemite of the Halaban crust within the fault zone was heated by an increment of frictional heat within the highly sheared fault zone and was additionally heated in part by mantle-derived magma. The magmatic heat is implied by small synkinematic plutons of alkalic granite that intruded along the margins of the gneiss antiform well within the fault zone. Mantle magmatism is implied by the very abundant (hence, voluminous) diabasic dikes of Najd age that intruded large areas between the major faults, by the mafic volcanism in the Jubaylah Group, and by strong magnetic anomalies over most Najd fault zones. The anomalies may imply the presence of gabbroic intrusions at depth. The Najdage magmatism was bimodal.

A few dated gabbro plugs and granite plutons within the Najd fault zones and a few dated andesitic basalt flows in the Jubaylah Group average about $530 \pm 20$ m.y. by the K-Ar whole-rock method. Such a date for the Najd faulting event is too young and may represent crustal cooling after uplift and erosion. A high-quality, whole-rock $\mathrm{Rb}-\mathrm{Sr}$ isochron of $577 \pm 15$ m.y. (Baubron and others, 1976, p. 58) on granite is probably only slightly older than the Jubaylah Group, because the granite intrudes the Murdama Group and disconformably underlies the basal Jubaylah conglomerate. The granite date agrees with the suggestion that algal fossils in limestone of the Jubaylah Group are about the age of 


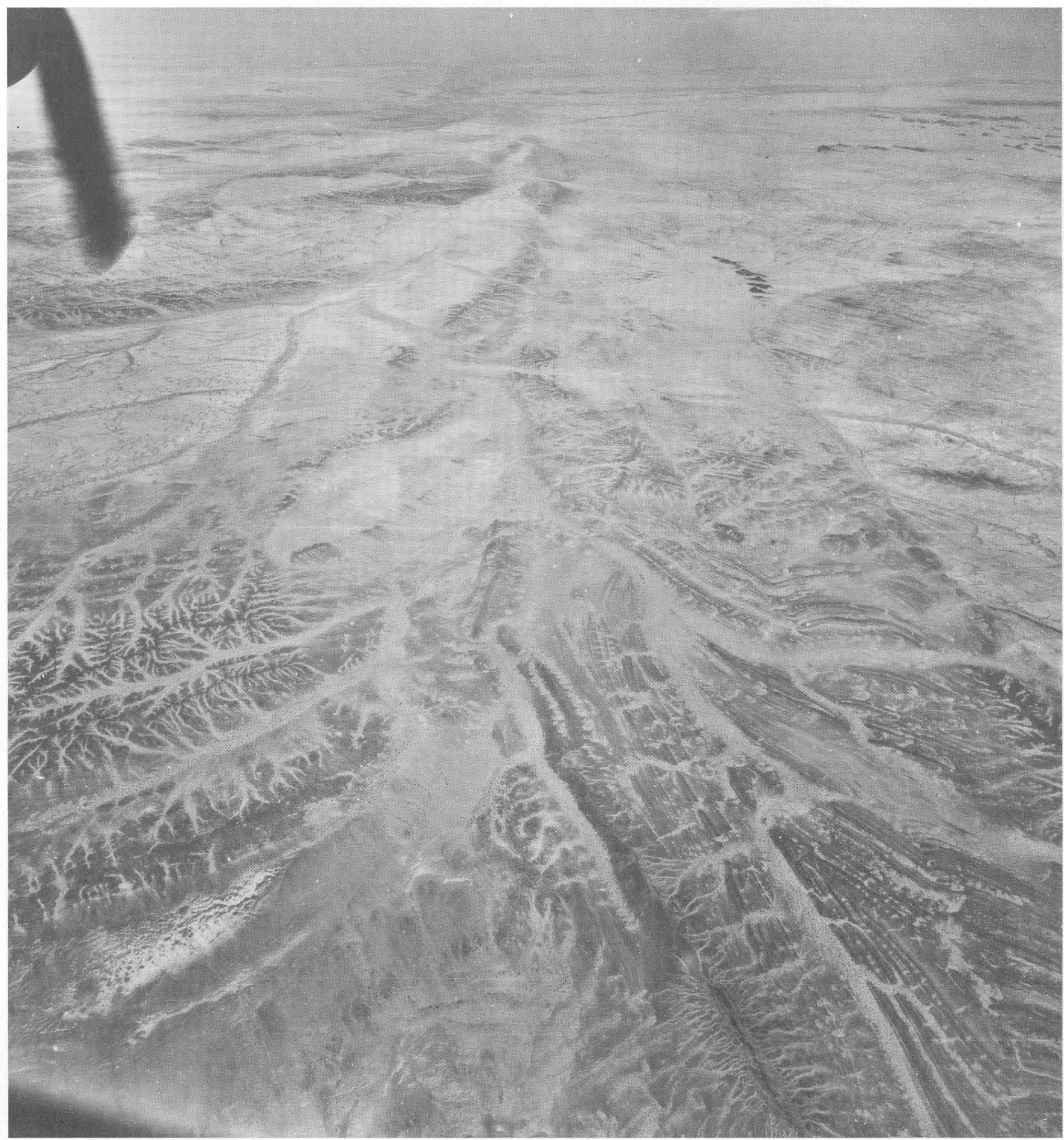

FIGURE 36.-Aerial view, looking northwest, of the Najd fault zone southwest of 'Afif. The infolded beds at near right are believed to be of Jubaylah and Shammar age; Murdama Group clastics at near left are infolded within metaclastic rocks of Halaban age (Letalenet, 1979). 


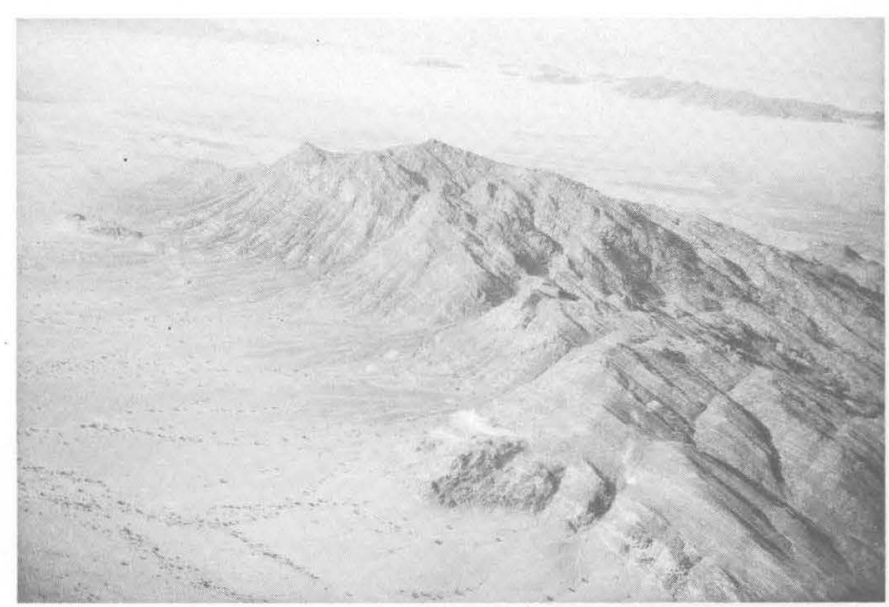

Figure 37.-Jabal Adhqān al 'Ațshān (lat $22^{\circ} 41^{\prime}$ N., long $44^{\circ} 06^{\prime}$ E.). A structure in the northeast flank of a gneiss dome of Najd age. Granite intruded into the Halaban Group, at the southwestern base of the mountain, later sheared in the Najd fault zone near the end of shield cratonization. Aerial view to the northwest.

the Precambrian-Cambrian boundary (about 570 m.y.; Cloud and others, 1979; Binda and Ramsay, 1980). The Jubaylah was folded into asymmetric synclines during the final Najd fault movement, and K-Ar biotite dates averaging about 550 m.y. (table 8) may represent this last spasmodic movement of the cratonization. Minor plugs and sills of gabbro and syenite may be subsequent to last synclinal folding of the Jubaylah, as evidenced by the K-Ar biotite dates. A gabbro plug intruded into the Najd fault zone at Wādī ar Rikā' (lat $28^{\circ} 28^{\prime}$ N., long $44^{\circ} 34^{\prime}$ E.) gave a whole-rock K-Ar age of $513 \pm 17$ m.y. for the chilled edge of the plug (sample $156 \mathrm{a}$, table 8) and $458 \pm 15$ m.y. for the deuterically altered core (sample $156 \mathrm{~b}$, table 8 ). Likewise, gabbro and diorite interpreted as a dike or sill at Khashm Qa'in (lat $24^{\circ} 46^{\prime} \mathrm{N}$., long $40^{\circ} 40^{\prime}$ E.) has a possible age of 502-512 m.y. (Delfour, 1981). In the northwestern extension of the Najd fault zone, a quartz syenite plug on the Red Sea coast (lat $27^{\circ} 28^{\prime}$ N., -long $35^{\circ} 07^{\prime}$ E.) yielded a whole-rock K-Ar age of $487 \pm 17$ m.y. (sample $12 \mathrm{~b}$, pl. 1, table 8 ). This is considered a minimum age by the analyst, Richard Marvin (oral commun., 1974). These younger dates are comparable to the youngest $\mathrm{K}$-Ar ages in southwest Jordan (Lenz and others, 1972) and are to be compared with fission track ages from sphene in diorite in southwest Arabia which range from 450 to 576 m.y. and average $510 \pm 52$ m.y. as analyzed by C.W. Naeser (written commun. to R.G. Coleman, 1969). Taken with Sutter's age estimate of 465 m.y. as the last time the Jubaylah quartz andesite or dacite passed through $100-150{ }^{\circ} \mathrm{C}$, it would appear that the shield went through an epeirogenic epoch toward the end of the Cambrian and early during the Ordovician. At least the northwestern part of the Najd fault system was covered by stable continental quartz sandstones by Late Cambrian time. Nearby in Jordan, trilobites in limestone within the stable sandstones are dated as early Middle Cambrian (Bender, 1975, p. 16).

\section{AGE AND STRONTIUM EVOLUTION}

An overview of the evolution of the Arabian Shield in terms of Rb-Sr whole-rock geochronology is succinctly shown in figure 38 (adapted from Fleck and others, 1980). In figure 38 , the primary crusts of the several volcanic-magmatic ares of Baish-Bahah, Jiddah, and Halaban ages are well represented by low initial strontium ratios for the volcanic and plutonic rocks between 900 and 680 m.y. The partial cratonization of Ablah age (early restricted collisional orogeny) is represented by granodioritic rocks between about 775 and 740 m.y. These rocks have initial strontium ratios greater than those of the primary crusts. The final cratonization of the entire shield is represented by granodioritic and granitic rocks between 660 and 580 m.y. These abundant rocks again have initial strontium ratios greater than those of the primary crust.

The granitic rocks of the craton are products of melts of the primary crust in which isotopic strontium decayed within this crust during only 100 to 300 m.y. and resulted in low initial strontium ratios (0.703 to 0.704$)$ in the granitic melts. These low ratios are distinctly crustal (by Arabian Shield standards), but they also indicate that old (greater than $1 \mathrm{Ga}$ ) continental basement may not be found in the Arabian Shield, except possibly in the easternmost part.

\section{PALEOZOIC SEDIMENTARY COVER ROCKS AT EDGE OF THE ARABIAN SHIELD}

\section{SIQ SANDSTONE}

The landward edge of the Arabian Shield is covered by sandstone beds of Cambro-Ordovician age, except for a gap of $130 \mathrm{~km}$ along the eastern end of the northern flank, which is occupied by the Great Nefud, and $420 \mathrm{~km}$ along the eastern rim, where the Khuff Limestone Formation of Permian age overlaps the shield. Of the rim rocks, the Siq Sandstone in northwest Arabia is probably the oldest. It is a reddish-brown massive and arkosic arenite exposed immediately above the crystalline rocks at Sha 'îb as Siq, lat $28^{\circ} 04^{\prime}$ N., long $35^{\circ} 40^{\prime}$ E., where Leopold Kober first saw it in 1910 (Kober, 1919). There the Siq Sandstone is exposed in a 65-m vertical cliff below a thinner section of reddishbrown, somewhat more stratified, flaggy sandstone on 

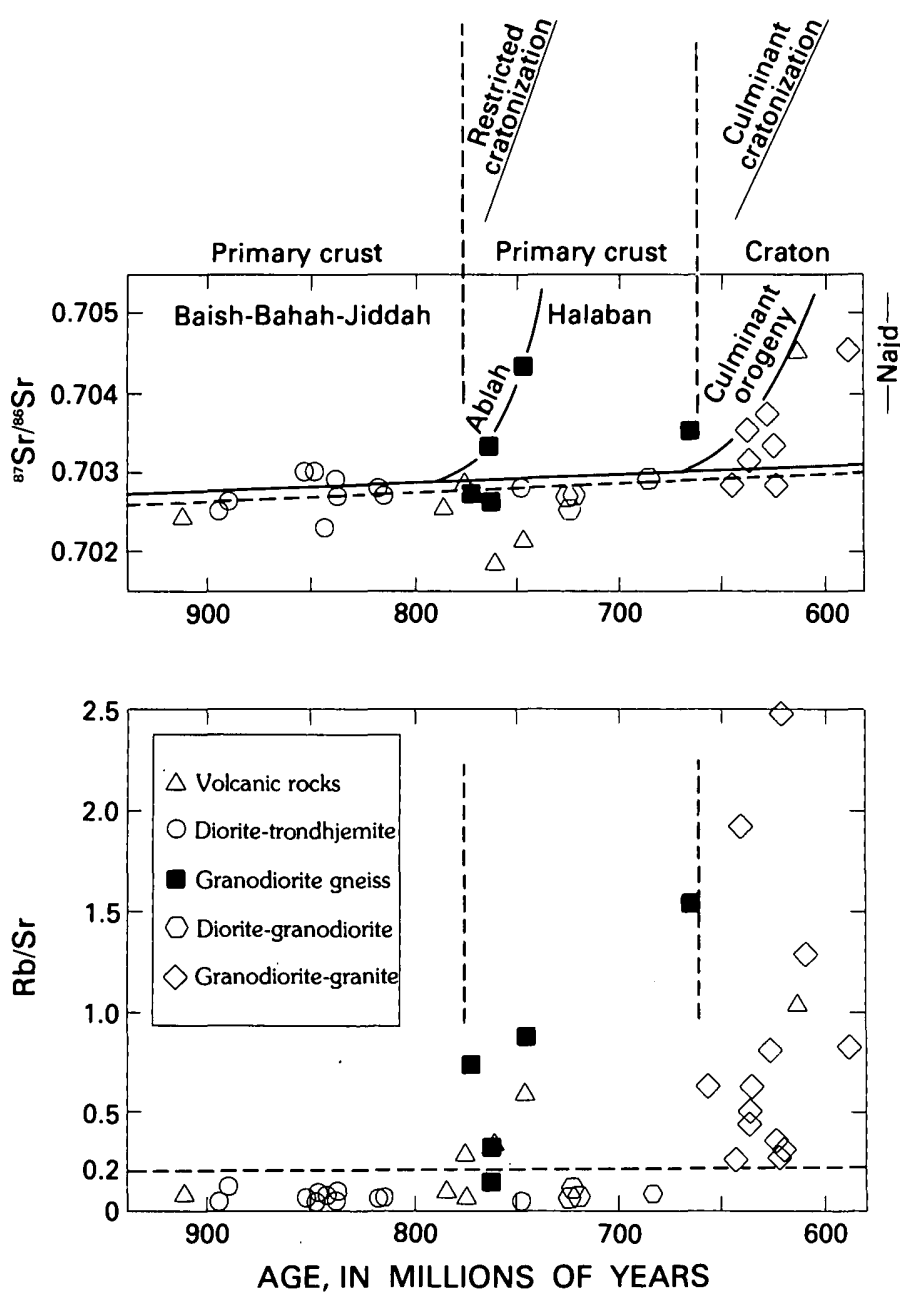

FIGURE 38.-Diagrams showing variations in initial ${ }^{87} \mathrm{Sr} /{ }^{86} \mathrm{Sr}$ (upper) and $\mathrm{Rb} / \mathrm{Sr}$ (lower) ratios with age (adapted from Fleck and others, 1980, fig. 26). Events and rocks on upper diagram added for this report. Vertical dashed lines show major changes in strontiumisotope composition and $\mathrm{Rb} / \mathrm{Sr}$ ratio of magmas corresponding to an earlier, regionally restricted orogenic event of partial cratonization of Ablah age and to a later, shieldwide culminant orogeny and cratonization. Strontium-evolution line (solid line; Faure and Powell, 1972) representing single-stage evolution from meteorite to average modern island arc is compared with least squares fit (upper dashed line) for Arabian Shield data having $\mathrm{Rb} / \mathrm{Sr}$ ratios less than 0.2 (lower dashed line).

the plain above (figs. $39 A-39 C$ ). From here the Siq extends eastward beneath the upland surface of the Hुismā Plateau to the isolated stacks and pinnacles on mesas of the stratigraphically higher white and buff exposures of the Ram-Umm Sahm Sandstone.

The basal few meters are gritty arkosic sandstone and conglomerate containing pebbles from the underlying crystalline rocks, but the more massive vertical walls of the Siq are composed of fine- to medium- grained, occasionally coarse-grained, quartz sandstone, channel crossbedded with most foresets dipping from northwest to northeast. The beds extend northward into Jordan, where Bender (1974a) considered them to be nonmarine and Lower Cambrian, the Quweira Sandstone of Quennell (1951). The flaggy upper member, which forms distinctive plains and benches, contains lenses and layers of silt and clay and some manganiferous and hydrated iron-oxide concretions which on weathering leave a cavernous surface (figs. $39 A-39 C$ ). Irregular calcareous cement also causes a cavernous weathering similar to that of the oxides.

The reddish-brown-weathering Siq Sandstone thickens to $115 \mathrm{~m}$ above Wādi Hुujīl $50 \mathrm{~km}$ to the southeast, and to $223 \mathrm{~m} 10 \mathrm{~km}$ farther at Wādi Amadan, where it overlies the Jubaylah Group across a basement graben. There the top of a massive middle section forms a bench $135 \mathrm{~m}$ above the shield rocks, and a second flagstone unit of the upper section extends above to the base of the younger cliff-forming and massively crossbedded Ram-Umm Sahm Sandstone (figs. 12, 39B). The sandstone thins southeastward and becomes more thin bedded; $275 \mathrm{~km}$ distant (lat $26^{\circ} 24^{\prime} \mathrm{N}$., long $38^{\circ} 42^{\prime} \mathrm{E}$.), in the Qal'at aş Şawrah quadrangle, the Siq is about $90 \mathrm{~m}$ thick at a 50 -m cliff $7 \mathrm{~km}$ north of the northern rim of the shield near Jabal Abadiyah. The lower section there is massive, red and tan, tabular, and trough-crossbedded sandstone under a 5-m-thick slab of finer grained silty sandstone whereon are trace fossils.

The Siq Sandstone questionably extends eastward along the northern edge of the shield from the Qal'at as S Sawrah area as far as long $40^{\circ} 25^{\prime}$ E., where outliers of flat-surfaced sandstone cap mesas in the easternmost of three basement grabens extending southeastward into the shield along the structural direction of the Najd fault system. West of long $40^{\circ} 25^{\prime}$ E., other outliers extend southward intermittently to the latitude of $\mathrm{Al}$ Madinah (lat $24^{\circ} 30^{\prime} \mathrm{N}$.). Most are small except in the vicinity of Bi'r Suwaydarah, east of Al Madinah, where about $250 \mathrm{~km}^{2}$ of sandstone underlies the plain along a major Najd fault zone. The sandstone doubtless extends northwest under Hुarrat Khaybar (Bigot and Chapelain, 1973; Delfour, 1977). The lithology is that of the Siq Sandstone farther northwest, and we have shown it as such although no fossils were found except at Qal'at as Şawrah which were identified and considered by A. Seilacher to be of Late Cambrian age (written commun., 1978). The fossils are Cruziana sp. (not Cruziana aff. C. furcifera d'Orbigny nor Cruziana huberi (Meunier)) and what appear to be radula marks of a large grazing gastropod, possibly Climactichnites sp. (fig. 39C), which is found elsewhere in the Potsdam Sandstone of Late Cambrian age. At Sha' $1 \mathrm{~b}$ as Siq the flaggy sandstone a few meters above the basement crystalline rocks contains limonite and psi- 


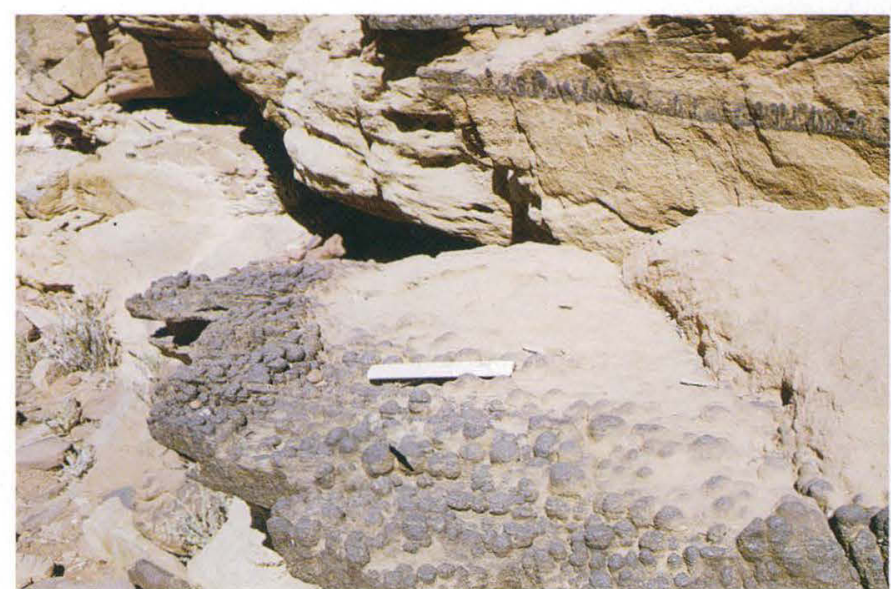

A

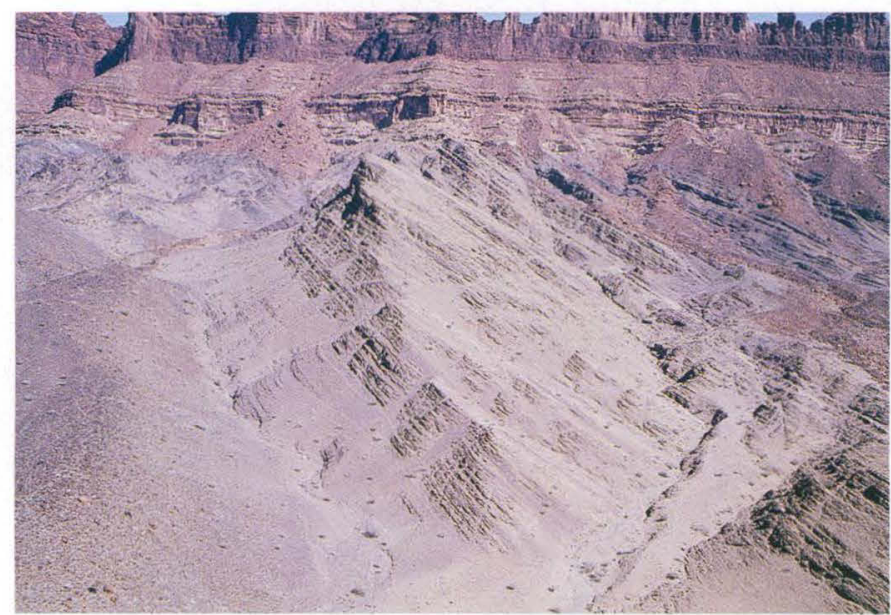

B

lomelane as well as calcareous concretions (fig. 39A) reminiscent of the manganiferous and cupiferous sediments in the upper part of the Middle Cambrian Burj Limestone Group in southwest Jordan (Bender, 1965, 1974a). If this correlation is valid and if the Cruziana sp. and Climactichnites(?) beds $275 \mathrm{~km}$ southeast are Upper Cambrian (Potsdamian), the sandstone becomes progressively younger toward the southeast and represents an onlapping shallow marine tongue.

\section{SAQ SANDSTONE AND RAM-UMM SAHM SANDSTONE}

The Saq Sandstone of Arenigian Age (uppermost Lower Ordovician) occupies the northeast flank of the shield, where it is stratigraphically below the Hanadir Shale of Llanvirhian Age (Bender, 1963) (lowermost Middle Ordovician) and above the crystalline rocks. There it represents a younging of the sandstone on the overlap along the north edge of the shield, probably the eastern extension of the Ram-Umm Sahm Sandstone. Farther south the sandstone crops out between the crystalline rocks and the Permian Khuff Limestone,

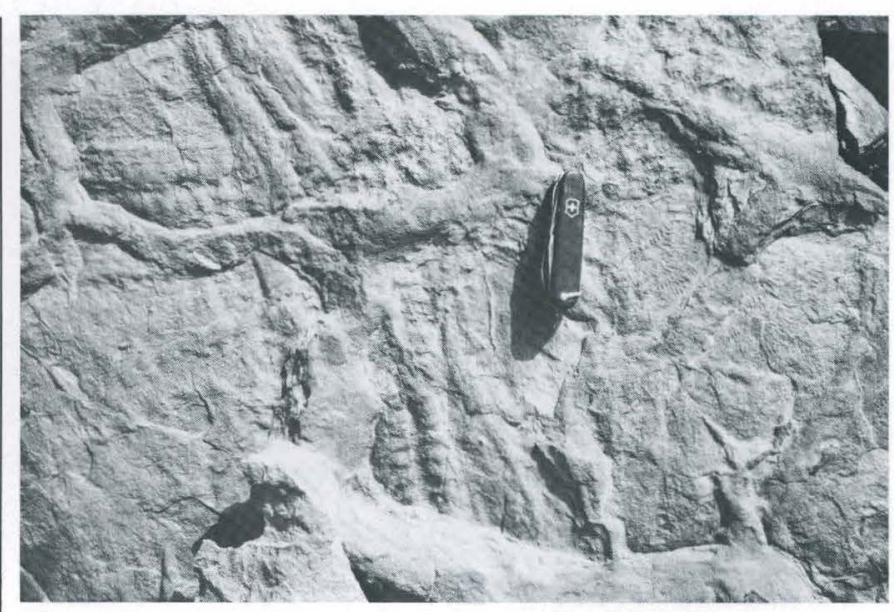

C

Figure 39.-The Siq Sandstone. $A$, Ferric and manganiferous concretions is lenses and seams within the basal beds of the Siq Sandstone at the upper rim at Shaib as Siq, the type locality. $B$, Siq Sandstone resting horizontally on Precambrian Hadiyah sedimentary rocks and beneath pinnacled Ram-Umm Sahm Sandstone. Headwaters of Wādī Shawāq. C, Cruziana sp. track in Siq Sandstone. Markings to the right of the knife appear to be grazing marks of a gastropod, probably Climactichnites(?) sp., of Late Cambrian(?) age. Near Jabal Abadiyah (lat $26^{\circ} 24^{\prime}$ N., long $38^{\circ} 42^{\prime}$ E.).

which overlaps the Hanadir Shale as well. The largescale, planar-crossbedded, white, tan, and gray Saq Sandstone resembles and is stratigraphically equivalent to the Ram-Umm Sahm Sandstone of northwest Arabia and southwest Jordan in that it is a mature arenite, in many places lacking arkosic beds. In places it rests on basement rocks without a basal conglomerate, although commonly there are lenses of grit and the basal few meters are reddish with iron cement. Cement in the basal beds may also be gypsiferous as well as calcareous, but the nonferrous cement is generally somewhat above the basal beds. Rare well-rounded pebbles, mostly quartz, some citrine, form local lenses in large-scale planar crossbedding of finer sand $(\mathrm{N}$. Layne, written commun., 1959-60; Powers and others, 1966).

The only evidence of age reported heretofore is trace fossils collected from near the southern end of the outcrop near the top of the sandstone at Jabal Haqil, 37 $\mathrm{km}$ northeast of Ad Dawādimī. These were identified by P.E. Cloud, Jr., as Cruziana aff. C. furcifera d'Orbigny and $C$. huberi (Meunier), which belong to the Cruziana rugosa group of Seilacher (1970). The $C$. rugosa traces are found in shale lenses in northwest Arabia and in Jordan in the upper part of the RamUmm Sahm Sandstone, where they are considered to be of Arenigian Age (uppermost Lower Ordovician) (Bender, 1975). 


\section{WAJID SANDSTONE}

The southeast edge of the shield is covered by the Wajid Sandstone, which crops out beneath the Permian Khuff Formation at Wādī ad Dawāsir, $420 \mathrm{~km}$ south of the southernmost exposure of the Saq Sandstone. Flatlying or gently arched, unmetamorphosed, and broken only by late Tertiary and Quaternary high-angle tension faults, and earlier considered devoid of fossils, it could heretofore be assigned only a pre-Permian and post-shield age.

The type locality is Jabal al Wajid at lat $19^{\circ} 06^{\prime} \mathrm{N}$., long $44^{\circ} 27^{\prime}$ E., where a thickness of $950 \mathrm{~m}$ was calculated (Powers and others, 1966). More recently, the sandstone has been drilled downdip to the east of the northern end of the outcrop, where a thickness of $500 \mathrm{~m}$ was measured beneath a disconformity, a thickness comparable to an estimate of $600 \mathrm{~m}$ by Alabouvette and Villemur (1973). Shaly beds above the disconformity contain three chitinozoan species of Silurian age, Conochitina latifrons, C. micracantha subsp. robusta, and Ancyrochitina nodosa (D. Hemer, oral commun., 1969). In addition, a recent study of the outcrop belt has yielded Scolithus (Tigillites), arthropod trails (Cruziana sp.?), and conical or circular structures at the base of the Wajid Sandstone (Alabouvette and Villemur, 1973), forms that are similar to those in the upper Saq Sandstone and, farther north, in the Ram-Umm Sahm Sandstone. Thus the Wajid in Jabal al Wajid appears to be uppermost Lower Ordovician or possibly somewhat younger, as the Tigillites trace fossils are most abundant in the Tabuk Formation of Lower Ordovician to Lower Devonian age exposed farther north in Arabia (Powers and others, 1966).

A Cambrian-Ordovician age for the Wajid Sandstone was first suggested by Darwin O. Hemer, who found algal forms in well cuttings similar to those described from the lower Paleozoic in the Russian Baltic region (Hemer, 1968).

The lithology of the Wajid Sandstone also tends to make such an age reasonable. The bulk of the sandstone is composed of mature grains and pebbles of quartz and displays large-scale planar crossbedding. Indeed, the disconformity reported by Alabouvette and Villemur (1973) may be equivalent to the top of the Saq Sandstone and the bottom of the Tabuk on the northern flanks of the shield, although the basal Tabuk Hanadir Shale with the Didymograptus index graptolite (Powers and others, 1966) is missing. Intermittent exposures and subcrop in the $\mathrm{Al}$ 'A rid region east of Jabal al Wajid, including sandstone, boulder beds, and fossiliferous shale ranging in age up to Lower or Middle Permian, have recently been included in the Wajid Sandstone (McClure, 1980). More recently, an Ordovi- cian age was strengthened by the discovery of the Ordovician Hydrozoan Disophyllum cf. peltatum associated with glaciogene sediments in Tigray, northern Ethiopia (Saxena and Assefa, 1983), beds that were correlated with the Wajid Sandstone (Dow and others, 1971; Beyth, 1973). Also, if a glacial origin is accepted for the exposures in uppermost Ordovician or lowermost Silurian in north-central Arabia, they would be coeval with glacial rocks in Algeria and Mali (Dow and others, 1971; McClure, 1978). However, the dropstones at Khashm Khaţmah and Jabal Umm Ghīrān, easternmost outcrops of what have been considered Wajid Sandstone, are now known from flora in nearby drill holes to be uppermost Carboniferous or lowermost Permian (McClure, 1980). This flora correlates with the flora in the glaciogene Haushi Group in Oman (Hudson, 1958) and the tillites in Yemen (Roland, 1978; Kruck and Thiele, 1983). Thus it appears that there was widespread glaciation during youngest Ordovician, and possibly oldest Silurian, in northern Arabia and north Africa, extending as far south as northern Ethiopia, and a late Paleozoic widespread glaciation (Gondwana Ice Age) extending into southern and southeastern areas of the Arabian Peninsula.

Occasional outliers of basement igneous and metamorphic rocks are kopjes or bornhardts, as at Bi'r Idima, where the bornhardt serves as a ground-water dam. Exposures of gabbro and metamorphosed mafic rocks in knobs as much as $50 \mathrm{~m}$ wide lie in disturbed lower Wajid Sandstone at Hijmah (lat $19^{\circ} 07^{\prime}$ N., long $44^{\circ} 05^{\prime}$ E.; Stoeser, oral commun., 1976), indicating movement during Wajid time and possibly disturbance during the Late Cambrian or Early Ordovician epeirogeny, suggested by the radiometric dates described above.

The descriptions of Alabouvette and Villemur (1973) show no organic forms in the south such as occur farther north near Wādī ad Dawāsir (Jackson and others, 1963). Furthermore, the calcareous cement and trough crossbedding resemble features described in the Siq Sandstone (Hadley, 1973), but by no means prove correlation.

A few widely scattered outliers of sandstone are deep within the shield. A small sandstone crest at Jabal Tinn, lat $22^{\circ} \mathrm{N}$., near Khurmah, $600 \mathrm{~km}$ south of the northern rim of the shield and $260 \mathrm{~km}$ northwest of the nearest outcrops of the Wajid, resembles the Wajid. The crest is composed of $16 \mathrm{~m}$ of white, fine-grained, massive sandstone, flat lying above basement gneiss. Similarly, a prominent outlier capping Jabal Tamīyah, $425 \mathrm{~m}$ above the desert floor at lat $25^{\circ} 36^{\prime} \mathrm{N}$., long $42^{\circ} \mathrm{E}$., and $80 \mathrm{~km}$ west of the Saq Sandstone, at the eastern edge of the shield, is $82 \mathrm{~m}$ thick, flat lying, and crossbedded. The base of the Saq Sandstone near Ḩā'il also lacks a 
basal conglomerate. A Saq age at Jabal Tamīyah and a Wajid age at Jabal Tin fits the concept of the younging of the lower Paleozoic fringe sandstones toward the southeast:

\section{KHUFF FORMATION}

The eastern edge of the shield between outcrops of the Saq Sandstone and the Wajid Sandstone is nonconformably overlain by the Permian Khuff Formation. Outcrops directly overlying the basement and overlapping the Saq Sandstone are mostly in the area east of the $\mathrm{Al}$ Amar-Idsas fault, extending from lat $22^{\circ} 40^{\prime} \mathrm{N}$. to lat $24^{\circ} 28^{\prime} \mathrm{N}$. Farther south, the Khuff probably rests on the basement, but the exposures are poor and may be those of an older Paleozoic paleosol.

The Khuff ranges in thickness to as much as $320 \mathrm{~m}$, but only $171.4 \mathrm{~m}$ was measured at the reference section between Wādī ar Rayn and Jabal ath Thuwayr (Powers, 1968). The base is a lateritic duricrust only a few meters thick at most, resulting from Paleozoic weathering beneath the basal conglomerate and sandstone of the Khuff. The bulk of the formation above the paleosol is neritic dolomite, shale or claystone, and limestone that dip gently eastward. These beds were deposited during a widespread marine transgression at the end of the Paleozoic. The Khuff Formation has been divided into five informal members on the basis of lithofacies changes both vertically and along the strike (Delfour and others, 1982).

\section{MESOZOIC SEDIMENTARY ROCKS}

\section{KHUMS FORMATION}

\section{DEFINITION}

The Khums Formation takes its name from Wādi Khums, where it was first mapped in the foothills east of the Tihāmat al Yemen (coastal plain) near the Yemen border (lat $16^{\circ} 50^{\prime} \mathrm{N}$., long $43^{\circ} 01^{\prime}$ E.) (Brown and Jackson, 1959). At the type locality, crossbedded and graded grit of the Khums nonconformably overlies Precambrian crystalline rocks where the contact is not a fault. Elsewhere it overlies the Wajid Sandstone of Cambro-Ordovician age. The uppermost quartzitic sandstone of the formation underlies Upper Jurassic limestone, apparently comformably (fig. 40).

\section{OCCURRENCE AND THICKNESS}

The Khums Formation crops out in a belt $3 \mathrm{~km}$ or less wide and extends for $35 \mathrm{~km}$ parallel to the Red Sea rift zone to the north and south of Wādi Khums. A similar belt lies $10 \mathrm{~km}$ southeast and extends into the Yemen.

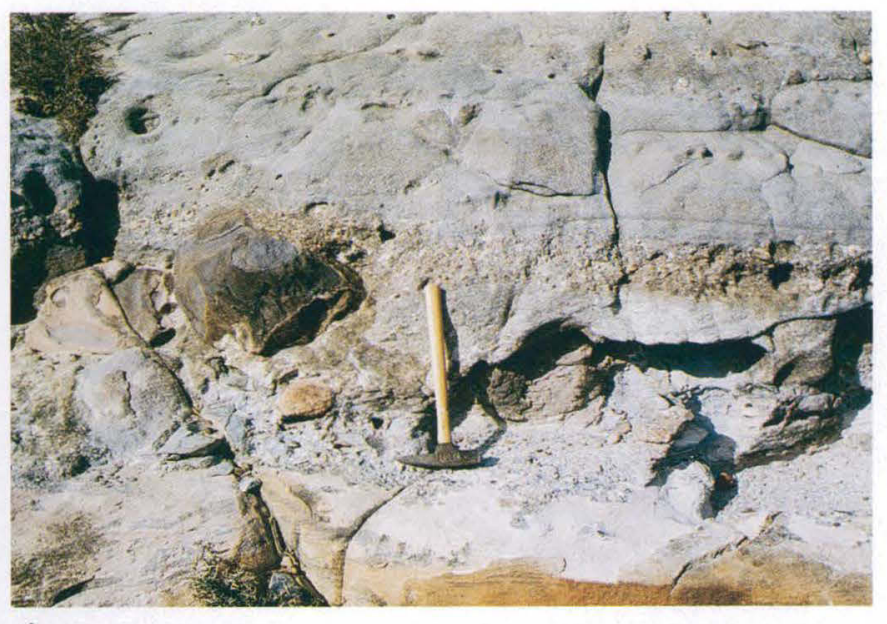

A

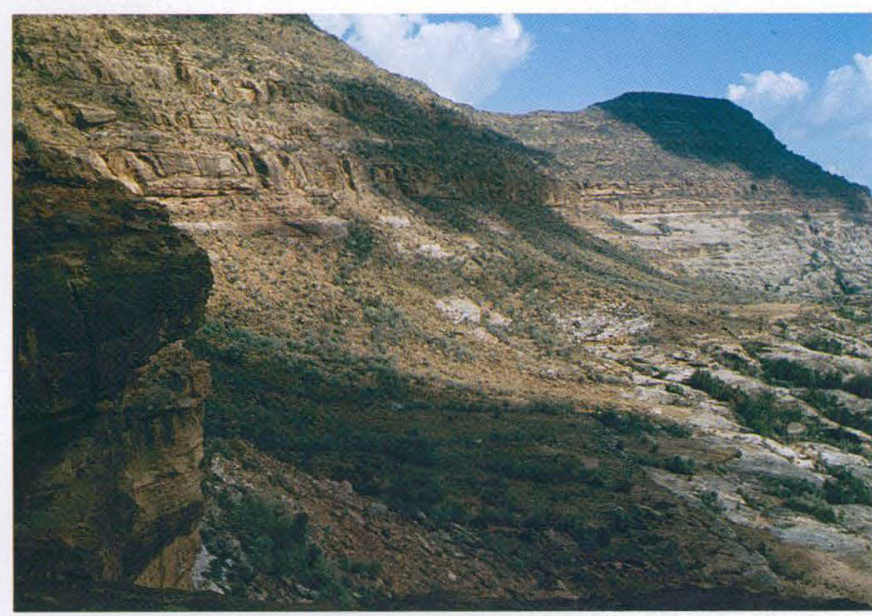

B

Figure 40.-The Triassic Khums Sandstone. $A$, Contact at base of Khums Sandstone marked by grit. Hammer rests on top of Wajid Sandstone. B, View of the south slope of Jabal Abū Hasan showing contact between the Cambro-Ordovician white Wajid Sandstone below and the tan Triassic Khums Sandstone above.

Both belts are partly bound by faults. Outliers occur on the crest of the scarp mountains to the east. The formation is of variable thickness, and because it is highly block faulted in places along the flank of the Red Sea rift, the thickness is somewhat difficult to estimate. Gillmann (1968) measured a maximum of 880 $m$ of sandstone in the faulted section of the Wādī Khums belt where we suggest that $400 \mathrm{~m}$ is Khums Sandstone, underlain by $480 \mathrm{~m}$ of Wajid Sandstone and overlain by the Jurassic Amran Formation. The Khums narrows to $10 \mathrm{~m}$ at the north end of the belt on the banks of Wādi Jizañn, but its top is a fault. Thirty kilometers north of Wādī Jīzān, an 
isolated outcrop of the Khums Formation measures $100 \mathrm{~m}$ in thickness. About $145 \mathrm{~km}$ northwest of the Wādi Khums locality and $15 \mathrm{~km}$ northwest of Ad Darb, tectonized and quartzitic sandstone is associated with calcareous and kaolinitic beds that contain rare molluscan steinkerns common to the Amran Series, a unit of the Jurassic marine beds of Yemen (Geukens, 1960). However, the bulk of the small outcrop northwest of Ad Darb could be the lower Paleozoic Wajid Sandstone, which caps the mountains to the east; a sliver of upper Khums containing Amran-type fossils may overlie the Wajid in this downdropped, shattered block. On the crest of the scarp mountains east of the Tihāmat, the Khums clastic deposits overlie the Wajid Sandstone on the mesa crest of Jabal Abū Hasan (al Qahar) $75 \mathrm{~km}$ north of Wādī Khums (figs. $40 A, 40 B$ ). At Jabal Abū Hasan, between altitudes of 1,541 to $1,861 \mathrm{~m}$, we measured $320 \mathrm{~m}$ where the beds are relatively undisturbed. Subsequently, D.G. Hadley and D.L. Schmidt (written commun., 1974) measured a thickness of $160 \mathrm{~m}$ at nearby Jabal al Qahar on the south side of Jabal Abū Hasan. R.E. Anderson measured another outlier of the Khums Formation near Alb Pass $40 \mathrm{~km}$ east of Jabal Abū Hasan on the high plateau of Al Yemen in Saudi Arabia near the Yemen border. There he found a thickness of $125 \mathrm{~m}$ (Anderson, 1979, p. 20), the same as the thickness of clastic rocks (Khohlan Series) above the basement and below the Upper Jurassic Amran Series measured by Geukens (1966, p. 8) 90 km southeast near Sa'dah in Yemen.

\section{LITHOLOGIC CHARACTER}

The Khums Formation in the rift zone is mostly a hard quartzitic sandstone containing minor beds of siltstone and occasional pebbles and boulders of quartzite. Granitoid and metamorphosed basement rocks are well rounded in the basal grits. The siltstone is gray green or gray and reddish brown and contains pebbles. The beds are highly disturbed and intruded by Tertiary basalt dike swarms that have contact-metamorphosed the sediments. Michel Gillmann (1968) examined the Khums outcrops along the foothills and divided the formation into three members: a lower crossbedded and coarse sandstone $(480 \mathrm{~m})$; a middle member of finegrained phosphatic sandstone, conglomerate, and ferruginous shale (violet colored) with some tuffaceous beds $(110 \mathrm{~m})$; and an upper member of fine-grained siliceous sandstone $(290 \mathrm{~m})$. We suggest, however, that the lower sandstone probably is Wajid Sandstone. Our section on Jabal Abū Hasan, where the bedding is approximately horizontal, was measured in 1965 and is as follows:

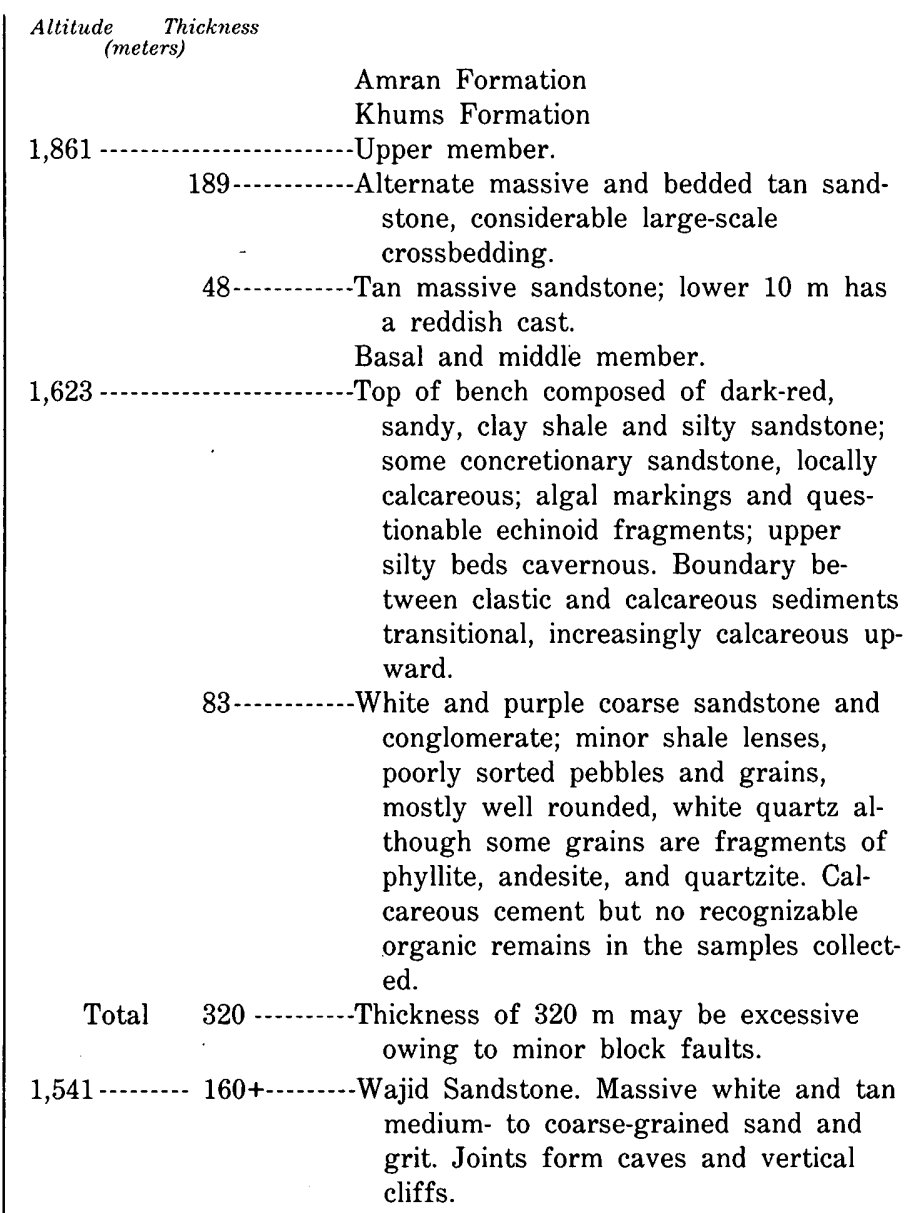

Anderson (1979) divided the Khums Formation near 'Alb Pass into a lower $35 \mathrm{~m}$ of alternating sandstone and pebbly ferruginous shale, a middle $80 \mathrm{~m}$ of massive buff and crossbedded buff sandstone and gritstone, and an upper $10 \mathrm{~m}$ of grayish-red, red-purple, and paleyellow sandstone with ferruginous beds.

Lithology considerably dissimilar to the Khums Formation but cropping out in Yemen between basement rocks or the Wajid Sandstone and the Amran Series was described by P. Lamare (1930b) as $215 \mathrm{~m}$ thick near Amran, $280 \mathrm{~m}$ thick at Jabal Ashmur $20 \mathrm{~km}$ to the west (quoting C. Rathjens), and $700 \mathrm{~m}$ thick near At Tur, 40 $\mathrm{km}$ still farther west, and near the western edge of the scarp mountains. This gives an increase in thickness toward the Red Sea graben similar to that in Saudi Arabia. The beds change from alternating sandy marl and sandstone and lignitic beds in the upper part near Amran to increasingly arenaceous outcrops westward toward the Red Sea. These rocks in the Yemen are called the Khohlan Series (Geukens, 1960). ${ }^{6}$

\footnotetext{
${ }^{6}$ Since this paper was written, a Carboniferous section of glaciogene sedimentary rocks was mapped above the basement or Wajid Sandstone and below the Khohlan Sandstone (Kruck and Thiele, 1983).
} 
The Khums Formation has only plant fossils, rare in outcrop, and the trace fossil Arthrophycus (Anderson, 1979 , p. 20). This trace fossil, a trail made by arthropods or worms, is generally considered OrdovicianSilurian, although similar furrows are reported from Cretaceous and upper Tertiary beds (Hantzschel, 1975, p. W38, W39). The beds containing questionable algal impressions and echinoid spines at Jabal Abū Hुasan may belong in the overlying marine Amran Formation. In Yemen, Geukens (1960) found worm borings, possibly Arthrophycus, in the uppermost beds at the same stratigraphic position as that described by Anderson near 'Alb Pass. P. Lamare and C.A. Carpentier (1932) reported a flora in lignitic lenses in the Yemen that they considered to be Lower Jurassic (Lias) or possibly uppermost Triassic (Rhaetian), which could be coeval with the Khums Formation. However, $115 \mathrm{~km}$ southeast of the Khums locality, carbonaceous beds occur in marl and sandstone near the village of Amran, where the lithology is considerably different.

The Khums Formation can be any age from CambroOrdovician to Early Jurassic on the basis of adjacent formations. However, the bulk of the evidence suggests that the Khums is paraconformable on the Cambro-Ordovician sandstone (Anderson, 1979) and possibly equivalent to part or all of the nonmarine Late Triassic Minjur Sandstone of central Arabia (Powers and others, 1966). The Minjur contains lignite beds in the subsurface at Riyadh, and this is similar to the lithology at the Amran locality. Samples from below $1,110 \mathrm{~m}$ in a test water well at Riyadh contained three new species of the conifer Brachyphyllum that were considered possibly to be middle Liassic, entirely on the basis that the overlying Marrat Formation is upper Liassic (Toarcian) (Boureau, 1956). Owing to the fragmentary nature and absence of stomata in the descriptions of the only conifer identified from the Yemen, Pagiophyllum peregrinum Sch., Boureau (1956) could not compare it with the Minjur fossils. However, in central Arabia the Minjur is truncated by a regional unconformity (Dubay, 1969), and Cameron (1974), in a more recent study of palynomorphs from the Minjur Sandstone, has proven it to be of Late Triassic age. Thus, the Khums Formation is here regarded as Upper Triassic (Rhaetian) or Lower Jurassic.

\section{AMRAN FORMATION}

\section{DEFINITION}

A blue-gray lithographic limestone (figs. 41, 42), silicified and brecciated in places, crops out above the

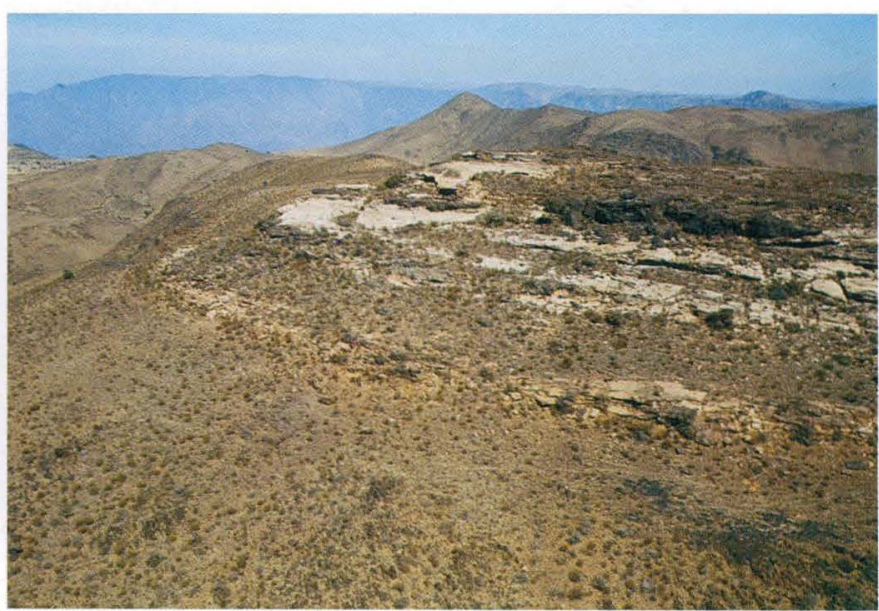

$\boldsymbol{A}$

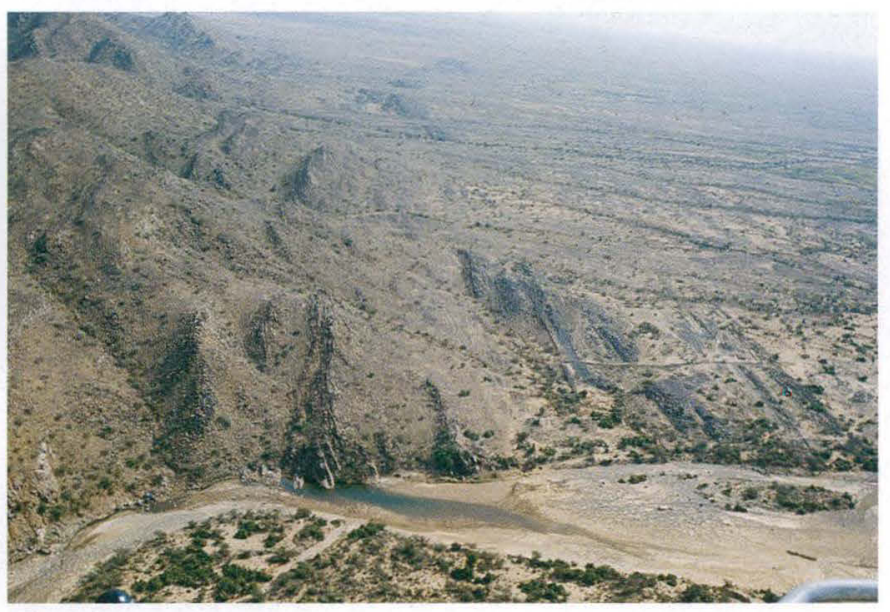

B

Figure 41.-Limestone of the Amran Formation. A, The Amran Limestone above Khums Sandstone on the top of Jabal Abū Ḩasan. B, Toe of 'Asīr Mountains east of Jizān with the Jurassic Amran Limestone dipping west beneath the coastal plain and overlying the Khums Sandstone, which crops out in foothills on the left. The sediments have been downfaulted to the west. At Wādī Khums, east of Jīzān.

Khums Formation in the foothills at the east edge of the coastal plain south of Wādī Khums. It was mapped in 1951 (Brown and Jackson, 1959) and named for the Hanifa Formation of Late Jurassic age in central Arabia on the basis of the similarity of its fossils to those of the Hanifa (R.A. Bramkamp, written commun., 1951). Subsequent work has established that the fauna of this limestone represents a greater timespan than that of the Hanifa, as the Hanifa has later been more precisely defined, and that the rocks are homologous to the Amran Series of Yemen (Lamare, 1930b). 


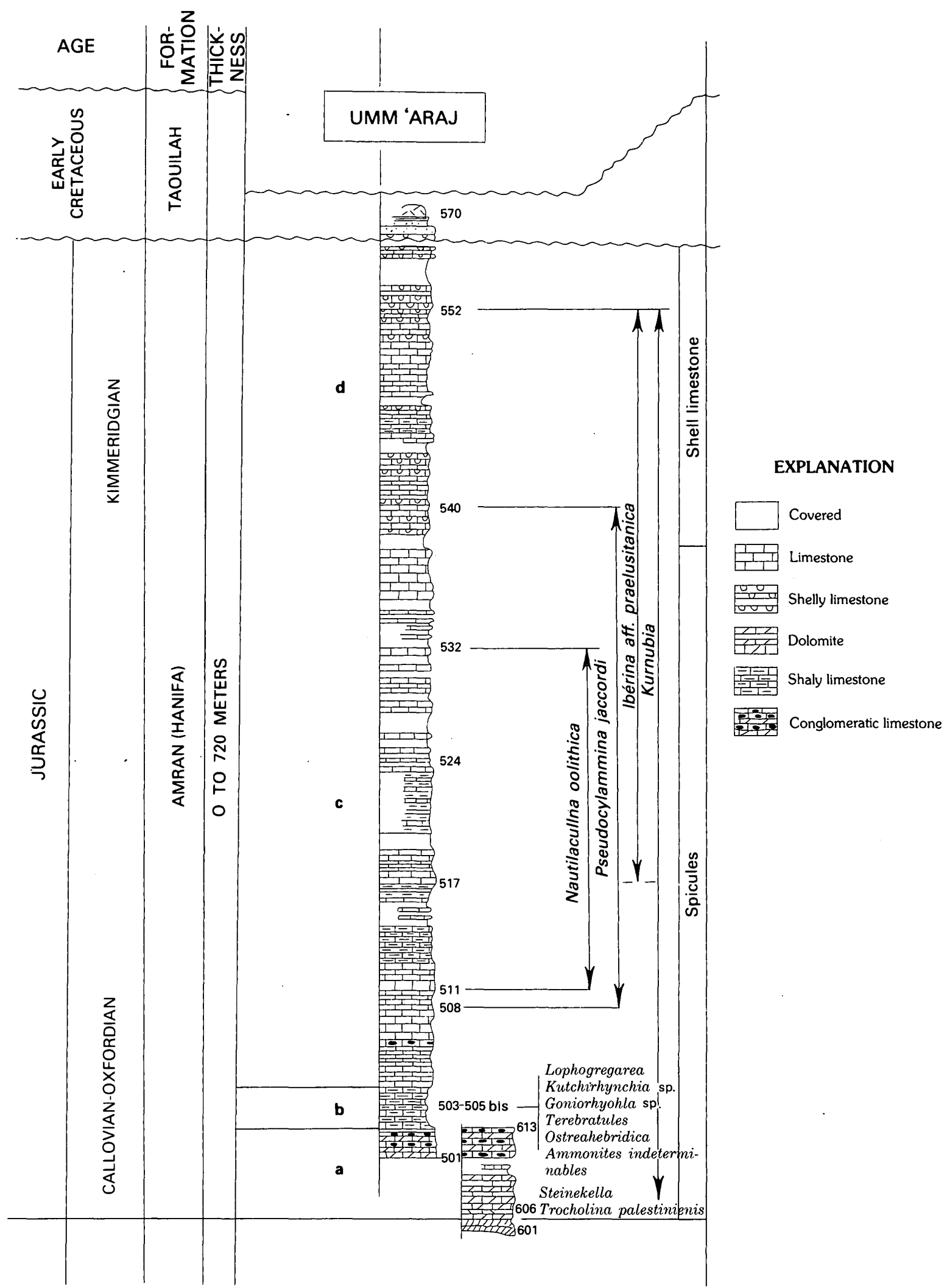

FIGURE 42. - Type section of the Amran Formation at Umm 'Araj. (Modified from Gillmann, 1968.) 


\section{OCCURRENCE AND THICKNESS}

The Amran Formation shown as the Hanifa Formation on the geologic maps of the 'Asīr quadrangle (Brown and Jackson, 1959) and of the Arabian Peninsula (USGS-ARAMCO, 1963) crops out at Wādī Fija, 3 $\mathrm{km}$ north of Wādī Khums, and in faulted blocks about $10 \mathrm{~km}$ southeast of Wādi Khums near Umm 'Araj (lat $16^{\circ} 46^{\prime}$ N., long $43^{\circ} 06^{\prime}$ E.). Ten kilometers farther southeast, a more extensive outcrop extends $20 \mathrm{~km}$ along the Yemen boundary and into Yemen. Smaller outliers are present on Jabal Abū Ḩasan (al Qahar) and near 'Alb Pass, where calcareous beds apparently disconformably overlie the Khums Formation. The Amran Formation is extensively exposed in northern Yemen, where the name is taken from outcrops near the town of Amran, $45 \mathrm{~km}$ northwest of Şan'ā' (Lamare, 1930b; Geukens, 1960, 1966; Grolier and Overstreet, 1978). Near Umm 'Araj, Gillmann (1968) divided the formation, there $720 \mathrm{~m}$ thick, into two major divisions, a lower unit $(500 \mathrm{~m})$ composed of three members and an upper unit $(220 \mathrm{~m})$ of phosphatic limestone.

Farther east on the crest of the 'Asīr escarpment, 82 $\mathrm{m}$ of sandstone and interbedded limestone and sandy limestone contain marine fossils.

\section{Section of Amran Formation on the southern slope of Jabal Abü Hasan (al Qahar) (elev. 1,861 to $1,942 \mathrm{~m}$ ), top of mesa}

Limestone, fossiliferous, tan, sandy, echinoid spines, small gastropods and pelecypods -..........................

Sandstone, massive, tan

Sandstone, moderately massive and bedded, mediumgrained

Sandstone, alternating brown, calcareous, and white, medium-grained

Limestone, thin-bedded, tan, sandy, in three benches: indeterminate small pelecypods and oyster fragments;

Topha sp., Turritella sp.; Naticid indet. Total thickness

Khums Formation at base of section

At the small mesa near 'Alb Pass, Anderson (1979) found $20 \mathrm{~m}$ of calcareous sandstone and thin interbedded strata of sandy fossiliferous limestone which he compared to the Amran Series that crops out $30 \mathrm{~km}$ southeast in Yemen.

The Amran Series of Yemen crops out extensively in northwest Yemen, where the thickness is given as 100-265 m (Basse and others, 1954), thickening to 550 $\mathrm{m}$ to the southwest (Geukens, 1960) but thinning northward toward the isolated outcrops in Saudi Arabia (Geukens, 1960). Geukens measured $105 \mathrm{~m}$ near Sa'dah, $90 \mathrm{~km}$ southeast of the easternmost outcrop in Saudi Arabia.

\section{LITHOLOGIC CHARACTER}

In the only extensive outcrop in Saudi Arabia near the Yemen border at the southeast corner of the Jizan coastal plain, most of the Amran is crystalline bluegray limestone and dolomite. Minor beds of shale and tuff are near the top. The rocks have been dropped down in the Red Sea graben and intruded by Tertiary dikes and sills of basalt, gabbro, and related igneous rocks of the early oceanic coastal rock of the Red Sea.

Gillmann (1968, p. 195) described the lower $500 \mathrm{~m}$ of sponge-spicule limestone as follows: "It includes $70 \mathrm{~m}$ at the base of dark-gray crystalline or sublithographic limestone and dark-gray dense dolomite with chert nodules at the top. In the middle, $30 \mathrm{~m}$ of gray, argillaceous, very fossiliferous limestone. Last, $400 \mathrm{~m}$ of dark-blue sublithographic limestone in thick massive layers with some intercalated beds of brown shelly limestone."

The upper shelly limestone is "formed of $220 \mathrm{~m}$ of violaceous fragmental limestone, gray-blue lithographic limestone, and marl and shale (with chlorite)" (Gillmann, 1968, p. 195).

In Yemen the limestone grades into a sandy facies, which farther east grades to interbedded gypsum and, in the eastern extension of Wãdi Jauf, to salt beds that crop out in diapiric structures at the throat of Wādī Hadramaut (Brown, 1972).

\section{NATURE OF CONTACT}

The Amran Formation rests parallel to the bedding of the underlying Khums Formation, but as the Amran contains Upper Jurassic fossils and the Khums Formation is most likely Upper Triassic (Rhaetian) or lower to middle Liassic (lowermost Jurassic), there appears to be a substantial hiatus or a period of extremely slow deposition prior to deposition of the marine Amran. Geukens (1960) considered this an epeirogenic period.

The only occurrence of overlying beds of Mesozoic age in Saudi Arabia is reported by Gillmann (1968, p. 195), who described $10 \mathrm{~m}$ of "coarse sandstone, breccia with limestone fragments, and brown shale" which could be compared to the sandstone in the Lower Cretaceous Tawilah Group in Yemen. However, the Early Cretaceous age may be open to question, as the Tawilah Group of sandstone beds cannot be separated with certainty from the overlying, lithologically similar Medj-zir Series, which contain Paleocene or Eocene fossils (Geukens, 1960; Grolier and Overstreet, 1978).

\section{PALEONTOLOGY AND AGE}

On the 'Asīr 1:500,000-scale geologic map (Brown and Jackson, 1959), the Amran Formation was named the 
"Hanifa Formation" on the basis of the similarity of the megafossils and lithology to the Hanifa Formation in central Arabia. These fossils, as identified by R.A. Bramkamp (written commun., 1951), are as follows:

Basal fauna north and south of Wādĩ Khums, east of the Red Sea coastal plain:

Somalirhynchia (two species)

Gryphaea balli Stefanini

Lopha aff. solitaria Sowerby

Parallelodon sp.

Lower part of limestone, north bank of Wādì Khums: Terebratula sp.

Rhynchonella sp.

Middle portion of the limestone section:

Cidaroid sp.

Ostrea sp.

Bramkamp at that time considered this fauna to be Hanifa, which was then questionably correlated with the Oxfordian stage of the European Jurassic, but he had reservations that it might be in part somewhat younger and of Jubaila (Upper Jurassic) Limestone equivalency (lower Kimmeridgian) of eastern Arabia (Steineke and others, 1958). Later, the limestone of the Amran Formation was studied more intensively by Michel Gillmann (1968, p. 195), who identified the following foraminifera and pelecypods:

Lower $70 \mathrm{~m}$ of limestone and dolomite:

Steinekella (Redmond)

Trocholina palestiniensis (Henson)

Kurnubia aff. jurassica-wellengsi

Pseydocyclammina sp.

Next $30 \mathrm{~m}$ of argillaceous limestone:

Lopha gregarea Sowerby

Kutchirhynchia sp.

Goniorhynchia sp.

Terebratulas

Ostrea hibridica Sowerby

Third unit of $400 \mathrm{~m}$ of limestone:

Pseudocyclammina jaccardi

Iberina spirocyclina sp. Henson

Nauticulina oolithica

Upper $220 \mathrm{~m}$ of limestone, marl, and shale: Iberina aff. praelusitanica (Maync)

These fossils range in age from Bathonian (upper Middle Jurassic) to Kimmeridgian (Upper Jurassic) (Gillmann, 1968). The Amran Series in Yemen has been identified from at least four collections as Malm, the epoch that includes the Upper Jurassic stages, Oxfordian, Kimmeridgian, and Portlandian, whereas the Amran Formation at Umm 'Araj in Saudi Arabia seemingly does not include the younger Portlandian fossils but extends downward into the Middle Jurassic. As the
Hanifa Formation has more recently been restricted to the lower Kimmeridgian (Powers, 1968), it seems desirable to apply the formational name from Yemen to the outcrops along the border in Saudi Arabia.

\section{KHURMA FORMATION}

The Khurma Formation consists of a quartzitic sandstone, locally including conglomerate and red shale, that is exposed on the Rakbah plain $150 \mathrm{~km}$ northeast of At Tā'if and northwest of Al Khurmah, where it rests on Precambrian shield rocks and underlies early Tertiary lakebeds and early Miocene basalt (Brown, Jackson, Bogue, and MacLean, 1963). The sandstone contains numerous Tigillites borings associated with small pelecypod casts. Although the outcrops are poorly exposed and mostly bound by faults, the sandstone is at least $50 \mathrm{~m}$ thick. Similar sandstone, tentatively correlated with the Khurma Formation (Madden and others, 1980), crops out beneath the Paleocene Umm Himar Formation $100 \mathrm{~km}$ east of At Tā'if and $75 \mathrm{~km}$ southeast of the widespread outcrops northwest of $\mathrm{Al}$ Khurmah. There the clastic rocks include a basal quartz-pebble conglomerate resting on the crystalline basement. The sandstone is crossbedded with foresets indicating a northerly transport direction at the time of deposition. It is generally friable and is locally cemented by secondary silica and iron oxides (Baghanem, 1972). Similar small outcrops of sandstone containing vertical borings are exposed at the edge of the crystalline shield on the coastal plain $70 \mathrm{~km}$ south of Jiddah. However, nearby sediments of Oligocene age suggest that these beds are younger than those exposed at Jabal Umm Himar. Likewise, sandstone beneath the Paleocene(?) Usfan Formation northeast of Jiddah should probably be identified with the Khurma Formation.

A precise age for the mature Khurma Formation is not possible to determine with the information available to us. The age could range from early Paleozoic to Tertiary, as the Sabellarifex dufrenoyi (Tigillites dufrenoyi Rouault) occurs in Ordovician sandstone in Jordan (which should be named Tigillites because it differs from Sabellarifex by its distinct annulation (Hantzschel, 1975, p. W38-39)). Tigillites(?) borings occur in all the lower Paleozoic sandstones fringing the shield in Saudi Arabia, though many are not annulated as are the borings in the Khurma Formation. The lower Paleozoic sandstone extends south onto the shield in numerous small, isolated outliers as far south as the latitude of $\mathrm{Al}$ Madinah (lat $24^{\circ} 30^{\prime} \mathrm{N}$.), $270 \mathrm{~km}$ northwest of the Khurma Formation, as well as in a single outcrop at Jabal Tinn northeast of At Tā'if. However, these sandstones are massive, evenly bedded, and 
devoid of Tigillites vertical boreholes. Certainly the Khurma clastics are pre- or early Paleocene, as they are disconformable below the Paleocene Umm Himar Formation (Madden and others, 1980). General evidence, including lithology and stratigraphic position, suggests a Cretaceous age similar to the fluviatile (Nubian-type) clastics widespread in east Africa, Yemen, and Jordan.

These beds were first observed by Richter-Bernburg and Schott (1954, p. 44, 45), who described silicified limestone with red sandy shale, greenish-gray, marly, somewhat silicified shale, coarse-grained sandstone, and purple and green variegated shale over a distance of about $21 \mathrm{~km}$ beginning $13 \mathrm{~km}$ west of $\mathrm{Al}$ Khurmah, to which they gave the name "Khurma Series." Most of these lithologies can be assigned to the Umm Himar Formation. As these beds strike N. $30^{\circ}-80^{\circ} \mathrm{E}$. and are underlain by greenschist and granitic gneiss striking $\mathrm{N}$. $30^{\circ}-60^{\circ}$ E., Richter-Bernburg and Schott (1954) considered them younger than the basement. The only vertical non-Precambrian beds we have seen in this area are marginal to feeder necks and hypabyssal intrusions of basalt. In a western belt, gastropod molds, undoubtedly from the overlying lakebeds, are associated with the Umm Himar deposits. Because of the vertical dips, it is problematic that those writers saw the quartzitic sandstone underlying the Paleocene. This sandstone we have tentatively identified with the Lower Cretaceous Nubian Sandstone of Egypt and the Sudan. An alternative explanation would require tectonism in the $\mathrm{Al}$ Khurmah area during late Mesozoic or early Tertiary, for which we have seen no evidence elsewhere on the upland plateau of the shield, although there is normal faulting of the sandstone.

\section{MESOZOIC-CENOZOIC SEDIMENTARY ROCKS}

\section{USFAN FORMATION}

\section{DEFINITION}

Tertiary fossiliferous sediments in western Saudi Arabia were first shown on a map prepared as part of a search for a water supply for Jiddah (Steineke and others, 1944). Cropping out in the low hills east and north of Jiddah, the continental and marine clastics contain bivalve and gastropod fossils that were identified as of Eocene age (R.A. Bramkamp, oral commun., 1944).

Subsequently, Karpoff (1956) published a description of the sediments. He divided them into two units: the Usfan Series, a red continental series of clastic rocks including a light-colored lacustrine limestone with rare leaf imprints as exposed at 'Usfān Pass (lat $21^{\circ} 54.8^{\prime}$ N., long $39^{\circ} 21.2^{\prime}$ E.) $50 \mathrm{~km}$ northeast of Jiddah, and the
"Shumaysi Sandstone," a light-colored quartz-pebble sandstone, locally iron-stained and calcareous with a silicious limestone at the top, seen $45 \mathrm{~km}$ east of Jiddah on the Makkah road. A year later, Karpoff (1957b) published a more comprehensive account following identification of a marine fauna considered to be of Maestrichtian (Late Cretaceous) age from 'Usfān Pass which led him to assign that series to a MaestrichtianEocene(?) span. The overlying red clastics of continental origin, the "Shumazi (Shumadi) Series," he thought might be Oligocene-Miocene, based on resemblance to the Tertiary of north Africa (Karpoff, 1956).

On the 1963 geologic map of the Southern Hejaz quadrangle (Brown, Jackson, Bogue, and Elberg, 1963), the Usfan Formation is shown in grabens trending more or less en echelon, parallel to the Red Sea rift, the Tertiary sediments flanked by Precambrian rock and overlain by Pliocene basalt. The nonmarine upper beds were split off and assigned to the Shumaysi Formation, following Karpoff.

\section{OCCURRENCE AND THICKNESS}

The Usfan Formation crops out in steeply dipping beds for an exposed thickness of 75 to $100 \mathrm{~m}$ across the Al Madīnah-Makkah road at 'Ufān Pass. Additional exposures rim the basalt northwest of the pass for 20 $\mathrm{km}$, and marine or littoral sediments $170 \mathrm{~m}$ thick, which may be an extension, crop out in a secondary graben at Hुaddat ash Shām, $45 \mathrm{~km}$ southeast of 'Usfān and 60 $\mathrm{km}$ northeast of Jiddah. Intermittent exposures extend north and south of Ḩaddat ash Shām for $32 \mathrm{~km}$ and locally contain some marine fossils, but the sediments are almost entirely sandstone and were shown on the 1963 geologic map as part of the overlying Shumaysi. They were mapped by AUXERAP (1967) as an extension of the Usfan Formation, perhaps on the basis of marine foraminifera, but if the lower part of the Shumaysi is Eocene, these outcrops should not be assigned to the Usfan.

\section{LITHOLOGIC CHARACTER}

The Usfan at the type locality (fig. 43) at 'Usfān Pass $4 \mathrm{~km}$ northwest of the village of 'Usfān is mostly finegrained sandstone, cemented by iron in some layers. It has a fossiliferous and nodular gray limestone near the base and some glauconitic fine sandstone near the top. The uppermost bed is $6 \mathrm{~m}$ of bedded phosphatic chert that is weathered; elsewhere it is lateritic (fig. 43). Poorly exposed sandstone beneath the lower fossiliferous limestone may be lowermost Usfan, but the beds have tubular borings across the bedding and contain plant fossils and ferruginous layers which may be 


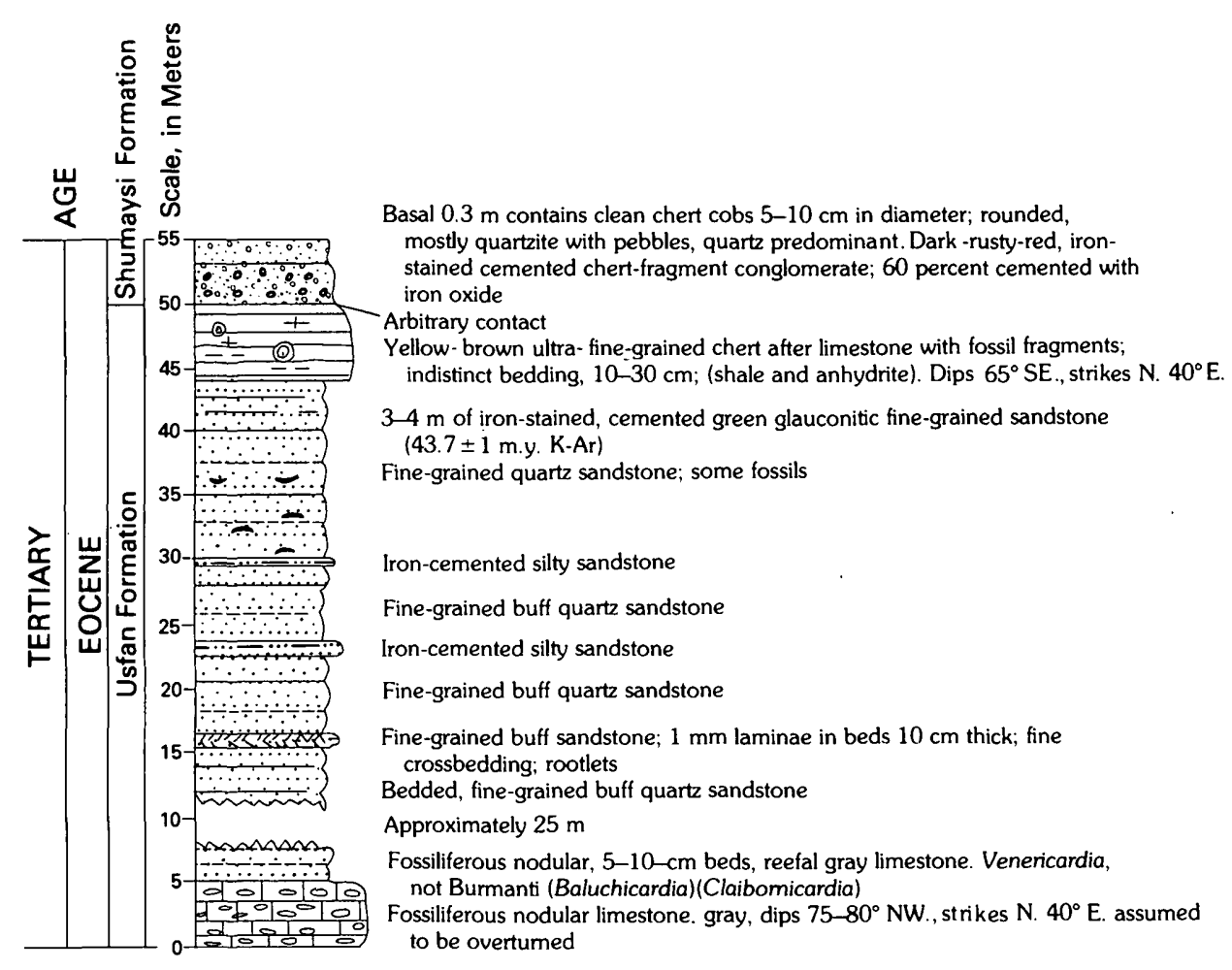

Figure 43.-Type section of the Usfan Formation at 'Usfān Pass.

either younger or older than the marine beds that are designated Usfan. The structure is obscure.

\section{NATURE OF CONTACT}

The Usfan Formation rests nonconformably on Precambrian crystalline rocks where the nonfaulted basal surface can be observed. It has been dropped down in a narrow graben, so sandstone or conglomerate adjacent to the crystalline rocks may be part of the overlying Shumaysi or, more likely, equivalent to the older Khurma Formation. The nonmarine Shumaysi disconformably rests on the Usfan; the basal sediment is a ferrous, iron-cemented, fragmental conglomerate composed of clasts of Usfan chert, limestone, shale, and pebbles and boulders of basement rocks.

\section{PALEONTOLOGY AND AGE}

The bivalves determined by $\mathrm{D}$. Mongin on which Karpoff (1957b) based his Maestrichtian age are as follows:

Cardita (Venericardia) ameliae Peron

Cardita (V.) ameliae var. orfellensis Rossi-Ronchetti

Corbula striatuloides Forbes

Lucina cf. L. desioli Ch-Rispoli
This fauna came from about $10 \mathrm{~m}$ above the base of a dark-gray limestone $3 \mathrm{~km}$ north of the village of 'Usfän (Karpoff, 1957b). Because the Maestrichtian age did not agree with the earlier determination by Bramkamp, we visited the outcrops and collected both bivalves and gastropods.

From two localities: the type outcrop, $3 \mathrm{~km}$ north of 'Usfān, and another exposure $9 \mathrm{~km}$ to the northwest: Venericardia sp. large and small (possibly two species)

Phacoides (Miltha?)

\section{Mesalia sp.}

Turritella sp.

These were studied by F.S. MacNeil (USGS, written commun., 1954), who reported as follows:

If these fossils were from North America, I would say that a Paleocene or lower Eocene age is indicated. The large Venericardia recalls $V$. wilcoxensis Dall, V. smithi Aldrich, and V. bulla Dall, species from the Paleocene of the Gulf Coast. The later is related to "Cardita" beaumonti from the Paleocene of India. The Arabian species looks to me more like these Paleocene species than it does the middle and upper Eocene species, V. alticostata (Courad). Even these likenesses might not be significant, however, considering that Arabia is nearly half way around the world.

Large Mesalia of the type found in the Arabian material are also characteristic of the Paleocene and lower Eocene of the Gulf Coast. The Arabian Mesalia is very similar to $M$. mavericki Gardner from the Paleocene of Texas and to $M$. wilcoxiana (Aldrich) from the 
Paleocene of Alabama. A smaller species with more spirals, $M$. alabamiensis (Whitfield), occurs in the lower Eocene (Wilcox) of Alabama.

The Turritella also appears to be close to an Alabama Midway species, T. alabamensis Whitfield.

The Phacoides is also similar to one described from the Paleocene of Texas by Gardner as Phacoides (Miltha?) albaripa.

In spite of these similarities with the Gulf Coast Paleocene, I would not trust it entirely until comparison with faunas from Egypt, India, and east Africa has been made. It is certainly true that some species groups and some generic assemblages which characterize the lower Tertiary of North America, are found in much younger beds, and even Recent, in the Indo-Pacific region."

Subsequently, MacNeil commented further:

Finlay and Marwick [New Zealand Geological Survey Paleontology Bulletin 15, 1937] figures a gastropod as Kaitangata hendersoni which may be close to the Mesalia in the Arabian material. The New Zealand fossil is from the Wangaloan beds, dated as Danian (supposed to be between our Paleocene and our Upper Cretaceous).

[L.R.] Cox (Annals and Mag., ser. 11, v. 1, 1938) described a species as Venericardia daviesi. This, as he says, is related to "Cardita" beaumonti. It comes from the Hangu shale of northwestern India which he assigns to the "Lower Eocene, Montian?. The Montian is the lower part of the Paleocene of Belgium."

Dr. Ralph Stewart examined the same collection and agreed with MacNeil's evaluation of Paleocene or early Eocene age. However, because of the long distances from North American fossils, he suggested that L.R. Cox of the British Museum could offer a valuable opinion. Cox's study, in which he concentrated on the gastropods, led him to comment as follows:

Turritella delettrei Coquand. A single broken Turritella is referred to this species; originally described [Coquand, 1862, Geology and paleontology of the southern region of the Province of Constantine, p. 266, pl. 30, figs. 1 and 2] from beds considered to be Lower Eocene (Suessonian) in age. The species is characterized by the presence of a single prominent, rounded cord adjoining the adapical suture of each whorl. The growth lines form a sinus just below the cord. The surface of the last whorl is quite flat between the latter and the abapical suture.

Calyptraea cf. aperta (Solander). A single eroded cast of a Calyptraea does not seem distinguishable from Solander's species. The European range of $C$. aperta is from Ypresian to Priabonian, and in Egypt it occurs in the Upper Libyan and Mokattam stages of the Eocene. No species of Calyptraea has been reported either from the Venericardia beaumonti beds or from the Eocene of India and Pakistan. The genus occurs in the Midway group of Texas. Its identification in Upper Cretaceous beds in England and elsewhere is doubtfully correct. (Locality A). Locality A is the type locality for the Usfan."

Phacoides sp. indet. A crushed, orbicular Phacoides of medium size is not clearly identifiable with any species described from the Eocene of the Middle East or Mediterranean area (Locality A).

Commenting on the age of the fauna, L.R. Cox (written commun., 1957) states:

There is insufficient evidence for arriving at a definite conclusion as to the stage of the Eocene represented. Of the species definitely identified, Mesalia fasciata is long ranging, and the evidence of Turritella delettrei rests upon the Suessonian (Lower Eocene) age assigned (on unknown grounds) to the formation in which it was originally found. The smaller Venericardia has been referred, with qualification, to Venericardia sindensis Cox, authentic modern records of which are from the Middle Eocene (Lutetian), although it may possibly also occur in the Lower Eocene (Ypresian), the stage to which the Laki beds belong. It is probable that the Calyptraea is not earlier than Ypresian. As the large Venericardia is a new species, it throws no light on geological age.

I am inclined to dismiss the suggestion that the formation is as old as Paleocene. The presence of Turritella delettrei, if this species is correctly stated to have been found in the Lower Eocene in northern Africa, rather suggests that the age of the formation is lower Eocene (Ypresian).

More recently, Dr. Druid Wilson (written commun., 1979) examined specimens from the 5 -m bed of gray limestone near the base of the exposure at 'Usfān and identified the larger bivalve as Venericardia (claibornicardia) sp., which is known with certainty only from the middle Eocene of Eastern North America and the Paris basin, but he feels that this occurrence in Saudi Arabia should not be used as a basis for a firm age determination.

In connection with the paleontological age of the Usfan, an ostracod fauna has been described at a depth of 1,637-1,638.7 $\mathrm{m}$ from an exploration well drilled on Maghersum Island off the coast of Sudan $220 \mathrm{~km}$ southwest of 'Usfān (Masoli, 1969). The ostracods include uppermost Cretaceous and Paleocene species according to Masoli, who also identified three foraminifera. Of the foraminifera, one, Cibicides sp., was found in the Haddat ash Sham graben southeast of 'Usfān and was also identified by Ruth Todd (USGS, written commun., 1959). She stated that no age determination could be made as the form occurs in both Cretaceous and Tertiary beds. Furthermore, the external shells seem to have been replaced, suggesting redeposition.

Thus, it appears from the available fossil evidence that the Usfan Formation can be either middle or lower Eocene or Paleocene but most likely is not Maestrichtian. This view is further strengthened by two K-Ar ages, $43.7 \pm 1.0$ and $56.4 \pm 1.2$ m.y., obtained from glauconite near the top of the exposure at 'Usfān Pass (samples 34a and 34b, table 10; pl. 2). These ages are middle Eocene and latest Paleocene; as argon can be lost from the mica by sufficient burial, the Paleocene age seems more reasonable, but the $56.4 \pm 1.2 \mathrm{~m}$.y. age may represent a Paleocene detrital component in a middle Eocene deposit (Dalrymple and Lanphere, 1969, p. 172, 173). The percentage of potassium is between 3.02 and 3.60 , values that are lower than ideal for K-Ar ages (Hunziker, 1979, p. 60-62).

\section{CENOZOIC ROCKS}

\section{UMM HIMAR FORMATION (PALEOCENE)}

\section{DEFINITION}

Lakebeds questionably assigned to the Miocene on the 1963 geologic map (Brown, Jackson, Bogue, and MacLean, 1963), on the basis of freshwater gastropods, 


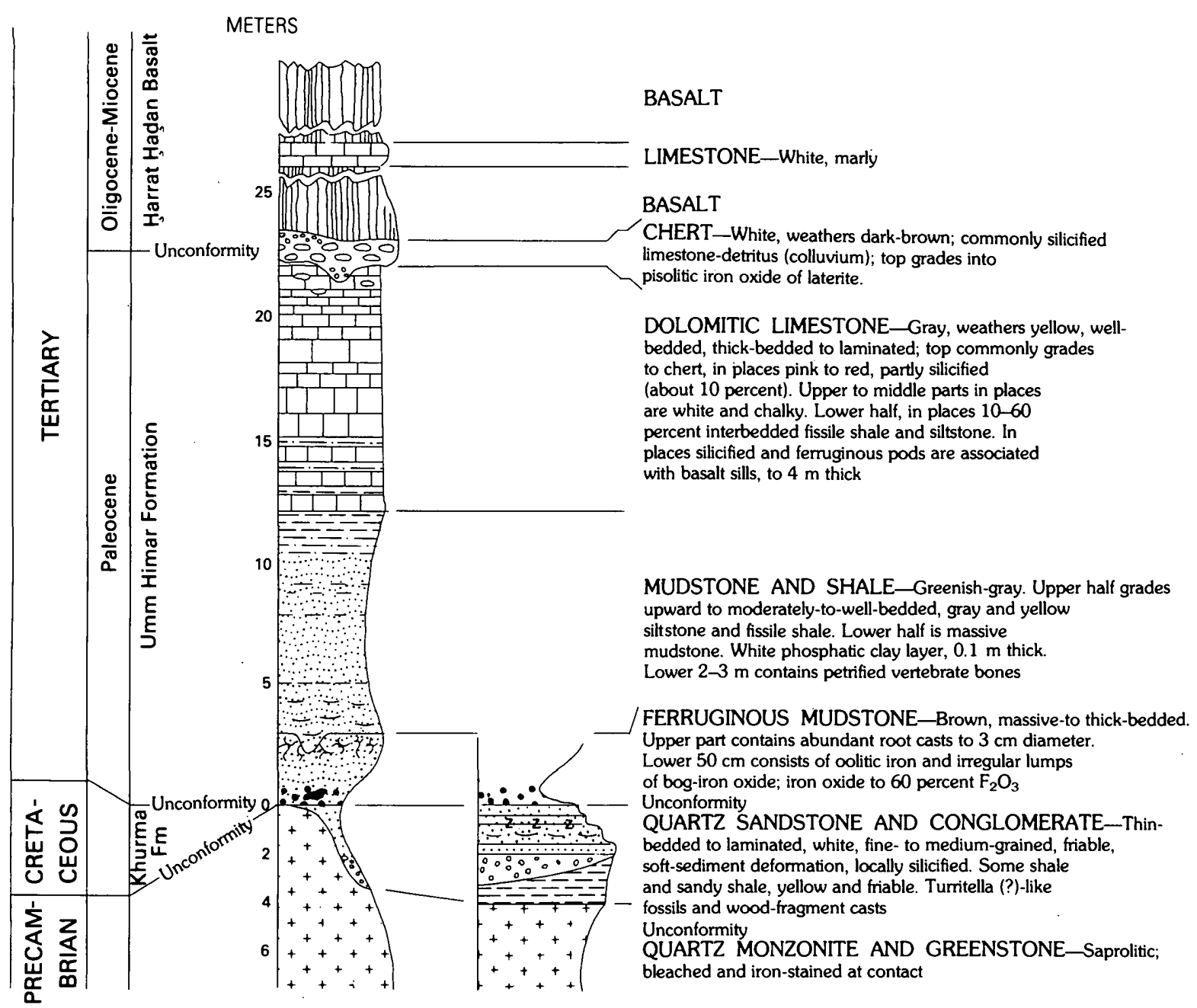

FIGURE 44.-Composite columnar section of the Umm Himar Formation and overlying and underlying rocks in the Jabal Umm Himar area. (From Madden and others, 1980.)

have since been studied and more extensively mapped (Baghanem, 1972; Baghanem and Mickelson, 1972; Gonzales, 1973). The lowermost beds now constitute the Umm Himar Formation as exposed on the eastern flank of Jabal Umm Himar, $100 \mathrm{~km}$ east of At Ta'if (Madden and others, 1980). Madden and others divided the Umm Himar into three members: a basal ferruginous mudstone; a middle greenish-gray and yellow siltstone and shale containing fossil vertebrate bones; and an upper dolomitic limestone (fig. 44). The middle member contains numerous vertebrate fossils including sharks, rays, fish, turtles, primitive crocodiles, and a primitive lung fish, a fauna considered mid-Paleocene and indicative of a coastal marine or estuarine environment (Madden and others, 1980).

\section{OCCURRENCE AND THICKNESS}

The lacustrine (and estuarine) beds are exposed intermittently within an elliptical ring enclosing an area
$145 \mathrm{~km}$ north-south by $60 \mathrm{~km}$ east-west within a wide, shallow basin on the Rakbah plain east of At Tà'if and surrounding the Harrat Hadan lava fields. The basalt beds rest on the older estuarine beds and are interbedded with younger sediments. Most of the older (Umm Himar) beds are exposed in an area of about $200 \mathrm{~km}^{2}$ along the southern and southeastern edge of the lava fields west and north of Turabah, but sporadic small outcrops lie within the lava fields or along wadi banks within the Hुarrat Hुaḑan and on the plains in low hills throughout the outer ring. The formation's average thickness is $22 \mathrm{~m}$.

\section{LITHOLOGIC CHARACTER}

The lowermost $3 \mathrm{~m}$ of ferruginous mudstone contains abundant root casts and oolitic and bog-iron ore. The middle $9 \mathrm{~m}$ includes vertebrate fossils in the lower 2 to $3 \mathrm{~m}$ of mudstone and grades upward into bedded gray and yellow siltstone and fissile shale. The upper $10 \mathrm{~m}$ is 


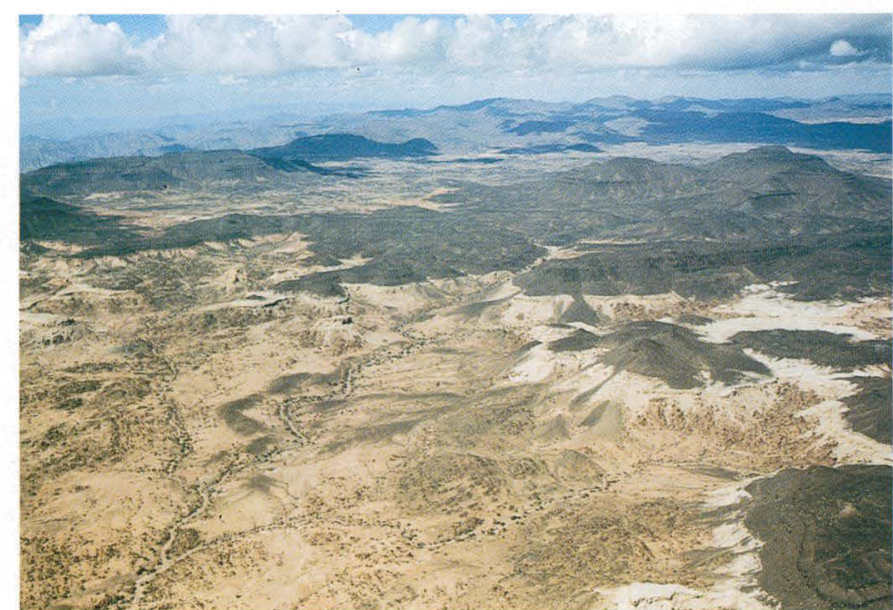

A

Figure 45.-Laterite under As Sarāt lavas. $A$, Laterite (white saprolite zone) weathered from underlying basement rocks and beneath As Sarāt lava (black); view to northeast near southwest end of lava plateau. Laterite is early Tertiary in age. $B$,

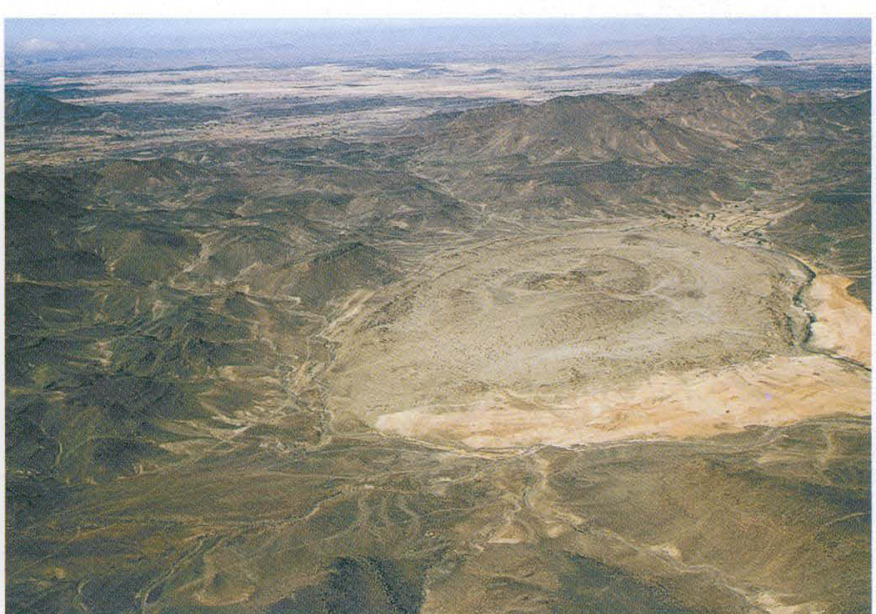

B

Calcareous feldspathoidal syenite plug in the midst of the As Sarāt lava plateau at $\mathrm{Al}$ Wārah. The syenite, with an early Miocene age of $22 \mathrm{~m} . \mathrm{y}$., transects the laterite and the lower beds of the As Sarāt basalt. (See table 10, samples 67, 68, 69; pl. 2.) dolomitic limestone and includes some interbedded shale and siltstone in the lower part; it grades into cherty limestone at the top.

\section{NATURE OF CONTACTS}

The Umm Himar unconformably overlies either deeply weathered quartz monzonite, andesitic greenstone, or the Khurma Formation, all beveled by the Hejaz pediplain to topography conducive to low coastal marshes and lacustrine sedimentation. The beds overlying the Umm Himar are likewise iron-rich soils corresponding to the widespread pre-Miocene saprolite and laterite found in the Sudan, Ethiopia, and the southwestern uplands of the Arabian Peninsula. Latest Oligocene or earliest Miocene latite and phonolite (22.2 \pm 3.5 m.y., sample 40 , table 10 ; pl. 2) intrude the Umm Himar Formation, and late Oligocene lower basalt flows (27.8 $\pm 1.4,26.6 \pm 1.3$ m.y., Arno and others, 1980b) overlie the Umm Himar, whereas younger, probably earliest Miocene, lakebeds are interlayered with the lavas.

\section{LATERITE AND SAPROLITE}

A deeply weathered saprolitic and lateritic profile representing a post-Mesozoic erosion interval underlies the As Sirat basalt on the crest of the 'Asir Mountains in southwest Arabia (fig. 45) (Overstreet and others, 1973, 1977). Of varying thicknesses ranging from a few centimeters to more than $50 \mathrm{~m}$, but mostly 20 to $30 \mathrm{~m}$, it is missing in places where the plateau basalt rests on the Wajid Sandstone and is thickest over Precambrian crystalline rocks rich in feldspar. The profiles are typical of those developed under tropical conditions, and they have a surface zone of pisolitic material, here goethite or kaolinite, grading downward to bleached clays, above a zone of enrichment in silica (silcrete); the lower section customarily is a saprolite (Overstreet and others, 1977 , p. 5). The most important clay minerals belong to the kaolinite group, with montmorillonite minor. Minor amounts of alunite were also discovered by Overstreet (1973). Hydrated ferrous minerals have concentrated in the upper lateritic zones where the underlying rocks contain ferromagnesian minerals or have been transported.

The age of the weathered zone is somewhat problematic. A minimum age is that for the base of the basalt, on the plateau of As Sirāt, $30.1 \pm 1.0$ m.y. (sample 69, table 10; pl. 2), using $\mathrm{K}-\mathrm{Ar}$ decay rates established in 1976 by the International Union of Geological Sciences. A similar situation exists on the northern Ethiopian plateau, where an age of $37.1 \pm 1.2$ m.y. (table 10) was obtained on a dike at Asmara, Ethiopia, which appeared to be intrusive into the lateritic red soils there underlying the plateau basalts. A recent analysis for a basal flow at Adigrat $130 \mathrm{~km}$ south of Asmara in northern Ethiopia, using the new decay constants, gave $30.0 \pm 0.7$ m.y. (Jones and Rex, 1974). In that area the laterite has been described as the upper part of the Amba Aradam Formation of Cretaceous age (Arkin and others, 1971, quoting M.M. Shumburo, 1968). Likewise, the Trap Series in Yemen (plateau basalts) have recently yielded ages of 29 to 20 m.y., and volcanic rock overlying the Medj-zir Series (Eocene) northwest of Şan'a' ${ }^{\prime}$ and in south-central Yemen have been dated at about 25 m.y. 
(Civetta and others, 1978). However, the lateritic profile is not shown in those areas, as it is limited to the basalt outliers northwest of the Jawf graben in extreme northwestern Yemen. Thus, it would appear that the As Sirat laterite could range in age from Cretaceous to mid-Oligocene (to about $30 \mathrm{~m} . \mathrm{y}$.). If the saprolite laterite in the Harrat Hadan area represents the same epoch as the As Sirat, the age span would be reduced to Paleocene-mid-Oligocene.

\section{SHUMAYSI FORMATION}

\section{DEFINITION}

The Shumaysi Formation was named by Karpoff $(1956,1957 a)$ for clastic sediments, mostly sandstone of continental and lacustrine origin, exposed along the western flank of Wādī Shumaysi, a southern distributary of Wādī Fāţimah $50 \mathrm{~km}$ east of Jiddah. On the southern Hijaz geologic map (Brown, Jackson, Bogue, and MacLean, 1963), the Shumaysi Formation is shown extending intermittently beneath the Tertiary basalt flows in the Usfan graben from Wādi Khulays south to lat $21^{\circ} \mathrm{N}$. mostly as outliers on the crystalline basement. It is exposed farther east in the Haddat ash Sham graben and questionably includes sediments beneath basalt in the Tihāmat foothills as far north as lat $22^{\circ} \mathrm{N}$. The type section at Wādi Shumaysi was divided by AlShanti (1966) into three members, a lower unit of mostly sandstone and conglomerate, a middle member including two oolitic iron-ore beds, and an upper member dominantly composed of shale and siltstone. A reference section has been designated on the west flank of Wādī al Fajj $20 \mathrm{~km}$ northwest of Wādī Shumaysi and north of Wādī Fâţimah, where the lithology is similar, where underlying crystalline basement and overlying basalt flows are likewise exposed, and where the identified fossils have been found.

\section{OCCURRENCE AND THICKNESS}

The widely scattered and separated outcrops of the Shumaysi beds range in thickness from 77 to $191 \mathrm{~m}$ and are $148 \mathrm{~m}$ thick at the type locality (fig. 46), as measured by Al-Shanti (1966). They are faulted, ramped, and skewed in a clockwise direction, the dips mostly to the northeast within the Usfan graben and to a lesser degree in the Haddat ash Sham graben, where nearly $200 \mathrm{~m}$ of sandstone crops out above a thin sandy shale containing ferrous oolites, Tigillites borings, and marine fossils, possibly reworked. Below the fossiliferous beds, which are $170 \mathrm{~m}$ thick, a coarse crossbedded sandstone may be either part of the Usfan Formation or sandstone of the Khurma Formation (AUXERAP, 1967).

\section{LITHOLOGIC CHARACTER}

The three members of the Shumaysi have been described and mapped in detail by A.M.S. Al-Shanti (1966) and M.A. Yamani (1968). The lower beds are clastics ranging from pebbly to conglomeratic and from siltstone to fine-grained sandstone. Considerable brown, thinly laminated shale is also present, especially in the outcrops north of Wādi Fäţimah. The middle member includes two beds of oolitic iron ore interbedded in shale and siltstone. Yamani (1968) has shown that the iron-ore beds, which range in thickness from a thin layer to $5 \mathrm{~m}$, are best developed in the vicinity of the Precambrian Fatimah Group, which crops out in the hills along the north side of Wādi Fàtimah. His measurements of foresets and ripple marks in the Shumaysi Formation indicate that current directions for the water-laid clastics radiate from the outcrops of the Fatimah Group, which he considers to be the source of the iron. However, the immediate source is laterization of a paleosol. In addition to the clastic material, the upper beds include volcanic ash, which crops out about $40 \mathrm{~m}$ below the top of the formation, and calcareous sediments. The beds above the volcanic ash are especially fossiliferous.

\section{NATURE OF CONTACTS}

In most outcrops where the basal sandstone of the Shumaysi Formation is exposed, it rests unconformably on Precambrian crystalline rocks, except where the Usfan Formation can be identified below. Where both formations can be seen, a lateritic breccia containing numerous vertical borings above glauconitic and phosphatic beds of the Usfan may represent a time breaka diastem-or a longer disconformity between the Usfan and Shumaysi Formations. Similar lateritic and siliceous beds overlie the Umm Himar Formation of Paleocene age $175 \mathrm{~km}$ east of Wādi Shumaysi. The overlying beds above the Shumaysi Formation are basaltic and andesitic lava flows of late Oligocene age, based on $\mathrm{K}$-Ar age determinations.

\section{PALEONTOLOGY AND AGE}

The Shumaysi Formation contains terrestrial, lacustrine, and marine fossils. Most of the pelecypods and gastropods are steinkerns. However, L.R. Cox (written commun., 1957) was able to identify a marine gastropod, Turritella (Protoma) cathedralis Brongniart, var. suprainflata Sacco of Oligocene-Miocene age in sandstone, near the top of the Shumaysi a few meters below a basalt flow dated at $32.6 \pm 2$ m.y. (K-Ar whole-rock; sample 35 , table $10 ;$ pl. 2). Samples submitted by Al- 


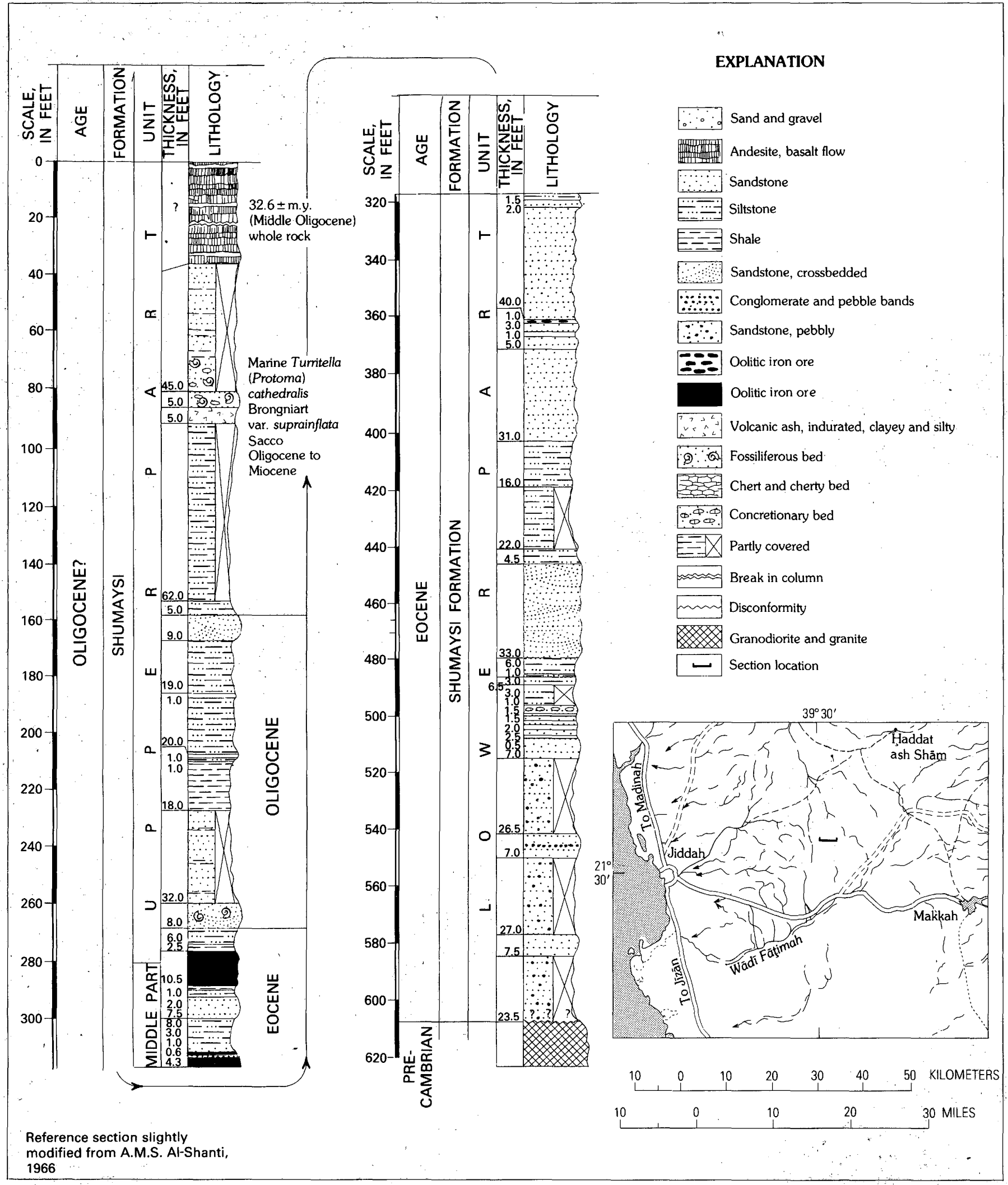

FIGURE 46.-Type referenced section slightly modified from locality of the measured Shumaysi Formation. (From Al-Shanti, 1966.) 
Shanti and identified by Cox as Lanistes, late EoceneHolocene age in northeastern Africa, Cox considered "differing slightly from the upper Eocene L. antiqus, but on the other hand not identical with any described form of later date." Cox also identified freshwater gastropods Melanoides (Tarebia) aff. M. barjacensis (Fontannes) as of Oligocene age and Ischurestoma sp. as of Eocene to Oligocene age.

Fossils from the Haddat ash Sham graben, including Haplophragmoides sp. and Cibicides sp., could not be assigned an age (Ruth Todd, USGS, written commun., 1960). Todd stated that "the Cibicides seemed to have been replaced, and the Haplophragmoides was laterally compressed and probably filled. The rarity of the specimens together with their replaced and filled conditions suggest they may be redeposited fossils." Likewise I.G. Sohn (USGS, written commun., 1960) reported that no age assignment could be made from very poorly preserved ostracodes from the outcrops in the Haddat ash Shām area. However, the foraminifera Operculina alpina multseptata Silvestri reported in the sedimentary section at Ar Rawdah on the north bank of Wādi Fāţimah in the Haddat ash Sham graben (AUXERAP, 1967) was originally assigned to the lower Eocene of Libya (Silvestri, 1937). In Arabia it may represent uppermost Usfan or may have been redeposited in the Shumaysi Formation during late Eocene or Oligocene. More recent work includes identification of pollen grains considered of early Eocene age (Moltzer and Binda, 1981). Fossiliferous and arenaceous beds 8 $m$ above the upper oolitic iron stratum are possibly the base of the Oligocene beds which carry Oligocene gastropods higher in the section. These fossiliferous beds in turn are capped by basalt of late Oligocene age (32.6-25.8 m.y., K-Ar).

\section{BAID FORMATION}

\section{DEFINITION}

The continental Baid Formation was named after exposures seen along Wādī Bayd (lat $17^{\circ} 37^{\prime}$ N., long $42^{\circ} 22^{\prime}$ E.) while mapping the Tihāmat 'Asīr in 1951 (Brown and Jackson, 1959). Later it was also found farther north on the Tihāmat ash Shām (Brown and Jackson, 1958). Most of the exposures of the siliceous nonmarine and volcaniclastic sedimentary beds and associated eruptive rocks are exposed along the banks of the lower courses of wadis flowing toward the Red Sea on the coastal plain, and at the eastern edge of the coastal lowlands in more-or-less continuous belts between wadis. Following our reconnaissance survey, the formation was studied in much more detail by AUXERAP (1967). As the discovery exposure repre- sents only a fraction $(40 \mathrm{~m})$ of the distinctive Baid lithology, a better stratotype was designated $15 \mathrm{~km}$ northwest of Wādī Bayd near Ad Darb along the south side of Wādi 'Itwad, where dissection of the coastal alluvium has exposed 1,200 $\mathrm{m}$ of the underlying Baid (fig. 47) (Gillmann, 1968). The Baid Formation has since been studied in detail from $10 \mathrm{~km}$ south of Wādī Qununah to north of Wādī ad Duqah, the northernmost $75 \mathrm{~km}$ of outcrop (Greenwood, 1975d; Hadley, 1975a, 1979).

\section{OCCURRENCE, THICKNESS, AND LITHOLOGY}

Intermittent exposures of the Baid Formation extend from south of Wādī Jìzān at lat $16^{\circ} 50^{\prime} \mathrm{N}$. northwestward to lat $19^{\circ} 48^{\prime} \mathrm{N}$., a distance of $370 \mathrm{~km}$ along the strike parallel to the coast. The exposures are in belts as much as $10 \mathrm{~km}$ wide and dip generally $30^{\circ}$ toward the Red Sea underneath surficial alluvial and coralline deposits. The exposure on Wādì Jīzān was first, and correctly in our opinion, interpreted as septa within hypabyssal dikes and interbedded flows of dacite, diabase, and obsidian (Gillmann, 1968). More recently, the siliceous argillite (silicite) of the Baid Formation has been described as resting discomformably on a dike swarm of the igneous Miocene Tihamat-Asir Complex, which is considered part of an ophiolitic suite (Coleman and others, 1979). Certainly the siliceous and volcaniclastic rocks are discomformable above the Khums Formation and are intruded by diabase dikes that are part of a Miocene igneous complex (fig. 48).

At Wādi Bayd, $40 \mathrm{~m}$ of the Baid Formation is exposed, striking N. $30^{\circ} \mathrm{W}$. and dipping $14^{\circ}$ southwest, $2 \mathrm{~km}$ downstream from agglomerate and metamorphosed mafic igneous rocks, all intruded by diabase. The Baid Formation is here cut by normal faults which also shear the diabase dikes (fig. 48). At Wadi 'Itwad near Ad Darb, the most complete section of the Baid Formation is exposed between 5 and $8 \mathrm{~km}$ west of the Precambrian rock on the coastal plain. According to Gillmann (1968), it is " $1,200 \mathrm{~m}$ of chiefly gray, buff, red, or green silicite with fish fossils and intercalated green or violaceous shale (chlorite, illite and montmorillonite), volcanic tuffs, and diabase sills" (fig. 47).

Crystalline schists of the basement extend to the coast north of Ad Darb, where they are nearly covered with Quaternary basalt and ash of the Al Birk lava fields. The Baid is faulted off, apparently as a result of sinistral movement on a Red Sea transform fault at Ad Darb (Coleman and others, 1979), but another coastal plain reentrant, the Tihāmat ash Shām, extends north of the Al Birk harrat and the Baid Formation appears again along the eastern edge of the coastal plain. At Wādī Halì, about $60 \mathrm{~m}$ of argillite, silicite, conglomer- 


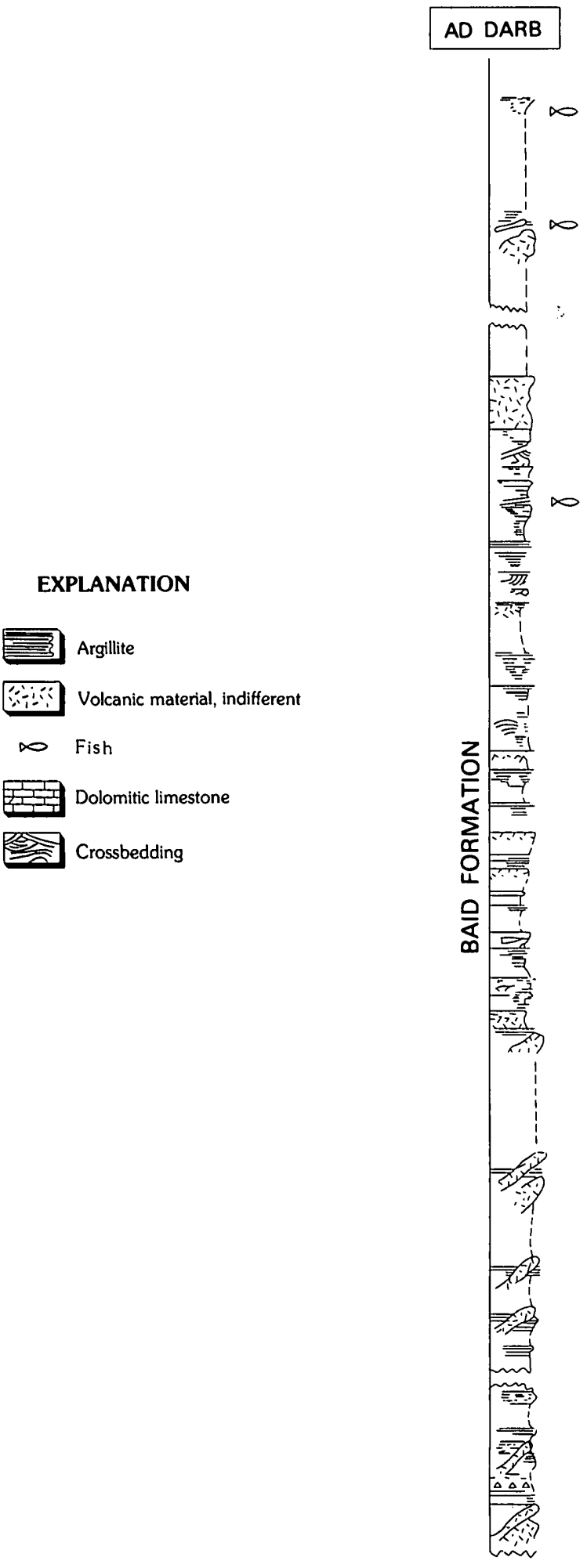

FIGURE 47. - Stratotype section of the Baid Formation, near Ad Darb. (Modified from Gillmann, 1968.) ate, and related volcaniclastic rocks are exposed in low hills on the coastal plain north of the wadi. The Baid Formation is also exposed along the southern flank of Wādi Yiba, $20 \mathrm{~km}$ farther north, where the beds are more calcareous, especially in the upper part. Still farther north, on the south flank of Wādi Qununah, the exposed lower $20 \mathrm{~m}$ of the Baid Formation is a polymict conglomerate with a thin bed of limestone near the top; it is in fault contact with Precambrian quartz diorite. The upper $17 \mathrm{~m}$ is argillite, the remainder, except $2 \mathrm{~m}$ of sandstone at the base, being covered with scree (Hadley, 1975a). The volcaniclastic rocks continue northward across Wādì Lumah, Wādī Ahsibah, and Wādī ad Duqah (Brown and Jackson, 1958). The lower part of the northernmost exposure 15 $\mathrm{km}$ north of Wādi ad Duqah is composed of tuff below tuffaceous siltstone and limestone (Hadley, 1979). Although a total thickness cannot be measured because of the alluvial cover and the faulting of the formation, the northern extension may be as much as $3,000 \mathrm{~m}$ thick, of which $275 \mathrm{~m}$ in the midportion consists of basalt flows and diabase dikes and sills.

The rock, mostly volcaniclastic, ranges from coarse conglomerate, in places agglomeratic, through graywacke sandstone to silt and clay, the last in many places being indurated to shale and argillite, which make up the majority of the exposures. Considerable tuff, both lithic and vitric, and chert are present, and most sediments are siliceous, the silica probably derived ultimately from ash. The southernmost outcrops in the Jizan coastal plain include flows and dikes of diabase, dacite, and obsidian (Gillmann, 1968).

The Baid Formation rests disconformably on the Jurassic Khums Formation at Wādī Jīzān, according to Gillmann (1968). Everywhere it is intruded by the Tihamat-Asir dike swarm, indicating that dikes represent a younger hypabyssal intrusive episode. The Baid is disconformably overlain by alluvial deposits, mostly outwash from the 'Asīr scarp mountains.

PALEONTOLOGY AND AGE

The Baid Formation contains few fossils, Gillmann reporting only ostracods and fish impressions difficult to identify. Fish remains from the outcrops near Ad Darb were examined by D.H. Dunkle, U.S. National Museum (written commun., February 10, 1953), who commented as follows:

Included among this suite of specimens are representatives of two families of freshwater fishes of widespread distribution in Asia and Africa today. One of these fishes is a cyprimid or minnow, which falls within the structural range of the recent genus Barbus. The other is of a cichlid, close, if not identical to, the living Tilapia. The incompleteness of the present specimens makes specific comparisons unfeasible. The past history, although very incompletely known, suggests a middle Tertiary age for this occurrence-late Oligocene or Miocene (Brown, 1970). 


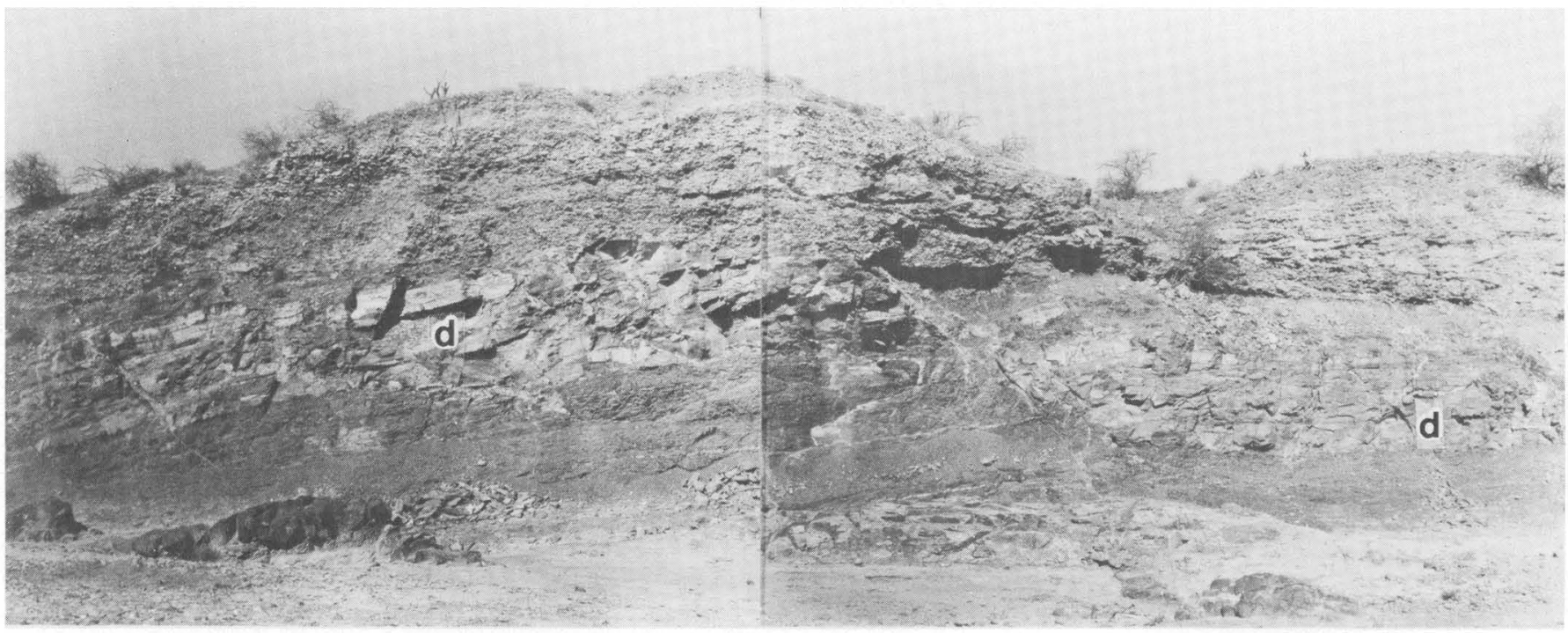

FIgURE 48.-Baid Formation at Wādī Bayd. About $40 \mathrm{~m}$ of tuffaceous sandstone and concoidal fracturing, siliceous, red, green, and gray shale containing calcareous concretions is exposed over an older agglomerate. Section is cut by a diabase sill (d) of the Tihamat-Asir Complex.

A K-Ar age of $19 \pm 0.6$ m.y. (sample 54, table 10, fig. 49) for the diabase sill in the middle Baid of the Tihamat ash Sham coastal plain agrees within instrumental error with three other ages of these sills reported by Coleman and others (1979), that is, an early Miocene age.

\section{JIZAN GROUP}

Recently, the middle Tertiary stratigraphy of the southern Red Sea coastal plain has been clarified and revised (Schmidt and others, 1983). The siliceous nonmarine and volcaniclastic sedimentary rocks and associated eruptive rocks exposed along the coastal plain between Jiddah and the Yemen border are assembled into the Jizan Group. Named for the Jizān coastal-plain region, the Jizan Group is divided into five formations, of which one is the Baid Formation, now redefined and restricted to rocks of a siliceous lakebed facies.

The five formations of the Jizan Group are as follows: a thin, local, basal sandstone formation; a thick formation of mafic volcanic rocks; a silicic, commonly welded tuff, volcanic formation; the widely recognized lakebeds of the Baid Formation; and an upper, thick, mafic to felsic volcanic, largely pyroclastic formation. The upper volcanic formation is everywhere covered across an angular unconformity by younger coastalplain sediments. The redefined Baid Formation is buff to light-reddish-brown, thin-bedded to laminated, siliceous, tuffaceous siltstone and claystone constituting a freshwater lakebed facies of the predominantly volcanic Jizan Group. The Baid rocks form a contiguous unit and in part interdigitate with the volcanic rocks of the overlying and underlying formations of the Jizan Group.

The 1:1,000,000 scale of the geologic map (pl. 1) is appropriate only to show the Jizan Group, but where the Baid Formation is predominant, as in the $\mathrm{Al}$ Qunfudhah region, the group is represented on the map by the Baid Formation. Where the volcanic formations either above or below the Baid are predominant, as in

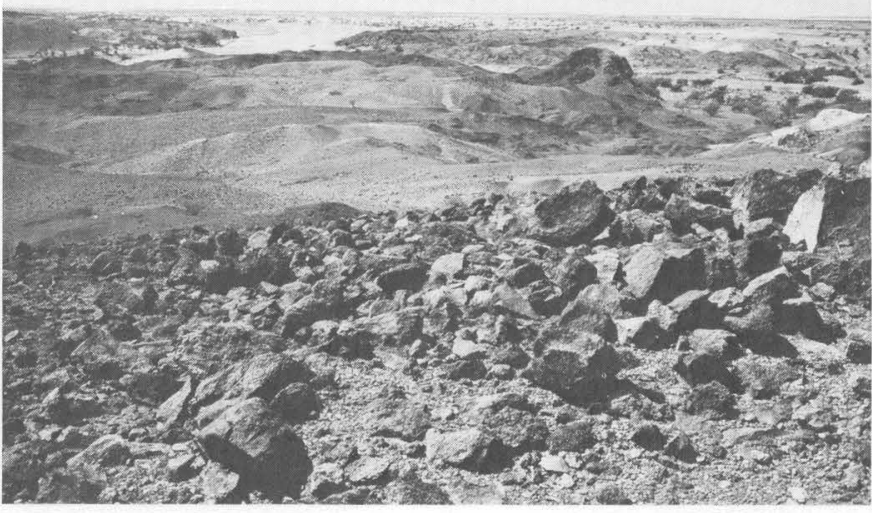

Figure 49.-Exposure of the Bathan boulder conglomerate in the foreground overlying the Miocene Jizan Group in the middle distance. The Jizan Group here is altered siliceous and limonitic tuff of the Baid Formation. View downstream toward the Red Sea and along Wādī ad Duqah in the Jabal Shada quadrangle. 
the Jīzann region, the group is shown as a volcanic formation.

The Jizan Group variably overlies the Wajid Sandstone, Khums Formation, and Amran Formation in the Jīzān region. It overlies the Precambrian shield rocks in the Al Qunfudhah region and overlies rocks as young as the Shumaysi Formation at Jabal Sitā', south of Jiddah. The Jizan rocks are everywhere intruded by the mafic rocks of the Tihamat-Asir Complex. Accordingly, the Jizan Group is of late Oligocene to early Miocene age (about 30 to 20 m.y.; Schmidt and others, 1983).

\section{BATHAN FORMATION (MIOCENE)}

\section{DEFINITION}

The Bathan Formation at its type locality is named for Wādī Bathān, a tributary of Wādī Lìth, $15 \mathrm{~km}$ northeast of Al Lith (Hadley and Fleck, 1980a). The exposure adjacent to Wādī Bathāan was first described, though not named, by Brown, Jackson, Bogue, and MacLean (1963) as "dissected and not obviously related to present drainages." They believed that this boulder conglomerate may be much older than Quaternary. Brown mapped similar deposits east and northeast of - Jiddah (Brown, Jackson, Bogue, and Maclean, 1963), and Nebert and others (1974) described one of these deposits north of Wādī Fāţimah.

\section{LITHOLOGY AND THICKNESS}

The Bathan is a terrigenous, polymictic clastic deposit consisting of boulder, cobble, and pebble conglomerate, sparse beds of coarse-grained sandstone, and rare, thin beds of red siltstone. Clasts consist of many types of Precambrian rocks; especially conspicuous are Precambrian granite and clasts of silicic tuffs and flows of the Baid Formation. The conglomerate is poorly sorted and commonly chaotically immature; some thick, massive beds contain boulders as much as $2 \mathrm{~m}$ across. Many clasts are angular to subrounded. The thickness of the Bathan at its type locality probably well exceeds $700 \mathrm{~m}$.

\section{OCCURRENCE AND NATURE OF CONTACTS}

The Bathan, where mapped, underlies the Red Sea coastal plain or the adjacent low foothills. In most places it is poorly exposed; in fact, it is mostly identified by a flat surface of residual lag of coarse, resistant boulders (fig. 49). Its upper surface everywhere is an erosional surface and its base, where rarely exposed, rests on a compound erosion surface that cuts across the Baid Formation, early Tertiary laterite, and Precambrian basement as at Wādī Bathān. At Harrat
Tuffil (Shamā), the Bathan rests on rhyolitic volcanic deposits associated with the Baid Formation (Pallister, 1983). Where bedding is visible, the beds dip gently as much as $15^{\circ}$ either southwest or northeast in blockfault structures.

\section{PALEONTOLOGY AND AGE}

No fossils have been found in the Bathan. It is younger than the stratigraphically underlying early Miocene Baid Formation and tholeiitic dikes of the Tihamat-Asir Complex. The Bathan Formation is probably of about middle Miocene age, as it is the erosional product of rapid uplift of the Red Sea Escarpment (Hadley and Fleck, 1980a) during the middle Miocene (Schmidt and others, 1983). During future mapping the Bathan probably will be identified between Jiddah and the Gulf of Aqaba, and it will probably be found to lie beneath the Raghama Formation.

\section{RAGHAMA FORMATION (MIOCENE)}

\section{DEFINITION}

The Raghama Formation was mapped on three USGS Miscellaneous Geologic Investigations Maps: Geology of Wādī as Sirḩān (I-200A, Bramkamp, Brown, and others, 1963), Geology of the Northwestern Hijaz (I-204A, Brown, Jackson, Bogue, and Elberg, 1963), and Geology of the Arabian Peninsula (I-270A, USGS-ARAMCO, 1963). The type locality was chosen for the area where the most complete exposure of Miocene reef limestone, gypsum, and related clastics was found during the reconnaissance mapping.

Jabal ar Raghamah forms the western flank of the lower alluvial plain of Wādī 'Ifāl in the Ash Shifā' region east of the Gulf of Aqaba at lat $28^{\circ} 20^{\prime} \mathrm{N}$. The Raghama Formation is exposed from lat $28^{\circ} 10^{\prime} \mathrm{N}$. to $28^{\circ} 35^{\prime} \mathrm{N}$. and extends westward from the alluvial plain of Wādi 'Ifāl to the coast of the Gulf of Aqaba, the Maqnā-Al Bad' area (Bramkamp, Brown, and others, 1963).

These sediments were first described in 1952 by Mustafa Sadek, who investigated the occurrence of native sulphur earlier reported by Burton (1878). The outcrops were revisited the following year by H.J. Philby and R.G. Bogue (Bogue, 1953) to further study the occurrence of the sulphur in the gypsiferous beds. Bogue described the area as basal coarse grit and conglomerate overlain by "sandstone, siltstone, and shale with interbeds of limestone and shale which often contains some volcanic tuff." Sulphur was identified with gypsiferous sandstone in the upper beds. The next year W. Schott of the German Geological Survey 
described the following section (Richter-Bernburg and Schott, 1954, p. 32):

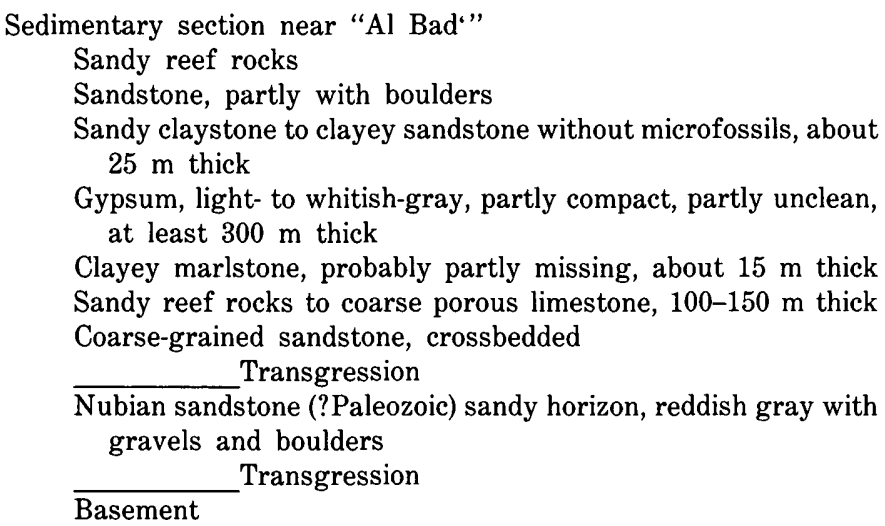

He questionably assigned a Miocene age to the beds above the Nubian Sandstone, including the gypsum.

In 1966, V.A. Trent and R.F. Johnson briefly investigated the economic possibilities of the area (Trent and Johnson, 1966). A study of the sulphur deposits was made by Bodenlos and Lari (1970), who divided the Raghama Formation into three major units: a basal coarse-grained clastic section; a middle unit of green marl, siltstone, and interbedded gypsum, with dolomite and sandstone in the upper part; and an upper unit of red sandstone containing a few thin beds of gypsum and siltstone.

A more detailed columnar section given by Bigot and Alabouvette, French Bureau des Recherches Geologiques et Minieres, of the outcrops in the Maqnā-Al Bad' area could be considered a reference section (Bigot and Alabouvette, 1976, fig. 6). However, they state that the Miocene in the Jabal ar Raghamah region (Maqnā-Al Bad' area) cannot be correlated with the Miocene detrital rocks of the "Jabal Dhaylan Series," exposed on the coastal plain $350 \mathrm{~km}$ southeast of Jabal ar Raghamah. At Jabal Dhaylān, the Tertiary beds have been studied in connection with mineralization at the base of the middle Miocene. This latest study divides the Raghama Formation, shown as a single formation unit on the 1963 USGS maps, into three units: a lower continental clastic section; a middle section of marine and littoral deposits, including reef limestones, gypsum, marl, and dolomite; and an upper section of clastics, oyster reefs, and gypsum.

\section{OCCURRENCE, THICKNESS, AND LITHOLOGY}

The Raghama Formation was shown on the 1963 USGS maps as outcropping intermittently from Yanbu' at lat $24^{\circ} \mathrm{N}$. to the Maqnā-Al Bad' area at lat $28^{\circ} 35^{\prime} \mathrm{N}$. The localities at Yanbu', Umm Lajj, Jabal Dhaylān, and north from $\mathrm{Al}$ Wajh were considered of
Miocene age on the basis of meager formal evidence. Thickness is variable, as might be expected from the marine transgressive reef and lagoonal evaporite type of sedimentation.

The basal conglomerate and sandstone are not present in all the outcrops, and the thickness where the basal conglomerate and sandstone have been measured ranges from less than a meter to more than $100 \mathrm{~m}$ (Bodenlos and Lari, 1970; Bigot and Alabouvette, 1976), with the maximum in the northern part of Jabal ar Raghamah near $\mathrm{Al} \mathrm{Bad}$. As much as $1,500 \mathrm{~m}$ of partly arkosic sandstone has been reported from Tīrān Island (Goldberg, 1963) in the northern Red Sea, $30 \mathrm{~km}$ south of the Raghama outcrop area east of the Gulf of Aqaba.

The middle member section is $400 \mathrm{~m}$ thick at the type locality at Jabal ar Raghamah (Bodenlos and Lari, 1970), but it ranges from $25 \mathrm{~m}$ to several hundred meters in thickness at various places along the Tihāmat (Bigot and Alabouvette, 1976). On Tiran Island the middle member is $250-450 \mathrm{~m}$ thick. According to Bodenlos and Lari (1970), the upper member of 100-200 $\mathrm{m}$ in the lowlands in the southwest corner of the peninsula south of Maqnā is comparable to the $250 \mathrm{~m}$ reported at Tirān Island off the peninsular point.

The section on Tīrān Island resembles the submarine Miocene of the Red Sea, as reported in various drilling records. The submarine section includes a midpart of evaporite more than $1,200 \mathrm{~m}$ thick consisting mostly of halite but including anhydrite above and below the salt beds that overlie $1,000 \mathrm{~m}$ of middle Miocene marl characterized by Globigerina. The sediments above the evaporite section proper are mixed beds of conglomerate, calcareous sandstone, limestone, clay shale, anhydrite, and salt, as would be expected above a dynamic sea floor.

\section{NATURE OF CONTACTS}

The Raghama Formation overlaps the crystalline basement in many places (fig. 50). More commonly it rests on sandstone and conglomerate of doubtful age, probably Oligocene, as near Jiddah, but farther north it overlies the possible Eocene or Cretaceous Nubian-type sandstone. At Jabal ar Raghamah, the sandstone beneath the marine beds was questionably considered to be Paleozoic by W. Schott (Richter-Bernburg and Schott, 1954). However, sandstone of Early Cretaceous Senonian age is faulted down in a small graben $5 \mathrm{~km}$ east oi Aqaba in Jordan (Bender, 1968), and similar beds occupy a similar graben in Arabia $5 \mathrm{~km}$ southeast of the graben in Jordan. The beds in Arabia consist of $10 \mathrm{~m}$ of conglomerate and coarse arkosic sandstone below $5 \mathrm{~m}$ of fine-grained sandstone, variegated in color and crossbedded (Trent and Johnson, 1966). This 


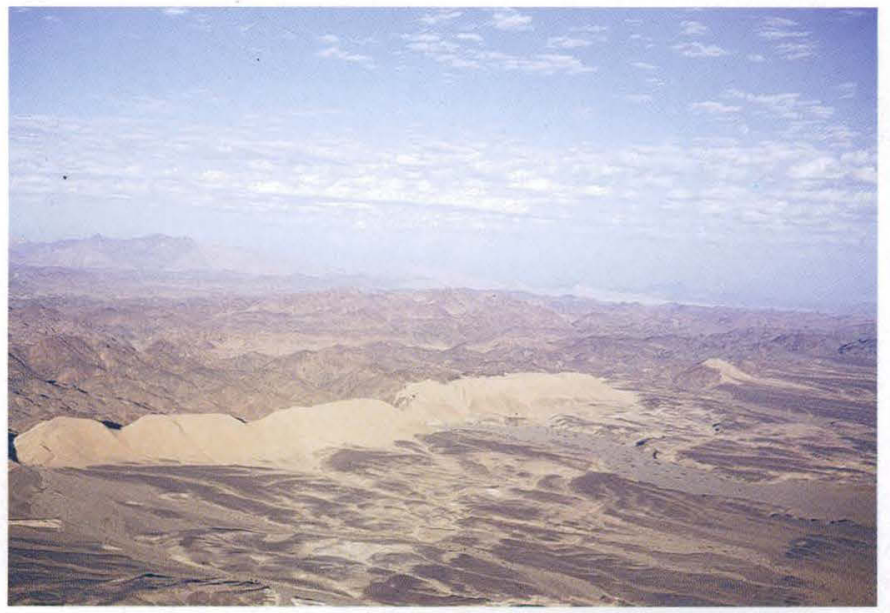

FiguRE 50.-The Raghama Formation, along the coast southeast of Dubā where Raghama carbonate beds dip southwest about $45^{\circ}$ along the northeast side of the coastal plain, reflecting the ramping of Miocene rocks. The underlying Precambrian crystalline rocks of the Ḩismá (Midian) block were rotated and elevated in a counterclockwise direction during the opening of the Red Sea rift.

is very likely composed of the same clastic material described by Bender (1968). These deposits may also be coeval with the basal sandstone at Jabal ar Raghamah, but without fossil evidence such a correlation is tenuous.

The Raghama underlies Quaternary terrace deposits, including Pliocene, Pleistocene, and Holocene coral reefs, in contrast to reefal coral of Miocene age that overlies the volcaniclastic sediments of the Baid Formation east of Al Qunfudhah. Northwest of Yanbu' the Raghama is overlain by Quaternary basalt flows.

\section{PALEONTOLOGY AND AGE}

The extensive exposures of Miocene reefs in the Yanbu' basin and northwestward toward Sharm al Khawr contain the coral Montastrea sp. cf. M. pedunculata (Duncan), previously known from the Miocene Gaj Formation of Pakistan, according to J.W. Wells (written commun., 1953), who identified it. Farther south and $10 \mathrm{~km}$ inland from Tuwwal, $80 \mathrm{~km}$ north of Jiddah, a dolomitic reef yielded the following foraminifera and algae (collected by B. Steenstra and identified by H.A. McClure):

Foraminifera

Borelis melo (common)

Heterostegina sp.

Taberina? malabarica

Peneroplis? farsensis

?Cycloclypeus
Algae

Halimeda cf. H. monile or incrassata

McClure (ARAMCO, written commun., 1975) considers the Borelis melo a widespread guide fossil for the upper Burdigalian, thus marking the approximate lower boundary of the middle Miocene (about 15 m.y. ago). The Tuwwal reef is correlated tentatively with the Globigerina Marl of the Gulf of Suez region.

Still farther south and east of Al Qunfudhah, $320 \mathrm{~km}$ south of Jiddah and $15 \mathrm{~km}$ inland near Sūq al Ahad, a coralline reef yielded the following corals, identified by J.W. Wells (USGS, written commun., 1953):

Favites sp. cf. F. profunda (Michelotti)

Montastrea or Plesiastrea sp.

Montastrea sp. cf. M. reussiana (M.E. \& H.) var. minor (Zuffardi)

The Favites sp. cf. F. profunda is known from the Miocene of Italy and Red Sea areas, and the Montastrea sp. cf. $M$. reussiana var. minor is known from the Miocene of Cyrenaica (Libya) and Red Sea areas, according to Wells.

In addition, from the fossils found at $\mathrm{Al}$ Qunfudhah F.S. MacNeill reported "several specimens of Cerithi$u m$ belonging to the group of $C$. jonkeri Martin and $C$. poetjanganensis Altena. C. jonkeri has been reported from the upper Miocene to the Pleistocene in the East Indies and the Philippines, the latter possibly only a subspecies of $C$. jonkeri from the Pleistocene of Nias and Java."

The oyster Ostrea crasissima Lamarck, which is synonymous with $O$. gryphoides, was found by us north of Umm Lajj. It was also found later near $\mathrm{Al}$ Wajd and south of Al Muwaylih by W. Schott, who reported (Richter-Bernburg and Schott, 1954) the opinion of K. Staesche that the source beds were Miocene, based on the associated microfauna. Although a review of post-Miocene Ostreidae by L.R. Cox (1929) indicates that the form extends into the Pliocene in the Indian Ocean affinity, it became extinct in the Mediterranean fauna at the end of the Miocene. The end of the Miocene coincides with the top of the evaporite section in the Raghama. In the sediments of the Red Sea, this datum is marked by an accoustic reflector-now dated at 5 m.y., near the beginning of the Pliocene when the Red Sea opened anew to allow ingress of Indian Ocean fauna.

As these limestone beds at $\mathrm{Al}$ Qunfudhah overlie the Baid Formation, which contains a diabase sill (19.1 \pm 0.6 m.y., K-Ar; sample 54, table 10, pl. 2), most of the evidence points to a middle to younger Miocene age for the Raghama Formation, with the possibility that some of the upper reefs may be Pliocene.

In this connection, the basalt flow east of Tuwwal, which caps the ridge containing the approximate lower 
boundary of the middle Miocene, was dated by K-Ar methods at $4.4 \pm 0.4$ m.y. (sample 30 , table 10 , pl. 2). An adjacent flow to the south in an identical physiographic setting was dated at $4.2 \pm 0.8$ m.y. (sample 32 , table 10 ). Thus, a minimum age is established.

\section{CENOZOIC IGNEOUS ROCKS ${ }^{7}$}

Igneous activity in western Saudi Arabia appears to have been dormant from late Proterozoic or earliest Cambrian until mid-Tertiary, when the opening of the Afro-Arabian rift system renewed magmatic upwelling which has continued until the present. The distribution of Tertiary intrusive rocks, represented by various dikes, plugs, stocks, and small plutons, and Cenozoic extrusive rocks, represented by the extensive floodbasalt fields, are shown on plate 2. Chemical and normative analyses (table 9) and K-Ar age determinations (table 10) for these igneous rocks are discussed in the appropriate sections that follow. The time distribution of the K-Ar ages of the Cenozoic igneous rocks of this report as well as of some other Cenozoic rocks from around the Red Sea is shown in the histogram of figure 51. Although some K-Ar radiometric ages greater than about $30 \mathrm{~m} . \mathrm{y}$. are reported for the Tertiary igneous rocks that flank the rift system, they are suspect, especially where the magma has penetrated thick Precambrian terrane rich in argon (Megrue and others, 1972). Various plots of the chemical and normative data are given in figures 52-56 and are briefly discussed in the text.

The Cenozoic rocks comprise flood basalt on the Arabian Shield, basaltic to rhyolitic volcanic rocks in a continental rift valley along the proto-Red Sea, tholeiitic dikes and gabbro and granophyre plutons likewise in the rift valley, and continental dikes inland from the rift valley (table 11).

Outpouring of alkali-olivine basalt marked the beginning of mid-Tertiary volcanism in western Saudi Arabia. Well within the craton these late Oligocene-early Miocene flows spread out as large flood-basalt fields upon a lowland of low altitude as well as low relief. The basal eruptions about 30 m.y. old were undersaturated picrite and ankaramite fed mostly from volcanic centers (feeder pipes) to form shield volcanoes. Overlying flows were mostly basanite, including some hawaiite that formed the great composite flood-basalt fields. Late products formed plugs of latite (phonolite), analcime syenite, and rhyolite (comendite) with ages of about 22 m.y.

\footnotetext{
${ }^{7}$ For a more complete discussion of the Cenozoic volcanic rocks, the reader is referred to USGS Open-File Report 83-788 by R.G. Coleman, R.T. Gregory, and G.F. Brown (1983), which is based on this report plus later field and laboratory work by Coleman and Gregory.
}

During the same time interval along the present-day coastal plain of the Red Sea, bimodal volcanics of the Jizan Group were deposited in a continental rift valley along the proto-Red Sea. About 20 m.y. ago, as seafloor spreading began, dike swarms of tholeiitic basalt and diabase intruded the Jizan Group volcanics in the rift valley. At about the same time and place, plutons of tholeiitic gabbro and granophyre were intruded through the thinned continental crust of the rift valley. Slightly inland along the continental margin, long, thick dikes (pl. 2) ranging from gabbro to quartz syenite (fig. 52 ) intruded the thicker, less extended, less thinned cratonic crust. A second pulse of flood basalt beginning during middle-late Miocene was superposed on the older basalt fields on the craton, but the lavas erupted along the continental margin as well. The flows continued to erupt to Recent time, and again were predominantly alkali olivine basalt that evolved to minor silicic phases, especially in the latest eruptions (fig. 60).

\section{TERTIARY HYPABYSSAL IGNEOUS ROCKS}

Intrusive igneous rocks associated with the Tertiary opening of the Red Sea include dikes, pipes or plugs, small stocks, and layered-gabbro plutons (Brown, 1960; Brown and Hase, 1971; Blank, 1977; Coleman, Fleck, and others, 1977). Dikes predominate although they are areally insignificant compared with the coeval harrats (flood-lava fields). The dikes consist of a swarm of hydrothermally altered diabase sheets associated with granophyre and cumulative gabbro plutons which together make up the Tihamat-Asir Complex. The complex extends intermittently $150 \mathrm{~km}$ north from the Yemen border as far as lat $17^{\circ} 45^{\prime} \mathrm{N}$., where it is terminated by the Ad Darb Red Sea transform fault. ${ }^{8}$ Northward beyond the Ad Darb fault, the individual dikes, which there transect continental rocks in the scarp mountains, are much larger, are separated by Precambrian screens, and are designated continental dikes by Blank and Coleman (Blank, 1977; Coleman and others, 1979). They extend northward $1,375 \mathrm{~km}$ to lat $28^{\circ} 30^{\prime}$ N., in Ash Shifā', east of the Gulf of Aqaba, and thence across southern Sinai (pl. 2) (Bartov and others, 1980). Whereas the Tihamat-Asir dike swarm extends inland $50 \mathrm{~km}$, the continental dikes are found as much as $100 \mathrm{~km}$ from the Red Sea coast. They are characterized by remarkably linear and predominantly reversely polarized magnetic anomalies (Blank, 1977), range in width from 50 to $300 \mathrm{~m}$, and are mostly gabbro,

${ }^{8}$ Since this was written, J.S. Pallister mapped a Tertiary dike swarm at Wādī Ad Damn $100 \mathrm{~km}$ southeast of Jiddah having composition and age similar to the Tihamat-Asir Complex (Pallister, 1983). 


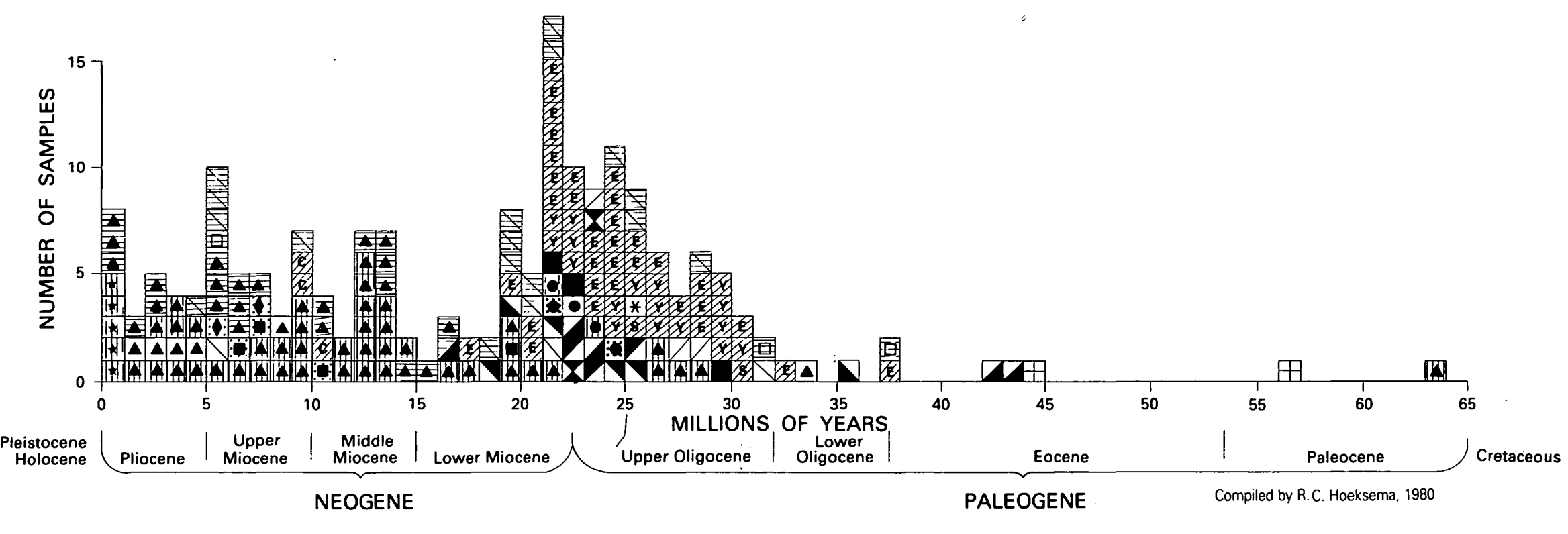

EXPLANATION

Saudi Arabia flood
lavas (harrats)
Labradorite analyzed from
layered gabbro

s. Aş Sirät, Saudi Arabia

E] Ethiopian Plateau (Jones, 1976)

c Cassam, Ethiopian Plateau

$\checkmark$ Yemen Plateau (Clvetta

(a) Basalt/andesite dike

- Tihamat Asir ophiolite
Dhyolite

$\Delta$ Alkaline olivine basalt (Saudi Arabia) basalt (elsewhere)

Trachyte

$\nabla$ Basaltrandesite plug

$\square$ Syenite/gabbro/diorite plug

Felsic stock

$\boxplus$ Glauconite in sandstone

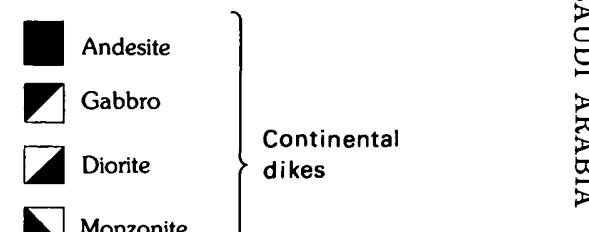

$\triangle$ Granodiorite \# Hormfels

$₫$ Radiogenic argon could not be measured. This is thought to be caused by the very young
age of the basalt

\footnotetext{
"K decay constants: $\quad \begin{aligned} \gamma \theta=0.581 \times 10^{-10} \mathrm{yr}-1 \\ \gamma \beta\end{aligned}$

Isotopic abundance: ${ }^{\infty} \mathrm{K} / \mathrm{K} / \mathrm{K}=1.167 \times 10^{-2}$ atom $\%$
}

FiguRE 51.-Histogram showing ages of selected Tertiary igneous rocks and glauconite from around the Red Sea. Most ages are from table 10; other sources are referenced in explanation of the figure. 
TABLE 9.-Chemical and normative analyses of Cenozoic igneous rocks from western Saudi Arabia ${ }^{1}$

\begin{tabular}{|c|c|c|c|c|c|c|c|c|c|c|c|c|}
\hline $\begin{array}{l}\text { Sample (location } \\
\text { given on pl. 2) } \\
\text { Field no. } \\
\text { Type of source rock }\end{array}$ & $\begin{array}{c}1 \\
47000 \\
\mathrm{X} \\
\end{array}$ & $\begin{array}{c}2 \\
47001 \\
X \\
\end{array}$ & $\begin{array}{c}3 \\
47002 \\
X \\
\end{array}$ & $\begin{array}{c}5 \\
47004 \\
\mathrm{X} \\
\end{array}$ & $\begin{array}{c}6 \mathrm{~T} \\
917 \mathrm{~T} \\
\mathrm{X} \\
\end{array}$ & $\begin{array}{c}6 \mathrm{~B} \\
917 \mathrm{~B} \\
\mathrm{X} \\
\end{array}$ & $\begin{array}{c}7 \mathrm{~T} \\
916 \mathrm{~T} \\
\mathrm{X} \\
\end{array}$ & $\begin{array}{c}7 \mathrm{~B} \\
916 \mathrm{~B} \\
\mathrm{X} \\
\end{array}$ & $\begin{array}{c}8 \\
\text { B1818 } \\
0\end{array}$ & $\begin{array}{c}9 \mathrm{~T} \\
915 \mathrm{~T} \\
\mathrm{X} \\
\end{array}$ & $\begin{array}{c}9 \mathrm{~B} \\
915 \mathrm{~B} \\
\mathrm{X} \\
\end{array}$ & $\begin{array}{c}10 \\
913 \\
\mathrm{X} \\
\end{array}$ \\
\hline \multicolumn{13}{|c|}{ Unadjusted oxides (wt. percent): } \\
\hline $\mathrm{SiO}_{2}$ & 46.30 & 46.30 & 47.40 & 51.50 & 46.60 & 45.70 & 42.50 & 43.70 & 49.30 & 43.70 & 46.60 & 45.70 \\
\hline $\mathrm{Al}_{2} \mathrm{O}_{3}$ & 14.60 & 13.20 & 15.60 & 14.60 & 17.10 & 16.10 & 14.00 & 14.40 & 15.30 & 15.00 & 16.10 & 16.90 \\
\hline $\mathrm{Fe}_{2} \mathrm{O}_{3}$ & 6.40 & 3.91 & 3.80 & 2.59 & 3.20 & 4.20 & 6.70 & 3.60 & 1.70 & 4.00 & 1.70 & 3.80 \\
\hline $\mathrm{FeO}-$ & 4.90 & 7.90 & 7.40 & 7.30 & 8.40 & 6.40 & 4.40 & 7.30 & 10.20 & 7.10 & 10.00 & 6.80 \\
\hline $\mathrm{MgO}-$ & 8.10 & 8.87 & 8.50 & 6.60 & 4.50 & 8.00 & 9.20 & 9.50 & 5.80 & 8.60 & 6.90 & 4.50 \\
\hline $\mathrm{CaO}$ & 11.20 & 12.30 & 10.60 & 10.40 & 7.60 & 10.30 & 11.80 & 11.20 & 9.00 & 10.00 & 7.50 & 10.80 \\
\hline $\mathrm{Na}_{2} \mathrm{O}$ & 3.70 & 3.62 & 3.30 & 4.53 & 4.20 & 2.60 & 4.40 & 2.80 & 2.70 & 3.70 & 3.80 & 4.30 \\
\hline $\mathrm{K}_{2} \mathrm{O}-$ & 0.96 & 0.78 & 0.54 & 0.92 & 1.40 & 0.35 & 0.67 & 0.80 & 1.00 & 1.40 & 1.20 & 1.40 \\
\hline $\mathrm{H}_{2} \mathrm{O}-$ & - & 2.40 & 1.80 & 0.40 & 2.70 & 3.00 & 1.72 & 2.50 & 0.97 & 1.65 & 2.58 & 1.10 \\
\hline $\mathrm{TiO}_{2} \ldots$ & 1.80 & 1.60 & 1.60 & 2.00 & 2.60 & 1.50 & 2.30 & 1.90 & 2.50 & 2.40 & 2.10 & 2.20 \\
\hline $\mathrm{P}_{2} \mathrm{O}_{5}$ & 0.27 & 0.17 & 0.16 & 0.23 & 0.68 & 0.28 & 0.58 & 0.40 & 0.37 & 0.50 & 0.41 & 0.44 \\
\hline $\mathrm{MnO}$ & 0.12 & 0.13 & 0.12 & 0.11 & 0.18 & 0.15 & 0.18 & 0.16 & 0.18 & 0.18 & 0.15 & 0.15 \\
\hline $\mathrm{CO}_{2}$ & - & - & - & - & 0.14 & 0.46 & 0.81 & 0.67 & 0.02 & 0.46 & 0.06 & 0.84 \\
\hline Total & 98.35 & 101.18 & 100.82 & 101.18 & 99.30 & 99.04 & 99.26 & 98.93 & 99.04 & 98.69 & 99.10 & 98.93 \\
\hline \multicolumn{13}{|l|}{ Adjusted oxides: } \\
\hline $\mathrm{SiO}_{2}-$ & 47.08 & 45.76 & 47.01 & 50.90 & 46.93 & 46.14 & 42.82 & 44.17 & 49.78 & 44.28 & 47.02 & 46.19 \\
\hline $\mathrm{Al}_{2} \mathrm{O}_{3}$ & 14.84 & 13.05 & 15.47 & 14.43 & 17.22 & 16.26 & 14.10 & 14.56 & 15.45 & 15.20 & 16.25 & 17.08 \\
\hline $\mathrm{Fe}_{2} \mathrm{O}_{3}$ & 6.51 & 3.86 & 3.77 & 2.56 & 3.22 & 4.24 & 6.75 & 3.64 & 1.72 & 4.05 & 1.72 & 3.84 \\
\hline $\mathrm{FeO}$ & 4.98 & 7.81 & 7.34 & 7.21 & 8.46 & 6.46 & 4.43 & 7.38 & 10.30 & 7.19 & 10.09 & 6.87 \\
\hline $\mathrm{MgO} \longrightarrow$ & 8.24 & 8.77 & 8.43 & 6.52 & 4.53 & 8.08 & 9.27 & 9.60 & 5.86 & 8.71 & 6.96 & 4.55 \\
\hline $\mathrm{CaO}$ & 11.39 & 12.16 & 10.51 & 10.28 & 7.65 & 10.40 & 11.89 & 11.32 & 9.09 & 10.13 & 7.57 & 10.92 \\
\hline $\mathrm{Na}_{2} \mathrm{O}$ & 3.76 & 3.58 & 3.27 & 4.48 & 4.23 & 2.63 & 4.43 & 2.83 & 2.73 & 3.75 & 3.83 & 4.35 \\
\hline $\mathrm{K}_{2} \mathrm{O}-$ & 0.98 & 0.77 & 0.54 & 0.91 & 1.41 & 0.35 & 0.67 & 0.81 & 1.01 & 1.42 & 1.21 & 1.42 \\
\hline $\mathrm{H}_{2} \mathrm{O}$ & - & 2.37 & 1.79 & 0.40 & 2.72 & 3.03 & 1.73 & 2.53 & 0.98 & 1.67 & 2.60 & 1.11 \\
\hline $\mathrm{TiO}_{2}$ & 1.83 & 1.58 & 1.59 & 1.98 & 2.62 & 1.51 & 2.32 & 1.92 & 2.52 & 2.43 & 2.12 & 2.22 \\
\hline $\mathrm{P}_{2} \mathrm{O}_{5}$ & 0.27 & 0.17 & 0.16 & 0.23 & 0.68 & 0.28 & 0.58 & 0.40 & 0.37 & 0.51 & 0.41 & 0.44 \\
\hline $\mathrm{MnO}$ & 0.12 & 0.13 & 0.12 & 0.11 & 0.18 & 0.15 & 0.18 & 0.16 & 0.18 & 0.18 & 0.15 & 0.15 \\
\hline $\mathrm{CO}_{2}$ & - & - & - & - & 0.14 & 0.46 & 0.82 & 0.68 & 0.02 & 0.47 & 0.06 & 0.85 \\
\hline
\end{tabular}


TABLE 9.-Chemical and normative analyses of Cenozoic igneous rocks from western Saudi Arabia 1 -Continued

\begin{tabular}{|c|c|c|c|c|c|c|c|c|c|c|c|c|}
\hline $\begin{array}{l}\text { Sample (location } \\
\text { given on pl. } 2)^{2}- \\
\text { Field no. } \\
\text { Type of source rock } \\
\text { Tyen }\end{array}$ & $\begin{array}{c}1 \\
47000 \\
X \\
\end{array}$ & $\begin{array}{c}2 \\
47001 \\
X \\
\end{array}$ & $\begin{array}{c}3 \\
47002 \\
\times \\
\end{array}$ & $\begin{array}{c}5 \\
47004 \\
\times \\
\end{array}$ & $\begin{array}{r}6 \mathrm{~T} \\
917 \mathrm{~T} \\
\mathrm{X} \\
\end{array}$ & $\begin{array}{c}6 \mathrm{~B} \\
917 \mathrm{~B} \\
\mathrm{X} \\
\end{array}$ & $\begin{array}{c}7 \mathrm{~T} \\
916 \mathrm{~T} \\
\mathrm{X} \\
\end{array}$ & $\begin{array}{c}7 \mathrm{~B} \\
916 \mathrm{~B} \\
\times \\
\end{array}$ & $\begin{array}{c}8 \\
\text { B1818 } \\
0 \\
\end{array}$ & $\begin{array}{c}9 \mathrm{~T} \\
915 \mathrm{~T} \\
\mathrm{X} \\
\end{array}$ & $\begin{array}{c}9 \mathrm{~B} \\
915 \mathrm{~B} \\
\mathrm{X} \\
\end{array}$ & $\begin{array}{c}10 \\
913 \\
\times \\
\end{array}$ \\
\hline \multicolumn{13}{|c|}{ Normative minerals: } \\
\hline Quartz - & - & - & - & - & - & - & - & - & 0.107 & - & - & - \\
\hline Corundum - & - & -- & - & - & - & - & -- & - & - & -- & - & - \\
\hline Orthoclase-- & 5.768 & 4.555 & 3.165 & 5.373 & 8.331 & 2.088 & 3.989 & 4.779 & 5.967 & 8.383 & 7.156 & 8.362 \\
\hline Albite- & 21.225 & 13.740 & 24.350 & 31.008 & 32.285 & 22.214 & 14.712 & 17.622 & 23.068 & 15.739 & 28.141 & 22.349 \\
\hline Anorthite- & 20.736 & 17.261 & 25.945 & 16.590 & 23.838 & 31.528 & 16.594 & 24.624 & 26.933 & 20.454 & 23.541 & 22.922 \\
\hline Nepheline- & 5.747 & 8.957 & 1.813 & 3.725 & 1.898 & - & 12.350 & 3.428 & - & 8.660 & 2.333 & 7.817 \\
\hline Wollastonite- & 14.182 & 17.516 & 10.512 & 13.744 & 3.660 & 6.381 & 13.949 & 10.279 & 6.505 & 9.837 & 4.559 & 9.588 \\
\hline Enstatite -- & 11.828 & 11.593 & 7.050 & 8.620 & 1.978 & 14.055 & 12.055 & 7.140 & 14.585 & 6.986 & 2.448 & 5.890 \\
\hline Ferrosilite -- & 0.564 & 4.658 & 2.674 & 4.283 & 1.557 & 4.292 & - & 2.291 & 13.664 & 1.991 & 1.961 & 3.150 \\
\hline Forsterite - & 6.085 & 7.176 & 9.774 & 5.344 & 6.523 & 4.248 & 7.728 & 11.756 & - & 10.313 & 10.436 & 3.811 \\
\hline Fayalite - & 0.320 & 3.177 & 4.086 & 2.926 & 5.659 & 1.430 & - & 4.157 & $\because$ & 3.239 & 9.216 & 2.246 \\
\hline Magnetite- & 9.435 & 5.603 & 5.465 & 3.711 & 4.672 & 6.149 & 8.163 & 5.276 & 2.489 & 5.877 & 2.487 & 5.569 \\
\hline Hematite $\ldots$ & - & - & - & $\ldots$ & - & - & 1.120 & - & - & - & - & - \\
\hline Ilmenite & 3.476 & 3.003 & 3.014 & 3.754 & 4.973 & 2.876 & 4.401 & 3.648 & 4.794 & 4.619 & 4.025 & 4.223 \\
\hline Apatite - & 0.650 & 0.398 & 0.376 & 0.538 & 1.622 & 0.670 & 1.384 & 0.958 & 0.885 & 1.200 & 0.980 & 1.053 \\
\hline Calcite - & - & - & - & - & 0.321 & 1.056 & 1.856 & 1.540 & 0.046 & 1.060 & 0.138 & 1.931 \\
\hline Total _- & 100.016 & 97.638 & 98.224 & 99.618 & 97.319 & 96.987 & 98.300 & 97.496 & 99.042 & 98.357 & 97.420 & 98.913 \\
\hline Sálic- & 53.476 & 44.513 & 55.273 & 56.697 & 66.353 & 55.830 & 47.644 & 50.452 & 56.075 & 53.235 & 61.170 & 61.451 \\
\hline Femic - & 46.540 & 53.125 & 42.951 & 42.921 & 30.965 & 41.157 & 50.655 & 47.044 & 42.968 & 45.122 & 36.250 & 37.462 \\
\hline Diopside ${ }^{4}$ & 26.574 & 33.768 & 20.237 & 26.647 & 7.196 & 12.222 & 26.004 & 19.710 & 12.863 & 18.814 & 8.968 & 18.627 \\
\hline DiWo-_ & 14.182 & 17.516 & 10.512 & 13.744 & 3.660 & 6.381 & 13.949 & 10.279 & 6.505 & 9.837 & 4.559 & 9.588 \\
\hline $\mathrm{DiEn} \longrightarrow$ & 11.828 & 11.593 & 7.050 & 8.620 & 1.978 & 4.475 & 12.055 & 7.140 & 3.282 & 6.986 & 2.448 & 5.890 \\
\hline DiFs & 0.564 & 4.658 & 2.674 & 4.283 & 1.557 & 1.367 & - & 2.291 & 3.075 & 1.991 & 1.961 & 3.150 \\
\hline Hypersthene $^{5}$ & - & - & - & - & - & 12.506 & - & - & 21.891 & - & - & - \\
\hline HyEn - & - & - & - & - & - & 9.580 & - & - & 11.303 & -- & - & - \\
\hline $\mathrm{HyFs}$ & -- & - & $\ldots$ & -- & - & 2.926 & - & - & 10.589 & - & -- & - \\
\hline Olivine $^{6}$ & 6.405 & 10.353 & 13.859 & 8.271 & 12.182 & 5.678 & 7.728 & 15.912 & - & 13.552 & 19.653 & 6.058 \\
\hline $\mathrm{OlFo}_{-}$ & 6.085 & 7.176 & 9.774 & 5.344 & 6.523 & 4.248 & 7.728 & 11.756 & - & 10.313 & 10.436 & 3.811 \\
\hline $\mathrm{OlFa}-$ & 0.320 & 3.177 & 4.086 & 2.926 & 5.659 & 1.430 & -- & 4.157 & - & 3.239 & 9.216 & 2.246 \\
\hline
\end{tabular}


TABLE 9.-Chemical and normative analyses of Cenozoic igneous rocks from western Saudi Arabia-Continued

\begin{tabular}{|c|c|c|c|c|c|c|c|c|c|c|c|c|}
\hline $\begin{array}{l}\text { Sample (location giv- } \\
\text { en on pl. } 1)^{2} \\
\text { Field no. } \\
\text { Type of source rock }{ }^{3} \text {. }\end{array}$ & $\begin{array}{c}12 \\
912 \\
\mathrm{X} \\
\end{array}$ & $\begin{array}{c}13 \\
1843 \\
0 \\
\end{array}$ & $\begin{array}{c}14 \\
919 \\
\mathrm{X} \\
\end{array}$ & $\begin{array}{c}15 \\
920 \\
\mathrm{X} \\
\end{array}$ & $\begin{array}{c}16 \\
921 \\
\mathrm{X} \\
\end{array}$ & $\begin{array}{c}17 \\
911 \\
\mathrm{X} \\
\end{array}$ & $\begin{array}{r}18 \\
910 \\
\mathrm{X} \\
\end{array}$ & $\begin{array}{c}19 \\
922 \\
\mathrm{X} \\
\end{array}$ & $\begin{array}{c}19 a \\
1309 \\
\mathbf{P} \\
\end{array}$ & $\begin{array}{c}22 \\
907 \\
\mathrm{X} \\
\end{array}$ & $\begin{array}{c}23 \\
909 \\
\mathrm{X} \\
\end{array}$ & $\begin{array}{c}24 \\
908 \\
\mathrm{X} \\
\end{array}$ \\
\hline \multicolumn{13}{|c|}{ Unadjusted oxides (wt. percent): } \\
\hline $\mathrm{SiO}_{2}$ & 43.40 & 46.50 & 46.60 & 46.20 & 44.10 & 47.40 & 44.80 & 45.30 & 39.80 & 46.70 & 43.20 & 45.00 \\
\hline $\mathrm{Al}_{2} \mathrm{O}_{3}$ & 15.20 & 14.90 & 16.70 & 16.90 & 15.20 & 16.50 & 16.70 & 15.80 & 13.40 & 16.50 & 14.60 & 14.90 \\
\hline $\mathrm{Fe}_{2} \mathrm{O}_{3}$ & 3.00 & 5.00 & 3.60 & 2.10 & 4.10 & 2.40 & 3.90 & 2.30 & 4.60 & 1.20 & 5.30 & 2.20 \\
\hline $\mathrm{FeO}$ & 8.40 & 9.80 & 8.90 & 10.30 & 7.40 & 9.20 & 9.70 & 8.20 & 8.20 & 9.10 & 5.60 & 9.20 \\
\hline $\mathrm{MgO}-$ & 9.80 & 4.90 & 7.20 & 6.50 & 9.50 & 8.00 & 6.00 & 9.00 & 9.90 & 9.20 & 9.80 & 10.50 \\
\hline $\mathrm{CaO}$ & 9.90 & 7.60 & 8.60 & 8.60 & 9.50 & 7.50 & 7.50 & 9.90 & 11.80 & 10.80 & 11.70 & 9.80 \\
\hline $\mathrm{Na}_{2} \mathrm{O}$ & 2.70 & 2.90 & 3.80 & 3.70 & 2.70 & 4.20 & 3.50 & 2.50 & 3.80 & 2.70 & 2.80 & 2.40 \\
\hline $\mathrm{K}_{2} \mathrm{O} \cdots$ & 1.10 & 1.10 & 0.89 & 0.86 & 0.86 & 0.74 & 0.67 & 0.74 & 0.90 & 0.25 & 0.64 & 0.67 \\
\hline $\mathrm{H}_{2} \mathrm{O}$ & 2.58 & 2.86 & 0.87 & 0.46 & 2.39 & 1.23 & 2.86 & 2.34 & 2.19 & 0.75 & 1.10 & 1.41 \\
\hline $\mathrm{TiO}_{2}$ & 2.40 & 3.90 & 2.10 & 2.40 & 2.40 & 2.20 & 2.60 & 1.70 & 2.80 & 1.50 & 1.70 & 1.80 \\
\hline $\mathrm{P}_{2} \mathrm{O}_{5}$ & 0.41 & 0.69 & 0.57 & 0.40 & 0.51 & 0.38 & 0.32 & 0.42 & 0.94 & 0.14 & 0.36 & 0.17 \\
\hline $\mathrm{MnO}$ & 0.18 & 0.17 & 0.18 & 0.18 & 0.15 & 0.18 & 0.18 & 0.15 & 0.20 & 0.15 & 0.15 & 0.19 \\
\hline $\mathrm{CO}_{2}-$ & 0.04 & 0.04 & 0.08 & 0.04 & 0.08 & 0.04 & 0.08 & 0.35 & 0.06 & 0.21 & 2.30 & 0.26 \\
\hline Total - & 99.11 & 100.36 & 100.09 & 98.64 & 98.89 & 99.97 & 98.81 & 98.70 & 98.59 & 99.20 & 99.25 & 98.50 \\
\hline \multicolumn{13}{|l|}{ Adjusted oxides: } \\
\hline $\mathrm{SiO}_{2}$ & 43.79 & 46.33 & 46.56 & 46.84 & 44.60 & 47.41 & 45.34 & 45.90 & 40.37 & 47.08 & 43.53 & 45.69 \\
\hline $\mathrm{Al}_{2} \mathrm{O}_{3}$ & 15.34 & 14.85 & 16.68 & 17.13 & 15.37 & 16.50 & 16.90 & 16.01 & 13.59 & 16.63 & 14.71 & 15.13 \\
\hline $\mathrm{Fe}_{2} \mathrm{O}_{3}$ & 3.03 & 4.98 & 3.60 & 2.13 & 4.15 & 2.40 & 3.95 & 2.33 & 4.67 & 1.21 & 5.34 & 2.23 \\
\hline $\mathrm{FeO}-$ & 8.48 & 9.76 & 8.89 & 10.44 & 7.48 & 9.20 & 9.82 & 8.31 & 8.32 & 9.17 & 5.64 & 9.34 \\
\hline $\mathrm{MgO}$ & 9.89 & 4.88 & 7.19 & 6.59 & 9.61 & 8.00 & 6.07 & 9.12 & 10.04 & 9.27 & 9.87 & 10.66 \\
\hline $\mathrm{CaO}$ & 9.99 & 7.57 & 8.59 & 8.72 & 9.61 & 7.50 & 7.59 & 10.03 & 11.97 & 10.89 & 11.79 & 9.95 \\
\hline $\mathrm{Na}_{2} \mathrm{O}$ & 2.72 & 2.89 & 3.80 & 3.75 & 2.73 & 4.20 & 3.54 & 2.53 & 3.85 & 2.72 & 2.82 & 2.44 \\
\hline $\mathrm{K}_{2} \mathrm{O} \longrightarrow$ & 1.11 & 1.10 & 0.89 & 0.87 & 0.87 & 0.74 & 0.68 & 0.75 & 0.91 & 0.25 & 0.64 & 0.68 \\
\hline $\mathrm{H}_{2} \mathrm{O}$ & 2.60 & 2.85 & 0.87 & 0.47 & 2.42 & 1.23 & 2.89 & 2.37 & 2.22 & 0.76 & 1.11 & 1.43 \\
\hline $\mathrm{TiO}_{2}-$ & 2.42 & 3.89 & 2.10 & 2.43 & 2.43 & 2.20 & 2.63 & 1.72 & 2.84 & 1.51 & 1.71 & 1.83 \\
\hline $\mathrm{P}_{2} \mathrm{O}_{5}$ & 0.41 & 0.69 & 0.57 & 0.41 & 0.52 & 0.38 & 0.32 & 0.43 & 0.95 & 0.14 & 0.36 & 0.17 \\
\hline $\mathrm{MnO}-$ & 0.18 & 0.17 & 0.18 & 0.18 & 0.15 & 0.18 & 0.18 & 0.15 & 0.20 & 0.15 & 0.15 & 0.19 \\
\hline $\mathrm{CO}_{2}-$ & 0.04 & 0.04 & 0.08 & 0.04 & 0.08 & 0.04 & 0.08 & 0.35 & 0.06 & 0.21 & 2.32 & 0.26 \\
\hline
\end{tabular}

Footnotes at end of table 
TABLE 9.-Chemical and normative analyses of Cenozoic igneous rocks from western Saudi Arabia-Continued

\begin{tabular}{|c|c|c|c|c|c|c|c|c|c|c|c|c|}
\hline $\begin{array}{l}\text { Sample (location giv- } \\
\text { en on pl. } 1)^{2}- \\
\text { Field no. } \\
\text { Type of source rocks. }\end{array}$ & $\begin{array}{c}12 \\
912 \\
\mathrm{X} \\
\end{array}$ & $\begin{array}{c}13 \\
1843 \\
0 \\
\end{array}$ & $\begin{array}{c}14 \\
919 \\
\mathrm{X} \\
\end{array}$ & $\begin{array}{c}15 \\
920 \\
\mathrm{X} \\
\end{array}$ & $\begin{array}{c}16 \\
921 \\
\mathrm{X} \\
\end{array}$ & $\begin{array}{r}17 \\
911 \\
\mathrm{X} \\
\end{array}$ & $\begin{array}{r}18 \\
910 \\
\mathrm{X} \\
\end{array}$ & $\begin{array}{c}19 \\
922 \\
\mathrm{X} \\
\end{array}$ & $\begin{array}{c}19 \mathrm{a} \\
1309 \\
\mathrm{P}\end{array}$ & $\begin{array}{r}22 \\
907 \\
\mathrm{X} \\
\end{array}$ & $\begin{array}{c}23 \\
909 \\
\mathrm{X} \\
\end{array}$ & $\begin{array}{c}24 \\
908 \\
\mathrm{X} \\
\end{array}$ \\
\hline \multicolumn{13}{|c|}{ Normative minerals: } \\
\hline Quartz & - & 2.203 & - & - & - & - & - & - & - & - & - & - \\
\hline Corundum - & - & - & - & - & - & - & - & - & - & - & - & - \\
\hline Orthoclase- & 6.559 & 6.477 & 5.255 & 5.152 & 5.139 & 4.374 & 4.007 & 4.430 & 5.394 & 1.489 & 3.811 & 4.020 \\
\hline Albite- & 14.862 & 24.451 & 28.088 & 26.548 & 22.264 & 30.692 & 29.973 & 21.433 & 2.974 & 23.029 & 23.872 & 20.492 \\
\hline Anorthite-- & 26.341 & 24.302 & 25.858 & 27.337 & 27.116 & 23.991 & 28.214 & 30.096 & 17.089 & 32.423 & 25.570 & 28.329 \\
\hline Nepheline- & 4.437 & - & 2.187 & 2.813 & 0.455 & 2.632 & - & - & 16.057 & 0.001 & - & 0.068 \\
\hline Wollastonite- & 8.458 & 3.558 & 5.237 & 5.432 & 6.957 & 4.381 & 2.845 & 6.114 & 14.896 & 8.070 & 6.636 & 7.613 \\
\hline Enstatite - & 5.664 & 12.160 & 3.155 & 2.868 & 4.971 & 2.623 & 3.268 & 7.083 & 10.585 & 4.813 & 6.163 & 4.829 \\
\hline Ferrosilite -- & 2.163 & 7.714 & 1.801 & 2.401 & 1.369 & 1.529 & 2.325 & 3.359 & 3.008 & 2.840 & 0.853 & 2.300 \\
\hline Forsterite-- & 13.288 & -- & 10.343 & 9.491 & 13.283 & 12.128 & 8.307 & 10.951 & 10.108 & 12.813 & 12.914 & 15.220 \\
\hline Fayalite & 5.591 & - & 6.507 & 8.755 & 4.032 & 7.791 & 6.514 & 5.723 & 3.165 & 8.333 & 1.969 & 7.991 \\
\hline Magnetite- & 4.389 & 7.224 & 5.215 & 3.087 & 6.011 & 3.481 & 5.723 & 3.379 & 6.765 & 1.754 & 7.743 & 3.238 \\
\hline Hematite - & - & - & - & - & - & - & - & - & - & - & - & - \\
\hline Ilmenite- & 4.599 & 7.380 & 3.985 & 4.621 & 4.609 & 4.180 & 4.997 & 3.271 & 5.394 & 2.872 & 3.253 & 3.471 \\
\hline Apatite - & 0.980 & 1.628 & 1.349 & 0.960 & 1.222 & 0.900 & 0.767 & 1.008 & 2.258 & 0.334 & 0.859 & 0.409 \\
\hline Calcite & 0.092 & 0.091 & 0.182 & 0.092 & 0.184 & 0.091 & 0.184 & 0.806 & 0.138 & 0.481 & 5.270 & 0.600 \\
\hline Total & 97.421 & 97.188 & 99.162 & 99.557 & 97.612 & 98.792 & 97.125 & 97.653 & 97.831 & 99.253 & 98.912 & 98.580 \\
\hline Salic-___ & 52.198 & 57.433 & 61.388 & 61.849 & 54.974 & 61.689 & 62.193 & 55.959 & 41.514 & 56.942 & 53.253 & 52.908 \\
\hline Femic-- & 45.223 & 39.755 & 37.774 & 37.708 & 42.638 & 37.103 & 34.931 & 41.694 & 56.316 & 42.311 & 45.659 & 45.672 \\
\hline Diopside $^{4}$ & 16.285 & 6.948 & 10.193 & 10.701 & 13.297 & 8.532 & 5.576 & 11.839 & 28.488 & 15.724 & 12.543 & 14.742 \\
\hline DiWo- & 8.458 & 3.558 & 5.237 & 5.432 & 6.957 & 4.381 & 2.845 & 6.114 & 14.896 & 8.070 & 6.636 & 7.613 \\
\hline DiEn - & 5.664 & 2.074 & 3.155 & 2.868 & 4.971 & 2.623 & 1.595 & 3.883 & 10.585 & 4.813 & 5.189 & 4.829 \\
\hline DiFs & 2.163 & 1.316 & 1.801 & 2.401 & 1.369 & 1.529 & 1.135 & 1.841 & 3.008 & 2.840 & 0.718 & 2.300 \\
\hline Hypersthene $e^{5}$ & - & 16.483 & - & - & - & - & 2.863 & 4.717 & - & - & 1.109 & - \\
\hline $\mathrm{HyEn}$ & - & 10.086 & - & - & - & - & 1.673 & 3.200 & - & - & 0.974 & - \\
\hline HyFs- & -- & 6.398 & - & - & - & - & 1.190 & 1.517 & - & $\ldots$ & 0.135 & - \\
\hline Olivine $^{6}$ & 18.879 & - & 16.850 & 18.246 & 17.315 & 19.919 & 14.821 & 16.674 & 13.273 & 21.146 & 14.883 & 23.211 \\
\hline $\mathrm{OlFo}-$ & 13.288 & - & 10.343 & 9.491 & 13.283 & 12.128 & 8.307 & 10.951 & 10.108 & 12.813 & 12.914 & 15.220 \\
\hline $\mathrm{OlFa}-$ & 5.591 & - & 6.507 & 8.755 & 4.032 & 7.791 & 6.514 & 5.723 & 3.165 & 8.333 & 1.969 & 7.991 \\
\hline
\end{tabular}

ollastonite

DiWo

Footnotes at end of table. 
TABLE 9.-Chemical and normative analyses of Cenozoic igneous rocks from western Saudi Arabia-Continued

\begin{tabular}{|c|c|c|c|c|c|c|c|c|c|c|c|}
\hline $\begin{array}{l}\text { Sample (location giv- } \\
\text { en on pl. } 1)^{2} \\
\text { Field no. } \\
\text { Type of source } \text { rock }^{3} \text {. }\end{array}$ & $\stackrel{25}{1314}$ & $\underset{\mathrm{X}}{26}$ & $\stackrel{27}{905}$ & $\underset{\mathrm{X}}{28}$ & $\underset{\mathrm{X}}{\mathrm{GFB} 1}$ & $\underset{\mathrm{X}}{\mathrm{GFB2}}$ & $\stackrel{36}{902}$ & $\begin{array}{c}37 \\
47027 \mathrm{~B} \\
\mathrm{X}\end{array}$ & $\stackrel{38}{\mathrm{P}}$ & $\begin{array}{c}40 \\
47024 \\
P\end{array}$ & $\begin{array}{c}41 \\
47023 \\
\mathrm{X}\end{array}$ \\
\hline \multicolumn{12}{|c|}{ Unadjusted oxides (wt. percent): } \\
\hline $\mathrm{SiO}_{2}-\ldots$ & 44.30 & 46.60 & 47.40 & 47.00 & 48.50 & 45.50 & 44.40 & 47.30 & 46.50 & 61.40 & 43.70 \\
\hline $\mathrm{Al}_{2} \mathrm{O}_{3}$ & 17.38 & 15.20 & 16.20 & 16.60 & 14.20 & 14.20 & 16.40 & 16.50 & 13.60 & 15.30 & 14.50 \\
\hline $\mathrm{Fe}_{2} \mathrm{O}_{3}$ & 4.54 & 4.00 & 3.60 & 1.70 & 3.03 & 6.01 & 5.60 & 3.40 & 7.29 & 6.50 & 6.20 \\
\hline $\mathrm{FeO}-$ & 6.70 & 7.40 & 7.40 & 9.50 & 7.90 & 5.90 & 5.30 & 7.60 & 4.60 & 0.28 & 5.60 \\
\hline $\mathrm{MgO}-$ & 7.29 & 9.50 & 8.60 & 8.10 & 9.12 & 8.22 & 6.50 & 8.10 & 8.60 & 0.12 & 8.50 \\
\hline $\mathrm{CaO}-$ & 11.61 & 9.80 & 10.30 & 10.80 & 10.10 & 10.60 & 12.00 & 10.60 & 7.84 & 1.90 & 10.50 \\
\hline $\mathrm{Na}_{2} \mathrm{O}$ & 3.56 & 2.90 & 3.10 & 2.70 & 4.31 & 4.18 & 2.70 & 3.00 & 5.05 & 5.80 & 3.60 \\
\hline $\mathrm{K}_{2} \mathrm{O} \ldots$ & 0.80 & 0.60 & 0.48 & 0.22 & 1.13 & 0.94 & 0.35 & 0.65 & 1.78 & 4.80 & 1.20 \\
\hline $\mathrm{H}_{2} \mathrm{O}$ & 0.63 & 0.85 & 0.79 & 0.56 & 0.60 & 2.40 & 2.13 & 1.40 & 0.60 & 2.14 & 1.48 \\
\hline $\mathrm{TiO}_{2}$ & 1.50 & 1.60 & 1.40 & 1.40 & 1.50 & 2.00 & 1.70 & 1.60 & 2.40 & 0.38 & 2.40 \\
\hline $\mathrm{P}_{2} \mathrm{O}_{5}$ & 0.18 & 0.32 & 0.13 & 0.12 & 0.18 & 0.38 & 0.21 & 0.31 & 0.53 & 0.11 & 0.63 \\
\hline $\mathrm{MnO}$ & 0.12 & 0.18 & 0.15 & 0.15 & 0.14 & 0.15 & 0.15 & 0.14 & 0.13 & 0.18 & 0.20 \\
\hline $\mathrm{CO}_{2}$ & $\ldots$ & 0.30 & 0.12 & 0.08 & -- & -- & 0.16 & $\ldots$ & - & 0.86 & 0.95 \\
\hline Total & 98.61 & 99.25 & 99.67 & 98.93 & 100.71 & 100.48 & 97.60 & 100.60 & 98.92 & 99.77 & 99.46 \\
\hline \multicolumn{12}{|l|}{ Adjusted oxides: } \\
\hline $\mathrm{SiO}_{2}$ & 44.92 & 46.95 & 47.56 & 47.51 & 48.16 & 45.28 & 45.49 & 47.02 & 47.01 & 61.54 & 43.94 \\
\hline $\mathrm{Al}_{2} \mathrm{O}_{3}$ & 17.62 & 15.31 & 16.25 & 16.78 & 14.10 & 14.13 & 16.80 & 16.40 & 13.75 & 15.34 & 14.58 \\
\hline $\mathrm{Fe}_{2} \mathrm{O}_{3}$ & 4.60 & 4.03 & 3.61 & 1.72 & 3.01 & 5.98 & 5.74 & 3.38 & 7.37 & 6.51 & 6.23 \\
\hline $\mathrm{FeO}-$ & 6.79 & 7.46 & 7.42 & 9.60 & 7.84 & 5.87 & 5.43 & 7.55 & 4.65 & 0.28 & 5.63 \\
\hline $\mathrm{MgO}$ & 7.39 & 9.57 & 8.63 & 8.19 & 9.06 & 8.18 & 6.66 & 8.05 & 8.69 & 0.12 & 8.55 \\
\hline $\mathrm{CaO}-$ & 11.77 & 9.87 & 10.33 & 10.92 & 10.03 & 10.55 & 12.30 & 10.54 & 7.93 & 1.90 & 10.56 \\
\hline $\mathrm{Na}_{2} \mathrm{O}$ & 3.61 & 2.92 & 3.11 & 2.73 & 4.28 & 4.16 & 2.77 & 2.98 & 5.11 & 5.81 & 3.62 \\
\hline $\mathrm{K}_{2} \mathrm{O}-$ & 0.81 & 0.60 & 0.48 & 0.22 & 1.12 & 0.94 & 0.36 & 0.65 & 1.80 & 4.81 & 1.21 \\
\hline $\mathrm{H}_{2} \mathrm{O}-$ & 0.64 & 0.86 & 0.79 & 0.57 & 0.60 & 2.39 & 2.18 & 1.39 & 0.61 & 2.14 & 1.49 \\
\hline $\mathrm{TiO}_{2}$ & 1.52 & 1.61 & 1.40 & 1.42 & 1.49 & 1.99 & 1.74 & 1.59 & 2.43 & 0.38 & 2.41 \\
\hline $\mathrm{P}_{2} \mathrm{O}_{5}$ & 0.18 & 0.32 & 0.13 & 0.12 & 0.18 & 0.38 & 0.22 & 0.31 & 0.54 & 0.11 & 0.63 \\
\hline $\mathrm{MnO}-$ & 0.12 & 0.18 & 0.15 & 0.15 & 0.14 & 0.15 & 0.15 & 0.14 & 0.13 & 0.18 & 0.20 \\
\hline $\mathrm{CO}_{2}$ & - & 0.30 & 0.12 & 0.08 & - & - & 0.16 & - & - & 0.86 & 0.96 \\
\hline
\end{tabular}

Footnotes at end of table. 
TABLE 9.-Chemical and normative analyses of Cenozoic igneous rocks from western Saudi Arabia-Continued

\begin{tabular}{|c|c|c|c|c|c|c|c|c|c|c|c|}
\hline $\begin{array}{l}\text { Sample (location giv- } \\
\text { en on plo. } \text { ent }^{2}- \\
\text { Field no. } \\
\text { Type of source rock }\end{array}$ & $\begin{array}{l}25 \\
1314 \\
X\end{array}$ & $\begin{array}{l}26 \\
923 \\
\mathrm{X}\end{array}$ & $\begin{array}{c}27 \\
905 \\
\mathrm{x}\end{array}$ & $\begin{array}{c}28 \\
904 \\
X\end{array}$ & $\underset{\mathrm{GFB}}{30}$ & $\begin{array}{l}33 \\
\text { GFB2 } \\
\mathrm{X}\end{array}$ & $\begin{array}{l}36 \\
902 \\
\mathrm{X}\end{array}$ & $\begin{array}{c}37 \\
47027 \mathrm{~B} \\
\mathrm{X}\end{array}$ & $\begin{array}{c}38 \\
47026 \\
P\end{array}$ & $\begin{array}{c}40 \\
47024 \\
P\end{array}$ & $\begin{array}{c}41 \\
47023 \\
X\end{array}$ \\
\hline \multicolumn{12}{|c|}{ Normative minerals: } \\
\hline Quartz & - & - & - & - & - & - & - & - & - & 8.096 & - \\
\hline Corundum - & - & - & - & - & - & - & - & -- & - & - & - \\
\hline Orthoclase- & 4.794 & 3.572 & 2.846 & 1.314 & 6.630 & 5.528 & 2.119 & 3.818 & 10.633 & 28.430 & 7.130 \\
\hline Albite- & 13.390 & 24.724 & 26.318 & 23.094 & 19.951 & 19.404 & 23.407 & 24.692 & 24.304 & 49.191 & 21.270 \\
\hline Anorthite- & 29.490 & 26.887 & 28.966 & 32.877 & 15.949 & 17.125 & 32.372 & 29.459 & 9.284 & 1.540 & 19.969 \\
\hline Nepheline- & 9.295 & - & - & - & 8.809 & 8.558 & 0.001 & 0.293 & 10.235 & - & 5.069 \\
\hline Wollastonite-- & 11.577 & 7.550 & 8.638 & 8.342 & 13.627 & 13.670 & 10.932 & 8.685 & 11.079 & 0.726 & 9.281 \\
\hline Enstatite & 7.916 & 8.407 & 5.768 & 6.431 & 8.869 & 10.685 & 8.427 & 5.641 & 9.575 & 0.300 & 7.593 \\
\hline Ferrosilite - & 2.746 & 2.834 & 2.311 & 4.465 & 3.822 & 1.485 & 1.342 & 2.451 & - & - & 0.563 \\
\hline Forsterite--- & 7.355 & 10.814 & 11.017 & 9.783 & 9.589 & 6.790 & 5.718 & 10.099 & 8.463 & - & 9.594 \\
\hline Fayalite - & 2.812 & 4.018 & 4.865 & 7.486 & 4.554 & 1.040 & 1.003 & 4.836 & - & -- & 0.784 \\
\hline Magnetite-_- & 6.675 & 5.843 & 5.237 & 2.491 & 4.362 & 8.672 & 8.319 & 4.900 & 8.384 & 0.390 & 9.038 \\
\hline Hematite - & - & - & - & - & - & - & - & - & 1.587 & 6.246 & - \\
\hline Ilmenite-..- & 2.889 & 3.062 & 2.668 & 2.688 & 2.829 & 3.780 & 3.308 & 3.021 & 4.608 & 0.723 & 4.583 \\
\hline Apatite - & 0.432 & 0.764 & 0.309 & 0.287 & 0.423 & 0.896 & 0.510 & 0.730 & 1.269 & 0.261 & 1.500 \\
\hline Calcite - & - & 0.687 & 0.274 & 0.184 & - & - & 0.373 & - & $\ldots$ & 1.960 & 2.172 \\
\hline Total —_ & 99.372 & 99.163 & 99.216 & 99.442 & 99.415 & 97.633 & 97.831 & 98.626 & 99.423 & 97.863 & 98.547 \\
\hline Salic_-_ & 56.969 & 55.184 & 58.130 & 57.285 & 51.340 & 50.614 & 57.899 & 58.263 & 54.457 & 87.257 & 53.438 \\
\hline Femic --- & 42.403 & 43.979 & 41.086 & 42.157 & 48.075 & 47.019 & 39.932 & 40.363 & 44.966 & 10.606 & 45.110 \\
\hline Diopside $^{4}$ & 22.239 & 14.493 & 16.652 & 16.334 & 26.318 & 25.840 & 20.701 & 16.777 & 20.655 & 0.646 & 17.437 \\
\hline DiWo-_- & 11.577 & 7.550 & 8.638 & 8.342 & 13.627 & 13.670 & 10.932 & 8.685 & 11.079 & 0.347 & 9.281 \\
\hline DiEn $\ldots$ & 7.916 & 5.193 & 5.721 & 4.717 & 8.869 & 10.685 & 8.427 & 5.641 & 9.575 & 0.300 & 7.593 \\
\hline DiFs - & 2.746 & 1.751 & 2.292 & 3.275 & 3.822 & 1.485 & 1.342 & 2.451 & - & - & 0.563 \\
\hline Hypersthene $e^{5}$ & - & 4.297 & 0.065 & 2.904 & - & - & - & -- & --- & - & - \\
\hline HyEn -.-. & - & 3.214 & 0.046 & 1.714 & - & - & - & -- & --- & -- & - \\
\hline $\mathrm{HyFs}-$ & - & 1.084 & 0.019 & 1.190 & - & - & - & - & -- & $\ldots$ & $\ldots$ \\
\hline Olivine $^{6}$ & 10.167 & 14.832 & 15.882 & 17.270 & 14.143 & 7.830 & 6.721 & 14.935 & 8.463 & -- & 10.379 \\
\hline OlFo & 7.355 & 10.814 & 11.017 & 9.783 & 9.589 & 6.790 & 5.718 & 10.099 & 8.463 & - & 9.594 \\
\hline $\mathrm{OlFa}-$ & 2.812 & 4.018 & 4.865 & 7.486 & 4.554 & 1.040 & 1.003 & 4.836 & - & - & 0.784 \\
\hline \multicolumn{12}{|l|}{$\begin{array}{l}\text { Wollastonite } \\
\text { minus }\end{array}$} \\
\hline DiWo- & -- & - & - & - & - & -- & - & - & - & 0.379 & - \\
\hline
\end{tabular}


TABLE 9.-Chemical and normative analyses of Cenozoic igneous rocks from western Saudi Arabia-Continued

\begin{tabular}{|c|c|c|c|c|c|c|c|c|c|c|c|c|}
\hline $\begin{array}{l}\text { Sample (location giv- } \\
\text { en on pl. } 1)^{2}- \\
\text { Field no. } \\
\text { Type of source rock } \\
\end{array}$ & $\begin{array}{c}42 \\
47022 \\
\mathrm{X} \\
\end{array}$ & $\begin{array}{c}43 \\
47021 \\
\quad \mathrm{X}\end{array}$ & $\begin{array}{c}44 \\
47025 \\
\mathrm{X}\end{array}$ & $\begin{array}{c}45 \\
926 \\
\mathrm{P} \\
\end{array}$ & $\begin{array}{r}46 \mathrm{a} \\
927 \mathrm{~A} \\
\mathrm{P} \\
\end{array}$ & $\begin{array}{c}46 \mathrm{~b} \\
927 \mathrm{~B} \\
\mathbf{P}\end{array}$ & $\begin{array}{r}51 \mathrm{a} \\
623 \mathrm{~A} \\
0 \\
\end{array}$ & $\begin{array}{c}51 \mathrm{~b} \\
623 \mathrm{~B} \\
0\end{array}$ & $\begin{array}{c}51 \mathrm{c} \\
623 \mathrm{C} \\
0 \\
\end{array}$ & $\begin{array}{c}52 \mathrm{a} \\
928 \mathrm{~A} \\
0 \\
\end{array}$ & $\begin{array}{c}52 \mathrm{~b} \\
928 \mathrm{~B} \\
0\end{array}$ & $\begin{array}{c}52 \mathrm{c} \\
928 \mathrm{C} \\
0 \\
\end{array}$ \\
\hline \multicolumn{13}{|c|}{ Unadjusted oxides (wt. percent): } \\
\hline $\mathrm{SiO}_{2}-$ & 43.20 & 44.20 & 44.50 & 70.30 & 46.20 & 46.60 & 52.50 & 51.50 & 51.50 & 53.80 & 48.40 & 61.90 \\
\hline $\mathrm{Al}_{2} \mathrm{O}_{3}$ & 14.30 & 14.50 & 14.50 & 14.70 & 22.10 & 22.90 & 17.60 & 15.02 & 16.90 & 18.20 & 13.40 & 14.60 \\
\hline $\mathrm{Fe}_{2} \mathrm{O}_{3}$ & 6.60 & 4.20 & 2.90 & 2.50 & 2.90 & 2.00 & 3.78 & 4.45 & 3.83 & 5.40 & 7.00 & 4.80 \\
\hline $\mathrm{FeO}-$ & 5.10 & 7.70 & 8.80 & 0.20 & 3.60 & 3.30 & 5.50 & 8.60 & 7.00 & 3.80 & 8.70 & 2.50 \\
\hline $\mathrm{MgO}$ & 9.00 & 9.80 & 11.00 & 0.28 & 3.80 & 3.40 & 4.38 & 3.32 & 3.98 & 1.60 & 4.30 & 1.50 \\
\hline $\mathrm{CaO}$ & 11.10 & 9.20 & 10.00 & 1.10 & 13.30 & 12.50 & 8.90 & 8.95 & 8.95 & 6.10 & 7.00 & 3.70 \\
\hline $\mathrm{Na}_{2} \mathrm{O} \ldots$ & 3.20 & 2.90 & 2.80 & 4.70 & 2.60 & 2.90 & 3.53 & 3.57 & 4.96 & 4.00 & 3.10 & 3.80 \\
\hline $\mathrm{K}_{2} \mathrm{O}$ & 1.20 & 1.00 & 0.81 & 4.20 & 0.32 & 0.38 & 0.92 & 0.92 & 1.41 & 1.30 & 1.30 & 2.30 \\
\hline $\mathrm{H}_{2} \mathrm{O} \cdots$ & 1.55 & 1.97 & 0.90 & 1.84 & 2.80 & 3.94 & 1.50 & 0.90 & 1.30 & 2.61 & 2.84 & 3.12 \\
\hline $\mathrm{TiO}_{2}$ & 2.30 & 2.60 & 2.00 & 0.18 & 1.10 & 0.88 & 1.20 & 2.80 & 1.40 & 1.60 & 3.00 & 1.10 \\
\hline $\mathrm{P}_{2} \mathrm{O}_{5}$ & 0.58 & 0.51 & 0.33 & 0.05 & 0.17 & 0.19 & 0.30 & 0.40 & 0.34 & 0.56 & 0.30 & 0.31 \\
\hline $\mathrm{MnO}$ & 0.20 & 0.20 & 0.20 & 0.03 & 0.11 & 0.07 & 0.12 & 0.16 & 0.14 & 0.15 & 0.22 & 0.11 \\
\hline $\mathrm{CO}_{2}$ & 1.00 & 0.30 & 0.98 & 0.02 & 0.02 & 0.02 & - & -- & - & 0.02 & 0.02 & 0.02 \\
\hline Total - & 99.33 & 99.08 & 99.72 & 100.10 & 99.02 & 99.08 & 100.23 & 100.59 & 101.71 & 99.14 & 99.58 & 99.76 \\
\hline \multicolumn{13}{|l|}{ Adjusted oxides: } \\
\hline $\mathrm{SiO}_{2}$ & 43.49 & 44.61 & 44.62 & 70.23 & 46.66 & 47.03 & 52.38 & 51.20 & 50.63 & 54.27 & 48.60 & 62.05 \\
\hline $\mathrm{Al}_{2} \mathrm{O}_{3}$ & 14.40 & 14.63 & 14.54 & 14.69 & 22.32 & 23.11 & 17.56 & 14.93 & 16.62 & 18.36 & 13.46 & 14.64 \\
\hline $\mathrm{Fe}_{2} \mathrm{O}_{3}$ & 6.64 & 4.24 & 2.91 & 2.50 & 2.93 & 2.02 & 3.77 & 4.42 & 3.77 & 5.45 & 7.03 & 4.81 \\
\hline $\mathrm{FeO}-$ & 5.13 & 7.77 & 8.82 & 0.20 & 3.64 & 3.33 & 5.49 & 8.55 & 6.88 & 3.83 & 8.74 & 2.51 \\
\hline $\mathrm{MgO}-$ & 9.06 & 9.89 & 11.03 & 0.28 & 3.84 & 3.43 & 4.37 & 3.30 & 3.91 & 1.61 & 4.32 & 1.50 \\
\hline $\mathrm{CaO}-$ & 11.17 & 9.29 & 10.03 & 1.10 & 13.43 & 12.62 & 8.88 & 8.90 & 8.80 & 6.15 & 7.03 & 3.71 \\
\hline $\mathrm{Na}_{2} \mathrm{O}$ & 3.22 & 2.93 & 2.81 & 4.70 & 2.63 & 2.93 & 3.52 & 3.55 & 4.88 & 4.03 & 3.11 & 3.81 \\
\hline $\mathrm{K}_{2} \mathrm{O}$ & 1.21 & 1.01 & 0.81 & 4.20 & 0.32 & 0.38 & 0.92 & 0.91 & 1.39 & 1.31 & 1.31 & 2.31 \\
\hline $\mathrm{H}_{2} \mathrm{O}-$ & 1.56 & 1.99 & 0.90 & 1.84 & 2.83 & 3.98 & 1.50 & 0.89 & 1.28 & 2.63 & 2.85 & 3.13 \\
\hline $\mathrm{TiO}_{2}$ & 2.32 & 2.62 & 2.01 & 0.18 & 1.11 & 0.89 & 1.20 & 2.78 & 1.38 & 1.61 & 3.01 & 1.10 \\
\hline $\mathrm{P}_{2} \mathrm{O}_{5}-$ & 0.58 & 0.51 & 0.33 & 0.05 & 0.17 & 0.19 & 0.30 & 0.40 & 0.33 & 0.56 & 0.30 & 0.31 \\
\hline $\mathrm{MnO}-$ & 0.20 & 0.20 & 0.20 & 0.03 & 0.11 & 0.07 & 0.12 & 0.16 & 0.14 & 0.15 & 0.22 & 0.11 \\
\hline $\mathrm{CO}_{2} \longrightarrow$ & 1.01 & 0.30 & 0.98 & 0.02 & 0.02 & 0.02 & - & - & - & 0.02 & 0.02 & 0.02 \\
\hline
\end{tabular}

Footnotes at end of table. 
TABLE 9.-Chemical and normative analyses of Cenozoic igneous rocks from western Saudi Arabia-Continued

\begin{tabular}{|c|c|c|c|c|c|c|c|c|c|c|c|c|}
\hline $\begin{array}{l}\text { Sample (location giv- } \\
\text { en on pl. 112 } \\
\text { Field no. } \\
\text { Type of source rock }\end{array}$ & $\begin{array}{c}42 \\
47022 \\
\mathrm{X} \\
\end{array}$ & $\begin{array}{c}43 \\
47021 \\
X \\
\end{array}$ & $\begin{array}{c}44 \\
47025 \\
X \\
\end{array}$ & $\begin{array}{c}45 \\
926 \\
P\end{array}$ & $\begin{array}{l}46 \mathrm{a} \\
927 \mathrm{~A} \\
\mathrm{P}\end{array}$ & $\begin{array}{l}46 \mathrm{~b} \\
927 \mathrm{~B} \\
\mathrm{P}\end{array}$ & $\begin{array}{c}51 \mathrm{a} \\
623 \mathrm{~A} \\
0 \\
\end{array}$ & $\begin{array}{c}51 \mathrm{~b} \\
623 \mathrm{~B} \\
0 \\
\end{array}$ & $\begin{array}{c}51 \mathrm{c} \\
623 \mathrm{C} \\
0\end{array}$ & $\begin{array}{c}52 \mathrm{a} \\
928 \mathrm{~A} \\
0 \\
\end{array}$ & $\begin{array}{c}52 \mathrm{~b} \\
928 \mathrm{~B} \\
0 \\
\end{array}$ & $\begin{array}{c}52 \mathrm{c} \\
928 \mathrm{C} \\
0 \\
\end{array}$ \\
\hline \multicolumn{13}{|c|}{ Normative minerals: } \\
\hline Quartz & - & - & - & 24.286 & - & - & 4.057 & 4.861 & - & 11.768 & 5.323 & 21.859 \\
\hline Corundum - & - & - & - & 0.588 & - & - & - & - & - & 0.513 & - & - \\
\hline Orthoclase- & 7.139 & 5.964 & 4.800 & 24.794 & 1.910 & 2.266 & 5.424 & 5.405 & 8.192 & 7.749 & 7.714 & 13.624 \\
\hline Albite- & 19.204 & 22.619 & 20.315 & 39.730 & 22.218 & 24.767 & 29.801 & 30.031 & 34.309 & 34.140 & 26.342 & 32.232 \\
\hline Anorthite--- & 21.253 & 23.813 & 24.673 & 4.999 & 48.158 & 48.794 & 29.393 & 22.111 & 19.354 & 26.707 & 18.888 & 16.026 \\
\hline Nepheline- & 4.364 & 1.163 & 1.866 & - & - & - & - & - & 3.768 & - & - & - \\
\hline Wollastonite- & 10.024 & 7.088 & 6.974 & - & 7.193 & 5.183 & 5.304 & 8.113 & 9.235 & - & 5.800 & 0.091 \\
\hline Enstatite - & 8.522 & 5.061 & 4.633 & 0.697 & 5.989 & 3.969 & 10.883 & 8.220 & 5.031 & 4.019 & 10.754 & 3.745 \\
\hline Ferrosilite- & 0.185 & 1.399 & 1.832 & - & 1.647 & 1.446 & 5.206 & 7.744 & 3.877 & 0.155 & 5.672 & - \\
\hline Forsterite - & 9.841 & 13.716 & 16.005 & - & 2.501 & 3.208 & - & - & 3.304 & - & - & - \\
\hline Fayalite-_ & 0.235 & 4.179 & 6.975 & - & 0.758 & 1.288 & - & - & 2.806 & - & - & -- \\
\hline Magnetite-_- & 9.634 & 6.146 & 4.217 & 0.221 & 4.246 & 2.927 & 5.468 & 6.414 & 5.460 & 7.897 & 10.192 & 5.241 \\
\hline Hematite - & - & - & - & 2.345 & - & - & - & - & - & - & - & 1.197 \\
\hline Ilmenite-_ & 4.398 & 4.984 & 3.809 & 0.342 & 2.110 & 1.687 & 2.274 & 5.287 & 2.614 & 3.065 & 5.722 & 2.094 \\
\hline Apatite - & 1.383 & 1.219 & 0.784 & 0.118 & 0.407 & 0.454 & 0.709 & 0.942 & 0.792 & 1.338 & 0.714 & 0.736 \\
\hline Calcite - & 2.290 & 0.689 & 2.235 & 0.045 & 0.046 & 0.046 & - & - & - & 0.046 & 0.046 & 0.046 \\
\hline Total & 98.472 & 98.041 & 99.117 & 98.165 & 97.183 & 96.034 & 98.520 & 99.128 & 98.741 & 97.398 & 97.166 & 96.890 \\
\hline Salic-_-_ & 51.960 & 53.560 & 51.654 & 94.397 & 72.286 & 75.827 & 68.676 & 62.407 & 65.623 & 80.878 & 58.267 & 83.741 \\
\hline Femic - & 46.512 & 44.481 & 47.463 & 3.768 & 24.897 & 20.207 & 29.845 & 36.720 & 33.118 & 16.521 & 38.899 & 13.149 \\
\hline Diopside $^{4}-$ & 18.731 & 13.548 & 13.439 & - & 13.748 & 9.969 & 10.272 & 16.045 & 18.143 & - & 11.263 & 0.169 \\
\hline DiWo- & 10.024 & 7.088 & 6.974 & - & 7.193 & 5.183 & 5.304 & 8.113 & 9.235 & - & 5.800 & 0.091 \\
\hline DiEn - & 8.522 & 5.061 & 4.633 & - & 5.141 & 3.508 & 3.361 & 4.084 & 5.031 & - & 3.577 & 0.078 \\
\hline DiFs & 0.185 & 1.399 & 1.832 & - & 1.414 & 1.278 & 1.608 & 3.848 & 3.877 & - & 1.887 & - \\
\hline Hypersthene $\mathrm{e}^{5}$ & - & - & - & 0.697 & 1.081 & 0.629 & 11.122 & 8.032 & - & 4.174 & 10.963 & 3.666 \\
\hline HyEn - & - & - & - & 0.697 & 0.848 & 0.461 & 7.523 & 4.136 & - & 4.019 & 7.177 & 3.666 \\
\hline HyFs- & - & - & - & - & 0.233 & 0.168 & 3.599 & 3.896 & - & 0.155 & 3.785 & - \\
\hline Olivine $^{6}$ & 10.077 & 17.895 & 22.980 & - & 3.259 & 4.496 & - & - & 6.110 & - & - & - \\
\hline $\mathrm{OlFo}-$ & 9.841 & 13.716 & 16.005 & - & 2.501 & 3.208 & - & - & 3.304 & - & - & - \\
\hline $\mathrm{OlFa}-$ & 0.235 & 4.179 & 6.975 & - & 0.758 & 1.288 & - & - & 2.806 & -- & - & - \\
\hline \multicolumn{13}{|l|}{$\begin{array}{l}\text { Wollastonite } \\
\text { minus }\end{array}$} \\
\hline DiWo-_- & - & - & - & - & - & - & - & - & - & - & - & - \\
\hline
\end{tabular}

Footnotes at end of table. 
TABLE 9.-Chemical and normative analyses of Cenozoic igneous rocks from western Saudi Arabia-Continued

\begin{tabular}{|c|c|c|c|c|c|c|c|c|c|c|c|c|}
\hline $\begin{array}{l}\text { Sample (location giv- } \\
\text { en on pl. } 1)^{2} \\
\text { Field no. } \\
\text { Type of source rock } \\
\end{array}$ & $\begin{array}{c}52 \mathrm{~d} \\
928 \mathrm{D} \\
0\end{array}$ & $\begin{array}{c}55 \mathrm{a} \\
930 \\
0\end{array}$ & $\begin{array}{c}56 \mathrm{a} \\
933 \mathrm{~A} \\
0\end{array}$ & $\begin{array}{c}56 \mathrm{~b} \\
933 \mathrm{~B} \\
0\end{array}$ & $\begin{array}{c}56 \mathrm{c} \\
933 \mathrm{C} \\
0\end{array}$ & $\begin{array}{c}58 \mathrm{a} \\
\text { BRK4A } \\
0\end{array}$ & $\begin{array}{c}58 \mathrm{~b} \\
\text { BRK4B } \\
0\end{array}$ & $\begin{array}{c}58 \mathrm{c} \\
\mathrm{BRK} 4 \mathrm{C} \\
0\end{array}$ & $\begin{array}{c}60 \\
935 \\
0\end{array}$ & $\underset{\mathrm{X}}{\stackrel{61}{93 \mathrm{~A}}}$ & $\begin{array}{c}62 \\
937 \\
\mathrm{X}\end{array}$ & $\begin{array}{c}63 \\
936 \\
0\end{array}$ \\
\hline \multicolumn{13}{|c|}{ Unadjusted oxides (wt. percent): } \\
\hline $\mathrm{SiO}_{2}-$ & 63.40 & 55.00 & 46.80 & 47.00 & 56.50 & 48.30 & 51.60 & 50.20 & 59.90 & 45.40 & 47.90 & 51.00 \\
\hline $\mathrm{Al}_{2} \mathrm{O}_{3}$ & 14.60 & 17.50 & 14.90 & 15.80 & 18.20 & 17.40 & 16.80 & 16.80 & 14.60 & 16.20 & 16.40 & 17.40 \\
\hline $\mathrm{Fe}_{2} \mathrm{O}_{3}$ & 2.70 & 2.70 & 1.40 & 3.10 & 2.10 & 3.00 & 3.90 & 3.30 & 4.20 & 5.40 & 3.80 & 3.80 \\
\hline $\mathrm{FeO}-$ & 4.20 & 5.60 & 10.40 & 9.60 & 5.20 & 7.30 & 6.80 & 6.80 & 5.60 & 6.00 & 6.80 & 6.70 \\
\hline $\mathrm{MgO}$ & 1.50 & 1.50 & 5.40 & 5.00 & 1.80 & 3.40 & 2.30 & 3.50 & 1.50 & 7.40 & 7.60 & 3.00 \\
\hline $\mathrm{CaO}$ & 3.70 & 6.50 & 8.50 & 8.20 & 6.20 & 9.20 & 6.40 & 7.80 & 2.60 & 10.40 & 8.90 & 7.00 \\
\hline $\mathrm{Na}_{2} \mathrm{O}$ & 4.00 & 4.00 & 3.50 & 3.40 & 4.30 & 3.10 & 4.00 & 3.80 & 4.40 & 3.40 & 3.50 & 3.60 \\
\hline $\mathrm{K}_{2} \mathrm{O} \ldots$ & 2.60 & 1.80 & 0.89 & 1.20 & 1.70 & 0.95 & 1.30 & 1.20 & 2.90 & 1.00 & 1.20 & 1.60 \\
\hline $\mathrm{H}_{2} \mathrm{O}$ & 2.30 & 2.81 & 5.25 & 2.55 & 2.59 & 2.30 & 4.00 & 2.37 & 2.60 & 1.03 & 0.85 & 2.52 \\
\hline $\mathrm{TiO}_{2}$ & 0.98 & 1.30 & 2.80 & 3.10 & 1.20 & 2.60 & 1.40 & 2.20 & 0.96 & 2.60 & 2.00 & 2.20 \\
\hline $\mathrm{P}_{2} \mathrm{O}_{5}$ & 0.29 & 0.46 & 0.58 & 0.58 & 0.42 & 0.83 & 0.77 & 0.82 & 0.15 & 0.50 & 0.36 & 0.58 \\
\hline $\mathrm{MnO}$ & 0.11 & 0.15 & 0.18 & 0.23 & 0.15 & 0.21 & 0.26 & 0.20 & 0.15 & 0.19 & 0.15 & 0.19 \\
\hline $\mathrm{CO}_{2}$ & 0.02 & 0.04 & 0.06 & 0.06 & 0.02 & 0.08 & 0.08 & 0.25 & 0.04 & 0.36 & 0.06 & 0.08 \\
\hline Total & 100.40 & 99.36 & 100.66 & 99.82 & 100.38 & 98.67 & 99.61 & 99.24 & 99.60 & 99.88 & 99.52 & 99.67 \\
\hline \multicolumn{13}{|l|}{ Adjusted oxides: } \\
\hline $\mathrm{SiO}_{2}$ & 63.15 & 55.35 & 46.49 & 47.08 & 56.29 & 48.95 & 51.80 & 50.58 & 60.14 & 45.45 & 48.13 & 51.17 \\
\hline $\mathrm{Al}_{2} \mathrm{O}_{3} \cdots$ & 14.54 & 17.61 & 14.80 & 15.83 & 18.13 & 17.63 & 16.87 & 16.93 & 14.66 & 16.22 & 16.48 & 17.46 \\
\hline $\mathrm{Fe}_{2} \mathrm{O}_{3}$ & 2.69 & 2.72 & 1.39 & 3.11 & 2.09 & 3.04 & 3.92 & 3.33 & 4.22 & 5.41 & 3.82 & 3.81 \\
\hline $\mathrm{FeO}-$ & 4.18 & 5.64 & 10.33 & 9.62 & 5.18 & 7.40 & 6.83 & 6.85 & 5.62 & 6.01 & 6.83 & 6.72 \\
\hline $\mathrm{MgO}$ & 1.49 & 1.51 & 5.36 & 5.01 & 1.79 & 3.45 & 2.31 & 3.53 & 1.51 & 7.41 & 7.64 & 3.01 \\
\hline $\mathrm{CaO}$ & 3.69 & 6.54 & 8.44 & 8.21 & 6.18 & 9.32 & 6.43 & 7.86 & 2.61 & 10.41 & 8.94 & 7.02 \\
\hline $\mathrm{Na}_{2} \mathrm{O}-$ & 3.98 & 4.03 & 3.48 & 3.41 & 4.28 & 3.14 & 4.02 & 3.83 & 4.42 & 3.40 & 3.52 & 3.61 \\
\hline $\mathrm{K}_{2} \mathrm{O}-$ & 2.59 & 1.81 & 0.88 & 1.20 & 1.69 & 0.96 & 1.31 & 1.21 & 2.91 & 1.00 & 1.21 & 1.61 \\
\hline $\mathrm{H}_{2} \mathrm{O}$ & 2.29 & 2.83 & 5.22 & 2.55 & 2.58 & 2.33 & 4.02 & 2.39 & 2.61 & 1.03 & 0.85 & 2.53 \\
\hline $\mathrm{TiO}_{2}$ & 0.98 & 1.31 & 2.78 & 3.11 & 1.20 & 2.64 & 1.41 & 2.22 & 0.96 & 2.60 & 2.01 & 2.21 \\
\hline $\mathrm{P}_{2} \mathrm{O}_{5}$ & 0.29 & 0.46 & 0.58 & 0.58 & 0.42 & 0.84 & 0.77 & 0.83 & 0.15 & 0.50 & 0.36 & 0.58 \\
\hline $\mathrm{MnO}$ & 0.11 & 0.15 & 0.18 & 0.23 & 0.15 & 0.21 & 0.26 & 0.20 & 0.15 & 0.19 & 0.15 & 0.19 \\
\hline $\mathrm{CO}_{2} \longrightarrow$ & 0.02 & 0.04 & 0.06 & 0.06 & 0.02 & 0.08 & 0.08 & 0.25 & 0.04 & 0.36 & 0.06 & 0.08 \\
\hline
\end{tabular}


TABLE 9.-Chemical analyses and normatives of Cenozoic igneous rocks from western Saudi Arabia-Continued

\begin{tabular}{|c|c|c|c|c|c|c|c|c|c|c|c|c|}
\hline $\begin{array}{l}\text { Sample (location giv- } \\
\text { en on ploc. } \\
\text { Field no. } \\
\text { Type of source rock }\end{array}$ & $\begin{array}{r}52 \mathrm{~d} \\
928 \mathrm{D} \\
0 \\
\end{array}$ & $\begin{array}{l}55 a \\
930 \\
0\end{array}$ & $\begin{array}{c}56 \mathrm{a} \\
933 \mathrm{~A} \\
0 \\
\end{array}$ & $\begin{array}{c}56 \mathrm{~b} \\
933 \mathrm{~B} \\
0 \\
\end{array}$ & $\begin{array}{c}56 \mathrm{c} \\
93 \mathrm{C} \\
0 \\
\end{array}$ & $\begin{array}{c}58 \mathrm{a} \\
\text { BRK4A } \\
0\end{array}$ & $\begin{array}{c}58 \mathrm{~b} \\
\text { BRK4B } \\
0\end{array}$ & $\begin{array}{c}58 \mathrm{c} \\
\text { BRK4C } \\
0\end{array}$ & $\begin{array}{c}60 \\
935 \\
0\end{array}$ & $\begin{array}{c}61 \\
938 \mathrm{~A} \\
\mathrm{X}\end{array}$ & $\begin{array}{c}62 \\
937 \\
\mathrm{X}\end{array}$ & $\begin{array}{c}63 \\
936 \\
0\end{array}$ \\
\hline \multicolumn{13}{|c|}{ Normative minerals: } \\
\hline Quartz- & 19.422 & 8.298 & - & - & 7.991 & 3.189 & 5.711 & 3.041 & 13.511 & - & - & 4.485 \\
\hline Corundum - & - & - & - & - & - & - & - & - & - & - & - & - \\
\hline Orthoclase-- & 15.303 & 10.705 & 5.225 & 7.104 & 10.008 & 5.689 & 7.712 & 7.145 & 17.206 & 5.916 & 7.125 & 9.486 \\
\hline Albite- & 33.712 & 34.065 & 29.422 & 28.822 & 36.248 & 26.585 & 33.979 & 32.401 & 37.381 & 24.349 & 29.044 & 30.563 \\
\hline Anorthite- & 14.147 & 24.637 & 22.170 & 24.350 & 25.242 & 31.171 & 24.140 & 25.432 & 11.568 & 26.019 & 25.617 & 26.681 \\
\hline Nepheline - & - & - & - & - & - & - & - & - & - & 2.414 & 0.387 & - \\
\hline Wollastonite- & 0.886 & 1.895 & 6.505 & 5.106 & 1.061 & 3.790 & 0.909 & 2.743 & 0.060 & 8.388 & 6.682 & 1.608 \\
\hline Enstatite & 3.721 & 3.760 & 5.387 & 6.981 & 4.466 & 8.582 & 5.751 & 8.784 & 3.751 & 6.542 & 4.605 & 7.496 \\
\hline Ferrosilite - & 4.052 & 6.225 & 5.468 & 5.817 & 6.088 & 7.119 & 7.466 & 6.550 & 5.529 & 0.929 & 1.538 & 5.904 \\
\hline Forsterite - & - & - & 5.588 & 3.850 & - & - & - & - & - & 8.346 & 10.101 & - \\
\hline Fayalite & - & - & 6.251 & 3.536 & - & - & - & - & - & 1.306 & 3.719 & - \\
\hline Magnetite- & 3.899 & 3.940 & 2.017 & 4.503 & 3.033 & 4.408 & 5.677 & 4.821 & 6.114 & 7.839 & 5.536 & 5.528 \\
\hline Hematite --_ & - & -- & - & - & - & - & -- & - & - & -- & - & -- \\
\hline Ilmenite-- & 1.854 & 2.485 & 5.283 & 5.898 & 2.270 & 5.005 & 2.669 & 4.210 & 1.831 & 4.944 & 3.817 & 4.192 \\
\hline Apatite - & 0.684 & 1.097 & 1.365 & 1.376 & 0.991 & 1.992 & 1.831 & 1.957 & 0.357 & 1.186 & 0.857 & 1.378 \\
\hline Calcite - & 0.045 & 0.092 & 0.136 & 0.137 & 0.045 & 0.184 & 0.183 & 0.573 & 0.091 & 0.820 & 0.137 & 0.183 \\
\hline Total & 97.725 & 97.198 & 94.816 & 97.478 & 97.443 & 97.715 & 96.028 & 97.657 & 97.399 & 98.997 & 99.166 & 97.504 \\
\hline Salic_-_ & 82.584 & 77.705 & 56.817 & 60.275 & 79.488 & 66.635 & 71.543 & 68.020 & 79.666 & 58.698 & 62.174 & 71.215 \\
\hline Femic - & 15.141 & 19.493 & 37.999 & 37.203 & 17.955 & 31.080 & 24.485 & 29.638 & 17.733 & 40.299 & 36.993 & 26.289 \\
\hline Diopside $^{4}$ & 1.761 & 3.819 & 12.897 & 10.056 & 2.124 & 7.464 & 1.817 & 5.383 & 0.121 & 15.859 & 12.826 & 3.162 \\
\hline DiWo- & 0.886 & 1.895 & 6.505 & 5.106 & 1.061 & 3.790 & 0.909 & 2.743 & 0.060 & 8.388 & 6.682 & 1.608 \\
\hline DiEn $\longrightarrow$ & 0.419 & 0.725 & 3.172 & 2.700 & 0.450 & 2.008 & 0.395 & 1.512 & 0.025 & 6.542 & 4.605 & 0.869 \\
\hline DiFs - & 0.456 & 1.200 & 3.220 & 2.250 & 0.613 & 1.666 & 0.513 & 1.128 & 0.036 & 0.929 & 1.538 & 0.685 \\
\hline Hypersthene $^{5}$ & 6.898 & 8.060 & 4.463 & 7.847 & 9.491 & 12.027 & 12.308 & 12.693 & 9.219 & - & - & 11.846 \\
\hline HyEn -- & 3.302 & 3.035 & 2.215 & 4.280 & 4.016 & 6.574 & 5.355 & 7.271 & 3.726 & - & - & 6.627 \\
\hline HyFs-- & 3.596 & 5.025 & 2.248 & 3.567 & 5.475 & 5.453 & 6.953 & 5.422 & 5.493 & -- & - & 5.219 \\
\hline Olivine $^{6}$ & -- & - & 11.839 & 7.386 & - & - & - & - & - & 9.652 & 13.820 & - \\
\hline $\mathrm{OlFo}_{0}$ & - & - & 5.588 & 3.850 & $\ldots$ & - & - & - & -- & 8.346 & 10.101 & - \\
\hline $\mathrm{OlFa}-$ & - & - & 6.251 & 3.536 & - & - & - & - & - & 1.306 & 3.719 & - \\
\hline \multicolumn{13}{|l|}{$\begin{array}{l}\text { Wollastonite } \\
\text { minus }\end{array}$} \\
\hline DiWo-_- & - & - & - & - & - & - & - & - & -- & - & - & - \\
\hline
\end{tabular}

Footnotes at end of table. 
TABLE 9.-Chemical and normative analyses of Cenozoic igneous rocks from western Saudi Arabia-Continued

\begin{tabular}{|c|c|c|c|c|c|}
\hline $\begin{array}{l}\text { Sample (location given on } \\
\text { pli. 1) } \\
\text { Field no. } \\
\text { Type of source rock }{ }^{3}-\end{array}$ & $\begin{array}{c}64 \\
939 \\
0\end{array}$ & $\begin{array}{c}65 \\
941 \\
\mathrm{X} \\
\end{array}$ & $\begin{array}{c}66 \\
940 \\
\mathrm{X}\end{array}$ & $\begin{array}{r}67 \\
520 \\
\times \\
\end{array}$ & $\begin{array}{c}73 \\
514-5 \\
\mathrm{~T}\end{array}$ \\
\hline \multicolumn{6}{|c|}{ Unadjusted oxides (wt. percent) } \\
\hline $\mathrm{SiO}_{2}$ & 51.50 & 47.00 & 47.00 & 52.50 & 47.70 \\
\hline $\mathrm{Al}_{2} \mathrm{O}_{3} \ldots$ & 18.60 & 15.50 & 17.10 & 11.80 & 15.70 \\
\hline $\mathrm{Fe}_{2} \mathrm{O}_{3}$ & 2.90 & 3.20 & 3.90 & 4.75 & 4.90 \\
\hline $\mathrm{FeO}-$ & 5.80 & 8.90 & 7.60 & 7.20 & 7.20 \\
\hline $\mathrm{MgO}$ & 2.40 & 9.20 & 5.60 & 4.64 & 7.10 \\
\hline $\mathrm{CaO} \ldots$ & 7.00 & 8.90 & 8.70 & 7.00 & 10.30 \\
\hline $\mathrm{Na}_{2} \mathrm{O}$ & 4.40 & 3.30 & 4.00 & 6.00 & 2.90 \\
\hline $\mathrm{K}_{2} \mathrm{O}-$ & 1.40 & 0.96 & 1.10 & 0.10 & 0.37 \\
\hline $\mathrm{H}_{2} \mathrm{O}-$ & 2.82 & 0.69 & 0.89 & 0.80 & 2.06 \\
\hline $\mathrm{TiO}_{2}$ & 1.60 & 1.90 & 2.40 & 2.80 & 1.40 \\
\hline $\mathrm{P}_{2} \mathrm{O}_{5}$ & 0.76 & 0.32 & 0.41 & 0.62 & 0.21 \\
\hline $\mathrm{MnO}$ & 0.15 & 0.18 & 0.18 & 0.13 & 0.21 \\
\hline $\mathrm{CO}_{2}$ & 0.08 & 0.04 & 0.06 & - & 0.05 \\
\hline Total & 99.41 & 100.09 & 98.94 & 98.34 & 100.10 \\
\hline \multicolumn{6}{|l|}{ Adjusted oxides: } \\
\hline $\mathrm{SiO}_{2}$ & 51.81 & 46.96 & 47.50 & 53.39 & 47.65 \\
\hline $\mathrm{Al}_{2} \mathrm{O}_{3}$ & 18.71 & 15.49 & 17.28 & 12.00 & 15.68 \\
\hline $\mathrm{Fe}_{2} \mathrm{O}_{3}$ & 2.92 & 3.20 & 3.94 & 4.83 & 4.90 \\
\hline $\mathrm{FeO}-$ & 5.83 & 8.89 & 7.68 & 7.32 & 7.19 \\
\hline $\mathrm{MgO}-$ & 2.41 & 9.19 & 5.66 & 4.72 & 7.09 \\
\hline $\mathrm{CaO}$ & 7.04 & 8.89 & 8.79 & 7.12 & 10.29 \\
\hline $\mathrm{Na}_{2} \mathrm{O}$ & 4.43 & 3.30 & 4.04 & 6.10 & 2.90 \\
\hline $\mathrm{K}_{2} \mathrm{O}$ & 1.41 & 0.96 & 1.11 & 0.10 & 0.37 \\
\hline $\mathrm{H}_{2} \mathrm{O}$ & 2.84 & 0.69 & 0.90 & 0.81 & 2.06 \\
\hline $\mathrm{TiO}_{2}$ & 1.61 & 1.90 & 2.43 & 2.85 & 1.40 \\
\hline $\mathrm{P}_{2} \mathrm{O}_{5}$ & 0.76 & 0.32 & 0.41 & 0.63 & 0.21 \\
\hline $\mathrm{MnO}-$ & 0.15 & 0.18 & 0.18 & 0.13 & 0.21 \\
\hline $\mathrm{CO}_{2}$ & 0.08 & 0.04 & 0.06 & - & 0.05 \\
\hline
\end{tabular}


TABLE 9.-Chemical and normative analyses of Cenozoic igneous rocks from western Saudi Arabia-Continued

\begin{tabular}{|c|c|c|c|c|c|c|}
\hline $\begin{array}{l}\text { Sample (location given on } \\
\text { pli. p) } \\
\text { Field no. } \\
\text { Type of source rock }\end{array}$ & $\begin{array}{c}64 \\
939 \\
0\end{array}$ & $\begin{array}{r}65 \\
941 \\
\mathrm{X}\end{array}$ & $\begin{array}{c}66 \\
940 \\
\mathrm{X}\end{array}$ & $\begin{array}{c}57 \\
520 \\
\mathrm{X}\end{array}$ & $\underset{T}{713}$ & \\
\hline \multicolumn{7}{|l|}{ Normative minerals: } \\
\hline Quartz & 2.181 & - & - & 0.371 & - & \\
\hline Corundum & - & - & - & - & - & \\
\hline Orthoclase- & 8.322 & 5.668 & 6.570 & 0.601 & 2.184 & \\
\hline Albite- & 37.453 & 24.820 & 29.781 & 51.627 & 24.514 & \\
\hline Anorthite- & 27.026 & 24.623 & 25.728 & 5.053 & 28.700 & \\
\hline Nepheline & - & 1.668 & 2.399 & - & - & \\
\hline Wollastonite & 1.004 & 7.160 & 6.182 & 10.915 & 8.627 & \\
\hline Enstatite- & 6.013 & 4.544 & 3.850 & 11.751 & 14.242 & \\
\hline Ferrosilite- & 5.927 & 2.161 & 1.961 & 4.998 & 5.841 & \\
\hline Forsterite & - & 12.858 & 7.180 & - & 2.399 & \\
\hline Fayalite & - & 6.739 & 4.032 & - & 1.084 & \\
\hline Magnetite & 4.230 & 4.636 & 5.715 & 7.003 & 7.097 & \\
\hline Hematite & - & - & - & - & - & \\
\hline Ilmenite & 3.057 & 3.605 & 4.607 & 5.408 & 2.656 & \\
\hline Apatite- & 1.811 & 0.757 & 0.982 & 1.493 & 0.497 & \\
\hline Calcite- & 0.183 & 0.091 & 0.138 & - & 0.114 & \\
\hline Total & 97.205 & 99.329 & 99.124 & 99.221 & 97.956 & \\
\hline Salic $\longrightarrow$ & 74.981 & 56.778 & 64.478 & 57.652 & 55.399 & \\
\hline Femic & 22.223 & 42.551 & 34.646 & 41.569 & 42.557 & \\
\hline Diopside $^{4}$ & 1.988 & 13.865 & 11.993 & 21.073 & 16.640 & \\
\hline DiWo & 1.004 & 7.160 & 6.182 & 10.915 & 8.627 & \\
\hline DiEn- & 0.496 & 4.544 & 3.850 & 7.127 & 5.682 & \\
\hline $\mathrm{DiFs}$ & 0.489 & 2.161 & 1.961 & 3.031 & 2.330 & \\
\hline Hypersthene $^{5}$ & 10.955 & - & - & 6.592 & 12.070 & \\
\hline HyEn- - & 5.517 & - & - & 4.624 & 8.560 & \\
\hline HyFs & 5.438 & - & - & 1.967 & 3.511 & \\
\hline Olivine $^{6}$ & - & 19.597 & 11.212 & - & 3.483 & \\
\hline OlFo - & - & 12.858 & 7.180 & - & 2.399 & \\
\hline $\mathrm{OlFa}$ & - & 6.739 & 4.032 & - & 1.084 & \\
\hline Wollastonite mi- & & & & & & \\
\hline nus DiWo & - & - & - & - & - & \\
\hline
\end{tabular}

b, $c$, and and $B$ indicate top and bottom flow, respectively, of thick section of many flows at same sample locality; $a$,

HyEn, enstatite content of hypersthene; $\mathrm{HyFs}$, ferrosilite content of hypersthen 
TABLE 10.-K-Ar ages for Cenozoic igneous rocks collected from western Saudia Arabia

[All material analyzed was whole rock except samples $34 \mathrm{a}$ and $34 \mathrm{~b}$, which were glauconite, and sample 70 , which was labradorite. Decay constants: $\lambda \beta=4.963 \times 10^{-111} / y \mathrm{r}$; $\lambda \zeta=0.581 \times 10^{-10} / \mathrm{yr} ; \kappa-40 / \mathrm{K}=1.167 \times 10^{-4}$ atomic percent $]$

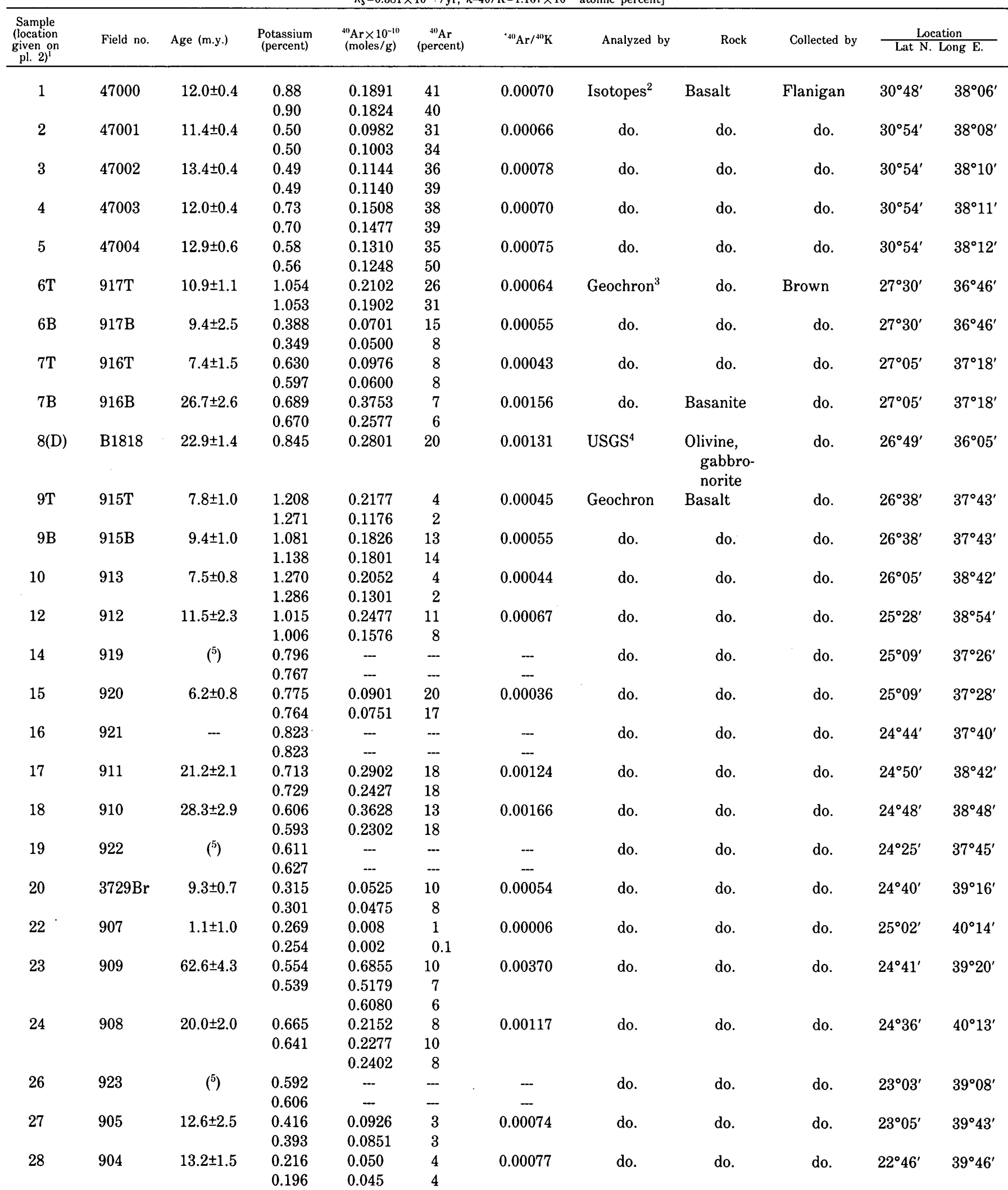


TABLE 10.-K-Ar ages for Cenozoic igneous rocks collected from western Saudia Arabia-Continued

[All material analyzed was whole rock except samples $34 \mathrm{a}$ and $34 \mathrm{~b}$, which were glauconite, and sample 70 , which was labradorite. Decay constants: $\lambda \beta=4.963 \times 10^{-10} / \mathrm{yr}$; $\lambda \zeta=0.581 \times 10^{-10} / \mathrm{yr} ; \kappa-40 / \mathrm{K}=1.167 \times 10^{-4}$ atomic percent]

\begin{tabular}{|c|c|c|c|c|c|c|c|c|c|c|c|}
\hline $\begin{array}{l}\text { Snmple } \\
\text { (location } \\
\text { given on } \\
\text { pl. } 2)^{1}\end{array}$ & Field no. & Age (m.y.) & $\begin{array}{c}\text { Potassium } \\
\text { (percent) }\end{array}$ & $\begin{array}{c}{ }^{40} \mathrm{Ar} \times 10^{-10} \\
\text { (moles/g) }\end{array}$ & 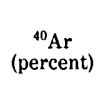 & ${ }^{\cdot 40} \mathrm{Ar} /{ }^{40} \mathrm{~K}$ & Analyzed by & Rock & Collected by & $\frac{\text { Lor }}{\text { Lat } N .}$ & $\frac{\text { jon }}{\text { ong E. }}$ \\
\hline 29 & $4702 B$ & $2.4 \pm 0.8$ & $\begin{array}{l}1.184 \\
1.207\end{array}$ & $\begin{array}{l}0.0375 \\
0.0826 \\
0.0275\end{array}$ & $\begin{array}{l}2 \\
5 \\
1\end{array}$ & 0.00014 & do. & do. & Flanigan & $22^{\circ} 40^{\prime}$ & $41^{\circ} 24^{\prime}$ \\
\hline 30 & GFB1 & $4.4 \pm 0.4$ & $\begin{array}{l}0.35 \\
0.37\end{array}$ & $\begin{array}{l}0.0270 \\
0.0283\end{array}$ & $\begin{array}{l}12 \\
10\end{array}$ & 0.00026 & Isotopes & do. & Brown & $22^{\circ} 14^{\prime}$ & $39^{\circ} 27^{\prime}$ \\
\hline 33 & GFB2 & $19.1 \pm 0.6$ & $\begin{array}{l}0.61 \\
0.61\end{array}$ & $\begin{array}{l}0.2019 \\
0.2056\end{array}$ & $\begin{array}{l}48 \\
59\end{array}$ & 0.00112 & do. & do. & do. & $21^{\circ} 58^{\prime}$ & $39^{\circ} 18^{\prime}$ \\
\hline $34 a$ & USFAN & $43.7 \pm 1.0$ & $\begin{array}{l}3.60 \\
3.56\end{array}$ & $\begin{array}{l}2.758 \\
2.738\end{array}$ & $\begin{array}{l}53 \\
44\end{array}$ & 0.00257 & do. & $\begin{array}{l}\text { Sandstone } \\
\quad \text { (glauconite) }\end{array}$ & do. & $21^{\circ} 58^{\prime}$ & $39^{\circ} 21^{\prime}$ \\
\hline $34 \mathrm{~b}$ & USFAN & $56.4 \pm 1.2$ & $\begin{array}{l}3.02 \\
3.04\end{array}$ & $\begin{array}{l}2.961 \\
3.059\end{array}$ & $\begin{array}{l}60 \\
66\end{array}$ & 0.00333 & do. & do. & do. & $21^{\circ} 58^{\prime}$ & $39^{\circ} 21^{\prime}$ \\
\hline 36 & 902 & $25.8 \pm 5.0$ & $\begin{array}{l}0.381 \\
0.351\end{array}$ & $\begin{array}{l}0.1776 \\
0.1526\end{array}$ & $\begin{array}{l}3 \\
2\end{array}$ & 0.00151 & do. & do. & do. & $21^{\circ} 50^{\prime}$ & $39^{\circ} 42^{\prime}$ \\
\hline 37 & $47027 \mathrm{~B}$ & $8.7 \pm 2.0$ & $\begin{array}{l}0.493 \\
0.494\end{array}$ & $\begin{array}{l}0.0450 \\
0.0851 \\
0.0926\end{array}$ & $\begin{array}{l}2 \\
7 \\
2\end{array}$ & 0.00050 & do. & do. & Flanigan & $21^{\circ} 51^{\prime}$ & $40^{\circ} 44^{\prime}$ \\
\hline 38 & 47026 & $8.0 \pm 0.5$ & $\begin{array}{l}1.121 \\
1.150\end{array}$ & $\begin{array}{l}0.1676 \\
0.1501\end{array}$ & $\begin{array}{l}9 \\
8\end{array}$ & 0.00047 & do. & do. & do. & $21^{\circ} 40^{\prime}$ & $40^{\circ} 58^{\prime}$ \\
\hline 39 & Well 3 & $25.9 \pm 3.0$ & $\begin{array}{l}0.395 \\
0.385\end{array}$ & $\begin{array}{l}0.1826 \\
0.1701\end{array}$ & $\begin{array}{l}21 \\
22\end{array}$ & 0.00152 & do. & do. & Brown & $21^{\circ} 24^{\prime}$ & $39^{\circ} 39^{\prime}$ \\
\hline $40(\mathrm{P})$ & 47024 & $22.2 \pm 3.5$ & $\begin{array}{l}4.187 \\
4.166\end{array}$ & $\begin{array}{l}1.596 \\
1.841 \\
1.409\end{array}$ & $\begin{array}{l}14 \\
14 \\
11\end{array}$ & 0.00130 & do. & $\begin{array}{l}\text { Horn- } \\
\text { blende, } \\
\text { latite- } \\
\text { phonolite }\end{array}$ & Flanigan & $21^{\circ} 18^{\prime}$ & $41^{\circ} 10^{\prime}$ \\
\hline 41 & 47023 & $16.6 \pm 1.5$ & $\begin{array}{l}0.854 \\
0.861\end{array}$ & $\begin{array}{l}0.2477 \\
0.2477\end{array}$ & $\begin{array}{l}18 \\
19\end{array}$ & 0.00097 & do. & Basalt & do. & $21^{\circ} 21^{\prime}$ & $41^{\circ} 20^{\prime}$ \\
\hline 43 & 47021 & $16.2 \pm 1.8$ & $\begin{array}{l}0.796 \\
0.774\end{array}$ & $\begin{array}{l}0.2702 \\
0.1927 \\
0.2027\end{array}$ & $\begin{array}{l}24 \\
21 \\
31\end{array}$ & 0.00095 & do. & do. & do. & $21^{\circ} 20^{\prime}$ & $41^{\circ} 25^{\prime}$ \\
\hline 44 & 47025 & $(5)$ & $\begin{array}{l}0.599 \\
0.595\end{array}$ & - & - & - & do. & Basalt & do. & $21^{\circ} 05^{\prime}$ & $41^{\circ} 35^{\prime}$ \\
\hline $45(\mathrm{P})$ & 926 & $19.3 \pm 0.9$ & $\begin{array}{l}3.785 \\
3.843\end{array}$ & $\begin{array}{l}1.284 \\
1.286\end{array}$ & $\begin{array}{l}28 \\
33\end{array}$ & 0.00113 & do. & $\begin{array}{c}\text { Rhyo- } \\
\text { lite }\end{array}$ & Brown & $20^{\circ} 56^{\prime}$ & $39^{\circ} 31^{\prime}$ \\
\hline $46 \mathrm{a}(\mathrm{P})$ & $927 \mathrm{~A}$ & $26.8 \pm 4.5$ & $\begin{array}{l}0.272 \\
0.263\end{array}$ & $\begin{array}{l}0.1351 \\
0.1151\end{array}$ & $\begin{array}{l}6 \\
4\end{array}$ & 0.00157 & do. & Gabbro & do. & $20^{\circ} 58^{\prime}$ & $39^{\circ} 35^{\prime}$ \\
\hline $46 \mathrm{~b}(\mathrm{P})$ & $927 \mathrm{~B}$ & $27.9 \pm 5.5$ & $\begin{array}{l}0.282 \\
0.266\end{array}$ & $\begin{array}{l}0.1576 \\
0.1101\end{array}$ & $\begin{array}{l}6 \\
4\end{array}$ & 0.00164 & do. & do. & do. & $20^{\circ} 58^{\prime}$ & $39^{\circ} 35^{\prime}$ \\
\hline 47 & $6 \mathrm{MX} 68$ & $7.0 \pm 4.3$ & $\begin{array}{l}0.17 \\
0.19\end{array}$ & $\begin{array}{l}0.0295 \\
0.01415\end{array}$ & $\begin{array}{l}1 \\
1\end{array}$ & 0.00041 & Isotopes & Basalt & do. & $20^{\circ} 56^{\prime}$ & $39^{\circ} 36^{\prime}$ \\
\hline $48(\mathrm{P})$ & 66 & $21.3 \pm 2.1$ & $\begin{array}{l}3.40 \\
3.37\end{array}$ & $\begin{array}{l}1.101 \\
1.376 \\
1.301\end{array}$ & $\begin{array}{l}7 \\
9 \\
8\end{array}$ & 0.00125 & Geochron & $\begin{array}{l}\text { Tra- } \\
\text { chyte }\end{array}$ & Gaskill & $20^{\circ} 53^{\prime}$ & $39^{\circ} 38^{\prime}$ \\
\hline 49 & $5 \mathrm{MX} 68$ & $2.9 \pm 0.2$ & $\begin{array}{l}1.00 \\
0.96\end{array}$ & $\begin{array}{l}0.0516 \\
0.0480\end{array}$ & $\begin{array}{l}22 \\
24\end{array}$ & 0.00017 & Isotopes & Basalt & Brown & $20^{\circ} 44^{\prime}$ & $39^{\circ} 40^{\prime}$ \\
\hline 50 & $\mathrm{HT}$ & $2.8 \pm 0.1$ & $\begin{array}{l}1.33 \\
1.31\end{array}$ & $\begin{array}{l}0.0652 \\
0.0623\end{array}$ & $\begin{array}{l}32 \\
35\end{array}$ & 0.00016 & do. & do. & do. & $20^{\circ} 42^{\prime}$ & $39^{\circ} 42^{\prime}$ \\
\hline
\end{tabular}


TABLE 10.-K-Ar ages for Cenozoic igneous rocks collected from western Saudia Arabia-Continued

[All material analyzed was whole rock except samples $34 \mathrm{a}$ and $34 \mathrm{~b}$, which were glauconite, and sample 70 , which was labradorite. Decay constants: $\lambda \beta=4.963 \times 10^{-10} / \mathrm{yr}$; $\lambda \zeta=0.581 \times 10^{-10} / \mathrm{yr} ; \kappa-40 / \mathrm{K}=1.167 \times 10^{-4}$ atomic percent $]$

\begin{tabular}{|c|c|c|c|c|c|c|c|c|c|c|c|}
\hline $\begin{array}{l}\text { Sample } \\
\text { (location } \\
\text { given on } \\
\text { pl. 2) }\end{array}$ & Field no. & Age (m.y.) & $\begin{array}{c}\text { Potassium } \\
\text { (percent) }\end{array}$ & $\begin{array}{l}{ }^{40} \mathrm{Ar} \times 10^{-10} \\
(\mathrm{moles} / \mathrm{g})\end{array}$ & $\begin{array}{c}{ }^{40} \mathrm{Ar} \\
\text { (percent) }\end{array}$ & ${ }^{40} \mathrm{Ar} /{ }^{40} \mathrm{~K}$ & Analyzed by & Rock & Collected by & $\frac{\text { Lo }}{\text { Lat } N}$ & tion \\
\hline $51 \mathrm{a}(\mathrm{D})$ & $623 \mathrm{~A}$ & $177 . \pm 13$ & $\begin{array}{l}0.50 \\
0.51\end{array}$ & 1.632 & 53 & 0.0108 & USGS & Gabbro & do. & $20^{\circ} 26^{\prime}$ & $40^{\circ} 13^{\prime}$ \\
\hline $51 \mathrm{~b}(\mathrm{D})$ & $623 \mathrm{~B}$ & $21.5 \pm 2.3$ & $\begin{array}{l}0.91 \\
0.90\end{array}$ & 0.3397 & 26 & 0.00126 & do. & do. & do. & $20^{\circ} 26^{\prime}$ & $40^{\circ} 13^{\prime}$ \\
\hline $51 \mathrm{c}(\mathrm{D})$ & $623 \mathrm{C}$ & $273 . \pm 20$ & $\begin{array}{l}0.54 \\
0.53\end{array}$ & 2.731 & 41 & 0.0171 & do. & do. & do. & $20^{\circ} 26^{\prime}$ & $40^{\circ} 13^{\prime}$ \\
\hline $52 \mathrm{a}(\mathrm{D})$ & $928 \mathrm{~A}$ & $18.0 \pm 1.3$ & $\begin{array}{l}1.364 \\
1.317\end{array}$ & $\begin{array}{l}0.4153 \\
0.4253\end{array}$ & $\begin{array}{l}17 \\
16\end{array}$ & 0.00105 & Geochron & Monzonite & do. & $20^{\circ} 22^{\prime}$ & $40^{\circ} 21^{\prime}$ \\
\hline $52 \mathrm{~b}(\mathrm{D})$ & $928 \mathrm{~B}$ & $25.3 \pm 1.8$ & $\begin{array}{l}1.008 \\
1.022\end{array}$ & $\begin{array}{l}0.4504 \\
0.4479\end{array}$ & $\begin{array}{l}26 \\
26\end{array}$ & 0.00148 & do. & do. & do. & $20^{\circ} 22^{\prime}$ & $40^{\circ} 21^{\prime}$ \\
\hline $52 \mathrm{c}(\mathrm{D})$ & $928 \mathrm{C}$ & $23.6 \pm 1.3$ & $\begin{array}{l}2.221 \\
2.210\end{array}$ & $\begin{array}{l}0.9107 \\
0.9032\end{array}$ & $\begin{array}{l}24 \\
24\end{array}$ & 0.00137 & do. & do. & do. & $20^{\circ} 22^{\prime}$ & $40^{\circ} 21^{\prime}$ \\
\hline $52 \mathrm{~d}(\mathrm{D})$ & $928 \mathrm{D}$ & $20.8 \pm 1.2$ & $\begin{array}{l}2.246 \\
2.307\end{array}$ & $\begin{array}{l}0.7981 \\
0.8532\end{array}$ & $\begin{array}{l}10 \\
11\end{array}$ & 0.00121 & do. & do. & do. & $20^{\circ} 22^{\prime}$ & $40^{\circ} 21^{\prime}$ \\
\hline 53 & 601 & $1.8 \pm 1.2$ & $\begin{array}{l}0.68 \\
0.66\end{array}$ & 0.0204 & 14 & 0.00010 & USGS & Basalt & do. & $20^{\circ} 51^{\prime}$ & $42^{\circ} 17^{\prime}$ \\
\hline $54(\mathrm{D})$ & Qun & $19.1 \pm 0.6$ & $\begin{array}{l}0.49 \\
0.48\end{array}$ & $\begin{array}{l}0.1615 \\
0.1617\end{array}$ & $\begin{array}{l}39 \\
28\end{array}$ & 0.00112 & Isotopes & Diabase & do. & $19^{\circ} 15^{\prime}$ & $41^{\circ} 19^{\prime}$ \\
\hline $55 \mathrm{a}(\mathrm{D})$ & 930 & $22.4 \pm 1.4$ & $\begin{array}{l}1.723 \\
1.705\end{array}$ & $\begin{array}{l}0.6855 \\
0.6530\end{array}$ & $\begin{array}{l}14 \\
14\end{array}$ & 0.00131 & Geochron & Diorite & do. & $18^{\circ} 45^{\prime}$ & $41^{\circ} 32^{\prime}$ \\
\hline $55 \mathrm{~b}$ & 931 & $12.4 \pm 1.2$ & $\begin{array}{l}0.917 \\
0.923\end{array}$ & $\begin{array}{l}0.1977 \\
0.1977\end{array}$ & $\begin{array}{l}1 \\
4\end{array}$ & 0.00072 & do. & Basalt & do. & $18^{\circ} 45^{\prime}$ & $41^{\circ} 32^{\prime}$ \\
\hline $56 \mathrm{a}(\mathrm{D})$ & $933 \mathrm{~A}$ & $43.1 \pm 3.0$ & $\begin{array}{l}0.880 \\
0.855\end{array}$ & $\begin{array}{l}0.6180 \\
0.6931\end{array}$ & $\begin{array}{l}31 \\
33\end{array}$ & 0.00253 & do. & Diorite & do. & $18^{\circ} 32^{\prime}$ & $41^{\circ} 38^{\prime}$ \\
\hline $56 \mathrm{~b}(\mathrm{D})$ & $933 \mathrm{~B}$ & $41.7 \pm 2.8$ & $\begin{array}{l}0.847 \\
0.836\end{array}$ & $\begin{array}{l}0.6105 \\
0.6205\end{array}$ & 23 & 0.00245 & do. & do. & do. & $18^{\circ} 32^{\prime}$ & $41^{\circ} 38^{\prime}$ \\
\hline $56 \mathrm{c}(\mathrm{D})$ & $933 \mathrm{C}$ & $34.7 \pm 2.1$ & $\begin{array}{l}1.245 \\
1.280\end{array}$ & $\begin{array}{l}0.7731 \\
0.7606\end{array}$ & $\begin{array}{l}34 \\
33\end{array}$ & 0.00203 & do. & $\begin{array}{l}\text { Grano- } \\
\text { diorite }\end{array}$ & do. & $18^{\circ} 32^{\prime}$ & $41^{\circ} 38^{\prime}$ \\
\hline $57 a$ & 932 & $2.1 \pm 1.0$ & $\begin{array}{l}0.691 \\
0.694\end{array}$ & $\begin{array}{l}0.025 \\
0.025\end{array}$ & $\begin{array}{l}3 \\
4\end{array}$ & 0.000121 & do. & Basalt & do. & $18^{\circ} 28^{\prime}$ & $41^{\circ} 31^{\prime}$ \\
\hline $57 \mathrm{~b}$ & 932 & $2.8 \pm 1.5$ & $\begin{array}{l}0.669 \\
0.685\end{array}$ & $\begin{array}{l}0.0450 \\
0.0200\end{array}$ & $\begin{array}{l}6 \\
3\end{array}$ & 0.00016 & do. & do. & do. & $18^{\circ} 28^{\prime}$ & $41^{\circ} 31^{\prime}$ \\
\hline 59 & 934 & $12.4 \pm 3$ & $\begin{array}{l}0.549 \\
0.509\end{array}$ & $\begin{array}{l}0.0926 \\
0.135\end{array}$ & $\begin{array}{l}3 \\
4\end{array}$ & 0.00072 & do. & do. & do. & $18^{\circ} 08^{\prime}$ & $41^{\circ} 34^{\prime}$ \\
\hline $60(\mathrm{D})$ & 935 & $19.2 \pm 1.1$ & $\begin{array}{l}2.753 \\
2.696\end{array}$ & $\begin{array}{l}0.9458 \\
0.8807\end{array}$ & $\begin{array}{l}42 \\
42\end{array}$ & 0.00112 & do. & $\begin{array}{l}\text { Grano- } \\
\text { diorite }\end{array}$ & do. & $18^{\circ} 09^{\prime}$ & $41^{\circ} 40^{\prime}$ \\
\hline 61 & $938 \mathrm{~A}$ & $12.1 \pm 1.5$ & $\begin{array}{l}0.798 \\
0.799\end{array}$ & $\begin{array}{l}0.160 \\
0.175\end{array}$ & $\begin{array}{l}9 \\
9\end{array}$ & 0.00070 & do. & Basalt & do. & $18^{\circ} 07^{\prime}$ & $41^{\circ} 44^{\prime}$ \\
\hline 62 & 937 & $4.3 \pm 0.6$ & $\begin{array}{l}1.090 \\
1.084\end{array}$ & $\begin{array}{l}0.0901 \\
0.0726\end{array}$ & $\begin{array}{l}8 \\
8\end{array}$ & 0.00025 & do. & do. & do. & $18^{\circ} 04^{\prime}$ & $41^{\circ} 45^{\prime}$ \\
\hline $63(\mathrm{D})$ & 936 & $23.0 \pm 1.5$ & $\begin{array}{l}1.375 \\
1.367\end{array}$ & $\begin{array}{l}0.5755 \\
0.5254\end{array}$ & $\begin{array}{l}26 \\
25\end{array}$ & 0.00135 & do. & Diorite & do. & $18^{\circ} 04^{\prime}$ & $41^{\circ} 46^{\prime}$ \\
\hline $64(\mathrm{D})$ & 939 & $25.3 \pm 1.7$ & $\begin{array}{l}1.298 \\
1.313\end{array}$ & $\begin{array}{l}0.5680 \\
0.5855\end{array}$ & $\begin{array}{l}35 \\
28\end{array}$ & 0.00148 & do. & Gabbro & do. & $18^{\circ} 02^{\prime}$ & $41^{\circ} 53^{\prime}$ \\
\hline 65 & 941 & $5.1 \pm 1.5$ & $\begin{array}{l}0.881 \\
0.885\end{array}$ & $\begin{array}{l}0.0776 \\
0.0776\end{array}$ & $\begin{array}{l}7 \\
7\end{array}$ & 0.00029 & do. & Basalt & do. & $18^{\circ} 30^{\prime}$ & $42^{\circ} 02^{\prime}$ \\
\hline 66 & 940 & $5.1 \pm 1.5$ & $\begin{array}{l}1.049 \\
1.082\end{array}$ & $\begin{array}{l}0.0776 \\
0.113\end{array}$ & $\begin{array}{l}7 \\
8\end{array}$ & 0.00030 & do. & do. & do. & $18^{\circ} 01^{\prime}$ & $42^{\circ} 05^{\prime}$ \\
\hline 67 & 520 & $25.3 \pm 0.5$ & $\begin{array}{l}1.34 \\
1.32\end{array}$ & $\begin{array}{l}0.5901 \\
0.5865\end{array}$ & $\begin{array}{l}92 \\
93\end{array}$ & 0.00148 & Isotopes & do. & do. & $18^{\circ} 10^{\prime}$ & $43^{\circ} 10^{\prime}$ \\
\hline 68 & $519 \mathrm{~B}$ & $22.5 \pm 0.7$ & $\begin{array}{r}3.865 \\
\text { avg. }\end{array}$ & 1.262 & 84 & 0.00129 & USGS & Syenite & do. & $18^{\circ} 05^{\prime}$ & $43^{\circ} 11^{\prime}$ \\
\hline 69 & 518 & $30.1 \pm 1.0$ & $\begin{array}{l}0.52 \\
0.54\end{array}$ & $\begin{array}{l}0.2773 \\
0.2799\end{array}$ & $\begin{array}{l}36 \\
32\end{array}$ & 0.00176 & Isotopes & do. & do. & $17^{\circ} 57^{\prime}$ & $43^{\circ} 12^{\prime}$ \\
\hline $70(\mathrm{~L})$ & $103 \mathrm{~B}$ & $21.8 \pm 1.4$ & $\begin{array}{l}0.23 \\
0.20\end{array}$ & $\begin{array}{l}0.0830 \\
0.0808\end{array}$ & $\begin{array}{l}14 \\
20\end{array}$ & 0.00128 & do. & Diorite & do. & $17^{\circ} 26^{\prime}$ & $42^{\circ} 36^{\prime}$ \\
\hline $72 \mathrm{a}(\mathrm{L})$ & $516 \mathrm{C}$ & $20.5 \pm 2$ & $\begin{array}{l}0.093 \\
0.080\end{array}$ & $\begin{array}{l}0.0290 \\
0.0327\end{array}$ & $\begin{array}{l}13 \\
18\end{array}$ & 0.00119 & do. & Gabbro & do. & $17^{\circ} 03^{\prime}$ & $42^{\circ} 55^{\prime}$ \\
\hline
\end{tabular}


TABLE 10.-K-Ar ages for Cenozoic igneous rocks collected from western Saudia Arabia-Continued [All material analyzed was whole rock except samples $34 \mathrm{a}$ and $34 \mathrm{~b}$, which were glauconite, and sample 70 , which was labradorite. Decay constants: $\lambda \beta=4.963 \times 10^{-10} / \mathrm{yr}$; $\lambda \zeta=0.581 \times 10^{-10} / \mathrm{yr} ; \kappa-40 / \mathrm{K}=1.167 \times 10^{-4}$ atomic percent $]$

\begin{tabular}{|c|c|c|c|c|c|c|c|c|c|c|c|}
\hline $\begin{array}{l}\text { Sample } \\
\text { (location } \\
\text { given on } \\
\text { pl. } 2)^{1}\end{array}$ & Field no. & Age (m.y.) & $\underset{\text { (percent) }}{\text { Potassium }}$ & $\begin{array}{c}{ }^{40} \mathrm{Ar} \times 10^{-10} \\
(\mathrm{moles} / \mathrm{g})\end{array}$ & $\begin{array}{c}{ }^{40} \mathrm{Ar} \\
\text { (percent) }\end{array}$ & ${ }^{400} \mathrm{Ar} /{ }^{410} \mathrm{~K}$ & Analyzed by & Rock & Collected by & $\frac{\text { Lo }}{\text { Lat } N .}$ & ion \\
\hline $72 \mathrm{~b}(\mathrm{H})$ & $517 \mathrm{C}$ & $24.9 \pm 1.0$ & $\begin{array}{l}3.12 \\
3.15\end{array}$ & $\begin{array}{l}1.351 \\
1.371\end{array}$ & $\begin{array}{l}26 \\
27\end{array}$ & 0.00145 & do. & Hornfels & do. & $16^{\circ} 57^{\prime}$ & $42^{\circ} 57^{\prime}$ \\
\hline $73(\mathrm{~L})$ & $514-5$ & $23.4 \pm 2$ & $\begin{array}{l}0.14 \\
0.14\end{array}$ & $\begin{array}{l}0.0051 \\
0.0595\end{array}$ & $\begin{array}{l}19 \\
15\end{array}$ & 0.00137 & do. & Gabbro & do. & $16^{\circ} 58^{\prime}$ & $42^{\circ} 57^{\prime}$ \\
\hline $74 \mathrm{a}(\mathrm{G})$ & $515 \mathrm{~A}$ & $21.1 \pm 0.6$ & $\begin{array}{l}3.17 \\
3.13\end{array}$ & $\begin{array}{l}1.171 \\
1.143\end{array}$ & $\begin{array}{l}43 \\
43\end{array}$ & 0.00123 & do. & $\begin{array}{l}\text { Grano- } \\
\text { phyre }\end{array}$ & do. & $16^{\circ} 56^{\prime}$ & $42^{\circ} 58^{\prime}$ \\
\hline $74 \mathrm{~b}(\mathrm{G})$ & $515 \mathrm{~B}$ & $23.9 \pm 1.0$ & $\begin{array}{l}1.71 \\
1.70\end{array}$ & $\begin{array}{l}0.7209 \\
0.7048\end{array}$ & $\begin{array}{l}26 \\
29\end{array}$ & 0.00140 & do. & do. & do. & $16^{\circ} 56^{\prime}$ & $42^{\circ} 58^{\prime}$ \\
\hline $\begin{array}{c}\text { Yemen } \\
(\mathrm{G})\end{array}$ & ROJ1 & $23.3 \pm 0.9$ & 1.26 & 0.4247 & 61 & 0.00136 & do. & Granite & Jackson & $13^{\circ} 30^{\prime}$ & $44^{\circ} 02^{\prime}$ \\
\hline $\begin{array}{l}\text { Ethiopia } \\
\text { (D) }\end{array}$ & Asmara & $37.1 \pm 1.2$ & 0.29 & $\begin{array}{l}0.1884 \\
0.1826\end{array}$ & $\begin{array}{l}32 \\
32\end{array}$ & 0.00218 & Isotopes & Basalt & Brown & $15^{\circ} 17^{\prime}$ & $38^{\circ} 56^{\prime}$ \\
\hline
\end{tabular}

Nore $-40 \mathrm{Ar}$ is radiogenic argon.

${ }^{1} \mathrm{~T}$ and $\mathrm{B}$ indicate top and bottom flows, respectively, of thick section of many flows at same sample locality. (D) indicates dike; (P), plug; (L), layered gabbro; (H), hornfels; (G), granophyre; absence of letter in parentheses indicates lava flow. Lowercase letters (a, b, $c$ d) indicate multiple samples from same locality.

\author{
"Isotopes, Inc., later Teledyne Isotope, Westwood, N.J. \\ ${ }^{3}$ Geochron Laboratories, Inc., Cambridge, Mass. \\ ${ }^{4}$ USGS analysts, R.F. Marvin, H.H. Mehnert, and Violet Merritt. \\ "Radiogenic argon could not be measured, owing, it is believed, to the very young age \\ of the basalts.
}

anorthosite, and diorite or quartz monzogabbro, although a few are as alkaline as quartz syenite (fig. 52).

The chemical composition of the sheeted dikes, cumulative gabbro, granophyre, and associated rocks of the Tihamat-Asir Complex resemble the ophiolites of $\mathrm{Cy}$ prus, Oman, and Newfoundland and are considered to be ophiolitic in the Tihamat-Asir by Coleman and others (1979). These writers found that a plot of $\mathrm{FeO}^{*}$ (total iron as $\mathrm{FeO}$ ), $\mathrm{SiO}_{2}$, and $\mathrm{TiO}_{2}$ against $\mathrm{FeO} / \mathrm{MgO}$ places the gabbro, granophyre, and sheeted dikes as well as the continental dikes of the complex within the tholeiitic series rather than the calc-alkalic series. The continental dikes between Jiddah and Ad Darb range in $\mathrm{K}$-Ar age from $273 \pm 20$ to $18.0 \pm 1.3$ m.y. (table 10, pl. 2). The older ages undoubtedly represent additional argon derived from the invaded Precambrian terrane, a situation similar to that in Liberia, where tholeiitic dikes parallel to the Atlantic coastline gave anomalously old ages in the Precambrian but concordant and younger ages in the Paleozoic sedimentary rocks (Dalrymple and others, 1975). One dike $175 \mathrm{~km}$ southeast of Jiddah, near Al Lith (sample 51, table 10, pl. 2), gave ages of $177 \pm 13$ and $273 \pm 20$ m.y. (K-Ar whole-rock, normalized to the Sydney decay rate) from the midzone, but an age of $21.5 \pm 2.3$ m.y. from the chilled edge, most likely near the age of emplacement. Nine whole-rock samples from five other dikes range in age from $18.0 \pm 1.3$ to $25.3 \pm 1.7$ m.y. (table 10), with an arithmetic mean of 22.1 m.y. A gabbroic body (normative anorthosite) in a small outcrop $70 \mathrm{~km}$ south of Jiddah at Jabal Sitā' gave ages of $26.8 \pm 4.5$ and $27.9 \pm 5.5$ m.y. (sample 46 , table 10 , pl. 2 ); it is probably to be correlated with the continental dikes. If so, this marks the northern end of the southern continental dikes, there being a distinct break in the continental dike set about the latitude of Jiddah (pl. 2). A linear magnetic low in the eastern Red Sea west of Jiddah strikes S. $63^{\circ}$ E. and passes near the leucocratic gabbro (normative anorthosite) outcrop (Kabbani, 1970). The magnetic anomalies associated with the southern continental dikes reappear $80 \mathrm{~km}$ to the northeast of Makkah, suggesting either a transverse fault with horizontal movement, possibly a transform zone, or a major en echelon offset in the northwesttrending fracture pattern, as suggested by Blank (1977). The only age determination on the northern continental dikes is of an olivine gabbronorite northwest of Al Wajd, which gave an age of $22.9 \pm 1.4$ m.y. (sample 8 , table 10). This age is concordant with the ages of the southern continental dikes, but the dike lacks a magnetic signature. The ages of five whole-rock samples from the layered gabbro and granophyre plutons that are part of the Tihamat-Asir ophiolite at Jabal at Tirf range from $20.5 \pm 2$ to $24.9 \pm 1$ m.y. (samples $72-74$, table 10 ) with a mean of $22.7 \pm 2$ m.y. A comparable age of $21.8 \pm 1.4 \mathrm{~m} . \mathrm{y}$. (sample 70 , table 10) for labradorite is from a layered diorite at Wādi Baysh 60 $\mathrm{km}$ northwest of Jabal at Tirf.

The Tihamat-Asir Complex intrudes the Precambrian basement, the overlying Paleozoic and Mesozoic sedimentary rocks, and the early Miocene Jizan Group, including the freshwater Baid Formation. Diabase sills intruded into the Baid Formation at lat $19^{\circ} 15^{\prime} \mathrm{N}$. gave a $\mathrm{K}$-Ar age of $19.1 \pm 0.6 \mathrm{~m} . \mathrm{y}$. (sample 54 , table $10, \mathrm{pl}$. 2), concordant with the ages measured by Fleck (Coleman and others, 1979), and this confirms that the tholeiitic rocks are younger than the Baid Formation. 
TABLE 11.-Locations and rock types for Cenozoic igneous rocks for which chemical analyses are given in table 9 and isotopic ages in table 10

[Petrographic names are based on CIPW normative calculations, after Irvine and Baragar; modal names are based on petrographic studies by Salman Bloch, Robert Coleman, and Richard Blank. m, meters]

\begin{tabular}{l}
$\begin{array}{l}\text { Sample (location } \\
\text { given on pl. 2) }\end{array} \quad$ Field no. \\
\hline
\end{tabular}

1 Alkaline basalt. Al Harrah (Ḩarrat ash Shama).

$2-47001-$ do.

$3-47002-10$ do.

$4-47003-47004$ do.

5 - 57004 Hawaiite.

6T-_-_- Hawaiite. Uppermost flow, Ḩarrat ar Rahāh, west edge.

6B_-_alkaline basalt. Basal flow, Harrat ar Rahāh, west edge.

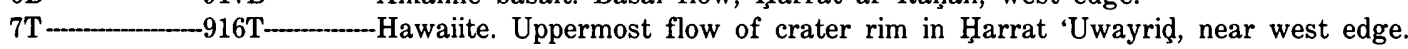

7B-_._._. Alkaline basalt. Basal flow, Harrat 'Uwayrid.

8(D) -_-_-Olivine-gabbro norite. Nonmagnetic dike, Wādì Marrā.

$9 \mathrm{~T}-915 \mathrm{~T}-\mathrm{H}$-_._._. Hawaiite. Uppermost flow, Harrat 'Uwayrid, south tip.

9B-_ Hawaiite. Basal flow, Harrat 'Uwayriḑ, south tip.

10 - Hawaiite. Upper of four flows, northwest outlier of Harrat Khaybar.

12 Alkaline basalt. Lowermost flow, west side Harrat al Kūrá. Vesicular.

13D-Gabbro, altered, minor interstitial quartz. Dike Wādī Umm Natash. Questionable Tertiary.

14 -Hawaiite. Lower 6-m flow in Harrat Lunayyir.

15 - Hawaiite. Recent black aa; uppermost Harrat Lunayyir.

16 - Basanite. Recent flow, $10 \mathrm{~m}$ thick. Jabal Salajah.

17 - 911 -____._._._. Hawaiite. Uppermost 15-m flow, Harrat I'shara.

18 Alnaline basalt. Lowermost of 12 or more flows resting on early Tertiary silt, Ḩarrat al Jarf.

19 Basanite. Base of Holocene or Pleistocene 10-m flow, Harrat an Nabah, on coastal plain.

$19 \mathrm{a}(\mathrm{P})$ Hawaite. Plug intruded into Aja' Granite, Jabal Shammar.

20 -3729B - Basanite. Basal flow at Khaybar, Harrat Khaybar.

21 - 206 Rhyolitic crystal tuff. Abyad wa Ubayyid. Holocene eruption, east side Harrat Khaybar.

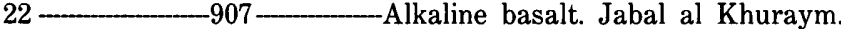

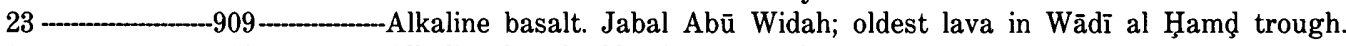

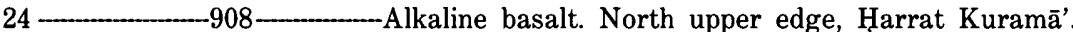

25 -B1314 --alkaline basalt. Harrat al Hutaymah. 12-m flow containing basaltic hornblende.

26 - Alkaline basalt. Uppermost Holocene flow, Harrat Rahat.

27 - Alkaline basalt. Western overflow from Harrat Rahat.

28 - Alkaline basalt. Western edge, Ḩarrat Rahat, lowermost flow in Wādī Thamrah.

29 -Alkaline basalt. Southeast edge of Harrat al Kishb.

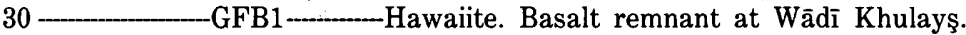

32 - Alkaline basalt. Eroded lava stream north of Al Kurā'.

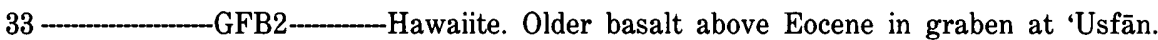

34a -................ Sandstone. Glauconite bearing.

$34 \mathrm{~b}$-USFAN _- Sandstone. Glauconite bearing.

35 -Well 4, Andesite. Overlies or intrudes Lower Eocene Shumaysi Formation. Area 1 (Al-Shanti, 1966).

36 -_._-Alkaline basalt. On graben of Ḩaddat ash Shām.

$37-47027 \mathrm{~B}$-..-Alkaline basalt. Harrat Biss.

38 - Hawaiite. Jabal al Barz. Isolated outlier on Sahl Rakbah. Ha_

39 Well 3,___ Basalt-andesite. Overlies or intrudes Lower Eocene Shumaysi Formation. Area 2A (Al-Shanti, 1966).

40(P)_______ Trachyte-phonolite plug. Jabal 'Ān.

41 Hawaiite. West side of Harrat Hadan, lowermost exposure.

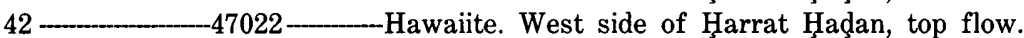

43 - 47021 - Alkali basalt. West side of Harrat Hadan.

Near basal outcrop $186 \mathrm{~m}$ above lowermost buried flow. Zeolitic.

Do.
Do.
Do.
Do. 
TABLE 11.-Locations and rock types for Cenozoic igneous rocks for which chemical analyses are given in table 9 and isotopic ages in table 10 - Continued

[Petrographic names are based on CIPW normative calculations, after Irvine and Baragar; modal names are based on petrographic studies by Salman Bloch, Robert Coleman, and Richard Blank. m, meters]

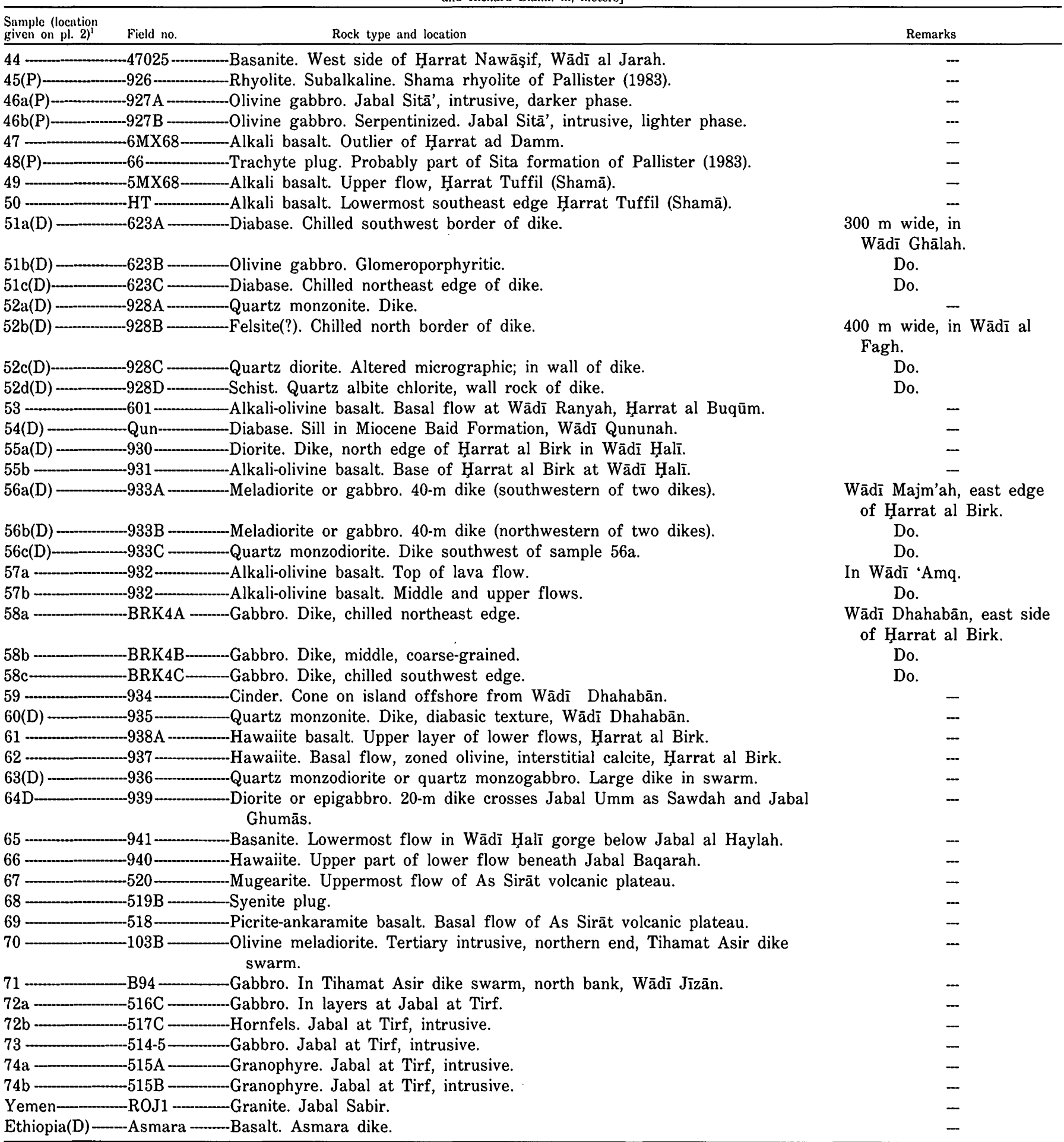

Ethiopia(D) --.-Asmara --..--Basalt. Asmara dike. ${ }^{\mathrm{I} T}$ and $\mathrm{B}$ indicate top and bottom flows, respectively, of thick section of many flows at same sample locality. (D) indicates dike; (P), plug. Lowercase letters (a, b, c, $d$ ) 


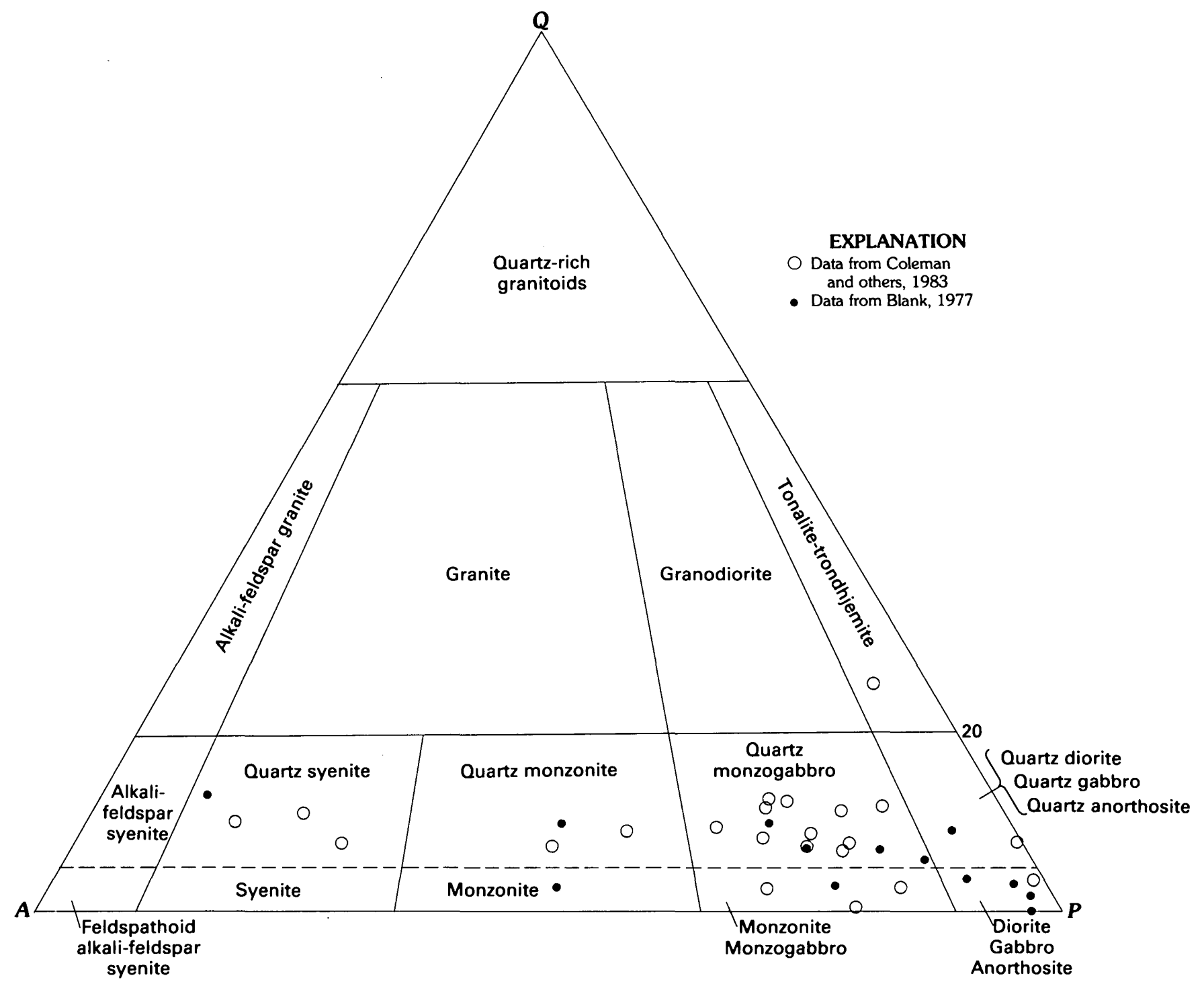

FIGURE 52.-Modal analyses of Tertiary continental dikes plotted on quartz (Q)-alkali feldspar (A)-plagioclase (P) diagram (Streckeisen, 1976).

In addition to the continental dikes, numerous isolated plugs and feeder pipes are exposed $100-500 \mathrm{~km}$ inland on the crystalline shield (Brown, 1972). Most of these can be identified, both chemically and by isotopic ages, with nearby plateau flood-basalts, especially in the As Sarāt region where the Jabal as Sarāt basalts have been eroded and where 26 feeder pipes are exposed (pl. 2; Coleman, Fleck, and others, 1977) in an area extending as far as $70 \mathrm{~km}$ northwest of the flows. Similar pipes exposed east of At Ta' if at lat $21^{\circ} \mathrm{N}$. are either associated with the Harrat Hadan flood basalts or are isolated intrusions; from lat $26^{\circ} 15^{\prime}$ to $26^{\circ} 35^{\prime} \mathrm{N}$., outlying pipes may be associated with the growth of Hुarrat al Ithnayn (Hutaym) and Ḩarrat Khaybar; and at lat $26^{\circ} 45^{\prime}$ to $27^{\circ} 20^{\prime} \mathrm{N}$., in a belt extending northeastward across the northeastern corner of the shield in the Jabal Shammar region, similar pipes probably were associated with an entirely eroded harrat, perhaps similar to the accumulation of local basalt flows and ash centers east of Jabal Salmá at lat $27^{\circ} \mathrm{N}$., long $42^{\circ} 25^{\prime}$ E., the Hुarrat al Hutaymah.

The As Sarāt feeder pipes are alkaline, either nepheline basalt or basanite (Coleman, Fleck, and others, 1977), as are pipes at or near other lava fields farther north. One isolated volcanic neck forming a spine in the Jabal Shammar belt, within the southern part of the granite batholith of Jabal Aja' at the north edge of the shield, is normative nepheline basanite (sample 19a, table 9). The basanite pipes near As Sirāt are $24.7 \pm 2$ and 25.4 \pm 2.7 m.y. old, based on K-Ar ages (Coleman, Fleck, and others, 1977).

Coeval felsic intrusives form stocks as much as $2 \mathrm{~km}$ in diameter. One stock in Jabal as Sarāt near the Yemen border at Al Wārah (sample 68, pl. 2) is analcite- 
151
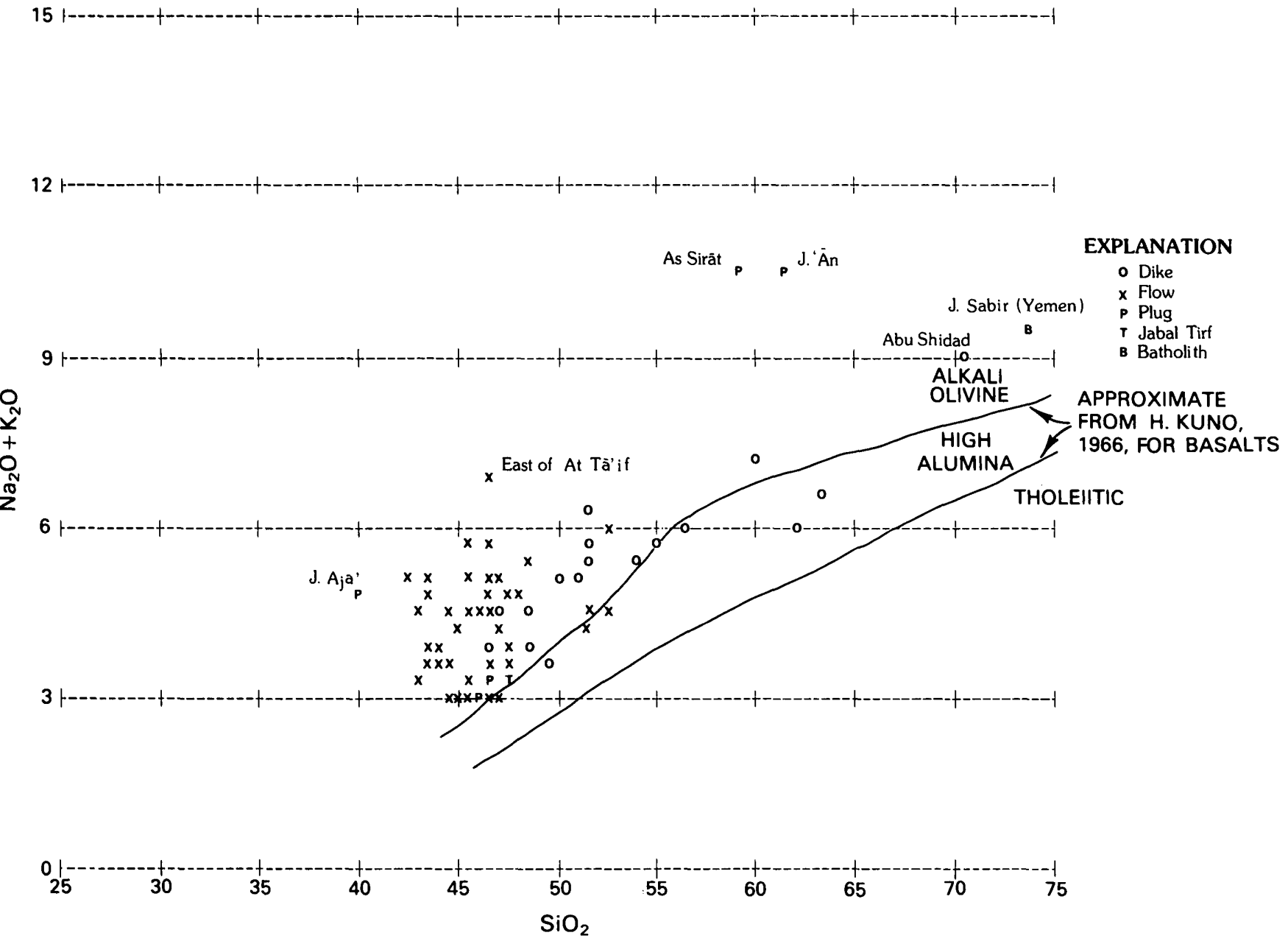

FIGURE 53.-Harker diagram showing alkalies plotted against silica for Tertiary igneous rocks.

bearing syenite surrounded by an analcite-trachyte rim, whereas a contemporary plug at Jabal 'Ān $410 \mathrm{~km}$ northwest and adjacent to Harrat Hadan has a core of trachyte or hornblende latite with a rim of phonolite (sample 40, table 10) (Richter-Bernburg and Schott, 1954; Gonzales, 1973). The As Sarāt stock is 81 percent orthoclase, 5 percent augite, 7 percent opaque minerals, 2 percent calcite, and 5 percent analcite (Salman Bloch, written commun., 1976), whereas the chilled edge is 71 percent albite and anorthoclase, 15.5 percent aegirineaugite, 7.5 percent opaque, and 6 percent analcite. The Jabal 'Ān stock, one of four in the neighborhood, is 45 percent potassium feldspar, 35 percent plagioclase, 15 percent hornblende, and 5 percent magnetite (Gonzales,

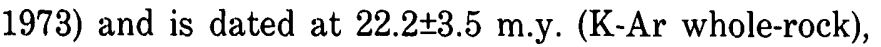
compared with $22.5 \pm 0.7 \mathrm{~m}$.y. for the As Sarāt stock (sample 68, table 10, pl. 2). A coeval age of $23.3 \pm 0.9$ m.y. (Yemen, table 10) from the granite of Jabal Sabir at lat $13^{\circ} 30^{\prime} \mathrm{N}$. near Ta'izz is one of many Tertiary alkalic granite and diorite plugs, stocks, and plutons continuing southward in the Yemen Arab Republic, many of which are concentrated along the rift scarp (Grolier and Overstreet, 1978).

\section{CENOZOIC BASALTIC LAVA FLOWS}

Lava fields (harrats) are scattered along the eastern seaboard of the Red Sea and on the western Arabian highlands. They extend intermittently northward from the Yemen Volcanics ("Trap Series"), which cover as much as $35,000 \mathrm{~km}^{2}$ of the southwestern Yemen Highlands to the Haurān of Jabal ed Drouz of comparable size in Syria. From there the lavas extend across eastern Jordan and into Saudi Arabia, where they are known as Al Harrah, or Harrat ash Shama. Within Saudi Arabia the larger harrats, in order of size, are as follows (pl. 2):

$$
\text { Harrat }
$$

Approximate area $\left(\mathrm{km}^{2}\right)$

\section{Khaybar-al Ithnayn (Hutaym)-al} Kurā‘ 21,400

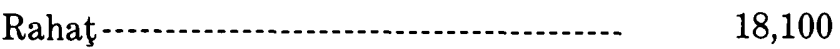

Al Hुarrah (ash Shama) -............... 15,200




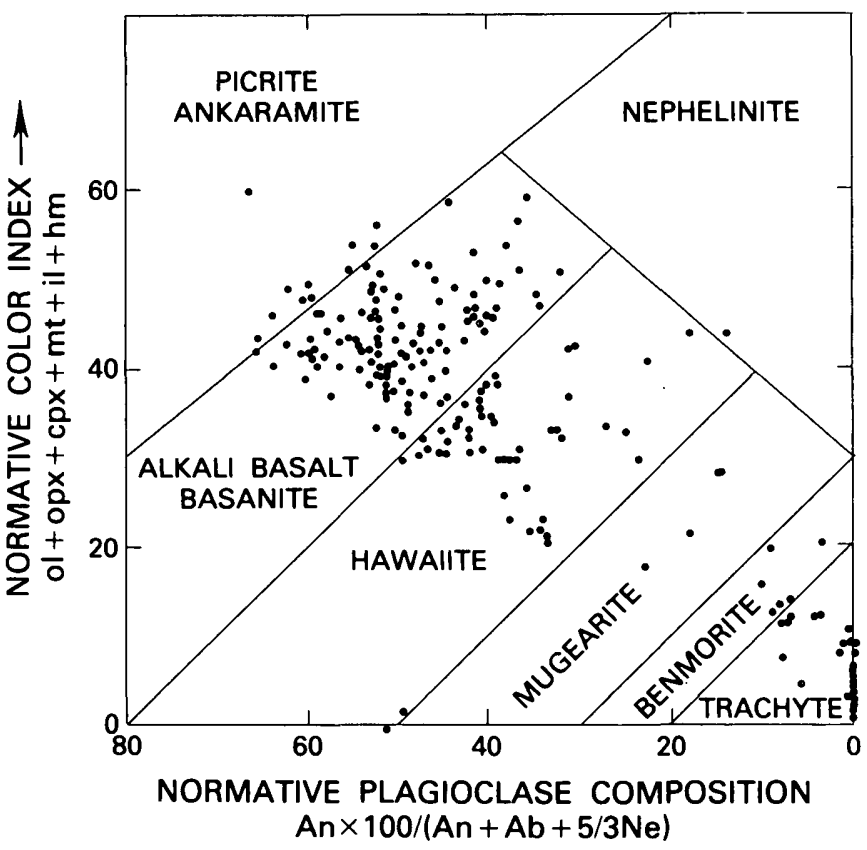

FIgURE 54.-Irvine and Baragar (1971) classification diagram. Normative color index versus normative plagioclase for analyzed samples from the harrats of Saudi Arabia. Values for minerals derived from CIPW normative calculation. (From Coleman and others, 1983.)
Harrat

Nawāşīf-al Buqūm Approximate area $\left(\mathrm{km}^{2}\right)$

10,800

Ar Rahāāh-'Uwayriḑ

7,150

6,700

3,700

Hadan

1,800

Al Birk

$<1,800$

I'shara-Khirsāt

1,750

Luna'yir

Harairah

$<1,530$

1,100

Al Hutaymah .............................. 900

As Sirāt ...................-

Jibāl al Khațibah -...................... 320

Malaki -.....................................-. 115

Besides the above, there are numerous spatter or cinder cones and small flows or ash fields. K-Ar dates on the lavas range from $62.6 \pm 4.3 \mathrm{~m} . \mathrm{y}$. to historical times. Most dates fall between 29 and $18 \mathrm{~m} . \mathrm{y}$. and between 14 and <1 m.y. (fig. 51, table 10; Gregory and others, 1982; Coleman and others, 1983). The most widespread lower flows of the lava fields between Makkah and $\mathrm{Al}$ Madinah are 12-13 m.y. old (middle Miocene), the same age span as the lower flows in the Al Hुarrah of Wādĩ as Sirhān and the basalt flows of the Hebron Formation in the Jordan Valley. These
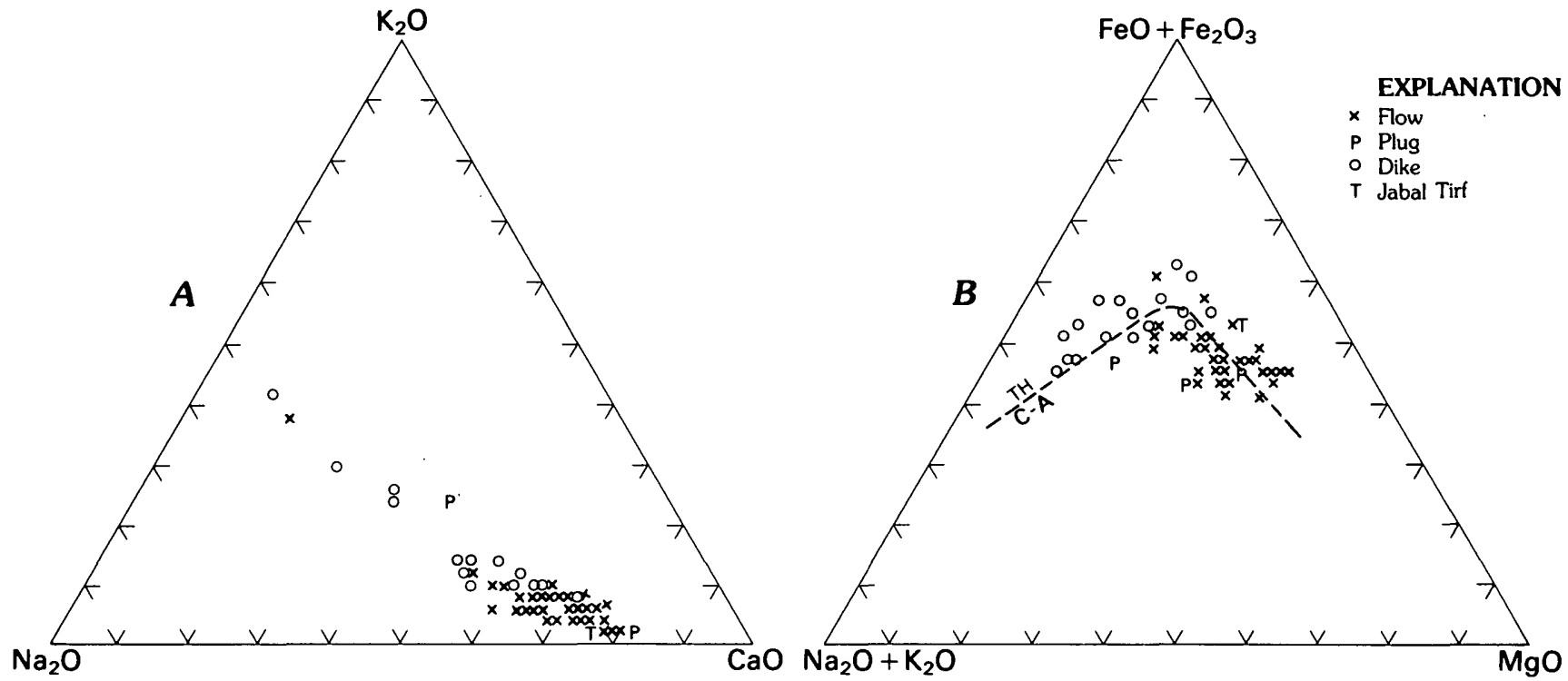

FIGURE 55.-Ternary diagrams showing distribution of Tertiary igneous rocks from western Saudi Arabia. $A, \mathrm{Na}_{2} \mathrm{O}-\mathrm{K}_{2} \mathrm{O}-\mathrm{CaO}$ ternary diagram. $B$, AFM diagram. Dashed line separates tholeiitic (above) and calc-alkalic (below) compositions according to Irvine and Baragar (1971) 


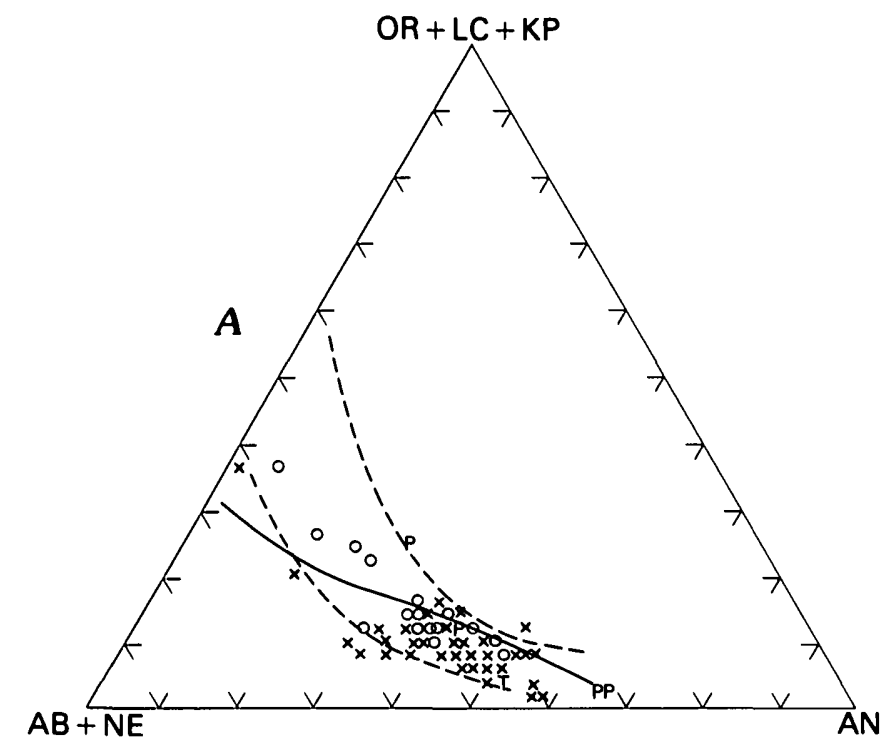

FIGURE 56.-Ternary diagrams showing distribution of Tertiary igneous rocks from western Saudi Arabia. $A$, Normative albite+nepheline-orthoclase-anorthite diagram. Subdivisions are according to Irvine and Baragar (1971); solid line separates potassic (above) from sodic (below) rocks of alkali olivine basalt series, and dashed lines separate K-poor (below) and K-rich

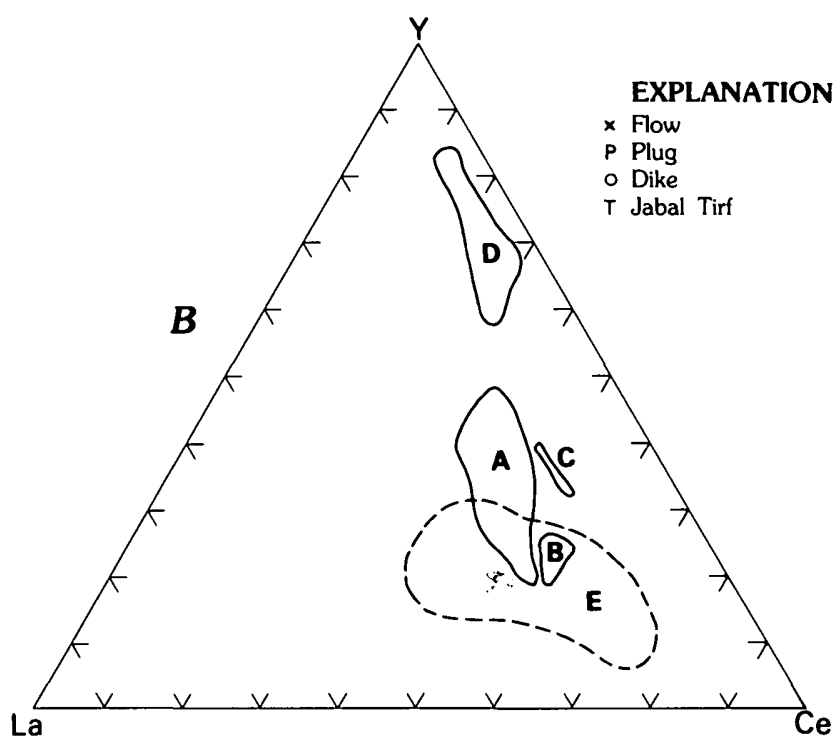

variants from "average" subalkaline rocks. $B$, Y-La-Ce ternary diagram. A, field of calc-alkaline andesites and basaltic andesites; $B$, field of ocean island alkali basalts; C, field of ocean island tholeiitic basalts; D, field of ocean-floor basalts and island-arc tholeiites; E, Tertiary igneous rocks of Saudi Arabia. (Fields except Arabian Tertiary igneous rocks from R.S. Thorpe, 1972.) lower flows are interbedded with lacustrine marls. Where the lava has ascended via narrow vents through basement rocks, the apparent ages determined from $\mathrm{K} / \mathrm{Ar}$ ratios tend to be older, because of argon enrichment, than physiographic evidence and degree of weathering would indicate. The larger flows seem to have escaped much of this contamination. The early flows erupted from fissures parallel to the Red Sea, are composed of picrite-ankaramite, and commonly are interbedded with shallow-water sediments (Gregory and others, 1982; figs. 53, 54, this report). Younger, more widespread flows are alkali-olivine basalts (pl. 2, table 11) containing sparse peridotitic inclusions. The most recent become bimodal, with hawaiite devoid of mantle inclusion and intermingled, sparse silicic flows and ash. The composition of only the most silicic may reflect minor melting of the sialic Arabian plate (Coleman and others, 1983, p. 68).

\section{THE HARRATS}

\section{AL HARRAH}

The northernmost harrat, Al Ḩarrah, or Harrat ash Shama, extends south from Jabal ed Drouz in Syria, where it is known as the Haurān, thence across Jordan and along the northeastern flank of Wādī as Sirhān into Arabia. Al Hुarrah covers $15,200 \mathrm{~km}^{2}$ in Saudi
Arabia, which is one-third of the total area of the combined lava fields of Jabal ed Drouz and Al Harrah. In Saudi Arabia, the basalt flows extend southeastward $210 \mathrm{~km}$ in a belt averaging $75 \mathrm{~km}$ wide with a maximum relief of $300 \mathrm{~m}$ and reaching altitudes of as much as $1,128 \mathrm{~m}$ in Jabal Liss. Al Harrah is characterized by numerous flows and many craters, calderas, and cones. In Jordan, six major emissions culminated with outpourings of basaltic tuffs and fissure eruptions (dikes) parallel to the Red Sea rift (Bender, 1974b). Of these, the lower three are known only in drill cores.

The surface rocks of $\mathrm{Al}$ Harrah in Jordan are considered to range in age from Miocene to Pleistocene and have been divided into four units, the lower three of which are separated by lacustrine sediments (Bender, 1974b). In Arabia the lower flows are interbedded with calcareous lacustrine sediments. These lower flows were sampled in outcrops along the north side of the Sabkhat Haz̧awz̧ā' (lat $30^{\circ} 50^{\prime}$ N., long $38^{\circ} 10^{\prime}$ E.) and were found to range in $\mathrm{K}-\mathrm{Ar}$ age from $13.4 \pm 0.4$ to $11.4 \pm 0.4$ m.y. (samples $1-5$, table 10, fig. 51). Test drilling to depths of $319 \mathrm{~m}$ beneath the sabkhah near the sample localities disclosed seven basalt flows interbedded in the lacustrine sediments, the lowermost 186 $\mathrm{m}$ of flow rocks being below the dated flows on the surface (C.L. Smith, 1980). The K-Ar ages of the outcrops on the north side of Sabkhat Haz̧awz̧ā' fall within the upper middle Miocene. The sampled rocks 
are comparable in age and stratigraphic position to the "lower basalts" on the shores of Lake Tiberias in Jordan $360 \mathrm{~km}$ to the northwest. At Lake Tiberias, basalt flows interbedded in the Hebron (Horodus) Formation of fluviolacustine origin (Michelson, 1978) have K-Ar ages of $15.6 \pm 1.6$ to $10.3 \pm 2.0$ m.y., whereas an upper interbedded flow elsewhere in the Hebron Formation has a K-Ar age of $4.9 \pm 1.3$ m.y. (Steinitz and others, 1978). This timespan extends from middle Miocene to Pliocene and includes at least four periods of weathering and soil development, as well as lacustrine sedimentation. The presence of northwest-trending faults in the lower lava, as mapped by Gettings (1979) in the Sabkhat Hुaz̧awz̧ā' area, strengthens the concept that the lower lavas in Saudi Arabia predate the last period of epeirogeny of the Red Sea-Jordan rift, that is, they are more than 5 m.y. old.

The unnamed lacustrine beds at Sabkhat Hुaz̧awz̧ā' were shown to be $150 \mathrm{~m}$ thick in a test well drilled by the Arabian-American Oil Company near the axis of the trough occupied by the sabkhah. These lacustrine beds disconformably overlie the Paleocene-Eocene Hibr Formation (Meissner and Ankary, 1972).

Following the older series of interbedded flows in Jordan, a younger flow exhibits late Pleistocene erosion followed by the accumulation of tuff and cinder cones. Final eruptions from basaltic cones along fissures parallel to the Red Sea have continued in Jordan to historic times (Bender, 1975). In Saudi Arabia, the southeasternmost lava fields in $\mathrm{Al}$ Hुarrah are considered to be Pleistocene to Holocene, but the possibility is recognized that some of the lowermost flows may be Pliocene. Numerous craters, some eroded, mark the landscape, although dikes along the fault flank of the Khawr Umm Wu'âl graben at the southeast edge of the harrat suggest fissure eruptions. A total of 527 plugs or necks, cones, craters, dikes, and tholoids or domes have been mapped, of which 286 are plugs or necks (Donald Holm, ARAMCO, written commun., 1960).

Most flows are a few meters thick; the maximum total measured is $100 \mathrm{~m}$ at the southeastern extension in Khwar Umm Wu'āl. In places, flows are scoriaceous and vesicular and include pyroclastic lenses and thin leucocratic dikes. Lava surfaces are blocky and weather into large boulders, for the most part making traversing difficult (Donald Holm, ARAMCO, written commun., 1960).

The chemical analyses of the dated samples are those of typical alkalic continental flood basalts (samples 1-5, table 9; fig. 54) and are very similar to the average for similar flows in Jordan (Bender, 1974b). The predominant mineral is andesine-labradorite; titaniferous augite and olivine are major components, and calcite and opaque oxides are minor constituents. The samples from Sabkhat Ḩaz̧awz̧ā' are amygdaloidal and contain secondary zeolite fillings.

\section{HSARRAT AR RAHĀA-'UWAYRID}

Ḩarrat ar Raḩāh-'Uwayriḑ forms the crest of the northwestern scarp mountains, extending from lat $28^{\circ} 05^{\prime} \mathrm{N}$. in Al Ḩismá southeastward to lat $26^{\circ} 30^{\prime} \mathrm{N}$., a distance of $225 \mathrm{~km}$. The two harrats are continuous; however, Harrat ar Raḩāh has been nearly split by erosion, leaving only a narrow divide occupied by a number of isolated volcanic plugs. Ar Raḩāh is widest $(35 \mathrm{~km})$ at the western front, narrowing to less than a kilometer at the Matar divide. The terrain is nearly flat, standing 150-300 $\mathrm{m}$ above Al Hुismá, and is composed of as many as 20 flows, increasing to as much as 25 flows for a total thickness of $515 \mathrm{~m}$ to the southwest. This thickness includes many weathering zones. Within the northwestern segment of the southern lobe where flows are most numerous, the lava shield rises $550 \mathrm{~m}$ above the plain. The flows were extruded from pipes (or perhaps from fissures that are no longer discernible). One such volcanic throat, Al Batra, stands on the sandstone plain west of the northern lobe of the lava field. The flows are of three distinct ages. The lower flows, of Miocene age (K-Ar determination), form the cliffs facing the Red Sea and rise above the Cambrian Siq Sandstone. The upper flows are Pleistocene and Holocene. Both the sandstone and the harrat are deeply dissected along the southern and southwestern fronts. Most of the younger lava flowed in a northeasterly direction, some along wadi channels no longer extant, suggesting eruption following regional tilting. The lava-filled channels now form interstream divides. More recent drainage dissects the flows or is parallel to them. An explosion crater at the source of the longest of these flows north of the harrat attests to continuing volcanic activity in the recent past.

The lava shield southeastward rises to a maximum altitude of $1,950 \mathrm{~m}$, compared with $1,750 \mathrm{~m}$ for the northwestern block (fig. 57).

The southeastern extension of Hुarrat ar Rahāh, Harrat 'Uwayrid, is comparable in extent to Harrat ar Raḩāh, rises to a reported height of $1,920 \mathrm{~m}$ (fig. 58), and is somewhat more deeply eroded. However, the region of $\mathrm{Al} \mathrm{Jaww}$, a depression partly separating the harrat, was the site of the most recent eruption in the lava plateau. A dome in the Tabuk Formation at the northwestern corner of this belt appears to be a volcanic diapir of Tertiary age that failed to reach the surface (Brown, Jackson, Bogue, and Elberg, 1963). Even though wind scour from sandblasting through the gap has cut lineations trending N. $45^{\circ} \mathrm{E}$., the flows and cinder cones are little eroded. Indeed, an eruption at 


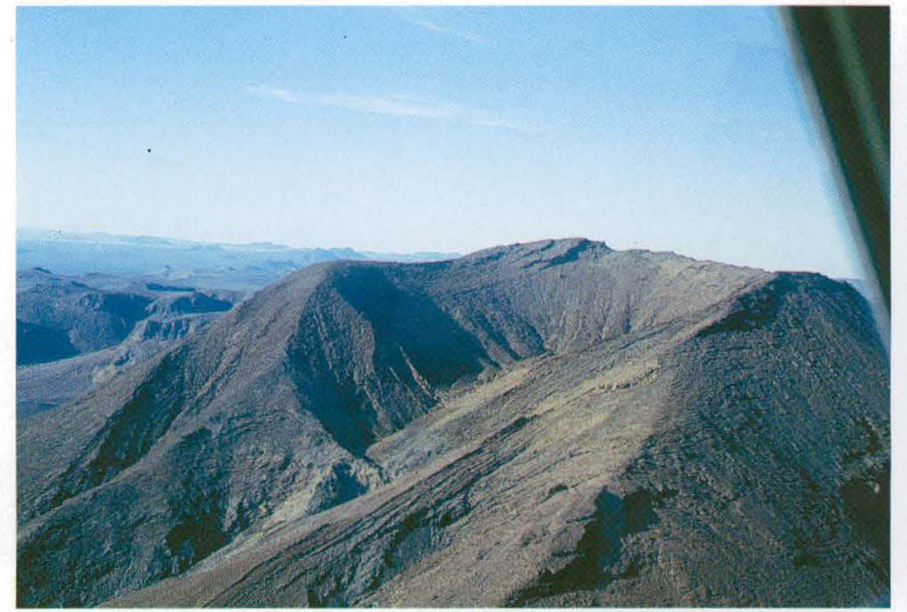

FIGURE 57.-The ruptured crater at the crest of Harrat al 'Uwayrid at an elevation of about $1,900 \mathrm{~m}$ above the Red Sea. A sample of hawaiite from the steeply westward dipping tuff gave an age of 7.4 m.y. (K-Ar whole rock). As the tuff came from a Holocene eruption at the eastern edge of the harrat, the sample may be an inclusion from a lower and older flow through which the explosion passed. View to the northwest.

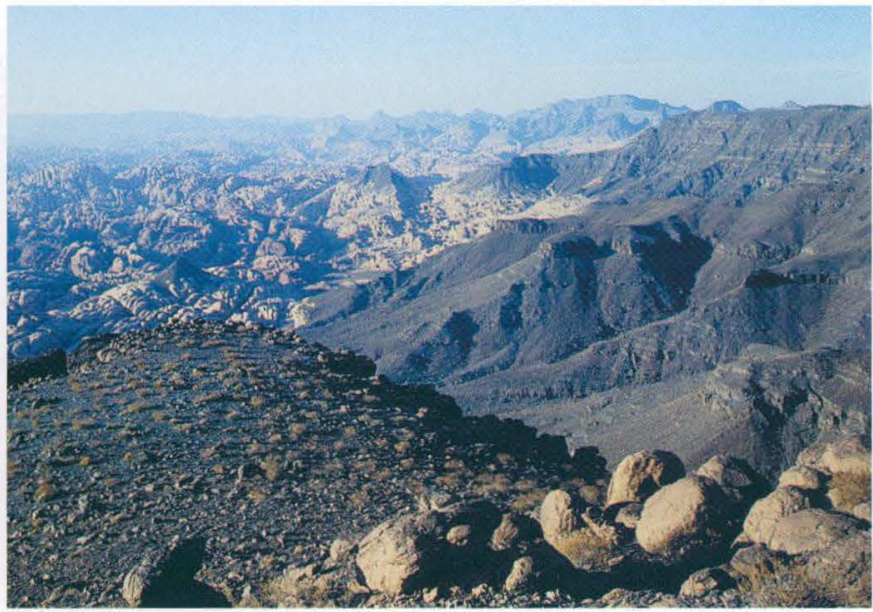

Figure 58.-Basalt erosional front of Ḩarrat ar Raḩāh on the right above the Ram-Umm Sahm Sandstone bench. View from the base of Haarrat al 'Uwayrid where the lower basalt flow of picriteankaramite gave a late Miocene age of $9.4 \pm 2.5$ m.y. (K-Ar whole rock). A Holocene eruption formed silicic domes and necks on the skyline (Coleman and others, 1983). Boulders in the foreground compose one of the discontinous earlier Tertiary clastic outliers. The Ordovician Ram-Umm Sahm Sandstone forms white pinnacles in the middleground; the darker reddish Siq Sandstone below is probably Upper Cambrian age. View to the northwest.

channels, suggesting a middle to late Pleistocene age. Thus the latest eruptions are undoubtedly Holocene.

Estimation of age from the erosion rate of the basalt is difficult because of climatic change, and also because of the erosive differences of sandblasting by wind and erosion by water, especially where there is a wide range of rock-fragment size in bed load. Doughty (1888, p. 419 , v. 1) described conditions in Harrat 'Uwayrid thus:

Viewing the great thickness of lava floods, we can image the very old beginning of the Harra-those streams upon streams of basalt, which appear in the walls of some wady-breaches of the desolate Aueyrid. Seeing the hillian are no greater, we may suppose that many of them (as the Averine Monte Nuovo) are the slags and the powder cast up in one strong eruption. The earlier over-streaming lavas are older than the configuration which is now of the land: - We are in an amazement, in a rainless country, to see the lava-basalt pan of the Harra, cleft and opened to a depth of a hundred fathoms to some valley-grounds as Thirba. Every mass is worn in grooves in the infirmer parts by aught that moves upon it; but what is this great outwearing of "stones of iron," indomitable and almost indestructible matter. We see in the cliff-inscriptions at Medain, that the thickness of your nail is not wasted from a face of soft sandstone, under this climate, in nearly 2,000 years.

Doughty's observations are accurate where the inscriptions on the sandstone tomb at Madā' in Sālih are a few meters above land surface, but where the facades bearing dated Nabatean inscriptions are exposed to the wind near land surface, sandblasting and spalling has undercut as much as a meter into the lower 1 or $2 \mathrm{~m}$ of the present drainage system was established, al the older incised meanders to form more recent flood 


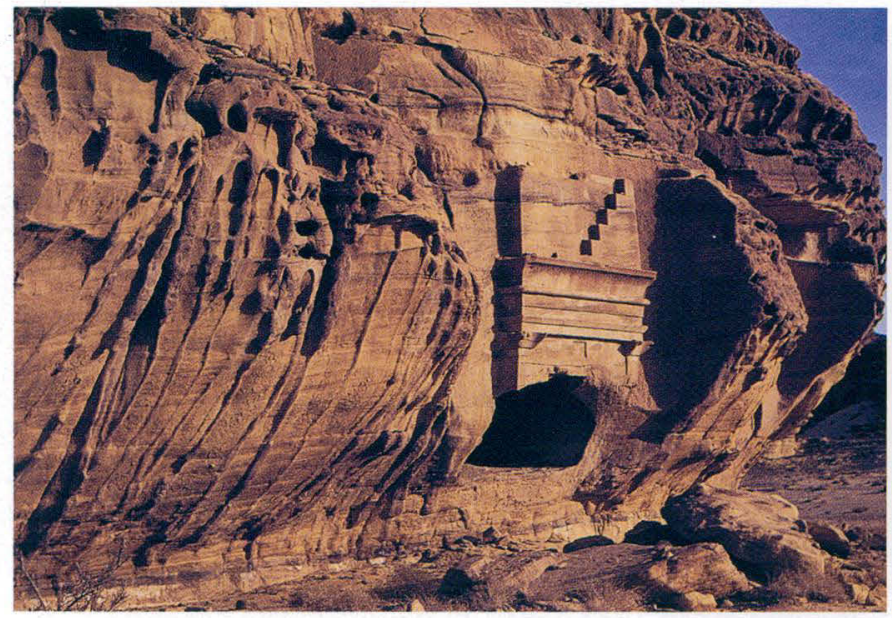

FIgURE 59.-Eolian undercutting of Ram-Umm Sahm Sandstone at a Nabatean tomb (65 A.D.) at Madā'in Şālih showing $1 \mathrm{~m}$ of windsand scour and spalling during two millennia.

the face during the last 2,000 years (fig. 59) (Brown, 1960). This amounts to $500 \mathrm{~m} / \mathrm{m} . \mathrm{y}$. As sandstone underlies the lavas all along the western front of Hुarrat ar Raḩāh-'Uwayriḑ, a timespan of 9 m.y. (the KAr age of the earliest volcanic eruption) would allow a scarp retreat of $4.5 \mathrm{~km}$ under present climatic conditions. If this process functioned alone, the lava cliffs should stand directly above the sandstone face, spalling off as the sandstone face retreated. Instead, we see the lava removed ahead of the sandstone, all indented by a dendritic drainage pattern among isolated sandstone buttes, then sandstone buttes capped with basalt, and finally, in the headwaters, the basalt front along the toe of the mountain face above a sandstone bench (fig. 58). Evidently insolation and the wide diurnal temperature range cause the lava to fracture so that most exposures are great piles of boulders often faceted into windkanter from sandblast. The boulders and cobbles are removed down the slopes by gravity, especially during the occasional desert rainstorms, to form the present terrain. Isolated lava-capped buttes suggest that the harrat scarp has receded as much as $5 \mathrm{~km}$ during the desert erosion cycle. As this rate must be more rapid than that of the present-day desert cycle, a late Miocene age for the oldest flows would be maximum.

The lavas we sampled from the base and top of the volcanic pile of Ḩarrat 'Uwayriḑ are alkali-olivine basalts falling within the hawaiite field on the plagioclasecolor index normative diagram (fig. 54); they contain phenocrysts of forsteritic olivine, some zoned toward fayalite rims, clinopyroxene, and opaque oxides, as well as some interstitial calcite and chlorite (Salman Bloch, written commun., 1976). Sample 7B (table 10) is unusually rich in olivine phenocrysts, as is the youngest flow

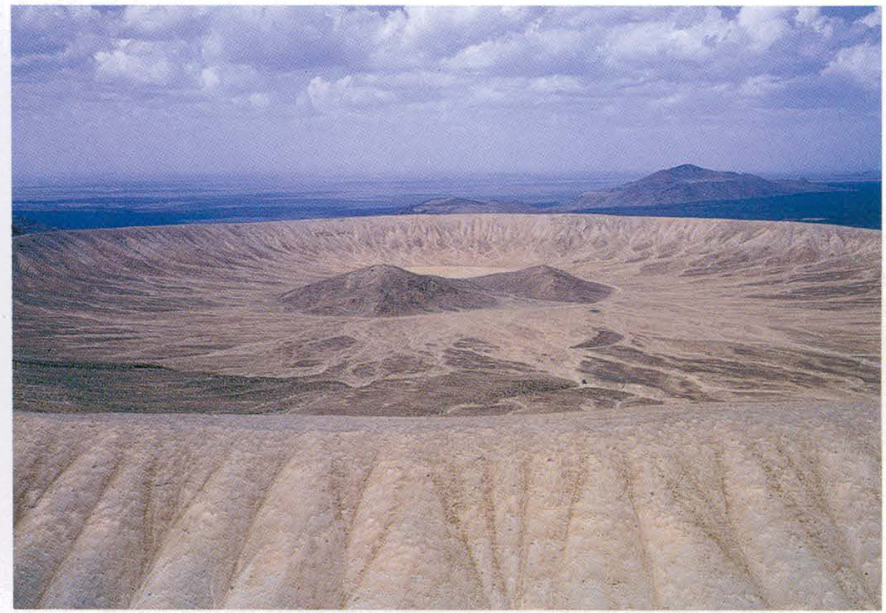

FIgURE 60.-The Holocene crater of white rhyolite tuff and lapilli of Abyad wa Ubayyid in Hुarrat Khaybar. The crater is $1.6 \mathrm{~km}$ wide, and the cone of rhyolite and pumice breccia in the center is $300 \times 500 \mathrm{~m}$ across.

at the crater crest near the center of the harrat where the olivine is glomeroporphyritic and zoned; some phenocrysts of fayalite are rimmed with iron oxides, and rare picotite is rimmed with opaque ore minerals. Amygdules contain calcite and stilbite.

\section{HARRAT KHAYBAR}

Hुarrat Khaybar, the adjoining Hुarrat al Ithnayn (Hutaym) on the northeast, and the southwestern extension, known locally as Hुarrat al Kurā', together form the largest plateau-basalt field in Saudi Arabia, although it is only slightly larger than Harrat Rahaţ between Al Madīnah and Makkah. Harrat Khaybar extends from long $38^{\circ} 30^{\prime} \mathrm{E}$. to long $40^{\circ} 46^{\prime} \mathrm{E}$. and from near Al Madīnah at lat $24^{\circ} 39^{\prime} \mathrm{N}$. to lat $26^{\circ} 11^{\prime} \mathrm{N}$. The Hुarrat al Ithnayn segment extends an additional $75 \mathrm{~km}$ north and as much as $85 \mathrm{~km}$ farther east. The basalt plateau rises from an altitude of $475 \mathrm{~m}$ at the crystalline basement along Wādī al Ḩamḑ to 2,015 $\mathrm{m}$ in Jabal al Abyaḑ in the center of the pile $115 \mathrm{~km}$ to the east. Boreholes have proven basalt thicknesses of more than $500 \mathrm{~m}$ (Delfour and Dhellemmes, 1980).

The older flows cover the western part, extending at least as far as the Al Madinah-Khaybar road; K-Ar ages normalized to the Sydney constants range from $11.5 \pm 2.3$ to $7.5 \pm 0.8$ m.y. (samples 12 and 10 , table 10 , pl. 2) for the part of the harrat called Al Kurā'. The age of the base of a flow at the south edge of the Khaybar oasis is $9.3 \pm 0.7$ m.y. (sample 20, table 10). Thus the timespan for the Harrat al Kurā' flows is similar to that for the lower flows of the Harrat 'Uwayrids. The degree of erosion appears similar, and there is some evidence that the lavas flowed westward prior to the 


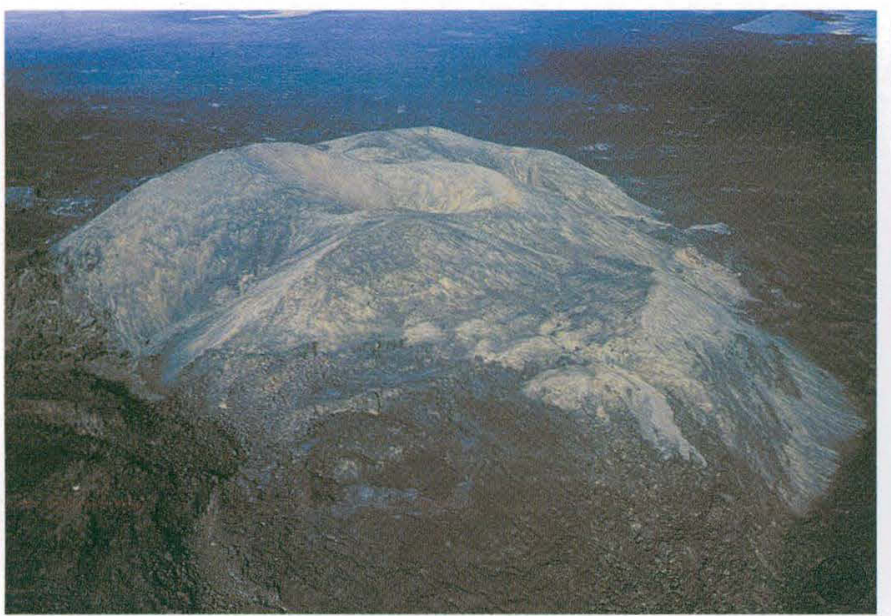

Figure 61.-The tholoid of Jabal Ithnayn, Harrat al Ithnayn.

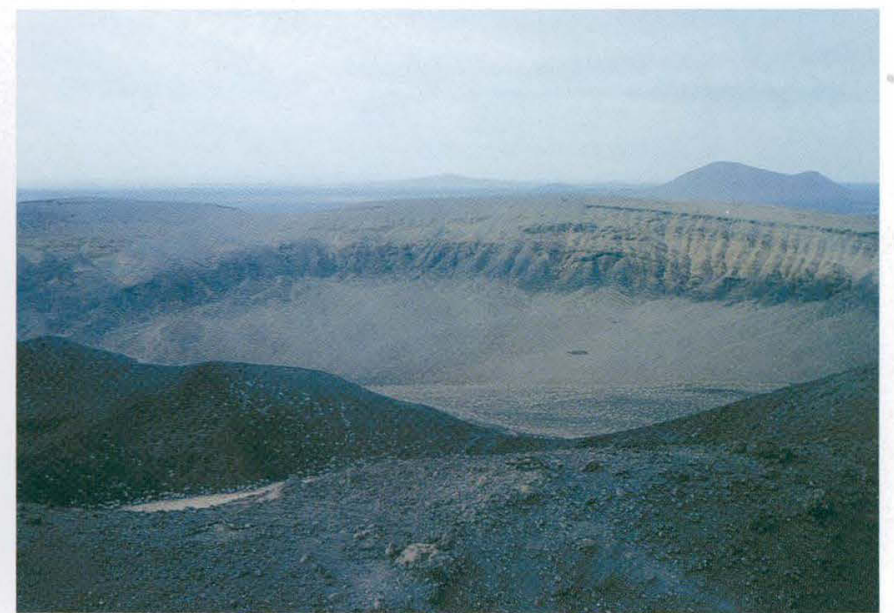

Figure 62.-The crater of Jabal Hibran, Ḩarrat al Ithnayn. elevation and tilting of the Red Sea flank. The basalt outlier west of the elbow of capture of Wādī al Jizl at the junction with Wādī al Hुamḑ suggests, but does not prove, that the lava streamed down the ancestral Wādi al Jizl valley to the northwest. All more recent flows constituting the eastern half of Khaybar and Harrat al Ithnayn (Hutaym) flowed radially from voleanic centers, but the general trend during the Quaternary is southeastward down wadis into the Wādī ar Rimah drainage (alkali basalt, $1.1 \pm 1.0$ m.y., Jabal al Khuraym, sample 22, table 10, pl. 2), most recent volcanic activity being within historical time.

The older lavas are alkali-olivine basalt, where sampled. Sample 12 (table 10, 11.5 \pm 2.3 m.y., K-Ar) at the western base may be considered a basanite from the normative analysis which disclosed an exceptionally low content of silica (table 9). Sample 10, from an outlier northwest of Ḩarrat Khaybar in the Qal'at aş Şawrah quadrangle, falls within the basanite or hawaiite field. Similar lavas in the lower part of Hुarrat Khaybar and around and near Abyaḑ wa Ubayyid (fig. 60) are reported to be largely hawaiite but also to include such minor rock types as nephelinite, trachybasalt, trachyandesite, phonolite, trachyte, and rhyolite (Baker and others, 1973). The more alkalic flows are generally the youngest, and at Abyaḑ wa Ubayyid (the "white mountain") peralkaline rhyolite and rhyolitic crystal tuff is concurrent with or slightly older than the youngest alkali basalts. The peralkaline rocks of Abyad wa Ubayyid are unique among the great bulk of the Tertiary and Quaternary alkaline basaltic volcanic rocks on and near the Arabian Shield in Saudi Arabia, although the latest eruptions in all the harrats are ash cinder cones, generally indicating a more silicious termination. Recently, the chemical variation from basanite to peralkaline rhyolite has been confirmed (Delfour and Dhellemmes, 1980)

Hुarrat al Ithnayn (Hutaym), the northeast extension of Harrat Khaybar, is made up of rocks similar to the volcanic deposits around Abyaḑ wa Ubayyid. The largest volcanic cone, Jabal Ithnayn (fig. 61), on the meridian of the north-south line of cones through Abyaḑ wa Ubayyid and $22 \mathrm{~km}$ north, is a gray, domeshaped cinder cone or tholoid surmounting a black Holocene basalt flow at an altitude of $1,416 \mathrm{~m}$. Recent volcanic activity was suggested to Doughty (1888) in 1883 by bedouin who described a warm, smoking vapor around the crest after winter rains. Numerous other cones of cinder, ash, and agglomeratic and scoriacious flows trend northwesterly parallel to $\mathrm{Al} \mathrm{Hुarrah} \mathrm{at}$ Wādī as Sirḩān and to a northwesterly extension of the Najd fault system (Brown, 1972). Jabal Hibran, the largest crater in Hुarrat al Ithnayn, is composed of layered ash, scoria, and bombs of welded trachyte or rhyolite (fig. 62).

\section{HaARAT LUNAYYIR}

Hुarrat Lunayyir (lat $25^{\circ} \mathrm{N}$.), on the lower slopes of the scarp mountains north of Yanbu' al Bahrr, was formed during two principal volcanic episodes during Pleistocene, including possibly late Pliocene and Holocene, time and has been mapped by Kemp (1981) and Pellaton (1982). Recent seismic studies in the Yanbu' region yielded microseismicity with epicenters clustered in the Harrat Lunayyir area (Merghelani and Irvine, 1981). The lava fields lie mostly at $1,000 \mathrm{~m}$ above the Red Sea, but flows spread down wadi channels in 
every direction, reaching the Red Sea at two places. Although parts are covered with alluvium on the coastal plain, the coastal lava overlies Pleistocene terraces, and individual flows are both younger and older than coralline benches on the coast (Bigot, 1975). The more than 50 vents whence came the flows lie as much as 60 or more kilometers inland where the harrat overlies Precambrian crystalline rocks. The K-Ar age of $6.2 \pm 0.8 \mathrm{~m}$.y. determined for sample 15 (table 10) is undoubtedly too old, as the sample came from the most recent flow, a black lava of the younger volcanic episodes. Samples 14 and 16 (table 10), also from the most recent eruptions, contain insufficient radiogenic argon to measure, which indicates a young age in keeping with the physiographic evidence. Kemp (1981) has divided the younger volcanic episode into five eruptions; the last possibly took place in the 10th century or earlier (von Wissman, 1963). Sample 16 comes from a small isolated eruption at Jabal Salajah (pl. 2) which terminated with an only slightly eroded cinder ash cone $20 \mathrm{~km}$ south of Hुarrat Lunayyir. The same volcanism continued intermittently farther south to near Yanbu' al Bahr and is also too young to measure by K-Ar methods (sample 19, table 10).

A series of small, very recent, perhaps historical, flows and cinder cones are widely scattered from Hुarrat Lunayyir (lat $25^{\circ} \mathrm{N}$.) to Hुarrat al Birk (lat $18^{\circ}$ N.) along the coastal plain and in the foothills and are apparently contemporaneous or penecontemporaneous with the scores of cinder and ash cones above the source fissures of the larger flood-basalt harrats. The young flows are black and scoriaceous, in contrast to the reddish-brown and gray weathered older flows. Chemically, the Holocene flows are all alkali-olivine basalts where sampled (sample 14, tables 9, 10, 11, approaches hawaiite from the normative andesine). Some of the scoriaceous and vesicular flows contain secondary calcite; ophitic to subophitic textures are formed by phenocrysts of olivine and labradorite in a groundmass of clinopyroxene, labradorite, olivine, and opaque iron-bearing minerals. Inclusions of the underlying crystalline shield rocks and ultramafic xenoliths are fairly common.

\section{HARRAT I'SHARA-KHIRSĀT AND HSARRAT HARAIRAH}

Remnants of older flows cap ridges along the flanks of Wādī al Ḩamḑ and Wādì al Jizl northwest of Al Madinah. The mesas and buttes range in thickness from $5 \mathrm{~m}$ to more than $400 \mathrm{~m}$, and $\mathrm{K}-\mathrm{Ar}$ ages range from $21.2 \pm 2.1$ and $62.6 \pm 4.3$ m.y. (samples 17,18 , and 23 , table 10). These ages contrast with $7.7 \pm 0.7$ and $11.1 \pm 0.8$ m.y. (Baubron and others, 1976, reported in Pellaton, 1979), the latter being coeval with the oldest flows of
Hुarrat Raḩat, which extends south of Al Madinah. The oldest sample came from the uppermost of three flows west of Jabal al Bayda', $35 \mathrm{~km}$ northwest of $\mathrm{Al}$ Madīnah, where olivine basalt contains zoned xenocrysts of forsterite rimmed by fayalite in a groundmass of plagioclase $\left(\mathrm{An}_{60}\right)$, clinopyroxene, and accessory opaques. The content of calcite is high in amygdules and in the groundmass (Salman Bloch, written commun., 1976). The normative values of sample 23 are similar to those of the lower flows of Harrat Khaybar in being especially low in silica (43.2 percent). As the generally accepted K-Ar age for the oldest flows of the shield is about 30 m.y., the 62.6-m.y. age seems excessive, especially in light of the fact that the lavas forming butte tops at Al Jarf $50 \mathrm{~km}$ northwest gave ages of $21.2 \pm 2.1$ and $28.3 \pm 2.9$ m.y. (samples 17 and 18 , table 10) and are comparable in age to lavas at Harrat Hुaḑan and Jabal as Sirāt farther south. The 28.3-m.y. age is from the basal flow of hydrothermally altered basalt containing olivine that is partially altered to bowlingite, and the clinopyroxene is partially changed to chlorite and calcite; the labradorite is unaltered (Salman Bloch, written commun., 1976). The 21.2-m.y. age is from the topmost $15 \mathrm{~m}$ flow, which is 12 or more flows above the lower sample; it is typical alkaliolivine basalt. The deeply eroded basalt pile at Harrat I'shara-Khirsāt is $820 \mathrm{~m}$ thick (some of this thickness may be attributed to fault repetition) and rests locally on flat-lying varvelike silt and fine sand of lacustrine or deltaic origin. Farther northwest at Harrat Harairah, a conglomerate bed above the basement and below the lowermost flow contains chert boulders with casts of Eocene fossils (Brown, 1970). However, the fossiliferous boulders were not seen at the northernmost outcrop of the harrat at the head of Wādi Tharīb (ash Schism). There, a small mesa rises above $1,500 \mathrm{~m}$ at the crest of the peninsular divide where 12 flows aggregate about $200 \mathrm{~m}$ of amygdaloidal and olivinerich basalt. Two beds of gravel are interbedded in the flows above the basal gravel, which overlies Precambrian basement.

The occurrence of post-Eocene gravel and conglomerate (similar to that shown in fig. 49), the retilting flow direction, and the extensive erosion suggest that Ḩarrat I'shara-Khirsāt and Ḩarrat Harairah are at least as old as Hुarrat 'Uwayrid northward and possibly are coeval with Hुarrat Hुaḑan and As Sirāt southward (Coleman and others, 1983). Indeed, the general sequence of picrite-ankaramite below peridotite-nodulebearing olivine basalt and overlying diktytaxitic alkaliolivine basalt is common to widespread outcrops (Coleman and others, 1983), suggesting a general correlation for the older volcanism. Most evidence points to two general eruptive episodes, late Oligocene-early Miocene (29-20 m.y.) and middle to late Miocene (10-7 m.y. timespans). 
HARRAT KURAMA $\bar{\prime}$

This relatively small harrat, which lies directly south of Hुarrat Khaybar and due east of the north end of Hुarrat Rahat at $\mathrm{Al}$ Madinah, is also known as Hुarrat Hirmah (Pellaton, 1981). The area drains west through Wādi Shaqrah and its tributaries, terminating at the Q $\bar{a}$ ' Hazawz̧ā', which is a sabkhah formed by the lava dam of Hुarrat Rahaţ. The wadi nearly divides the harrat into two lobes, the westernmost part apparently the older and the only part sampled. An eroded and cratered cone rises $90 \mathrm{~m}$ above the harrat surface near the northwestern edge of the lobe and is the source of the surrounding flows. The scoriaceous and subophitic olivine basalt (sample 24, table 9) contains amygdules of calcite; the olivine is not zoned, in contrast to other samples, but some grains are altered to iddingsite (Salman Bloch, written commun., 1976). The K-Ar analysis yielded an early Miocene age of $20.0 \pm 2.0 \mathrm{~m} . \mathrm{y}$. (sample 24, table 10), which may be excessive. However, the weathering of the basalt and the well-developed drainage on a general southwesterly sloping surface suggests preramping flowage into the ancestral Wâdi al Hुamd̆ valley and an eruptive epoch older than that of adjoining, much larger olivine-basalt floods.

The harrat lobe east of Wādi Shaqrah is marked by hummocks forming irregular lineaments similar to those in the southern portion of Al Harrah near the Jordan frontier. They were evidently formed by younger, very fluid flows. The eruption came from a crest of $960 \mathrm{~m}$ in the central part of the harrat, the highest part surrounding the collapsed Hirma crater in the westcentral part. The crater is $6 \times 4 \mathrm{~km}$ in area and has vertical walls at least $25 \mathrm{~m}$ in height. Although the crater floor is a saline silt plain and the lower walls are sandstone, similar to the Siq Sandstone exposed southeast of the harrat, the entire thickness of the basalt flows is exposed.

\section{HARRAT RAHAT}

Ḩarrat Rahat extends from $\mathrm{Al}$ Madinnah at lat $24^{\circ} 30^{\prime}$ N. to Wādī Fāţimah north of Makkah (lat $21^{\circ} 40^{\prime}$ N.), a distance of $310 \mathrm{~km}$. Hुarrat Rahat has an average width of $60 \mathrm{~km}$. The basalt has filled the upper valley of Wādī al Hुamḑ, whose ancestral thalweg probably extended from the headwaters of Wādī Fātimah northward to Al Madinnah, thence beyond to Wādi al Jizl where the present channel sought a more direct course to the Red Sea through a narrow gap in the foothills at lat $26^{\circ} \mathrm{N}$. The harrat surface rises southward from $650 \mathrm{~m}$ above the Red Sea at Al Madinah to a maximum altitude of $1,640 \mathrm{~m}$ at lat $23^{\circ} \mathrm{N}$., decreasing somewhat in the southern part, where the crest is 1,570 $\mathrm{m}$. The high points are linear clusters of cinder and ash cones (many breached) or Holocene domes and repre- sent four centers of late volcanicity, so Hुarrat Rahat is actually four coalescing harrats: Hुarrat Madinah, which is essentially contiguous with Hुarrat abu Rashid to the south; Harrat Bani Abdullah still farther south; Hुarrat Turrah, which includes the crest; and Hुarrat ar Rukhā', the southernmost. The cone and dome eruptions occur as segments of the north-south volcanic central linear ridge that continues northward en echelon across Harrat Khaybar and probably represent fissure (fault) control (Brown, Jackson, Bogue, and MacLean, 1963). The youngest peaks are near Al Madinah, where the latest flow erupted in A.D. 1256 (Doughty, 1888, v. 1, p. 593). Numerous cones in the segment some $75 \mathrm{~km}$ south, Hुarrat abu Rashid, likewise have young black flows, mostly aa-type and similar to Ḩarrat Madinah flows, with somewhat scattered eruptive centers trending N. $20^{\circ} \mathrm{W}$. compared with a trend of $\mathrm{N} .10^{\circ} \mathrm{W}$. for Harrat Bani Abdullah. Many of the black scoriaceous flows erupted after a late period of more siliceous cinder and ash eruptions, a sequence similar to that at Abyad wa Ubayyid in the Ḩarrat Khaybar (Pellaton, 1981). Another segment of medial cones and craters was erupted on the older flows of Harrat Turrah, with the eruptive centers trending N. $08^{\circ} \mathrm{W}$., very similar to the trend of Ḩarrat ar Rukhā'. The weathering and fracturing of the blocky basalt and the accumulation of ventifacts developed an extremely rough terrain. Lava flowed centrifugally from each center, abutted flows from adjoining centers, and then flowed either east or west onto the flanks of the ancestral Hुamdु valley. On the west, numerous lavas from the three southernmost harrats flowed through passes in the scarp mountains and down the broad wadis draining the westward front of the Hejaz Scarp. The fluid basalt streams then flowed out onto the coastal plain; most of the flows lie on a pediplain that was about $75 \mathrm{~m}$ above the present surface (Brown, Jackson, Bogue, and MacLean, 1963). Hुarrat Rahat has been drilled at numerous places for water supply, notably south of Al Madīnah and along its northeast border. A thickness of $70 \mathrm{~m}$ was drilled at Dhumariyah, $85 \mathrm{~km}$ southeast of $\mathrm{Al}$ Madinah near the north edge of Hुarrat abu Rashid, where three lower flows are separated by white marl (Durozoy, 1972). Most flows are only a few meters thick. The decrease of maximum altitude toward the southern edge of the harrat suggests that the younger flows total at least $170 \mathrm{~m}$ in thickness, assuming that the ancestral Wādì al Hुamd̆ valley slopes uniformly northward.

The drilling at Dhumariyah (Durozoy, 1972) penetrated a carbonaceous marl overlying basement rocks. Red marl, sand, and gravel overlie the basal marl and underlie the basal lava. The carbonaceous marl contains a microflora of probable Oligocene age which may, however, correlate with the uppermost nonmarine and carbonaceous sediments of the Shumaysi Formation 
west of Makkah. There, basalt flows or sills overlying the Shumaysi are dated at $25.9 \pm 3.0$ and $32.6 \pm 2$ m.y. from bore samples (samples 35 and 39, table 10) and $20.1 \pm 0.7$ m.y. from a basalt outcrop (Coleman and others, 1979). The Shumaysi Formation contains freshwater fossils of Eocene and Oligocene age, the middle and lower carbonaceous beds containing Eocene spores below an upper and meager Oligocene fauna (AlShanti, 1966; Moltzer and Binda, 1981). Another surface sample from Hुaddat ash Shām, west of the southern Ḩarrat Rahat, gave an age of $25.8 \pm 5.0$ m.y. (sample 36 , table 10). This basalt overlies sediments containing early Tertiary, apparently Eocene, marine foraminifera. The sediments are locally capped by a laterite zone under the basalt. Thus, it appears that the earliest lavas adjacent to and possibly underlying westernmost Hुarrat Rahat lavas are near Oligocene in age and belong to the continental rift volcanism prior to uplift. Indeed, two K-Ar whole-rock ages from the lowermost of the upper lava streams overflowing the scarp divide, and thus representing upper flows of Hुarrat Rahaţ, are $12.6 \pm 2.5$ and $13.2 \pm 1.5 \mathrm{~m}$.y. (samples 27 and 28 , table 10 ), or middle Miocene in age. Younger flows at Khulays and at the harrat north of Sharm Abhur $40 \mathrm{~km}$ north of Jiddah (samples 30 and 32, table 10), dated at $4.4 \pm 0.4$ and $4.2 \pm 0.8$ m.y., are remnants of tongues that spilled over the rising Hejaz Escarpment from the upper Ḩamḑ valley.

All analyzed lavas are normative alkali basalt, including the sample from locality 37 (table 9) at Hुarrat Biss ('Ushayrah), which is separated from but near the southeast corner of Ḩarrat ar Rukhā' and which yielded an age of $8.7 \pm 2.0$ m.y. (table 10). However, flow rock from locality 38 , a small lava field at Jabal al Barz, $35 \mathrm{~km}$ southeast of 'Ushayrah, gave a coeval age of $8.0 \pm 0.5$ m.y. (table 10) and an analysis of 6.83 percent $\mathrm{K}_{2} 0+\mathrm{Na}_{2} 0$ and 10.24 percent normative nepheline (table 9) composition of greatly increased alkali that trends toward the nearby and older Jabal 'Ān compound plug (sample 40, pl. 2), which is hornblende latite-phonolite. Sample 33, from lava overlying the early Tertiary Usfan sediments in the Usfan graben north of Jiddah, contains 5.12 percent $\mathrm{K}_{2} 0+\mathrm{Na}_{2} 0$ and 8.56 normative nepheline (table 9). The K-Ar age is $19.1 \pm 0.6$ m.y. (table 10). The youngest flows in Hुarrat Rahat are alkaliolivine basalt which erupted soon after a late episode producing sodic lavas ranging from soda mugearite to phonolite (C.L. Smith, 1980; Kemp and others, 1982; J.W. Smith, 1982).

HARRAT AL KISHB

Harrat al Kishb lies east of the southern part of Ḩarrat Rahaţ. It appears to be Pleistocene and Holo- cene, but its radiogenic argon content was insufficient to measure age except for sample 29, from a $3-\mathrm{m}$ basal flow at the south edge of the harrat that gave an age of $2.4 \pm 0.8$ m.y. (table 10). A partial chemical analysis shows it to fall within the basanite field on the alkalisilica diagram, but many of the white lavas in this harrat are tholoids (domes) and doubtless are trachytic or dark rhyolitic. The earlier flows are dark gray, and more recent flows are vitreous. More than 150 cinder and scoriae cones (many breached with small lava flows) and tholoids rise above the lava field (fig. 63). A remarkable phreatic explosion caldera or crater, $\mathrm{Al}$ Wahbah, is $2 \mathrm{~km}$ wide and $270 \mathrm{~m}$ deep midway along the western margin of the harrat (fig. 64).

The walls are Precambrian granitoid, and the crater is rimmed with ash and other ejecta formed by a phreatic explosion. A more recent basalt flows around the ash rim. Ultramafic xenoliths, mostly dunitic, are scattered through the ash. On the harrat the cones and craters (fig. 63) often lie along lines following sublava fissures. The most prominent in the northwestern quadrant of the harrat trends N. $15^{\circ} \mathrm{W}$., about parallel to similar lines of cones on Hुarrat Rahaţ.

\section{HARRAT AL HUTAYMAH}

Craters similar to Al Wahbah are scattered on crystalline rocks east of Jabal Salmá in the Harrat al Hutaymah at the extreme northeast corner of the shield and, like Al Wahbah, expose crystalline basement rocks in the shattered walls of craters rimmed by ejecta and volcanic ash. One at Jabal Humayyan is 1.25 $\mathrm{km}$ wide and $110 \mathrm{~m}$ deep (Bramkamp, Ramirez, and others, 1963). According to J. Mytton (USGS, written commun., 1964), lava flows are of the pahoehoe type at Al Jubb (Na'ai), a village in a collapsed cone, that lies within the crags of Jabal Salmá. The ash at many craters contains large pyroxene-rich crystals and inclusions from the underlying Precambrian rocks. The chemical analysis of a $12-\mathrm{m}$-thick flow above $40 \mathrm{~m}$ of volcanic ash at Jabal Humayyan is that of an alkaline basalt approaching basanite (sample 25, table 9).

\section{HARRAT HADAN}

Hुarrat Hadan lies on the plain east of At Tă'if and south of Hुarrat al Kishb. The alkali-olivine basalt rests on a laterite above the Paleocene sedimentary rocks of the Umm Himar Formation. The Hadan section, gently dipping eastward and about $150 \mathrm{~m}$ thick, consists of a lower part of basalt flows, a middle part as much as 17 $\mathrm{m}$ thick of white, bedded, tufalike limestone with thin interlayers of basalt (D.G. Hadley, USGS, written commun., 1977), and an upper part of basalt flows. The flows are deeply weathered to saprolite and are deeply 




Figure 63.-Oblique aerial view east-northeast of Harrat al Kishb. Early flows were toward the basement peneplain to the east and northeast owing to ramping from Red Sea rifting. Jabal ash Sauwahah in middle distance centers Holocene centrifugal lava

eroded. Physiographically, Ḩarrat Ḩaḑan resembles the As Sirāt harrat (Madden and others, 1980), Harrat I'shara-Khirsāt, and Hुarrat Harairah. It should be of comparable age. streams resulting from the buildup of older eruptions, creating slopes opposing the regional ramp. Note meridional linearity of the Holocene craters and tholoids.

The lava sampled from the upper flows in the southwestern part of the harrat were dated at $16.6 \pm 1.5$ and 16.2 \pm 1.8 m.y. (samples 41 and 43 , table 10). Two flows sampled more recently from basal flows of the 


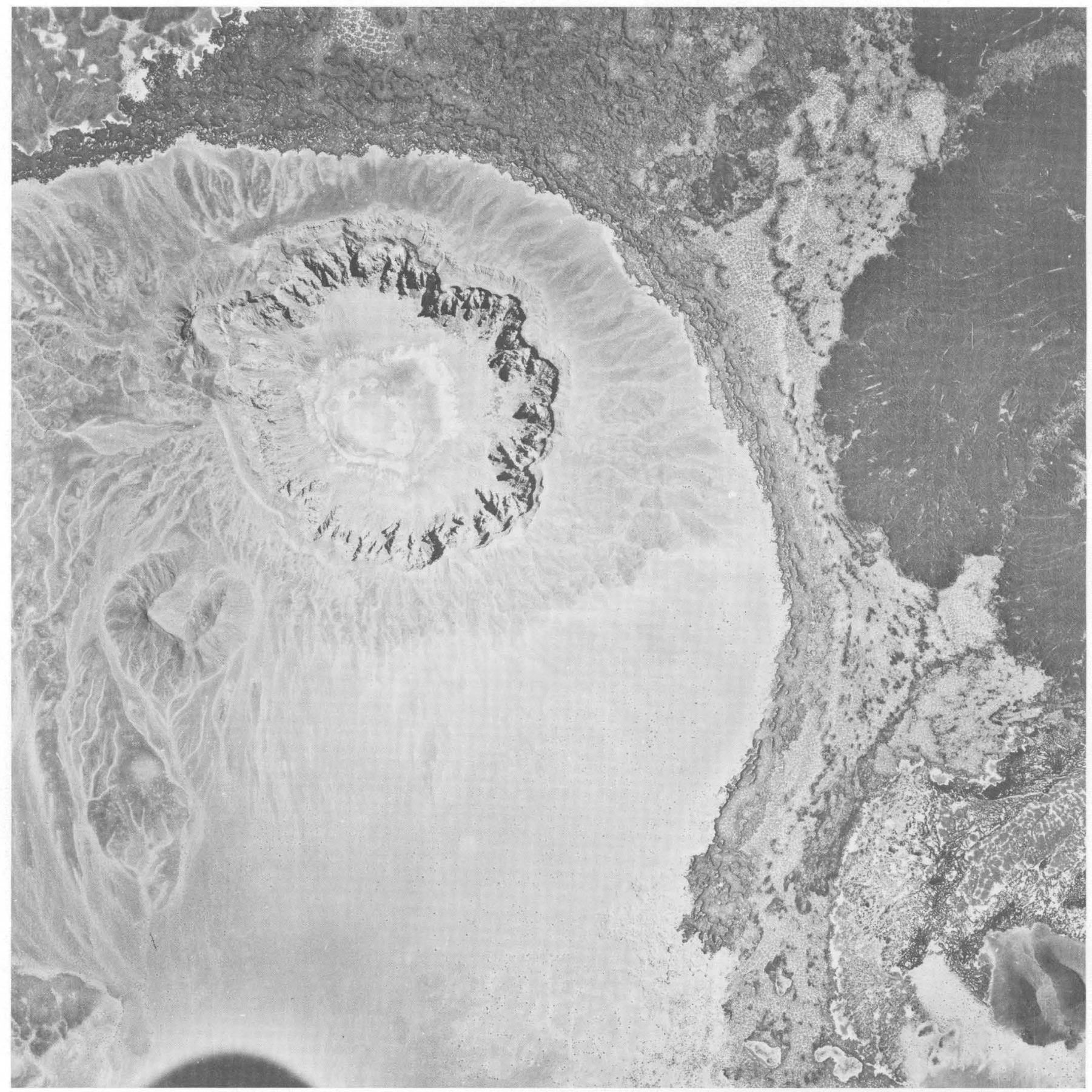

FIGURE 64.-Al Wahbah phreatic crater from a Holocene eruption at the northwestern corner of Harrat al Kishb. The crater is $2 \mathrm{~km}$ wide and $270 \mathrm{~m}$ deep and is blasted out of basement crystalline rocks. Vertical aerial photograph. North is to the left.

harrat by Arno and others (1980b) gave K-Ar wholerock ages of $27.8 \pm 1.4$ and $26.6 \pm 1.3$ m.y., which are well within the age range of the other late Oligocene-early Miocene harrats of the shield. One uppermost exposed flow gave an age of $3.4 \pm 0.5$ m.y. (sample 42 , table 10) but is probably too young according to the geomorphol- ogy. The chemical analyses of the three dated samples of the upper flows are similar and lie within the basanite field on the alkali-silica diagram (Cox and others, 1979).

The flows in part erupted from feeder pipes now exposed as isolated hills on the plain along the western, 
southern, and northern edges of the harrat; however, other isolated hills are lava-capped outliers. One of the isolated outlying hills, Jabal 'Ān southwest of Ḩarrat Hुadan, consists of a localized dike set or complex volcanic neck composed of a core of trachyte or hornblende latite and a chilled phonolitic rim (sample 40 , table 9) that has a K-Ar whole-rock age of $22.2 \pm 3.5$ m.y. (sample 40, table 10). By composition and age, the Jabal 'Ān neck is similar to, though somewhat more evolved than, the syenite-trachyte stock of the As Sirāt harrat (sample 75, pl. 2).

\section{HSARRAT NAWĀŞİF-AL BUQŪM}

The flood basalts of the large Ḩarrat Nawāşif-al Buqūm extend in a northeasterly direction southeast of Hुarrat Hुadan and form the fourth largest lava-covered region in western Saudi Arabia. The combined harrats form a remarkably uniform and youthful-appearing, nearly flat lava dome with tongues of basalt radiating outward from its edges. The relatively higher flattish central part is dotted with many Holocene scoria cones and ash rings that trend N. $20^{\circ} \mathrm{E}$. on a belt averaging $20 \mathrm{~km}$ wide. One flow tongue 2 to $4 \mathrm{~km}$ wide extends 40 $\mathrm{km}$ eastward beyond the edge of the harrat down Wādi Ranyah valley to Ranyah. At Ranyah this deeply weathered, mostly saprolitic basalt gave a K-Ar wholerock age of $3.5 \pm 0.3 \mathrm{~m}$.y., whereas a shorter tongue of 'ess weathered basalt $30 \mathrm{~km}$ west-northwest of Ranyah gave an age of $1.1 \pm 0.3$ m.y. (Hotzl and others, 1979). A sample of the lowest exposed basalt in the Wãdī Ranyah canyon, $75 \mathrm{~km}$ southwest of Ranyah, yielded an age of $1.8 \pm 1.2$ m.y. (sample 53 , table 10 ). On the west edge of the harrat, a youthful basalt flowed about $65 \mathrm{~km}$ down the Wādi Turabah valley, mostly as a tongue only $1 \mathrm{~km}$ wide. A sample of this flow contained insufficient argon to measure (sample 44, table 10), whereas about $2 \mathrm{~km}$ away Arno and others $(1980 \mathrm{~b})$ reported a sample probably from the same flow that give a K-Ar age of $2.7 \pm 0.4$ m.y. A sample of the northernmost tongue is reported by Arno and others (1980) to have an age of $4.4 \pm 1.0$ m.y.

Thus, the harrat is probably only about 5 m.y. old, although somewhat older flows may underlie its undissected central region. Arno and others (1980) reported three other ages from the harrat. One from a neck in basement in the southwestern part of the harrat gave an old K-Ar whole-rock age, $22.8 \pm 1.1$ m.y.; either this hypabyssal rock contains excess argon or, more likely, it is an erosional outlying plug of Hुarrat Hुaḑan, as the collection site lies only $40 \mathrm{~km}$ southeast of Hुarrat Hadan. Another sample reported from the neck of a youthful scoria cone in the northeastern part of the harrat gave an age of $15.8 \pm 0.8 \mathrm{~m}$.y., which is too old and may be attributable to excess argon. The underlying flow nearby gave an age of $7.3 \pm 1.8$ m.y., which also is much too old for its morphology.

One chemical analysis (sample 44, table 9) is that of an alkali-olivine basalt; Arno and others (1980b), using many analyses, found that the basalts are basanite and nepheline basanite.

\section{HARRAT AD DAMM AND HARRAT TUFFIL (SHAMĀ)}

From 70 to $105 \mathrm{~km}$ south of Jiddah, small lava flows overlie crystalline basement or Tertiary rhyolite on the coastal plain and the foothills of the scarp mountains. Harrat ad Damm, the oldest flow, consists of elongate erosional remnants sloping seaward on the crystalline foothills about $110 \mathrm{~m}$ above the modern wadi channels. A sample from the northwesternmost remnant gave an age of $7.0 \pm 4.3$ m.y. by K-Ar whole-rock analysis (sample 47 , table 10), an age comparable to ages from the upper lavas of Hुarrat 'Uwayrid and the erosional remnant west of Ḩarrat Khaybar in the Qal'at as Şawrah quadrangle. Harrat Tuffil (Shamā) flowed out on a younger ancestral coastal pediplain similar to the harrat at Yanbu' al Bahr and Al Birk. However, the Tuffil lava came from a single vent rising $100 \mathrm{~m}$ above the general altitude of the modern coastal plain. The dissected base of the lava lies about $50 \mathrm{~m}$ above the modern coastal plain and 15 to $20 \mathrm{~km}$ inland from the modern shoreline. Two samples (samples 49 and 50, table 10) gave ages of $2.9 \pm 0.2$ and $2.8 \pm 0.1$ m.y. by whole-rock K-Ar methods. This basalt overlies rhyolitic tuff of the Baid Formation along its west edge and Precambrian granite farther east. The sheared and brecciated tuff was considered to be Precambrian on USGS geologic map I-210A (Brown, Jackson, Bogue, and MacLean, 1963), but a sample from Jabal Abu Shidad, $30 \mathrm{~km}$ north of the harrat, gave a wholerock K-Ar age of $19.3 \pm 0.9$ m.y. (sample 45 , table 10 ). This is an early Miocene age compatible with the age of the Baid Formation elsewhere along the southern coast. The tuff is somewhat younger than the leucocratic gabbro intrusive nearby (sample 46, table 9). Subsequent work has extended the Baid rhyolite south to beneath the western edge of Harrat Tuffil (Laurent, 1976).

HGARRAT AL BIRK

The lavas of Harrat al Birk, also know as Harrat Hayil or Hubhub al Sheikh, cover the coastal plain from lat $18^{\circ} 45^{\prime}$ to lat $17^{\circ} 45^{\prime} \mathrm{N}$. and separate the Tihāmat ash Shām from the Tihāmat 'Asīr. Volcanic activity appears to be limited to an extensive Quaternary outpouring of alkaline basalt culminating with abundant Holo- 
cene cinder cones. Near Al Birk the older, bluish, olivine-rich basalt underlies a coquina bed which is $3 \mathrm{~m}$ above the sea; in turn, the coquina is overlain by $15-20$ $m$ of reddish-weathering basalt. Throughout Hुarrat al Birk, four samples representative of lower or older flows (samples 55b, 59, 61, and 62, table 10) gave K-Ar whole-rock ages of between 12.4 and 4.3 m.y., and two samples representative of upper or younger flows (samples 57a and 57b) gave ages of 2.8 and 2.1 m.y., but certainly some of these latter flows are as young as late Quaternary, judging by their geomorphic form. On the basis of comparison with 12 independent samples collected recently from throughout the harrat, which range in age from $1.51 \pm 0.39$ to $0.25 \pm 0.04$ m.y. (Coleman and others, 1983), and 4 widely spaced samples, which range in age from $1.9 \pm 0.05 \mathrm{~m} . \mathrm{y}$. to too young to determine (Arno and others, 1980a), our early determinations seem too old by an order of magnitude.

The Quaternary cinder cones are abundantly dispersed throughout the harrat and represent late feeder pipes. Farther east of the Al Birk lava fields, at Jabal Bă'à (Hadley, 1975c) and Jabal al Qishr (fig. 65), isolated patches of ash on the mountain slopes around a vent are freshly eroded and appear to represent an eruption during the last century. Elsewhere on the Tihāmat 'Asīr, both east of Harrat al Birk and south on the Jizan coastal plain, separate volcanoes ejected ash as well as alkali-olivine basalt. These volcanoes also appear to be young, especially on the Tihāmat 'Asīr. Lava from the two volcanoes east of Harrat al Birk, Jabal Baqarah and Jabal al Haylah, contain alkaliolivine basalt as well as hawaiite and the basanite variety of alkali-olivine basalt (samples 65 and 66 , table 11; Coleman, Fleck, and others, 1977). Samples from the basal flows yielded ages of $5.1 \pm 1.5$ m.y. (K-Ar wholerock, samples 65 and 66 , table 10 ), but later analysis of samples from Jabal al Haylah gave ages of $0.18 \pm 0.06$ and $0.25 \pm 0.04$ m.y. (Coleman and others, 1983). This is a more reasonable age, especially for the cone, which sits on a surface containing rolled Achulean axes in the valley of Wādī Tayyah (Overstreet, 1973). However, the basal flow that was originally sampled could be appreciably older than the youthful volcanic cone. Youthful volcanoes on the Jizan plain, Jibāl 'Akwatain and Jibāl Umm al Qummatain, have been dated by K-Ar whole-rock analyses at $0.31 \pm 0.32$ and $0.53 \pm 0.08$ m.y., and basalt flows in the foothills in Wādī Jĩzān gave an age of $0.99 \pm 0.23$ m.y. (another sample of basalt was too low in argon to yield a radiometric age) (Coleman and others, 1983).

A volcanic eruption occurred at Harrat Gar'atain between Jibāl 'Akwatain and Jibāl Umm al Qummatain on the east side of the Jizan plain near the Yemen border early in the last century, according to Lamare

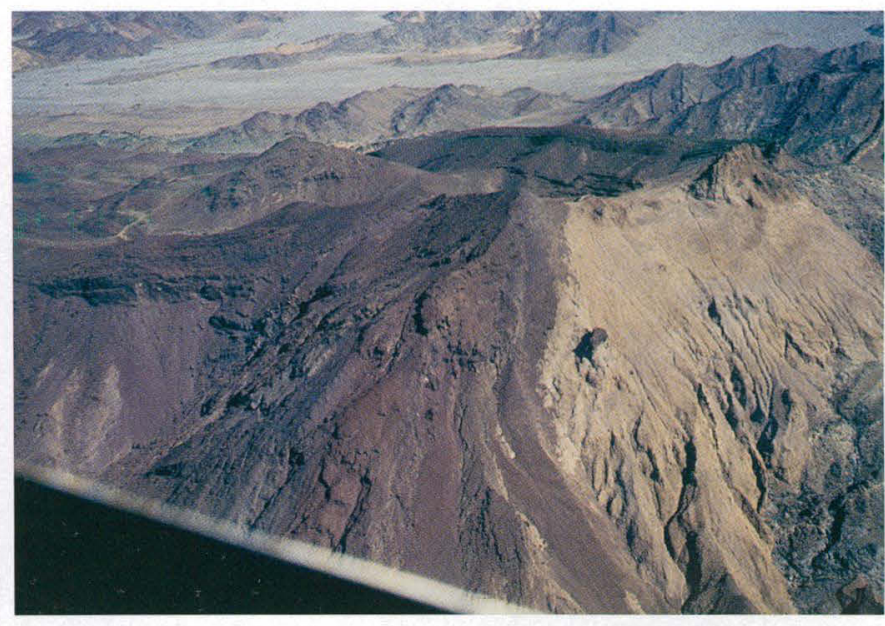

FIGURE 65.-Cinder-ash cone, Jabal al Qishr, an easternmost vent of Harrat al Birk, probably a historical eruption. Lat $18^{\circ} 31^{\prime} \mathrm{N}$., long $41^{\circ} 48^{\prime} \mathrm{E}$.

(Neumann Van Padang, 1963; pl. 2). Much volcanic ash in the rich soils and alluvium of the eastern Jizan coastal plain attest to the accuracy of the report. Further, more tenuous evidence are several nearby extinct and active hot springs. The northern and larger of the $\mathrm{Al}$ Waghrah springs discharges $1 \mathrm{~km}$ east of the southernmost cinder cones of the harrat. It has a reported temperature of $56.6{ }^{\circ} \mathrm{C}$, whereas an active spring $4 \mathrm{~km}$ distant measured $54.2{ }^{\circ} \mathrm{C}$ (Fairer, 1982). However, a higher temperature, $78{ }^{\circ} \mathrm{C}$, is reported at 'Ayn Khulab, $30 \mathrm{~km}$ southeast near the Yemen border. Both Al Waghrah and 'Ayn Khulab are in or near the faults of the Red Sea rift and associated volcanism, as is 'Ayn al Hुarrā, $450 \mathrm{~km}$ north near Al Lith, which has the highest reported temperature, 86 ${ }^{\circ} \mathrm{C}$ (Lopoukhine and Stieltjes, 1976). Other warm springs, as "Ayn ad Damad, $40 \mathrm{~km}$ northeast of $\mathrm{Al}$ Waghrah $\left(43^{\circ} \mathrm{C}\right)$, and 'Ayn al Junah, $31 \mathrm{~km}$ southeast of 'Ayn al Hुarrā $\left(46^{\circ} \mathrm{C}\right)$, may not have direct association with volcanism, the temperatures being somewhat elevated above the mean annual surface temperature because of deep circulation of meteoric water in the fractural Precambrian rocks exposed around the springs (Donald White, written commun., 1971; Lopoukhine and Stieltjes, 1976).

Ultramafic inclusions in the lavas at Al Birk, especially in the ejecta of the Quaternary cinder cones, consist of a mixture of harzburgite, websterite, and gabbro, which is in contrast to the composition of inclusions farther south in the Jizan coastal plain, where only harzburgite was found. The differences are attributed to magmatic penetration through a thick sialic crust in the Al Birk area, whereas the southern lava fields came 
from pipes that penetrated a thinner oceanic crust (Gettings, 1977; Ghent and others, 1980). The older K$\mathrm{Ar}$ ages of the $\mathrm{Al}$ Birk lavas could be attributed to excess argon derived from the underlying Precambrian crystalline rocks, which are argon-rich, or to the mafic inclusions in the younger ejecta.

\section{JABAL AS SARĀT}

The southernmost harrat on the 'Asīr highlands, Jabal as Sarāt, extends to the Yemen border (pl. 2). Jabal as Sarāt appears to be a northern remnant of the lower portion of the Yemen Volcanics (Trap Series), with ages from $30.1 \pm 1.0 \mathrm{~m} . \mathrm{y}$. in the lowermost flow to $25.3 \pm 0.5 \mathrm{~m} . \mathrm{y}$. in the uppermost flow (samples 67 and 69 , table 10). Ages from the Yemen Trap Series (29.7 \pm 0.9 to $20.8 \pm 1 \mathrm{~m} . \mathrm{y})$ and the Northern Ethiopian Plateau volcanics at Adigrat ( $30.0 \pm 0.7$ to $19.4 \pm 0.6 \mathrm{~m} . \mathrm{y}$.) all range in age from late Oligocene to early Miocene (Jones and Rex, 1974; Jones, 1976; Coleman, Fleck, and others, 1977; Civetta and others, 1978) (fig. 51). In addition to alkaliolivine basalt rocks, the Yemen volcanic rocks include hawaiite, mugearite, ignimbrite (rhyolite), andesite, trachyte porphyry, and tuff, whereas the As Sarat flows are picritic basalt at the base and grade up to alkali-olivine basalts (basanite) and to hawaiite. The 17 to 20 flows of Jabal as Sarāt total $580 \mathrm{~m}$ in thickness (Coleman, Fleck, and others, 1977), which is considerably less than half the maximum thickness of the Yemen Volcanics. Isolated feeder pipes of basalt and andesite crop out as far as $20 \mathrm{~km}$ east of the Sarāt harrat fields and as far as $70 \mathrm{~km}$ to the northwest at Jabal Qarn and Bani Thwar. These were isotopically dated at $24.7 \pm 2$ and 25.4 \pm 2.7 m.y. ago (Coleman, Fleck, and others, 1977), whereas the Al Wārah syenite plug in the midst of the harrat gave an age of $22.5 \pm 0.7 \mathrm{~m}$.y. (table 10 , sample 68), the same isotopic age as the Jabal 'A stock near Ḩarrat Haḑan, $410 \mathrm{~km}$ to the northwest.

\section{CENOZOIC HISTORY AND EVOLUTION OF THE RED SEA}

\section{EARLY TERTIARY SETTING}

Structural elements in the Red Sea area prior to development of the continental rift valley beginning in late Oligocene to early Miocene time are not evident. Early Tertiary events in the Red Sea region were presumably independent of later Red Sea evolution. Slight epeirogenic downwarping during Paleocene time resulted in a shallow marine sea extending from the Mediterranean through Egypt and Jordan south to the vicinity of Jiddah, where about $100 \mathrm{~m}$ of limestone and fine-grained sandstone were deposited as the Usfan Formation (Brown, 1970 , p. 80,81 ), and $200 \mathrm{~km}$ to the east in the vicinity of Harrat Hुaḑan, where more than $22 \mathrm{~m}$ of mudstone, shale, and limestone were deposited as the Umm Himar Formation (Madden and others, 1980). The last vestiges of this sea (arms of the dying Tethys Sea) probably lasted through middle Eocene time in northern Saudi Arabia (Kluyver and others, 1981) and are represented in the Jiddah area possibly by part of the Shumaysi Formation (Moltzer and Binda, 1981, p. 70). In North Yemen, more than $200 \mathrm{~m}$ of marine sandstone of the Paleocene Medj-zir Series was deposited (Geukens, 1966).

Cratonic stability during early Tertiary time is indicated by the thick lateritic soil (a kaolinite-ferruginous cap, from 1 to $2 \mathrm{~m}$ thick, underlain by from 20 to $30 \mathrm{~m}$ of saprolite; Overstreet and others, 1977, p. 6) that developed across the low-relief, low-altitude, crystalline rocks of the Precambrian Shield.

\section{CONTINENTAL RIFT-VALLEY STAGE}

Continental rifting along the proto-Red Sea began about $30 \mathrm{Ma}$ (fig. 66) as a mantle plume rose beneath the Afar triple junction and fracturing and rifting progressively extended along three axes: the proto-Red Sea, the Gulf of Aden, and the East African Rift. The continental rift valley progressively developed until about 20 m.y. ago. The heat regime at and near the triple junction in Ethiopia, Somalia, and especially Yemen was much greater than farther north along the proto-Red Sea axis. Near the triple junction, abundant silicic and mafic volcanic rocks were deposited in a wide continental rift valley as well as inland from the rift. Farther north along the continental rift valley (the proto-Red Sea axis), the volume of volcanic rocks decreased, the rift narrowed, and silicic volcanism was restricted to within the rift. Silicic volcanism within the rift extended as far north as Jiddah, 1,100 km northnorthwest of the triple junction. The distinct northward decrease in the size of the continental rift and in the intensity of volcanic activity seems directly related to a decreasing heat flow away from the triple junction and to the progressive northward development with time of mantle convection along the proto-Red Sea axis. North of Jiddah, volcanic rocks of the continental-rift-valley stage are limited to small remnants of basaltic flows that extended northward in the northwestern reaches of the Shumaysi and Wādi al Hamḑ-Jizl troughs. During the approximately $10 \mathrm{~m} . \mathrm{y}$. of the continental-riftvalley stage, both the mafic and silicic volcanic rocks seem to have evolved with time, that is, their $\mathrm{K}_{2} \mathrm{O}$ content increased with decreasing age, as the heat flow increased beneath the rift valley. 


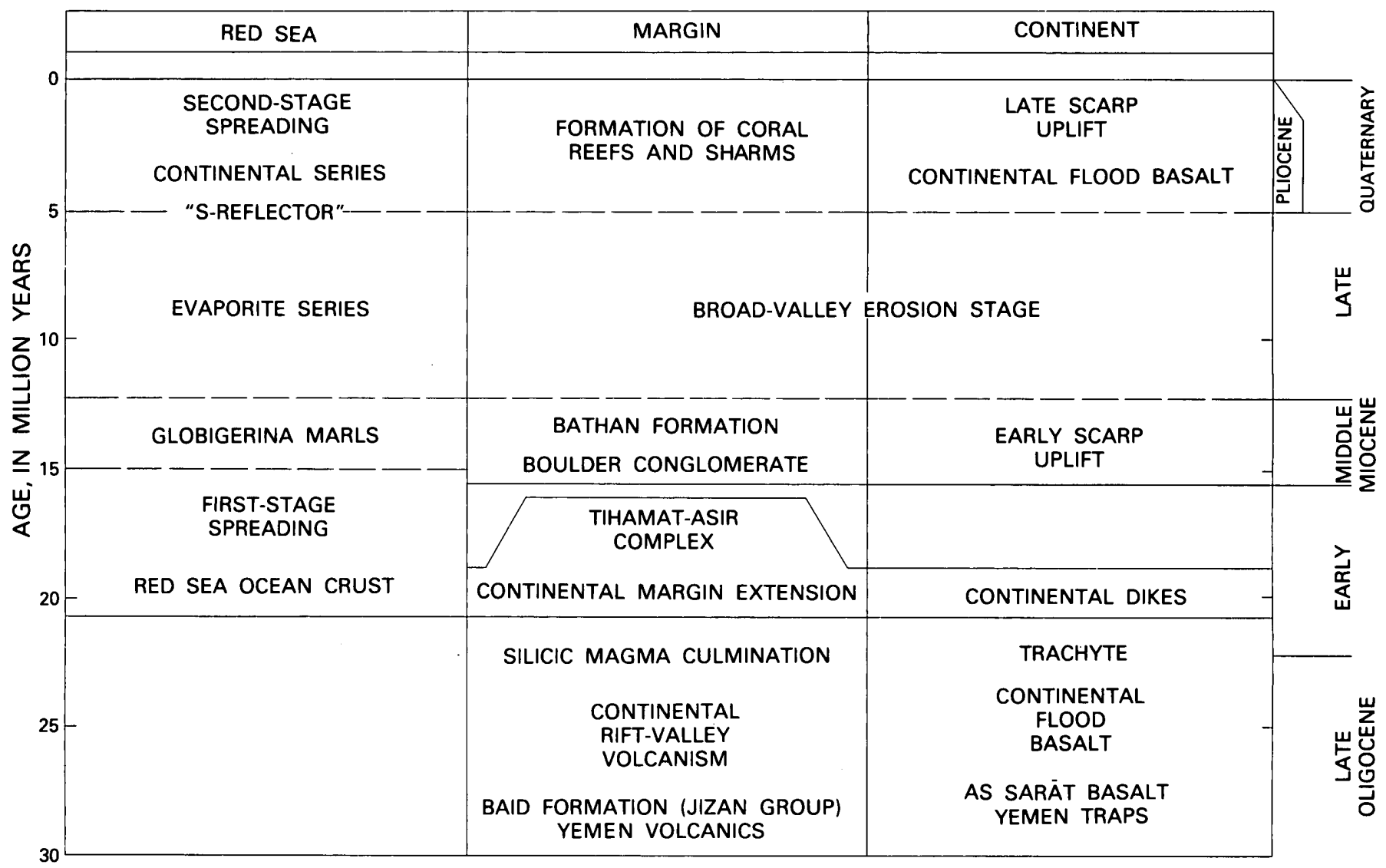

FIGURE 66.-Summary of the geologic history of the southern coastal plain area (continental margin) relative to that of the adjacent Red Sea and the adjacent continental area beginning with the formation of the continental rift valley through the present-day Red Sea. (After Schmidt and others, 1985.)

In southern Arabia, the oldest Tertiary volcanic rocks indicative of an active and rising mantle are dated at about 30 m.y. Inland from the Red Sea area, flows of alkali-olivine basalt are well preserved in the deeply eroded fields of As Sarāt (about $600 \mathrm{~m}$ thick, $100 \mathrm{~km}$ inland) and Ḩarrat Ḩaḑan (about $150 \mathrm{~m}$ thick, $200 \mathrm{~km}$ inland). In both places, early Tertiary lateritic deposits are well preserved beneath the basalt flows (Overstreet and others, 1977; Madden and others, 1980). The As Sarāt basalts and underlying laterite extend southward into the Yemen to the latitude of Sa'dah. South of Sa'dah, the volcanic pile, the Yemen Trap Series, is about $1.000 \mathrm{~m}$ thick and consists of peralkaline rhyolite (comendite and pantellerite), trachyte, trachyandesite, olivine basalt, and ankaramite (Shukri and Basta, 1955, p. 160). Laterite beneath the volcanic rocks south of Sa'dah has not been recorded, and its absence suggests some slight erosion that is represented by a few thin beds of Nubian-type sandstone reportedly at the base of or intercalated within the volcanic rocks. Some slight upwarping over a broad region may be suggested by the erosion of the laterite beneath the Yemen Volcanics (Trap Series) and by the related thin, intercalated quartz sandstones, but there is no evidence of erosion deeper than the thickness of the saprolite. A topographically high regional dome as implied by Gass (1970) did not exist. A similar large areal distribution of laterite on basement and beneath alkali-olivine basalt extends southward in Eritrea to where the basalt rests directly on sandstone, attesting to a prerift continuity across the Red Sea (Abul-Haggag, 1961).

The distribution of middle Tertiary volcanic rocks clearly indicates increased continental heat flow as the Afar triple junction is approached. North of Sa'dah (about $450 \mathrm{~km}$ north of the triple junction), mantlederived alkali-olivine basalt and subordinate trachyte (Coleman, Fleck, and others, 1977) was extruded through a full continental thickness (Healy and others, 1983). South of Sa'dah, similar basaltic rocks are interlayered in a one-to-one ratio with rhyolitic ignimbrites that were derived from thinned, hot, continental crust beneath the continental rift. The ignimbrites of the Yemen Volcanics (Trap Series) probably originated from abundant explosive volcanoes, now eroded down to their granite roots, in a highly active continental rift that had a half-width of $50 \mathrm{~km}$ or more. On eruption, 
these ignimbrites traveled more than $100 \mathrm{~km}$ east of their rift vents. The conspicuous increase in $\mathrm{K}_{2} \mathrm{O}$ with decreasing age (from 30 to 20 m.y.) for both the basaltic and rhyolitic volcanic rocks in the Yemen sequence implies an increase in crustal heat with time within the thinned continental crust beneath the rift valley and within the thicker crust marginal to the rift, for distances of as much as $200 \mathrm{~km}$.

Tertiary granite plutons in the wide continental rift of north Yemen are exposed as far as $350 \mathrm{~km}$ north of the triple junction (Grolier and Overstreet, 1978). Silicic volcanic rocks (without exposed granite plutons) within the narrow rift on the southernmost coastal plain of Saudi Arabia, from 450 to $600 \mathrm{~km}$ north-northwest of the triple junction, are subordinate to felsic and mafic rocks and imply that silicic magma production in the continental crust in this area was much less than in Yemen. Again, the heat flow from the convecting mantle ridge beneath the continental rift in southern Saudi Arabia was less than to the south.

The Baid Formation is the key and unifying feature in identification of the continental rift valley in Saudi Arabia. Its composition and distribution indicate that explosive volcanism produced siliceous ash throughout the rift to as far north as Jiddah. We have only two age determinations (19.3 and 21.3 m.y.; samples 45 and 48, table 10) on rhyolite and trachyte(?) from the Jabal Sitā' area and the major-element chemistry from the Jabal Shama (Harrat Tuffil) perlite (Laurent, 1976; Schmidt and others, 1982; Pallister, 1983) to suggest that the age and chemistry of silicic magmatism did not change significantly along the continental rift in spite of the apparent northward decrease in heat flow.

\section{FIRST-STAGE SEA-FLOOR SPREADING}

About this time the Tihamat-Asir Complex of tholeiitic diabase, basalt dikes, gabbro, and granophyre plutons was intruded into the rift volcanic rocks (Jizan Group) within the continental rift of the coastal plain of Saudi Arabia. The Tihamat-Asir Complex is inferred to be restricted to a narrow zone, probably about $12 \mathrm{~km}$ wide, at the thinned continental margin of the Arabian Shield.

The chemistry of the Tihamat-Asir dikes and the layered gabbro of Jabal at Tirf suggest a mantle origin from tholeiitic magma similar to that of modern Red Sea oceanic basalt (Coleman and others, 1979). The origin of the voluminous granophyre of the same age is less certain, and it could in fact be derived from the lower crust. However, the mafic-rock chemistry (including the rare-earth-element contents and $\mathrm{Rb} / \mathrm{Sr}$ ratios) has been modified considerably, probably by contamination with older continental crust as well as by differen- tiation at shallow crustal depths (Coleman and others, 1979). Both contamination and shallow differentiation are likely if these rocks intruded a complexly faulted and thinned continental crust in a continental rift, as we propose. A continental-rift environment, involving circulating nonmarine water, also helps to explain the large depletion in ${ }^{18} \mathrm{O}$ values in the Jabal at Tirf layered gabbro (Taylor and Coleman, 1977). As further evidence that at least a thin continental crust underlies the exposed rift belt, xenoliths of metamorphosed Wajid Sandstone and rare Precambrian gneisses are found in the basalts of Quaternary volcanoes located from 2 to 3 $\mathrm{km}$ west (seaward) of the Jabal at Tirf layered gabbro.

Once sea-floor spreading began, the Arabian-Nubian Shield parted along the full length of the Red Sea and the formation of oceanic crust was synchronous along this entire length, as predicted by rigid-plate tectonic theory and as shown by the magnetic-stripe anomalies of Hall (1980). In the Jīzān area, Blank and others (1981) showed that the easternmost magnetic-stripe anomalies of the Red Sea (Hall and others, 1977) are produced by the Tihamat-Asir Complex, that is, the oldest magnetic stripe actually is produced by the dikes that intrude continental crust. The easternmost anomaly is positive, and the reversed second anomaly appears on the western edge of the complex.

Modeling of magnetic-stripe anomalies of the Red Sea, exclusive of those of the axial trough, using synthetic anomalies generated from the Tertiary geomagnetic polarity time scale, shows a good match for the interval from 21 to 15 or 14 m.y. (M.E. Gettings, written commun., 1981). This model evidence, though not decisive, agrees with our geologic age assignments.

A brief continental-margin extensional episode accompanied the initiation of sea-floor spreading. Most deformation of the rift volcanic rocks (Jizan Group), the underlying Paleozoic and Mesozoic sedimentary rocks, and the underlying Precambrian crystalline rocks occurred at this time. The continental extension had the character of collapsing toward the new ocean crust. The relative age of this extension is well recorded by the geology. The Jizan Group as a whole was rotated about $30^{\circ}$ seaward, and the earlier, most intensely altered Tihamat-Asir dikes were similarly rotated and dip steeply eastward (Kellogg and Blank, 1982), whereas the younger, less altered Tihamat Asir dikes are typically vertical. This episode of initial spreading and crustal extension lasted perhaps 1 to 2 m.y. during the time that the first ocean crust was emplaced, consolidated, and cooled.

The thicker continental crust, from 50 to $100 \mathrm{~km}$ inland of the continental rift, was distended on vertical fractures that were filled with magma originating in the deep, underlying convecting mantle. The average 
age of these continental dikes is about $20 \mathrm{~m} . \mathrm{y}$. (Eyal and others, 1981) or 22 m.y. (Blank, 1977), which at least roughly agrees with the initiation of sea-floor spreading. Hence, the continental crust marginal to the continental rift was distended at the same time the continental rift was extended.

Initially, the continental margin extended and the sea floor spread while the world rigid-plate configuration and dynamics were such that the Arabian plate could rotate away from Africa, that is, the continental margin extended briefly until the linear rate of formation of new lithosphere equaled the rate of plate movement. When these two parameters were balanced, continental-margin extension ceased.

The present-day crustal structure across the extended continental rift at Ad Darb, from the Arabian Shield on the northeast to the oceanic crust at the Farasan Islands (pl. 1), has been determined using a seismic deep-refraction profile (Mooney, 1980; H.R. Blank and M.E. Gettings, written commun., 1981; Healy and others, 1983) and a gravity study in the Jīzān area (Gettings, 1977). The crust of the Arabian Shield is about $40 \mathrm{~km}$ thick, and the oceanic crust beneath the Farasan Islands is about $9 \mathrm{~km}$ thick. Several kilometers east of the exposed rift belt at Ad Darb, the continental crust thins to about $18 \mathrm{~km}$; hence, across the continental rift itself, a thinned continental crust 10 to $15 \mathrm{~km}$ thick is reasonable. The gravity study east of Jīzān suggests an oceanic-continental crust boundary east of $A b \bar{u}$ 'Arīsh within a steep 150-milligal (Mgal) step (4-5 $\mathrm{Mgal} / \mathrm{km}$ ) in the gravity data. Our field studies indicate that the entire exposed rift belt is underlain by continental crust; hence, the ocean-crust boundary actually lies a few kilometers west of the exposed rift belt but east of $A b \bar{u}$ 'Arish and entirely beneath the Quaternary cover.

\section{SUBSEQUENT EVENTS}

The first-stage opening of the Red Sea (Girdler and Styles, 1974) possibly extended from 20 to 15 or $14 \mathrm{Ma}$, a period of 5 or $6 \mathrm{~m} . \mathrm{y}$. This suggests a half-spreading rate of about $2.2 \mathrm{~cm} / \mathrm{yr}$. Four to six kilometers of clastic and evaporitic sedimentary rocks were deposited upon the newly formed oceanic crust. These include the middle Miocene Infra-Evaporite Series, the upper Miocene Evaporite Series, and the Continental Series of Gillmann (1968), as recorded in the Mansiyah drill hole (3,931.6 m deep) $40 \mathrm{~km}$ north of Jizān. The InfraEvaporite Series is correlated with the lower and upper Globigerina Marls of the Gulf of Suez (Tromp, 1950; Said, 1962, p. 19, 180, 313; Souaya, 1966) by way of comparable rocks in the Sudan coastal area (Sestini,
1965; Gillmann, 1968, p. 204; Whiteman, 1971, p. 205-211), as well as with reef limestone in the Jiddah area, where Vindobonian (middle Miocene) foraminifera have been reported (B. Steenstra and H.A. McClure, written commun., 1975). The Infra-Evaporite Series constrains a minimum age of middle Miocene for the end of the first-stage opening of the Red Sea. The top of the Evaporite Series is well dated by the " $S$ " anhydrite reflector throughout the Red Sea and presumably corresponds to the "M" reflector of the Mediterranean Sea at about 5 m.y. (Ross and Schlee, 1977, p. E13).

Gillmann (1968) suggested that the Baid Formation may correlate with his Infra-Evaporite Series, located at a depth of $4,000 \mathrm{~m}$ in the Mansiyah drill hole. By our interpretation, the Oligocene-lower Miocene tuffaceous Baid Formation will not be found in any of the Red Sea sedimentary rocks that overlie oceanic crust and also not, as suggested by Gillmann, on top of the Evaporite Series in the Jizan salt dome. The Baid Formation is restricted to the continental margin east of $\mathrm{Ab} \overline{\mathrm{u}}$ 'Arissh, and a sequence correlative with the Baid Formation should be found on the Sudan coastal plain in the western half of the continental rift.

One of the enigmas of the Red Sea history is the age of the Red Sea Escarpment. It has been postulated that an early, large dome rose over the mantle plume at the triple junction and above the convecting mantle ridges beneath the triple arms of the Red Sea, Gulf of Aden, and East African Rift (Gass, 1970). No polymictic sands or gravels are found beneath or within the Jizan Group, and no Precambrian detritus is found in the extensive Baid Formation, which contains only volcanic ash and erosional volcanic debris. Additionally, the early Tertiary lateritic paleosol is preserved today only beneath the Jizan volcanic rocks near sea level in the Al Lith and Ad Darb areas and beneath the flood-basalt flows on the inland plateau in the As Sarāt, Harrat Hadan, and Sa'dah (north Yemen) areas. This implies that the laterite was widespread and erosionally stable through the continental-rifting stage and that it was thoroughly eroded only upon uplift of the Red Sea Escarpment.

The Nubian-type basal sandstone of the Jizan Group and Yemen Volcanics (Trap Series) represents erosion of a thin saprolitic surface of low relief on stable Precambrian crystalline rocks near sea level. In contrast, the chaotic coarse conglomerate of the Bathan Formation is the first clue of the uplift of a Red Sea Escarpment at some time considerably after deposition of the Jizan Group. Possibly correlative terrigenous conglomerates and sandstones in the Infra-Evaporite Series suggest a middle Miocene age for the Bathan.

During a long nontectonic interlude, a broad-valley erosional stage developed on the early escarpment and 
the initial rugged relief of the scarp was greatly subdued. Two broad erosional valleys, transecting the escarpment west of Hुarrat Rahat northeast of Jiddah, are preserved beneath upper Miocene basalt flows from Harrat Rahaţ. In contrast, the present-day streams flow in steep canyons incised below the broad-valley level.

In the Biljurshī' area, Greenwood (1975b) suggested a two-stage uplift of the escarpment and identified an ancient elevated erosion surface that cuts across steeply dipping Precambrian structures and that probably represents the broad-valley erosional stage. These highly eroded, relict surfaces are vaguely recognizable in other places, such as south of Khamis al Bahr $(40 \mathrm{~km}$ north-northeast of Al Birk).

Most significantly, the broad-valley erosional stage (or the nontectonic interlude) is well documented by the late Miocene pause in shear movement on the Dead Sea Rift when Jordanian drainages flowed across the rift to the Mediterranean Sea (Zak and Freund, 1981).

During and since Pliocene time, the Red Sea Escarpment was rejuvenated in a second-stage scarp uplift. This late uplift is likely still in progress, as evidenced by the very rugged and steep topography of the modern scarp, by deeply incised wadis, and by the commonly reversed topographic relief of $100 \mathrm{~m}$ or more on latest Miocene basalt flows in the coastal plain north and south of Jiddah.

Approximate timing of the cyclic Red Sea Escarpment uplifts is suggested by the stratigraphy in the Mansiyah drill hole. Gillmann (1968, p. 199, 205) notes "some conglomerate" as a "regressive facies" in the middle Miocene(?) Upper Infra-Evaporite Series, and we suggest that this conglomerate facies correlates with the Bathan Formation. The apparent lack of clastic detritus in the thick upper Miocene Evaporite Series may correspond inland to the broad-valley erosional stage. The thick, post-Miocene Continental Series consists of clastic debris, including "sandstone and conglomerates" and "50 m of graywacke and polygenic conglomerate" (Gillmann, 1968, p. 196) that we suggest may correspond to the rejuvenated, second-stage scarp uplift.

During Pliocene-Pleistocene-Holocene time, that is, the last 5 m.y., a second stage of sea-floor spreading produced the $60-\mathrm{km}$-wide axial trough of the Red Sea and suggests a spreading half-rate of about $0.6 \mathrm{~cm} / \mathrm{yr}$. At the same time and earlier, abundant alkali-olivine basalt flows were extruded on the Red Sea coastal plain (150 km from the Red Sea axis), in the Hejaz Mountains (300 km for the axis), and on the upland plateau of the Najd (400-500 km inland from the axis). Some of these youthful volcanic fields may be as old as middle Miocene.

\section{GEOMORPHOLOGY}

\section{CYCLES OF EROSION}

To understand the terrain development in western Arabia, one should first visualize the morphology of landforms in a waterless desert, that is, in a true arid cycle (fig. 67). In general, subaerial physiographic features are produced by the degrading and weathering action of water, gravity, ice, winds, and organisms in opposition to the aggrading internal agencies of diastrophism and volcanicity. The action of weathering agents tends to decrease altitude and, although the end product may be a plain or may approach a plain, increase in relief is an early and normal result of the action of any of them. As the action of water and gravity are nearly universal, the so-called normal erosive process is the humid or fluvial cycle. In subfreezing temperatures, ice action is dominant (glacial cycle), and where both ice and water play a subordinate role, the wind produces the characteristic features of the true arid cycle.

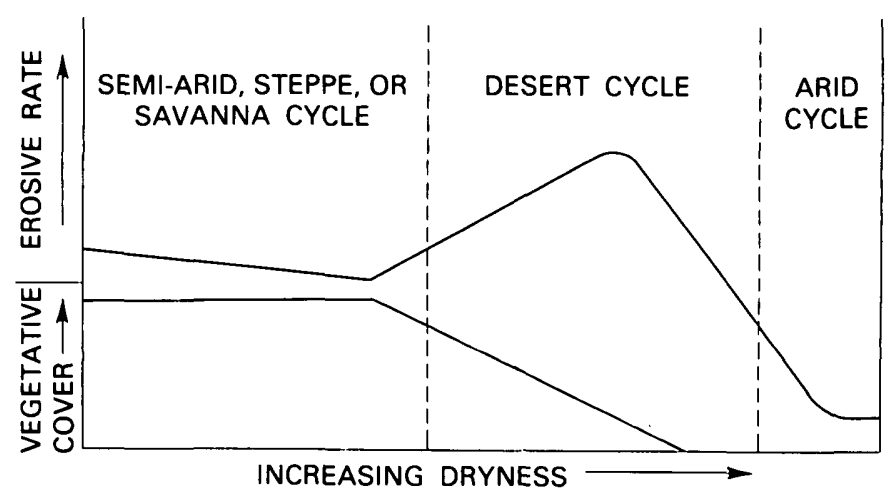

FIGURE 67.-The savanna, desert, and arid cycle.

\section{ARID CYCLE}

In an area of diverse rocks exposed only to the action of the wind, gravity, and temperature changes, the regional terrain must of necessity be initially tectonic. The wind readily attacks the softer beds wherever they are exposed; gravity works on the steeper slopes, fragments becoming available through fracturing due to pressure release and to insolation (the breakage of rocks due to surface changes of temperatures, which are great in such waterless areas). There is very little rock decomposition; rather, unstable minerals persist in the weathering products. Thus, sandstone and shale are carved out at a rate that depends on relative hardness 
and cementation; grains less than $0.08 \mathrm{~mm}$ in diameter are carried away from the region until they reach a place having sufficient moisture to hold them, whereas grains 0.08 to $1 \mathrm{~mm}$ in diameter are moved into sand masses, such as dunes, sand sheets, and ergs. The sand scours the bedrock (fig. 68) wherever it is carried over the exposed surfaces and impinges against the rock surfaces, abrading them and slowly releasing more material for transport. As the process continues, the harder beds form ridges of increasing vulnerability to attack by insolation and wind, and they too eventually are reduced until an extensive plain, cutting across all rock types, is formed, without reference to the usual concept of base level. Such a process of beveling must be many times slower than erosion by water or ice, in part because the sand forms a protective cover over an ever-increasing part of the terrain. Indeed, if the wind direction were constant, the sand probably would early form thin sheets or make sand shadows that protect the low areas on the lee side of the rock ridges. Eventually the sand would move out of the region until it reached a place of anchorage by vegetation or was blown into a distant sea. If there should be conflicting winds, and none dominant enough to carry the sand out of the region, the sand would probably form expanding ergs which would grow in depth and area, shifting from place to place, until the region would be covered with sand and the rock floor beneath the dunes would have an undulating pattern, hollows developing where wind erosion had been active longest. Under these conditions, the process might be thought of as operating under inverse control by an ever-moving base level at the surface of the sand.

Apparently no area has been rainless sufficiently long to allow such an eolian-dominated process to develop a plain - at any rate, such a plain has not been recognized. In most desert areas sufficient rain falls, usually of the torrential type, to impose special terrain features that are ultimately more subdued than those described above. The combination of wind and water together with the other less important agents produces distinctive landforms in a process termed the "desert cycle" by E. De Martonne (1926) and by von Engeln (1942).

\section{COMMON DESERT EROSION CYCLE}

The distinguishing features of the common desert erosion cycle, developed by infrequent desert torrential rainfall under conditions of meager vegetation, wide temperature fluctuation, and eolian as well as fluvial erosion and transportation, as evolved by earlier authors (Davis, 1905, 1933; Cotton, 1942) may be stated as follows:
1. Rock disintegration predominates over decomposition.

2. The accumulation of sand into dunes, ergs, and sand sheets by the wind is localized by the deposition of wadi alluvium.

3. Basins that do not have surface drainage to the sea may contain playa lakes which exert a base-level control.

4. The local base rises as the basin fills with debris, resulting in a buried rock floor that is convex upward.

5. The centripetal drainage lines into the basins are occupied by intermittent streams that are about the same size or shorter than the slopes of the basin flanks; the spottiness of desert rainfall prevents development of master valleys.

6. Pediments, or carved plains, are formed that rise on slopes of 0.5 to 7 percent from the local or regional base level to the base of desert mountains; they are cut with uniform grade across different structures and rock types.

7. As the region becomes more mature, the spreading basins intersect, resulting in coalescence of base levels and integration of drainage.

8. In maturity, wind action becomes more effective as fluvial power decreases, but if the deserts are in a rain shadow and the erosive processes lower the mountain fringe, rainfall may increase and the cycle shift into a more humid form.

The relative importance of wind and running water in desert regions has been debated at length. As a desert becomes drier, the vegetative cover decreases and the erosive action of the increasingly intermittent storms increases. When the periods between rains are sufficiently long and relative humidity becomes too low for effective dews, the vegetation, if any, is too sparse materially to retard the rate of erosion. This acceleration of erosion by water during increasing desiccation makes the effects of eolian erosion difficult to distinguish, except for the local and rather small-scale effects of corrasion such as polished and fluted rocks, pedestals, and yardangs. It is more difficult to recognize the larger forms of eolian erosion such as those caused chiefly or wholly by deflation. The problem is illustrated qualitatively by figure 67 , which shows that as vegetative cover decreases, the erosive rate by water increases, until there is no effective vegetative cover. Thereafter, erosion is largely a function of the concentration of rainfall and the effects of wind abrasion.

Most of the large features of deserts appear to result from water action, although wind-scoured hollows as much as $400 \mathrm{ft}$ deep and 10 or $12 \mathrm{mi}$ across in Mongolia have been described (Berkey and Morris, 1927). Their origin seems to require deflation on soft sands and silts 


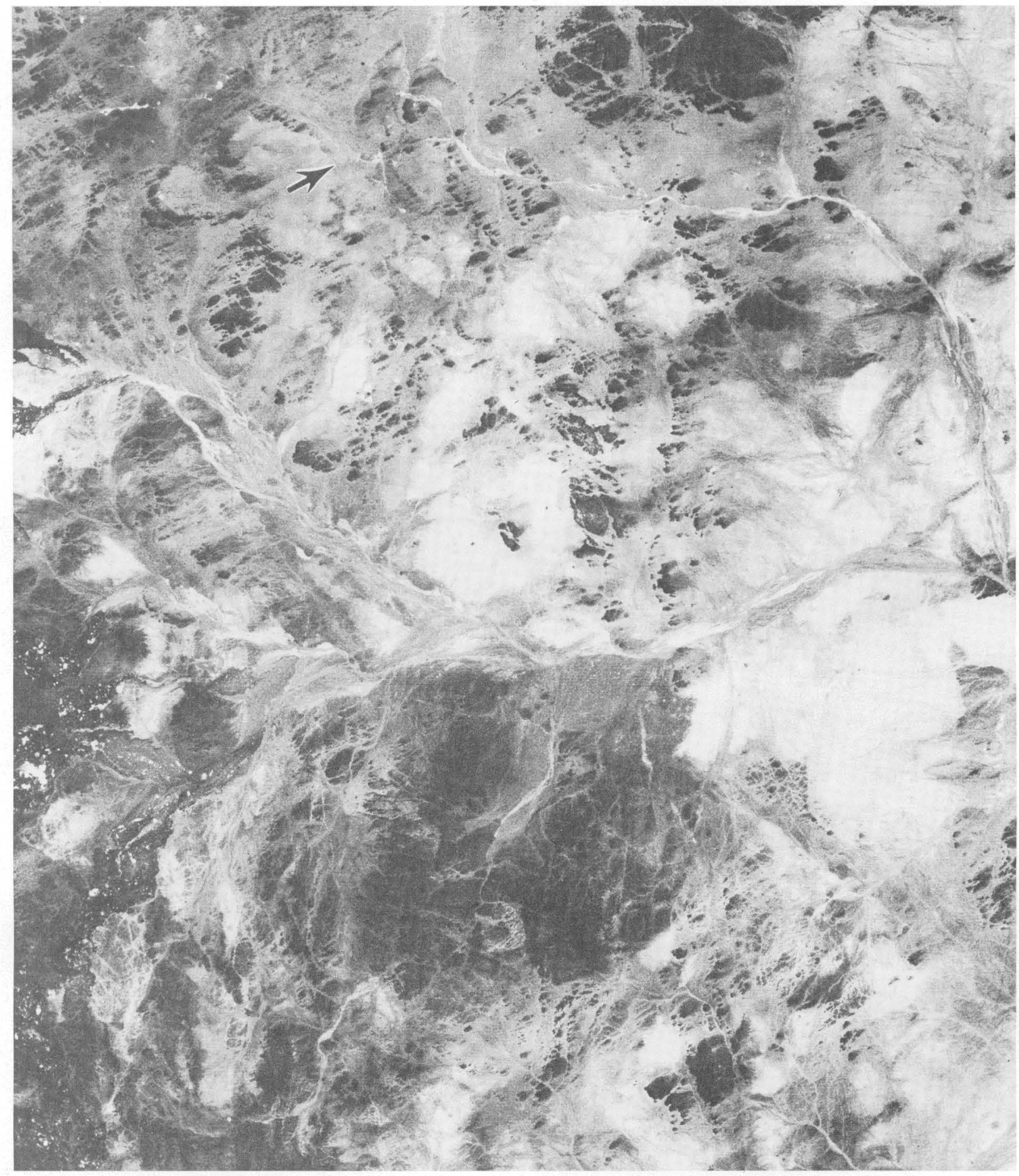

Figure 68.-Landsat image showing effects of wind erosion, north of Wādì ar Rimah and east of Harrat Khaybar and Harrat Ithnayn. Regional wind direction is toward about N. $70^{\circ}$ E. Note preponderance of yardang troughs upwind (from S. $70^{\circ}$ W.) from southeast-draining wadis, especially where there is a grus plain. There is a paucity of yardangs on the northeast flanks of these streams where the sand has been removed during floods, thus depriving the wind of a scouring tool (see arrow). 
(but some are on granites and other crystalline rocks in Arabia), aided by gullying in the short walls on the flanks. According to Berkey and Morris, the basins progress downward, possibly to the water table, then grow laterally at a rate faster than debris is brought in by the centripetal gullies. This vertical removal of debris is at approximate right angles to the removal of bedrock in the arid cycle and requires initiation by water. The concentration of wind action carrying sandblast leads to deeper and deeper cutting down the swales in a desert surface. This downcutting by wind scour is effectively measured by temperature variation past the dewpoint so that moisture trickles down flanking slopes to the bottoms of gullies. Such moisture concentration there leads to chemical decomposition, and subsequent floods or sandblast scour out the loose material. As a result, yardangs and yardang troughs are major features in the older deserts, especially in coarse-grained igneous rocks whose feldspars weather, forming grus (figs. 75, 76).

Not all deserts have interior drainage; about onethird of the world's deserts have drainage to the sea (De Martonne, 1926), either because streams flowing across them have headwaters in more humid regions or because of initial tectonic conditions. Furthermore, most, if not all, deserts, and in particular the Sahara and Arabian deserts, were more humid during episodes of the Pleistocene when the climate was at least semiarid or steppelike. In Arabia during the Pleistocene, Wādī as Shabā' seems to have reached the Arabian Gulf, or nearly so, as did Wādī ar Rimah and possibly Wādī ad Dawāsir, although the last may have debouched into lakes in the Rub al Khali basin.

Davis (1905) has pointed out that the evolution of the desert cycle is largely controlled by the initial relief, which may be of a wide valley and range, as in central Arabia, or of a massive mountain, as in the Hejaz. If the region is one of gently dipping sedimentary rock, as in the Najd, the cycle would begin in a manner similar to the ideal humid cycle operating on an uplifted peneplain formed either by former fluvial or marine planation or on a desert peneplain following a long period of cliff retreat. Without a substantial period of bahada accumulation, the harder beds would form cuestas. The wind would keep pace with the gullying of promontories, and the pediment would extend to near the cliff base by "direct replacement of one already well-planed rock floor by another at a lower level" (Cotton, 1942). The desert cycle in such sedimentary rocks has been described in Australia, Libya, the Kalahari, Mongolia, and the mesa country of Southwestern North America. The beveled surfaces that seem flattest in the drier areas are believed to have suffered greatest desiccation, in contrast to the forma- tion of coalescing alluvial fans and sloping pediments in areas where conditions have approached or entered the semiarid cycle.

\section{TIHĀMAH}

The coastal plain (pl. 3) along the eastern shore of the Red Sea, known as the Tihāmah, extends with few interruptions from the Gulf of Aqaba on the north to Bābal Mandab on the south of the Red Sea. The Tihāmat appears to have developed during alternations of desert and savanna cycles, with the Red Sea as a variable base level of erosion. The coastal plain ranges in width from narrow beaches to as much as $40 \mathrm{~km}$. In the southern part, the Tihāmat al Yemen and Tihamat 'Asīr, a 2- to 3-m elevated beach, extends northward as far as Al Wajh. It is marked by low headlands and overhanging scarps ascribed by Guilcher (1952) to chemical weathering (solution) activated by surf and eolian action. Near Jiddah, the surface rises eastward about $1.5 \mathrm{~m} / \mathrm{km}$ for about $5 \mathrm{~km}$ to altitudes of about 10 $\mathrm{m}$. Above $10 \mathrm{~m}$ the slopes are steeper, on the order of 10 $\mathrm{m} / \mathrm{km}$, until a general altitude of about $100 \mathrm{~m}$ is reached at the foot of the coastal mountain belt. Remnants of a $20-\mathrm{m}$ beach underlie a basalt flow north of Jiddah, and surfaces at $6,10,22$, and $31 \mathrm{~m}$, mostly on coralline rocks, have been measured from Umm Lajj northward to near the Gulf of Aqaba (fig. 69). The surface culminates at an altitude of $525 \mathrm{~m}$ on Tìrān Island at the mouth of the gulf $(500 \mathrm{~m}$ according to Goldberg, 1963), and marine benches extend up to at least $320 \mathrm{~m}$, facing southwest (Schick, 1958). The surfaces appear to be elevated primarily by vertical movement along the old transverse Najd fault system

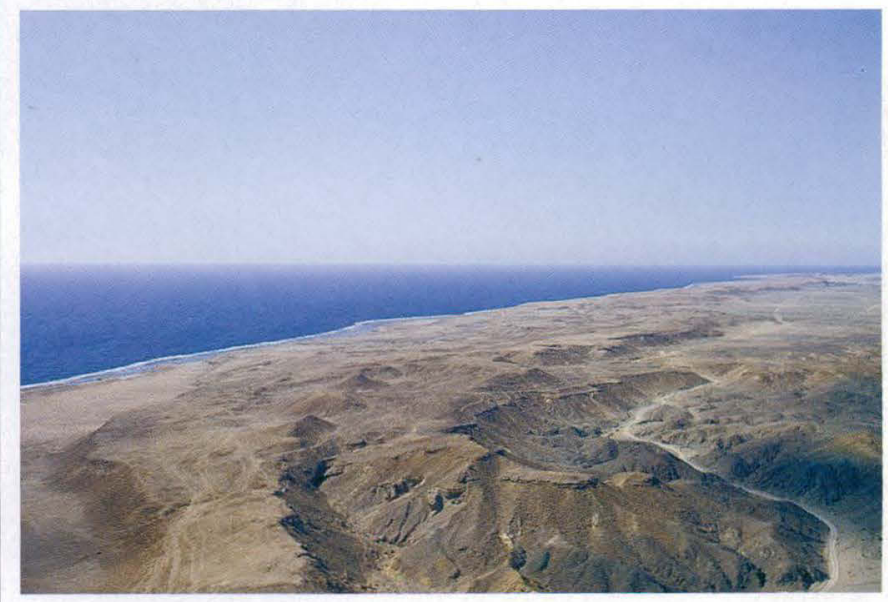

Figure 69.-Terrace benches at 6, 22, and $31 \mathrm{~m}$ above the northern Red Sea north of Al Wajh resulting from intermittent ramping of the Ḩismá (Ash Shifä') block of the peninsula during the Quaternary. View to the northwest. 
as part of the ramping of the northeast flank of the rift valley (Brown, 1972). However, relative movement of blocks between transform faults that cross the Red Sea in a northeast direction may also account for some of the uplift. The generally accepted two-stage opening of the Red Sea, with two poles of rotation as developed by Girdler and Styles (1974) and by Richardson and Harrison (1976), would cause vertical uplift from compression during the second, Pliocene-Pleistocene-Holocene stage, as the sea floor widened in an easterly direction and the peninsula rotated about $7^{\circ}$ counterclockwise.

The seaward, lower part of the Tihämah is, for the most part, a depositional or coralline surface that gradually merges eastward into an erosional pediment that extends across the rift fault zone of the eastern edge of the Red Sea rift. The coastal-plain surface in many places can be followed continuously from Tertiary sedimentary and igneous rocks eastward onto the pediment beveled across the crystalline rocks of the Precambrian basement. Thus, most of the pediment developed after the last large-scale movement on the easternmost margin and after the inception of a major rift opening at about $22 \mathrm{Ma}$ in the southern Tihāmah, when gabbro and related dike swarms were emplaced (see samples 72 and 73, table 10; Coleman and others, 1979). An ancestral remnant of an old pediment surface is preserved beneath the about 3-m.y.-old basalts, Hुarrat Tuffil, $100 \mathrm{~km}$ south of Jiddah, where the ancestral pediment, beveled on Precambrian rock and protected from erosion by its basalt cover, lies $50 \mathrm{~m}$ above the modern coastal plain midway between the coast and the foothills on a $30-\mathrm{km}$-wide coastal plain.

At intervals along the present shore are small inlets called sharms (locally, khawr or marsa). Some are connected to present drainage debouchment from the coastal mountains (pl. 4); others are not related to present drainage. Typically they are " $T$ " or bottleshaped, widening out landward from a narrow throat, about $30 \mathrm{~m}$ deep, and maintaining a depth of as much as $10 \mathrm{~m}$ behind the coralline ridges on the flanks of the throat entrances. The water bays behind the throats are commonly 5 to $9 \mathrm{~km}$ long parallel to the coral ridge and extend inland from 2 to $5 \mathrm{~km}$. There is now no active, or only very minor, erosion in the sharms. Also, as floods now rarely reach the Red Sea across the coastal plain except in a few of the largest wadis, there must have been more rainfall when the sharms were formed. The openings in the coralline fringing reefs suggest that less saline, perhaps silt-laden, water from runoff in wadis draining the western slopes of the uplands may have inhibited growth of coral across the throats before the present pediment was elevated $3 \mathrm{~m}$ or more. Thus, at first glance it would appear that the sharms are coeval with the time of coral growth now exposed in the flanks of the throats. However, much evidence points to a later breaching of the fossil reef. For instance, the bifurcation and trifurcation of the sharms behind the beach ridge, as noted by Gvirtzman and others (1977), was thought to represent lagoons that existed before the reefs were breached.

The age of the elevated coral reef making up the seaward edge of the sharms is greater than a minimum ${ }^{14} \mathrm{C}$ age of $40,000 \mathrm{yr}$, as determined by Meyer Rubin (in Brown, 1970), for the coral at Jiddah. Goldberg and Yaron (1978) assigned a ${ }^{230} \mathrm{Th} /{ }^{234} \mathrm{U}$ age of $146,000 \pm 16 \mathrm{yr}$ for the reef that is at $11-13 \mathrm{~m}$ altitude today on the southeastern coast of Sinai. The elevated reef, commonly beneath a 2-3-m terrace, along the central and southern Red Sea of Saudi Arabia is elevated as high as $50 \mathrm{~m}$ on the Jīzann salt dome and is possibly about $135,000 \mathrm{yr}$ old (uranium-series dating of the coral; J.W. Whitney and B. Szabo, USGS, oral commun., 1983). An elevated coralline reef in the Afar rift is $54,000 \pm 4,600$ yr ${ }^{230} \mathrm{Th} /{ }^{234} \mathrm{U}$ dating of the unrecrystallized mollusk Tridacna; Bonatti and others, 1971), but the Afar is considerably more active than the Red Sea coast and the age is probably younger than the Red Sea elevated coral. At any rate, the coral is old relative to the assumed youthful erosional age of the sharms, for any estuarine depression behind the coral reef would have been filled long before the formation of the sharms.

The $30-\mathrm{m}$ depth at the throat of some of the sharms suggests a period of downcutting when the Red Sea level was $30 \mathrm{~m}$ or more below present sea level. Low base levels existed during the various Quaternary glaciations, when large volumes of the Earth's water were stored in glacial ice. Recently described evidence from many sources indicates that epochs of maximum glaciation were coeval with aridity in the low-latitude deserts, at least during the late Pleistocene, even though the present interglacial epoch is arid (Bowler, 1976; Deuser and others, 1976; Wendorf and others, 1976; Sarnthien, 1978). Therefore, pluvial epochs seem to correspond to the interval between glacial and interglacial, that is, the more or less equivalent intervals of deglaciation on one hand and warming on the other. It is during these pluvial epochs when integrated drainage across the coastal plain to the Red Sea might be expected and when erosional cutting of the sharms probably took place.

Pluvial lakebeds were deposited in Ar Rub 'al Khālī, according to ${ }^{14} \mathrm{C}$ dates, between 36,000 and $17,000 \mathrm{yr}$ B.P., with a cluster of ages from 30,000 to $21,000 \mathrm{yr}$ B.P., and younger lakebeds were deposited from 9,000 to $6,000 \mathrm{yr}$ B.P. (McClure, 1977). The older pluvial epoch was about contemporary with the ${ }^{14} \mathrm{C}$ ages of $20,400 \pm 500$ to $24,630 \pm 500$ yr B.P., for the deep artesian 
water from widely scattered wells in central and eastern Arabia (Thatcher, Rubin, and Brown, 1961).

Farther afield, Sarnthien (1978) gives wet intervals for the Sahara of from 12,500 to $11,000 \mathrm{yr}$ B.P., from 10,000 to 7,500 yr B.P., and from 6,500 to 5,500-5,000 yr B.P. The oldest interval coincides with an extensive pluvial interval of the Nile (Fairbridge, 1977), although the Nile floods come from a different climatic zone that may not be synchronous with the desert cycle. Sarnthien (1978) states that prior to the moist interval, active sand dunes extended from lat $10^{\circ}$ to lat $37^{\circ} \mathrm{N}$. in the Sahara about 18,000 yr ago-synchronous with the polar ice maximum (about 20,000 yr B.P.; Peltier, 1980) and with maximum desert aridity. The pluvial chronology is further strengthened by an analysis of oxygen isotope ratios from the planktonic foraminiferal fauna of the Red Sea and the Gulf of Aden (Deuser and others, 1976) which showed that the cold intervals for the Red Sea coincided with intervals of major deglaciation. The last of these cold intervals, recorded in the cores from the Deep Sea Drilling Project (Scripps Institution of Oceanography, University of California), occurred from about 17,000 to $8,000 \mathrm{yr}$ B.P., that is, between the Wurm glacial maximum and the climatic optimum of the Paleolithic.

The pluvials of Arabia and the Sahara seem to be of much shorter duration than the interglacial epochs, so that if glacial expansion corresponds to desert-cycle aridity, interglaical does not entirely correspond to desert-cycle pluvial. Hence, perhaps the interval of change, that is, the interval of rapid ice retreat-rapid deglaciation, should be considered the dynamic climatic control for the pluvial interval. Perhaps the present aridity of Arabia during an interglacial epoch is explained by the correlation of the pluvial only with relatively short transitional time during rapid deglaciation.

The origin of the sharms requires a coincidence of low sea level and pluvial conditions such that the coastal-mountain water flowed to the Red Sea. Streamflow to a sea level lower than the present would allow the erosional breaching of the elevated reef and at the same time allow lateral erosion of some of the soft estuarian sediment fill behind the reef, thus forming the typical " $T$ " pattern of the sharms. The post-Wurm rise in sea level for the Red Sea can be extrapolated from data by Peltier (1980, fig. 8 ). Accordingly, the sea level rose from about $-75 \mathrm{~m}, 13,000 \mathrm{yr}$ ago, to $-30 \mathrm{~m}$, about 9,000 yr ago, and to about the present sea level, 5,000 yr ago.

The sharms can be estimated to have formed about 12,000 to $8,000 \mathrm{yr}$ ago, during the early half of the Sahara-Arabian pluvial and when sea level rose from about $-60 \mathrm{~m}$ to $-20 \mathrm{~m}$. At the maximum low sea level of about $-120 \mathrm{~m}, 18,000 \mathrm{yr}$ ago, climate was too arid, and after about 8,000 yr ago, sea level was too high for the sharms to form. Certainly after about 5,000 yr ago the climate also was too arid. A fossil coralline reef in the Gulf of Aqaba now at intertidal level has been dated at $4,770 \pm 140$ yr B.P. (Friedman, 1965)-the sharms must have been cut before this time. Similar sharms probably formed earlier during the early part of other interglacial epochs, for example, perhaps between 36,000 and 32,000 yr ago, during the early part of the last Wurm interglacial.

The eastern and higher Tihāmah is in places covered with eolian sand, generally accumulating transversely to the offshore-onshore winds, usually in barchan dunes. In several places where winds shift alternately from northwest to southwest and vice versa, long seif dune ridges accrete approximately perpendicular to the trend of the coastal plain. In general, the sand grains are 3 and $4 \mathrm{~mm}$ in diameter, much coarser than the bulk of the sand accumulated in the great interior nafuds. They accumulate in para-ripples. The smaller grains generally are deposited in dunes farthest from the shoreline against the base of the scarp mountains (Guilcher, 1952).

\section{SCARP MOUNTAINS}

The ramping of the eastern flank of the Red Sea rift and subsequent faulting has exposed a southwestfacing scarp (fig. 70) against which atmospheric weathering has been active, probably since the middle Miocene. This has formed a mountainous belt 40 to $140 \mathrm{~km}$ wide which rises eastward to the rim of the great interior plateau of Najd, the Hejaz-'Asīr highlands area in southern Arabia (pl. 3), and the Hismá Plateau in northern Arabia. The crest reaches a maximum 3,000 m in Jabal as Sūdah in the 'Asīr near the Yemen border. The crest line gradually declines northward to a minimum height of about $1,000 \mathrm{~m}$ in the mountains northwest of $\mathrm{Al}$ Madinah, where the mountainous belt is widest and where Wādì al Ḩamḑ has been captured and flows more directly to the Red Sea through a precipitous canyon.

Farther north, another block is tilted upward along a series of faults that cut obliquely across the belt, forming the mountains of Ash Shifā', which lie in front of the Hismá Plateau and extend into Jordan. The highest point of Ash Shifā' is Jabal al Lawz, 2,400 m above the Gulf of Aqaba, but five peaks making up the Ash Shifā' region as far as $100 \mathrm{~km}$ south are $2,000 \mathrm{~m}$ or more in altitude. A series of knife ridges and canyons marks the southwestern fronts of each block. These 


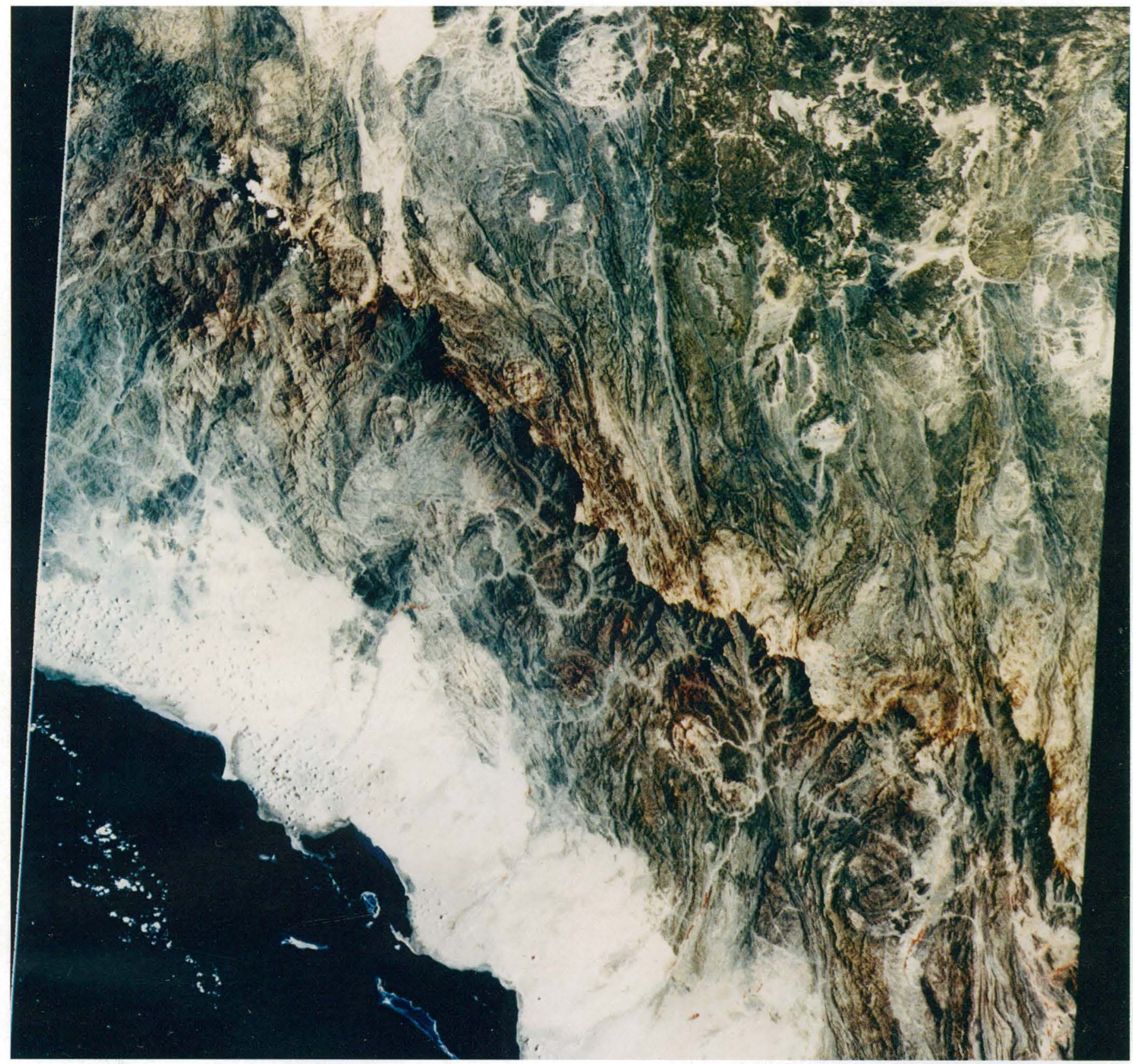

Figure 70.-Landsat image of the erosional scarp of 'Asīr at lat $20^{\circ}$ N. Red Sea on lower left, bordered by the Tihāmat 'Asīr (coastal plain) and the scarp mountains cut back to the Najd pediplain (upper right). The pediplain here has been ramped up to altitudes of as much as $2,600 \mathrm{~m}$. The southern end of the plateau lavas of Hुarrat Buqūm occupy the northeast corner of the scene. From Jet Propulsion Lab. 
southwest-facing slopes are in places transected by north-trending valleys which permit passage from one canyon to the next. Some north-trending valleys are subsequent erosional valleys carved parallel to the structural grain in basement schist. Narrow valleys trending northwest are underlain by the wide Tertiary mafic dikes that weather more rapidly than the Precambrian crystalline wall rocks. Some of these narrow valleys are flanked by thick walls representing the finegrained chilled margins of the mafic dikes. Subsequent erosion has developed the north-south tributaries, along which it is possible to cross watersheds at the heads of the subsequent streams without being aware of a reverse in slope, such was the flatness of the preuplift pedimentation.

Many lower courses of the larger wadis draining the scarp mountains show stream capture (pl. 3). Wādì al Hुamd is an outstanding example, with an elbow of capture at the junction of Wādi al Jizl. Evidently the wadi prior to the uplift of the Hejjāz Mountains flowed out to the coast north of the present mouth at lat $26^{\circ}$ N., perhaps as far north as the emergence of the major Najd fault into the Red Sea at lat $28^{\circ} \mathrm{N}$. The schists of the shear zones formed valleys on the shield toward the end of cratonization, a natural environment for the development of subsequent streams on the early Tertiary lowland of the northern Red Sea coast (Madden and others, 1980). If so, the ancestral wadi was captured progressively southward as the peninsula rotated counterclockwise and ramped upward in response to the compressional and sinistral stresses toward and along the Jordan (Dead Sea) rift. A subsequent outlet may have been via the Wādi Saluwah-Wādì Damā thalweg, debouching through upper Wādī as Surr, followed by a later capture southward via Wādī Bayda and the lower Wādi as Sirr trough. As the tilting continued along the Najd fault system, Wādi Thalbah and Wādi Azlam captured the lower As Sirr valley and Wādi al Hुamd reached the sea via its present course. Regardless of the precise channelways, the northeast tilting (elevation) of the Hisma block undoubtedly caused the wadi to seek an outlet to the sea in a southerly or westerly direction as the earlier lower course was elevated.

In a similar manner, wadis farther south were captured (pl. 3). Notably, Wādì al Far'ah from the east side of Jabal Radwá, near Yanbu' al Bahr, had a previous channel extending south and debouching through Wādī al Faqir onto the coastal plain $40 \mathrm{~km}$ south of the present mouth. This ancestral stream sought a more western outlet as the country south of Wādì al Faqir rose. Test drilling in Wādī al Faqir penetrated alluvium about $100 \mathrm{~m}$ thick, whereas the current drainage in Wādì al Far'ah has a shallow bed over granite. Similarly, Wādī as Safra', $30 \mathrm{~km}$ farther southeast, drains two meridional valleys-Musayj'id or Wādī as Safrā (3 km wide) and Tasḩah (1 km wide)through a 200-m-wide gorge, creating a flood hazard on the Al Madinah-Jiddah road.

Wādī Fāţimah between Jiddah and Makkah likewise debouched southward through Wādī Shumaysi, which has a deep alluvial valley in comparison to the nearly bare rock floor of the present lower Wādi Fāţimah whose debouchment is $30 \mathrm{~km}$ northwest of the ancestral valley. Farther south, Wādì Qununah, Wādì Yiba, and Wādī Ḩalī in particular seem to have changed drainage directions. Thus, the wadis draining the scarp mountains have been captured or have reversed flow toward the northwest as the Asir block ramped, tilted, and skewed in a counterclockwise direction under tensional conditions. Wadis on the southwest flank of the Hुismā block north of the Najd faults, however, moved southwest as the country rose to the northwest under compressional conditions.

Tertiary igneous rocks, both hypabyssal and extrusive, have been emplaced along the original rift zone at or near the toe of the scarp mountains. These rocks commonly give an initially deceptive rugged appearance to the lower foothills of the scarp-mountain terrain when viewed from the Tihāmah, but the prerift pedimentation surface is well developed below the dissected volcanic rocks.

\section{HEJAZ-ASĪR AND HISMĀ PLATEAUS}

The Hejaz-Asīr Plateau forms the uplifted and dissected southwestern corner of the Najd pediplain of western Arabia. Triangular in shape, it extends southward from At Tā'if to and beyond the Yemen border, an area of about $40,000 \mathrm{~km}^{2}$ above $2,100 \mathrm{~m}$ in altitude. Broadening and increasing in altitude southward, it culminates in Jabal as Sūdah at 3,000 m near Abhā, the capital of 'Asīr Province and the most salubrious region in Arabia. As the western lip next to the scarp mountains is approached, more abundant rainfall results from orographic convection over the scarp, especially during the late summer monsoon season. Great rainfall increases the amount of dissection, as do the fracturing and warping of the bedrock caused by ramping in connection with the evolution of the Red Sea rift. Rainfall greater than $20 \mathrm{~cm}$ annually is limited to a crest zone 20-30 km wide. Consequently, eastward and northward wadi flow decreases rapidly downstream and deposition is greater than erosion near the eastern edge of the plateau. Berms and strath surfaces indicate 


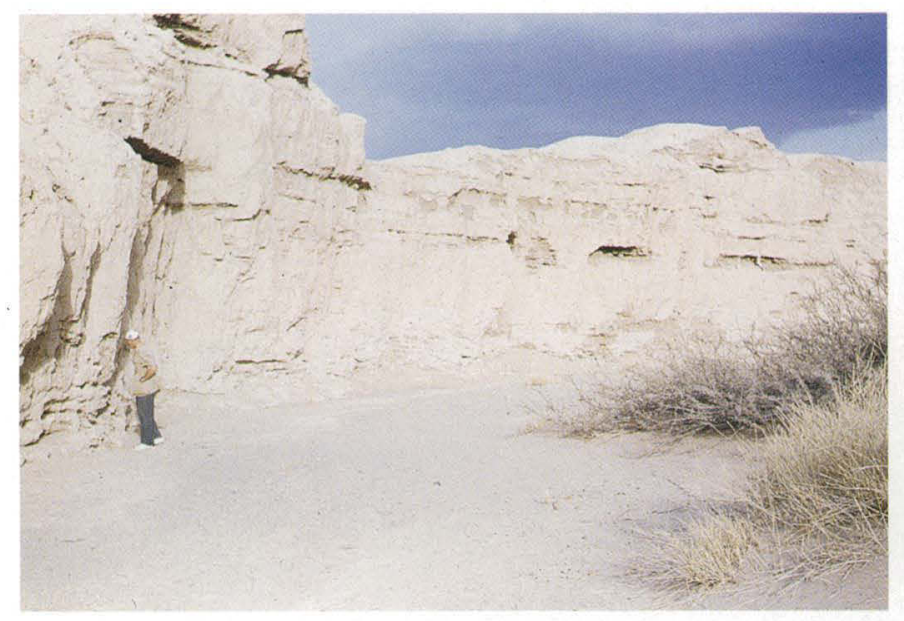

Figure 71.-Loessal silt in Wādī Tathlīth above Ḩamḑah. (Photograph by Thomas Smallwood.)

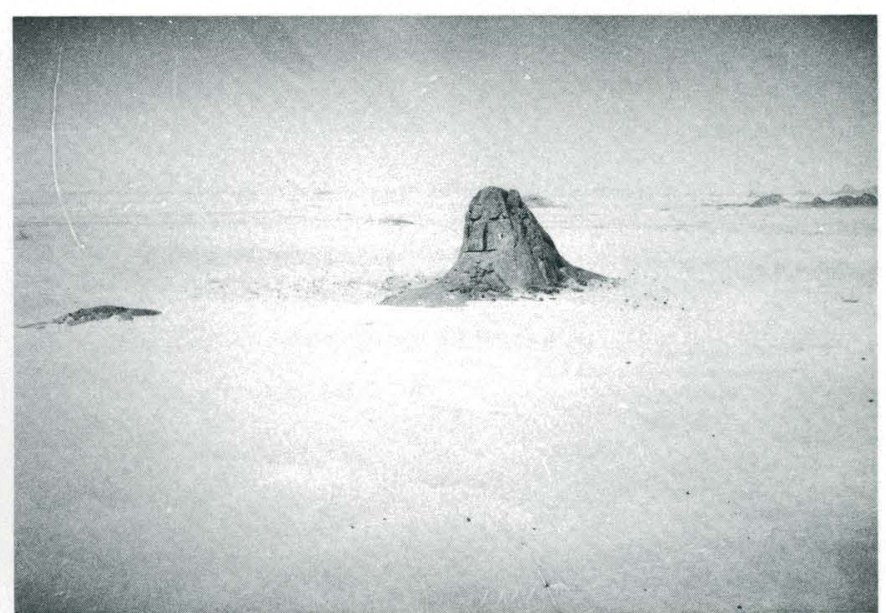

Figure $72 .-$ Bornhardt at Jabal Kursh, lat $22^{\circ} 25^{\prime}$ N., long $43^{\circ} 39^{\prime}$ E. intermittent uplift or climatic change, with at least one relatively static period.

The wadis widen in midcourse where runoff from tributaries coalesce, but many are constricted by narrow throats, particularly where reentering metamorphic terrain from a grus plain underlain by granite rock. Such constrictions have ponded floods and deposited loessal silt (fig. 71) upstream (behind them) during periods of excessive flooding (Brown, 1960). The paucity of grit and gravel suggests low relief and gentle stream gradients at the time of silt deposition. Charcoal from fire hearths on the upper part of the loessal silt have been dated by ${ }^{14} \mathrm{C}$ measurement at $6,350 \pm 350$ and $5,830 \pm 300$ yr B.P. (Schmidt and others, 1983), which is in excellent agreement with the age of the Holocene pluvial epoch $(9,000$ to $6,000 \mathrm{yr}$ B.P.) as determined by ${ }^{14} \mathrm{C}$ dates of lacustrine deposits in Ar Rub al Khālī (McClure, 1977). The accumulation upstream from constrictions was increased by the higher water table and longer moist periods so that vegetation accumulated, as evidenced by abundant root casts in the silt deposit. The absence of fossil pulmonate gastropod shells seen in present-day loess may be attributed to dissolution of the carbonate by humid acid from the vegetation (Schmidt and others, 1983).

In northwestern Arabia east of the Gulf of Aqaba, the Hismā Plateau is an upland comparable to the Hejaz-'Asīr, lying between the scarp mountains of Ash Shifa'' on the west and the great northern sandstone plains extending eastward to the Great Nafud. Likewise, the high position of the Hismā Plateau results from concomitant ramping in connection with the counterclockwise rotation of Arabia away from Africa. The sandstone plateau rim extends southward from the Jordan border at an altitude of $1,800 \mathrm{~m}$ and descends to $1,100 \mathrm{~m}$ at lat $28^{\circ} \mathrm{N}$., east of the mouth of the Gulf of Aqaba. The surface slopes eastward to the western edge of the Great Nafud basin to an altitude of about $800 \mathrm{~m}$, where the Hejaz Railroad crosses the plain. The southern rim of the Hुismā is higher south of lat $28^{\circ} \mathrm{N}$., where the multiple flows of plateau-flood basalt have built up the surface to 2,000 m in the Hुarrat ar Raḩāh. Except for the plateau basalts in the Southern Hुismā, sandstone buttes and mesas dot the plain, increasing in height and numbers toward the rim east of and somewhat below the crests of the crystalline mountains of Ash Shifā'.

\section{NAJD PEDIPLAIN}

The crystalline Najd pediplain formed from coalescing pediments begins at the northeastern edge of the Hejaz-'Asīr Plateau, where the ramping of the plateau flattens out toward the northeast. It is a vast reg or serir on which are scattered isolated inselbergs of more resistant igneous and metamorphic rocks, bornhardts and kopjes that are generally granitic, and lava fields present mostly along the western edge (figs. 72, 73, 74).

Where granitoid rocks crop out, most of the terrain is nearly flat and featureless, especially where the crystalline rocks have been tectonized and weathered to clay by periodic hydration of feldspars and where wind 


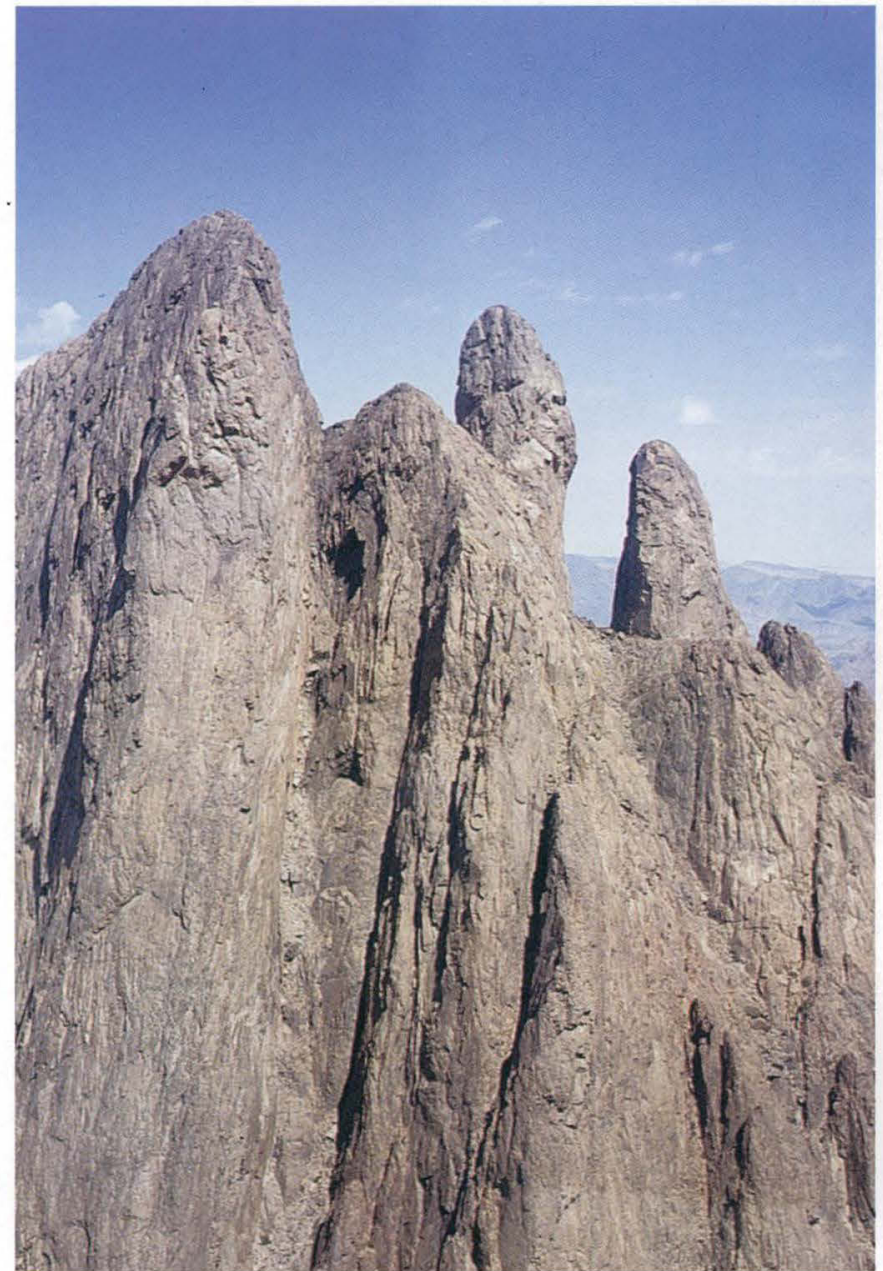

FIGURE 73.-Spines of Jabal Shār rising to an altitude of 1,990 m east of Al Muwaylih on the Red Sea coast at lat $27^{\circ} 39^{\prime} \mathrm{N}$. The small batholith is composed almost entirely of posttectonic graphic granite. A U/Pb age of $625 \pm 5$ m.y. was obtained by Hedge (1984).

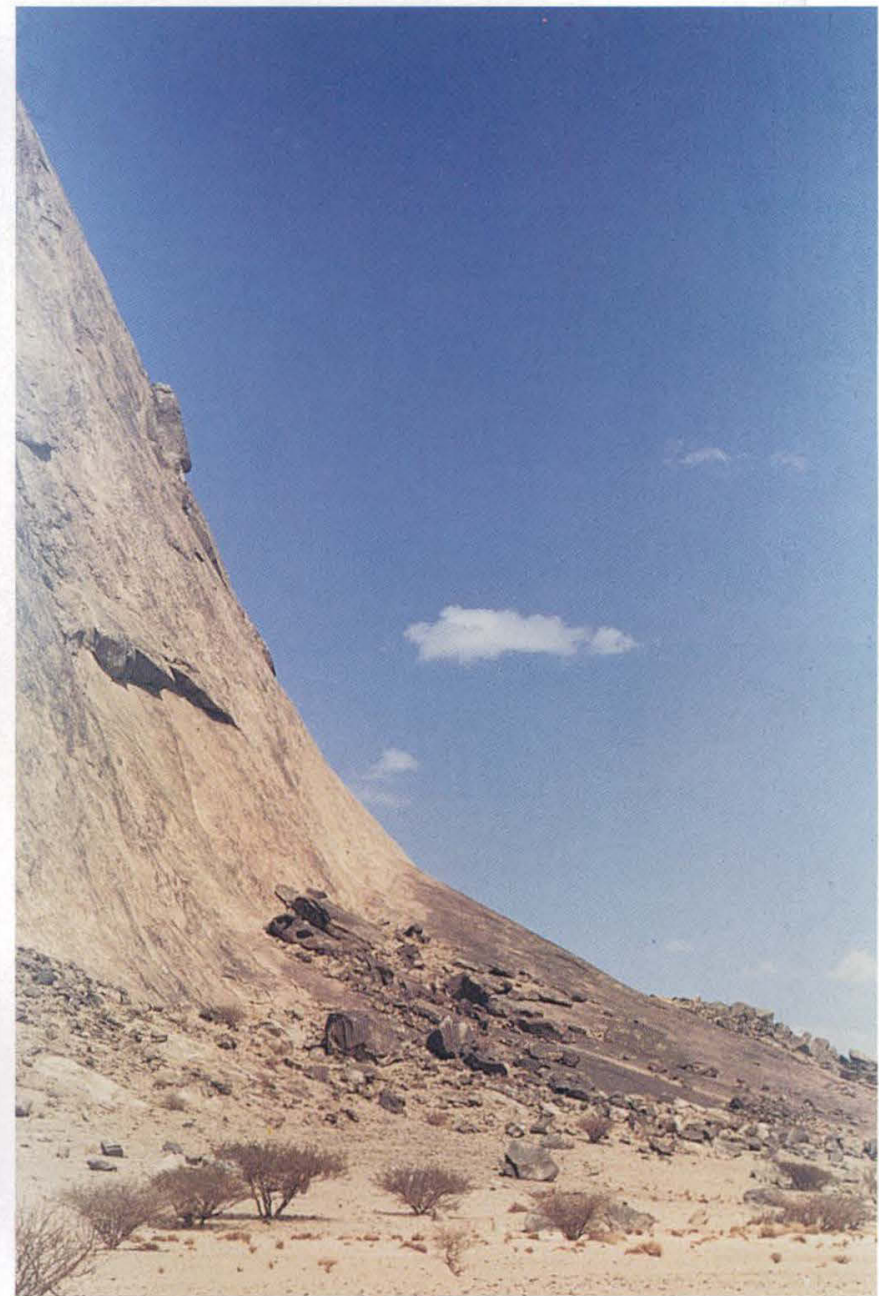

FIGURE 74.-Base of the conical inselberg of Jabal al Gharāmīl at lat $21^{\circ} 51^{\prime} \mathrm{N}$., long $42^{\circ} 54^{\prime} \mathrm{E}$. The jabal wall of posttectonic granite rises at an angle of $65^{\circ}$ from the horizontal; the lower slope rising at $21^{\circ}$ is underlain by contact-metamorphosed sediments of Murdamah age. Nearby the jabal is cut by a Najd fault. scour has removed the residual grains. The wide temperature range, especially when the cooling falls below the dewpoint and below freezing, hastens the disintegration. The differential rate of expansion and contraction of the polymineralic rocks also hastens the process, even where there has been little or no postemplacement tectonism. Thus, the ridges and higher hills are composed mostly of lavas and metasedimentary rocks, the isolated bornhardts being the exception.

From maximum altitudes of $2,100 \mathrm{~m}$, the plains decline to a minimum of $670 \mathrm{~m}$ where Wādi ar Rimah debouches onto the sedimentary Najd at Al Qaşim. The desert floor is not a uniform slope but is broken, hinge fashion, along the northwest-trending Najd fault system into four segments or blocks-the southwest, or Asir, block, two central blocks between the three Najd faults, and a northern, or Shammar, block.

The surface of the Asir block slopes evenly from about $1,400 \mathrm{~m}$ to $1,000 \mathrm{~m}$ in a northeasterly direction to the southernmost Najd fault.

The two central blocks each lie between two flanking Najd faults. Their surfaces are considerably flatter than the Asir block but are tilted somewhat, also to the northeast, and fall from a general altitude of $1,050 \mathrm{~m}$ 
along the northern edge of the southern Najd fault to a low region of sabkhahs (playas) at about $940 \mathrm{~m}$. Thence, they rise to $1,100 \mathrm{~m}$ northeastward toward the middle of the three principal Najd fault zones (USGSARAMCO, 1963). The elevation of the southern edge is dramatically shown at Wādī Subay‘. Ground water pumped up onto the elevated bank of Wādì Subay“ flows by gravity northward $(60 \mathrm{~km})$ to Zalim and to a sabkhah floor; yet the wadi, entrenched in crystalline rocks, flows east and south, to be lost in the 'Irq Subay' in and along the southern flank of the Najd fault. The central region, which falls between the flanking Najd faults, is the lowest part of the Najd pediplain exclusive of the lower reaches of Wādì ar Rimah. It is nearly devoid of drainage lines and is characterized by numerous small, irregular sabkhahs in desert sinks seemingly scoured by the wind. These sabkhahs are nearly devoid of alluvial debris, and their salt crust directly overlies decomposed bedrock.

The northern, or Shammar, block of the crystalline Najd pediplain falls from $1,100 \mathrm{~m}$ along the northernmost Najd fault to $950 \mathrm{~m}$ in the extreme northeast corner, where Wādī Ḩā'il flows northeast onto the sedimentary rocks, and to $670 \mathrm{~m}$ at Ar Rass, where Wādī ar Rimah flows northeast off the crystalline rocks. Along the western part of this crystalline Najd surface, the larger wadis follow the northwest direction of the Najd fault system where large horizontal movements have created schistose shear zones that are readily deflated or, where above grade, are scoured by floods to create thalwegs. Eastern portions drain northeastward into the well-integrated, dendritic, and slightly entrenched drainage system of Wādī ar Rimah.

The increased rainfall shown by the $10-\mathrm{cm}$ isohyetal lines in the Shammar block (fig. 2) is not easily explained from our limited knowledge of precipitation derived from interpretation of vegetation and from the short timespan of measured rainfall. However, satellite images show a strong wind direction from S. $70^{\circ} \mathrm{W}$., parallel to yardang-troughlike grooves in the landscape, a direction confirmed from general sand movement eastward from sand sources on grus plains and wadi floors (figs. 68, 75, 76). The western lava fields have increased in height from repeated eruptions and epeirogenic uplift to general altitudes of $1,300 \mathrm{~m}$ in Harrat Rahat between At Ţā'if and Al Madīnah and to a maximum elevation of $2,000 \mathrm{~m}$ at Abyad wa Ubayyid in the Harrat Khaybar north of Al Madinah. This barrier, together with the permanent low-pressure area generated from the rising thermal off the black lava fields and the southwest wind direction, causes orographic convection and precipitation. Farther north, away from the extensive lava fields, the rainfall de-

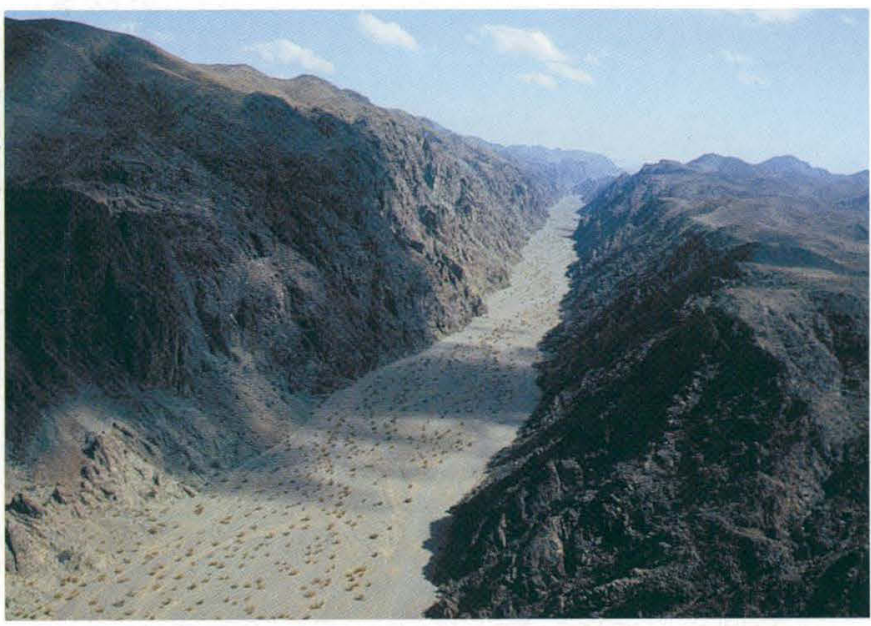

Figure 75.-Wādī Tharīb (Ash Schism) yardang valley (lat $26^{\circ} 30^{\prime}$ N., long $37^{\circ} 20^{\prime}$ E.) cut in metavolcanic-metasedimentary greenstone and parallel to a subsidiary Najd fault. View looking N. $85^{\circ} \mathrm{W}$.

clines to less than $5 \mathrm{~cm}$ until still farther north the westerlies of the Mediterranean climate begin to influence precipitation (fig. 2).

The Najd pediplain exhibits much evidence of the desert cycle of erosion. Besides accumulations of sand dunes and wind-scoured sinks (sabkhahs), windblown sand, moving along the desert surface, cuts channels parallel to wind direction wherever there is an initial low area such as a schist belt, joint, fault, or other lineation. Once a channel starts, the accumulation of rain, or even dew resulting from the wide diurnal temperature range of the desert, tends to chemically weather the thalweg of the groove or channel. Subsequent sandblast, concentrated in the lowest surfaces of the terrain, removes the chemically or frost-loosened debris, which, together with flash floods, deepens the channel, further concentrating the sandblast and creating streamlined valleys or "yardang troughs" (figs. 68, 76). On the Najd pediplain these features form valleys and ridges that are often at large angles to structural lineations in the basement rocks. Where a transverse wadi deposits coarse flood debris in the main stream, the trough ends because sandblast is missing downwind from the streambed. These yardang troughs are most abundant from lat $24^{\circ} \mathrm{N}$. north to the north edge of the shield, particularly east of the wind gaps between Harrat Rahat and Harrat Khaybar and at the north end of Harrat Khaybar, where the yardang troughs are often 10 to $20 \mathrm{~m}$ deep, especially in crystalline rocks (fig. 75). 


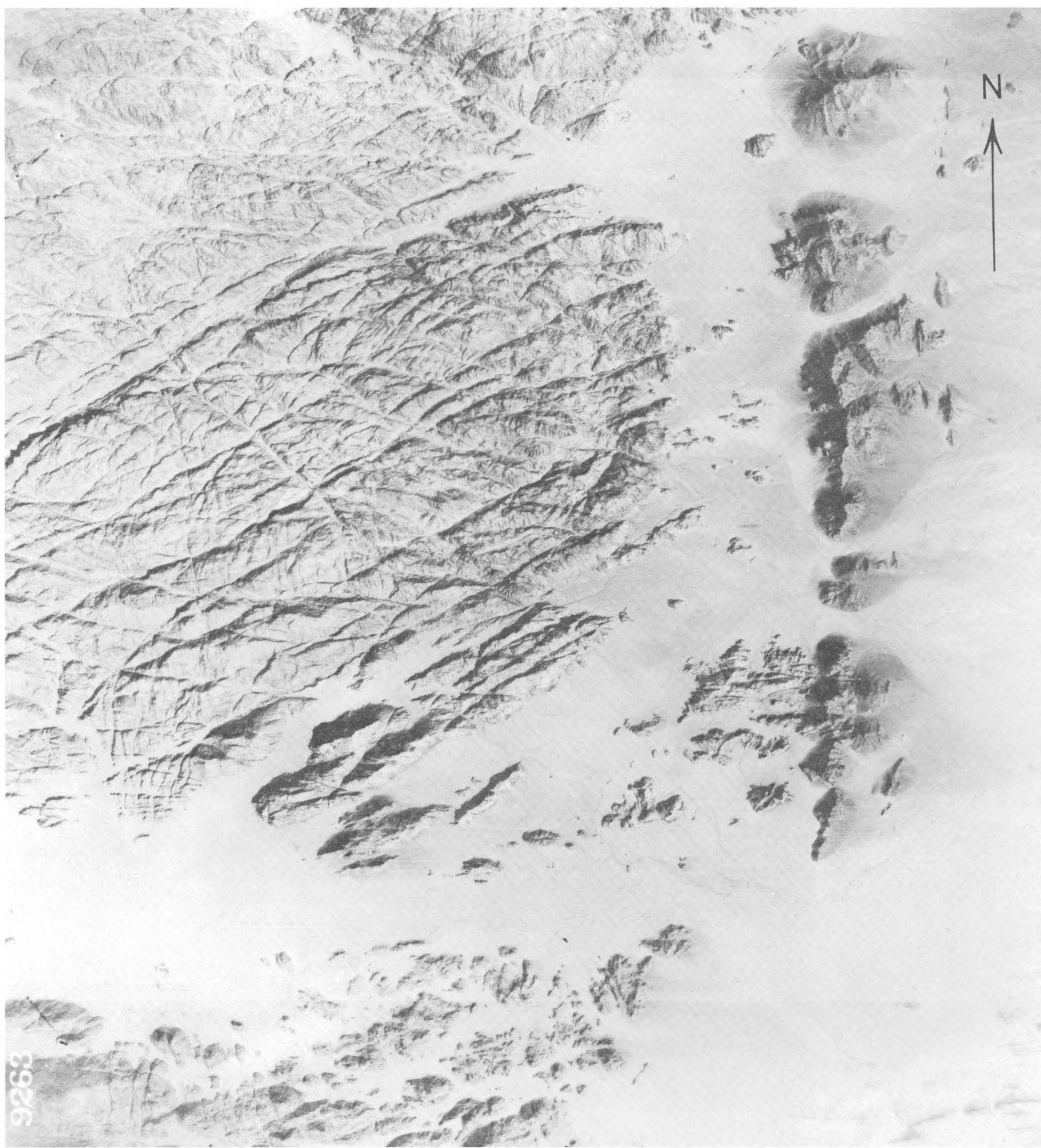

FIgURE 76.-Yardang troughs following one set of joints parallel to the wind direction to N. $70^{\circ}$ E. on southern end of Jabal Salmá. Rhyolite dike on the east side (discontinuous ridge on right) which has been dissected by sandblast is downwind from the major yardang troughs. Jabal Salmá is a complex late and posttectonic pluton where two sets of joint systems are apparent. Jabal Shammar region, northeast corner of the Arabian Shield. Vertical aerial photograph; width of aerial view is about $13 \mathrm{~km}$. 


\section{REFERENCES CITED}

Abul-Haggag, Y., 1961, A contribution to the physiography of northern Ethiopia: London, University of London, Athlone Press, 159 p., 64 figs.

Akaad, M.K., and Noweir, A.M., 1969, Lithostratigraphy of the Hammamat-Um Seleimat district, Eastern Desert, Egypt: Nature, v. 223, no. 5203, p. 284, 285.

1978, Geology and lithostratigraphy of the Arabian desert orogenic belt of Egypt between latitudes $25^{\circ} 35^{\prime}$ and $26^{\circ} 30^{\prime} \mathrm{N}$. [abs.]: Precambrian Research, v. 6, p. A6.

Alabouvette, B., and Pellaton, C., 1975, Geology and minerals exploration of the Wadi Kamal quadrangle, 24/37D: [France] Bureau des Recherches Geologiques et Minieres Technical Record 75-JED-18, 46 p., 1 fig., 3 maps.

Alabouvette, B., and Villemur, J.R., 1973, Reconnaissance of the Wajid sandstone: [France] Bureau des Recherches Geologiques et Minieres Open-File Report 73-JED-3, 22 p., 17 pls., map, app.

Aldrich, L.T., 1956, Measurement of radioactive ages of rocks: Science, v. 123 , p. 871-875.

Aldrich, L.T., Brown, G.F., Hedge, C.E., and Marvin, R.F., 1978, Geochronologic data for the Arabian Shield: U.S. Geological Survey Open-File Report IR (Saudi Arabia)-240, 20 p., 1 fig; also USGS Open-File Report 78-75, $22 \mathrm{p}$.

Aldrich, L.T., Davis, G.L., and James, H.L., 1965, Ages of minerals from metamorphic and igneous rocks near Iron Mountain, Michigan: Journal of Petrology, v. 6, p. 445-472.

Aldrich, L.T., Davis, G.L., Tilton, G.R., and Wetherill, G.W., 1956, Radioactive ages of minerals from the Brown Derby mine and the Quartz Creek granite near Gunnison, Colorado: Journal of Geophysical Research v. 61, p. 215-232.

Al-Rehaili, M.H., and Warden, A.J., 1980, Comparison of the Bir Umq and Hamdah ultrabasic complexes, Saudi Arabia, in Cooray, P.G., and Tahoun, S.A., eds., Evolution and mineralization of the Arabian-Nubian Shield: Oxford, Pergamon Press Ltd., Institute for Applied Geology Bulletin 3, v. 4, p. 143-156.

Al-Shanti, A.M.S., 1966, Oolitic iron ore deposits in Wadi Fatima between Jeddah and Mecca, Saudi Arabia: Saudi Arabian Directorate General of Mineral Resources Bulletin 2, 51 p., 13 pls., 33 figs., 8 tables.

1974, Al Ji'lani layered basin intrusion, Ad Dawādimī district, Kingdom of Saudi Arabia: Saudi Arabian Directorate General of Mineral Resources Bulletin 12, 45 p., 1 pl., 27 figs., 10 tables. 1976, Geology of Ad Dawādimī district, Kingdom of Saudi Arabia: Saudi Arabian Directorate General of Mineral Resources Bulletin 13, scale 1:100,000, 57 p., 33 figs.

Al-Shanti, A.M.S., and Gass, I.G., 1983, The Upper Proterozoic ophiolite melange zones of the easternmost Arabian Shield: Journal of the Geological Society of London, v. 140, p. 867-876, 4 figs., 1 table.

Al-Shanti, A.M.S., and Mitchell, A.H.G., 1976, Late Precambrian subduction and collision in the Al Amar-Idsas region, Arabian Shield, Kingdom of Saudi Arabia: Tectonophysics, v. 30, p. 41-47.

Anderson, R.E., 1977, Geology of Wadi Tarj quadrangle, sheet 19/ 42A, Kingdom of Saudi Arabia: Saudi Arabian Directorate General of Mineral Resources Geologic Map GM-29, scale 1:100,000, with text, 23 p., 7 figs., 1 table.

1979, Geology of the Wadi 'Atf and Mayza quadrangles, Kingdom of Saudi Arabia: Saudi Arabian Directorate General of Mineral Resources Bulletin 25, 33 p., 2 maps, scale 1:100,000, 6 illus., 3 tables.

Andreasen, G.E., and Petty, A.J., 1974, Total intensity aeromagnetic map of the northern Hijaz quadrangle and part of the Wadi As Sirhan quadrangle, Kingdom of Saudi Arabia: Saudi Arabian
Directorate General of Mineral Resources Geologic Map GM-9, scale $1: 500,000$.

Andrews, F.W., 1950, 1952, 1956, The flowering plants of the Sudan, v. 1, 2, 3: London, T. Buncle and Co., Ltd., p. 237, 485, 579.

Anonymous, 1972, Ophiolites, Penrose Field Conference: Geotimes, December, p. 24, 25.

Arkin, Y., Beyth, Michael, Dow, D.B., Levitte, D., Haile, Temesgen, and Hailu, Tsegaye, 1971, Geologic map of Mekele sheet, area ND 37-11 Tigre Province: [Addis Ababa] Imperial Ethiopian Government, Ministry of Mines, Geological Survey of Ethiopia Map ND 37-11, scale 1:250,000.

Arno, V., Bakashwin, M.A., Baker, A.Y., Barberi, F., Basahel, A., Dipaola, G.M., Ferrara, G., Gazzaz, M.A., Giuliani, A., Heikel, M., Marinelli, G., Nassief, A.O., Rosi, M., and Santacroce, R., 1980a, Recent basic volcanism along the Red Sea coast: The Al Birk lava field in Saudi Arabia, in Zanettin, Bruno, Geodynamic evolution of the Afro-Arabian rift system: Rome, Accademia Nazionale dei Lincei, p. 645-654.

$1980 \mathrm{~b}$, Recent volcanism within the Arabian plate, Preliminary data from Harrats Hadan and Nawasif-Al Buqum, in Zanettin, Bruno, Geodynamic evolution of the Afro-Arabian rift system: Rome, Accademia Nazionale dei Lincei, p. 629-643.

Auxiliar Enterprise de Recherches et l'Activities (AUXERAP), 1967: Unpublished report to the Ministry of Petroleum and Mineral Resources, Kingdom of Saudi Arabia, Jiddah.

Baghanem, A.M., 1972, Geology of the lake beds near Turabah, Saudi Arabia: M.S. thesis, South Dakota School of Mines and Technology, Rapid City, S. Dak.

Baghanem, A.M., and Mickelson, J.C., 1972, Geology of the lake beds near Turabah, Saudi Arabia [abs.]: Geological Society of America, Rocky Mountain Section Meeting, Denver, 1972, p. 363.

Baker, P.E., Brosset, R., Gass, I.G., and Neary, C.R., 1973, Jebel al Abyad: A recent alkalic volcanic complex in western Saudi Arabia: Lithos, v. 6 , no. 3 , p. 291-314.

Bakor, A.R., Gass, I.G., and Neary, C.R., 1976, Jabal al Wask, northwest Saudi Arabia: An Ecocambrian back-arc ophiolite: Earth and Planetary Science Letters, v. 30, no. 1, p. 1-9.

Bartov, Y., Steinitz, G., Eyal, M., and Eyal, Y., 1980, Sinistral movement along the Gulf of Aqaba-Its age and the relation to the opening of the Red Sea: Nature, v. 285, p. 220-222.

Basse, Elaine, Karrenberg, Herbert, Lehman, J.P., Alloiteau, James, and LeFranc, J.P., 1954, Fossiles du jurassique superierur et des "gres de Nubie" de la region de Sanaa (Yemen): Society of the Geology of France Bulletin, ser. 6, v. 4, p. 655-687.

Baubron, J.C., Delfour, J., and Vialette, Y., 1976, Geochronological measurements $(\mathrm{Rb} / \mathrm{Sr}$; K/Ar) on rocks of the Arabian Shield, Kingdom of Saudi Arabia: [France] Bureau des Recherches Geologiques et Minieres Open-File Report 76-JED-22, 152 p.

Bayley, R.W., 1972, Geologic map and section of the Wadi Yiba quadrangle, Tihamat ash Sham area [Kingdom of Saudi Arabia]: Saudi Arabian Directorate General of Mineral Resources Geolog. ic Map GM-1, scale 1:100,000.

Bender, Friedrich, 1963, Stratigraphic der "Nubiochen Sandsteine" in Sud-Jordanien: Geologie Jahrb., v. 81, p. 237-276, 11 figs., map. 1965, Zur geologie der kupfererzvorkommen am ostrand des Wadi Araba, Jordan: Geologie Jahrb., ser. B, v. 10, p. 16 1968, Geologic map of Jordan-Aqaba Ma'in sheet, Federal Republic of Germany, Bundesanstalt fur Bodenforschung, map, scale $1: 250,000$.

1974a, Explanatory notes on the geological map of the Wadi Araba, Jordan: Geologic Jahrb. ser. B, v. 19, 62 p., 12 figs., 4 pls. 1974b, Geology of Jordan: Contributions to the regional geology of the Earth: Berlin, Gebruider Borntraeger, supp. ed., v. 7, p. 107. 
1975, Geology of the Arabian Peninsula-Jordan: U.S. Geological Survey Professional Paper 560-I, scale 1:500,000, $36 \mathrm{p}$.

Berkey, C.P., and Morris, F.K., 1927, Geology of Mongolia: American Museum of Natural History, v. 2, p. 56, 58, 146, 201.

Beyth, Michael, 1973, Correlation of Paleozoic-Mesozoic sediments in northern Yemen and Tigre, northern Ethiopia: Bulletin of the American Association of Petroleum Geologists, v. 57, p. 2440-2443.

Bhutta, M.A., 1970, Geology and economic possibilities of the Wadi Hajir-Wadi al Fara area (23/39 D): Saudi Arabian Directorate General of Mineral Resources Report 363, 123 p. 5 figs. 5 pls.

Bigot, M., 1975, Geology and mineral exploration of the Umm Lajj quadrangle, 25/37C: [France] Bureau des Recherches Geologiques et Minieres Open-File Report 75-JED-7, scale 1:100,000 $47 \mathrm{p} ., 2 \mathrm{pls}$.

Bigot, M., and Alabouvette, B., 1976, Geology and mineralization of the Tertiary Red Sea coast of northern Saudi Arabia: [France] Bureau des Recherches Geologiques et Minieres Open-File Report 76-JED-5, 84 p. 7 pls., 11 figs.

Bigot, M., and le Chapelain, J.R., 1973, Geology and mineral exploration of the As Suwaydirah quadrangle, 24/40A: [France] Bureau des Recherches Geologiques et Minieres Open-File Report 73-JED-7, scale 1:100,000, 26 p., 2 pls.

Binda, P.L., 1981, The Precambrian boundary in the Arabian Shield: A review: Jiddah, King Abdul Aziz University, Faculty of Earth Science Bulletin 4, p. 107-120.

Binda, P.L., and Bokhari, M.M., 1980, Chitinozoan-like microfossils in a late Precambrian dolostone from Saudi Arabia: Geology, v. 8, p. $70,71$.

Binda, P.L., and Ramsay, C.R., 1980, Earliest Phanerozoic or latest Proterozoic fossils from the Arabian Shield: Precambrian Research, v. 13 , p. $375-377$.

Black, R., Morton, W.H., and Rex, D.C., 1975, Block tilting and volcanism within the Afar in the light of recent $\mathrm{K} / \mathrm{Ar}$ age data, in Pilger, A., and Rosler, A., eds., Afar depression of Ethiopia: International Symposium on the Afar Region and Related Rift Problems, Bad Bergzabern, 1974, Proceedings, v. 1: Stuttgart, E Schweizerbartsche Verlagsbuchhandlung (Nagele u. Obermiller), p. $296-300$.

Blank, H.R., Jr., 1977, Aeromagnetic and geologic study of Tertiary dikes and related structures on the Arabian margin of the Red Sea, in Hilpert, L.S., ed., Red Sea research 1970-1975: Saudi Arabian Directorate General of Mineral Resources Bulletin 22, p. G1-G18.

Blank, H.R., Jr., and Gettings, M.E., 1985, Geology of the Jizan quadrangle, sheet 16/42B, Kingdom of Saudi Arabia: U.S. Geological Survey Open-File Report 85-0724.

Blank, H.R., Jr., Gettings, M.E., and Kellogg, K.S., 1981, Linear magnetic anomalies onshore and offshore in southwest Saudi Arabia [abs.]: EOS, Transactions of the American Geophysical Union, v. 62 , no. 17, p. 407.

Blank, H.R., Jr., Gettings, M.E., Petty, A.J., and Andreasen, G.E., 1980, Total-intensity aeromagnetic map of the Precambrian Arabian Shield, Kingdom of Saudi Arabia: Saudi Arabian Deputy Ministry for Mineral Resources Geoscience map, Technical Record 6, SA-(IR)-344, scale 1:2,000,000. (in color)

Blodget, H.W., and Brown, G.F., 1982, Geological mapping by use of computer-enhanced imagery in western Saudi Arabia: U.S. Geological Survey Professional Paper 1153, 10 p., 2 pls.

Bodenlos, A.J., and Lari, Ahman, 1970, Possibilities of sulphur mineralization in Saudi Arabia: U.S. Geological Survey Open-File Report (IR)SA-113, 29 p., 2 figs.

Bogue, R.G., 1953, Report on geologic reconnaissance in northwestern Saudi Arabia: Saudi Arabian Directorate General of Mineral Resources Open-File Report 27, $30 \mathrm{p}$.
1954, Reconnaissance of mineral deposits in a part of western Saudi Arabia: Saudi Arabian Directorate General of Mineral Resources Open-File Report 31, 66 p.

Bois, J., 1971, Geology and mineral exploration of the northwestern part of the Jabal Damkh quadrangle (sheet 23/44 A): [France] Bureau des Recherches Geologiques et Minieres Open-File Report 71-JED-2, 27 p., 3 app., scale 1:100,000.

Bonatti, Enrico, Emiliani, Cesare, Ostlund, Gote, and others, 1971, Final desiccation of the Afar rift, Ethiopia: Science (AAAS), v. 172 , no. 3982 , p. 468,469 , sketch map.

Bounny, I., 1975, Geology and mineral exploration of the Wadi Dwayrah area, Kingdom of Saudi Arabia: [France] Bureau des Recherches Geologiques et Minieres Open-File Report 75-JED-25, 72 p., 6 pls., 2 figs.

Boureau, Edourad, 1956, Contribution a l'etude de flores Jurassiques d'Asie, II, Sur des coniferes noveaux d'Arabie Seoudite: Societe Geologique de France Bulletin 6, p. 653-657, 2 figs.

Bowen, R.W., 1971, Graphic normative analysis program: U.S. Geological Survey Computer Contribution 13, 80 p., 2 tables.

Bowler, J.M., 1976, Aridity in Australia: Age, origins, and expressions in aeolian land forms and sediments: Earth-Science Review, v. 12, p. $279-310$

Bramkamp, R.A., Brown, G.F., Holm, D.A., and Layne, N.M., Jr., 1963, Geologic map of the Wadi as Sirhan quadrangle, Kingdom of Saudi Arabia: U.S. Geological Survey Miscellaneous Geologic Investigations Map I-200A, scale 1:500,000 [1964].

Bramkamp, R.A., Gierhart, R.D., Owens, L.D., and Ramirez, L.F., 1963, Geologic map of the western Rub al Khali quadrangle, Kingdom of Saudi Arabia: U.S. Geological Survey Miscellaneous Geologic Investigations Map I-218A, scale 1:500,000 [1964]

Bramkamp, R.A., Ramirez, L.F., Brown, G.F., and Pocock, A.E., 1963, Geologic map of the Wadi ar Rimah quadrangle, Kingdom of Saudi Arabia: U.S. Geological Survey Miscellaneous Geologic Investigations Map I-206A, scale 1:500,000.

Breed, C.S., Fryberger, S.G., Andrews, Sarah, McCanley, Carmilla, Lennartz, Frances, Gebel, Dana, and Horstman, Kevin, 1979, Regional studies of sand seas, using Landsat (ERTS) imagery, in McKee, E.D., ed., A study of global sand seas: U.S. Geological Survey Professional Paper 1052, p. 305-397 [1980]

Brown, G.F., 1948, Geology and groundwater of Al Kharj district, Nejd, Saudi Arabia: Ph.D. thesis, Northwestern University, Evanston, Ill., 128 p., 43 pls., 1 fig.

1960, Geomorphology of western and central Saudi Arabia: International Geological Congress, 21st, Copenhagen, 1960, Proceedings, pt. 21, p. 150-159.

1970, Eastern margin of the Red Sea and the coastal structures in Saudi Arabia: Philosophical Transactions of the Royal Society of London, A-267, p. 75-87.

1972, Tectonic map of the Arabian Peninsula: Saudi Arabian Directorate General of Mineral Resources, Arabian Peninsula Map AP-2, scale 1:4,000,000.

Brown, G.F., Delfour, Jacques, and Coleman, R.G., 1972, Geologic formations in the Arabian Shield [summary]: International Geological Congress, 24th, Montreal, 1972, Proceedings, sec. 1, p. 333.

Brown, G.F., and Hase, D.H., 1971, Explanatory note of the tectonics of the Pre-Cambrian Arabian segment of the African Shield: Tectonics of Africa, UNESCO sciences de la terre, v. 6, p. 429, 430.

Brown, G.F., and Jackson, R.O., 1958, Geology of the Tihamat ash Sham quadrangle, Kingdom of Saudi Arabia: U.S. Geological Survey Miscellaneous Geologic Investigations Map I-216A, scale $1: 500,000$ [1959]. 
1959, Geology of the Asir quadrangle, Kingdom of Saudi Arabia: U.S. Geological Survey Miscellaneous Geologic Investigations Map I-217A, scale 1:500,000.

1960, The Arabian Shield: International Geological Congress, 21st, Copenhagen, 1960, Proceedings, pt. 9, p. 69-77.

1979, An overview of the geology of western Arabia, in Tahoun, S.A., ed., Evolution and mineralization of the ArabianNubian Shield: King Abdulaziz University, Institute of Applied Geology Bulletin 3 (Oxford-New York, Pergamon Press Ltd.), v. 1 , p. 3-10.

Brown, G.F., Jackson, R.O., Bogue, R.G., and Elberg, E.L., Jr., 1963, Geologic map of the northwestern Hijaz quadrangle, Kingdom of Saudi Arabia: U.S. Geological Survey Miscellaneous Geologic Investigations Map I-204A, scale 1:500,000.

Brown, G.F., Jackson, R.O., Bogue, R.G., and MacLean, W.H., 1963, Geologic map of the southern Hijaz quadrangle, Kingdom of Saudi Arabia: U.S. Geological Survey Miscellaneous Geologic Investigations Map I-210A, scale 1:500,000.

Bureau des Recherches Geologiques et Minieres (BRGM), 1966, Annual report, February 1965 to January 1966: [France] Bureau des Recherches Geologiques et Minieres Open-File Report SG-JED 66-A-7.

Burton, R.F., 1878, The gold mines of Midian and the ruined Midianite cities: London, C. Kegan Paul and Co., 395 p.

Calvez, J.Y., Alsac, C., Delfour, J., Kemp, J., and Pellaton, C., 1983, Geologic evolution of western, central and eastern parts of the northern Precambrian Shield, Kingdom of Saudi Arabia: Saudi Arabian Deputy Ministry for Mineral Resources Open-File Report BRGM-OF-03-17, 57 p., 1 app., geologic sketch map, scale $1: 1,000,000$.

Cameron, D.K., Jr., 1974, New Triassic palynomorphs from the Arabian Peninsula: Grana, v. 14, p. 4-10, 2 figs., 1 pl.

Chessex, R., Delaloye, M., Muller, J., and Weidmann, M., 1975, Evolution of the volcanic region of Ali Sabieh (T.F.A.I.), in the light of K-Ar age determinations, in Pilger, A., and Rosler, A., eds., Afar depression of Ethiopia: International Symposium on the Afar Region and Related Rift Problems, Bad Bergzabern, 1974, Proceedings, v. 1: Stuttgart, E. Schweizerbartsche Verlagsbuchhandlung (Nagele u. Obermiller), p. 221-227.

Church, B.N., 1975, Quantitative classification and chemical comparison of common volcanic rocks: Geological Society of America Bulletin, v. 86 , no. 2, p. 257-263.

Civetta, Lucia, La Volpe, Luigi, and Liere, Lucio, 1978, K-Ar ages of the Yemen Plateau: Journal of Volcanology and Geothermal Research, v. 4, p. 307-314.

Cloud, Preston, Awramik, S.M., Morrison, Karen, and Hadley, D.G., 1979, Earliest Phanerozoic or latest Proterozoic fossils from the Arabian Shield: Precambrian Research, v. 10 p. 73-93.

Coleman, R.G., Fleck, R.J., Hedge, C.E., and Ghent, E.D., 1977, The volcanic rocks of southwest Saudi Arabia and the opening of the Red Sea, in Hilpert, L.S., ed., Red Sea research, 1970-1975: Saudi Arabian Directorate General of Mineral Resources Bulletin 22, p. D1-D30

Coleman, R.G., Ghent, E.D., Fleck, R.J., and Griscom, Andrew, 1977, Jabal Shāyi gabbro in southwest Saudi Arabia: Saudi Arabian Deputy General for Mineral Resources Bulletin 17, 46 p., 1 pl., 32 figs., 16 tables.

Coleman, R.G., Gregory, R.T., and Brown, G.F., 1983, Cenozoic volcanic rocks of Saudi Arabia: U.S. Geological Survey Open-File Report OF-03-93, 86 p., 23 figs., 6 tables.

Coleman, R.G., Hadley, D.G., Fleck, R.G., Hedge, C.T., and Donato, M.M., 1979, The Miocene Tihama Asir ophiolite and its bearing on the opening of the Red Sea, in Tahoun, S.A., ed., Evolution and mineralization of the Arabian-Nubian Shield: Institute for Ap- plied Geology Bulletin 3, Oxford, Pergamon Press Ltd., v. 1, p. 173-186.

Cooper, J.A., Stacey, J.S., Stoeser, D.C., and Fleck, R.J., 1979, An evaluation of the zircon method of isotopic dating in the southern Arabian craton: Contributions to Mineralogy and Petrology, v. 68 , p. $429-439$.

Cotton, C.A., 1942, Climatic accidents in landscape-making: New York, John Wiley and Sons, p. 3-47.

Cox, K.G., Bell, J.D., and Parkhurst, R.J., 1979, The interpretation of igneous rocks: London, George Allen Unwin, p. 14.

Cox, L.R., 1929, Notes on the post-Miocene Ostreidae and Pectinidae of the Red Sea region, with remarks on the geological signifi-. cance of their distribution: Malacologia, Proceedings, v. 18, p. 165-185.

Dalrymple, G.B., Gromme, C.S., and White, R.W., 1975, Potassiumargon age and paleomagnetism of diabase dikes in Liberia: Initiation of central Atlantic rifting: Geological Society of America Bulletin, v. 86 , no. 3 , p. 399-411.

Dalrymple, G.B., and Lanphere, M.A., 1969, Potassium-argon dating: San Francisco, W.H. Freeman and Co., p. 172, 173.

Daniel, E.J., 1963, International lexicon of stratigraphy for Jordan: Centre National de la Recherche Scientifique International Geologic Congress, Commission de Stratigraphie, p. 360-369, and addendum, Bender, Friedrich, Litho-stratigraphic and time-stratigraphic subdivision of the Nubian sandstones in S. Jordan, p. 403-419.

Davies, F.B., 1980, Reconnaissance geology of the Duba quadrangle: Saudi Arabian Directorate of Mineral Resources Geologic Map GM-57, scale 1:100,000.

Davis, W.M., 1905, The geographical cycle in an arid climate: Journal of Geology, v. 13, p. 381-407.

1933, Geomorphology of mountainous deserts: International Geological Congress, 16th, Washington, D.C., Proceedings, v. 2, p. $703-714$.

Delfour, Jacques, 1967, Report on the mineral resources and geology of the Hulayfah-Musayna'ah region (sheet 78, zone 1 north): [France] Bureau des Recherches Geologiques et Minieres OpenFile Report JED-66, 138 p., scale 1:100,000.

1970, Le groupe de j'balah une nouvelle unite du bouclier Arabe: [France] Bureau des Recherches Geologiques et Minieres Bulletin, ser. 2, sec. 4, no. 4, p. 19-32, 5 figs., 2 tables; see also The J'Balah Group, A new unit of the Arabian Shield: [France] Bureau des Recherches Geologiques et Minieres Open-File Report 70-JED-4, 31 p., 5 figs.

1975, Vulcanism and mineral deposits of the Arabian-Nubian shield: [France] Bureau des Recherches Geologiques et Minieres Open-File Report 75-JED-24, 35 p., 6 illus., 2 tables.

1977, Geology of the Nuqrah quadrangle, sheet 25E, Kingdom of Saudi Arabia: Saudi Arabian Directorate General of Mineral Resources Geologic Map GM-28, scale 1:250,000 with text, 32 p., 2 figs.

1979a, Geologic map of the Halaban quadrangle, Kingdom of Saudi Arabia: Saudi Arabian Directorate General of Mineral Resources Geologic Map GM-46 A, scale 1:250,000, with text, 32 p., 22 figs.

$1979 \mathrm{~b}$, Upper Proterozoic volcanic activity in the northern Arabian Shield, Kingdom of Saudi Arabia, in Tahoun, S.A., ed., Evolution and mineralization of the Arabian-Nubian Shield: Institute for Applied Geology Bulletin 3, Oxford, Pergamon Press Ltd., v. 2, p. 59-75.

1981, Geologic map of the Al Hissu quadrangle, sheet $24 \mathrm{E}$, Kingdom of Saudi Arabia: Saudi Arabian Directorate General of Mineral Resources Map 58C, scale 1:250,000, with text, 47 p., 21 figs. 
Delfour, Jacques, and Dhellemmes, R., 1980, Geologic map of the Khaybar quadrangle, sheet 25D, Kingdom of Saudi Arabia: Saudi Arabian Directorate General of Mineral Resources Geologic Map GM-50-A, scale 1:250,000, with text, 24 p., 5 figs., 5 tables.

Delfour, Jacques, Dhellemmes, Robert, Elgass, Philippe, Vaslet, Denis, Brosse, Jean-Michel, Le Nindre, Yves-Michel, and Dottin, Olivier, 1982, Geologic map of the ad Dawadimi quadrangle, sheet $24 \mathrm{G}$, Kingdom of Saudi Arabia: Saudi Arabian Deputy Ministry for Mineral Resources Geologic Map GM-60-A, scale 1:250,000, with text, 36 p., 21 figs.

De Martonne, E., 1926, Geographie physique, v. 2: Paris, Payot, p. 965-967.

Deuser, W.G., Ross, E.H., and Waterman, L.S., 1976, Glacial and pluvial periods: Their relationship revealed by Pleistocene sediments of the Red Sea and Gulf of Aden: Science, v. 191, p. $1168-1170$.

Dodge, F.C.W., 1979, The Uyaijah ring structure, Kingdom of Saudi Arabia: U.S. Geological Survey Professional Paper 774-E, p. E1-E17.

Dodge, F.C.W., Fleck, R.J., Hadley, D.G., and Millard, H.T., Jr., 1979, Geochemistry and ${ }^{87} \mathrm{Sr} /{ }^{86} \mathrm{Sr}$ ratios of Halaban rocks of the Central Arabian Shield, in Tahoun, S.A., ed., Evolution and mineralization of the Arabian-Nubian Shield: Institute for Applied Geology Bulletin 3 (Oxford, Pergamon Press Ltd.), v. 2, p. 153-163.

Doughty, C.M., 1888, Travels in Arabia deserta: (2 v.): Cambridge, Cambridge University Press. Reprinted 1920, 1921, New York, Random House; v. 1, 621 p., v. 2, 690 p., map.

Dow, D.B., Beyth, Michael, and Hailu, Tsegaye, 1971, Paleozoic glacial rocks recently discovered in northern Ethiopia: Geologic Magazine, v. 108 , no. 1 , p. 53-60.

Dubay, Lavesloo, 1969, Water development surveys, geological investigations: Unpublished report, Italconsult, for Ministry of Agriculture and Water, Kingdom of Saudi Arabia.

Durozoy, G., 1972, Hydrogeologie des basaltes du Harrat Rahat: [France] Bureau des Recherches Geologiques et Minieres Bulletin, ser. 2 , sec. 3 , no. 2 , p. 37-50, 6 figs., 2 tables.

Duyverman, H.J., Harris, N.B.W., and Hawkesworth, C.J., 1982, Crustal accretion in the Pan African: $\mathrm{Nd}$ and $\mathrm{Sr}$ isotope evidence from the Arabian Shield: Earth and Planetary Science Letters, v. 59 , p. 315-326.

Eijkelboom, Garet, 1966, The mineral resources and geology of the Jibal Damkh-Arwah region (sheet 115, zone II): [France] Bureau des Recherches Geologiques et Minieres Open-File Report 66-A-13, 18 p., 1 fig., 9 app., scale 1:100,000.

1969, BRGM mineral exploration and geological mapping, in Mineral resources research 1967-68: Saudi Arabian Directorate General of Mineral Resources, p. 81-86.

Von Engeln, O.D., 1942, Geomorphology: New York, Macmillan Co., p. $399-438$.

Eyal, M., Eyal, Y., Bartov, Yosi, and Steinitz, G., 1981, The tectonic development of the western margin of the Gulf of Elat (Aqaba) Rift: Tectonophysics, v. 80 , p. 39-66.

Fairbridge, R.W., 1977, Global climate change during the 13,500 b.p. Gothenburg geomagnetic excursion: Nature, v. 265, February 1977 , p. 340, 341, 1 fig.

Fairer, G.M., 1982, Reconnaissance geology of the Sabya quadrangle, sheet 17/42 D, Kingdom of Saudi Arabia: Saudi Arabian Deputy Ministry for Mineral Resources: Geoscience Map GM-68, 26 p., scale 1:100,000, with text, $12 \mathrm{p}$.

1982, Reconnaissance geology of the Ad Darb quadrangle, sheet 17/42 A, Kingdom of Saudi Arabia: Saudi Arabian Deputy Ministry for Mineral Resources map series, USGS Open-File Report OF-02-87, scale 1:100,000.
Faure, Gunter, and Powell, J.L., 1972, Strontium isotope geology: Berlin, Springer-Verlag, $188 \mathrm{p}$.

Finlay, and Marwick, 1937, New Zealand Geological Survey Paleontology Bulletin 15 , p. 41 , figs. 4,5 , pl. 5 .

Fleck, R.J., 1985, Age of diorite-granodiorite gneisses of the JiddahMakkah region, Kingdom of Saudi Arabia: Saudi Arabian Deputy Ministry for Mineral Resources Professional Paper PP-2, p. 21-27.

Fleck, R.J., Coleman, R.G., Cornwall, H.R., Greenwood, W.R., Hadley, D.G., Schmidt, D.L., Prinz, W.C., and Ratte, J.C., 1976, Geochronology of the Arabian Shield, western Saudi Arabia, KAr results: Geological Society of America Bulletin, v. 87, p. 9-21.

Fleck, R.J., Greenwood, W.R., Hadley, D.G., Anderson, R.E., and Schmidt, D.L., 1980, Rubidium-strontium geochronology and plate-tectonic evolution of the southern part of the Arabian Shield: U.S. Geological Survey Professional Paper 1131, 38 p.

Fleck, R.J., and Hadley, D.G., 1985, Ages and strontium initial ratios of plutonic rock in a transect of the Arabian Shield: U.S. Geological Survey Open-File Report 85-0727, 43 p., 19 figs.

Frakes, L.A., Kemp, E.M., and Crowell, J.C., 1975, Late Paleozoic glaciation: Part 6, Asia: Geological Society of America Bulletin, v. 86, p. $454-464$

Freund, R., Garfunkel, Zri, Zak, I., Goldberg, M., Weissbrod, T., and Derin, B., 1970, The shear along the Dead Sea rift: Philosophical Transactions of the Royal Society of London, v. 267A, p. 107-130.

Friedman, G.M., 1965, A fossil shoreline reef in the Gulf of Elat (Aqaba): Israel Journal of Earth Science, v. 14, p. 86-90.

Frisch, W., and Al-Shanti, A.M.S., 1977, Ophiolite belts and the collision of island arcs in the Arabian Shield: Tectonophysics, v. 43 , p. $292-306$.

von Gaertner, H.R., and Schurenburg, H., 1954, Final report, Geological research in western Arabia: Hannover, Amt fur Bodenforschung, $103 \mathrm{p}$.

Gass, I.G., 1970, The evolution of volcanism in the junction area of the Red Sea, Gulf of Aden and Ethiopian rifts: Philosophical Transactions of the Royal Society of London, v. 267A, p. 369-381.

Gettings, M.E., 1977, Delineation of the continental margin in the southern Red Sea region from new gravity evidence, in Hilpert, L.S. ed., Red Sea research 1970-1975: Saudi Arabian Directorate General of Mineral Resources Bulletin 22, p. K1-K11.

1979, Preliminary results of the Sabkhah Hazawza gravity project, Wadi as Sirhan area, Kingdom of Saudi Arabia: U.S. Geological Survey Open-File Report 79-1660, 12 p., 4 figs.

1982, Heat-flow measurements at shot points along the 1978 Saudi Arabia seismic deep-refraction line, Part 2: Discussion and interpretation: U.S. Geological Survey Open-File Report 82-0794, $43 \mathrm{p}$.

Geukens, F.P.M., 1960, Contribution a la geologie du Yemen: Institut Géologique de L'Université de Louvain, Memoires, v. 21, p. 122-179, 8 pls., geol. map.

1966, Geology of the Arabian Peninsula, Yemen: U.S. Geological Survey Professional Paper 560-B., 23 p., 17 figs.

Ghent, E.D., Coleman, R.G., and Hadley, D.G., 1980, Ultramafic inclusions and host alkali olivine basalts of the southern coastal plain of the Red Sea, Saudi Arabia: American Journal of Science, v. $280 \mathrm{~A}$, p. $499-527$.

Gillmann, Michel, 1968, Primary results of a geological and geophysical reconnaissance of the Jizan coastal plain in Saudi Arabia: American Institute of Mining and Metallurgical Engineers Regional Technical Symposium, 2d, Dhahran, Saudi Arabia, 1968, Report, p. 159-209.

Girdler, R.W., and Styles, P., 1974, Two stage Red Sea floor spreading: Nature, v. 247, p. 7-11.

Goldberg, M., 1963, The geology of Tiran Island: Proceedings of the Israel Geological Society, v. 12 , p. 83,84 . 
Goldberg, M., and Yaron, F., 1978, The $\mathrm{Th}^{230} / \mathrm{U}^{234}$ ages of raised Pleistocene marine terraces on the Island of Tiran and the southeastern coast of Sinai with some tectonic implications: International Congress on Sedimentology, 10th, Jerusalem, July 9-14, 1978, Abstracts, v. 1, p. 258, 259.

Goldsmith, Richard, 1966, Section of the Fatimah Formation near Bahrah, Saudi Arabia: Saudi Arabian Directorate General of Mineral Resources-U.S. Geological Survey Open-File Report (IR)SA-65, 6 p., 1 fig.

Gonzales, Louis, 1973, Geologic map and sections of the Jabal 'In quadrangle: Saudi Arabian Directorate General of Mineral Resources Geologic Map GM-2, scale 1:100,000, with text, $7 \mathrm{p}$.

Greene, R.C., 1983, Reconnaissance geology of the Thaniyah quadrangle, sheet 20/42C, Kingdom of Saudi Arabia: U.S. Geological Survey Open-File Report 83-0448, 25 p., scale 1:100,000.

Greenwood, W.R., 1975a, Geology of the Al 'Aqiq quadrangle, sheet 20/41D: Saudi Arabian Directorate General of Mineral Resources Geologic Map GM-23, scale 1:100,000.

$1975 \mathrm{~b}$, Geology of the Biljurshi quadrangle, sheet 19/41B, with a section on Geophysical investigations by G.E. Andreasen and a section on Geochemical investigations and mineral resources by V.A. Trent and T.H. Kiilsgaard: Saudi Arabian Directorate General of Mineral Resources Geologic Map GM-25, scale $1: 100,000$.

$1975 c$, Geology of the Jabal Ibrahim quadrangle, sheet 20/41C, Kingdom of Saudi Arabia, with a section on Economic geology by R.G. Worl and W.R. Greenwood: Saudi Arabian Directorate General of Mineral Resources Geologic Map GM-22 with text, 18 p., scale $1: 100,000$.

1975d, Reconnaissance geology of the Jabal Shada quadrangle, sheet 19/41A, Kingdom of Saudi Arabia: Saudi Arabian Directorate General of Mineral Resources Geologic Map GM-20, scale 1:100,000.

1979, Geology of the An Nimas quadrangle, sheet 19/42C, Kingdom of Saudi Arabia: Saudi Arabian Directorate General of Mineral Resources Geologic Map GM-37, scale 1:100,000.

Greenwood, W.R., Anderson, R.E., Fleck, R.J., and Roberts, R.J., 1980, Precambrian geologic history and plate tectonic evolution of the Arabian Shield: Saudi Arabian Directorate General of Mineral Resources Bulletin 24, $35 \mathrm{p}$.

Greenwood, W.R., and Brown, G.F., 1973, Petrology and chemical analyses of selected plutonic rocks from the Arabian Shield, Kingdom of Saudi Arabia: Saudi Arabian Directorate General of Mineral Resources Bulletin 9, 9 p.

Greenwood, W.R., Hadley, D.G., Anderson, R.E., Fleck, R.J., and Schmidt, D.L., 1976, Late Proterozoic cratonization in southwestern Saudi Arabia: Philosophical Transactions of the Royal Society of London, A. v. 280 , p. 517-527.

Gregory, R.T., Coleman, R.G., and Brown, G.F., 1982, Cenozoic volcanic rocks of Saudi Arabia: Evidence from the continent for a two-stage opening of the Red Sea: Geological Society of America Abstracts, v. 14 , no. 7, p. 502 .

Grolier, M.J., and Overstreet, W.C., 1978, Geologic map of the Yemen Arab Republic (San'â'): U.S. Geological Survey Miscellaneous Geologic Investigations Map I-1143-B, scale 1:500,000.

Guilcher, Andre, 1952, Formes et processus d'erosion sur les recifs coralliens du nord du bac Farasan (Mer Rouge) [Erosion forms and processes on coral reefs of the northern part of the Farasan bank]: Revue de Geomorphologie Dynamique, An. 3, no. 6, p. 261-274 (French and English summaries).

Gvirtzman, Gdaliahu, Buchbinder, Binyamin, Sneh, Amihai, Nir, Yaacov, and Friedman, G.M., 1977, Morphology of the Red Sea fringing reefs: A result of the erosional pattern of the last-glacial low-stand sea level and the following Holocene recolonization:
Memoirs du Bureau des Recherches Geologiques et Minieres (France), no. 89, 12 p.

Hadley, D.G., 1973, Geology of the Sahl al Matran quadrangle, Northwestern Hijaz, Kingdom of Saudi Arabia: Saudi Arabian Directorate General of Mineral Resources Geologic Map GM-6, scale $1: 100,000$, with text, $11 \mathrm{p}$

1974, The taphrogeosynclinal Jubaylah group in the Mashhad area, Northwestern Hijaz, Kingdom of Saudi Arabia: Saudi Arabian Directorate General of Mineral Resources Bulletin 10, 18 p., 19 figs., 2 tables.

1975a, Geology of the Al Qunfudhah quadrangle, sheet 19/ 41C, Kingdom: of Saudi Arabia: Saudi Arabian Directorate General of Mineral Resources Geologic Map GM-19, scale 1:100,000, with text, $11 \mathrm{p}$.

1975b, Geology of the Qal'at as Sawrah quadrangle, sheet 26/ 38D: Kingdom, of Saudi Arabia: Saudi Arabian Directorate General of Mineral Resources Geologic Map GM-24, scale 1:100,000, with text, $28 \mathrm{p}$.

1975 c, Geology of the Wadi Hali quadrangle, sheet 18/41B, Kingdom of Saudi Arabia: Saudi Arabian Directorate General of Mineral Resources Geologic Map GM-21, scale 1:100,000, with text, $19 \mathrm{p}$.

1976, Geology of the Bi'r Juqjuq quadrangle, sheet 21/43D, Kingdom of Saudi Arabia: Saudi Arabian Directorate General of Mineral Resources Geologic Map GM-26, scale 1:100,000, with text, $30 \mathrm{p}$.

1979, Reconnaissance geology of the Musaylim quadrangle, sheet 19/40B, Kingdom of Saudi Arabia: Saudi Arabian Directorate General of Mineral Resources Geologic Map GM-34, scale 1:100,000, with text, $7 \mathrm{p}$.

1982, Reconnaissance geology of the Manjamah quadrangle, sheet 18/41A, Kingdom of Saudi Arabia: U.S. Geological Survey Open-File Report 82-0285, 13 p., scale 1:100,000.

Hadley, D.G., and Fleck, R.J., 1980a, Reconnaissance geologic map of the Al Lith quadrangle, sheet $20 / 40 \mathrm{C}$, Kingdom of Saudi Arabia: Saudi Arabian Directorate General of Mineral Resources Geologic Map GM-32, scale 1:100,000, with text, 10 p.

$1980 \mathrm{~b}$, Reconnaissance geologic map of the Jabal Afaf quadrangle, sheet 20/40D, Kingdom of Saudi Arabia: Saudi Arabian Directorate General of Mineral Resources Geologic Map GM-33, scale 1:100,000; with text, $10 \mathrm{p}$.

Hall, S.A., 1980, A total intensity magnetic anomaly map of the Red Sea and its interpretation: U.S. Geological Survey Open-File Report 80-0131, $271 \mathrm{p}$.

Hall, S.A., Andreasen, G.E., and Girdler, R.W., 1977, Total-intensity magnetic anomaly map of the Red Sea and adjacent coastal areas: A description and preliminary interpretation, in Red Sea research 1970-1975: Saudi Arabian Directorate General of Mineral Resources Bulletin 22, p. F1-F15.

Halpern, M., 1980, Rb-Sr "Pan-African" isochron ages of Sinai/ igneous rocks: Geology, v. 8, p. 48-50.

Hantzschel, Walter, 1975, Trace fossils and problematics: Geological Society of America, Treatise on Invertebrate Paleontology, pt. W., misc., supp. 1, p. W38, W39.

Hashad, A.H., 1980, Present status of geochronological data on the Egyptian basement complex, in Cooray, P.G., and Tahoun, S.A., eds., Evolution and mineralization of the Arabian-Nubian Shield: Institute for Applied Geology Bulletin 3, Oxford, Pergamon Press Ltd., v. 3, p. 31-46.

Hatcher, R.D., Jr., Zietz, Isidore, Regan, R.D., and Abu-Ajamieh, Muhammad, 1981, Sinistral strike-slip motion on the Dead Sea rift: Confirmation from new magnetic data: Geology, v. 9, p. 458-462.

Healy, J.H., Mooney, W.D., Blank, H.R., Gettings, M.E., Kohler, W.M., Lamson, R.J., and Leone, L.E., 1983, Saudi Arabian 
seismic deep-refraction profile: Final project report: Saudi Arabian Deputy Ministry for Mineral Resources Open-File Report USGS-0F-02-37; also U.S. Geological Survey Open-File Report 83-390, 429 p.

Hedge, C.E., 1984, Precambrian geochronology of part of northwestern Saudi Arabia, Kingdom of Saudi Arabia: U.S. Geological Survey Open-File Report 84-381, 12 p.

Hemer, D.0., 1968, Diagnostic palynologic fossils from Arabia: American Institute of Mining Engineers Regional Technical Symposium, 2d, Dhahran, Saudi Arabia, 1968 Report, p. 311-315.

Higazy, K.A., and El-Ramly, M.F., 1960, Potassium-argon ages of some rocks from the eastern desert of Egypt: United Arab Republic Geological Survey and Mining Department Paper 7, 19 p.

Hotzl, H., Lippolt, H.J., Maurin, V., Moser, H., and Rauert, W., 1979, Quaternary studies on the recharge area situated in crystalline rock regions, in Al-Sayari, S.S., and Zotl, J.G., eds., Quaternary period in Saudi Arabia: Vienna and New York, Springer-Verlag, p. $230-236$.

Huber, Charles, 1891, Journal d'un voyage en Arabie 1883-1884: Paris, Imprimerie Nationale, 778 p. (Illustrated atlas, with list and brief descriptions of Huber's rock collection)

Hudson, R.G.S., 1958, in Discussion on King's paper, Basic palaeography of Gondwanaland during the late Paleozoic and Mesozoic eras: Geological Society of London, Proceedings, v. 114, p. 70, 71.

Hunziker, J.C., 1979, Potassium-argon dating: Lectures in isotope geology; New York, Springer-Verlag, p. 58-63.

Irvine, T.N., and Baragar, W.R.A., 1971, A guide to the chemical classification of the common volcanic rocks: Canadian Journal of Earth Sciences, v. 8, no. 5, p. 523-548.

Jackaman, Barry, 1972, Genetic and environmental factors controlling the formation of the massive sulphide deposits of Wadi Bidah and Wadi Wassat, Saudi Arabia: Saudi Arabian Directorate General of Mineral Resources Technical Record TR 1972-1, p. 243.

Jackson, R.O., Bogue, R.G., Brown, G.F., and Gierhart, R.D., 1963, Geologic map of the southern Najd quadrangle, Kingdom of Saudi Arabia: U.S. Geological Survey Miscellaneous Geologic Investigations Map I-211 A, scale 1:500,000.

Japanese Geological Mission [Okuni, Shizuka; Komura, Kojiro; Hatanaka, Takefumi; Iso, Miyoji; and Kuwagata, Hisao], 1965 [rev. 1967 by Hirayama, Ken], Gold deposits in Al Wajh district with data on limestone and water resources: Japanese Geological Survey, Saudi Arabian Mission Open-File Report JGM-A-1, 216 p., 151 illus.

Japanese Geological Mission, 1967, Report on the iron ore deposits in the Wadi Sawawin district: Japanese Geological Survey, Saudi Arabian Mission Report JGM-A-2. (Later revised and published as Ministry of Petroleum and Mineral Resources Technical Record TR-1978-11, 62 p., 15 figs., 8 tables.)

Johnson, R.F., and Trent, V.A., 1967, Mineral reconnaissance of the Wadi as Surr quadrangle, Kingdom of Saudi Arabia: Saudi Arabian Directorate General of Mineral Resources Mineral Investigations Map MI-5, scale 1:100,000.

1968, Mineral reconnaissance of the Wadi al 'Ays quadrangle, Kingdom of Saudi Arabia: Saudi Arabian Deputy Ministry of Mineral Resources Open-File Report USGS-(IR)SA-45, map scale $1: 100,000,8$ p., 1 fig.

Jones, P.W., 1976, Age of the lower flood basalts of the Ethiopian Plateau: Nature, v. 261 , p. 567-569.

Jones, P.W., and Rex, D.C., 1974, New dates from the Ethiopian Plateau volcanics: Nature, v. 252, p. 218, 219, 1 fig.

Jordi, H.A., and Lonfat, F., 1963, Stratigraphic subdivision and problems in Upper Cretaceous-Lower Tertiary deposits in north- western Libya: Revue l'Institut Francais du Petrole, Revue, v. 18 , pt. 2, p. $1428-1436$

Kabbani, F.K., 1970, Geophysical and structural aspects of the central Red Sea rift valley: Philosophical Transactions of the Royal Society of London, v. A-267, p. 89-97.

Kahr, V.P., 1961, Geology north of Yanbu al Bahr: Saudi Arabian Directorate General of Mineral Resources Open-File Report 126, 213 p., 289 figs., 3 pls.

Kanaan, F.M., 1979, The geology, petrology, and geochemistry of the granitic rocks of Jabal al Hawshah and vicinity, Jabal al Hawshah quadrangle, Kingdom of Saudi Arabia: Saudi Arabian Directorate General of Mineral Resources Bulletin 23, 114 p., 1 pl., 18 tables.

Karpoff, Roman, 1955, Observations preliminaries sur le socle Ancien de L'Arabie: Societe de Geologique de France, Comptes rendus, p. $105,106$.

1956, Sur quelques series sedimentaires d'Arabie occidentale et centrale: Societe de Geologique de France, Comptes rendu Sommaire des Seances, no. 2, Sea. Jan 23, p. 17, 18.

1957a, Esquisse geologique de l'Arabie Seoudite: Geologique Societe de France, 6 ser., v. 7, p. 672-676.

1957b, Sur l'existence du Maestrichtien au nord de Djeddah (Arabie Seoudite): Academie des Sciences [Paris], Compter rendu, t. 245 , no. 16 , p. $1322-1324$.

1960, L'antecambrian de la peninsula Arabique: International Geologic Congress, 21st, Copenhagen, 1960, pt. 9, p. 80-93.

Kazmin, V., 1971, Precambrian of Ethiopia: Nature, Physical Science, v. 230 , no. 16 , p. 176,177 , sketch map.

Kellogg, K.S., and Blank, H.R., Jr., 1982, Paleomagnetic evidence bearing on Tertiary tectonics of the Tihamat Asir coastal plain, southwestern Saudi Arabia: U.S. Geological Survey Open-File Report 82-1047, 40 p.

Kemp, John, 1981, Geology of the Wadi Al 'Ays quadrangle, sheet 25C, Kingdom of Saudi Arabia: Saudi Arabian Deputy Ministry for Mineral Resources Geologic Map GM-53-A, scale 1:250,000, with text, $39 \mathrm{p}$.

Kemp, John, Gros, Yves, and Prian, Jean-Pierre, 1982, Geologic map of the Mahd adh Dhahab quadrangle, sheet 23E, Kingdom of Saudi Arabia: Saudi Arabian Deputy Ministry for Mineral Resources Geologic Map GM-64 A, scale 1:250,000, with text, $39 \mathrm{p}$.

Kemp, John, Pelleton, C., and Calvez, J.Y., 1980, Geochronological investigations and geologic history in the Precambrian of northwestern Saudi Arabia: [France] Bureau des Recherches Geologiques et Minieres Open-File Report 01-1, 120 p., 41 figs.

Kiilsgaard, T.H., Greenwood, W.R., Puffett, W.P., Haqvi, Mohammad, Roberts, R.J., Worl, R.G., Merghelani, Habib, Flanigan, V.J., and Gazzaz, A.R., 1978, Mineral exploration in the Wadi Bidah district, 1971-1976, Kingdom of Saudi Arabia: U.S. Geological Survey Open-File Report 78-771, 95 p., 17 figs.

Kingsley, Charles, 1873, Town geology: London, Strahan and Co., p. 224.

Kluyver, H.M., Bege, V.B., Smith, G.H., Ryder, J.M., and Van Eck, M., 1981, Sirhan-Turayf phosphate project: Results of work carried out under the phosphate agreement, 29th Dhual Hijjah 1398-30th Jumad Thani 1401 [29th November 1978-4th May 1981]: Saudi Arabian Deputy Ministry for Mineral Resources Technical Record RF-TR-01-5, 77 p.

Kober, Leopold, 1911, Report on a trip through Hijaz: Academy of Science, Vienna, Anzeiger, v. 48, p. 285, 286.

1919, The northern Hejaz: Academy of Natural Science and Mathematics, Vienna, K.L. Denkschiften, v. 96, p. 794-813.

Kroner, A., Roobol, M.J., Ramsay, C.R., and Jackson, N.J., 1979, PanAfrican ages of some gneissic rocks in the Saudi Arabian Shield: Journal of the Geological Society, London, v. 136, pt. 4, p. 455-461. 
Kruck, Wolfgang, and Thiele, Joachim, 1983, Late Paleozoic glacial deposits in the Yemen Arab Republic: Geol. Jahr. Reihe B. Heft. 46,32 p., 3 figs., 7 pls.

$\mathrm{Ku}$, T.L., 1969, Uranium series isotopes in sediments from the Red Sea hot-brine area, in Degens, E.T., and Ross, D.A., eds., Hot brines and recent heavy metal deposits in the Red Sea: New York, Springer-Verlag, p. 512-524.

Kuno, H., 1966, Lateral variation of basalt magma type across continental margins and island arcs: Volcanologique Bulletin, v. 29 , p. $195-222$.

Lamare, Pierre, 1923, Sur l'existence do granites alcaline dans le Shammar (Arabie), et sur la constitution geologique de cette region: Societe Geologique de France, Comp. Rend. Som. Seances, no. 16, p. 188-190.

1930 a, Etudes Geologie en Ethiopie, Somalie, et Arabe Meridionale: Societe Geologique de France Memoir 14, new ser., tome 6, p. 32,33 .

$1930 \mathrm{~b}$, Nature et extension des depots secondaires dans I'Arabie, l'Ethiopie, et les pays Somalia: Societe Geologique de France Memoir 14 new ser., tome 4, p. 49-55.

Lamare, Pierre, and Carpentier, C.A., 1932, Vegetaux fossiles du Yemen: Societe Geologique de France Bulletin, ser. 5, v. 2, p. 83-92.

Laurent, D., 1976, A perlite deposit at Jabal Shama: [France] Bureau des Recherches Geologiques et Minieres Open-File Report 76-JED-24, 18 p., sketch map.

Le Maitre, R.W., 1976, The chemical variability of some common igneous rocks: Journal of Petrology, v. 17, pt. 4, p. 589-633.

Lenz, H., Bender, F., Besang, C., Harre, W., Kreuzer, H., Muller, P., and Wendt, I., 1972, The age of early tectonic events in the zone of the Jordan Geosuture: International Geology Congress, 24th, Montreal, Proceedings, sec. 3, p. 371-379.

Letalenet, J., 1974, Geology and mineral exploration of the Jabal al Murdamah quadrangle, sheet 23/43A: [France] Bureau des Recherches Geologiques et Minieres Open-File Report 74-JED-10, 35 p., map, scale 1:100,000.

1979, Geologic map of the Afif quadrangle, sheet 23F: Saudi Arabian Directorate General of Mineral Resources Geologic Map GM-47-A, scale 1:250,000, with text, $20 \mathrm{p}$.

Liddicoat, W.K., 1975, A review of Wadi Sawawin diamond drill corelogs and interpretation (Interim report 1): Saudi Arabian Directorate General of Mineral Resources Technical Record TR-1975-5, 13 p.

Lopoukhine, M., and Stietjes, L., 1976, Geothermal reconnaissance in the Kingdom of Saudi Arabia: [France] Bureau des Recherches Geologiques et Minieres Open-File Report 76-JED-18, 39 p., 11 figs., 3 tables, 4 app.

Luce, R.W., Bagdady, A.Y., and Roberts, R.J., 1976, Geology and ore deposits of the Mahd adh Dhahab district, Kingdom of Saudi Arabia: U.S. Geological Survey Open-File Report 76-0865, 28 p., 1 pl., 13 figs.

McClure, H.A., 1977, Radiocarbon chronology of late Quaternary lakes in the Arabian desert: Nature, v. 263 , no. 5580, p. 755, 756.

1978a, Ar Rub' al Khali, in Al-Sayari, S.S., and Zotl, J.G., eds., Quaternary period in Saudi Arabia: Vienna and New York, Springer-Verlag, p. 252-263.

1978b, Early Paleozoic glaciation in Arabia; Paleogeography, Paleoclimatology, Paleoecology, v. 25, p. 315-326.

1980, Permian-Carboniferous glaciation in the Arabian Peninsula: Geological Society of America Bulletin, pt. 1, v. 91, p. 707-712.

McDougall, I., Morton, W.H., and Williams, M.A.J., 1975, Age and rates of denudation of Trap Series basalts at Blue Nile Gorge, Ethiopia: Nature, v. 254, p. 207-209.
Madden, C.T., Naqvi, I.M., Whitmore, F.C., Jr., Schmidt, D.L., Langston, Wann, Jr., and Wood, R.C., 1980, Paleocene vertebrates from coastal deposits in Harrat Hadan area, Aţ Tā'if region, Kingdom of Saudi Arabia: U.S. Geological Survey OpenFile Report 80-0227, 32 p., 5 figs., 4 tables.

Mandaville, J.P., Jr., 1973, A contribution to the flora of Asir, southwestern Arabia: Miami, Field Research Publications, 13 p.

Masoli, Mario, 1969, The Mukawwar Formation ostracofauna (Upper Cretaceous-Paleocene) of the Maghersum well 1 (Sudan): African Micropaleontological Colloquium, 3d, National Information and Documentation Center, Cairo, 1969, Proceedings, no. 5, p. 329-340.

Megrue, G.H., Norton, E., and Strangway, D.W., 1972, Tectonic history of the Ethiopian rift as deduced by K-Ar ages and paleomagnetic measurements of basaltic dikes: Journal of Geophysical Research, v. 77 , no. 29, p. 5744-5754.

Meissner, C.R., Jr., and Ankary, A.O., 1972, Phosphorite deposits in the Sirhan-Turayf basin, Kingdom of Saudi Arabia: Saudi Arabian Directorate General of Mineral Resources, Mineral Resources Report of Investigations 2, $27 \mathrm{p}$.

Merghelani, H.M., and Irvine, J.I., 1981, Seismicity of the Yanbu region: Available from Saudi Arabian Directorate General of Mineral Resources, $32 \mathrm{p}$.

Meunier, M.S., 1888, Fossiles noveau provenant d'Arabie: Le Naturaliste 10, p. 204, 205

1891, Charles Huber, Journal of a Journey in Arabia (1883-1884): Paris National Press.

Michelson, H., 1978, Stratigraphy of the lake area, in Serruya, C., ed., Lake Kinnert: The Hague, W. Junkby, Pub., p. 17-23.

Ministry of Petroleum and Mineral Resources, 1977, Annotated bibliography 1970-1975 A.D.: Saudi Arabian Directorate General of Mineral Resources Bulletin 20, 98 p.

1980, Annotated bibliography pre-1970 A.D.: Saudi Arabian Directorate General of Mineral Resources Bulletin 19, $126 \mathrm{p}$. 1981, Annotated bibliography 1396-1400 A.H. (1976-1980 A.D.): Saudi Arabian Deputy Ministry for Mineral Resources Bulletin 27, $102 \mathrm{p}$.

Miyashiro, Akiho, 1974, Volcanic rocks series in island arcs and active continental margins: American Journal of Science, v. 274, no. 4, p. 321-355.

Moltzer, J.G., and Binda, P.L., 1981, Micropaleontology and palynology of the middle and upper members of the Shamaysi Formation, Saudi Arabia: Bulletin of the Faculty of Earth Sciences, King Abdulaziz University, no. 4, p. 57-74, app.

Mooney, W.D., 1980, IASPEI workshop: Seismic modeling of laterally varying structures: EOS, Transactions of the American Geophysical Union, v. 62 , no. 2, p. 19, 20.

Moore, J.M., 1979, Tectonics of the Najd Transcurrent Fault System, Saudi Arabia: Journal of the Geological Society of London, v. 136, p. $441-454$

Musil, Alois, 1926, The Northern Hegaz, in Oriental explorations and studies, v. 1, no. 1: New York, American Geographical Society, $215 \mathrm{p}$.

Nasseef, A.D., and Gass, I.G., 1977, Granitic and metamorphic rocks of the Taif area, western Saudi Arabia: Geological Society of America Bulletin, v. 88 , no. 12, p. 1721-1730.

Nebert, Karl, 1969, Geology of the Jabal Samran and Jabal Farasan region: Saudi Arabian Deputy Ministry for Mineral Resources Bulletin 4, 32 p., 51 figs., 4 pls., 2 tables.

Nebert, K., Alshaibi, A.A., Awlia, M., Bounny, I., Nawab, Z.A., Sharief, O.H., Sherbini, O.A., and Yeslam, A.H., 1974, Geology of the area north of Wadi Fatima, Kingdom of Saudi Arabia: Centre for Applied Geology [Jiddah] Bulletin 1, 31 p., 38 figs., 5 pls.

Neuman Van Padang, M., 1963, Catalogue of the active volcanoes of the world including solfatara fields: Part 16, Arabia and the 
Indian Ocean: International Association of Volcanology, p. $3.1-14$.

Overstreet, W.C., 1973, Contributions to the prehistory of Saudi Arabia, 1: Miami, Field Research Projects, 11 p., 99 figs., 3 tables.

Overstreet, W.C., Overstreet, E.F., and Goudarzi, G.H., 1973, Mineralogical and chemical investigations of the laterite in the As Sarat Mountains, Kingdom of Saudi Arabia: U.S. Geological Survey Saudi Arabian Project Report 46, 66 p.

Overstreet, W.C., Stoeser, D.B., Overstreet, E.F., and Goudarzi, G.H., 1977, Tertiary laterite of the As Sarat Mountains, Asir Province: Saudi Arabian Directorate General of Mineral Resources Bulletin 21, 24 p., 1 pl., scale 1:100,000, 11 figs.

Pallister, J.S., 1983, Reconnaissance geologic map of the Harrat Tuffil quadrangle, sheet 20/39B, Kingdom of Saudi Arabia: U.S. Geological Survey Open-File Report $83-332,3$ pls., scale 1:100,000, 3 app.

Pallister, J.S., and Hopson, C.A., 1981, Samail ophiolite plutonic suite: Field relations, phase variation, cryptic variation, and layering, and a model of a spreading ridge magma chamber: Journal of Geophysical Research, v. 86, no. B4, p. 2593-2644.

Pellaton, Claude, 1976, Geology and mineral exploration of the Wadi Dahgalah quadrangle, sheet 26/37D, Kingdom of Saudi Arabia: [France] Bureau des Recherches Geologiques et Minieres Report 76-JED-10, scale 1:100,000, with text, $24 \mathrm{p}$.

1979, Geologic map of the Yanbu' al Bahr quadrangle, sheet ${ }_{24}$ C, Kingdom of Saudi Arabia: Saudi Arabian Directorate General of Mineral Resources Geologic Map GM-48-A, scale 1:250,000, with text, $24 \mathrm{p}$.

1981, Geologic map of the Al Madinah quadrangle, sheet 24D, Kingdom of Saudi Arabia: Saudi Arabian Deputy Ministry for Mineral Resources Geologic Map GM-52-A, scale 1:250,000, with text, $19 \mathrm{p}$.

1982, Geologic map of the Umm Lajj quadrangle, sheet 25B, Kingdom of Saudi Arabia: Saudi Arabian Deputy Ministry for Mineral Resources Geologic Map GM-61-A, scale 1:250,000, with text, $14 \mathrm{p}$.

Peltier, W.R., 1980, Models of glacial isostasy and relative sea level, in Bally, A.W., Bender, P.L., McGetchin, T.R., and Walcott, R.L., eds., Dynamics of plate interiors: American Geophysical Union and Geological Society of America, Geodynamic Series, v. 1, p. 111-128.

Powers, R.W., 1968, Lexique stratigraphique international, v. III, fas. 10bl, Arabie Saoudite: Paris, Centre national de la Recherche Scientifique, p. 76.

Powers, R.W., Ramirez, L.F., Redmond, C.D., and Elberg, E.L., Jr., 1966, Geology of the Arabian Peninsula-Sedimentary geology of Saudi Arabia: U.S. Geological Survey Professional Paper $560-\mathrm{D}, 147 \mathrm{p}$

Prinz, W.C., 1984, Geologic map of the Wādī Ḩalēy quadrangle, sheet 18E, Kingdom of Saudi Arabia: Ministry of Petroleum and Mineral Resources, Geoscience Map GM-74-C, scale 1:250,000, with text, 2 figs., 2 tables, $13 \mathrm{p}$.

Quennell, A.M., 1951, Geology and mineral resources of (former) Trans-Jordan: Colonial Geology and Mineral Resources [London] 2, p. 85-115.

Rathjens, Karl, 1942, Beobachtungen in Yemen: Geologische Rundschau, v. 33, p. 248-279.

Reischmann, Thomas, 1981, Petrographie and geochemie spat-Proterozoischer volkanite der Wadi Lith region, Saudi Arabia: Mainz, Germany, University of Mainz, Unpub. M.S. thesis, 172 p., 3 app.

Richardson, E.S., and Harrison, C.G.A., 1976, Opening of the Red Sea with two poles of rotation: Earth and Planetary Science Letters, v. 30, p. $135-142$.
Richter-Bernburg, G., and Schott, W. (Amt fur Bodenforschung, Hannover, Germany), 1954, Geological researches in western Saudi Arabia: Saudi Arabian Directorate General of Mineral Resources Open-File Report 28, 69 p., 25 illus.

Roland, N.W., 1978, Jung palaozoisch Glazialspuren auf dem Arubischen Schild: Eiszeitalter u. Gegen, v. 28, p. 133-138, 4 figs.

Ross, D.A., and Schlee, John, 1977, Shallow structure and geologic development of the southern Red Sea, in Red Sea research 1970-1975: Saudi Arabian Directorate General of Mineral Resources Bulletin 22, p. E1-E18.

Sahl, M., and Al Fatawi, B., 1981, Reconnaissance geology of the Tayyibal Ism quadrangle, sheet $28 / 34$ B, Kingdom of Saudi Arabia: Saudi Arabian Deputy Ministry for Mineral Resources Open-File Report 01-18, 24 p., 2 figs, 1 pl.

Said, Rushdi, 1962, The geology of Egypt: New York, Elsevier, 377 p., 71 figs., $10 \mathrm{pls}$.

Sarnthien, M., 1978, Sand deserts during glacial maximum and climatic optimum: Nature, v. 272 , p. $43-46$.

Saxena, G.N., and Assefa, G., 1983, New evidence on the age of the glacial rocks of northern Ethiopia: Geology Magazine, v. 120(6), p. 549-554.

Schick, A.P., 1958, Marine terraces on Tiran Island, northern Red Sea: Geografiska Annalen Stockholm, heft 1, XL, p. 63-66, 4 figs.

Schmidt, D.L., 1980, Geology of the Wadi al Miyah quadrangle, sheet 20/42B, Kingdom of Saudi Arabia: USGS Saudi Arabian Mission Technical Record 12 (IR352); Saudi Arabian Directorate General of Mineral Resources Map series, scale 1:100,000, with text, 87 p., 21 figs., 9 tables, 2 pls.

1981a, Geology of the Jabal al Qarah quadrangle, sheet 20/ 43C, Kingdom of Saudi Arabia: USGS Saudi Arabian Mission Miscellaneous Document 31 (IR367); Saudi Arabian Directorate General of Mineral Resources Map series, scale 1:100,000, 52 p., 12 figs., 4 tables, 3 pls.

1981b, Geology of the Yafikh quadrangle, sheet 20/43D, Kingdom of Saudi Arabia: USGS Saudi Arabian Mission Miscellaneous Document 39 (IR397); Saudi Arabian Directorate General of Mineral Resources Map series, scale 1:100,000, 99 p., 14 figs., 14 tables, 2 pls.

1985, Reconnaissance geologic map of the Al Junaynah quadrangle, sheet 20/42D, Kingdom of Saudi Arabia: Saudi Arabian Directorate General of Mineral Resources Geologic Map GM 71-A, scale 1:100,000, with text, $31 \mathrm{p}$.

Schmidt, D.L., and Brown, G.F., 1984, Major-element chemical evolution of the late Proterozoic shield of Saudi Arabia: Symposium on Pan-African Crustal Evolution in the Arabian-Nubian Shield, 1st, International Geological Correlation Program Project 164, Faculty of Earth Sciences, King Abdulaziz University Bulletin (Jeddah), no. 6 , p. 1-21, 13 figs.

Schmidt, D.L., and Hadley, D.G., 1985, Stratigraphy of the Miocene Baid Formation, southern Red Sea coastal plain, Kingdom of Saudi Arabia: U.S. Geological Survey Open-File Report 85-0241, $49 \mathrm{p}$.

Schmidt, D.L., Hadley, D.G., and Brown, G.F., 1983, Middle Tertiary continental rift and evolution of the Red Sea in southwestern Saudi Arabia: U.S. Geological Survey Open-File Report 83-0641, $60 \mathrm{p}$.

Schmidt, D.L., Hadley, D.G., Greenwood, W.R., Gonzalez, Louis, Coleman, R.G., and Brown, G.F., 1973, Stratigraphy and tectonism of the southern part of the Precambrian Shield of Saudi Arabia: Saudi Arabian Directorate General of Mineral Resources Bulletin 8, 13 p.

Schmidt, D.L., Hadley, D.G., and Stoeser, D.B., 1979, Late Proterozoic crustal history of the Arabian Shield, southern Najd Province, Kingdom of Saudi Arabia, in Tahoun, S.A., ed., Evolution and mineralization of the Arabian-Nubian Shield: King Abdulaziz 
University, Institute for Applied Geology Bulletin 3 (Oxford-New York, Pergamon Press Ltd.), v. 2, p. 41-48.

Schmidt, D.L., Puffett, W.P., Campbell, W.L., and Al-Koulak, Z.H., 1982 , Gold placer and Quaternary stratigraphy of the Jabal Mokhyat area, southern Najd Province, Kingdom of Saudi Arabia: U.S. Geological Survey Open-File Report 82-414, 76 p.

Schott, W., 1953, Geologische Studienreise nach Yemen: Zeitschrift der Deutschen Geologischen Geselleschaft, Hannover, bd. 105, pt. 3 , p. 548.

Schurmann, H.M.E., 1966, The Precambrian in north Africa: Leiden, E.J. Brill, 351 p., 88 pls.

Scott, Hugh, and Britton, E.B., 1941, British Museum [Natural History] expedition to south west Arabia 1937-38: v. 1, pt. repts. 1-8.

Seilacher, Adolf, 1970, Cruziana stratigraphy of "non-fossiliferous" Paleozoic sandstone, in Crimes, T.P., and Harper, J.C., Trace fossils: Liverpool, Seel House Press, p. 447-476.

Sestini, Guilano [Julian], 1965, Cenozoic stratigraphy and depositional history, Red Sea coast, Sudan: American Association of Petroleum Geologists Bulletin, v. 49, no. 9, p. 1453-1472.

Shanti, M.S., and Roobol, M.J., 1982, Guide books for an excursion to the Jabal Ess-Ash Shism area of northern Saudi Arabia: International Geological Correlation Program, 1st symposium, project 164, Pan-African Crustal Evolution in the Arabian-Nubian Shield, Facility of Earth Science Research series no. 14, 35 p.

Shapiro, Leonard, 1967, Rapid analysis of rocks and minerals by a single-solution method, in Geological Survey research 1967: U.S. Geological Survey Professional Paper 575-B, p. B187-B191.

Shimron, A.E., and Brookins, D.G., 1974, Rb-Sr radiometric age of late Precambrian fossil-bearing and associated rocks from Sinai: Earth and Planetary Science Letters, v. 24, p. 136-140.

Shimron, A.E., and Horowitz, Akaron, 1972, Precambrian organic microfossils from Sinai: Pollen et Spores 14, no. 3 (Museum National d' Histoire Naturelle, Paris), p. 333-342, 15 figs.

Shukri, N.M., and Basta, E.Z., 1955, Petrography of the alkaline volcanic rocks of Yaman; Egyptian University scientific expedition to SW. Arabia: L'Institute du Desert de'Egypte Bulletin, v. 36 , p. $129-163$.

Silvestri, Alfredo, 1937, Fossile eocenico singolare della Tripolitania: Societa Geologica Italiana Bulletin, v. 56, p. 203-208.

Simlin, Tom, Siebert, Lee, McClelland, Lindsay, Bridge, David, Newhall, Christopher, and Latter, J.H., 1981, Volcanoes of the World: Stroudsburg, Pa., Hutchinson Ross, 232 p.

Skiba, W.J., 1980, The form and evolution of late Precambrian plutonic masses in the Jiddah-Rabigh-Wadi Al-Quaha area, Saudi Arabia, in Cooray, P.G., and Tahoun, S.A., eds., Evolution and mineralization of the Arabian-Nubian Shield: Institute for Applied Geology Bulletin 3, Oxford, Pergamon Press Ltd., v. 3, p. 105-120.

Skiba, W.J., and Warden, A.J., 1969, Preliminary report on the geology of selected areas in the southern part of the Arabian Shield: Saudi Arabian Directorate General of Mineral Resources Open-File Report 325, 42 p., 2 maps.

Smith, C.L., 1980, Brines of Wadi as Sirhan, Kingdom of Saudi Arabia: U.S. Geological Survey Open-File Report 80-1261, 30 p., 7 figs., 3 tables.

Smith, J.W., 1980a, Reconnaissance geologic map of the Wadi Mahani quadrangle, sheet 22/40A, Kingdom of Saudi Arabia: Saudi Arabian Directorate General of Mineral Resources Geologic Map GM-35, scale 1:100,000, with text, $18 \mathrm{p}$.

$1980 \mathrm{~b}$, Reconnaissance geology of the At Ta'if quadrangle, sheet 21/40C. Kingdom of Saudi Arabia: Saudi Arabian Deputy Ministry for Mineral Resources Geologic Map GM-56, scale 1:100,000, with text, $33 \mathrm{p}$.
1982, Reconnaissance geologic map of the Wadi Hammah quadrangle, sheet 22/40C, Kingdom of Saudi Arabia: Saudi Arabian Deputy Ministry for Mineral Resources Geologic Map GM-65, scale 1:100,000, with text, 19 p.

Soliman, M.M., 1981, Petrology and geochemistry of some granitic rocks from the western Al-Quway'iyah region: Arabian Journal of Science and Engineering, F, no. 3, p. 289-297.

Souaya, F.J., 1966, Miocene foraminifera of the Gulf of Suez region, U.A.R.; Part 3, Biostratigraphy: Micropaleontology, v. 12, no. 2, p. $183-202$.

Stacey, J.S., Roberts, R.J., Doe, B.R., Delevaux, M.H., and Gramlich, J.W., 1981, A lead-isotope study of mineralization in the Saudi Arabian Shield: Contributions to Mineralogy and Petrology, v. 74, p. $175-188$.

Stacey, J.S., and Hedge, C.E., 1984, Geochronologic and isotopic evidence for early Proterozoic crust in the eastern Arabian Shield: Geology, v. 12, p. 310-313.

Steiger, R.H., and Jager, E., 1977, Subcommission on geochronology; Convention on the use of decay constants in geo- and cosmochronology: Earth and Planetary Science Letters, v. 36, p. 359-367.

Steineke, Max, Berg, E.L., and Wadsack, G., 1944, Preliminary map of the water resources and geology of the Jeddah-Usfan-Wadi Fatima area: U.S. Military Mission to Saudi Arabia in cooperation with the Arabian American Oil Co., unpub., scale 1:100,000.

Steineke, Max, Bramkamp, R.A., and Sander, J.J., 1958, Stratigraphic relations of Arabian Jurassic oil, in Habitat of oil: American Association of Petroleum Geologists symposium (Tulsa), p. 1294-1329, 6 figs.

Steinitz, G., Bartov, Y., and Hunziker, J.C., 1978, K-Ar age determinations of some Miocene-Pliocene basalts in Israel; Their significance to the tectonics of the Rift Valley: Geographic Magazine, $v$. 115 , no. 5 , p. $329-340$.

Stoeser, D.B., 1985, Plutonic rock distribution map of the southern Arabian Shield, Kingdom of Saudi Arabia: U.S. Geological Survey Open-File Report 85-264, map, scale 1:500,000.

Stoeser, D.B., and Elliott, J.E., 1980, Post-orogenic peralkaline and calc-alkaline granites and associated mineralization of the Arabian Shield, Kingdom of Saudi Arabia, in Cooray, P.G., and Tahoun, S.A., eds., Evolution and mineralization of the ArabianNubian Shield: Institute for Applied Geology Bulletin 3, Oxford, Pergamon Press Ltd., v. 4, 168 p.

1985, Plutonic rock distribution map of the northeastern Arabian Shield, Kingdom of Saudi Arabia: Saudi Arabian Deputy Ministry of Mineral Resources, USGS-OF-04-52; also U.S. Geological Survey Open-File Report 85-0255, map, scale 1:500,000.

Streckeisen, A.L., 1973, Plutonic rocks, classification and nomenclature recommended by the IUGS Subcommission on the Systematics of Igneous Rocks: Geotimes, v. 18, no. 10, p. 26-30.

1976, To each plutonic rock its proper name: Earth-Science Reviews, v. 12 , no. 1 , p. 1-33.

1979, Classification and nomenclature of volcanic rocks, lamprophyres, carbonatites, and melititic rocks; Recommendations and suggestions on the systematics of igneous rocks: Geology (Boulder), v. 7, p. 331-335, 1 table.

Stuckless, J.S., and VanTrump, George, Jr., 1979, A revised version of Graphic Normative Analysis Program (GNAP) with examples of petrologic problem solving: U.S. Geological Survey Open-File Report 79-1237, 115 p.

Taylor, H.P., Jr., and Coleman, R.G., 1977, Oxygen isotopic evidence for meteoric-hydrothermal alteration of the Jabal at Tirf igneous complex, Saudi Arabia [abs.]: EOS, Transactions of the American Geophysical Union, v. 58, no. 6, p. 316.

Thatcher, Leland, Rubin, Meyer, and Brown, G.F., 1961, Dating desert ground water: Science, v. 134, p. 104, 105. 
Theobald, P.K., and Thompson, C.E., 1966, Geology and geochemistry of a part of the Ablah Formation at Jabal Rumur, Kingdom of Saudi Arabia: U.S. Geological Survey Open-File Report (IR)SA-88, 15 p., 8 figs., 2 tables.

Thorpe, R.S., 1972, Ocean floor basalt affinity of Precambrian schist from Anglesey: Nature (Physical Science), v. 240, Dec. 18, p. 165.

Tothill, J.D., ed., 1948, Agriculture in the Sudan: Oxford; Oxford University Press, $974 \mathrm{p}$.

Trent, V.A., and Johnson, R.F., 1966, Reconnaissance mineral and geologic investigation in the Maqna quadrangle, Aqaba area, Saudi Arabia: U.S. Geological Survey Open-File Report (IR)SA-51, 5 p., 3 figs.

Tromp, S.W., 1950, The age and origin of the Red Sea graben: Geological Magazine, v. 87, p. 385-392.

Twitchell, K.S., 1958, Saudi Arabia, with an account of the development of its natural resources: Princeton, N.J., Princeton University Press, $281 \mathrm{p}$.

Uchimizu, Mamoru, 1966, Geology and petrology of alkali rocks from Dogo, Oki Islands: Tokyo University Faculty of Science Journal, sec. 2 , v. 16, pt. 1, p. 85-159, illus. (incl. geol. map, scale 1:80,000).

U.S. Geological Survey, 1972, Topographic map of the Arabian Peninsula: Saudi Arabian Directorate General of Mineral Resources Arabian Peninsula Map AP-1, scale 1:4,000,000.

U.S. Geological Survey-Arabian American Oil Company (USGS-ARAMCO), 1963, Geologic map of the Arabian Peninsula: U.S. Geological Survey Miscellaneous Geologic Investigations Map I-270-A, scale 1:2,000,000.

Vail, J.R.; 1971, Geological reconnaissance in part of Berber District, northern province, Sudan: Sudan Geological Survey Department Bulletin 18, Khartoum, 76 p., 2 pls., 6 figs., 2 tables, 1 map.

Vesey-FitzGerald, D.F., 1955, Vegetation of the Red Sea coast south of Jeddah, Saudi Arabia: Journal of Ecology, v. 43, p. 477-489. 1957, The vegetation of central and eastern Arabia: Journal of Ecology, v. 45, p. 779-798.

Vidal, Gonzalo, 1979, Acritarchs from the upper Proterozoic and lower Cambrian of East Greenland: Grønlands Geologiske Under søgelso, Bulletin 134, p. 8, 34, 35 .
Vincent, Gerard, 1968, Geology and mineral resources of the Halaban-Sabhah region (sheet 118, zone 2): [France] Bureau des Recherches Geologiques et Minieres Open-File Report 68-JED-1, 3 app., scale 1:100,000.

Warden, A.J., 1982, Reconnaissance geology of the Markas quadrangle, sheet 18/43B, Kingdom of Saudi Arabia: Available from Saudi Arabian Deputy Ministry for Mineral Resources, 58 p., scale $1: 100,000$.

Wendorf, Fred, Schild, Romuald, Said, Rushdi, Haynes, C.V., Gautier, A., and Kobusiewicz, M., 1976, The prehistory of the Egyptian Sahara: Science, v. 193 , no. 4248 , p. 103-114.

Wetzel, Rene, and Morton, D.M., 1959, Contribution a la geologie de la Transjordanie: Museum National d'Histoire Naturelle (Paris), Notes et memoires sur le Moyen Orient, t. 7, p. 107-120.

Whiteman, A.J., 1970, Nubian group: Origin and status [discussion]: American Association of Petroleum Geologists Bulletin, v. 54, no. 3 , p. $522-526$.

1971, The geology of the Sudan Republic: Oxford, Clarendon Press, $290 \mathrm{p}$.

von Wissman, Herman, 1963, Catalogue of the active volcanoes of the world including solfatara fields (Neumann van Padang, M., ed.): Rome, International Association of Volcanology Catalogue, v. 16, p. 3.1-1-3.1-12.

Worl, R.G., 1978, Ore controls at the Mahd adh Dhahab gold mine, Kingdom of Saudi Arabia, in Evolution and mineralization of the Arabian-Nubian Shield: King Abdulaziz University, Institute of Applied Geology Bulletin 3 (Oxford-New York, Pergamon Press Ltd.), v. 2, p. 93-106.

Wyllie, P.J., 1977, Crustal anatexis: An experimental review: Tectonophysics, v. 43 , p. $41-71$.

Yamani, M.A., 1968, Geology of the oolitic hematite of Wadi Fatima, Saudi Arabia, and the economics of its exploitation: Ph.D. thesis, Cornell University, Ithaca, N.Y., 10 p., 9 figs., 2 pls.

Zak, I., and Freund, R., 1981, Asymmetry and basin migration in the Dead Sea rift: Tectonophysics, v. 80 , no. 1-4, p. 27-38. 